Gerald Spindler

Rechtsprobleme und wirtschaftliche Vertretbarkeit einer Kulturflatrate

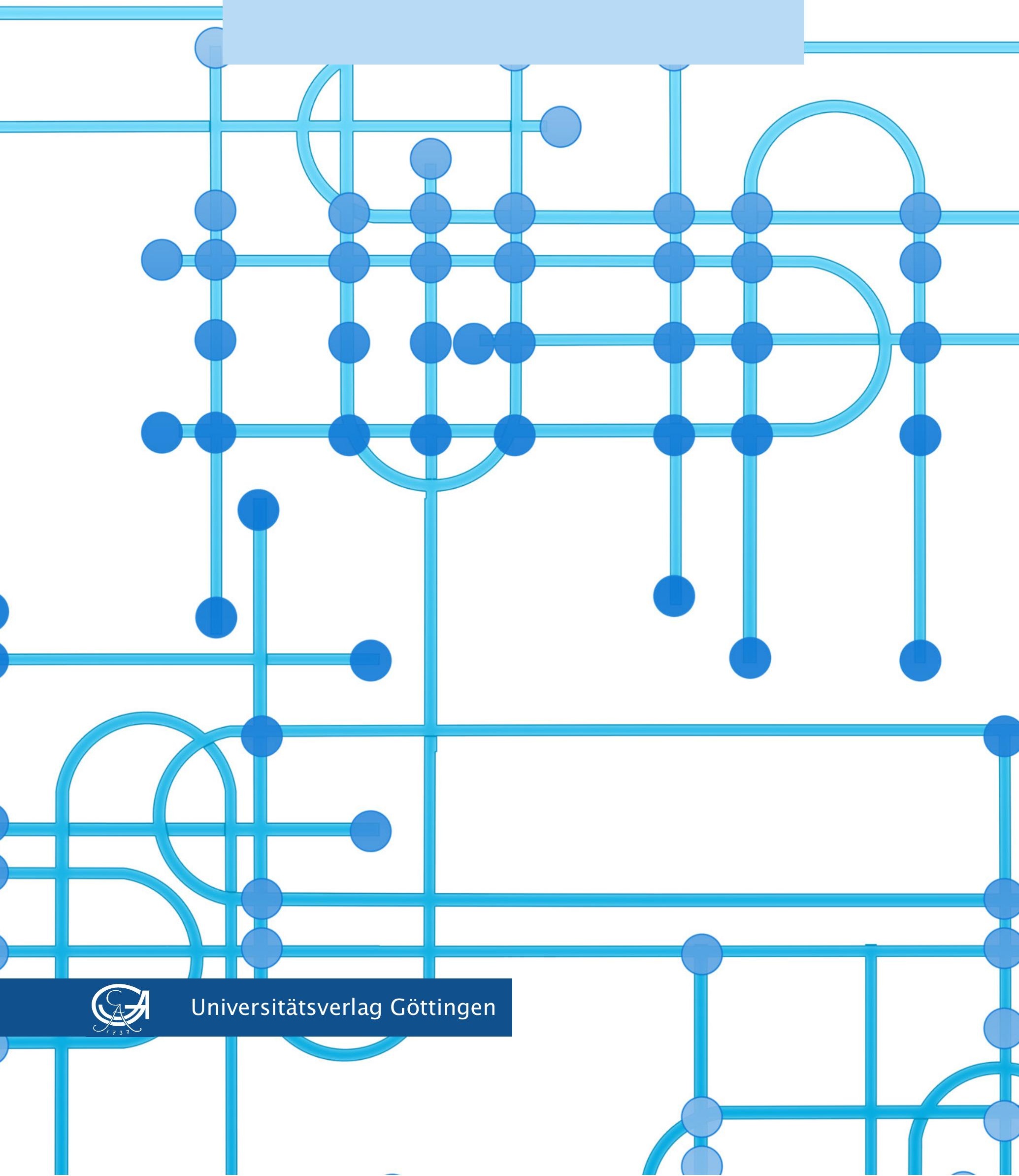



Gerald Spindler

Rechtsprobleme und wirtschaftliche Vertretbarkeit einer Kulturflatrate

This work is licensed under a

Creative Commons

Attribution-NonCommercial-NoDerivatives

4.0 International License.

(c) $(1) \ominus$ 
erschienen im Universitätsverlag Göttingen 2014 
Gerald Spindler

\section{Rechtsprobleme und wirtschaftliche Vertretbarkeit einer Kulturflatrate}

Überarbeitung des im Auftrag der Bundestagsfraktion Bündnis 90/Die Grünen erstellten Gutachtens Stand 08. Januar 2014

Unter Mitwirkung der wissenschaftlichen Mitarbeiter Michael Funke, Matti Rockenbauch und Jörn Wittmann

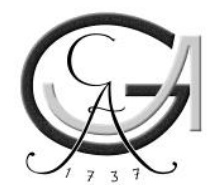

Universitätsverlag Göttingen 2014 


\section{Bibliographische Information der Deutschen Nationalbibliothek}

Die Deutsche Nationalbibliothek verzeichnet diese Publikation in der Deutschen Nationalbibliographie; detaillierte bibliographische Daten sind im Internet über < http://dnb.ddb.de> abrufbar

Prof. Dr. Gerald Spindler

Lehrstuhl für Bürgerliches Recht, Handels- und Wirtschaftsrecht, Rechtsvergleichung,

Multimedia- und Telekommunikationsrecht

Platz der Göttinger Sieben 6

37073 Göttingen

Dieses Buch ist auch als freie Onlineversion über die Homepage des Verlags sowie über den OPAC der Niedersächsischen Staats- und Universitätsbibliothek (http:/ /www.sub.uni-goettingen.de) erreichbar und darf gelesen, heruntergeladen sowie als Privatkopie ausgedruckt werden Es gelten die Lizenzbestimmungen der Onlineversion.

Umschlaggestaltung: Franziska Lorenz

Titelabbildungen: Gert Altmann, www.pixelio.de

\section{(C) 2014 Universitätsverlag Göttingen}

http://univerlag.uni-goettingen.de

ISBN: 978-3-86395-128-3 


\section{Inhalt}

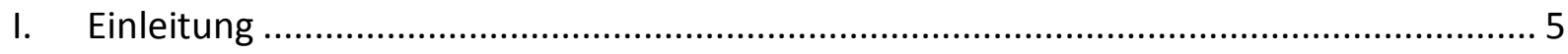

II. Gutachtenfragestellung und Gang der Untersuchung ……………………………..... 7

III. Das grundsätzliche Problem:

Schranken, effektive Rechtsdurchsetzung und Abgabenlösungen ......................................... 9

A. Abgaben als zweitbeste Lösung gegenüber individueller Rechtsdurchsetzung.......................... 9

B. Die Geräteabgabe als Korrelat für die Privatkopie im UrhG 1965 ........................................... 10

IV. Die gegenwärtige Rechtsdurchsetzung im Internet ..................................................... 15

A. Anonymität und Globalität als Hürden …………………………………………….... 15

B. Bisherige Reaktionen der Rechteinhaber und Folgeprobleme ............................................. 15

1. Rechtsverfolgungsmaßnahmen der Rechteinhaber gegenüber Nutzern .............................. 15

2. Rechtsverfolgungsmaßnahmen gegenüber Intermediären ................................................. 18

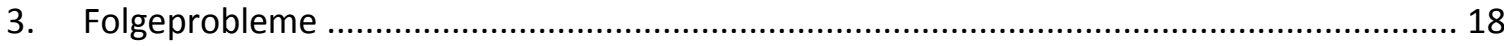

4. Bisherige Marktreaktionen.............................................................................................. 24

V. Das Grundmodell einer Kulturflatrate:

Erweiterte Privatkopieschranke mit Internetanschlussabgabe ............................................. 29

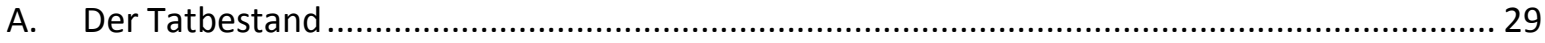

1. Privatkopien erweitert auf jeden Download .................................................................... 30

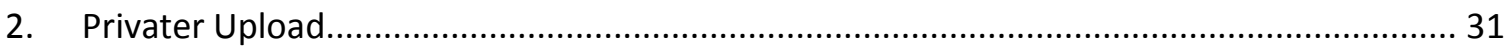

3. Beschränkung auf nicht-kommerzielle Nutzer und Anbieter ................................................. 31

4. Erweiterung auf Bearbeitungsschranken/-rechte (Remixes, Mashups etc.) - Schranke

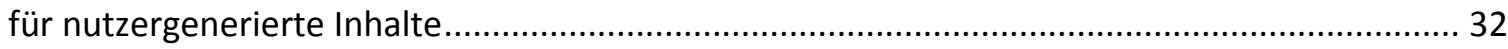

5. Beschränkung auf nicht DRM-geschützte Werke.............................................................. 35

6. Nicht erfasste Werkkategorien ................................................................................. 36

7. Erfasste Rechteinhaber ................................................................................................. 37

8. Beschränkung auf digitale Veröffentlichungen ................................................................. 37

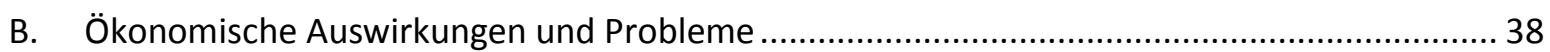

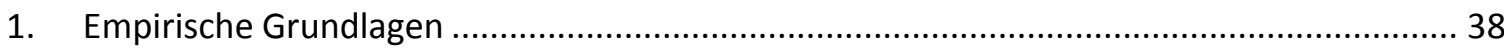

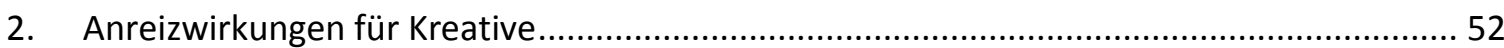

3. Anreize und Auswirkung auf Verleger bzw. Intermediäre …….......................................... 55

4. Anreizwirkungen für andere Marktteilnehmer und neue Geschäftsmodelle:....................... 55

5. Anreizwirkung für Nutzer: Ineffiziente Nutzung der Anschlusskapazitäten?......................... 57

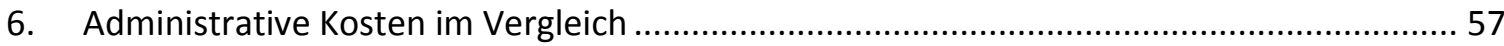

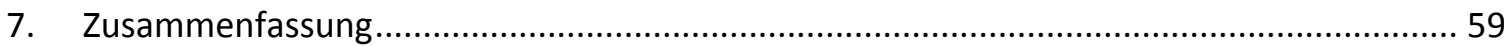




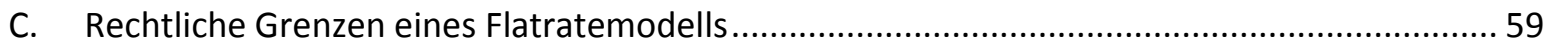

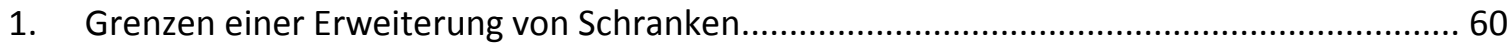

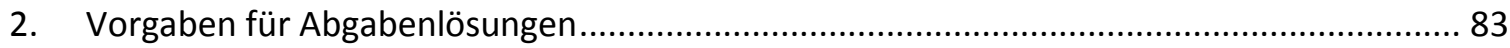

3. Transitorische Probleme: Eingriffe in bestehende Verträge und Rechte?........................... 94

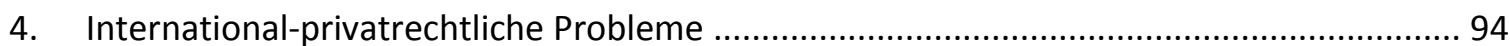

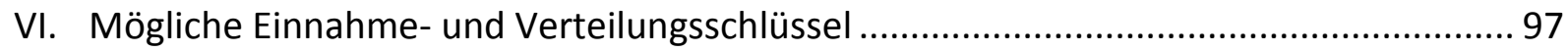

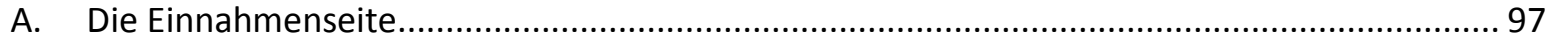

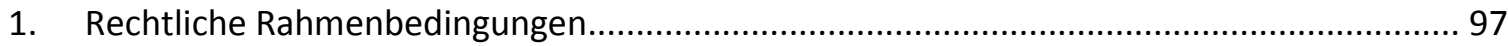

2. Die Geräteabgabe als Vorbild? Volumenbasierte Abgaben ohne Substitutionsrate ............. 98

3. Auf tatsächlicher Substitution basiertes Gegenmodell (Fisher)......................................... 109

4. Mischmodell: Volumenbasiert mit Substitutionsrate .................................................... 118

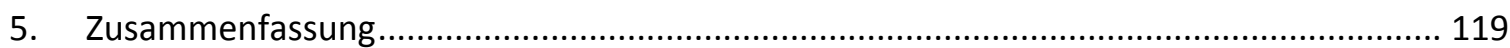

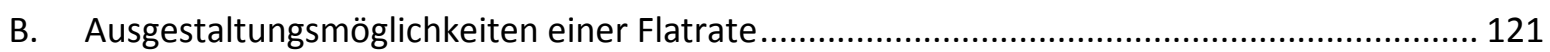

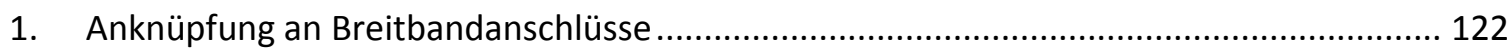

2. Personenbezogene Anknüpfung? (Vermeidung der Zahlung mehrerer

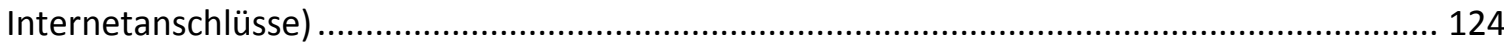

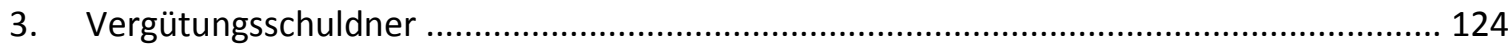

4. Verhältnis zu den traditionellen Geräteabgaben ........................................................ 125

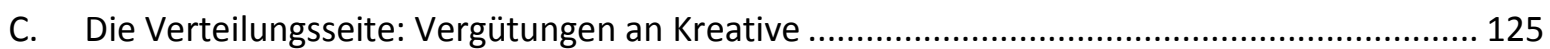

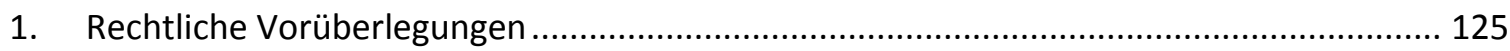

2. Überblick über die Verteilung der Einnahmen bei Verwertungsgesellschaften ................. 128

3. Erfassung nach Nutzungsintensität: Folgen für die Kulturflatrate …................................ 135

4. Progressionsverteilungsmodelle (Abflachung bei Stars etc.) - Förderungen ....................... 145

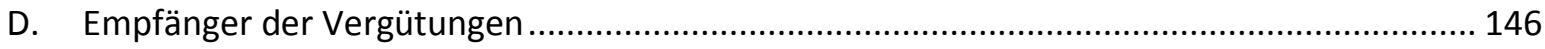

E. Einziehung und Verteilung: Verwertungsgesellschaften und andere Lösungen.................... 147

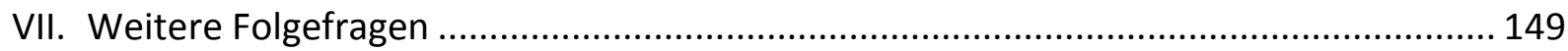

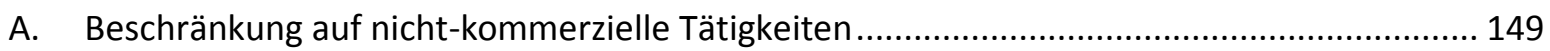

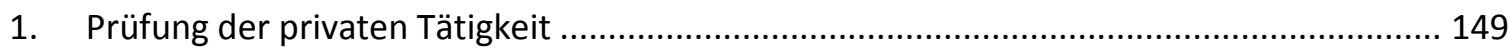

2. Behandlung von Resellern und Routern (Internet-Cafés, Schulen etc.)............................ 153

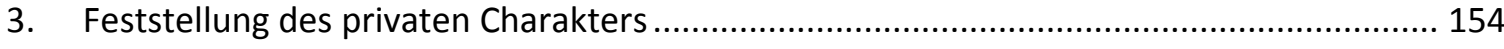

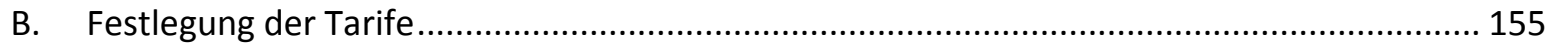

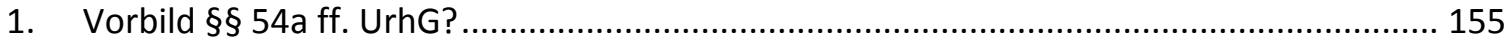

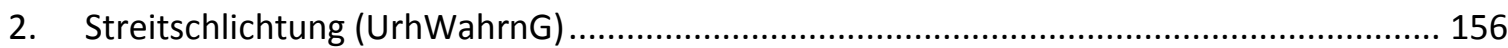

VIII. Auswirkungen auf die (Urheber-) Persönlichkeitsrechte ............................................. 157

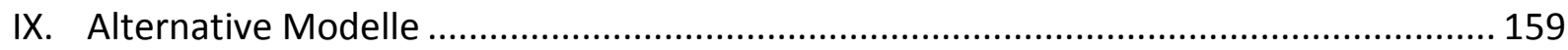


A. Freiwillige Vergabe von Vergütungen ................................................................ 159

1. Die Kulturwertmark ................................................................................... 159

2. Auktions- und Vorfinanzierungsmodelle mit Freigabe von Urheberrechten (Crowdfunding; Micropayment) ............................................................................. 160

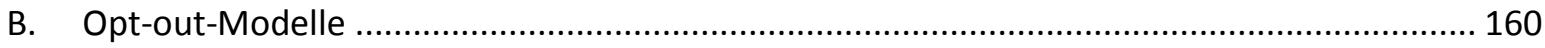

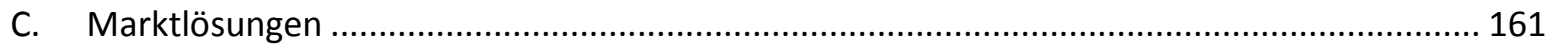

1. Beibehaltung der autonom vereinbarten Kollektivlizenzen (YouTube) .......................... 161

2. Entwicklung von vertikal integrierten Systemen (Apple/iTunes) sowie Streaming-

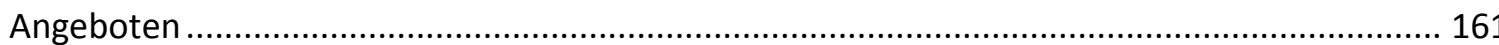

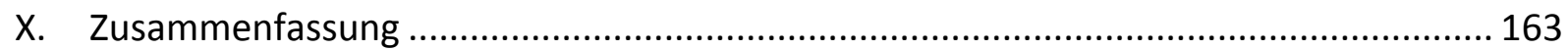

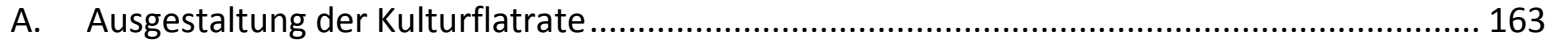

B. Rechtliche und ökonomische Beurteilung....................................................... 164

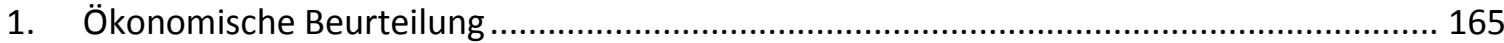

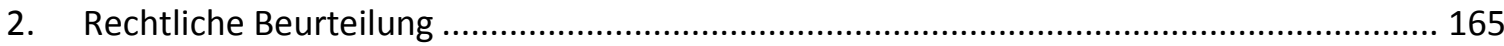

C. Berechnung und Verteilung............................................................................ 167

1. Höhe der Abgabe.......................................................................................... 167

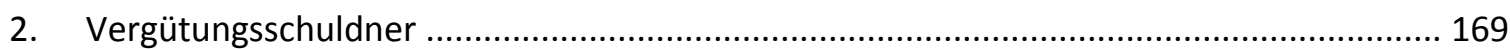

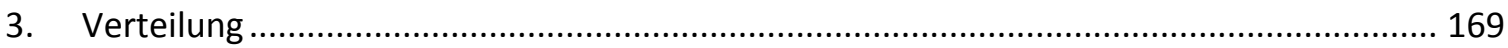

4. Erhebung und Festlegung der Tarife .................................................................. 169

D. Alternative Modelle und Auswirkungen auf Geschäftsmodelle ..................................... 170

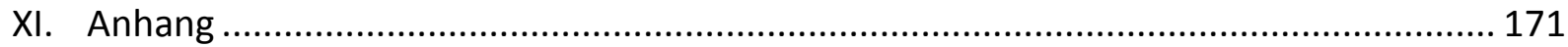

A. Übersicht über vorliegende empirische Studien aus Hargreaves-Report ........................... 171

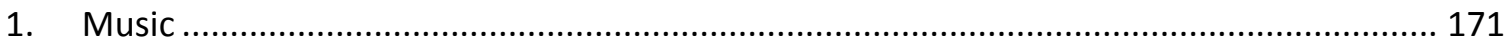

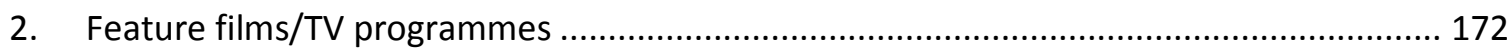

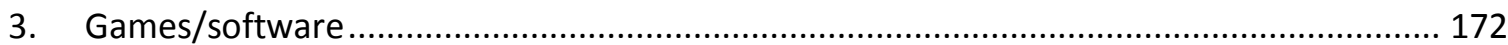

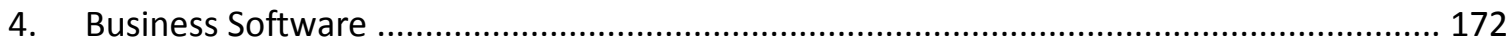

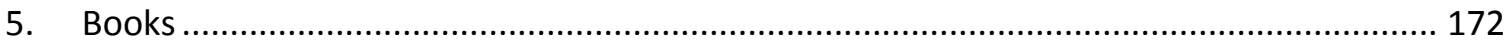

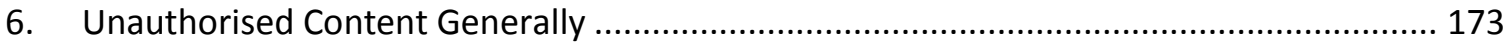

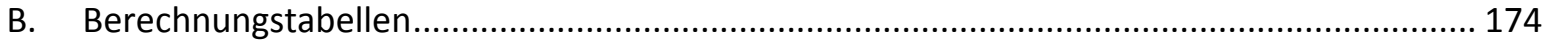

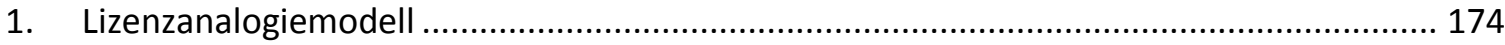

2. Auf tatsächlicher Substitution basiertes Gegenmodell (Fisher) ................................... 189

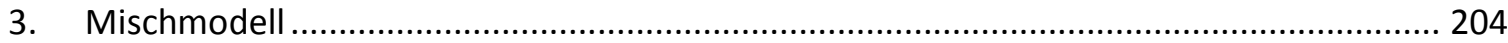

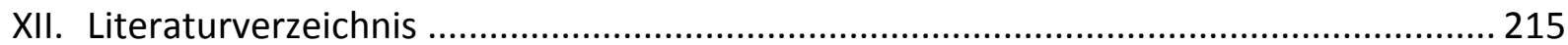





\section{Einleitung}

In den letzten zehn Jahren ist mit der Verbreitung des Internet, von verbesserten Breitbandverbindungen und Komprimierungstechnologien eine erhebliche Zunahme von Kopier- und Tauschvorgängen von Content-Files zu beobachten, sei es über P2P-Netzwerke, über Filehoster wie Rapidshare oder andere Modelle wie eDonkey etc. Ebenso ermöglicht intelligente Aufnahmesoftware, fast völlig automatisiert aus dem großen Angebot von Internetradios die passenden Inhalte nach den Wünschen des Nutzers herauszufiltern und aufzuzeichnen. ${ }^{1}$ Schließlich schaffen verschiedene Lösungen zum Aufzeichnen von Musik aus Angeboten wie YouTube oder Grooveshark auch hier die Möglichkeit, zum eigenen dauerhaften Gebrauch Musikkopien anzufertigen. ${ }^{2}$

Die Content- und hier insbesondere die Musikindustrie haben hierauf zum einen mit erheblichen Anstrengungen zur Rechtsverfolgung und -durchsetzung reagiert, zum anderen mit entsprechenden Medienkampagnen. Seitens der Gesetzgebung wurden die Bestimmungen zur legalen Privatkopie (Download) in $\S 53$ Abs. 1 UrhG $^{3}$ verschärft, aber auch in Umsetzung der Enforcement-Richtlinie ${ }^{4}$ Auskunftsansprüche gegenüber Providern eingeführt. Neben der Verfolgung von Nutzern primär für den Upload-Bereich werden in stets steigender Zahl auch Intermediäre wie Host-Provider bis hin zu Access-Providern in Anspruch genommen, vorrangig auf Unterlassung im Bereich der sog. Störerhaftung. ${ }^{5}$ Einher damit geht ein beständiger Streit um die Reichweite von Auskunftsansprüchen gegenüber Providern, einschließlich der dadurch verursachten Kosten. ${ }^{6}$ Nachdem zunächst im Wesentlichen die Musikindustrie betroffen war, danach die Filmindustrie, zeichnet sich auch für andere Content-Bereiche eine entsprechende Problemstellung ab, etwa für E-Books.

\footnotetext{
${ }^{1}$ Eingehend zu den verschiedenen Formen der Web-Radios und der Aufnahmesoftware, Prill, WebradioStreamripping: Eine neue Form der Musikpiraterie?, erscheint demnächst, S. 15 ff., bei Fn. 62; v. Zimmermann, MMR 2007, 553, 554; Schwartmann, K\&R Beihefter 2/2011, 1, 19 ff.

${ }^{2}$ So ermöglicht etwa die von DVDVideoSoft angebotene Freeware „Free YouTube to MP3 Converter“ das Extrahieren und separate Abspeichern der Audiospur aus einem YouTube-Video, s. dazu http://www.dvdvideosoft.com/products/dvd/Free-YouTube-to-MP3-Converter.htm; die Firma SciLor's bietet eine Open-Source-Lösung zum Herunterladen von Musik aus Grooveshark an (sogar ausgehend von Rechnern, die sich in der von Grooveshark blockierten Region Deutschland befinden),

http://www.scilor.com/grooveshark-downloader.html.

${ }^{3}$ Urheberrechtsgesetz vom 9. September 1965 (BGBI. I S. 1273), das zuletzt durch Artikel 1 des Gesetzes vom 14. Dezember 2012 (BGBI. I S. 2579) geändert worden ist.

${ }^{4}$ Richtlinie 2004/48/EG des Europäischen Parlaments und des Rates vom 29. April 2004 zur Durchsetzung der Rechte des geistigen Eigentums, ABI. Nr. L 157 v. 30.4.2004, S. 45, in der Fassung der Berichtigung vom 2.6.2004, ABI. Nr. L 195, S. 16.

${ }^{5}$ Als sog. Störer haftet, wer - ohne selbst Verletzer zu sein - „in irgendeiner Weise willentlich und adäquat kausal an der rechtswidrigen Beeinträchtigung mitgewirkt hat, sofern es ihm rechtlich und tatsächlich möglich und zumutbar ist, die unmittelbare Rechtsverletzung zu unterbinden bzw. zu verhindern", Spindler, in: Spindler/Schuster, Recht der elektronischen Medien, 2. Aufl. 2011, § 97 UrhG Rn. 18, unter Verweis auf BGH GRUR 1999, 418 ff. - Möbelklassiker; BGH GRUR 2001, 1038 ff. - ambiente.de; BGH GRUR 2004, 860 ff. - Internetversteigerung I; BGH GRUR 2007, 708 ff. - Internetversteigerung II; BGH GRUR 2008, 702 ff. - Internetversteigerung III; ausf. Überblick über die Rspr. zur Störerhaftung von Host- und Access-Providern bei Leupold/Glossner, in: Leupold/Glossner, Münchener Anwaltshandbuch IT-Recht, 2. Aufl. 2011, Teil 2 Rn. 478 ff., 569 ff.

${ }^{6}$ S. zu den urheberrechtlichen Auskunftsansprüchen Spindler, ZUM 2008, 640 ff.; ferner Bohne, in: Wandtke/Bullinger, UrhR, 3. Aufl. 2009, § 101 UrhG Rn. 30 ff.; Dreier, in: Dreier/Schulze, UrhG, 4. Aufl. 2013, § 101 UrhG Rn. 10 ff., 35 ff. jeweils mwNachw.
} 
Umgekehrt fühlen sich viele Nutzer überwacht und kriminalisiert. Dabei setzt die Rechtsverfolgung nicht nur bei Intermediären wie den Providern oder den einzelnen Nutzern an, sondern bezieht sich inzwischen auf jegliche Form der Bereitstellung von Internetanschlüssen, ob es etwa WLAN-Betreiber sind, ${ }^{7}$ Internet-Cafés, ${ }^{8}$ Schulen oder Arbeitgeber generell, über deren Netze Rechtsverletzungen stattfinden, bis hin zu Eltern, die wegen Aufsichtspflichtverletzungen gegenüber ihren minderjährigen Kindern im Rahmen der Internetnutzung für deren Rechtsverletzungen belangt werden. ${ }^{9}$

Nicht nur in Deutschland wird diese Situation als unbefriedigend empfunden. So hält der vielbeachtete Hargreaves-Report für Großbritannien, das keine Privatkopie und Geräteabgabe kennt, fest:

„A second and also significant problem is that we have in recent years witnessed a growing mismatch between what is allowed under copyright exceptions, and the reasonable expectations and behaviour of most people. Digital technology has enabled use and reuse of material by private individuals in ways that they do not feel are wrong - such as sharing music tracks with immediate family members, or transferring a track from a CD to play in the car. It is difficult for anyone to understand why it is legal to lend a friend a book, but not a digital music file. The picture is confused by the way some online content is now sold with permissions to format shift (iTunes tracks) or to "lend" files (Amazon ebooks) at no extra cost. This puts the law into confusion and disrepute. It is not a tenable state of affairs." ${ }^{\prime 10}$

\footnotetext{
${ }^{7}$ BGHZ 185, 330 ff. - Sommer unseres Lebens = MMR 2010, 565 ff., m. Anm. Mantz = NJW 2010, 2061, m. Anm. Nenninger; der BGH hat die Haftung des Betreibers eines ungesicherten WLAN bejaht und damit einen Schlusspunkt unter die von den unteren Instanzgerichten kontrovers geführte Debatte gesetzt; s. den Überblick über die dem BGH-Urteil vorangegangene Rspr. bei Leupold/Glossner, in: Leupold/Glossner, Münchener Anwaltshandbuch IT-Recht, 2. Aufl. 2011, Teil 2 Rn. 582 f.

${ }^{8}$ LG Hamburg MMR 2011, 475.

${ }^{9}$ Dabei wird die grundsätzliche Möglichkeit der Störerhaftung der Eltern durchweg bejaht, wenn auch die Gerichte im Einzelfall unterschiedliche Ausmaße der elterlichen Beaufsichtigungspflichten annehmen und so zu variierenden Ergebnissen kommen; Haftung verneint: LG Köln, Urt. v. 11.09.2012 - 33 O 353/11 = BeckRS 2012, 20714; OLG Frankfurt MMR 2008, 169, 170 f.; LG Mannheim MMR 2007, 267, 268, m. Anm. Solmecke; Haftung bejaht: OLG Köln MMR 2010, 281, 282, m. Anm. Solmecke; LG Hamburg CR 2006, 780, 781; LG Hamburg MMR 2007, 131, 132; LG München I, MMR 2008, 619, 620 f.; AG Frankfurt a.M., Urt. v. 04.02.2009 - 29 C 549/08 81; s. zur Rspr. auch Kirchberg, ZUM 2012, 544, 546 f.

${ }^{10}$ Hargreaves, Digital Opportunity, 2011, S. 43.
} 


\section{Gutachtenfragestellung und Gang der Untersuchung}

„ergebnisoffen die Möglichkeiten (...) ermitteln, wie eine Erlaubnis zum Online-Austausch, Down- und Upload/ öffentliche Zugänglichmachung von kulturellen Werken gesetzlich zugelassen werden kann, und eine Vergütung mittels pauschaler Einzahlung effektiv zu sichern (ist). Ziel ist die Sicherung einer angemessenen Vergütung unter Berücksichtigung der Urheberpersönlichkeitsrechte, die bürgerrechtskonform umgesetzt wird und eine klare Alternative zu repressiven Rechtsdurchsetzungsmodellen wie Two-/Three-Strikes eröffnet. Es sollen Pauschalvergütungsmodelle unter genannten Vorgaben auf ihre wirtschaftliche und rechtliche Machbarkeit geprüft werden."

Entsprechend diesem Auftrag werden zunächst das generelle Verhältnis von Schranken, effektiver Rechtsdurchsetzung und Abgaben (III.) und die derzeitige Rechtsdurchsetzung (in der gebotenen Kürze) erörtert (IV.). Vor diesem Hintergrund wird der mögliche Tatbestand einer neuen Schrankenregelung zur Einführung einer „Kulturflatrate“ dargelegt (V.), der sich zunächst an europa- und verfassungsrechtlichen Rahmenbedingungen messen lassen muss (V.C.). Eine der zentralen Fragen - und auch stets vorgebrachten Kritikpunkte - bezieht sich auf die Berechnung der Einnahmen (VI.A) ebenso wie diejenige der Verteilung unter den Kreativen (VI.C.). Neben der Frage der möglichen Indizes, die hier herangezogen werden können, gilt es vor allem, einen Korridor für die mögliche Höhe einer solchen Abgabe zu berechnen, soweit dies überhaupt aufgrund des vorliegenden empirischen Zahlenmaterials möglich ist.

Die vorliegende Untersuchung kann aus Zeit- und Kostengründen keine eigenständigen empirischen Erhebungen durchführen, sondern muss sich auf vorliegende Studien und deren Auswertung beschränken, so dass diesbezüglich bereits an dieser Stelle ein entsprechender Vorbehalt bezüglich der Berechnungsergebnisse getroffen werden muss. Auch können rechtsvergleichende Aspekte nur am Rande einfließen. Schließlich können die Auswirkungen einer Kulturflatrate auf die verschiedenen Open-Source- bzw. Creative-Commons-Lizenzen nicht ausführlicher behandelt werden. ${ }^{11}$

Dabei sind auch die ökonomischen Auswirkungen auf die Anreize für Kreative (V.B.2) ebenso wie die Folgen auf die verschiedenen Geschäftsmodelle in der Unterhaltungsindustrie (V.B.4.) auszuloten sofern dies aufgrund vorliegender empirischer Studien und Zahlen möglich ist. Abschließend werden kurz alternative Modelle diskutiert (IX).

\footnotetext{
${ }^{11}$ Denkbare wäre hier, dass durch den Charakter der Kulturflatrate als Schranke spezifische Verwendungsbeschränkungen in einer Creative-Commons-Lizenz wegfielen, damit auch die entsprechende Bindungen in der Lizenzkette. Ob dies angesichts der weitgehenden Ähnlichkeiten zwischen Creative-Commons-Lizenzen/OpenSource Lizenzen gegenüber den einer durch die Kulturflatrate eingeführten „Rechten“ wie Down- und Upload sowie Bearbeitung tatsächlich zu negativen Effekten führen würde, zumal die kommerzielle Nutzung auch bei einer Kulturflatrate ausgeschlossen ist, erscheint zwar mehr als zweifelhaft, müsste jedoch anhand der verschiedenen Lizenzen ausführlicher geprüft werden.
} 



\section{Das grundsätzliche Problem: Schranken, effektive Rechtsdurchsetzung und Abgabenlösungen}

\section{A. Abgaben als zweitbeste Lösung gegenüber individueller Rechtsdurchsetzung}

Der hinter einer "Kulturflatrate" stehende Gedanke ist keineswegs neu: Es handelt sich vielmehr um das Ökonomen schon seit mehr als einem Jahrhundert geläufige grundlegende Problem des Verhältnisses von Eigentumsrechten und ihrer effektiven Rechtsdurchsetzung, gleichzeitig ausbalanciert mit den Interessen Dritter (bzw. Nicht-Eigentümer) an deren Nutzung. Gerade das Urheberrecht ist seit jeher von diesem Konflikt aus der Zuweisung von (temporären) Ausschließlichkeitsrechten (Verwertungsrechten) einerseits und Schranken zugunsten bestimmter im Allgemeininteresse privilegierter Nutzungen andererseits gekennzeichnet. Korrelat dieser Einschränkungen der Verwertungsrechte und der damit verbundenen Duldung sind oftmals entsprechende Vergütungen, die die Nutzer zwangsweise an die Urheber (bzw. die Rechteinhaber) entrichten müssen. ${ }^{12}$

Nicht nur aus Allgemeininteresse privilegierte Nutzungen können indes derartige Schranken und Vergütungstatbestände begründen, sondern auch rein ökonomische Abwägungen. Denn jegliche zwangsweise Vergütung wirkt wie eine Steuer oder Abgabe, die zwar gegenüber einer privaten und individuellen Rechtsdurchsetzung immer nur als zweitbeste Lösung gelten kann - jedoch nur, sofern die Kosten der individuellen Rechtsdurchsetzung in der Gesamtabwägung geringer ausfallen als die negativen Auswirkungen einer solchen zwangsweisen Abgabe. Anders formuliert kann eine Abgabe für Eingriffe in Eigentumsrechte (bzw. Ausschließlichkeitsrechte) effektiver die Interessen der Eigentümer schützen als deren individuelle Rechtsdurchsetzung. Wenn etwa Schädiger des Eigentumsrechts schwer bzw. nur mit hohen Kosten zu ermitteln sind, selbst bei ihrer Ermittlung die Rechtsdurchsetzung mit erheblichen Kosten verbunden ist und schließlich eine Vielzahl von Schädigern auftritt, kann eine pauschale Abgabe, die an bestimmte abstrakte Tatbestände anknüpft, sinnvoller als eine individuelle Rechtsverfolgung sein, da sie hinsichtlich der Kompensation effektiver ist. ${ }^{13}$ Die Abgabe dient dazu zugunsten der Urheber das Marktversagen zu korrigieren. ${ }^{14}$ Dies kann auch aus Wohlfahrtsökonomischer Sicht sinnvoll sein. ${ }^{15}$ Allerdings wird teilweise eingewandt, dass im digitalen Bereich durch DRM-Technik kein Bedürfnis mehr nach Pauschalabgaben bestünde, da das Marktversagen durch Kontrollmechanismen beseitigt würde. ${ }^{16}$ Dies verkennt aber zum einen, dass DRM Mechanismen möglicherweise nicht allen Urhebern zugänglich sind, zum anderen, dass schutzwürdige Interessen der Allgemeinheit durch den Einsatz von DRM-Systemen tendenziell ausgeblendet wer-

\footnotetext{
${ }^{12}$ S. Melichar, in: Schricker/Loewenheim, UrhR, 4. Aufl. 2010, Vor §§ 44a ff. UrhG Rn. 23; Stieper, Rechtfertigung, Rechtsnatur und Disponibilität der Schranken des Urheberrechts, 2009, S. 137 ff.; aus ökonomischer Perspektive ferner ebd., S. 87, $89 \mathrm{f}$.

${ }^{13}$ Landes/Posner, The Economic Structure of Intellectual Property Law, 2003, S. 116; Stieper, Rechtfertigung, Rechtsnatur und Disponibilität der Schranken des Urheberrechts, 2009, S. 87; Loewenheim, in: Schricker/Loewenheim, UrhR, 4. Aufl. 2010, § 54 UrhG Rn. 1; s. auch Bornkamm, in: FS Nordemann, 2004, S. 299, $299 \mathrm{f}$.

${ }^{14}$ Dreier, in: Dreier/Schulze, UrhG, 4. Aufl. 2013, Vorb. §§ 44a ff. Rn. 4; Stieper, Rechtfertigung, Rechtsnatur und Disponibilität der Schranken des Urheberrechts, 2009, S. 84 f.

${ }^{15}$ Chen/Png, 2003, S. 118, die ermittelt haben, dass aus wohlfahrtsökonomischer Sicht eine Gebühr auf das Kopiermedium der individuellen Rechtsverfolgung überlegen ist.

${ }^{16}$ Bell, N. Carolina L. Rev., Vol. 76, 1997-1998, S. 557, 583, sowie Merges, BTLJ, Vol. 12, 1997, 115, 130.
} 
den, indem keine Interessenabwägung mehr möglich ist, sondern nur das Interesse des Urhebers durchgesetzt wird. ${ }^{17}$

Dies gilt erst recht vor dem Hintergrund, dass eine effektive Rechtsverfolgung mit externen Effekten bzw. Schäden für unbeteiligte Dritte verbunden sein kann.

\section{B. Die Geräteabgabe als Korrelat für die Privatkopie im UrhG 1965}

13 Vor diesem Hintergrund erscheint auch die vom Gesetzgeber des UrhG 1965 gewählte Lösung bei Einführung einer Schranke zugunsten von Privatkopien gekoppelt mit einer pauschalen Geräteabgabe verständlich. Vorausgegangen war der zunehmende Gebrauch von stetig technisch weiterentwickelten Tonbandgeräten, die Schallplatten in annehmbarer Tonqualität aufnehmen konnten. Dementsprechend sah sich die Rechtsprechung seit Mitte der 50er Jahre gezwungen, im Rahmen von Klagen von Rechteinhabern gegen Hersteller und Händler von Tonbandgeräten sowie gegen Hersteller von Tonbändern und auf der Grundlage der deutschen Störerhaftung verschiedene Pflichten zu entwickeln. Der BGH entwickelte dabei in mehreren Entscheidungen die Auffassung, dass nicht nur die Hersteller bei der Bewerbung und beim Verkauf, sondern auch die Händler vor Kaufabschluss den sog. GEMA-Hinweis erbringen mussten. Demnach war schriftlich darauf hinzuweisen, dass im Falle einer Benutzung des Tonbandes bzw. Tonbandgerätes innerhalb Deutschlands zur Aufnahme urheberrechtlich geschützter Werke die Einwilligung der Verwertungsgesellschaft GEMA einzuholen wäre. Den Grundstein für diese Rechtsprechung legte das „Magnettonband I“-Urteil aus dem Jahr 1955, in dem der BGH entschied, dass auch Vervielfältigungen mittels Magnettonband zum persönlichen Gebrauch ohne Erlaubnis des Urhebers unzulässig seien. ${ }^{18}$

14 Dies wirkte sich auch auf die Hersteller und Händler von Tonbändern und Tonbandgeräten aus, da diese dem BGH zufolge mittelbar für Urheberrechtsverletzungen der Nutzer verantwortlich seien und somit als Störer haften müssten, gleich ob die unmittelbare Verletzungshandlung selbstständig durch den Tonband- bzw. Gerätenutzer durchgeführt werde. ${ }^{19}$ Ausgangslage des grundlegenden Urteils des BGH war, dass der Tonbandgeräte-Hersteller Grundig Magnettonbandgeräte, die nicht nur zur Aufnahme über ein Mikrofon fähig waren, sondern auch Schallplatten auf Tonband überspielen konnten, in den Handel gebracht und vor allem auch entsprechend beworben hatte. ${ }^{20}$ Für die Hersteller von Tonbandgeräten galt nach Ansicht des BGH, dass derjenige, der Geräte auf den Markt bringe, „die ihrer technischen Natur und ihrer Zweckbestimmung nach auf eine Benutzung abgestellt sind, die einen Eingriff in Urheberrechte Dritter zur Folge haben kann, ohne daß dies den in Betracht kommenden Abnehmerkreisen allgemein bekannt ist“, verpflichtet sei, „durch geeignete Sicherungsmaßnahmen ernsthafte Vorsorge gegen eine rechtsverletzende Benutzung der Geräte zu treffen“. ${ }^{21}$ Als geeignet und zumutbar wurde der besagte "GEMA-Hinweis" erachtet, der in der Werbung selbst angebracht werden musste, da diese bereits die erste Störerhandlung darstellte. ${ }^{22}$ Diese Pflicht wurde kurz darauf auf die Hersteller von Tonbändern erweitert und bestätigt, und zwar gegen die Ansicht der Revision, die geltend machte, dass es sich bei Tonbändern um gleichsam neutrale Gegen-

\footnotetext{
${ }^{17}$ Stieper, Rechtfertigung, Rechtsnatur und Disponibilität der Schranken des Urheberrechts, 2009, S. 94 f.; dazu s. ferner unten Rn. $44 \mathrm{ff}$.

${ }^{18}$ BGHZ 17, $266 \mathrm{ff}$. - Magnettonband I.

${ }^{19}$ BGHZ 17, 266, 291 f. - Magnettonband I; 42, 118, 126 f. - Personalausweise; BGH NJW 1960, 771, 773; NJW 1963, 1736, 1736 f.; NJW 1963, 1739, 1740; NJW 1965, 746, 746 f.

${ }^{20}$ BGHZ 17, 266, 267 f. - Magnettonband I.

${ }^{21}$ BGH NJW 1960, 771, $773 \mathrm{f}$.

22 BGHZ 17, 266, 292 f. - Magnettonband I; BGH NJW 1960, 771, 773.
} 
stände handele, die nicht per se die Gefahr einer Urheberrechtsverletzung begründeten. ${ }^{23}$ Für den BGH war demgegenüber entscheidend, dass auch der Hersteller von Tonbändern Störer i.S.v. § 1004 $\mathrm{BGB}^{24}$ sei und die Anbringung eines GEMA-Hinweises in der Werbung für Tonbänder deren Benutzung für "neutrale“, also urheberrechtskonforme Zwecke nicht berühre, mithin zumutbar sei. ${ }^{25}$ In einem parallel verhandelten Verfahren übertrug der BGH die Hinweispflichten auch auf Händler von Tonbandgeräten, die somit verpflichtet waren, bei schriftlicher Werbung sowie in Kaufverhandlungen auf das Erfordernis der GEMA-Erlaubnis hinzuweisen. Im Ergebnis waren somit lediglich die Händler von Tonbändern von der Pflicht des GEMA-Hinweises ausgenommen.

Über den GEMA-Hinweis hinausgehende Pflichten wurden vom BGH zwar diskutiert, aber in jedem Fall abgelehnt. Als unzumutbar wurde bspw. erachtet, dass ein Händler dauerhaft ein entsprechendes DIN/A4-Plakat in seinen Verkaufsräumen aufhängen müsse. ${ }^{26}$ Darüber hinaus wurde insbesondere die Identitätsfeststellung der Käufer von Tonbandgeräten mittels Personalausweiskontrolle durch den Hersteller für unzumutbar erklärt. Denn die bloße Kenntnis von der Identität der Erwerber könne noch immer nicht gewährleisten, dass es zu unzulässigen Vervielfältigungen komme. Um dies zu überprüfen, seien vielmehr Kontrollmaßnahmen in deren „persönlicher häuslicher Sphäre“ notwendig, was grundrechtliche Probleme aufwerfe: „Da die Art der Verwendung der Geräte nur an Ort und Stelle festgestellt werden könnte und die Kl. bereits die Möglichkeit angekündigt hat, die erforderlichen Feststellungen auf Mitteilungen von Wohnungsnachbarn, Portiers usw. hin zu veranlassen, würde hierdurch die Gefahr unangemessener Eingriffe in die Unverletzlichkeit des häuslichen Bereichs heraufbeschworen (Art. $13 \mathrm{GG}$ ). ${ }^{\text {27 }}$

Die Unverhältnismäßigkeit der zu einer effektiven Verfolgung von Urheberrechtsverletzungen nötigen Eingriffe in die Grundrechte der Betroffenen, insbesondere der Wohnung, Art. 13 GG, und das Massenphänomen der privaten Kopie veranlassten schließlich den Gesetzgeber zur Suche nach einer salomonischen Lösung, die er in der Einführung einer neuen Schranke zugunsten von Privatkopien - und, damit verbunden, der Ablösung der individuellen Rechtsdurchsetzung - gekoppelt mit einer pauschalen Abgabe auf alle Geräte und Leermedien, die geeignet waren, zur Anfertigung von Privatkopien zu dienen. Die amtliche Begründung des Regierungsentwurfs dazu wörtlich:

„Ein Verbot der privaten Vervielfältigung kann in der Praxis nicht durchgesetzt werden. Eine wirksame Überprüfung könnte nur dann durchgeführt werden, wenn den Kontrolleuren der privaten Verwertungsgesellschaften gestattet werden würde, die Wohnung jedes einzelnen Staatsbürgers daraufhin zu überprüfen, ob er ein Magnettongerät besitzt, mit diesem urheberrechtlich geschützte Werke aufnimmt und hierfür eine Genehmigung des Urhebers bzw. der Verwertungsgesellschaften nachweisen kann. Eine solche Kontrolle würde jedoch dem in Artikel 13 des Grundgesetzes ausgesprochenen Grundsatz der Unverletzlichkeit der Wohnung widersprechen. ${ }^{128}$

Die vor diesem Hintergrund im Regierungsentwurf noch vorgesehene Vergütungspflicht des Gerätenutzers wurde vom Rechtsausschuss allerdings wieder verworfen und durch die bis heute

\footnotetext{
${ }^{23}$ BGH NJW 1963, 1736, 1737.

${ }^{24}$ Bürgerliches Gesetzbuch in der Fassung der Bekanntmachung vom 2. Januar 2002 (BGBI. I S. 42, 2909; 2003 I

S. 738), das zuletzt durch Artikel 7 des Gesetzes vom 19. Oktober 2012 (BGBI. I S. 2182) geändert worden ist.

${ }^{25}$ BGH NJW 1963, 1736, 1737.

${ }^{26}$ BGH NJW 1963, 1739, $1740 \mathrm{f}$.

${ }^{27}$ BGHZ 42, 118, 131 - Personalausweise.

${ }^{28}$ BT-Drs. IV/270, S. 71.
} 
gültige Geräteabgabe ersetzt. Eine Vergütungspflicht des Nutzers wurde als ebenso wenig durchsetzbar angesehen wie ein Verbot. Der Rechtsausschuss berief sich dabei auf die spärlichen Erfolge der GEMA bei der Aufklärung der schon 1955 vom BGH für unzulässig erklärten privaten Anfertigung von Tonbandkopien. ${ }^{29}$ Der Rückgriff auf die Hersteller entsprechender Geräte sei hingegen sowohl erfolgversprechend (im Sinne einer angemessenen Vergütung der Urheber) als auch systemkonform:

„Die Lösung entspricht dem auch sonst im Urheberrecht vorherrschenden System, nach dem der gewerbliche Verwerter, wie z.B. der Verleger, der Schallplattenhersteller oder der Konzertveranstalter die Vergütung an den Urheber zahlt und sie dann im Rahmen der Preisgestaltung auf den privaten ,Endverbraucher' abwälzt. Der Unterschied zu diesen Fällen besteht darin, daß der Hersteller von Tonbandgeräten nicht selbst unmittelbar das Werk nutzt, sondern lediglich die Vorrichtung dafür zur Verfügung stellt. Wirtschaftlich gesehen ist jedoch auch der Hersteller von Tonbandgeräten Nutznießer des Urhebergutes, denn er nutzt bewußt die Eignung der Geräte zur privaten Überspielung geschützter Werke aus [...]. Ohne diese Eignung der Geräte würde er auch nicht annähernd die gleichen Umsätze erzielen können. “30

Interessanterweise - und wenig beachtet in der derzeitigen Diskussion - blieb der Gesetzgeber aber auch später nicht bei dieser Koppelung aus Wahrnehmung von Schranken und Geräteabgaben stehen. Denn grundsätzlich bestand jenseits der Schranke nach wie vor die Möglichkeit für die Rechteinhaber, gegen die Schädiger individuell vorzugehen, etwa bei Kopien zu kommerziellen Zwecken. Dennoch hat für bestimmte eng umgrenzte Bereiche der Gesetzgeber selbst für nicht privilegierte Verletzungen der Verwertungsrechte eine Abgabe eingeführt: So stellt §54c UrhG nur auf die tatsächliche Handlung ab, nicht aber wie § 54 UrhG (für die Geräteabgabe) auf die (berechtigte) Privatkopie. §54c UrhG zielt vor allem auf die durch Kopiergeräte angefertigten Vervielfältigungen von Büchern etc. ab - gerade hier hatte der I. Zivilsenat des BGH Anfang der 80er Jahre entschieden, dass der Betreiber eines Kopierladens (Copyshop) nicht dazu verpflichtet sei, bei jedem Kunden zu überprüfen, zu welchen Zwecken der Kunde Vervielfältigungen anfertigt bzw. ob er dazu berechtigt sei. Der BGH rechtfertigte diese Einschränkung der Störerhaftung schon damals vor allem mit den überwiegenden Persönlichkeitsinteressen der Nutzer und den damit eingeschränkten Prüfungs- bzw. Kontrollpflichten der Betreiber:

\begin{abstract}
„Als weitere Maßnahme, Rechtsverletzungen der Kl. auszuschließen, könnte eine generelle Kontrolle des Fotokopierguts zu Beginn und während des Fotokopiervorgangs in Betracht kommen. Mit Recht hat jedoch das BerGer. ausgeführt, daß eine solche generelle Kontrollpflicht im Allgemeinen durchgreifenden Bedenken begegnet. Es weist darauf hin, daß die Fotokopiergeräte der Bekl. auch zur Vervielfältigung privater Aufzeichnungen, Urkunden und dergleichen benutzt werden, deren Inhalt vielfach vertraulich und nicht zur Kenntnisnahme durch dritte Personen bestimmt ist. Eine umfassende Kontrolle - und nur sie käme überhaupt als eine wirksame Maßnahme in Betracht - würde den Anspruch des einzelnen Kunden auf Vertrau-
\end{abstract}

\footnotetext{
${ }^{29}$ BT-Drs. IV/3401, Schriftlicher Bericht des Rechtsausschusses (12. Ausschuss) über den von der Bundesregierung eingebrachten Entwurf eines Gesetzes über Urheberrecht und verwandte Schutzrechte (Urheberrechtsgesetz), S. 8.

${ }^{30}$ BT-Drs. IV/3401, Schriftlicher Bericht des Rechtsausschusses (12. Ausschuss) über den von der Bundesregierung eingebrachten Entwurf eines Gesetzes über Urheberrecht und verwandte Schutzrechte (Urheberrechtsgesetz), S. 9.
} 
lichkeit, der seine Grundlage in den verfassungsrechtlich geschützten persönlichen Freiheitsrechten (Art. 1, $2 \mathrm{GG}$ ) hat, in unerträglicher Weise beeinträchtigen. ${ }^{\prime 31}$

22 Diese Lösung - Einführung einer Schranke zugunsten privater Kopien verknüpft mit einer Geräteabgabe - setzte sich in der Folgezeit in den meisten kontinentaleuropäischen Ländern durch. Lediglich das Vereinigte Königreich, Irland, Luxemburg, Malta und Zypern kennen bis heute keine umfassende Privatkopieschranke und auch keine Geräteabgabe. ${ }^{32}$

\footnotetext{
${ }^{31}$ BGH NJW 1984, 1106, 1107.

${ }^{32}$ S. dazu Kretschmer, 2011, S. 10, unter Hinweis darauf, dass im Vereinigten Königreich zumindest das sog. Time-Shifting bei Übertragungen zulässig ist.
} 



\section{Die gegenwärtige Rechtsdurchsetzung im Internet}

\section{A. Anonymität und Globalität als Hürden}

23 Da das Internet ermöglicht, ohne Angabe der Identität Informationen zu verbreiten oder zu erhalten, ${ }^{33}$ sind einer effektiven Rechtsverfolgung von vornherein Grenzen gesetzt. Zwar erlaubt die Kommunikationsstruktur des Internet eine eindeutige Identifizierung jedes einzelnen Rechners durch die Vergabe der sog. IP-Adresse; ${ }^{34}$ auch erlaubt die Umstellung auf die nächste Generation der IPAdressenvergabe, die sog. IPv6, eine deutlich weitere Bandbreite von Adressen, ${ }^{35}$ wodurch eine statische Vergabe von IP-Adressen ermöglicht wird, ${ }^{36}$ so dass daraus eine einfachere Identifizierung resultieren könnte. ${ }^{37}$ Abgesehen davon, dass ein Betroffener bzw. ein Rechteinhaber darauf angewiesen ist, dass der Access-Provider die entsprechenden Daten gespeichert hat, und vom Provider diese Daten erst im Wege des Auskunftsanspruchs herausverlangen muss (§ $101 \mathrm{UrhG}$ ), können durch verschiedene Dienste bzw. Techniken die Identifizierung eingeschränkt werden, etwa durch sog. ProxyServer, ${ }^{38}$ oder durch echte Anonymisierungsdienste. ${ }^{39}$

24 Hinzu kommt die Globalität und dezentrale Struktur des Netzes, die oft die Durchsetzung von Ansprüchen leer laufen lässt, da Urheber rechtswidriger Inhalte und Aktivitäten im Ausland nur schwer ermittelbar sind. Zwar ist innerhalb der EU die Vollstreckbarkeit von rechtskräftigen Titeln erheblich vereinfacht worden; doch bleibt nach wie vor das Vollstreckungsrisiko vor Ort, das gerade bei Ansprüchen mit geringer Höhe erheblich zu Buche schlägt.

\section{B. Bisherige Reaktionen der Rechteinhaber und Folgeprobleme}

\section{Rechtsverfolgungsmaßnahmen der Rechteinhaber gegenüber Nutzern}

Ungeachtet dieser Hürden hat vor allem die Musikindustrie anfangs in den USA, aber auch in den europäischen Ländern mit Rechtsverfolgungsmaßnahmen zunächst gegen zentrale FilesharingBetreiber wie Napster begonnen, um dann ca. seit 2004 auch gegen Nutzer vorzugehen, was in Deutschland zu dem bekannten Phänomen der Massenabmahnungen geführt hat. ${ }^{40}$ Nach einer nicht amtlichen, sondern vom „Verein gegen den Abmahnwahn“ stammenden Schätzung sollen 2011 allein in Deutschland ca. 218.000 Abmahnungen mit einem Streitwert von rund 165 Mio. Euro anhängig gewesen sein, ${ }^{41}$ wobei die Abmahnungen allerdings rückläufig zu sein scheinen. ${ }^{42}$ Nach einer vom

\footnotetext{
${ }^{33}$ Zur Definition der Anonymität Hansen in: Roßnagel, Handbuch Datenschutzrecht, 2003, Kap. 3.3 Rn. 50; dabei werden verschiedene Grade der Anonymität unterschieden, vgl. Brunst, Anonymität im Internet, 2009, S. 24 ff.

${ }^{34}$ Dazu Köhntopp/Köhntopp, CR 2000, 248.

${ }^{35}$ Das IPv6 verwendet 128 Bit, gegenüber bloß 32 Bit vom IPv4.

${ }^{36}$ So etwa Hoeren, ZRP 2010, 251, 252 f.; Schaar, Datenschutz im Internet, 2002, Rn. 176.

${ }^{37}$ Zum Aufbau der IPv6-Adressen s. Wegener/Heidrich, CR 2011, 479.

${ }^{38}$ Federrath/Golembiewski, DuD 2004, 486, 487.

${ }^{39}$ Zur genauen Funktionsweise von JAP s. Federrath, in: Bäumler/v. Mutius, Anonymität im Internet, 2003, S. 172, ferner ders., DuD 2003, 169.

${ }^{40}$ Fitzner, Von Digital-Rights-Management zu Content Identification, 2011, S. 46 f.; zur wohlfahrtsökonomischen Unterlegenheit von individueller Rechtsverfolgung ggü. pauschalen Abgaben: Chen/Png, 2003, S. 118.

${ }^{41}$ S. dazu die nicht repräsentative Statistik des „Vereins zur Hilfe und Unterstützung gegen den Abmahnwahn e.V.“, 2011, S. 5, abrufbar unter: http://www.verein-gegen-den-

abmahnwahn.de/zentrale/download/statistiken/2011/Jahresstatistik\%202011.pdf, wobei daraus ebenfalls
} 
Verbraucherzentrale Bundesverband e.V. (vzbv) in Auftrag gegebenen Umfrage durch Infratestdimap sollen rund zwei Drittel der Bundesbürger ab 14 Jahren von solchen Abmahnungen gehört haben und rund 6 Prozent selbst einmal abgemahnt worden sein. Nach Einschätzung des vzbv sollen die Kosten in der Regel zwischen 500 und 1.000 Euro variieren. ${ }^{43}$

Auch die bisherigen Versuche der Gesetzgebung, durch eine Einschränkung der Abmahnkosten in § 97a UrhG der massenhaften Abmahnungen Herr zu werden, haben offenbar nicht verfangen, so dass zur Zeit Überlegungen angestellt werden, in einer weiteren Urheberrechtsnovelle die Kosten der Abmahnungen weiter zu begrenzen. Das Bundesjustizministerium hat dazu einen Referentenentwurf $^{44}$ erstellt, der u.a. die Ergänzung des $\S 97$ a UrhG um einen dritten Absatz vorsieht, wonach dem zu Unrecht Abgemahnten ein Ersatzanspruch bzgl. „der für die Rechtsverteidigung erforderlichen Aufwendungen" zustehen soll. Zudem würde die im Entwurf vorgesehene Einfügung eines neuen $\S$ $49 \mathrm{GKG}^{45}$ den Streitwert im ersten Verfahren zwischen einer Privatperson und einem bestimmten Rechteinhaber auf 500 Euro beschränken. Zu dem Referentenentwurf konnte innerhalb der Koalition allerdings noch kein Konsens erzielt werden, so dass die Problematik weiter offen in der Diskussion ist. $^{46}$

Probleme rufen auch die seit Umsetzung der Enforcement-Richtlinie eingeführten Auskunftsansprüche nach § 101 UrhG gegen Internet-Provider auf Offenlegung der Identitätsdaten der Nutzer hervor. Denn bislang war innerhalb der Rechtsprechung umstritten, ob der Auskunftsanspruch gegen den Provider nur bei gewerblichem Ausmaß der Rechtsverletzung geltend gemacht werden oder ob auf diese Voraussetzung verzichtet werden kann. Ein gewerbliches Ausmaß der Rechtsverletzung ist zumindest gem. § 101 Abs. 1 UrhG für den Auskunftsanspruch gegen den Verletzer erforderlich. Dies wurde von den meisten Gerichten - teilweise im Erst-recht-Schluss - auch für den Auskunftsanspruch gem. § 101 Abs. 2 UrhG, mithin gem. dessen Nr. 3 gegenüber Internetprovidern angenommen. ${ }^{47}$ Dies entsprach auch der ausdrücklichen Intention des Gesetzgebers. ${ }^{48}$ Da sich das „gewerbliche Ausmaß" gem. § 101 Abs. 1 S. 2 UrhG sowohl aus dem Ausmaß an Rechtsverletzungen als auch der Schwere der Rechtsverletzung ergeben kann, führte dies vor allem in Bezug auf die Schwere der Rechtsverletzung zu erheblichen Auslegungsproblemen im Rahmen von Filesharing; die Gerichte haben hier zunehmend dazu tendiert, bereits bei einem Upload einer gerade erschienen CD oder

hervorgeht, dass im Vorjahr 2010 noch 575.800 Abmahnungen mit einem Gesamtforderungsvolumen von 412 Mio. Euro ergangen sein sollen.

${ }^{42}$ S. dazu http://www.initiative-abmahnwahn.de/wp-content/uploads/2012-_1._-Halbjahr.pdf, S. 4.

${ }^{43}$ http://www.vzbv.de/10162.htm\#.

${ }^{44}$ Der Text ist abrufbar unter: http://www.textintern.de/Bilder/Referentenentwurf.pdf.

${ }^{45}$ Gerichtskostengesetz vom 5. Mai 2004 (BGBI. I S. 718), zuletzt geändert durch Artikel 5 des Gesetzes vom 17. August 2012 (BGBI. I S. 1726).

${ }^{46}$ S. dazu http://www.cdu.de/archiv/2370_34345.htm; http://www.heise.de/newsticker/meldung/Koalitionstreitet-auch-ueber-Gesetz-gegen-Abmahn-Missbrauch-1542523.html; http://www.fr-

online.de/politik/urheberrecht-cdu-haelt-anti-abzocke-gesetz-auf,1472596,14950034.html; s. auch die Stellungnahme der Bundesrechtsanwaltskammer, abrufbar unter: http://www.brak.de/zur-

rechtspolitik/stellungnahmen-pdf/stellungnahmen-deutschland/2012/mai/stellungnahme-der-brak-2012-

27.pdf.

${ }^{47}$ OLG Zweibrücken MMR 2009, 43, 44; OLG Oldenburg MMR 2009, 188, 189; OLG Schleswig GRUR-RR 2010, 239, 239 f.; OLG Hamburg ZUM 2010, 893, 897; OLG München GRUR-RR 2012, 68, 69; LG Köln MMR 2009, 645; LG Frankfurt a.M. MMR 2008, 829, $829 \mathrm{f}$.

${ }^{48}$ BT-Drs. 16/5048, S. 49, 65; zu den Auseinandersetzungen im Gesetzgebungsverfahren s. Wimmers, in: Schricker/Loewenheim, UrhR, 4. Aufl. 2010, § 101 UrhG Rn. 33 f. 
DVD von einer erheblichen Schwere auszugehen. ${ }^{49}$ Damit näherten sich die entsprechenden Gerichte derjenigen Auffassung an, die vertrat, dass es gar nicht erst eines gewerblichen Ausmaßes der Rechtsverletzung bedürfe, um einen Auskunftsanspruch gem. § 101 Abs. 2 UrhG zu begründen. ${ }^{50}$

Der BGH hat sich jüngst in seiner „Alles kann besser werden“-Entscheidung zugunsten letzterer Ansicht entschieden und verlangt somit kein gewerbliches Ausmaß der Rechtsverletzung. ${ }^{51}$ Zur Begründung führte der BGH an, das Kriterium des „gewerblichen Ausmaßes“ beziehe sich dem Wortlaut nach gerade nicht auf die am Anfang des § 101 Abs. 2 S. 1 UrhG stehende „Rechtsverletzung“, sondern - im Falle von Nr. 3 - auf die Erbringung von Dienstleistungen, die für rechtsverletzende Tätigkeiten genutzt werden. Dieses Ergebnis der Wortlautauslegung unterstrich der BGH zusätzlich damit, der Auskunftsanspruch nach § 101 Abs. 2 UrhG sei gegenüber dem aus § 101 Abs. 1 UrhG selbstständig, da er nicht lediglich ein Hilfsanspruch zur Vorbereitung des Auskunftsanspruchs gegen den Verletzer sei. Da sich beide Ansprüche somit in Ziel und Inhalt unterschieden und voneinander unabhängig seien, verbiete sich auch ein Erst-recht-Schluss, dass für einen Anspruch gegenüber einer nicht selbst verletzend tätig werdenden Person erst recht die Voraussetzungen gegeben sein müssten, die für einen Anspruch gegenüber dem Verletzer gelten. ${ }^{52}$ Der Anspruch nach $\S 101$ Abs. 2 UrhG setze lediglich das Vorliegen der Voraussetzungen des Unterlassungs- und Schadensersatzanspruchs gem. $\S 97$ UrhG voraus, mithin gerade kein gewerbliches Ausmaß der Rechtsverletzung. ${ }^{53}$ Die subjektive Intention des Gesetzgebers sei hingegen nicht maßgeblich, da es bei der Auslegung allein auf den im Gesetzeswortlaut objektiviert zum Ausdruck kommenden Willen des Gesetzgebers ankomme. ${ }^{54}$ Diese Entscheidung des BGH bedeutet für die Praxis, dass de lege lata in Ermangelung einer gesetzlichen Erheblichkeitsschwelle jegliche widerrechtliche Verletzung eines nach dem UrhG geschützten Rechts ausreicht, um einen Auskunftsanspruch gem. § 101 Abs. 2 S. 1 Nr. 3 UrhG gegen den Internetprovider zu begründen.

Offenbar haben aber die Rechtsverfolgungsmaßnahmen nur zu einer Art Hase-und-Igel-Wettrennen geführt, indem Filesharer Ausweichstrategien entwickelt haben, sei es durch Verschlüsselungen, Anonymisierungsdienste oder neue Angebote wie Filehoster, die nicht mehr dem alten P2PFilesharing entsprechen. So soll nach Angaben des Medienforschungsunternehmens BigChampagne

\footnotetext{
${ }^{49}$ S. OLG Köln MMR 2008, 820, 822, unter Verweis auf die Empfehlung des Rechtsausschusses im Bundestag: „Wer ein gesamtes Musikalbum, zudem in der relevanten Verkaufsphase, der Öffentlichkeit zum Erwerb anbietet, tritt wie ein gewerblicher Anbieter auf [...]. Er kann und will nicht mehr kontrollieren, in welchem Umfang von seinem Angebot Gebrauch gemacht wird und greift damit in die Rechte des Rechteinhabers in einem Ausmaß ein, das einer gewerblichen Nutzung der fremden Rechte durch den Verletzer entspricht“; bestätigt in OLG Köln MMR 2009, 334, 335; ebenso OLG Schleswig GRUR-RR 2010, 239, 240; LG Frankfurt a.M. MMR 2008, 829, 830; LG Köln MMR 2008, 761; die Gegenansicht verlangt eine „erhebliche Qualität“ der Rechtsverletzung, d.h. einen Umfang, „der über das hinausgeht, was einer Nutzung zum privaten oder sonstigen eigenen Gebrauch entsprechen würde“, OLG Zweibrücken MMR 2009, 43, 45; bestätigt in OLG Zweibrücken MMR 2009, 702: vier Jahre alte Software mit einem Marktwert von 400 Euro; ebenso LG Kiel MMR 2009, 643, 644; LG Darmstadt MMR 2009, 52, 53 f.: „mehrstündige Session und [...] Bereithalten von 620 Audio-Dateien überwiegend gängiger Titel“; s. auch einschränkend OLG Oldenburg MMR 2009, 188, 189; differenzierend unter Ablehnung beider Extremansichten Wimmers, in: Schricker/Loewenheim, UrhR, 4. Aufl. 2010, § 101 UrhG Rn. 41 mwNachw.

${ }^{50}$ LG Bielefeld MMR 2009, 870; ebenso Bohne, CR 2010, 104, 106 ff.; i.E. auch Dreier, in: Dreier/Schulze, UrhG, 4. Aufl. 2013, § 101 UrhG Rn. 12.

${ }^{51}$ BGH MMR 2012, 689, 690 Rn. 11.

52 BGH MMR 2012, 689, 691 Rn. 21.

${ }^{53}$ BGH MMR 2012, 689, 691 Rn. 20.

${ }^{54}$ BGH MMR 2012, 689, 692 Rn. 30, unter Verweis auf BVerfGE 1, 299, 312 sowie BGHZ 13, 265, 277.
} 
die Zahl der Filesharer nach wie vor ansteigen. ${ }^{55}$ Genaue, durch amtliche Statistiken oder unabhängige Forschung belegte Zahlen sind allerdings nicht ersichtlich.

\section{Rechtsverfolgungsmaßnahmen gegenüber Intermediären}

Aber nicht nur Internetnutzer sahen sich entsprechenden Rechtsverfolgungsmaßnahmen gegenüber, sondern auch Internetintermediäre und Hersteller von P2P-Tauschsoftware. Anders als bei den Auskunftsbegehren richten sich die Klagen hier im Wesentlichen auf die Unterbindung von Verbindungen der Nutzer zu illegalen Angeboten oder gegen bestimmte Plattformen selbst, die etwa Files zum Download bereithalten. Während die Verfahren gegen Suchmaschinen oder Access-Provider eher vereinzelt und überwiegend auch erfolglos blieben, ${ }^{56}$ erst recht vor dem Hintergrund der maßgeblichen Entscheidung des EuGH in Sachen SABAM, ${ }^{57}$ richteten sich die Bemühungen vor allem gegen die Filehoster, vor allem Rapidshare. Nachdem ursprünglich ein Verfahren vor dem OLG Hamburg zum Erfolg führte, insbesondere eine Identifizierungspflicht für Rapidshare vorgeschrieben wurde, ${ }^{58}$ gab derselbe Senat des OLG Hamburg seine Rechtsprechung kürzlich unter dem Eindruck eines gegensätzlichen Urteils des OLG Düsseldorf auf. Im Zentrum der Entscheidungen stand wiederholt die Frage, ob Rapidshare ein noch von der Rechtsordnung gebilligtes Geschäftsmodell verfolgt und die Prüfungspflichten sich im Rahmen des Zumutbaren halten. ${ }^{59}$ Jüngst hat der BGH eine letztinstanzliche Entscheidung in der zuvor vom OLG Düsseldorf entschiedenen Sache Rapidshare gefällt. ${ }^{60}$ Der BGH hat das Urteil des OLG aufgehoben und zur erneuten Verhandlung zurückverwiesen. Damit zeichnet sich insgesamt ab, dass zwar - allein schon vor dem Hintergrund des europäischen Rechts - keine proaktiven Überwachungspflichten für Host-Provider gelten, diese aber zu mehr als nur einem einfachen „Notice-and-Take-Down“-Verfahren verpflichtet sind.

\section{Folgeprobleme}

\section{a) Datenschutz und Persönlichkeitsrechte der Nutzer}

31 Die Rechtsverfolgung gegenüber Nutzern impliziert notwendigerweise, dass ihre Identität festgestellt werden kann. Eine solche Feststellung und anschließend Auskunftserteilung gegenüber den Rechteinhabern bedingt aber ihrerseits, dass die sensiblen Verkehrsdaten der Nutzer jeweils für eine gewisse Zeit gespeichert werden, was gerade bei Access-Providern, die Flatrates anbieten, häufig nicht der Fall ist, da diese Flatrates keine kontinuierliche Speicherung von Verkehrsdaten erfordern, so dass sich die Access-Provider oftmals auf datenschutzrechtliche Pflichten zur Löschung der entsprechenden Daten berufen. ${ }^{61}$ Diese Schwierigkeiten haben ebenfalls zu Diskussionen darüber geführt,

\footnotetext{
${ }^{55}$ S. die Präsentation von Präsentation von Garland von BigChampagne, From Napster to Now, 2010, S. 16, allerdings nur bis einschl. 2009.

${ }^{56}$ Gegen die Zumutbarkeit von technischen Sperrmaßnahmen durch Access-Provider LG Hamburg ZUM 2009, 587, 589 f.; MMR 2010, 488, 489 f.; anders aber später LG Hamburg, Beschl. v. 06.05.2010 - 3100 154/10= BeckRS 2010, 12237, wo das Gericht es in einem einstweiligen Verfügungsverfahren für zumutbar erachtete, einem Access-Provider aufzuerlegen, die Abrufbarkeit der für Filesharing häufig genutzte Webseite „The Pirate Bay" zumindest nach Abmahnung durch den Verletzten zu verhindern.

${ }^{57}$ EuGH MMR 2012, 174 ff. - Scarlet Extended SA/SABAM; s. dazu auch Spindler, JZ 2012, $308 \mathrm{ff}$.

${ }^{58}$ OLG Hamburg MMR 2008, 823, 826 - Rapidshare I.

${ }^{59}$ So noch OLG Hamburg MMR 2008, 823, 825 f. - Rapidshare I; anders nunmehr OLG Hamburg MMR 2012, 393, 395 - Rapidshare II; zuvor schon OLG Düsseldorf MMR 2010, 483, 484 - Rapidshare; s. ferner OLG Düsseldorf MMR 2010, 702 ff. - Rapidshare II; MMR 2011, 250 ff. - Rapidshare III.

${ }^{60}$ BGH MMR 2013, 185 ff. - Alone in the Dark, m. Anm. Hoeren; zuvor OLG Düsseldorf MMR 2011, 250 ff. Rapidshare III.

${ }^{61}$ S. OLG Frankfurt a.M. MMR 2009, 542, 544; LG München I MMR 2010, 111, m. Anm. Petersdorff-Campen; LG Hamburg MMR 2009, 570, 572 f.; LG Darmstadt MMR 2006, 330, 331 f.; AG Bonn MMR 2008, 203; s. dazu Boh-
} 
ob etwa ein Quick-Freeze-Verfahren eingeführt werden sollte, bei dem quasi auf "Zuruf" durch den Verletzten der Provider die relevanten Daten vorerst speichern muss, um sie dann im Rahmen eines Auskunftsverfahrens dem Verletzten offenzulegen. ${ }^{62}$

32 Die datenschutzrechtlichen Vorbehalte und Gefährdungen für das informationelle Selbstbestimmungsrecht liegen hier auf der Hand: Denn eine derartige Speicherung ist nicht weit entfernt von der sog. Vorratsdatenspeicherung nach $\S 113 a$ TKG $^{63}$ welche das BVerfG in dieser konkreten Ausgestaltung als nicht vereinbar mit der Verfassung erklärt hat. ${ }^{64}$ Diesbezüglich betonte das Gericht, dass eine staatlich angeordnete anlasslose Speicherung von Daten bei Telekommunikationsdiensteanbietern über einen Zeitraum von sechs Monaten nur verfassungskonform sei, wenn das Gesetz gleichzeitig strenge Anforderungen hinsichtlich der Datensicherheit vorsehe und die Verwendung der Daten u.a. nur zwecks der Verfolgung schwerer Straftaten erfolge, „(...) die überragend wichtige Rechtsgüter bedrohen oder zur Abwehr von Gefahren für solche Rechtsgüter" ${ }^{65}$ Das gelte jedoch nur für den unmittelbaren Abruf der anlasslos gespeicherten Daten. Bei einer nur mittelbaren Verwendung der Daten, wie etwa im Wege des Auskunftsersuchens bei einem Telekommunikationsdiensteanbieter zur Identifizierung von Anschlussinhabern anhand der IP-Adressen, lässt das BVerfG hingegen eine Rechtsgutbeeinträchtigung genügen, „(...) der von der Rechtsordnung auch sonst ein hervorgehobenes Gewicht beigemessen wird“. ${ }^{66}$ Die mittelbare Verwendung der Vorratsdaten beschränkt das BVerfG dabei aber ausdrücklich auf Auskunftsersuchen von Strafverfolgungsbehörden, womit direkte zivilrechtliche Auskunftsansprüche zwischen Privaten - wie etwa zwischen Rechteinhaber und Access-Provider nach $\S 101$ Abs. 2 S. 1 Nr. 1 UrhG - diesbezüglich ausgeschlossen sind; die Möglichkeiten der Rechteinhaber sind in diesen Fällen auf ein Auskunftsersuchen durch die Strafverfolgungsbehörden und anschließende Einsichtnahme in die Strafakten beschränkt. ${ }^{67}$ Der zivilrechtliche Auskunftsanspruch aus $\S 101$ Abs. 2 S. 1 Nr. 1 UrhG bei Urheberrechtsverletzungen bleibt damit - genau wie die Auskunftsersuchen der Strafverfolgungsbehörden ohne verfassungskonforme Umsetzung einer Vorratsdatenspeicherung - auf die Daten beschränkt, die ein Telekommunikationsdiensteanbieter aufgrund der in den $\S \S 96$ bis 101 TKG genannten Zwecke speichern darf. ${ }^{68}$ Bei einer derzeitigen Speicherdauer der relevanten Daten von ca. sieben Tagen ${ }^{69}$ läuft dieser Anspruch allerdings in praxi häufig ins Leere, sofern man nicht entgegen der aktuellen Rechtsprechung die Möglichkeit einer Verlängerung der Speicherdauer auf Zuruf (Quick Freeze) annimmt. ${ }^{70}$

ne, in: Wandtke/Bullinger, UrhR, 3. Aufl. 2009, § 101 UrhG Rn. 35; Nägele/Nitsche, WRP 2007, 1047, 1052; s. auch Czychowski/Nordemann, NJW 2008, 3095, 3097 f. mwNachw.

${ }^{62}$ S. dazu das Eckpunktepapier des BMJ, abrufbar unter:

http://www.bmj.de/SharedDocs/Downloads/DE/pdfs/eckpunktepapr_zur_sicherung_vorhandener_verkehrsda ten.pdf?_blob=publicationFile; dazu auch MMR-Aktuell 2011, 319439; ablehnend Möstl, ZRP 2011, 225, 228; befürwortend Graulich, NVwZ 2008, 485, 490; Gola/Klug/Reif, NJW 2007, 2599, 2600; s. auch BVerfG MMR 2010, 356, 360 Rn. 208.

${ }^{63}$ Telekommunikationsgesetz vom 22. Juni 2004 (BGBI. I S. 1190), das zuletzt durch Artikel 1 des Gesetzes vom 3. Mai 2012 (BGBI. I S. 958) geändert worden ist.

${ }^{64}$ BVerfG NJW 2010, 833.

${ }^{65}$ BVerfG NJW 2010, 833, 840 ff.; zusammenf. Schramm/Wegener, MMR 2011, 9, $10 \mathrm{ff}$.

${ }^{66}$ BVerfG NJW 2010, 833, 844 f. Rn. 254 ff., insb. 262.

${ }^{67}$ BVerfG ZUM-RD 2010, 181, 184 Rn. 46 mwNachw.

${ }^{68}$ S. dazu ausf. Wehr/Ujica, MMR 2010, 667, $669 \mathrm{f}$.

${ }^{69}$ Ausf. dazu Wehr/Ujica, MMR 2010, 667, 669 f. m. Nachw. aus der Rspr.

${ }^{70}$ OLG Hamm MMR 2011, 193; s. auch den Überblick zu der aktuellen Rspr. zu § 101 UrhG bei Czychowski/Nordemann, GRUR-RR 2012, 233, 240 f. mwNachw.; s. auch Spindler, in: Spindler/Schuster, Recht der elektronischen Medien, 2. Aufl. 2011, § 101 UrhG Rn. 23; s. aber auch Wehr/Ujica, MMR 2010, 667, 669 f. mit Nachw. zu älterer Rspr., die eine Datenspeicherung auf Zuruf bejahte. 
Doch selbst eine verfassungskonforme Umsetzung der Vorratsdatenspeicherung und entsprechend zulässiger Datenabrufinstrumente für Strafverfolgungsbehörden stellt keineswegs das Allheilmittel hinsichtlich der Rechtsverfolgung dar. Denn die hier in Rede stehenden Urheberrechtsverletzungen sind nicht als "schwere Straftaten“ entsprechend den Ausführungen des BVerfG einzustufen, wodurch der unmittelbare Abruf der Vorratsdaten an dieser verfassungsrechtlichen Hürde scheitert. ${ }^{71}$ Damit verbleibt allenfalls ein mittelbarer Zugriff auf die anlasslos gespeicherten Daten. Ein Auskunftsersuchen bei einem Access-Provider zur Identifizierung eines Teilnehmers anhand der IPAdresse kann dann aber auch nur bedingt von Nutzen sein: Denn inzwischen findet ein beträchtlicher Teil des illegalen Filesharing im Internet nicht mehr nur in sog. P2P-Netzen statt, ${ }^{72}$ bei denen der Dateiaustausch durch direkte Verbindung der einzelnen gleichartigen Teilnehmer (Peers), bei gleichzeitiger Zurverfügungstellung bereits heruntergeladener Dateifragmente an alle anderen Peers, erfolgt. ${ }^{73}$ Vielmehr werden inzwischen - wie bereits erwähnt - urheberrechtlich geschützte Werke häufig über sog. Filehoster verbreitet. ${ }^{74}$ Dabei wird ein Werk in digitaler Form auf dem Server eines Internet-Speicherplatzanbieters (Host-Provider) hochgeladen. Der Link zu der Datei wird dann über unterschiedliche Verbreitungswege anderen Internetnutzern zur Verfügung gestellt. ${ }^{75}$ Der Download der Datei erfolgt hierbei direkt von dem Hoster, eine direkte Verbindung zwischen Peers findet also nicht statt. Dadurch können Strafverfolgungsbehörden und Rechteinhaber auch nicht die direkte Verbindung der Peers nutzen, um an die in den Datenpaketen eingebetteten IP-Adressen der herunterladenden und - vor allem - hochladenden Nutzer zu gelangen. Für einen Zugriff auf die IPAdressen müsste in dieser Konstellation der Umweg über die Logfiles der Filehoster gegangen werden. $^{76}$

34 Nur wenn Provider und Filehoster über längere Zeit die jeweiligen Nutzer und die von Ihnen genutzten Dienste, Webseiten etc. speicherten, könnten also überhaupt effektiv Rechtsverfolgungsmaßnahmen eingeleitet werden. Gleichzeitig können diese Daten aber auch für die Nachverfolgung sämtlicher Aktivitäten verwandt werden, was einen erheblichen Eingriff in die Persönlichkeitsrechte der Nutzer zur Folge hätte. Dementsprechend hat das BVerfG auch hervorgehoben, dass eine anlasslose Datenspeicherung verfassungsrechtlich nur zulässig sei, wenn diese nicht gleichzeitig eine praktische Rekonstruierbarkeit aller Aktivitäten der Bürger zur Folge hätte. ${ }^{77}$ Daher nimmt das Gericht aus-

\footnotetext{
${ }^{71}$ Krit. daher zu den vom BVerfG herausgearbeiteten Voraussetzungen für ein Auskunftsersuchen Czychowski/Nordemann, NJW 2008, 3095, $3097 \mathrm{f}$.

${ }^{72}$ S. dazu die Studie von Sandvine - Fall 2010 Global Internet Phenomena Report, S. 33 f.

${ }^{73}$ Schwartmann, K\&R Beihefter 2/2011, 1, 9; Solmecke, K\&R 2007, 138, 138 ff.; Brinkel, Filesharing, 2006, S. 14 ff.

${ }^{74}$ Bekanntestes Beispiel ist wohl der Anbieter Rapidshare. Inzwischen existiert aber eine Vielzahl gleichartiger Anbieter. Das Geschäftsmodell sieht dabei vor, dass zwar jeder Nutzer grds. auf die hochgeladenen Dateien zugreifen kann. Allerdings werden dabei die Bandbreite und das Datenkontingent für nicht zahlende Nutzer stark beschränkt. Die zahlenden „Premiumnutzer“ können dann je nach Zahlungshöhe von einem Tag bis zu mehreren Monaten hohe Datenmengen von den Servern der Anbieter herunterladen. Lädt ein Nutzer Dateien auf die Server hoch, erhält er zudem Bonuspunkte für jeden Download der Dateien. Mit diesen Bonuspunkten lässt sich dann etwa ab einer gewissen Anzahl der sog. Premiumzugang verlängern. Interessant an diesem Geschäftsmodell ist, dass wohl anscheinend durchaus die Bereitschaft zumindest bei einem Teil der Filesharer existiert, für den Konsum der Werke ein Entgelt zu entrichten. So kosten etwa 30 Tage Premiumzugang bei Rapidshare 9,90 Euro (https://rapidshare.com/\#!buyrapids); s. zu Filehostern: Röhl/Bosch, NJOZ 2008, 1197, 1199 f.; Härting, Internetrecht, 4. Aufl. 2010, Kap. H., IV., Rn. 1775.

${ }^{75}$ Schwartmann, K\&R Beihefter 2/2011, 1, 12.

${ }^{76}$ Wobei auch hier für die Internetnutzer die Möglichkeit besteht, ihre vom Access-Provider zugewiesene IPAdresse mittels Anonymisierungstools wie etwa eines TOR-Netzwerkes zu verschleiern, s. dazu etwa https://www.torproject.org.

${ }^{77}$ BVerfG ZUM-RD 2010, 181, 202 f. Rn. 218.
} 
drücklich die Kommunikationsinhalte und die von den Kunden aufgerufenen Internetseiten durch kommerzielle Diensteanbieter generell von einer Vorratsdatenspeicherung aus. ${ }^{78}$ Hinzu kommt, dass Filehoster in aller Regel ihr Angebot und ihre Rechenzentren außerhalb der Jurisdiktion der Bundesrepublik Deutschland betreiben, womit selbst eine verfassungskonforme nationale Datenspeicherungsverpflichtung ins Leere laufen würde.

\section{b) Erweiterte Haftung und schwer zu kalkulierende Risiken}

Neben diesen Risiken hinsichtlich der Wahrung des Datenschutzes ergeben sich aus der im Ansatz weit gefassten Rechtsprechung zur Störerhaftung aber weitere Probleme, die bereits vereinzelt in der Praxis aufgetreten sind:

\section{(1) Anschlussinhaber (WLAN etc.)}

Dass ein Anschlussinhaber für selbst begangene Rechtsverletzungen zu haften hat, ist selbstverständlich. Ist ein Anschlussinhaber jedoch nicht Täter oder Teilnehmer einer rechtsverletzenden Handlung, ergibt sich ein weniger eindeutiges Bild. Grundsätzlich kann auch derjenige als sogenannter Störer auf Unterlassung in Anspruch genommen werden, der in irgendeiner Weise willentlich und adäquat kausal zur Verletzung eines Rechtsgutes beiträgt. ${ }^{79}$ Aufgrund dieser weiten Definition des Störerbegriffs verwundert es nicht, dass die Rechtsprechung auch den Anschlussinhaber selbst hinsichtlich der in die Zukunft gerichteten Unterlassungsansprüche für jedenfalls im Ansatz verpflichtet gehalten hat, rechtswidrige Handlungen über seinen Anschluss zu unterbinden. So muss ein WLAN-Inhaber für eine marktübliche Verschlüsselung seines WLANs sorgen, damit nicht Dritte über sein Netz entsprechende unberechtigte Verwertungsvorgänge durchführen können. ${ }^{80}$ Für gesicherte WLANs ist allerdings noch nicht erkennbar, ob der Anschlussinhaber auch für dritte Teilnehmer seines Netzes grundsätzlich haften soll; Ansätze lassen sich allerdings vereinzelten Entscheidungen zur Haftung für Minderjährige und andere Familienangehörige entnehmen:

\section{(2) Haftung für Familienangehörige}

37 Zunächst müssen Eltern grundsätzlich nach § 832 BGB für das Verhalten ihrer minderjährigen Kinder auch im Hinblick auf Filesharing etc. einstehen, sofern eine Verletzung der Aufsichtspflicht bejaht werden kann. ${ }^{81}$ Was jedoch die Haftung als Störer angeht, ist entscheidend, ob eine Verletzung von Prüfpflichten seitens des Anschlussinhabers vorliegt, was der BGH wiederum davon abhängig macht, inwieweit solche Pflichten einem Anschlussinhaber zuzumuten sind. ${ }^{82}$ Bei der konkreten Festlegung dieser Pflichten divergiert die Rechtsprechung jedoch, ${ }^{83}$ so dass die Rechtslage sich hier keineswegs klar feststellen lässt. Problematisch erscheint vor allem die Zumutbarkeit von Prüfungspflichten der Eltern, die oftmals nicht über die gleichen technischen Kenntnisse wie ihre Kinder verfügen, zudem nur im begrenzten Umfang bestimmte Webseiten sperren können. Das OLG Frankfurt a.M. hat dazu vertreten, dass der Grundsatz, dass der Anschlussinhaber die Nutzung des Anschlusses durch den Ehepartner nicht zu überwachen habe, solange keine konkreten Anhaltspunkte für eine Rechtsverlet-

\footnotetext{
${ }^{78}$ BVerfG ZUM-RD 2010, 181, 202 f. Rn. 218.

${ }^{79}$ S. zum Störerbegriff BGH NJW 2010, 2061, 2062 - Sommer unseres Lebens m. zahlr. Nachw. aus der Rspr.

${ }^{80}$ BGH NJW 2010, 2061; allerdings verkennt der BGH hier, dass der WLAN-Inhaber letztlich wie ein AccessProvider zu behandeln ist, s. dazu Spindler, CR 2010, 592, 595 ff.; s. auch die kritische Auseinandersetzung mit dieser Entscheidung des BGH bei Mantz, MMR 2010, 565, 568 ff. mwNachw. aus der Rspr.; s. auch bereits zur Störerhaftung bei einem ungesicherten WLAN-Netz LG Hamburg MMR 2006, 764 m. Anm. Mantz.

${ }^{81}$ LG Düsseldorf ZUM-RD 2011, 698, 699; OLG Köln MMR 2012, 387, 389 f.

82 BGH NJW 2010, 2061, 2062 - Sommer unseres Lebens, m. zahlr. Nachw. aus der Rspr.

${ }^{83}$ Ausf. zum Thema Kirchberg, ZUM 2012, 544, 546 ff.; Peter, K\&R 2007, 371 ff.; ferner Leistner/Stang, WRP $2008,533,548 \mathrm{ff}$.
} 
zung bestehen, auch im Verhältnis zu den Kindern des Anschlussinhabers gelte. ${ }^{84}$ Insgesamt zeichnet sich eine Linie in der Rechtsprechung ab, die unterschiedliche Pflichten des Anschlussinhabers annimmt, je nachdem, ob es sich bei den Kindern noch um Minderjährige oder bereits um Volljährige handelt.

Auch können sich durchaus Grauzonen hinsichtlich der urheberrechtlichen Qualifizierung ergeben, etwa bei Downloads von Musikvideos über YouTube oder das Mitschneiden von gestreamten Inhalten.

Ein weiterer Sonderfall ergibt sich zudem, wenn zum Haushalt des Anschlussinhabers nicht nur (minderjährige) Kinder, sondern auch ein Ehepartner gehört. Das LG Köln hat jüngst dazu ausgeführt, dass eine Störerhaftung eines Elternteils wegen Verletzung der sog. Prüfpflichten gegenüber den minderjährigen Kindern ausscheide, wenn der andere Ehepartner im selben Haushalt wohne. Zur Begründung verwies das LG Köln darauf, dass dem Ehepartner gegenüber keine vergleichbaren Prüfpflichten gälten. ${ }^{85}$ Da somit nicht auszuschließen sei, dass die Urheberrechtsverletzung von dem Ehepartner begangen wurden, ohne dass den Anschlussinhaber eine Verantwortung dafür träfe, könne auch nicht ohne weiteren Kausalitätsnachweis eine Störerhaftung aus der Verletzung der in Bezug auf die Kinder bestehenden Prüfpflicht angenommen werden. ${ }^{86}$

(3) Organisationen (Arbeitgeber, Schulen, Universitäten etc.)

Anhand dieser Beispiele wird bereits deutlich, dass grundsätzlich jede Organisation, die den Zugang zum Internet ermöglicht, unter Umständen im Rahmen der Störerhaftung gehalten sein kann, die Aktivitäten ihrer Teilnehmer zu kontrollieren, sofern sich überhaupt nur eine Aufsichtspflicht ergibt. Bislang ist wenig geklärt, wie die grundsätzliche Privilegierung der Access-Provider sich zur Aufsichtspflicht derartiger Organisationen verhält - denn im Prinzip ist jedes Zugänglichmachen eines Netzes ein Sachverhalt, der der Privilegierung nach § 8 TMG unterfällt, was letztlich auch zu einer nur äußerst eingeschränkten Störerhaftung führt. ${ }^{87}$ So lassen sich über die Störerhaftung keine Schadensersatzansprüche geltend machen, sondern allein Unterlassungsansprüche. ${ }^{88}$ Andererseits ist weitgehend anerkannt, dass Organisationen grundsätzlich im Rahmen ihrer Möglichkeiten und ihrer Überwachungspflichten auch für rechtswidrige Handlungen ihrer Angestellten/Angehörigen haften können. ${ }^{89}$ Der Arbeitgeber kann sich von diesen Pflichten auch nicht durch den arbeitsvertraglichen Ausschluss der privaten Nutzung des Internet über den Arbeitsrechner freizeichnen, da ihm dies die Möglichkeit eröffnen würde, entgegen den Grundsätzen der Störerhaftung seinen eigenen Haftungsbereich zu definieren. ${ }^{90}$ Maßgeblich ist daher auch hier die Frage nach der Zumutbarkeit von Beaufsichtigungs- und Instruktionspflichten. Angesichts der Tatsache, dass die Internetnutzung am Ar-

\footnotetext{
${ }^{84}$ OLG Frankfurt a.M. MMR 2008, 169, 170.

${ }^{85}$ LG Köln, Urt. v. 11.09.2012 - 33 O 353/11, Rn. 45, 52 unter Verweis auf OLG Köln MMR 2011, 396, 397.

${ }^{86}$ LG Köln, Urt. v. 11.09.2012 - 330 353/11, Rn. 53 f.

${ }^{87}$ Spindler, CR 2010, 592, 595; im Übrigen ist ein Vertragsverhältnis zwischen dem Zugangsvermittler und dem Sender oder Empfänger der übermittelten Daten nicht erforderlich, so dass es allein auf die technische Zugangsvermittlung ankommt, s. Hoffmann, in: Spindler/Schuster, Recht der elektronischen Medien, 2. Aufl. 2011, § 8 TMG Rn. 18.

${ }^{88}$ BGH GRUR 2008, 702, 705 Rn. 38 - Internetversteigerung III; GRUR 2008, 708, 710 Rn. 17; GRUR 2004, 860, 862 f.; s. Spindler, in: Spindler/Schuster, Recht der elektronischen Medien, 2. Aufl. 2011, § 97 UrhG Rn. 33 mwNachw.

${ }^{89}$ LG München I MMR 2008, 422, 423, dort allerdings unter Verneinung der Haftung, wenn keine Überwachungspflicht ohne konkrete Anhaltspunkte für eine Rechtsverletzung beständen; Leistner/Stang, WRP 2008, $533,551$.

${ }^{90}$ So zu Recht Leistner/Stang, WRP 2008, 533, 553.
} 
beitsplatz häufig bereits für die Arbeit selbst erforderlich ist, erscheint es unzumutbar, entsprechende Pflichten ohne konkrete Anhaltspunkte für Rechtsverletzungen anzunehmen, zumindest solange eine eindeutige Dienstanweisung besteht, die die urheberrechtswidrige Nutzung untersagt. ${ }^{91}$ Weitergehende Pflichten könnten zudem in Widerspruch zu arbeitnehmerdatenschutzrechtlichen Vorgaben stehen. ${ }^{92}$

\section{c) Kriminalisierung und Rechtsbewusstsein}

41 Aber nicht nur zivilrechtliche Rechtsverfolgungen stehen im Fokus: Vielmehr haben sich die Rechteinhaber gerade vor der Einführung von Auskunftsersuchen oftmals der Strafbewehrung einer vorsätzlichen Urheberrechtsverletzung mit der Anzeigeerstattung und der Einleitung von Ermittlungsmaßnahmen beholfen, da über die Akteneinsicht der Strafverfolgungsbehörden die Identität der Beteiligten ermittelbar war - was allerdings auch zu deutlichen Überlastungen der Ermittlungsbehörden führte, so dass teilweise Anweisungen ergingen, bestimmte Straftaten nicht mehr zu verfolgen. ${ }^{93}$ Bekannt wurden etwa Fälle, in denen beispielsweise40.000 Strafanzeigen durch ein Unternehmen innerhalb eines halben Jahres erstattet wurden. ${ }^{94}$

Diesen teilweise intensiven Strafverfolgungsmaßnahmen steht offenbar eine verbreitete Auffassung in der Bevölkerung gegenüber, die Urheberrechtsverletzungen zwar als illegal begreift, aber sie doch als eine Art "Kavaliersdelikt" ansieht - wobei die wohl früher verbreiteten Meinung, dass die entsprechenden Handlungen legal seien, inzwischen durch die entsprechenden Kampagnen der Rechteinhaber einem Rechtsbewusstsein gewichen ist, das die Rechtswidrigkeit laienhaft richtig einschätzen kann. So sind gerade einmal 6\% der Deutschen noch der Ansicht, dass die Weitergabe eines kopierten E-Books rechtlich erlaubt sei, beim Anbieten von Medieninhalten über Sharehoster und P2PNetzwerke sind es sogar nur noch $2 \% .{ }^{95}$ Zwischen diesen Zahlen und dem nach wie vor verbreiteten Filesharing besteht eine offensichtliche Diskrepanz. Allerdings fehlen hier eingehende und unabhängige kriminologische Untersuchungen noch weitgehend bzw. befinden sich erst in den Kinderschuhen. ${ }^{96}$ Auffällig ist zumindest, dass bei einer Befragung Jugendlicher durch den Börsenverein des Deutschen Buchhandels e.V. aus dem Jahr 2010 zwar 86,8\% angaben, von der Illegalität von Filesharing zu wissen, aber nur 55,3\% Filesharing auch als "falsch“ empfanden. ${ }^{97}$ Angesichts des trotz teilweise massiver Rechtsverfolgungen offenbar anhaltenden Phänomens stellt sich naturgemäß die Frage, ob eine Kriminalisierung im Sinne einer ultima ratio des Strafrechts nach wie vor angemessen ist oder nicht vielmehr eine Reduktion auf zivilrechtliche Ansprüche oder eben eine generelle Abgabe sinnvoller ist. Allerdings wird auch auf die jüngsten Ergebnisse in Frankreich anhand des „Loi HA-

\footnotetext{
${ }^{91}$ Leistner/Stang, WRP 2008, 533, 553; s. auch Mengel, in: Mengel, Compliance und Arbeitsrecht, 2009, Kap. 6 Rn. 64 f. mwNachw.

${ }^{92}$ Mengel, in: Mengel, Compliance und Arbeitsrecht, 2009, Kap. 6 Rn. 64.

${ }^{93}$ S. http://www.heise.de/newsticker/meldung/67918; die Generalstaatsanwaltschaften Celle und Berlin sahen kein öffentliches Interesse an einer Verfolgung und lehnten die Auskunftsansprüche daher ab, $\mathrm{s}$. http://www.heise.de/newsticker/meldung/93693; s. entsprechend den Beschl. des AG Offenburg MMR 2007, 809, m. Anm. Bär, wonach ein Auskunftsanspruch aufgrund von „Bagatellkriminalität“ unverhältnismäßig und daher abzulehnen sei.

${ }^{94}$ S. http://www.heise.de/newsticker/meldung/68882.

${ }^{95}$ GfK, DCN-Studie 2012, Vollversion, S. $37 \mathrm{f}$.

${ }^{96}$ S. bspw. Kerner/Bott/Reich, in: FS Schwind, S. 963, $982 \mathrm{ff}$

97 Börsenverein des Deutschen Buchhandels e.V., Illegal aber egal?, 2010, S. 10; siehe hierzu aber auch The American Assembly, Copy Culture in the US and Germany, 2013, S. 39, die zwar nicht Jugendliche sondern 18 29-jährige erfasst, aber dort nur zu einer weitgehenden Akzeptanz hinsichtlich des Teilens im Familien- und Freundeskreis kommt.
} 
DOPI“ zurückzukommen sein, das ein abgestuftes Warn- und Unterbindungssystem gegenüber hartnäckigen rechtsverletzenden Internetteilnehmern vorsieht. ${ }^{98}$

\section{Bisherige Marktreaktionen}

Da die Rechtsverfolgungsmaßnahmen zwar gewisse Erfolge erzielen konnten, ihr Gesamteffekt aber fraglich bleibt, ist es nicht verwunderlich, dass die Rechteinhaber auch andere Instrumente einzusetzen versuchten. Daneben sind Marktreaktionen bzw. neue Geschäftsmodelle von Interesse, die auch im Rahmen der Gesamtabwägung eine Rolle spielen können:

\section{a) DRM-Systeme}

Eine der ersten Reaktionen der Musikindustrie auf den zunehmenden Tausch von Musikstücken bestand in der Einführung bzw. Verwendung von Digital Rights Management (DRM) Systemen. Aus Sicht der Rechteinhaber sollten diese DRM-Systeme dazu dienen, ihre geschützten Inhalte, die im Zuge der Digitalisierung den Charakter öffentlicher Güter angenommen hatten, wieder kontrollierbar zu machen, um so die wirtschaftlichen Anreize zu erhalten, weiterhin Inhalte zu produzieren. Als „öffentliche Güter" werden in der Wirtschaftstheorie solche Güter bezeichnet, die durch sog. Nichtrivalität und Nichtausschließbarkeit gekennzeichnet sind. Während Nichtrivalität meint, dass ein Gut nicht knapp ist, sondern von einer Vielzahl von Personen genutzt werden kann, ohne die Nutzung durch andere Personen zu beeinträchtigen, wird unter Nichtausschließbarkeit die fehlende Möglichkeit verstanden, andere von der Nutzung des Gutes auszuschließen..$^{99}$ Durch die Digitalisierung einerseits und den Ausbau der Möglichkeiten der Verbreitung digitaler Inhalte andererseits haben die Inhalte der Kreativindustrie inzwischen den Charakter öffentlicher Güter angenommen, weshalb DRM-Systeme zumindest die Nichtausschließbarkeit beseitigen sollten, indem sie die rechtekonforme Nutzung erzwingen. ${ }^{100}$ Genau betrachtet, würden eigentlich bereits die an den jeweiligen Werken bestehenden Urheber- und Leistungsschutzrechte diese Funktion einnehmen. ${ }^{101}$ Doch in dem Moment, in dem die herkömmliche Rechtsdurchsetzung versagt, bedarf es anderer Instrumente. Was (wie eingangs beschrieben ${ }^{102}$ ) in den 50er-Jahren vor der Einführung der Geräteabgabe aus praktischen und rechtlichen Gründen noch nicht möglich war - eine automatisierte Kontrolle der Nutzungsberechtigung -, ließe sich mithilfe von DRM-Systemen auf technischem Wege grundsätzlich durchführen. ${ }^{103}$

\footnotetext{
${ }^{98}$ S. unten Rn. 96 ff.; s. ferner GfK, DCN-Studie 2012, Vollversion, S. 46 f., wonach 72\% der Bevölkerung Warnhinweise vor rechtlichen Sanktionen für sinnvoll und 57\% für potentiell wirksam halten; beachte aber: The American Assembly, Copy Culture in the US and Germany, 2013, S. 42, nach der unter den 59\% der Befragten die Strafen befürworten eine Sperrung des Internetzugangs nur zwischen 24\% (18-29 Jahre) und 44\% (70+ Jahre) befürworten.

${ }^{99}$ Stieper, Rechtfertigung, Rechtsnatur und Disponibilität der Schranken des Urheberrechts, 2009, S. 75 f.; Mestmäcker/Schweitzer, Europäisches Wettbewerbsrecht, 2. Aufl. 2004, § 28 Rn. 2 jeweils mwNachw; Ünlü, Content Protection, 2005, S. 36.

${ }^{100}$ Fitzner, Von Digital-Rights-Management zu Content Identification, 2011, S. 62 f.

${ }^{101}$ Ausf. Stieper, Rechtfertigung, Rechtsnatur und Disponibilität der Schranken des Urheberrechts, 2009, S. 78

ff.; Fitzner, Von Digital-Rights-Management zu Content Identification, 2011, S. 63 f.; Ünlü, Content Protection, 2005, S. $42 \mathrm{ff}$

102 S. oben Rn. $13 \mathrm{ff}$.

${ }^{103}$ Daraus wurden bereits gegensätzliche Schlussfolgerungen für das Urheberrecht gezogen: Auf der einen Seite wurde argumentiert, dass DRM-Systeme urheberrechtliche Schranken entbehrlich machten, da sie eine automatisierte, individuelle Lizenzierung ermöglichen, s. Bell, N. Carolina L. Rev., Vol. 76, 1997-1998, S. 557, 583, sowie Merges, BTLJ, Vol. 12, 1997, 115, 130, auf der anderen Seite wurde aus denselben Gründen vertreten, dass DRM-Systeme die Nichtexklusivität der entsprechend geschützten Werke vollständig beseitigten und somit bereits das ausschließliche Verwertungsrecht des Urhebers entbehrlich sei, s. Dowell, Cal. L. Rev., Vol. 86,
} 
DRM-Systeme fungieren, vereinfacht formuliert, als Kopierschutz für digitale Werke, lassen gleichzeitig aber auch die Kontrolle über die Nutzung der Musikstücke bis hin zur Zahlungsabwicklung zu. ${ }^{104}$ Möglich ist damit auch darüber hinausgehend die Beschränkung der Nutzung eines Werkstückes in der vom Rechteinhaber festgelegten Form; ${ }^{105}$ also etwa der Anzahl von Kopien, der Nutzungsdauer, der Anzahl der Nutzungsvorgänge oder auch von deren Koppelung mit bestimmten Abspielgeräten. ${ }^{106}$ DRM-Systeme sind dabei keineswegs auf den Musiksektor oder den Vertrieb über das Internet beschränkt, ${ }^{107}$ sondern finden in extenso sowohl im Online- als auch Offline-Bereich Anwendung bei Computerspielen, ${ }^{108}$ Filmen ${ }^{109}$ bis hin zu E-Books ${ }^{110}$ oder auch Software. ${ }^{111}$

Anders als in anderen Branchen wandte sich die Musikindustrie indes seit 2007 von dem Einsatz von DRM-Systemen $a b^{112}$ - wohl aus verschiedenen Gründen: Zum einen weil auch DRM-Systeme trotz hohen Kostenaufwands umgehbar sind ${ }^{113}$ und die entsprechenden Musikfiles sich dann, um die DRMSysteme "bereinigt", wiederum in den jeweiligen Tauschbörsen befanden, zum anderen weil die Kompatibilität von etlichen, insbesondere älteren Abspielgeräten mit den eingesetzten DRMSystemen nicht gewährleistet war. ${ }^{114}$ Auch können die DRM-geschützten Files abgespielt und als analoges Signal direkt an der Soundkarte „abgegriffen“ werden, so dass fast kaum ein Qualitätsverlust eintritt und eine (bislang) legale Privatkopie hergestellt werden kann. ${ }^{115}$ Insgesamt konnte der Einsatz von DRM-Systemen die Menge der über P2P-Netzwerke getauschten Musikfiles offenbar nicht redu-

1998, S. 843, 854, Fn. 45; dass aber beides nicht der Fall ist, hat Stieper, Rechtfertigung, Rechtsnatur und Disponibilität der Schranken des Urheberrechts, 2009, S. 92 ff. mwNachw, überzeugend dargelegt.

${ }^{104}$ S. Enders, ZUM 2004, 593, der DRM-Systeme wie folgt beschreibt: „Inhaltlich geht es um Techniken und Methoden zum Schutz von urheberrechtlich geschützten Werken und sonstigen Leistungsschutzrechten für digitale Dokumente“; s. auch die begriffliche Definition bei Pfennig, ZUM 2004, 198, wonach es sich bei DRMSystemen um „Systeme zur elektronischen Lieferung, Verwaltung und automatisierten Abrechnung von geschützten Werken in digitaler Form aus dem Server des Rechteinhabers Urhebers in den Server des Empfängers" handelt; s. ferner Schulz, GRUR 2006, 470 f.; Schippan, ZUM 2004, 188, 189 f.; eingehend und mwNachw auch zu den technischen Hintergründen von DRM-Systemen Fitzner, Von Digital-Rights-Management zu Content Identification, 2011, S. 51 ff., die überdies zwischen DRM-Systemen „der ersten und zweiten Generation“ unterscheidet: Die erste Generation richtete sich demnach in erster Linie auf die Verhinderung von Vervielfältigungen, während erst die zweite Generation über das beschriebene erweiterte Funktionsspektrum verfügte. ${ }^{105}$ Dreier, in: Dreier/Schulze, UrhG, 4. Aufl. 2013, § 95a UrhG Rn. 2; Schulz, GRUR 2006, 470, 471; Enders, ZUM 2004, 593; Pfennig, ZUM 2004, 198.

${ }^{106}$ Schippan, ZUM 2004, 188, 190; Dreier, in: Dreier/Schulze, UrhG, 4. Aufl. 2013, § 95a UrhG Rn. 2.

${ }^{107}$ Enders, ZUM 2004, 593.

${ }^{108}$ S. etwa Grützmacher, in: Wandtke/Bullinger, UrhR, 3. Aufl. 2009, § 69f UrhG Rn. 14, der auf einen Schutz von Software mittels ROMs, EPROMs und USB-Dongles hinweist.

${ }^{109}$ S. etwa zu Schutzmechanismen von Filmen auf einem DVD-Datenträger: Schulz, GRUR 2006, 470, 473, die etwa "Content Scrambling Systeme“ (CSS) oder „Regional Playback Control-Verfahren“ nennt.

${ }^{110}$ S. zur Funktionalität von DRM-System bei E-Books Guggemos, ZUM 2004, 183, wonach etwa mittels DRM „(...) die Anzahl der erlaubten Ausdrucke, der zulässigen Textentnahmen und die Möglichkeit zum Einfügen von Kommentierungen, aber auch die zulässige Nutzungsdauer des Textes" festgelegt werden kann, was mittels spezieller Software-Player oder -Reader zur ausschließlichen Nutzung der digitalen Inhalte realisiert wird.

${ }^{111}$ Hier wird in der Regel mit Internet-Produktaktivierungscodes gearbeitet, um die Funktionen einer Software freizuschalten, s. dazu etwa die Produktaktivierung von Microsoft Windows 7, http://windows.microsoft.com/de-DE/windows7/activate-windows-7-on-this-computer.

112 Näher Grassmuck, 2010, S. 31 f.; Fitzner, Von Digital-Rights-Management zu Content Identification, 2011 , S. $80 \mathrm{ff}$. , je mwNachw.

${ }^{113}$ S. zur Umgehbarkeit von DRM zusammenf. Stickelbrock, GRUR 2004, 736, 739; s. zur Umgehbarkeit bei Computerprogrammen Grützmacher, in: Wandtke/Bullinger, UrhR, 3. Aufl. 2009, § 69f UrhG Rn. 15.

${ }^{114}$ Fitzner, Von Digital-Rights-Management zu Content Identification, 2011, S. 86 mwNachw.

${ }^{115}$ Teilweise auch automatisiert wie im Fall der Software „Tunebite“, s. dazu

http://audials.com/de/tunebite/index.html. 
zieren. ${ }^{116}$ Hinzu kamen IT-Sicherheits- und Datenschutzskandale, etwa durch eingesetzte Spysoftware bei Sony/BMG, die sich beim Einlegen einer Musik-CD in einen PC selbst installierte und für den Nutzer unbemerkt Veränderungen am System vornahm. Diese führten unter anderem dazu, dass die Nutzer auch andere CDs nur noch mit dieser Software konsumierten, Dritte neu geschaffene Sicherheitslücken ausnutzten sowie Informationen über gehörte Musik mit einem Server abglichen und dabei zwangsläufig personenbezogene Daten übermittelt werden konnten. ${ }^{117}$ Die Akzeptanz von DRM-Systemen durch die Kunden war daher offensichtlich nicht gegeben; ${ }^{118}$ selbst wichtige Download-Angebote, wie z.B. von Amazon ${ }^{119}$, Musicload der Deutschen Telekom AG $^{120}$ und mittlerweile auch iTunes von Apple ${ }^{121}$ bieten heute digitale Musikwerke ohne DRM-Systeme an. ${ }^{122}$

\section{b) Entwicklung von vertikal integrierten Systemen (Apple/iTunes)}

47 Eine der außerhalb der eigentlichen Rechteinhaber bzw. der Verwerter angesiedelten Marktreaktionen lässt sich an dem Erfolg von iTunes bzw. der Strategie von Apple ablesen. Zum einen zeichnet sich der Erfolg von iTunes dadurch aus, dass die mit der Digitalisierung und dem Internetvertrieb mögliche „Unbundling"-Strategie konsequent umgesetzt wurde, indem der Nutzer nicht mehr gezwungen war, ein ganzes Album oder eine im Verhältnis überteuerte Single im Musikbereich zu erwerben, sondern einzelne Songs kaufen konnte. Zum anderen bestand Apple auf einer Art Einheitspreis von 0,99 Euro, die eine starke Standardisierung ermöglichte. Der eigentliche Geschäftserfolg scheint aber - mangels empirisch verlässlicher Zahlen allerdings mehr oder minder spekulativ - auf dem Vertrieb der dazugehörigen Hardware im Rahmen eines gesamten Systems zu beruhen, ob iPod, iPhone oder iPad etc., ${ }^{123}$ was gewisse Parallelen zum Aufkommen des Radios und dessen mehr hardwarebasierten Geschäftserfolgen zeigt. Wie dargelegt, basierte diese vertikale Integration auf DRMgestützten Systemen, die nur beschränkt die Vervielfältigung zuließen; offensichtlich hat aber die Aufgabe des DRM-Schutzes dem Erfolg von iTunes und der vertikalen Integration nicht geschadet, wobei allerdings ausdrücklich darauf hinzuweisen ist, dass mangels entsprechender Zahlen kaum Aussagen darüber getroffen werden können, ob der Downloadservice für sich genommen rentabel ist oder nur in Verbindung mit der vertriebenen Hardware.

\footnotetext{
${ }^{116}$ Martin, Loy. L.A. Ent. L. Rev., Vol. 28, No. 3, 2007-2008, 265, 289; Grassmuck, ZUM 2005, 104, 109.

${ }^{117}$ S. dazu ausf. Hansen, DuD 2006, 95, unter anderem auf S. 97 unter Hinweis darauf, dass die Virenschutzsoftware von Microsoft diese Software als Spyware klassifizierte.

${ }^{118}$ Ausf. zu den Akzeptanzproblemen der Nutzer Fitzner, Von Digital-Rights-Management zu Content Identification, 2011, S. 86 ff. m. zahlr. wNachw.

${ }^{119}$ http://www.amazon.de/gp/help/customer/display.html?nodeld=200319960\#drm; Bundesverband Musikindustrie, Musikindustrie in Zahlen 2009, S. 61.

${ }^{120}$ http://www.musicload.de/hilfe/musik/dateiformatewmaundmp3.ml.

${ }^{121}$ http://support.apple.com/kb/HT1711.

122 IFPI, Digital Music Report 2010, S. 8; Fitzner, Von Digital-Rights-Management zu Content Identification, 2011, S. 82 f.

${ }^{123}$ Die Einnahmen aus den Verkäufen über den iTunes Store werden von Apple im Quartalsbericht Q4/2012 zusammen mit den Einnahmen aus dem Apple App Store, dem iBookstore und anderen Quellen aufgeführt, so dass eine exakte Auskunft über die iTunes-Erträge nicht möglich ist. Doch stehen den Einnahmen in Höhe von 2,296 Mrd. Dollar aus der soeben genannten Sparte immerhin Einnahmen in Höhe von 25,455 Mrd. Dollar aus den Bereichen „iPod“, „iPhone and Related Products and Services“ sowie „iPad and Related Products and Services" gegenüber, s. http://images.apple.com/pr/pdf/q4fy12datasum.pdf. Der Anteil pro Sparte am Gesamtgewinn von 8,2 Mrd. Dollar ist der Aufstellung nicht zu entnehmen, doch wenn man konzerneigenen Aussagen aus dem Jahr 2010 Glauben schenken darf, sind die digitalen Vertriebswege iTunes und App Store nur minimal rentabel, s. http://allthingsd.com/20100225/apple-billions-of-songs-billions-of-apps-not-much-profit/; s. ferner: http://www.asymco.com/2011/06/13/itunes-now-costs-1-3-billionyr-to-run/ sowie http://www.billboard.biz/bbbiz/content_display/magazine/upfront/e3ia99a189e2d22c6c7e6b9697d33f76984.
} 


\section{c) Entwicklung von Musik-Abo-Diensten (Spotify etc.)}

Eine andere Marktreaktion scheint angesichts des zunehmenden Ausbaus von Breitbanddiensten und besseren Übertragungskapazitäten im Netz in der Entwicklung von Streaming-Angeboten zu bestehen, die für eine pauschale Gebühr dem Kunden den temporären Zugriff auf ein breites Musikangebot erlauben: Mit Diensteanbietern wie etwa Spotify ${ }^{124}$ und Simfy ${ }^{125}$ sind Plattformen entstanden, die ihren Kunden neue Gratis- sowie Premium-Modelle für die legale Nutzung von digitalen Musikwerken anbieten. Grundsätzlich handelt es sich dabei um Dienste, die das Streamen von Musik auf PC, Mac oder Smartphone über spezielle Abspielsoftware ermöglichen; allerdings ist für zahlende „Premium-Nutzer" auch der Musikgenuss offline möglich, jedoch DRM-geschützt, so dass nach Beendigung des Abonnements die Stücke nicht mehr gehört werden können. ${ }^{126}$ Neben dieser Einschränkung kommt es in den Gratisversionen der Angebote zu Werbeeinblendungen und Limitierungen etwa hinsichtlich der Qualität, der Gesamtnutzungsdauer oder der Abspielhäufigkeit einzelner Musikstücke. ${ }^{127}$ Die Anzahl der „Premium-Nutzer“ bei diesen Angeboten wächst bislang stetig an. ${ }^{128}$ Die Zukunft dieser Modelle wird allerdings ambivalent beurteilt, da die zahlreichen Web-RadioAngebote, die auch eine Personalisierung erlauben, eine ernsthafte und unentgeltliche Konkurrenz zu den Streaming-Angeboten darstellen. ${ }^{129}$

In gleicher Weise etablieren sich offenbar im Filmbereich temporäre Nutzungsangebote wie Lovefilm von Amazon. ${ }^{130}$

\footnotetext{
${ }^{124}$ www.spotify.com.

${ }^{125}$ www.simfy.de.

${ }^{126} \mathrm{http}: / /$ www.spotify.com/de/get-spotify/premium/.

${ }^{127} \mathrm{~S}$. dazu etwa den Überblick der unterschiedlichen Angebote von Spotity, http://www.spotify.com/de/getspotify/premium/, sowie die Nutzungsbedingungen von Spotify: „Der Spotify-Service steht in folgenden Versionen zur Verfügung: als eine durch Werbung finanzierte, für den Nutzer kostenfreie Serviceleistung, bei der es während der ersten 6 Monate nach Einrichtung Ihres Spotify-Kontos keine monatliche Obergrenze hinsichtlich der Spieldauer oder der Anzahl der Abspielungen eines bestimmten Titels gibt und die Spieldauer danach auf maximal 10 Stunden monatlich und maximal 5 Abspielungen pro einzelnem Titel begrenzt ist (der „Free Service"), als eine werbefreie Version des Spotify-Services, für die Sie ein monatliches oder jährliches Abonnement bezahlen (der "Unlimited Service”); als werbefreie Version des Spotify-Services einschließlich eines OfflineModus, der das Anhören von Musik ohne bestehende Internetverbindung mittels bedingter Downloads ermöglicht sowie weiterer zusätzlicher Funktionen, für die Sie ein monatliches oder jährliches Abonnement bezahlen (der "Premium Service”) und als Version, die den Zugriff auf die Funktionen des Spotify-Services mittels eines getragenen, mobilen Handsets erlaubt (der "mobile Service”)", http://www.spotify.com/de/legal/end-useragreement/.

${ }^{128}$ IFPI, Digital Music Report 2012, S. 8, 10 f.; s. auch den BLM-Webradiomonitor 2012, S. 51 ff.

${ }^{129}$ So ist allein die Zahl der in Deutschland verfügbaren personalisierten Webradio-Angebote zwischen 2011 und 2012 von 9 auf 16 gestiegen, s. den BLM-Webradiomonitor 2011, S. 7, und 2012, S. 7; zurückhaltend noch die Prognose der Zukunft personalisierter Webradio-Angebote bei ARD-Forschungsdienst, Media Perspektiven 2011, 617; eingehend zu Web-Radios Prill, Webradio-Streamripping: Eine neue Form der Musikpiraterie?, erscheint demnächst, S. 15 ff.; instruktiv ferner Malcher, Personalisierte Webradios - Sendung oder Abruf, 2011, S. $13 \mathrm{ff}$.

${ }^{130}$ Dazu www.lovefilm.de/.
} 



\section{Das Grundmodell einer Kulturflatrate: \\ Erweiterte Privatkopieschranke mit Internetanschlussabgabe}

Angesichts dieser nicht von der Hand zu weisenden Probleme der Rechtsdurchsetzung einerseits, den Reaktionen in der Bevölkerung und dem gewandelten Rechtsverständnis in der Laiensphäre andererseits liegt es nahe, nach Alternativlösungen zu suchen. Dabei ist nochmals zu betonen, dass bei ineffektiver individueller Rechtsdurchsetzung Abgabenlösungen aus rein ökonomischen Gründen als bessere Lösungen in Betracht kommen können - ohne dass damit in irgendeiner Weise eine moralisch-ethische Bewertung des "geistigen Eigentums" oder des mangelnden Respekts etwa vor einer Schöpfung durch Kreative verbunden wäre. Es handelt sich allein um die effizientere Bewältigung eines allgemeinen Phänomens, das unter Umständen die Interessen von Urhebern, Verwertern und Nutzern besser zum Ausgleich bringen kann als die derzeitigen Methoden einer Rechtsdurchsetzung. Eine Abgabenlösung, wie sie schon bei der herkömmlichen Privatkopie in Gestalt der Geräteabgabe zum Einsatz kommt, könnte verbunden mit Messverfahren im Internet zu einer vorteilhafteren Lösung gelangen als die individuelle Rechtsdurchsetzung.

In rechtsvergleichender Hinsicht gibt es bislang wenige Vorbilder: Zwar hatte das französische Parlament am 20. Dezember 2005 das Gesetz „Loi sur le droit d'auteur et les droits voisins dans la société de l'information (DADVSI)“ um einen Artikel ergänzt, der eine globale Lizenz für privates Filesharing vorsah, bezogen auf Film und Musikwerke; ${ }^{131}$ allerdings wurde nach nur wenigen Monaten und heftigen Diskussionen der Artikel wieder aufgehoben. ${ }^{132}$ Andere Vorstöße, wie etwa die Vorhaben auf der Isle of Man sind bislang lediglich Zukunftsprojekte ohne konkrete Konturen. ${ }^{133}$

52 Dennoch sind die grundlegenden Umrisse einer "Kulturflatrate“ oder "compulsory license“ weitgehend konsentiert, insbesondere die Verknüpfung von Schranken zugunsten des Down- und Uploads sowie der entsprechenden Vergütungen für Kreative:

\section{A. Der Tatbestand}

53 Kurz gefasst, soll die neue Schranke einer Kulturflatrate die nicht-kommerzielle Weitergabe und Vervielfältigung von digitalen, urheberrechtlich geschützten Werken rechtfertigen, ${ }^{134}$ wobei auch Bearbeitung (Mashups, Remixes etc.) erfasst werden sollen. Gleichzeitig soll hierfür eine pauschale Vergütung auf jeden Internetanschluss erhoben werden, der dann allerdings noch weiteren Differenzierungen unterliegt. Für diesen Tatbestand oder vergleichbare Modelle hat sich allerdings international noch kein einheitlicher Sprachgebrauch durchgesetzt. So wird oft von auch von „alternative

\footnotetext{
${ }^{131}$ Die (identischen) Änderungsvorschläge der konservativen Partei UMP und der Sozialistischen Partei sind abrufbar unter: http://www.assemblee-nationale.fr/12/amendements/1206/120600153.asp und http://www.assemblee-nationale.fr/12/amendements/1206/120600154.asp.

${ }^{132}$ Die am 30. Juni 2006 endgültig vom Parlament beschlossene Fassung ist abrufbar unter: http://www.legifrance.gouv.fr/affichTexte.do?cidTexte=JORFTEXT000000266350\&dateTexte=\&categorieLien=i d.

${ }^{133} \mathrm{~S}$. zum ursprünglichen Vorhaben

http://www.nytimes.com/2009/01/26/business/worldbusiness/26music.html?_r=1\&partner=rss\&emc=rss; der monatliche Betrag pro Internetnutzer sollte demnach zwischen 1 und 1,38 britischen Pfund liegen; der Regierung der Isle of Man zufolge sollte dem Gesetzesvorhaben eine Testphase vorangehen, s. dazu http://www.isleofman.com/businessandfinance/ebusiness/Interview.aspx.

${ }^{134}$ Roßnagel/Jandt/Schnabel, MMR 2010, 8, 8 f.; Roßnagel et al., Gutachten, 2009, S. 11.
} 
compensation system “135, „noncommercial use levy“136 oder „compulsory blanket license“ gesprochen. ${ }^{137}$

54 Betroffen sind in erster Linie die Verwertungsrechte der Vervielfältigung ( $\S 16$ UrhG) und des öffentlich Zugänglichmachens ( $\S 19 a$ UrhG), aber auch das Bearbeitungsrecht nach $\S 23$ UrhG. $^{138}$

Bislang greift im deutschen Recht allein hinsichtlich der Vervielfältigung die Schranke der Privatkopie nach $\S 53$ Abs. 1 UrhG ein, die zudem an die Voraussetzung geknüpft ist, dass die Kopie von einer nicht offensichtlich rechtswidrigen Vorlage gefertigt wurde - was im zweiten Korb präzisiert wurde, indem als offensichtlich rechtswidrig auch die zugänglich gemachte Vorlage gelten kann. ${ }^{139}$ Ferner ist die Vervielfältigung ausschließlich zu privaten Zwecken erlaubt, wobei die Rechtsprechung als Richtschnur eine Zahl von ca. 4-7 Kopien zulässt, ohne sich endgültig festgelegt zu haben. ${ }^{140}$ Die Privatkopieschranke greift dann aber auch im Onlinebereich, sofern noch ein hinreichend persönlicher Kontakt bejaht werden kann. ${ }^{141}$ Dabei gilt keine feste zahlenmäßig Obergrenze, da allein die persönliche Verbindung maßgeblich ist. ${ }^{142}$ Pauschal kann aber nicht davon ausgegangen werden, dass es sich bspw. bei den „Freunden“ auf Facebook ausschließlich um Personen handelt, zu denen eine hinreichende Verbundenheit besteht. Im Gegensatz dazu bestehen weder für das Recht zum öffentlichen Zugänglichmachen ( $§ 19 a$ UrhG) noch für das Bearbeitungsrecht ( $\$ 23$ UrhG) gesetzliche Schranken für den Privatgebrauch. Als Pendant zur Privatkopie und als Ausgleich für die damit gerechtfertigten Eingriffe in die Verwertungsrechte führte der Gesetzgeber die Geräte- und Leermedienabgabe ein, § 54 Abs. 1 UrhG, ${ }^{143}$ die nur über Verwertungsgesellschaften erhoben und verteilt wird, § 54h UrhG.

56 Demgemäß wären zur Legalisierung freier Tauschvorgänge und Bearbeitungen neue Schranken erforderlich, die zum einen die Privatkopie nach § 53 Abs. 1 UrhG erweitern, indem jede Vervielfältigung zum privaten Gebrauch unter die Schranke fiele, unabhängig davon, ob die Vorlage rechtswidrig ist, zum anderen auch das öffentliche Zugänglichmachen eine neue Schranke der privaten Veröffentlichung erhielte, ebenso das Bearbeitungsrecht. Parallel dazu müssten die Abgabentatbestände erweitert werden, indem sie nicht nur Geräte- und Leermedienabgaben erfassen, sondern auch andere Tatbestände einbeziehen, etwa Breitbandanschlüsse.

\section{Privatkopien erweitert auf jeden Download}

57 Für Downloads als Vervielfältigungsvorgänge kommt vor allem die Privatkopieschranke nach § 53 Abs. 1 UrhG in Betracht. Während diese früher breitflächig im Rahmen des Filesharing eingreifen konnte, sofern man nicht als Quelle der Vervielfältigung ein rechtmäßiges Original verlangte, hat sich diese Rechtslage seit dem 1. Januar 2008 geändert. Gerade um das Filesharing einzuschränken, wur-

\footnotetext{
${ }^{135}$ Fisher, 2004, S. 199 ff.; Grassmuck, 2009, S. 11.

${ }^{136}$ Netanel, Harvard JOLT, Vol. 17, 2003, 1 ff.; Grassmuck, 2009, S. 10.

${ }^{137}$ Roßnagel et al., Gutachten, 2009, S. 58 mwNachw.

${ }^{138}$ Statt vieler Roßnagel et al., Gutachten, 2009, S. 8 mwNachw.; Runge, GRUR Int. 2007, 130, 131; Braun, GRUR 2001, 1106, 1107.

${ }^{139}$ Einzelheiten bei Dreier, in: Dreier/Schulze, UrhG, 4. Aufl., 2013, § 53 UrhG, Rn. 11, mwNachw.; Brinkel, Filesharing, 2006, S. $147 \mathrm{ff}$.

${ }^{140}$ BGH GRUR 1978, 474, 476; krit. dazu etwa Dreier, in Dreier/Schulze, UrhG, 4. Aufl., 2013, § 53 UrhG Rn. 9 , der nur ein Exemplar für gerechtfertigt hält.

${ }^{141}$ S. dazu am Bsp. neuerer Musikstreamingdienste, bei denen der Zugriff auf die Musikdateien eines Nutzers ausschließlich vernetzten „Freunden“ des bereitstellenden Nutzers vorbehalten ist, Schapiro, ZUM 2008, 273 ff., insb. $277 \mathrm{ff}$.

${ }^{142}$ Schapiro, ZUM 2008, 273, 278.

${ }^{143}$ S. oben Rn. $13 \mathrm{ff}$.
} 
de in $\S 53$ Abs. 1 UrhG der Zusatz aufgenommen, dass die Vervielfältigung nicht von einer offensichtlich rechtswidrig hergestellten oder öffentlich zugänglich gemachten Vorlage stammt. Will man daher das Filesharing weitgehend erlauben, muss dieser Zusatz wieder gestrichen werden, so dass eine Rechtslage etwa vergleichbar den Niederlanden hergestellt wird, dass jeder Download unabhängig von der Rechtmäßigkeit der Vorlage erlaubt ist, sofern er zu privaten Zwecken erfolgt. ${ }^{144}$

\section{Privater Upload}

Eine Kulturflatrate, die breitflächig das Filesharing ermöglichen soll, muss den Tatbestand der Privatkopie erweitern um das öffentliche Zugänglichmachen, den Upload. Selbst wenn auch nur kleine Teile bzw. Fragmente eines Werkes öffentlich zugänglich gemacht werden, wie dies z.B. durch BitTorrent-Filesharing oder eDonkey geschieht, liegt bereits ein Eingriff in die Verwertungsrechte nach § 19a UrhG vor. ${ }^{145}$ Daher müsste eine Schranke, die einen freien Austausch ermöglichen will, den Upload, also das öffentliche Zugänglichmachen zu privaten Zwecken, ebenfalls erfassen.

\section{Beschränkung auf nicht-kommerzielle Nutzer und Anbieter}

Eines der zentralen Kriterien im Rahmen einer Kulturflatrate stellt die Beschränkung auf private bzw. nicht-kommerzielle Nutzer und Anbieter dar, vergleichbar der bereits bestehenden Privatkopieschranke nach $\S 53$ Abs. 1 UrhG. So einfach dieses Kriterium auf den ersten Blick erscheinen mag, kann es in konkreten Fällen doch Probleme aufwerfen, die für das Filesharing relevant sein können:

So ist die Frage aufgeworfen worden, inwieweit Diensteanbieter wie P2P-Softwareanbieter oder Host-Provider wie Rapidshare als eigene Anbieter von Inhalten qualifiziert werden können, indem sie Inhalte öffentlich gem. § 19a UrhG zugänglich machen. ${ }^{146}$ Damit würde jedoch übersehen, dass diese Dienstleister selbst nicht Täter sind, sondern nur Gehilfen - ihre Tätigkeiten (und auch die Frage, ob kommerziell oder nicht) sind rein akzessorisch zu den Handlungen der jeweiligen Nutzer. Mit anderen Worten spielt es für die Kulturflatrate keine Rolle, ob Anbieter wie Rapidshare einen wirtschaftlichen Nutzen aus den Aktivitäten ihrer Kunden ziehen, da es für die Schranke nur auf die nichtkommerzielle Eigenschaft der Kunden ankommt. Sowie hier die Schranken eingreifen würden, entfiele auch jede Verantwortlichkeit dieser Dienstanbieter, da sie nur akzessorisch haften. Anders ist dies zu beurteilen, wenn die Diensteanbieter sich die Inhalte selbst zu eigen machen, oder in der Terminologie des EuGH in der Entscheidung L'Oréal/eBay ${ }^{147}$ sich nicht mehr lediglich neutral verhalten hierauf ist zurückzukommen. ${ }^{148}$

61 Eine andere Frage ist, ob diejenigen, die selbst Inhalte anbieten, als kommerzielle Anbieter zu qualifizieren sind, wenn sie andere Nutzer von BitTorrent-Portalen auf ihre eigenen, mit Werbung betriebenen Webseiten etc. versuchen umzuleiten. Dafür werden die Torrents einfach im Dateinamen um die jeweiligen URLs erweitert und dauer- und massenhaft in Umlauf gebracht. Alternativ werden die URLs in Textfenstern angezeigt, die mit dem Content verknüpft werden, oder auch in

\footnotetext{
${ }^{144}$ S. die offizielle Antwort des niederländischen Justizministeriums auf eine parlamentarische Anfrage Annex Parliamentary Papers II 2006/07, no. 2060719410; Parliamentary Papers II, 2007/08, 28.482, no. 5, p. 33 et seq.; Parliamentary Papers II, 2007/08 28.482, no. 8, S. 13; s. auch Haarlem District Court, 12 May 2004, 85489/HA ZA 02-99 (Techno Design v Stichting Brein), para. 6.18; ferner Huygen et al., 2009, S. 50 f. mwNachw. ${ }^{145}$ Dreier, in: Dreier/Schulze, UrhG, 4. Aufl. 2013, § 19a UrhG Rn. 6; v. Ungern-Sternberg, in: Schricker/Loewenheim, UrhR, 4. Aufl. 2010, § 19a UrhG Rn. 43; Brinkel, Filesharing, 2006, S. 87 ff.; differenzierend nach der Qualität der übertragenen Fragmente Solmecke/Bärenfänger, MMR 2011, 567, 570 f.; s. auch Heinemeyer et al., MMR 2012, 279, 280; für die Rechtslage in den Niederlanden Huygen et al., 2009, S. 52 f.

${ }^{146}$ Bauer, Das Konzept der Musikflatrate, Dipl. Arbeit, 2011, S. 47.

${ }^{147}$ EuGH MMR 2011, 596, 602 Rn. 113 - L'Oréal SA u.a./eBay International AG u.a.

${ }^{148}$ S. unten Rn. $470 \mathrm{ff}$.
} 
separaten Textdateien. ${ }^{149}$ Einer Studie zufolge soll dieses Geschäftsmodell hinter $26 \%$ aller über BitTorrent vertriebenen Inhalte und $40 \%$ aller Downloads stehen. ${ }^{150}$ Etwa die Hälfte der verantwortlichen Anbieter versucht auf diese Weise, torrentbezogene Produkte zu vermarkten, beispielsweise sog. „private tracker“, mit denen sich höhere Download-Geschwindigkeiten erreichen lassen. Die andere Hälfte verfolgt andere Geschäftsmodelle, dies sind überwiegend Anbieter von PornografiePortalen. ${ }^{151}$ In diesen Fällen liegt in der Tat eine eher kommerzielle Anwendung nahe - dies könnte aber insoweit der Rechtsprechung und der entsprechenden Ausdifferenzierung im Rahmen einer Schranke überlassen werden.

\section{Erweiterung auf Bearbeitungsschranken/-rechte}

(Remixes, Mashups etc.) - Schranke für nutzergenerierte Inhalte

Angesichts der Möglichkeiten des sog. Web 2.0 - der Verwendung von Inhalten Dritter, um diese zu verändern, zu erweitern, zu kürzen etc. - liegt es nahe, auch eine Schrankenerweiterung auf die Bearbeitungsrechte vorzusehen, um nutzergenerierte Inhalte zu erfassen. ${ }^{152}$ Denn Anwendungen wie Facebook oder gerade YouTube, aber auch andere Plattformen, ermöglichen die Kombination von verschiedenen Inhalten und deren Bearbeitung, um neue Werke zu erstellen, etwa eigene Videos mit bekannter Musik. Eine solche Erweiterung hat demnach zum Vorteil, dass zahlreiche gerade im Web 2.0 üblichen Vorgänge erfasst werden, etwa die Verbindung von Musik mit (eigenen) Videos (YouTube), und damit für die Nutzer die rechtlichen Risiken weiter verringert werden. ${ }^{153}$

Gem. $\S 23$ S. 1 UrhG dürfen Bearbeitungen und andere Umgestaltungen eines urheberrechtlich geschützten Werkes nur mit Einwilligung des Urhebers veröffentlicht oder verwertet werden. Dabei ist das Tatbestandsmerkmal der Veröffentlichung bereits dann erfüllt, wenn das Werk einer Mehrzahl an Personen zugänglich gemacht wird, die mit dem Bearbeiter oder Umgestalter nicht persönlich verbunden sind. ${ }^{154}$ In dem gewählten Beispiel eines eigenen YouTube-Videos mit urheberrechtlich geschützter Musik eines Dritten wäre das also bereits dann der Fall, sobald das Video auf der Plattform für andere Nutzer zum Abruf verfügbar ist. Dem steht $§ 24$ UrhG gegenüber, wonach ein selbstständiges Werk, das in freier Benutzung des Werkes eines anderen geschaffen worden ist, ohne Zustimmung des Urhebers des benutzten Werkes veröffentlicht und verwertet werden darf. Beide Vorschriften sind zusammen zu betrachten, ${ }^{155}$ sodass alle Stufen der Bearbeitung, Änderung oder sonstigen Umgestaltung einwilligungsbedürftig sind, bis die Charakteristika des benutzten Werkes hinter denen des neuen Werkes „verblassen“, mithin eine freie Benutzung i.S.v. § 24 UrhG vorliegt. ${ }^{156} \mathrm{Da}$ § 24 UrhG dem Zweck dient, die freie Werkschöpfung auch in Anlehnung an bestehende Werke zu ermöglichen, ${ }^{157}$ kommt es für die Frage der Zustimmungsbedürftigkeit entscheidend darauf an, wel-

${ }^{149}$ Cuevas et al., 2010, S. 8.

${ }^{150}$ Cuevas et al., 2010, S. 9.

${ }^{151}$ Cuevas et al., 2010, S. 8 f.

152 S. auch den Dritten Zwischenbericht der Projektgruppe Urheberrecht der Enquete-Kommission „Internet und digitale Gesellschaft" des Bundestages, BT-Drs. 17/7899, S. 20.

${ }^{153}$ Aigrain, 2012, S. 85 f.

${ }^{154}$ Schulze, in: Dreier/Schulze, UrhG, 4. Aufl. 2013, § 15 UrhG Rn. 39 ff., § 23 UrhG Rn. 17; Bullinger, in: Wandtke/Bullinger, UrhR, 3. Aufl. 2009, § 23 UrhG Rn. 7; s. aber auch Vianello, MMR 2009, 90, 91, der mit dem Willen des Gesetzgebers argumentiert und eine Veröffentlichung erst dann bejaht, wenn eine gewerbliche Nutzung beabsichtigt ist.

155 Bullinger, in: Wandtke/Bullinger, UrhR, 3. Aufl. 2009, § 24 UrhG Rn. 7.

${ }^{156}$ BGH GRUR 2002, 799, 800 - Stadtbahnfahrzeug; BGH GRUR 1994, 208; Schulze, in: Dreier/Schulze, UrhG, 4. Aufl. 2012, § 23 UrhG Rn. 4; Wiebe, in: Spindler/Schuster, Recht der elektronischen Medien, 2. Aufl. 2011, § 24 UrhG Rn. 3; Bullinger, in: Wandtke/Bullinger, UrhR, 3. Aufl. 2009, § 24 UrhG Rn. 12.

${ }^{157}$ Schulze, in: Dreier/Schulze, UrhG, 4. Aufl. 2012, § 24 UrhG Rn. 1. 
chen Abstand das neue zum benutzten Werk hält, mithin auf die Individualität des neuen Werkes. Dabei ist ein strenger Maßstab anzulegen. ${ }^{158}$ Entscheidend sind nicht die Unterschiede zwischen den Werken, sondern ihre Übereinstimmungen. ${ }^{159}$ Das bearbeitete oder umgestaltete Werk darf lediglich eine Anregung für das eigene Schaffen sein. ${ }^{160}$ Nicht ausreichend für eine freie Benutzung ist etwa die bloße Existenz anderer Elemente im neuen Werk, ebenso wenig das Entfernen einzelner Passagen des alten Werkes. ${ }^{161}$ Die Verwendung einzelner isolierter Passagen in einem anderen Kontext kann aber als Zitat nach $\S 51$ UrhG zulässig sein, soweit der Zitatzweck dies gebietet. ${ }^{162}$ Parodien sind hingegen von $\S 24$ UrhG gedeckt, mithin zustimmungsfrei, solange sie sich nicht auf das Kopieren der Charakteristika des benutzten Werkes beschränken, sondern sich „antithematisch“ mit innen auseinandersetzen. ${ }^{163}$ Für Musikstücke besonders relevant ist $\S 24$ Abs. 2 UrhG, wonach die Zustimmungsfreiheit nicht für die Benutzung eines Werkes der Musik gilt, durch welche eine Melodie erkennbar dem Werk entnommen und einem neuen Werk zugrunde gelegt wird. Eine Melodie ist der Rechtsprechung zufolge eine geschlossene Tonfolge, die dem Werk seine individuelle Prägung gibt. ${ }^{164} \mathrm{Al}-$ lerdings fallen charakteristische Rhythmen und Klangeffekte nicht unter $\S 24$ Abs. 2 UrhG, weshalb das sog. Sampling grds. zustimmungsfrei i.S.v. § 24 Abs. 1 UrhG ist. ${ }^{165}$ Ebenfalls von Relevanz ist, dass die bloße Übertragung eines Werkes in eine andere Kunstform - z.B. die Vertonung eines Sprachwerkes - nicht zustimmungsbedürftig ist. ${ }^{166}$ Daraus kann aber nicht der Schluss gezogen werden, dass die Hinterlegung eines Musikstücks mit (Stand-) Bildern, um es auf eine Videoplattform hochladen zu können, eine freie Benutzung in diesem Sinne darstellt. Dies würde die Rechteinhaber an Musikstücken praktisch schutzlos stellen, da mithilfe von Tools zum Mitschneiden des Videotons die digitale Verbreitung ihrer Werke schon jetzt rechtlich gestattet wäre. Richtigerweise liegt in diesem Fall eine Bearbeitung oder sonstige Umwandlung i.S.v. § 23 S. 1 UrhG vor, die ohne eine entsprechende Lizenz des Urhebers nicht zulässig ist.

$\S 24$ UrhG ist zudem auch auf das in § 85 UrhG normierte Leistungsschutzrecht des Tonträgerherstellers entsprechend anwendbar. ${ }^{167} \S 85$ Abs. 1 S. 1 UrhG untersagt grundsätzlich die Entnahme bereits kleinster Tonfetzen eines Tonträgers - unabhängig von quantitativen oder qualitativen Gesichtspunkten - und macht damit etwa deren Nutzung in einem eigenen Werk (sog. Sampling) von der Zustimmung des Rechteinhabers abhängig. ${ }^{168}$ Neben der aufgezeigten Ausnahme des $§ 24$ Abs. 2 UrhG gilt die Schranke des $\S 24$ UrhG hier jedoch nach Auffassung des BGH nicht in solchen Fällen, in denen

\footnotetext{
158 BGH GRUR 1994, 191, 193 - Asterix-Persiflagen; BGH GRUR 2002, 799, 800 f. - Stadtbahnfahrzeug; Wiebe, in: Spindler/Schuster, Recht der elektronischen Medien, 2. Aufl. 2011, § 24 UrhG Rn. 2.

159 BGH GRUR 1981, 267, 269 - Dirlada; BGH GRUR 1965, 45, 48 - Stadtplan; BGH GRUR 1961, 635, 638 - Stahlrohrstuhl; BGH GRUR 1960, 251, 253 - Mecki Igel; BGH GRUR 2006, 53, 54 f. - Wagenfeld-Tischleuchte; Bullinger, in: Wandtke/Bullinger, UrhR, 3. Aufl. 2009, § 24 UrhG Rn. 9; Wiebe, in: Spindler/Schuster, Recht der elektronischen Medien, 2. Aufl. 2011, § 24 UrhG Rn. 5.

160 BGH NJW 2004, 3633, 3635; BGH NJW 2011, 761, 764; Schulze, in: Dreier/Schulze, UrhG, 4. Aufl. $2012, \S 24$ UrhG Rn. 7; Vianello, MMR 2009, 90, 91.

${ }^{161}$ Bullinger, in: Wandtke/Bullinger, UrhR, 3. Aufl. 2009, § 24 UrhG Rn. 9.

162 Lüft, in: Wandtke/Bullinger, UrhR, 3. Aufl. 2009, § 51 UrhG Rn. 3 mwNachw.

163 BGH GRUR 1971, 588, 589 - Disney-Parodie, unter Verweis auf BGHZ 26, 57; GRUR 1994, 191, 193 - AsterixPersiflagen; GRUR 1994, 206, 208 - Alcolix; Bullinger, in: Wandtke/Bullinger, UrhR, 3. Aufl. 2009, § 24 UrhG Rn. 14.

${ }^{164}$ BGH GRUR 1988, 810, 811 - Fantasy; BGH GRUR 1988, 812, 814 - Ein bisschen Frieden; OLG München ZUM 2000, 408, 409 - Melodieentnahme; OLG Dresden GRUR 1999, 332, 335 - Kaleidoskop.

${ }^{165}$ Wiebe, in: Spindler/Schuster, Recht der elektronischen Medien, 2. Aufl. 2011, § 24 UrhG Rn. 8; Loewenheim, in: Schricker/Loewenheim, UrhR, 4. Aufl. 2010, § 24 UrhG Rn. 35.

${ }^{166}$ Bullinger, in: Wandtke/Bullinger, UrhR, 3. Aufl. 2009, § 24 UrhG Rn. 6 mwNachw.

${ }^{167}$ S. dazu ausf. BGH ZUM 2009, 219, 222 f. m. zahlr. Nachw.

${ }^{168}$ S. dazu ausf. BGH ZUM 2009, 219, 220 ff. m. zahlr. Nachw.
} 
der Verwender imstande ist, die betroffenen Teile des Tonträgers selbst herzustellen bzw. einzuspielen. ${ }^{169}$

Die Erweiterung der Schranken im Rahmen eines Abgabensystems auf Bearbeitungsrechte (Remixes) wurde bereits von Fisher vorgeschlagen. ${ }^{170}$ Allerdings erzeugt eine solche Erweiterung Folgeprobleme zum einen auf der Vergütungsseite, zum anderen im Rahmen der Urheberpersönlichkeitsrechte: Hinsichtlich der Vergütung müssten derartige neu geschaffene Werke ihrerseits als Werke begriffen werden und damit auch an der Vergütung partizipieren - was indes schwer ermittelbar sein dürfte, da weder Urheber dieser neuen Werke identifizierbar noch die Werke selbst ohne weiteres bzw. einfach im Netz zu finden sein werden. Darüber hinaus könnten dadurch Anreize geschaffen werden, bestehende Werke einfach als "Remixes“ zu deklarieren, um selbst in den Genuss von Vergütungen zu kommen. ${ }^{171}$ Allerdings könnten diese Probleme durch digitale „identifier“, die den Werken wie digitale Fingerabdrücke beigefügt werden, überwunden werden.

Bedenken resultieren daraus auf den ersten Blick auch hinsichtlich der Wahrung der Urheberpersönlichkeitsrechte, insbesondere für den Schutz vor Verunstaltung der eigenen Werke durch die Bearbeitung Dritter. § $14 \mathrm{UrhG}^{172}$ untersagt Entstellungen oder andere Beeinträchtigungen eines Werkes, welche die Eignung aufweisen, die berechtigten Interessen des Urhebers zu gefährden. Für Filmwerke und zu deren Herstellung benutzte Werke gilt dies gem. § 93 Abs. 1 UrhG allerdings nur bei gröblichen Beeinträchtigungen, was erst angenommen werden kann, wenn eine völlige Verkehrung des ursprünglichen Sinngehaltes des Filmwerkes bzw. des ihm zugrundeliegenden Werkes oder eine völlige Verunstaltung von urheberrechtlich wesentlichen Teilen des Films oder Werks entgegen den Intentionen des Urhebers stattfindet. ${ }^{173}$ Allerdings handelt es sich hier letztlich um eine Frage der möglichen Verfolgung von unzulässigen Verunstaltungen; denn auch nach geltendem Recht kann der Urheber selbst bei einer Lizenzierung ${ }^{174}$ der Bearbeitung auf Basis der genannten Vorschriften sowie nach $\S 39$ UrhG gegen gewisse Beeinträchtigungen wie etwa eine Verunstaltung vorgehen, ${ }^{175}$ so dass es sich nur um die Frage "Schranke oder Lizenzierung" handelt, nicht aber um die Wahrung der Persönlichkeitsrechte überhaupt. Anders formuliert erleichtert das Erfordernis einer Lizenzierung bei Bearbeitungen die Rechteverfolgung bei Verunstaltungen, es ist aber keine notwendige Bedingung zur Sicherstellung der Urheberpersönlichkeitsrechte.

\footnotetext{
${ }^{169}$ BGH ZUM 2009, 219, 222.

${ }^{170}$ Fisher, 2004, S. $30 \mathrm{ff}$.

${ }^{171}$ Darauf weist zu Recht Aigrain, 2012, S. 86 hin.

${ }^{172}$ S. etwa auch $\S 75$ UrhG für den Schutz vor einer Beeinträchtigung der Darbietung eines ausübenden Künstlers.

${ }^{173}$ S. dazu grundlegend OLG München GRUR 1986, 460, 461; s. weiter Schwarz/Reber, in: Loewenheim, Handbuch des Urheberrechts, 2. Aufl. 2010, § 74 Rn. 30.

${ }^{174} \S 62$ UrhG normiert darüber hinaus ein Änderungsverbot für Werke, deren Nutzung aufgrund der Schrankenregelungen der $\S \S 44 a \mathrm{ff}$. UrhG zulässig ist.

${ }^{175}$ Wiebe, in: Spindler/Schuster, Recht der elektronischen Medien, 2. Aufl. 2011, § 14 UrhG Rn. 1, § 39 UrhG Rn. 1 ff.; s. auch Schulze, in: Dreier/Schulze, UrhG, 4. Aufl. 2013, § 39 UrhG Rn. 1 ff., insb. Rn. 3 zum Verhältnis von $\S 39$ zu § 14 UrhG; für den Fall der Verfilmung eines Romans s. OLG München GRUR 1986, 460, 462.
} 
Schließlich müssen derartige Schranken ähnlich wie das Zitatrecht (§ 51 UrhG i.V.m. § 63 UrhG) oder wie die Bedingungen in Open Source- bzw. Creative Commons Lizenzen ${ }^{176}$ mit der Pflicht verbunden werden, die ursprüngliche Quelle bzw. den Urheber zu nennen. ${ }^{177}$

\section{Beschränkung auf nicht DRM-geschützte Werke}

Die Vorschläge zur Einführung einer Schranke für die nicht-kommerzielle freie Vervielfältigung und den Upload (öffentliches Zugänglichmachen) haben bislang weitgehend darauf verzichtet, die Schranke auch auf DRM-geschützte Systeme zu erstrecken. Hierfür sprechen vor allem pragmatische Gründe, die auf dem strengen Verbot der InfoSoc-Richtlinie ${ }^{178}$ gem. deren Art. 6 fußen, der die Schranken nach Art. 5 Abs. 2, 3 der Richtlinie für DRM-geschützte Inhalte weitgehend ausschließt. Zudem könnten durch DRM-Systeme die typischen Durchsetzungsprobleme und damit die Gründe für die komplexen Rechtsverfolgungsmaßnahmen vermieden werden, da durch die technologisch implementierten Kopierbeschränkungen eine freie Verbreitung verhindert werden könnte.

Allerdings schränken DRM-Systeme bestimmungsgemäß ein Werk in seiner Nutzbarkeit ein und weisen damit grundsätzlich einen Minderwert gegenüber nicht DRM-geschützen Werken gleicher Art auf. ${ }^{179}$ Dieser Minderwert müsste in jedem Fall bei der Verteilung der Einnahmen aus einer Kulturflatrate Berücksichtigung finden. Dabei ist wiederum zu beachten, dass es nicht „das" DRM-System gibt. Die Einschränkungen durch die eingesetzte Technik können extrem divergieren. So kann ein digitales Werk etwa auf 10 oder aber auch auf 100 Kopier-, Brenn- oder Konsumvorgänge beschränkt sein. Innerhalb einer Kulturflatratelösung würde das zu einer starken Erhöhung der Abgabenverteilungskomplexität führen, da jede denkbare Form der DRM-Systeme und des jeweiligen Minderwertes berücksichtigt werden müsste.

Fraglich ist daher, ob DRM-geschützte Werke grundsätzlich nicht berücksichtigt werden sollten - wie dies bereits in der Geräteabgabe der Fall ist, § 54a Abs. 1 S. 2 UrhG. Damit würden derartig geschützte Werke nicht an den Abgaben aus einer Flatrate partizipieren, obwohl de facto wohl dennoch um das DRM-System bereinigte Werkexemplare per Filesharing verteilt werden würden. Hierbei wirkt das in der Praxis durchaus zu beobachtende Hase-und-Igel-Spiel zwischen Hackern und Industrie verstärkend, ebenso wie die von Konsumenten in einigen Sektoren zu Tage tretende Abneigung gegenüber DRM-Systemen, insbesondere in der Musikbranche. ${ }^{180}$ Auch könnte die Herausnahme von DRM-Systemen aus einer "Kulturflatrate“ die Anreize zum Einsatz von DRM-Systemen steigern und damit gerade den Zielsetzungen der allgemeinen Schrankensetzung im Interesse eines weitgehenden Austauschs widersprechen. ${ }^{181}$ Die Software- und die Filmbranche zeigen, dass in einigen Bereichen DRM-Systeme unter bestimmten Rahmenbedingungen (relativ einheitliche Hardware, OnlineAktivierungen etc.) durchaus in der Lage sind, die Rechte effektiv durchzusetzen - auch wenn hier

\footnotetext{
${ }^{176}$ S. § 4 der GPL; die Namensnennung des Urhebers ist Bestandteil jeder der sechs aktuellen Creative Commons Lizenzen, zu den einzelnen Lizenzen siehe die Homepage von Creative Commons Deutschland, abrufbar unter: http://de.creativecommons.org/was-ist-cc/.

${ }^{177}$ Aigrain, 2012, S. 86.

${ }^{178}$ Richtlinie 2001/29/EG des Europäischen Parlaments und des Rates vom 22. Mai 2001 zur Harmonisierung bestimmter Aspekte des Urheberrechts und verwandter Schutzrechte in der Informationsgesellschaft, ABI. Nr. L 167 v. 22.6.2001, S. 10.

${ }^{179}$ S. dazu die Darstellung diesbezüglicher Aussagen der ehem. Justizministerin Zypries bei Grassmuck, ZUM 2005, 104, $105 \mathrm{f}$.

180 Darauf verweist Aigrain, 2012, S. 121 f., der hierin eine Zwangsjacke für Kreative erblickt.

${ }^{181}$ Runge, GRUR Int. 2007, 130, 136.
} 
nicht verschwiegen soll, dass beide Branchen ebenfalls mitunter in zahlreiche Verfolgungsmaßnahmen involviert sind.

71 Im Folgenden wird für die Ausgestaltung der Schranke auch wegen des strengen europarechtlichen Schutzes der DRM-geschützten Werke (Art. 6 InfoSoc-RL) von einer Ausnahme zugunsten der DRMgeschützten Werke ausgegangen. Dies kann auch im Hinblick auf die verfassungsrechtlichen Fragen sowie den Dreistufen-Test relevant sein, da in diesem Fall Rechteinhaber nach wie vor über den DRM-Schutz individuelle Lizenzierungen durchführen könnten, somit eine Wahlmöglichkeit zwischen Kulturflatrate und DRM-Schutz bzw. individueller Lizenzierung bestünde.

\section{Nicht erfasste Werkkategorien}

72 Aus der Schranke einer Kulturflatrate sollen zudem zunächst Software und Spiele ausgenommen werden. Dies kann ebenfalls aus pragmatischer Sicht damit gerechtfertigt werden, dass zurzeit vor allem im Bereich der Musikindustrie wohl die meisten Verwertungsvorgänge durch Private stattfinden und zudem hier zahlreiche empirische Untersuchungsergebnisse zu den Auswirkungen des Filesharing vorliegen. ${ }^{182}$ Abgesehen davon sind einer privaten Vervielfältigung bei Computerprogrammen (und damit auch Spielen) de lege lata auf europäischer Ebene noch wesentlich engere Grenzen gezogen als für andere Werke; so sieht die (rekodifizierte) Software-RL der EU ${ }^{183}$ - in Deutschland umgesetzt in den $\S \S 69$ a bis 69g UrhG ${ }^{184}$ - in Art. 5 Abs. 2 das Recht zur Anfertigung einer Sicherungskopie nur unter der restriktiven Bedingung vor, dass die Kopie für die Benutzung erforderlich ist. Stellt der Softwarehersteller also etwa eine Sicherungskopie der Software zur Verfügung, entfällt die Erforderlichkeit für die Erstellung einer eigenen Sicherungskopie. ${ }^{185}$ Eine generelle Vervielfältigungsprivilegierung für Software zu privaten Zwecken existiert hingegen nicht.

Abgesehen von der unterschiedlichen Rechtslage de lege lata zeichnet sich Software aber auch durch weitere Besonderheiten aus, angefangen bei den wesentlich komplexeren und im Markt (im Gegensatz zur Musikindustrie) durchgesetzten Kopierschutzsystemen, wie etwa Produktaktivierungen, bis hin zu umfangreichen und differenzierten Lizenzierungen. Schließlich handelt es sich bei Software nicht immer um "Stangenware", so dass sich jenseits der massenhaft gehandelten Software wie z.B. Windows-Software auch Business-Software findet. Anders als bei Musik, E-Books oder Filmen fällt hier eine einheitliche Lösung daher schwerer. ${ }^{186}$

Schließlich dürfte für Software nicht im gleichen Ausmaß wie für andere Werkkategorien ein Bedürfnis nach freiem Tausch und Bearbeitung bestehen, da sie zum einen nicht unabdingbar ist, um am kulturellen und meinungsbildenden Leben in einer Gesellschaft teilhaben zu können, zum anderen auch etablierte Open-Source-Lösungen wie Linux und Open-Office vorliegen. Allerdings kann sich diese Sachlage ändern, je mehr Inhalte und Filme etwa in Videospiele eingebunden werden oder die Grenzen zwischen Software und interaktiven Inhalten verschwimmen.

\footnotetext{
182 S. den Überblick bei Tschmuck, Economics, 2010, S. 16 ff.; Grassmuck, 2010, S. 25 ff.

${ }^{183}$ Richtlinie 2009/24/EG des Europäischen Parlaments und des Rates vom 23. April 2009 über den Rechtsschutz von Computerprogrammen, ABI. Nr. L 111 v. 5.5.2009, S. 16.

${ }^{184}$ S. weiterführend zur Entwicklungsgeschichte des Rechtsschutzes von Software in Europa Kilian, GRUR Int. 2011, 895.

${ }^{185}$ Wiebe, in: Spindler/Schuster, Recht der elektronischen Medien, 2. Aufl. 2011, § 69d UrhG Rn. 24; Lehmann, in: Loewenheim, Handbuch des Urheberrechts, 2. Aufl. 2010, § 76 Rn. 18; Dreier, in: Dreier/Schulze, UrhG, 4. Aufl. 2013, § 69d UrhG Rn. 16.

186 Ähnliche Einschätzung bei Roßnagel et al., Gutachten, 2009, S. 5.
} 
Ferner ist der Einwand berechtigt, dass eine Ausnahme zugunsten bestimmter Werkkategorien bzw. Branchen nur dann möglich ist, wenn damit nicht die gleichen Einschränkungen für die Nutzung des Internet einhergehen wie bislang. Anders ausgedrückt wäre der Mehrwert einer Kulturflatrate aus Sicht der Nutzer eher beschränkt, wenn die Rechtsdurchsetzungsmethoden und die damit einhergehenden Fragen der Grundrechtseingriffe wie Überwachung von Anschlüssen etc. weiterhin in einigen Sektoren bestehen blieben, da nicht alle Werkkategorien erfasst werden. ${ }^{187}$

Dadurch, dass neben Musik auch E-Books sowie Filme umfasst werden, sind aber auch Werke erfasst, die gesellschaftspolitisch unter Umständen nicht erwünscht sind, insbesondere erotische und pornografische Filmwerke. Sofern hier Urheberrechte sowie Leistungsschutzrechte entstehen, müssen diese ebenso von einer Kulturflatrate erfasst werden, wie dies schon im Bereich der Geräteabgabe seit deren Einführung der Fall ist. ${ }^{188}$ Ein Ausschluss von erotischen und pornografischen Darstellungen bedürfte einer eingehenden verfassungsrechtlichen Analyse vor dem Hintergrund der Kunstfreiheit nach Art. 5 Abs. 3 GG, ${ }^{189}$ die den Umfang dieses Gutachtens sprengen würde. Hinzuweisen ist dabei insbesondere darauf, dass sich im Hinblick auf die Eröffnung des Schutzbereichs der Kunstfreiheit eine Niveaukontrolle verbietet. ${ }^{190}$

\section{Erfasste Rechteinhaber}

77 Von einer Kulturflatrate als Schranke mit Abgabe würden entsprechend dem Vorbild der Geräteabgabe ( $\S \S 54 \mathrm{ff}$. UrhG) alle Urheber sowie alle Leistungsschutzrechteinhaber wie etwa Tonträgerhersteller oder ausübende Künstler etc. einbezogen. Da die Verwertungsvorgänge wie Vervielfältigungen oder das Recht auf öffentliches Zugänglichmachen nicht nur die Urheber selbst, sondern auch die Leistungsschutzberechtigten betrifft, wäre es nicht zu rechtfertigen, diese von der Verteilung einer Abgabe auszuschließen, ebenso wenig, eine Schranke nur für Urheber aufzustellen. Dementsprechend sehen bereits jetzt die jeweiligen Leistungsschutzrechte pauschal die Geltung des Abschnitts 6 des ersten Teils des UrhG vor, der alle Schranken, aber auch die Geräteabgaben umfasst, z.B. §§ 72 Abs. 1, 83, 85 Abs. 4, 94 Abs. 4 UrhG.

\section{Beschränkung auf digitale Veröffentlichungen}

78 Ferner sollte die Schranke auf digital veröffentlichte Werke beschränkt sein. Damit würden etwa privat gesandte Werke, die der Urheber nicht zur Veröffentlichung vorgesehen hatte, von vornherein ausgenommen werden ${ }^{191}$ - ansonsten würden die Persönlichkeitsrechte des Urhebers unverhältnismäßig eingeschränkt, da sie nicht mehr darüber bestimmen könnten, wann ein Werk an die Öffent-

\footnotetext{
${ }^{187}$ Darauf weist insoweit zutr. Aigrain, 2012, S. 82 hin.

${ }^{188}$ Für diesen Bereich hat sich eine eigene Verwertungsgesellschaft etabliert, die GÜFA (Gesellschaft zur Übernahme und Wahrnehmung von Filmaufführungsrechten $\mathrm{mbH}$ ), die die Rechte im Bereich des erotischen und pornografischen Films wahrnimmt, s. dazu www.guefa.de.

${ }^{189}$ S. dazu nur grundlegend BVerfGE 83, 130, 138 f. - Josefine Mutzenbacher, sowie BGHSt 37, 55, 57 ff. - Opus Pistorum; die vormals vertretene These von der strikten Trennung von Kunst und Pornographie ist seitdem überholt; eingehend Schroeder, Pornographie, Jugendschutz und Kunstfreiheit, 1992, S. 47 ff.; Schreibauer, Das Pornographieverbot des $\S 184$ StGB, 1999, S. 155 ff.; sowie Hörnle, in: Münchener Kommentar zum StGB, 2. Aufl. 2012, § 184 Rn. 24 ff., jeweils m. zahlr. wNachw.; selbst für "harte“ Pornographie i.S.v. §§ 184a ff. StGB, s. BGHSt 37, 55, 61 - Opus Pistorum, wenngleich die Kunstfreiheit zumindest bei der Kinderpornographie gem. § 184b StGB prinzipiell hinter die bei der Herstellung beeinträchtigten Individualgrundrechte zurücktreten muss, s. Beisel, Die Kunstfreiheitsgarantie des Grundgesetzes und ihre strafrechtlichen Grenzen, 1997, S. 328; ebenso Laufhütte/Roggenbuck, in: Leipziger Kommentar, StGB, 12. Aufl. 2010, § 184b Rn. 18.

${ }^{190}$ BVerfGE 75, 369, 377; Laufhütte/Roggenbuck, in: Leipziger Kommentar, StGB, 12. Aufl. 2010, § 184 Rn. 9; Hörnle, in: Münchener Kommentar zum StGB, 2. Aufl. 2012, § 184 Rn. 25; Perron/Eisele, in: Schönke/Schröder, StGB, 28. Aufl. 2010, § 184 Rn. 5a.

${ }^{191}$ Ebenso Aigrain, 2012, S. 84.
} 
lichkeit gelangt. Ebenso sollten nur in digitaler Weise veröffentlichte Werke erfasst werden, so dass etwa Aufnahmen von Konzerten oder Mitschnitte von Filmen in Kinos weiterhin nicht zulässig wären, nicht zuletzt, um hier bestehende Geschäftsmodelle der zeitversetzten Releases nicht zu gefährden. ${ }^{192}$ Allerdings ist einzuräumen, dass die Grenze in der Praxis zwischen berechtigten Uploads von Filmen oder Livemusik und unzulässigen Mitschnitten schwer zu ziehen sein wird. Auch kann die Rechtsverfolgung in diesen Fällen nicht erschwert werden, etwa durch Versagen von Auskunftsansprüchen. ${ }^{193}$

\section{B. Ökonomische Auswirkungen und Probleme}

Eine solche Schrankenerweiterung und die Einführung einer Abgabe als Äquivalent hierfür werfen sowohl in ökonomischer als auch rechtlicher Hinsicht zahlreiche Fragen auf, denen es im Folgenden gilt nachzugehen. Dabei stehen zunächst die ökonomischen, durch empirische Untersuchungen untermauerten Effekte des Filesharing im Vordergrund, da sie auch für die rechtliche Analyse eine erhebliche Rolle spielen, ebenso wie für die Frage der konkreten Berechnung einer Abgabenhöhe. Empirische Untersuchungen können hier einen Fingerzeig für die europa- und verfassungsrechtliche Beurteilung, etwa der potentiellen Eingriffe in Rechte und der Reichweite der Einschätzungsprärogative des Gesetzgebers geben. Daher wird zunächst der Stand der empirischen Forschung beleuchtet, soweit er für die Zwecke der Untersuchung relevant ist, um sich anschließend den rechtlichen Implikationen zuzuwenden:

\section{Empirische Grundlagen}

Trotz (oder gerade wegen) dieser Verzahnung von empirischer Forschung mit rechtlicher Beurteilung ist zuvor auf einige methodische Probleme der meisten empirischen Studien hinzuweisen, die die Frage der Einschätzungsprärogative des Gesetzgebers in einem besonderen Licht erscheinen lassen:

\section{a) Methodische Probleme}

81 Zunächst ist festzuhalten, dass verlässliche Daten offenbar nur in begrenztem Maße zur Verfügung stehen. Wie auch andere Studien feststellen mussten, liegen gerade bei neueren Technologien und Geschäftsmodellen wenige empirische Daten vor. ${ }^{194}$ Hinzu kommt, dass zwar für die Musikindustrie aufgrund des zunehmenden Tauschs seit der Einführung der Tauschzentrale "Napster" 1999/2000 und der Verbreitung von P2P-Filesharing einige empirische Studien vorliegen, aber nur wenige für verwandte Bereiche wie Filme, Spiele, Software oder E-Books. Zahlreiche in die Diskussion eingeführte Daten stammen von Lobbyismus-Organisationen und können daher nur mit aller Vorsicht als Grundlage für Aussagen herangezogen werden. ${ }^{195}$

Die viel beachtete Hargreaves-Studie im Auftrag der britischen Regierung führt hierzu aus:

"Given its importance, you would think that we would have a very clear picture of the scale and dynamics of online piracy, but this is not so. There is no doubt that a great deal of piracy is taking place, but reliable data is surprisingly thin on the ground. There is no shortage of claims about levels of infringement, but in the Review's four months of evidence gathering, we have

\footnotetext{
192 Zutr. Aigrain, 2012, S. 84.

${ }^{193}$ So aber Aigrain, 2012, S. 84, bei dem völlig offen bleibt, wie Urheber dann ihre Ansprüche realisieren können sollten.

${ }^{194}$ S. die Kritik an den vorhandenen Studien und Untersuchungsmethoden bei Oberholzer-Gee/Strumpf, 2010, S. 17 ff.; eine ausführliche Übersicht über vorhandene Studien im Musikbereich enthält Grassmuck, 2010, passim mwNachw, ebenso Tschmuck, Creativity, 2012, S. 188 ff., ders., Teil 18, 2009.

195 Ähnlich die Einschätzung von Hargreaves, Digital Opportunity, 2011, S. 18 f.
} 
failed to find a single UK survey that is demonstrably statistically robust. For many surveys, methodology is not available for peer review."196

"With the exception of the Industry Canada study, we have either not been able to examine the methodology of the studies or, where we have, we have discovered problems with the methodology. Consequently, we have not found either a figure for the prevalence and impact of piracy worldwide or for the UK in which we can place our confidence"197

Auch das US Government Accounting Office (GAO) musste in seinem Bericht "Intellectual Property: Observations on Efforts to Quantify the Economic Effects of Counterfeit and Pirated Goods" konstatieren:

"According to experts we spoke with and literature we reviewed, estimating the economic impact of IP infringements is extremely difficult, and assumptions must be used due to the absence of data. Assumptions, such as the rate at which consumers would substitute counterfeit goods for legitimate products, can have enormous impacts on the resulting estimates and heighten the importance of transparency. [...] Most experts we spoke with and the literature we reviewed observed that despite significant efforts, it is difficult, if not impossible, to quantify the net effect of counterfeiting and piracy on the economy as a whole." ${ }^{198}$

87 Hierfür sind verschiedene Faktoren verantwortlich: Hinsichtlich der auf Interviews oder Fragebögen beruhenden Studien ${ }^{199}$ stellt sich häufig das Problem, dass die Antworten der Teilnehmer auf bestimmten Voreinstellungen oder -überlegungen beruhen, sei es dass wahrheitswidrig ein Mitwirken an Filesharing geleugnet wird, z.B. nach entsprechenden Publizitäts- oder Rechtsverfolgungskampagnen der Musikindustrie, ${ }^{200}$ oder schlicht Unkenntnis der Befragten besteht, ob sie überhaupt Urheberrechtsverletzungen begangen haben, ${ }^{201}$ oder sei es dass es gerade als "schick" in bestimmten Kreisen gilt, an Filesharing teilzunehmen, obwohl dies nicht der Realität entspricht. ${ }^{202}$ Diese ergebnisbeeinträchtigenden Faktoren in den Umfragen sind schwer bis gar nicht identifizierbar. ${ }^{203}$ Zudem bestehen erhebliche Zweifel an der Repräsentativität der Befragten in etlichen Umfragen, etwa wenn Befragungen nur unter Studenten einer Universität durchgeführt werden. ${ }^{204}$ Insgesamt begegnen

\footnotetext{
${ }^{196}$ Hargreaves, Digital Opportunity, 2011, S. 69.

${ }^{197}$ Hargreaves, Digital Opportunity, 2011, S. 73.

198 US Government Accountability Office (GAO), Intellectual Property - Observations on Efforts to Quantify the Economic Effects of Counterfeit and Pirated Good, 2010, S. 15 f.

${ }^{199}$ S. etwa die Studien von Zentner, Measuring the Effect of File Sharing on Music Purchases, Journal of Law \& Economics, Vol. 49, No. 1, 2006, S. 63 ff.; Andersen/Frenz, 2007; Bahanovich/Collopy, 2009.

${ }^{200}$ S. die Kritik von Liebowitz, 2011, an einer kanadischen Studie von Andersen/Frenz, 2007.

${ }^{201}$ Oberholzer-Gee/Strumpf, 2010, S. 16 f. in einer Analyse verschiedener US-amerikanischer empirischer Studien; s. kritisch zu dieser Studie US Government Accountability Office (GAO), Intellectual Property - Observations on Efforts to Quantify the Economic Effects of Counterfeit and Pirated Good, 2010, S. 25: "While this is an enviable data set of actual illegal downloads, the study has two main limitations: first, the study uses a static model which does not reflect the effect of downloads apart from the week the download occured. Second, the study only observed the supply side of music. Thus, it is not clear if consumers who are illegally downloading music would have purchased the genuine albums".

${ }^{202}$ S. die Kritik des US Government Accountability Office (GAO), Intellectual Property - Observations on Efforts to Quantify the Economic Effects of Counterfeit and Pirated Good, 2010, S. 21.

${ }^{203}$ US Government Accountability Office (GAO), Intellectual Property - Observations on Efforts to Quantify the Economic Effects of Counterfeit and Pirated Good, 2010, S. 21.

${ }^{204}$ So etwa in den Studien von Rob/Waldfogel, 2004; Bounie/Bourreau/Waelbroeck, 2005; Leung, 2009.
} 
daher die auf derartigen Befragungen beruhenden Studien erheblichen Zweifeln, ob sie tatsächlich das Nutzerverhalten richtig erfassen können. ${ }^{205}$

Aber auch Verzerrungen durch interessengeleitete Angaben von verschiedenen Organisationen ${ }^{206}$ oder fehlende Verifizierbarkeit erschüttern die Glaubwürdigkeit mancher Studie: So wird etwa die für das Vereinigte Königreich vielbeachtete TERA-Studie ${ }^{207}$ dahingehend kritisiert, dass ihre Aussagen über die Ausmaße des Filesharing und die daraus resultierenden Schäden der Kreativindustrie sich in großen Teilen nicht aufgrund der angegebenen Quellen verifizieren ließen. ${ }^{208}$ Von den für das Vereinigte Königreich angenommenen Schäden in Höhe von 1,4 Mrd. Euro lassen sich Hargreaves zufolge lediglich 490 bis 538 Mio. Euro durch verlässliche Daten bestätigen. Die Schäden im Bereich Musik sind demnach etwa zur Hälfte belegbar, im Bereich Film fast zur Gänze. Die Angaben seitens TERA, der Schaden der Software-Industrie belaufe sich im Vereinigten Königreich auf 742 Mio. Euro pro Jahr, lassen hingegen sogar jedweden Nachweis vermissen und sind somit nicht überprüfbar. ${ }^{209}$

89 Eine oft als Beleg herangezogene Studie von Liebowitz aus dem Jahr 2006 verwendet die Zahl der Internet-Verbreitung, ${ }^{210}$ um auf dieser Basis die Auswirkungen des Filesharing auf die Albumverkäufe der Musikindustrie zu ermitteln. ${ }^{211}$ Liebowitz untersucht zunächst, welche Auswirkungen Radio und Fernsehen auf die Musikverkäufe hatten und haben, unterstellt vergleichbare Auswirkungen durch die Verbreitung des Internet und berücksichtigt diesen Anteil umgerechnet auf durchschnittliche Alben pro Kopf bei der Berechnung der Auswirkungen des Filesharing auf ausgebliebene Musikverkäufe. ${ }^{212}$ Um die konkrete Masse an Filesharing festzustellen, wird der Faktor der „FilesharingAffinität ${ }^{\text {“213 }}$ der Internetnutzer ermittelt, wofür entscheidend sei, wie viele Personen Filesharing betreiben und mit welcher Intensität sie dies tun. ${ }^{214}$ Da diese Affinität von vielen Faktoren wie etwa der technologischen und demografischen Entwicklung, der Marktpreise usw. abhängig sei, müsse dieser Wert für jede zeitliche Periode gesondert ermittelt werden. ${ }^{215}$ Liebowitz macht dabei insbesondere das Alter der User als eine der wesentlichen Ursachen für das Ausbleiben von Albumverkäufen aus, da gerade junge Menschen die intensivsten Musikkäufer und gleichzeitig die intensivsten Filesharer seien. ${ }^{216}$ Insgesamt kommt die Studie zu dem Ergebnis, dass im Jahr 2003 aufgrund des Filesharing durchschnittlich nur 2,44 Alben statt 3,74 Alben pro Kopf gekauft worden seien, was einen Unterschied von 1,29 Alben pro Kopf ausmache. ${ }^{217}$ Insgesamt gelangt Liebowitz zu dem Ergebnis, dass File-

\footnotetext{
${ }^{205}$ S. auch US Government Accountability Office (GAO), 2010, S. 16; zusammenfassend Grassmuck, 2010, S. 16 mwNachw.

${ }^{206}$ Hargreaves, Digital Opportunity, 2011, S. 69, der zudem darauf verweist, dass die Studien, die sich allein an dem Datenvolumen orientieren, zu Fehlschlüssen verleiten, da auch Spam und Videostreaming zu höheren Auslastungen geführt haben.

${ }^{207}$ Zu dieser Studie s. unten ausf. Rn. $96 \mathrm{ff}$.

${ }^{208}$ S. zur Kritik im Detail Hargreaves, Digital Opportunity, Supporting Document CC, Data on the Prevalence and Impact of Piracy and Counterfeiting, 2011, S. $3 \mathrm{ff}$.

${ }^{209}$ Zu den Zahlen s. Hargreaves, Digital Opportunity, Supporting Document CC, Data on the Prevalence and Impact of Piracy and Counterfeiting, 2011, S. 10.

210 "Internet penetration", was Liebowitz als den Anteil der Internetnutzer einer Stadt definiert, Liebowitz, 2006, S. 20.

${ }^{211}$ Liebowitz, 2006, S. 10.

212 Liebowitz, 2006, S. 14 ff., 23 f.

213 „Filesharing prospensity“.

${ }^{214}$ Liebowitz, 2006, S. 15.

${ }^{215}$ Liebowitz, 2006, S. $15 \mathrm{ff}$.

${ }^{216}$ Liebowitz, 2006, S. 17 ff., insb. 20.

${ }^{217}$ Liebowitz, 2006, S. 23.
} 
sharing nicht nur für den Rückgang, sondern auch für ein nicht entstandenes Wachstum in der Musikindustrie verantwortlich sei. ${ }^{218}$

90 Dass indes weder die Zahl der Internet-Nutzer bzw. deren prozentualer Anteil an der Bevölkerung noch die Qualität der Internetanschlüsse noch die Rechtmäßigkeit von Privatkopien aufgrund von Filesharing-Angeboten mit dem Anteil an Filesharing zu tun hat, zeigt eine Studie im Auftrag niederländischer Ministerien, die trotz einer gegenüber der USA wesentlich höheren Durchdringung mit Internetanschlüssen keine signifikanten Unterschiede zwischen den USA und den Niederlanden hinsichtlich der Filesharing-Aktivitäten feststellen konnte. ${ }^{219}$

91 Die offenbar einzige verlässliche Methode - mit der teilweise die Content-Industrie selbst arbeitet, etwa in Gestalt der Ermittlungen der Fa. BigChampagne - ist die eigene Teilnahme an FilesharingNetzwerken, um zu ermitteln, welche Inhalte angeboten werden. Demgemäß versuchen einige Studien, mit diesen „harten“ Fakten zu arbeiten. ${ }^{220}$ Eine reine Untersuchung von Datenströmen stößt angesichts der teilweise verschlüsselten Inhalte jedoch auf Probleme; hinzukommen verschiedene Protokolle, die von diversen Filesharing-Methoden verwandt werden. ${ }^{221}$ Hierauf ist im Rahmen der Messung für die Verteilung von Abgaben zurückzukommen. ${ }^{222}$ Selbst bei eigener Teilnahme an den Netzwerken erscheint es aber fraglich, ob tatsächlich das Ausmaß an Nachfrage nach bestimmten Titeln bestimmt werden kann, da nur das Angebot abgefragt werden kann; allerdings spricht einiges dafür, dass das Angebot in etwa der Nachfrage entspricht, etwa wenn bestimmte Werke oft angeboten werden.

92 Den vorliegenden Untersuchungen mit Hilfe der Daten aus den Filesharing-Netzwerken zufolge scheinen aber keine (ausschließlich) positiven Korrelationen zwischen Filesharing-Aktivität und Umsatzrückgang jedenfalls in der Musikindustrie zu bestehen, ${ }^{223}$ zumindest nicht für alle Teile des Marktes. ${ }^{224}$ So wird bspw. in der Studie von Blackburn zwischen unbekannten und bekannten Künstlern differenziert, wonach letztere Umsatzeinbußen hinnehmen müssen, erstere aber durch Filesharing eher zu profitieren scheinen. ${ }^{225}$ Allerdings sind auch diese Studien nicht frei von Kritik geblieben. ${ }^{226}$ Kritisiert wird ferner, ${ }^{227}$ dass nur wenige Studien sich auf ein breites, verlässliches statistisches Material stützen können.

\footnotetext{
${ }^{218}$ Liebowitz, 2006, S. 24.

${ }^{219}$ Huygen et al., 2009, S. $86 \mathrm{f}$.

${ }^{220}$ Tanaka, 2004; Bhattacharjee et al., Management Science, Vol. 53, 2007, 1359 ff.; Balázs/Lakatos, International Journal of Communication, Vol. 6, 2012, $413 \mathrm{ff}$.

${ }^{221}$ S. dazu Grassmuck, 2010, S. 17.

${ }^{222}$ S. unten Rn. $393 \mathrm{ff}$.

${ }^{223}$ So auch das Fazit von Oberholzer-Gee/Strumpf, 2007, S. 24 f.; Grassmuck, 2010, S. 17; zum Video-/DVDMarkt ebenso Smith/Telang, 2008, S. 30 ff. die allerdings nur solche Filme untersucht haben, die schon im Fernsehen ausgestrahlt wurden und explizit vorherige Verwertungsstufen ausnehmen.

${ }^{224}$ Nach Blackburn, On-line Piracy and Recorded Music Sales, 2004, S. 47 seien Schätzungen, welche innerhalb der heterogenen Gruppe aller Künstler nicht differenzierten „naïve“ und es sei unrealsistisch, dass File-Sharing auf alle Künstler die gleichen Auswirkungen habe.

${ }^{225}$ Blackburn, On-line Piracy and Recorded Music Sales, 2004, S. 47 f.; ähnlich Mortimer/Nosko/Sorensen, 2010, S. 19.

${ }^{226}$ S. etwa die Kritik von Liebowitz, 2005, an Oberholzer-Gee/Strumpf, 2004 - Studie; dies wiederum kritisiert von Tschmuck, Teil 3, 2009.

${ }^{227}$ Tschmuck Economics, 2010, passim.
} 


\section{b) Nutzungsverhalten in Deutschland}

93 Die aktuelle, im Auftrag der Rechteinhaber erstellte Studie zur digitalen Content-Nutzung (DCNStudie 2012, ${ }^{228}$ bis 2010 sog. „Brennerstudie“) hält fest, dass im Jahr 2011 mehr als ein Drittel der Deutschen (22,1 Mio. Personen bzw. 35\%) Medieninhalte entweder online genutzt, z.B. durch Streaming, oder aus dem Internet heruntergeladen hat. ${ }^{229} \mathrm{Ca}$. ein Viertel von diesen nutzte Medieninhalte ausschließlich online, der Rest lud diese ausschließlich oder zumindest auch herunter. Dabei stellten Musikstücke die meistgenutzte bzw. -geladene Werkkategorie dar: 18,5 Mio. Menschen (im Vorjahr 15,6 Mio.). Dahinter liegen TV-Serien (9,7 Mio.), Spiel- und Kinofilme (7,1 Mio.), Hörbücher (4,0 Mio.) und E-Books (3,4 Mio.). Dies beinhaltet sowohl die legale wie auch die illegale Nutzung.

Von den 14,8 Mio. Menschen, die Medieninhalte 2011 zumindest auch online nutzten, taten dies 7,9 Mio. über Videostreaming-Plattformen wie YouTube, 6,1 Mio. über Online-Radios und Mediatheken und immerhin 2,5 Mio. über Streaming-Portale für Filme, wie z.B. „movie2k“ ${ }^{230}$ Das Streaming gewinnt insbesondere für audiovisuelle Medieninhalte (TV-Serien, Spiel- und Kinofilme) an Bedeutung: Ca. drei Viertel derjenigen, die Inhalte dieser Art unter Verwendung des Internet konsumierten, nutzen dafür überwiegend Streaming-Portale, während nur 6\% (für TV-Serien) bzw. 10\% (für Filme) angaben, die Inhalte überwiegend herunterzuladen. Bei Musik verwendeten 52\% der Nutzer StreamingAngebote, 22\% hingegen überwiegend Download-Angebote. ${ }^{231}$ Auf alle Medieninhalte bezogen, haben ca. 90\% der Streaming-Nutzer angegeben, Streaming-Angebote im Jahr 2011 im Vergleich zum Vorjahr gleich häufig oder sogar häufiger verwendet zu haben. ${ }^{232}$ Insbesondere die audiovisuellen Medieninhalte sind es, die häufig über von der DCN-Studie als „illegal“ gekennzeichneten StreamingPortale wie "movie2k" konsumiert werden: $41 \%$ derjenigen, die Filme online anschauen, haben dies 2011 über Portale dieser Art getan, bei TV-Serien sind es noch 19\%. ${ }^{233}$ Demgegenüber verläuft die Online-Nutzung von Hörbüchern mit $43 \%$ zum größten Teil legal über die offiziellen Webseiten von Künstlern oder Verlagen. Nur 30\% der Online-Nutzer von Hörbüchern konsumierten diese über Videostreaming-Plattformen wie YouTube, wobei unklar bleibt, ob die Studie diese Form der Nutzung (bzw. das Anbieten auf diesen Plattformen) als legal oder illegal einstuft. Bei Musik werden diese Videostreaming-Plattformen sogar mit 54\% mehrheitlich genutzt.

Von den 16,3 Mio. Deutschen, die 2011 Medieninhalte aus dem Internet heruntergeladen haben, verwendeten 44,3\% ausschließlich Quellen, die von der DCN-Studie als legal eingestuft ${ }^{234}$ wurden. ${ }^{235}$ 19\% der Downloader nutzten wiederum illegale Quellen wie P2P-Netzwerke oder Sharehoster, 3,6\%

\footnotetext{
${ }^{228}$ Durchgeführt von der Gesellschaft für Konsumforschung (GfK) im Auftrag des Bundesverbands Musikindustrie e.V., der Gesellschaft zur Verfolgung von Urheberrechtsverletzungen e.V. (GVU) und des Börsenvereins des Deutschen Buchhandels e.V., Presseversion abrufbar unter:

http://www.musikindustrie.de/fileadmin/piclib/presse/Dokumente_zum_Download/DCN-

Studie_2012_neu_Presseversion_Final.pdf.

${ }^{229}$ Bemessungsgrundlage: 63,6 Mio. private Deutsche ab 10 Jahre, s. GfK, DCN-Studie 2012, Vollversion, S. 12.

${ }^{230}$ GfK, DCN-Studie 2012, Vollversion, S. 14.

${ }^{231}$ GfK, DCN-Studie 2012, Vollversion, S. 16.

${ }^{232}$ GfK, DCN-Studie 2012, Vollversion, S. 17.

${ }^{233}$ GfK, DCN-Studie 2012, Vollversion, S. 18.

${ }^{234}$ Als legal zählten kostenpflichtige Downloadmöglichkeiten über Online-Shops wie iTunes, über offizielle Künstler- und Verlagsseiten sowie über Plattformen für lizenzfreie Inhalte; als illegal zählten Downloadmöglichkeiten über Tauschbörsen, Peer-to-Peer-Netzwerke, ftp-Server, Newsgroup Services, Blogs, Foren, Boards, Sharehoster und Cyberlocker; als Grauzone wurde das Mitschneiden auf Videostreaming-Plattformen wie YouTube sowie das individuelle oder automatisierte Mitschneiden von Online-Radios oder Podcasts gewertet, s. GfK, DCN-Studie 2012, Vollversion, S. 19.

${ }^{235}$ GfK, DCN-Studie 2012, Vollversion, S. 21.
} 
sogar ausschließlich. Die von der DCN-Studie als Graubereich bewerteten Downloadmöglichkeiten wurden von mehr als einem Drittel (37\%) der Downloader genutzt. Innerhalb dieses sog. Graubereichs beziffert die DCN-Studie die Zahl der Personen, die Musik von Webradios oder Podcasts mithilfe spezieller Aufnahmesoftware mitgeschnitten haben, mit 2,6 Mio. Die entsprechenden Aufnahmen von Musikvideos auf YouTube wurden der Studie zufolge von 4,4 Mio. Personen durchgeführt. ${ }^{236}$

\section{c) Ökonomische Auswirkungen des Filesharing}

Sowohl für die verfassungs- als auch europarechtliche Bewertung (im Rahmen des Dreistufen-Tests) können die Auswirkungen des Filesharing auf Umsatz- und Erlösrückgänge in den Content-Industrien (Musik, Film, E-Books und andere) eine Rolle spielen, so dass hier ein Blick auf die verschiedenen empirischen Studien zu werfen ist. Allerdings ergibt sich hier kein einheitliches Bild - wobei nochmals auch die methodologischen Probleme zu betonen sind.

\section{(1) Der Substitutionseffekt}

97 Zahlreiche Untersuchungen führen für den US-amerikanischen Markt im „worst case“ nicht mehr als $20 \%{ }^{237}$ oder $30 \%{ }^{238}$ der Umsatzeinbußen auf Filesharing zurück.

Im deutschen Markt sollen im Rahmen der bereits zitierten DCN-Studie bzw. den Untersuchungen der GfK zufolge 73\% derjenigen, die Musik ausschließlich aus illegalen Quellen heruntergeladen haben, nicht bereit sein, Geld für Musik auszugeben, die übrigen 27\% derselben Gruppe nur ca. 18 Euro pro Jahr, während jeder Deutsche im Durchschnitt 56 Euro pro Jahr für Musik ausgibt. ${ }^{239}$ Ferner wird berichtet, dass die Umsätze der deutschen Musikindustrie von 2,4 Mrd. Euro im Jahre 2001 auf 1,5 Mrd. Euro im Jahr 2010 zurückgegangen seien, während die Zahl der illegal heruntergeladenen Musikstücke von 429 Mio. auf 900 Mio. gestiegen sei. ${ }^{240}$ Die Umsätze der Musikindustrie sollen von 2,4 Mrd. Euro im Jahre 2001 auf 1,5 Mrd. Euro im Jahr 2010 zurückgegangen sein, dabei von 429 Mio. illegaler Musik-Downloads auf 900 Mio. Downloads. ${ }^{241}$ Die sog. TERA-Studie kommt zu dem Ergebnis, dass illegale Downloads normale Angebote zu 10 bis $30 \%$ substituieren; demnach habe die deutsche Musikindustrie einen Verlust von 86 Mio. Euro im Jahre 2008 erlitten. ${ }^{242}$ Andere von der (ausländischen) Musikindustrie in Auftrag gegebene Studien sprechen etwa von 180 Mio. Pfund Schaden im Jahr 2008. ${ }^{243}$ Dies deckt sich in etwa mit den Ergebnissen der TERA-Studie, die für das Vereinigte Königreich einen Schaden von 230 Mio. Euro durch illegale Downloads errechnet hat. ${ }^{244}$ In Spanien sollen es sogar 413 Mio. Euro gewesen sein, in Italien 262 Mio. Euro. ${ }^{245}$ Der internationale Musikverband IFPI gibt an, dass seit 1997 die Umsätze um mehr als 55\% zurückgegangen sind, wobei zwar das

\footnotetext{
${ }^{236}$ GfK, DCN-Studie 2012, Vollversion, S. 53.

${ }^{237}$ Rob/Waldfogel, 2004, S. 3; allerdings stößt die Studie von Rob/Waldfogel auf erhebliche Vorbehalte, da sie nicht repräsentativ ist, vgl. Tschmuck, Teil 4, 2009; s. auch Oberholzer-Gee/Strumpf, 2010, S. 16 mwNachw.

${ }^{238}$ So die Studie von Zentner, Measuring the Effect of File Sharing on Music Purchases, Journal of Law \& Economics, Vol. 49, No. 1, 2006, 63, 66, der allerdings in einer ersten Regression genau den umgekehrten Effekt feststellte, dass Filesharing zu mehr CD-Käufen führte, dies dann aber verwarf; krit. daher Tschmuck, Teil 8, 2009.

${ }^{239}$ Bundesverband Musikindustrie, Musik im digitalen Wandel, 2012, S. 8; s. auch GfK, DCN-Studie 2011, Presseversion, S. 22.

${ }^{240}$ Bundesverband Musikindustrie, Musik im digitalen Wandel, 2012, S. 13 f., 15.

${ }^{241}$ Bundesverband Musikindustrie, Musik im digitalen Wandel, 2012, S. 13 f., 15.

242 TERA Consultants, 2010, S. 26; die Studie wurde durchgeführt von der Firma TERA Consultants und ist Teil der „Initiative Business Action to Stop Counterfeiting and Piracy“ (BASCAP) der Internationalen Handelskammer, s. http://www.bascap.com/.

243 IFPI Digital Music Report 2009, S. 22.

${ }^{244}$ TERA Consultants, 2010, S. 32.

245 TERA Consultants, 2010, S. 28, 30.
} 
Download-Geschäft zugenommen hat, dieses aber beileibe nicht den gesamten Rückgang kompensieren konnte. ${ }^{246}$

Die Musikindustrie zieht dabei eine direkte kausale Verbindung zwischen dem urheberrechtswidrigen Nutzungsverhalten und den erlittenen finanziellen Einbußen, gestützt auf die Ergebnisse der dargestellten TERA-Studie. Die dort im März 2010 im Auftrag der Internationalen Handelskammer ermittelte Substitutionsrate zwischen 10 und 30\% differenziert nach der Art der Medieninhalte (Musik/Film/TV-Serien). Der Gesamtschaden der deutschen audiovisuellen Industrie belief sich der TERAStudie zufolge im selben Jahr auf 446 Mio. Euro, verbunden mit einem Verlust von 25.400 Arbeitsplätzen. ${ }^{247}$

100 Allerdings sind Zweifel an den Berechnungsmethoden sowie an der Nutzung veralteter Zahlen der Studien zu den Substitutionsraten nicht zu verhehlen, worauf zurückzukommen ist. ${ }^{248}$ Hinsichtlich der Methodik wird insbesondere an den kausalen Zusammenhängen zwischen Internetpenetration und Filesharing gezweifelt ${ }^{249}$ und darüber hinaus bei auf Befragungen basierenden Studien auf nicht repräsentative Personengruppen hingewiesen, die keine Grundlage für eine allgemeine Substitutionsrate bilden könnten. ${ }^{250}$

101 Insgesamt verwundern die höchst unterschiedliche Einschätzungen hinsichtlich der tatsächlichen Effekte daher nicht: ${ }^{251}$ So stellt die Hargreaves-Studie in einem Vergleich der verschiedenen für den angelsächsischen Raum (Vereinigtes Königreich und USA) vorliegenden Studien erhebliche Unterschiede in den Angaben fest. So wird etwa von Musikverbänden im Vereinigten Königreich auf einen Anteil von 76\% illegalen Downloads verwiesen für das Jahr 2010, ${ }^{252}$ während andere Studien nur auf $13 \%$ kommen. ${ }^{253}$ Die meisten Studien beruhen indes auf Befragungen von Internetteilnehmern, teilweise unterschiedlichen Alters. Zudem ist es schwer, die stets angegebenen Verluste in ein Verhältnis zur Marktentwicklung zu setzen. So hält Hargreaves etwa fest, dass sowohl im Musik- als auch Buchund auch Filmmarkt ein kontinuierliches Wachstum, zumindest aber ein konstant hohes Niveau an Umsatzzahlen von 2004 bis 2009 festzustellen sei. ${ }^{254}$ Dies steht im Einklang mit den Ergebnissen von Waldfogel, der keinen Rückgang der Anzahl an Neuerscheinungen seit dem Aufkommen von P2PFilesharing festgestellt hat, sondern vielmehr eine Verlagerung von den Major-Labels hin zu Independent-Labels, da diese auch schon mit geringeren Stückzahlen einen Gewinn erwirtschaften können. ${ }^{255}$

102 Eine direkte Kausalität zwischen dem Filesharing und den erlittenen Schäden, insbesondere aber auch deren Ausmaße werden in verschiedener Hinsicht in Frage gestellt. Einigkeit besteht schon nicht über den zugrunde zu legenden methodischen Ansatz bei der Überprüfung der Folgen von Filesha-

\footnotetext{
${ }^{246}$ S. dazu die Studie http://paidcontent.co.uk/image/set/recording-industry-in-numbers-2009/P2/.

${ }^{247}$ TERA Consultants, 2010, S. 26.

${ }^{248}$ Tschmuck, Ökonomische Folgen, 2011; Oberholzer-Gee/Strumpf, 2009, S. 16 ff.

${ }^{249}$ Tschmuck, Ökonomische Folgen, 2011; Oberholzer-Gee/Strumpf, 2009, S. 17 f.

250 Oberholzer-Gee/Strumpf, 2009, S. 16 f.; Tschmuck, Ökonomische Folgen, 2011.

${ }^{251}$ S. zu den unterschiedlichen möglichen Effekten des Filesharing auch den Überblick bei van Eijk/Poort/Rutten, Communication \& Strategies, Vol. 77, 1. Quart. 2010, S. 35, 46.

${ }^{252}$ So die Untersuchung der British Recorded Music Industry (BPI), Digital Music Nation 2010 - The UK's legal and illegal digital music landscape, S. 27.

${ }^{253}$ Hargreaves, Digital Opportunity, 2011, S. 70, unter Verweis auf Music Matters/Synovate/MidemNet, Global Survey of 8,500, aged $18+$ in 13 countries, 2010.

${ }^{254}$ Hargreaves, Digital Opportunity, 2011, S. 74.

${ }^{255}$ Waldfogel, 2011, S. 26 f.; siehe auch ders., J.Law \& Econ. 2013, 715, 736.
} 
ring. ${ }^{256}$ Dementsprechend variieren auch die Ergebnisse. ${ }^{257}$ Der Substitutionseffekt kann dabei zwar als Ausgangspunkt grundsätzlich als gesichert gelten; doch werden seine Ausmaße höchst unterschiedlich beurteilt. In Bezug auf Musik wird von den bisher durchgeführten Studien regelmäßig von einer Substitutionsrate von etwa $20 \%$ ausgegangen, ${ }^{258}$ so dass sich die TERA-Studie ebenfalls innerhalb dieser Schätzung bewegt. In Bezug auf CD-Verkäufe geht die TERA-Studie allerdings etwa für das Vereinigte Königreich von einer Substitutionsrate von $48 \%$ aus. ${ }^{259}$ Dieser Wert ist vom HargreavesReport aus methodischen Gründen in Zweifel gezogen worden. Denn TERA stützt sich dabei auf eine Untersuchung, die die Auswirkungen der „Musikpiraterie“ der Jahre 1994 bis 1998 in den Blick nimmt, in der der fragliche Wert mit $14 \%$ oder $42 \%$ angegeben wird, abhängig von der Berechnungsmethode. ${ }^{260}$ Dabei ist nicht nur der fehlende Beleg für den Aufschlag von $6 \%$ auf den Maximalwert der Vorbildstudie auffällig, auch deren Untersuchungszeitraum lässt an der Allgemeingültigkeit der Zahlen zweifeln. Untersucht wurde schließlich ein Zeitraum, in dem die Distributionskanäle für Musik sich noch gänzlich auf die Weitergabe von Datenträgern wie CDs beschränkten. Ob sich diese Aussagen eins-zu-eins auf die heutige Zeit übertragen lassen, ist fraglich. Vereinzelt wird aber auch von deutlich niedrigeren Substitutionsraten ausgegangen, die sich im Bereich von 5 bis $7 \%$ bewegen. ${ }^{261}$

\section{(2) Der Sampling-Effekt (Promotionseffekt, „Discovery“)}

103 Dieser Substitutionseffekt kann aber offenbar durch einen sog. Sampling-, ${ }^{262}$ Netzwerk- oder auch "discovery“- bzw. Promotions-Effekt ausgeglichen werden. ${ }^{263}$ Ob daher überhaupt der gesamte Turnover der Musikindustrie beeinträchtigt wird, erscheint offen: Insbesondere im Zusammenhang mit Musik, wo zumindest ein Teil der Nutzer das Filesharing in erster Linie dazu verwendet, sich Musik zunächst kostenlos zu beschaffen und probezuhören, um dann ggf. das oder die Musikstücke kostenpflichtig zu erwerben. ${ }^{264}$ Innerhalb der bisher durchgeführten Untersuchungen variiert die Einschätzung, wie stark der Sampling-Effekt durch Filesharing unterstützt wird und inwieweit er in der Lage ist, den negativen Substitutionseffekt auszugleichen. Eine besondere Position nimmt allerdings Liebowitz ein, der in seiner häufig von der Musikindustrie zitierten Studie den Sampling-Effekt im Zusammenhang mit Musiktauschbörsen als ausschließlich nachfragehemmend bewertet, so dass auch dieser ausschließlich negative Folgen für die Absätze der Musikindustrie nach sich ziehe. ${ }^{265}$ Die deutsche Musikindustrie sieht den Sampling-Effekt sogar als gänzlich widerlegt an und beruft sich dabei auf die oben bereits genannten Ergebnisse die GfK-Untersuchungen, dass 73\% der MusikDownloader kein Geld für Musik ausgeben wollen und der Rest lediglich bereit wäre, maximal 18 Euro pro Jahr zu bezahlen. ${ }^{266}$ Diese These kann allerdings nur sehr eingeschränkte Gültigkeit bean-

\footnotetext{
${ }^{256}$ S. dazu die Kritik bei Tschmuck, Ansätze, 2009.

${ }^{257}$ Tschmuck, Teil 18, 2009.

${ }^{258}$ S. Oberholzer-Gee/Strumpf, 2009, S. 16.

259 TERA Consultants, 2010, S. 54.

${ }^{260}$ Hargreaves, Digital Opportunity, Supporting Document CC, Data on the Prevalence and Impact of Piracy and Counterfeiting, 2011, S. 8.

${ }^{261}$ So Huygen et al., 2009, S. 105.

${ }^{262}$ Nicht zu verwechseln mit dem ebenfalls Sampling genannten Vorgang, bei dem bestimmte Melodieteile eines Musikwerkes diesem entnommen und in einem anderen Werk wiederverwendet werden, s. oben Rn. 62 ff.

${ }^{263}$ S. die Studien von Peitz/Waelbroeck, 2006; Bounie/Bourreau/Waelbroeck, 2005; Hendricks/Sorensen, Journal of Political Economy, Vol. 117, No. 2, 2009, S. 324 ff.; s. ferner die Zusammenfassung aller vorliegenden Studien bei Grassmuck, 2010, S. 19 ff. mwNachw.

${ }^{264}$ Statt vieler S. Blackburn, On-line Piracy and Recorded Music Sales, 2004, S. 9; ferner Liebowitz, 2005 , S. 15.

${ }^{265}$ Liebowitz, 2005, S. $16 \mathrm{ff}$.

${ }^{266}$ Bundesverband Musikindustrie, Musik im digitalen Wandel, 2012, S. 8.
} 
spruchen und darf nicht verallgemeinert werden. Denn diese Prozentsätze beziehen sich lediglich auf die Gruppe derjenigen Musik-Downloader, die ausschließlich illegale Quellen verwenden. Die GfK kam in der aktuellen DCN-Studie zu dem Ergebnis, dass dies lediglich 3,6\% aller deutschen MedienDownloader betrifft. ${ }^{267}$ Zusammen genommen bedeutet das, dass nur ca. 2,6\% derjenigen, die Musik herunterladen, nicht bereit sind, Geld für diese auszugeben.

104 Zudem führte Filesharing offenbar zu einer erhöhten Nachfrage nach Livekonzerten, ebenso offenbar zu einer Erhöhung der Konzertpreise, mit denen anscheinend die Verluste für den Verkauf von Tonträgern mehr als kompensiert wurden. Sowohl den Studien von Oberholzer-Gee/Strumpf als auch von Blackburn zufolge sind die positiven Auswirkungen des Sampling-Effekts in der Lage, den Substitutionseffekt vollständig auszugleichen, so dass Filesharing letztlich weder pauschal positive noch negative Folgen habe. ${ }^{268}$ Blackburn unterscheidet allerdings zusätzlich noch zwischen den Auswirkungen auf die Verkaufszahlen bereits etablierter, bekannter Künstler und Newcomern. Bei ersteren sei der Substitutionseffekt größer, während letztere besonders vom Sampling-Effekt profitierten. ${ }^{269}$ Zu dem Ergebnis, dass der Sampling-Effekt den Substitutionseffekt nicht nur aufwiege, sondern übertreffe, so dass Filesharing im Ergebnis der Musikindustrie nutze, kommen Andersen/Frenz in ihrer kanadischen Studie von 2007 . $^{270}$

105 Auch kommen empirische Studien in den USA zum Anstieg von Konzerten in der Zeit zwischen 1995 und 2004 zu einer indirekten Bestätigung der "Sampling“-Effekte, indem ab 2001 ein starker Anstieg hinsichtlich der Einkünfte aus Konzerten verzeichnet werden konnte. Sowohl die Preise für Eintrittskarten als auch die Häufigkeit von Konzerten haben hier überproportional zugenommen. ${ }^{271}$ Negative Effekte entstehen durch Filesharing offenbar vor allem für etablierte Stars, während eher unbekannte Musiker von Filesharing profitieren, indem ihr Bekanntheitsgrad und dadurch auch der Verkauf ihrer Musik steigen. ${ }^{272}$ Allerdings konnte dies in einer neueren Studie nicht belegt werden; hier führten illegale, vor dem eigentlichen Release-Datum im Internet herunterladbare Musiktitel dazu, dass eher die etablierten Stars erhöhte Verkaufszahlen verzeichnen konnten, am wenigstens dagegen die Independent-Labels mit unbekannten Musikern. ${ }^{273}$

106 Auch jüngere publizierte Studien, die allerdings auf Umfragen beruhen, weisen darauf hin, dass Filesharer wesentlich mehr Musik erworben haben als andere. ${ }^{274}$ Eine andere jüngst erschienene Studie zu Download-Zahlen im Filesharing-Bereich und kurz danach veröffentlichten Musiktiteln führt sogar zu dem Ergebnis, dass Filesharing positive Auswirkungen auf die Verkaufszahlen habe. ${ }^{275}$ In ähnlicher Weise gelangen Peukert/Claussen für den Filmbereich nach Schließung der Webseite „Megaupload" zu dem Ergebnis, dass eine größere Anzahl an Zuschauern (insbesondere solche, die ohnehin nichts bezahlt hätten) durch das Filesharing erschlossen worden sei, vor allem infolge einer vermehrten Informationsverbreitung über den Film, z.B. durch Postings in sozialen Netzwerken. Das

\footnotetext{
${ }^{267}$ GfK, DCN-Studie 2012, Vollversion, S. 21.

${ }^{268}$ Blackburn, On-line Piracy and Recorded Music Sales, 2004, S. 13.

${ }^{269}$ Blackburn, On-line Piracy and Recorded Music Sales, 2004, S. 13.

${ }^{270}$ Andersen/Frenz, 2007, S. 28 f.

${ }^{271}$ Oberholzer-Gee/Strumpf, 2010, S. 20; unter Verweis auf Mortimer/Nosko/Sorensen, 2010, S. 19 f.; s. auch

für Schweden die Studie von Johansson/Larsson, 2009; zusammenfassend Grassmuck, 2010, S. 37 f.

${ }^{272}$ So im Wesentlichen das Ergebnis der Studie von Blackburn, On-line Piracy and Recorded Music Sales, 2004, S. 5; in die gleiche Richtung gehend: Mortimer/Nosko/Sorensen, 2010, S. 19.

${ }^{273}$ Hammond, 2012, S. $21 \mathrm{ff}$.

274 The American Assembly, Copyright Infringement and Enforcement in the USA, Research Note, 2011.

${ }^{275}$ Hammond, 2012, S. 21 ff.
} 
wiederum habe einen positiven Effekt auf die Kinobesucherzahlen erzeugt, da so mehr zahlungswillige Konsumenten von dem Film erführen. ${ }^{276}$ Nach Schließung des großen Sharehosters Megaupload seien dagegen negative Auswirkungen auf die Kinoumsätze zu beobachten gewesen. Dem wird allerdings seitens der Gesellschaft zu Verfolgung von Urheberrechtsverletzungen e.V. (GVU) entgegengehalten, dass die Studie auf Umsätze und nicht Besucher abstelle und dass in Deutschland die Zuschauerzahlen in den letzten Jahren gestiegen seien, was auch weiterhin der Fall sei. ${ }^{277}$

107 Für Europa deuten neuere Studien auf vergleichbare Ergebnisse hin:

108 Eine Studie der University of Hertfordshire im Auftrag der British Music Rights im Jahr 2008 unter Jugendlichen von 14 bis 25 Jahren ergab, dass 60\% der Befragten Filesharing betrieben, um vor dem Kauf einer CD die Musik zur Probe zu hören. Interessanterweise spielt die Frage der Qualität und die Sicherheit eines CD-Kaufs gegenüber Filesharing eine geringere Rolle (im Gegensatz zu einer Umfrage in Schweden), ${ }^{278}$ vielmehr der Besitz eines physischen Tonträgers und die künstlerische Gestaltung etwa eines Booklets. Nur 45\% der befragten britischen Jugendlichen gaben den Vorteil der Soundqualität als Grund für einen Kauf an, während der Besitz des Tonträgers immerhin für fast zwei Drittel (64\%) und die Gestaltung noch für $57 \%$ ausschlaggebend ist. ${ }^{279}$ Auch verändert sich mit abnehmendem Alter die Art des Musikkonsums, indem zu Lasten von physischen Tonträgern die Downloads zunehmen. Ebenso ergibt sich ein deutliches Übergewicht zugunsten von Konzertkarten. ${ }^{280}$

109 Ähnliche Resultate ergab eine breit angelegte repräsentative Umfrage unter holländischen Internetnutzern im Auftrag der niederländischen Regierung. ${ }^{281}$ Demnach hatte das Filesharing offenbar keine Auswirkung auf das Kaufverhalten der Internetnutzer, indem diese gegenüber den NichtFilesharern in vergleichbarer Weise Musik und Filme kauften. ${ }^{282}$ Demgegenüber ist die Neigung zu bezahlten Download-Diensten wie iTunes oder Amazon eher gering und zeigt zudem eine abnehmende Tendenz; offensichtlich wird hier kein Unterschied hinsichtlich der Qualität der heruntergeladenen Werke gesehen. ${ }^{283}$ Verlusten der Musikindustrie in Höhe von 100 Mio. Euro stünde demnach ein Gesamtwohlfahrtszuwachs in Höhe von 200 Mio. Euro gegenüber. ${ }^{284}$

110 Umgekehrt liegen inzwischen die ersten Zahlen aus Frankreich hinsichtlich der Effektivität der dort eingeführten Three-Strikes-Abschreckung (HADOPI) als Rechtsverfolgungsmaßnahme mit Hilfe der Provider vor. Demnach soll der Abschreckungseffekt gegenüber den abgemahnten Internetteilnehmern fast mehr als $97 \%$ betragen haben. ${ }^{285}$ Insgesamt soll die Nutzung von illegalem Filesharing um $43 \%$ oder gar um $66 \%$ für Filme abgenommen haben, sofern „Network centric“-Analysen ${ }^{286}$ verwandt

\footnotetext{
${ }^{276}$ Peukert/Claussen, Piracy and Movie Revenues: Evidence from Megaupload, 2012, S. 2.

${ }^{277}$ Gesellschaft zur Verfolgung von Urheberrechtsverletzungen e.V., Pressemitteilung vom 26.11.2012.

${ }^{278}$ STIM, Pirates, file-sharers and music users. A survey of the conditions for new music services on the Internet, 2009, S. 11: 60,9\% gaben hier als Nachteil des Filesharing die Verseuchung mit Viren an, ebenso die schlechtere Filequalität, 66,2\% aber auch die Illegalität des Filesharing.

${ }^{279}$ Bahanovich/Collopy, 2009, S. 20.

${ }^{280}$ Bahanovich/Collopy, 2009, S. 27.

${ }^{281}$ Huygen et al., 2009.

${ }^{282}$ Huygen et al., 2009, S. 82: 68\% bei Musik, 61\% bei Filmen kauften dann auch Musik und DVDs ein.

${ }^{283}$ Huygen et al., 2009, S. 81.

${ }^{284}$ Huygen et al., 2009, S. 105, 107.

${ }^{285}$ HADOPI, 2012, S. 3.

${ }^{286}$ Hier wurden in P2P-Netzwerken ein Sample von bestimmten Werken wie Filmen auf die Häufigkeit ihres Angebots untersucht, nicht allerdings Filesharing außerhalb solcher Netzwerke, so dass unklar bleibt, wie etwa Rapidshare oder andere Filehoster einbezogen wurden, s. dazu HADOPI 2012, S. 7.
} 
wurden. ${ }^{287}$ Der Besuch von entsprechenden P2P-Webseiten soll um $17 \%$ bzw. $29 \%$ abgenommen haben. ${ }^{288}$

111 Die unterschiedlichen Ergebnisse sind darauf zurückzuführen, dass der Bewertung der Auswirkungen von HADOPI unterschiedliche Messsysteme zugrunde gelegt wurden. So liegt der Zahl von $17 \%$ die Auswertung des im „Digital Music Report 2012“ des Unternehmens Nielsen angegebenen Zeitraums von Januar 2011 bis Dezember 2011 zugrunde. Begutachtet wurde die Zahl der Besucher von Webseiten, die Links zu P2P-Inhalten bereithalten. Auf einen Rückgang von 29\% kam hingegen das Unternehmen Médiamétrie, das im gleichen Untersuchungszeitraum die Anzahl der Nutzer von P2PProgrammen ermittelte. Keine Rückschlüsse lassen sich aus diesen Zahlen allerdings auf die Inhalte ziehen, die getauscht bzw. nicht getauscht wurden. Mit anderen Worten, es lässt sich kein Rückschluss ziehen, ob es sich bei dem Rückgang der generellen Nutzungszahlen zugleich um einen entsprechend hohen Rückgang des derzeit illegalen Filesharing handelt.

112 Schon an dieser Stelle ist allerdings festzuhalten, dass eine Kulturflatrate als allgemeine Schranke jede Form des Down- und Uploads erfassen würde, unabhängig davon, welche Technologien zum Tausch eingesetzt werden, ob klassisches P2P-Filesharing, BitTorrents oder Filehoster.

\section{d) Zusammenfassung}

113 Versucht man ein Fazit zu ziehen, erscheint eine genaue ökonomische Einschätzung, ob die negativen oder positiven Effekte überwiegen, kaum möglich. ${ }^{289}$ Allerdings kann festgehalten werden, dass bis zu einem bestimmten Grad Substitutionseffekte stattfinden, aber ebenso wenig eine Gleichung aufgemacht werden kann "illegaler Download = Schaden“ , da nicht gesagt ist, dass bei entsprechend möglicher Kontrolle tatsächlich entgeltlich das Musikstück gekauft worden wäre. Anders formuliert ist in Studien der Effekt festgestellt worden, dass Nutzer niemals die gleiche Musik, die getauscht wurde, oder andere gekauft hätten, ja zum Teil diese Musik nicht einmal abgespielt wurde. Geschenktes wird anscheinend gerne einfach besessen, ohne ihm wirklich einen Nutzen zuzuordnen, so dass hieraus letztlich keine Verluste resultieren, da diese Musik nie erworben worden wäre. ${ }^{290} \mathrm{Zu}$ dem liegt bislang kein empirischer Nachweis dahingehend vor, zu welchem Preis ein illegal heruntergeladenes Werk ggf. gekauft worden wäre. So ist etwa denkbar, dass zwar ein bereits als Download vorhandenes Werk gekauft wird, aber erst zu einem Zeitpunkt, wo deutliche Rabatte gegenüber dem Erstveröffentlichungstermin gewährt werden, ${ }^{291}$ was wiederum bei der Substitutionsrate Berücksichtigung finden müsste.

114 Grundsätzlich scheint ein Kausalitätsproblem zu bestehen, indem die verschiedenen Faktoren bzw. Variablen nicht eindeutig in einen Ursache-Wirkungs-Zusammenhang gebracht werden können, selbst wenn andere Indikatoren wie etwa verschiedene Geschwindigkeiten bei Verbindungen benutzt werden. ${ }^{292}$ So hält die ausführliche Studie von TNO im Auftrag der niederländischen „Regierung fest:

\footnotetext{
${ }^{287}$ HADOPI, 2012, S. 3; ähnliche Werte finden sich bei Adermon/Liang, Piracy, Music and Movies: A Natural Experiment, 2010, S. 3, in einer Untersuchung der Auswirkung der schwedischen Urheberrechtsreform, durch welche die Wahrscheinlichkeit der Verfolgung gestiegen ist.

${ }^{288}$ HADOPI 2012, S. 4.

289 US Government Accountability Office (GAO), 2010, S. 14 f.

${ }^{290}$ Oberholzer-Gee/Strumpf, 2010, S. 3 f.

${ }^{291}$ Diesen Umstand sprechen auch van Eijk/Poort/Rutten, Communication \& Strategies, Vol. 77, 1. Quart. 2010, S. 35, 46 in ihrem Überblick über die Effekte des Filesharing an.

${ }^{292}$ S. dazu Grassmuck, 2010, S. 15.
} 
"All in all, file sharing seems to have only a moderate effect on physical audio format sales. This is in line with the observed global decline in turnover. That said, there does not appear to be a direct relationship between the downturn in sales and file sharing. The state of play in the film industry has been less researched to date, but available findings unanimously suggest a negative relationship." ${ }^{\prime 293}$

Die Studie betont auch:

"The degree of substitution is difficult to determine and controversial, yet we can state with certainty that there is no one-on-one correlation between file sharing and sales." ${ }^{294}$

118 Eine einfache Gleichung dergestalt, dass jedem heruntergeladenen Musikstück ein unterlassener Kauf entspräche, kann daher nicht aufgestellt werden.

119 In der Geschichte der Medienindustrie sind solche Verdrängungs-, aber auch Komplementäreffekte nicht unbekannt. Schon mit der Einführung des Radios in den 20er Jahren wurde eine weitgehende Verdrängung der damaligen Tonträger zugunsten des Radiohörens befürchtet; ${ }^{295}$ stattdessen trat das Gegenteil ein, das Radio diente dem zusätzlichen Absatz der verbreiteten Inhalte auf Ton- oder anderen Medienträgern. Ferner zeigt gerade die Entwicklung der Radio Corporation of America (RCA), dass die vertikale Integration einer der wichtigsten Motoren war, indem der Verkauf von Radiogeräten im Vordergrund stand - nicht die Verbreitung von Inhalten oder Werbung. ${ }^{296}$ Allerdings bestand gerade wegen der unterschiedlichen Nutzungsmöglichkeiten keine unmittelbare Substituierbarkeit. In ähnlicher Weise wurde mit der Einführung des Video- und des Kassettenrecorders eine Verdrängung der Musikindustrie befürchtet (,Home taping is killing music“). ${ }^{297}$

120 Auch können andere Faktoren für den Umsatzrückgang eine Rolle spielen, ${ }^{298}$ da mit der Möglichkeit, einzelne Titel nur zu kaufen und per Download zu erwerben, der frühere Absatz von ganzen Alben zugunsten des - allerdings monetär insgesamt weniger lukrativen - Absatzes von einzelnen Songtiteln (Singlekauf statt Album) abgenommen hat. ${ }^{299}$ Weitere Faktoren können in der Sättigung des Marktes mit CDs bestehen, ${ }^{300}$ so dass der Markt sich jetzt unter Umständen einem normalen Niveau nähern könnte, ferner die Umschichtung in den Budgets der kaufwilligen Nutzerschichten, insbesondere von Jugendlichen, ${ }^{301}$ die Änderung der Verkaufsstrategie der „Majors“ durch die Konzentration

\footnotetext{
${ }^{293}$ Huygen et al., 2009, S. 83.

${ }^{294}$ Huygen et al., 2009, S. 103.

${ }^{295}$ Tschmuck, Creativity, 2012, S. 53.

${ }^{296}$ Die RCA beschränkte sich bis 1926 auf den reinen Hardware-Verkauf, und betätigte sich auch danach nur mittelbar über eine Teilhaberschaft i.H.v. 50\% an der neugegründeten NBC (National Broadcasting Corporation) als Inhalteanbieter, Tschmuck, Creativity, 2012, S. 56 f.

${ }^{297}$ So lautete in den 80er Jahren ein Slogan der British Phonographic Industry, http://de.wikipedia.org/wiki/Home_Taping_Is_Killing_Music.

${ }^{298}$ Zusammenfassend Grassmuck, 2010, S. $31 \mathrm{ff}$. mwNachw.

${ }^{299}$ Grassmuck, 2010, S. 5; s. auch Tschmuck, Stellungnahme, 2010, S. 12; sowie ders., Ursachenanalyse, 2009; zum generellen Trend des Entbündelns von Inhalten im Markt für digitale Güter: Elberse, Journal of Marketing, Vol. 74 (May 2010), 108, $121 \mathrm{f}$.

${ }^{300}$ Tschmuck, Creativity, 2012, S. 216; Grassmuck, 2010, S. 5.

${ }^{301}$ Djordjevic et al., 2009, S. 90 f.
} 
auf publikumswirksame Stars, ${ }^{302}$ so dass andere Käuferschichten auf unabhängige Labels, aber auch auf Filesharing zurückgriffen. ${ }^{303}$

121 Oberholzer-Gee/Strumpf haben vor dem Hintergrund der beschriebenen Studien drei Bedingungen formuliert, unter denen Filesharing sich als volkswirtschaftlich unerwünscht darstellt:

"Three conditions need to hold for less-certain rights to undermine the incentives for artistic production: original works and copies on file-sharing networks must be reasonably close substitutes; artists and the entertainment industry must not be able to shift from previous sources of income to the (similarly profitable) sale of complements; and falling incomes must be an important-enough motivator for artists to reduce production." ${ }^{304}$

\section{e) Besonderheiten in Filmmärkten}

123 Auch wenn vergleichbare Daten für den Filmmarkt fehlen, der offenbar nach wie vor durch technologische Innovationen gekennzeichnet ist, können auch hier einige Aspekte festgehalten werden. So ist durch den Übergang von DVD zu BluRay und 3D-Technologie wiederum ein Vorsprung vor den Bandbreiten und Kompressionstechnologien im Internet festzustellen, die das Ausmaß an Filesharing verringern dürften, zumindest so lange, wie die technischen Rahmenbedingungen, insbesondere Breitbandkapazitäten, den Vorsprung nicht wieder aufholen, etwa durch VDSL oder LTE als neue Übertragungstechniken.

124 Allerdings unterscheidet sich das Konsumentenverhalten bezüglich des Produktes „Film“ in einigen Punkten wesentlich von Musikwerken: Anders als in der Musik spielt hier offenbar der „Probiereffekt" keine besondere Rolle, indem Konsumenten sich nicht etwa erst den Film herunterladen, um dann zu entscheiden, ob man ihn kaufen will. Auch werden offenbar Filme gegenüber Musikstücken seltener mehrfach konsumiert, so dass der Substitutionseffekt hier wesentlich größer sein dürfte, ${ }^{305}$ was sich auch in entsprechenden „Erfolgen“ von illegalen Streaming-Portalen wie kino.to etc. niedergeschlagen haben dürfte. Ferner zeichnet sich die Filmproduktion durch erhebliche produktspezifische Investitionen bei gleichzeitig späteren niedrigen Vertriebskosten aus (Projektfinanzierungen). Die hohen Projektkosten müssen im Falle des Misserfolges komplett abgeschrieben werden.

125 Auch ist nicht zu verkennen, dass die sog. Medienchronologie als entscheidendes Momentum zur Vermarktung von Filmen (Kino - Vertrieb als DVD oder BluRay oder per kommerziellen Download Pay-TV-Premiere - Free-TV-Premiere ${ }^{306}$ ) durch das Internet und Filesharing entscheidend verkürzt wird. Schon jetzt aber ist zu beobachten, dass die zeitliche Schere zwischen den einzelnen Schritten sich dramatisch verkürzt. Indes schließt eine Schranke für den nicht-kommerziellen Gebrauch nicht aus, dass nach wie vor kommerzielle Verwertungen entlang der Medienchronologie möglich bleiben, da weder das Mitschneiden von Filmen in einem Kino noch die TV-Ausstrahlung davon erfasst wären. Es hängt damit vom Nutzungsverhalten der potentiellen TV-Zuschauer ebenso wie Kino-Besucher ab, ob sie trotz Verfügbarkeit von Filmen im Netz noch die Angebote wahrnehmen; für eine nicht völlige Substitution spricht etwa die andersartige Ausstattung in einem Kino oder die Einbettung in ein Pro-

\footnotetext{
${ }^{302}$ Tschmuck, Ursachenanalyse, 2009.

${ }^{303}$ Zum Aufstieg von Independent Labels in den letzten Jahren s. nur Waldfogel, 2011, S. $26 \mathrm{ff}$.

${ }^{304}$ Oberholzer-Gee/Strumpf, 2010, S. 6.

${ }^{305}$ S. dazu Huygen et al., 2009, S. 5.

${ }^{306}$ Diese Vermarktungsstrategie wird aufgrund der schrittweisen Nutzung von Zeitfenstern für die Auswertung "windowing" genannt, s. dazu und zu den Auswirkungen des Internet auf die Verwertung durch Film- und Musikindustrie Bhatia/Gay/Honey, Journal of Interactive Marketing, Vol. 17, No. 2, 2003, 70, 73 f.
} 
gramm; ${ }^{307}$ allerdings bedarf es hier tatsächlich empirischer Untersuchungen, um die Effekte sicher einzuschätzen.

126 Auch darf die Filmproduktion nicht nur aus der Sichtweise der "Majors" bzw. HollywoodProduktionen gesehen werden; vielmehr hängt gerade die heimische Filmindustrie intensiv von der Kooperation mit TV-Sendeanstalten ab. ${ }^{308}$ Das völlig unabhängig von Filesharing zu beobachtende geänderte Mediennutzungsverhalten über die Generationen hinweg deutet jedoch darauf hin, dass die Finanzierung von Filmen über TV-Sendeanstalten abnehmen dürfte, sofern diesen verwehrt sein sollte, die neuen Medien auch zu nutzen.

127 Summa summarum ist festzuhalten, dass anscheinend die Substitutionseffekte im Filmmarkt wesentlich abgeschwächt sind, andererseits aber auch das finanzielle Risiko weit höher erscheint, so dass einiges hier für höhere Substitutionsfaktoren bzw. die Annahme von Schäden spricht. Letztlich bedarf es aber auch hier genauerer empirischer Erhebungen.

\section{f) Buchmärkte}

128 Was für Filmproduktionen gilt, ist für E-Books und Buchmärkte erst recht einschlägig, namentlich das weitgehende Fehlen verlässlicher Studien, die Aufschluss über das Verhältnis von Filesharing und Einkünfte im Buchmarkt geben. Bekannt ist, dass etwa im US-amerikanischen Markt der Verkauf von E-Books inzwischen den Verkauf von Papierbüchern übertroffen hat; ${ }^{309}$ ein deutlicher Aufwärtstrend zeichnet sich auch für den deutschen Markt ab. Zwar lag der Marktanteil von E-Books am Buchmarkt 2011 nur bei 1,4\%, doch wird bis 2015 ein kontinuierlicher Anstieg auf 6,3\% erwartet. ${ }^{310}$

129 Allerdings ist das Verhalten der Filesharer ebenfalls von Interesse: Nach einer Studie in Frankreich wurden nicht etwa hauptsächlich marktgängige Bestseller kopiert und geteilt, sondern fast zu $25 \%$ philosophische Werke. Ob damit tatsächlich Substitutionseffekte eintreten und nicht vielmehr wiederum Promotionseffekte zumindest gleichgewichtig sind, ist zweifelhaft. ${ }^{311}$ Zumal es auch hier naheliegt anzunehmen, dass entsprechend den Musikmärkten die Auswirkungen unterschiedlich ausfallen, bspw. für bekannte und unbekannte Autoren und/oder für unterschiedlicher Genres. ${ }^{312}$ Einige Studien sehen sogar positive Effekte zwischen einer freien Weiterverbreitung von E-Books und dem Verkauf in der Promotions-Phase sowie den nachfolgenden vier Wochen. ${ }^{313}$

130 Indes dürfte E-Book-Sharing noch nicht weit verbreitet sein; daher kann es auch Verzögerungseffekte geben. Wie bereits hervorgehoben, lassen sich aber derzeit kaum Aussagen über die jeweiligen Effekte, insbesondere der Substitution durch Filesharing treffen.

\footnotetext{
${ }^{307}$ Dies führt im Wesentlichen Aigrain, 2012, S. 114 an.

${ }^{308}$ Für Frankreich teilweise sogar gesetzlich vorgeschrieben, vgl. Aigrain, 2012, S. 114 f.

${ }^{309}$ S. dazu die Pressemitteilung des Onlinehändlers Amazon mit der Überschrift: „Amazon.com Now Selling More Kindle Books Than Print Books", abrufbar unter: http://phx.corporate-

ir.net/phoenix.zhtml?c=176060\&p=irol-newsArticle\&ID=1565581\&highlight; sowie Wischenbart, The Global eBook Market, S. 6.

${ }^{310}$ S. PricewaterhouseCoopers, E-Books in Deutschland, S. 59, abrufbar unter http://www.pwc.de/de/technologie-medien-und-telekommunikation/e-books-in-deutschland-der-beginneiner-neuen-gutenberg-aera.jhtml.

${ }^{311}$ So ist bspw. der Verlagsgründer Tim O'Reilly der Ansicht, dass nicht wahrgenommen zu werden, oder in Vergessenheit zu geraten eine größere Bedrohung als Raubkopien seien, dazu: O'Leary, Impact of P2P and Free Distribution on Book Sales, 2009, S. 16.

${ }^{312}$ Entsprechend differenziert O'Leary, Impact of P2P and Free Distribution on Book Sales, 2009, S. $6 \mathrm{ff}$. bei der Weitergabe von Freiexemplaren als Promotion zwischen verschiedenen Zielgruppen.

${ }^{313}$ O'Leary, Impact of P2P and Free Distribution on Book Sales, 2009, S. 5.
} 


\section{Anreizwirkungen für Kreative}

\section{a) Wirtschaftlicher Erfolg und Anreizwirkungen}

131 Die Auswirkungen der Einführung einer „Kulturflatrate“ auf Kreative einzustufen, fällt aus verschiedenen Gründen schwer: Zum einen kann offenbar das klassische ökonomische Modell rationalen Verhaltens bzw. monetärer Anreize im Urheberrecht nur beschränkt angewandt werden. Oftmals spielen intrinsische Motive eine erhebliche Rolle, die sich nur bedingt durch die in der Ökonomie unterstellten Nutzenmaximierungen abbilden lassen; erfolgversprechender erscheint hier die Anwendung der Methode der Behavioral Economics auf Kreativakte, die allerdings - soweit ersichtlich noch in ihren Kinderschuhen zu stecken scheint, zumal weitreichende Feldstudien und Experimente erforderlich sind. ${ }^{314}$ Anders formuliert kann nicht immer von einem ökonomischen Erfolg auf die Motive eines Urhebers geschlossen werden, kreativ tätig zu werden. Leistner/Hansen bezweifeln die Anwendbarkeit des rein ökonomischen Anreizmodells insbesondere auf die Bereiche klassischen Werkschaffens und sehen allenfalls Raum dafür aus der Perspektive der Verwerter, deren Motivation naturgemäß weniger aus dem kreativen Schöpfungsprozess als solchem als vielmehr aus dem Gedanken des Investitionsschutzes gespeist wird. ${ }^{315}$ Die auf rein ökonomischen Motiven aufbauende Betrachtungsweise ignoriert vor allem den urheberpersönlichkeitsrechtlichen Aspekt kreativen Schaffens. Dieser zeigt sich schließlich nicht erst darin, dass dem Urheber Mittel gegen die Entstellung seiner Werke zustehen, sondern ist bereits für die Veröffentlichungsabsicht von Bedeutung, die gleichsam „persönlichkeitsrechtlich determiniert“ ist. ${ }^{316}$

132 Daher verwundert es nicht, dass nach wie vor umstritten ist, ob allein von Verkäufen von Musik (oder anderen Werken) generell (und nicht nur für wenige "Superstars“) Anreizwirkungen für Kreative ausgehen - und nicht vielmehr von anderen Faktoren, wie etwa einer empfundenen Wertschätzung. ${ }^{317}$ Denn offensichtlich ist die Aussicht auf ökonomische Erträge etwa von einem Musikalbum relativ gering. Empirische Studien weisen darauf hin, dass die große Mehrzahl von Musikern von einem Beruf außerhalb der Musikbranche lebt. ${ }^{318}$ Für den US-amerikanischen Markt wird von einem höchstens 8-12\%igen Anteil der Musiker an den Verkaufserlösen berichtet, zudem erst nach Erreichen einer bestimmten Schwelle an Verkäufen, da die Fixkosten von vornherein vom Gesamterlös abgezogen werden. ${ }^{319}$ Das ist auch vergleichbar mit dem Künstler-Anteil aus dem Erlös eines CD-Verkaufs in Deutschland, den die Musikindustrie mit 9,9\% beziffert. ${ }^{320}$ Auch erreichen offenbar nur wenige Alben die Erfolgsschwelle, gerade einmal 1.500 Alben waren 2011 verantwortlich für knapp 90\% aller Albenverkäufe, bei einer Erscheinungszahl von ca. 77.000 Alben (bezogen auf den US-amerikanischen

\footnotetext{
${ }^{314}$ S. Spindler, in: Riesenhuber/KIöhn, Das Urhebervertragsrecht im Lichte der Verhaltensökonomik, 2010, S. 195, 200.

${ }^{315}$ S. Leistner/Hansen, GRUR 2008, 479, 484, Fn. 44; ähnlich auch Engert, in: Riesenhuber/Klöhn, Das Urhebervertragsrecht im Lichte der Verhaltensökonomik, 2010, S. 167, 181; zust. Spindler, in: Riesenhuber/Klöhn, Das Urhebervertragsrecht im Lichte der Verhaltensökonomik, 2010, S. 195, $201 \mathrm{f}$.

${ }^{316}$ So zu Recht Wandtke, in: Riesenhuber/Klöhn, Das Urhebervertragsrecht im Lichte der Verhaltensökonomik, 2010, S. 153, 154, 157 f., der darauf hinweist, dass Superstars ihre Vertrags- und Vergütungsbedingungen ab einem bestimmten Popularitätsgrad maßgeblich diktieren können, während unbekannte Künstler vielmehr "fast alles" akzeptieren, um ihre Werke veröffentlicht zu sehen.

${ }^{317}$ Wandtke, in: Riesenhuber/Klöhn, Das Urhebervertragsrecht im Lichte der Verhaltensökonomik, 2010, S. 153, 154.

${ }^{318}$ Madden, 2004, S. 24.

${ }^{319}$ Belsky/Kahr/Berkelhammer/Benkler, Mich. Telecomm. \& Tech. L. Rev., Vol. 17, 2010-2011, S. 1, 13 ff. mwNachw.

${ }^{320}$ Bundesverband Musikindustrie, Musikindustrie in Zahlen 2010, S. 19.
} 
Markt). ${ }^{321}$ Wie diese Phänomene ökonomisch erklärt werden können, ist noch weitgehend offen, ob etwa sog. Turnier-Modelle, um den Status eines Superstars zu erwerben, oder eher Reputationsmärkte herangezogen werden können. ${ }^{322}$

133 Betrachtet man demgegenüber die Auswirkungen auf die Anreize für Musiker, neue Musik zu entwickeln und zu publizieren, so wird von den Verbänden beklagt, etwa dem Bundesverband Musikindustrie, dass sich die Aufstiegschancen von Newcomern verringern würden. ${ }^{323}$ Entsprechende Untersuchungen konnten jedoch keine signifikanten negativen Anreizwirkungen des Filesharing im Musikmarkt feststellen, insbesondere nicht hinsichtlich der Zahl von Neuerscheinungen oder Musikern, ${ }^{324}$ was auch jüngst erst bestätigt wurde; verantwortlich hierfür können trotz Filesharing die erheblich verringerten Kosten hinsichtlich der Produktion (mittels Softwaretools für jeden PC) und Distribution via Internet sein. ${ }^{325}$ Anderen Studien zufolge soll nach Einführung von Napster keine Änderung hinsichtlich der Quantität der veröffentlichten Musik festzustellen sein. ${ }^{326}$

134 Für die Filmindustrie ergeben sich in ähnlicher Weise Zahlen, die angesichts der Filesharingaktivitäten eher irritieren sind: So ist die weltweite Produktion von Filmen von 3.807 Filmen auf 4.989 in 2007 gestiegen, selbst in Ländern mit erheblichen Filesharingaktivitäten wie Korea oder Indien (als besonders filmorientierter Nation) ${ }^{327}$ mit einem Anstieg von 877 auf 1.164 Filme. Selbst in den USA ist ein leichter Zuwachs von 789 Filmproduktionen im Jahr 2007 hin zu 817 Produktionen im Jahr 2011 zu beobachten. ${ }^{328}$ Auch in Deutschland ist ein Anstieg zu verzeichnen. Seit 1999 ist die Zahl der hierzulande produzierten Filme von 124 auf $276 \mathrm{im} \mathrm{Jahr} 2010$ gestiegen. ${ }^{329}$

\section{b) Gegenläufige Entwicklungen}

135 Neben den offenbar vermehrt auftretenden Verlagerungen der Einkünfte auf Konzerte und Merchandising haben als eine Reaktion auf die veränderten Technologiebedingungen, aber auch Marktstrukturen, verschiedene Anbieter begonnen, mit veränderten Plattformen zu experimentieren, die es dem Nutzer überlassen, ob und wie viel er für einen Musiktitel ausgeben möchte, teilwei-

\footnotetext{
${ }^{321}$ The Nielsen Company \& Billboard's 2011 Music Industry Report, S. 7.

${ }^{322}$ S. Oberholzer-Gee/Strumpf, 2009, S. 22 f., wonach sich Superstars besonders in Märkten entwickeln, in denen niedrige Produktionskosten, geringe bis keine Qualitätsverluste bei hoher Produktionsrate sowie qualitätsbewusste Verbraucher zusammentreffen, unter Verweis auf Rosen, The American Economic Review, Vol. 71, No. 5, 1981, 845 ff.; in eine ähnliche Richtung Leistner, in: Riesenhuber/Klöhn, Das Urhebervertragsrecht im Lichte der Verhaltensökonomik, 2010, S. 119, 125 f. mwNachw, der den urheberrechtlichen Märkten „,ausgeprägte winner-takes-all Charakteristika“ bescheinigt, was dadurch noch mehr an Bedeutung erlange, dass für den Marktzutritt die „systematische Überschätzung der eigenen Leistungskraft, Fähigkeit oder Erfolgsaussichten" eine maßgebliche Rolle spiele.

${ }^{323}$ Bundesverband Musikindustrie, Musik im digitalen Wandel, 2012, S. 6.

${ }^{324}$ Tanaka, 2004, S. 8; Bhattacharjee et al., Management Science, Vol. 53, 2007, 1359, 1371 f.; OberholzerGee/Strumpf, 2007, S. 38 ff.; Waldfogel, 2011, S. 27; s. auch die unverändert hohen Neuerscheinungszahlen oben Fn. 321.

${ }^{325}$ Eingehend dazu Waldfogel, 2011, 23 ff. aufgrund einer Studie der Neuerscheinungen vor und nach dem Auftreten von Napster.

${ }^{326}$ Waldfogel, 2011, S. 22.

${ }^{327}$ Allerdings ist hier zweifelhaft und näher zu untersuchen, ob die technischen Bandbreiten in diesen Ländern tatsächlich das Teilen von Filmen ermöglichten.

${ }^{328}$ S. dazu auch Smith/Telang, 2009, S. 23 ff., die keinen negativen Effekt beobachtet haben.

${ }^{329}$ S. Motion Picture Association of America (MPAA), Theatrical Market Statistics 2011, S. 17; Formatt-Institut, Film- und Fernsehproduktion in Nordrhein-Westfalen im Vergleich zu anderen Bundesländern 2009 und 2010, Juni 2012, S. 137.
} 
se verbunden mit der Etablierung sozialer Normen. ${ }^{330}$ Diese alternativen Vertriebsformen, die freies Teilen von Musiktiteln erlauben, sind offenbar über den Appell an das Gewissen der Nutzer in der Lage, ausreichend Vergütungen zu generieren, die direkt an die Künstler ausgeschüttet werden. ${ }^{331}$ Wie Belsky et al. festhalten, spielt die Herstellung unmittelbarer Kontakte zwischen Nutzern und Künstlern offenbar eine wesentlich größere Rolle als bisher unter Zugrundelegung klassisch ökonomischer Modelle angenommen.

136 Neben diesen neuen Plattformen können als eine der Auswirkungen des fast kostenlosen Vertriebskanals Internet auf die Musikschaffenden die wesentlich herabgesetzten Barrieren des „market entry" gelten, der einfachen Verbreitung und größeren Chance auf Wahrnehmung auch zuvor unbekannter Musiker. Die Empfehlung durch Freunde über soziale Netzwerke, teilweise ihre Einbettung in entsprechende Profile als YouTube-Videos, aber auch durch Blog-Portale tritt zunehmend an die Stelle von Rezensionen oder Empfehlungen durch Musikverlage oder auch von Radiosendungen. Teilweise wird hier von „viraler“ Verbreitung von Musik gesprochen. ${ }^{332}$ Bestes Beispiel dieser Art von Entdeckungen dürfte der Pop-Sänger Justin Bieber sein, aber auch das Musikstück „Over the Rainbow“ von Israel Kamakawiwo'ole, das Jahre nach dessen Tod in Deutschland zum Erfolg wurde. ${ }^{333}$

137 Die mitunter im Rahmen des Dreistufen-Tests ${ }^{334}$ geäußerte Befürchtung, dass eine Kulturflatrate zu einem vermehrten Einsatz von DRM-geschützten Inhalten führen würde und daher insgesamt sowohl die Kreativität als auch die Verbreitung von Inhalten abnehmen würde, ${ }^{335}$ scheint daher unzutreffend; vielmehr ist trotz der nach wie vor zu beobachtenden Filesharing-Aktivitäten ein Rückgang der DRM-Angebote aus den verschiedensten Gründen zu beobachten.

138 Allerdings ist hervorzuheben, dass es hierfür nicht der Einführung einer Kulturflatrate bzw. einer Ausdehnung einer Schranke bedarf; auch ist zu bedenken, dass nach einem erfolgreichen Markteintritt oftmals die proprietäre Auswertung erfolgt, wie gerade bei den genannten Musikern zu beobachten.

\section{c) Verstärkte Kreativität durch Bearbeitungsrechte?}

139 Teilweise wird auch vorgebracht, dass freie Bearbeitungsrechte und der Tausch von Inhalten zu mehr Kreativität führen können, die zu einer neuen „Read-Write-Culture“ führen könne statt des früheren passiven Kulturkonsums. ${ }^{336}$ Allerdings ist es bislang wissenschaftlich kaum gesichert, ob dies tatsächlich zu mehr Kreativität mit entsprechender Qualität führt oder nicht vielmehr zu einem „Race to the bottom", indem gute Angebote angesichts einer Fülle von schlechten Inhalten verschwinden, analog dem Lemon-Markets-Theorem von Akerlof, wonach gute Qualität zunehmend schwierig signalisiert

\footnotetext{
${ }^{330}$ Etwa Magnatune, die allerdings mit einem "licensing" Modell arbeiten, s. www.magnatune.com; zu den verschiedenen Formen ausführlich mwNachw Belsky/Kahr/Berkelhammer/Benkler, Mich. Telecomm. \& Tech. L. Rev., Vol. 17, 2010-2011, S. 1. Inzwischen ist Magnatune offenbar zu einer pauschalen Gebühr pro Monat übergegangen.

${ }^{331}$ Eingehend Belsky/Kahr/Berkelhammer/Benkler, Mich. Telecomm. \& Tech. L. Rev., Vol. 17, 2010-2011, S. 1, insbesondere S. 63 ff. mit Verbesserungsvorschlägen auf der Grundlage von Erkenntnissen der Behavioral Economics.

${ }^{332}$ Wikström, The Music Industry, 2009, S. 162.

${ }^{333}$ S. http://www.billboard.com/news/justin-bieber-the-billboard-cover-story1004074692.story\#/features/justin-bieber-the-billboard-cover-story-1004074692.story?page=2; http://www.zeit.de/gesellschaft/zeitgeschehen/2010-10/israel-kamakawiwoole-rainbow.

${ }^{334}$ S. dazu unten Rn. $213 \mathrm{ff}$.

${ }^{335}$ So Runge, GRUR Int. 2007, 130, 136.

${ }^{336}$ Lessig, Remix. Making art and commerce thrive in the hybrid economy, 2008, S. $76 \mathrm{ff}$.
} 
werden kann. ${ }^{337}$ Andererseits sprechen empirische Daten im Musikbereich anscheinend dafür, dass nach wie vor Qualität auch bei Filesharing über Jahre hinweg sich durchsetzen kann. ${ }^{338}$

\section{Anreize und Auswirkung auf Verleger bzw. Intermediäre}

140 Wendet man den Blick auf Verleger bzw. Intermediäre (Musikverlage, Buchverlage, Filmhersteller und -vertriebe etc.) scheint die Einführung einer Kulturflatrate mitsamt Abgaben sich zunächst negativ auszuwirken, indem sie ihre zuvor exklusiv geltend gemachten Rechte jedenfalls gegenüber privaten Nutzern „verlieren“ durch die Einführung einer allgemeinen Schranke.

141 Allerdings stehen dem zwei gegenläufige Faktoren gegenüber:

142 Zunächst wird der wirtschaftliche Verlust durch eine Abgabe im Rahmen der Kulturflatrate weitgehend, wenn nicht sogar ganz kompensiert, da die Rechteinhaber als Leistungsschutzrechteinhaber in die Verteilung einbezogen werden müssen.

143 Zum anderen können sich durchaus andere Geschäftsmodelle entwickeln, die sich längst in Branchen abzeichnen, die keine Güter mit Exklusivrechten vertreiben und für die das Internet im erheblichen Masse zu einer Des-Intermediatisierung geführt hat. Mit anderen Worten hat das Internet dazu geführt, dass zahlreiche Zwischenhandelsstufen ihre Funktion verloren haben und neue Geschäftsfelder suchen müssen, um zu überleben, etwa in der Reisebranche. Angesichts der Flut an Informationen, was auch für digitale Inhalte mit Exklusivrechten (also Urheberrechten) gilt, sind Such- und Qualitätssiegelfunktionen umso wichtiger. ${ }^{339}$ Gerade Verlage können diese neuen Funktionen gegen Bezahlung als „Gatekeeper" übernehmen ${ }^{340}$ - ob dies unentgeltlich oder durch Werbung (mit Hilfe persönlicher Daten) geschieht, ist dabei zweitrangig und dem Wettbewerb überlassen.

144 Schließlich reagiert zumindest die Musikbranche offenbar längst auf die Verschiebungen zwischen den Einkunftsarten, indem sog. 360-Grad-Verträge mit Künstlern geschlossen werden, die sich nicht nur auf die Einnahmen aus dem Verkauf von Tonträgern (oder Downloads) beschränken, sondern sämtliche mit der Aktivität des Künstlers zusammenhängende Einnahmequellen wie Konzerte, Merchandising etc. erfassen. Teilweise werden diese Veränderungen in der Vertragspraxis bereits als Reaktion auf das vermehrte Filesharing gesehen, ${ }^{341}$ so dass durchaus zweifelhaft sein kann, ob tatsächlich die befürchteten negativen Effekte für die Musikindustrie bei Einführung einer Kulturflatrate entstehen würden - wohlgemerkt gleichzeitig abgesichert durch eine Abgabe.

\section{Anreizwirkungen für andere Marktteilnehmer und neue Geschäftsmodelle:}

145 Anders kann sich die Lage für neuere Geschäftsmodelle darstellen, insbesondere Streaming-Portale oder Download-Portale wie iTunes oder Amazon etc. Denn ihre unmittelbaren Leistungen würden durch privates Filesharing substituiert. Dies zeigen empirische Studien (Umfragen), wonach viele Teilnehmer auf Filesharing verzichten, wenn zu geringen Kosten legale Streaming-Dienste bereitgestellt werden. Andererseits wurde offenbar von vielen Nutzern auch kein besonderer Wert auf Qualität oder andere qualitätsorientierte Nebenleistungen gelegt. ${ }^{342}$ Der Erfolg von Diensten wie Spotify

\footnotetext{
${ }^{337}$ Darauf zielt im Wesentlichen das Argument von Schmalz, No Economy - Wie der Gratiswahn das Internet zerstört, 2009, S. 180 f., 185, ab, grundlegend dafür Akerlof, Quarterly Journal of Economics 1970, 488 ff.

${ }^{338}$ Ausführlich dazu Waldfogel, J.Law \& Econ. 2013, $715 \mathrm{ff}$.

${ }^{339}$ Dies erkennt im Ansatz auch Schmalz, No Economy - Wie der Gratiswahn das Internet zerstört, 2009, S. 182.

${ }^{340}$ Tschmuck Creativity, 2012, S. 231 f., 270 f.

${ }^{341}$ Huygen et al., 2009, S. 23 f., die zudem auf Bundling-Verträge hinweisen, also Download-Abonnements verbunden mit verbilligten Konzertkarten oder Crowdfunding von Musikern.

${ }^{342}$ S. oben Rn. $103 \mathrm{ff}$.
} 
oder Simfy aber auch T-Entertain (als Angebot der Deutschen Telekom AG im sog. Triple-Play, also der Verbindung von Internet, Telefonie, TV, Rundfunk und Video on Demand) ${ }^{343}$ zeigt, dass tatsächlich ein Bedarf an derartigen Modellen besteht. So hat auch eine der jüngsten Studien für den USamerikanischen Markt gezeigt, dass die Hälfte der Filesharer auf legale Streaming-Angebote umgestiegen sind, als sie zu erschwinglichen Preisen erhältlich waren - und dies noch, bevor der Dienst Spotify in den USA lanciert wurde. ${ }^{344}$

146 So hielt schon der OECD-Report zur Entwicklung digitaler Inhalte fest, ${ }^{345}$ dass vier Vertriebsmodelle durch das Internet forciert werden: Der direkte, per Download erfolgende Verkauf wie bei iTunes, die Streaming-Subskription aufgrund einer monatlichen Gebühr ohne Download wie etwa bei Spotify, der nur für eine begrenzte Zahl oder Zeit autorisierte Download und schließlich das Internetradio, sei es unentgeltlich über Werbemaßnahmen finanziert oder durch Abonnement. ${ }^{346}$

147 Andererseits ist es denkbar, dass sich trotz der kostenlosen „Konkurrenz" durch freies Filesharing die kommerziellen Angebote weiterhin aufrecht erhalten lassen, indem sie zum einen bessere Qualität, insbesondere Virenfreiheit, Suchfunktionen und zusätzliche Services, etwa auch verbilligte Konzertkarten (oder im Filmbereich Kinokarten) etc. anbieten, um sich gegenüber den privaten „Angeboten“ abzuheben.

148 Demgegenüber scheint es auf der Hand zu liegen, dass legale Streaming-Angebote wie Spotify, aber auch Triple-Play-Angebote wie T-Entertain durch eine Kulturflatrate erheblich in Mitleidenschaft gezogen würden, da Nutzer auf die kostenlosen Angebote ausweichen würden. Dem steht wiederum gegenüber, dass schon jetzt das Nebeneinander von Plattformen wie YouTube, für die zahlreiche sog. Musikripper existieren, die das Herunterladen der Musik erlauben, mit den Streaming-Portalen wie Spotify oder den Download-Portalen zeigt, dass eine freie Verfügbarkeit nicht von vornherein dazu führt, dass solche Portale verschwinden müssten. Schließlich darf bei Download-Portalen wie iTunes nicht vergessen werden, dass diese primär auch der Verkaufsförderung der Hardware dienen; so ist etwa der Verkauf von iPods (und anderer Musikabspielgeräte) gerade während der Zeit des Filesharing signifikant angestiegen. ${ }^{347}$ Hier kann wiederum eingewandt werden, dass das Nebeneinander von legalen Portalen mit Filesharing etc. im Wesentlich dadurch bedingt sein kann, dass es anscheinend genügend Nutzer gibt, die sich keiner Rechtsverfolgung aussetzen wollen und daher bei einem entsprechend niedrigen Preis diese Portale eher als ein Filesharing mit rechtlichem Risiko nutzen wollen.

149 Diese Fragen lassen sich im Rahmen eines wissenschaftlichen Gutachtens ohne ausführliche Feldforschungen nicht beantworten: An dieser Stelle muss nochmals betont werden, dass verlässliche empirische Grundlagen weitgehend fehlen, so dass Prognosen über die eintretenden Effekte äußerst schwierig und derzeit nur Spekulationen möglich sind. Insgesamt lassen sich die Effekte schwer endgültig negativ oder positiv einschätzen. Maßgeblich ist hier wiederum die Einschätzungsprärogative des Gesetzgebers, worauf zurückzukommen ist.

\footnotetext{
${ }^{343}$ Auch andere Access-Provider wie Kabel Deutschland bieten vergleichbare Modelle an.

${ }^{344}$ The American Assembly, Copyright Infringement and Enforcement in the USA, Research Note, 2011.

345 OECD (2005), OECD Report on Digital Music: Opportunities and Challenges, OECD Digital Economy Papers, No. 100, S. 49.

${ }^{346}$ S. dazu auch Huygen et al., 2009, S. 21.

${ }^{347}$ Oberholzer-Gee/Strumpf, 2010, S. 21 mwNachw.
} 


\section{Anreizwirkung für Nutzer: Ineffiziente Nutzung der Anschlusskapazitäten?}

150 Schließlich könnte eine Kulturflatrate zu einer Steigerung des Internetverkehrs aufgrund eines vermehrten Filesharing führen. Da die Abgabe alle Internetanschlüsse erfasst, könnten ineffiziente Anreizwirkungen für solche Nutzer entstehen, die bislang kein Filesharing durchgeführt haben, da sie in den Genuss der Schranken kommen wollen, anders formuliert, etwas für ihre Abgabe erlangen wollen. Damit könnte es letztlich zu einer Fehlallokation von Ressourcen kommen, indem an sich von Nutzern nicht gewünschte Aktivitäten ausgeübt würden, um die Abgabe „auszunutzen“. Derartigen Effekten kann aber mit einer Staffelung der Abgabe je nach Volumen und/oder Geschwindigkeit der Anschlüsse entgegengewirkt werden, ${ }^{348}$ da der Aktivitätslevel von Filesharern im erheblichen Maße von diesen beiden Parametern abhängt, ${ }^{349}$ mithin eine Steuerung der Internetaktivitäten auch innerhalb eines Abgabenmodells möglich bleibt. So könnte etwa für Anschlüsse mit hohen Geschwindigkeiten eine progressiv höher gestaffelte Abgabe vorgesehen werden, etwa im Sinne eines Zuschlags. Würde etwa eine Kulturflatrateabgabe für den normalen, "langsamen“ Anschluss 3 Euro betragen, könnte diese auf 9 Euro für Anschlussinhaber mit hohen Geschwindigkeiten aufgestockt werden, um deren unterschiedliches Nutzungsverhalten gegenüber normalen Nutzern zu erfassen - wobei diese Zahlenrelationen letztlich dem Ermessen derjenigen überlassen sind, die die Abgabenhöhe festsetzen oder verhandeln.

\section{Administrative Kosten im Vergleich}

151 Nicht zu vernachlässigen sind schließlich die administrativen Kosten im Vergleich, einerseits der traditionellen Rechtsverfolgungsmaßnahmen, andererseits der Einziehung und Durchsetzung einer Kulturflatrate. Für die Rechtsverfolgungsmaßnahmen lassen sich in Deutschland kaum belastbare Zahlen nennen - lediglich für die Vorratsdatenspeicherung hatte die Internetindustrie entsprechende Zahlen in Rechtsstreitigkeiten vorgelegt, ohne dass hier aber von einer objektiven Basis gesprochen werden kann. Denn an den Verfahren waren unterschiedliche Provider beteiligt, die jeweils mit verschiedenen Anschaffungen bzw. Ausgaben belastet waren, sodass sich die Zahlen nicht verallgemeinern lassen. In einem vom VG Berlin ausgesetzten und an das BVerfG verwiesenen Rechtsstreit wurden bspw. vom beteiligten Provider Hardware-Kosten i.H.v. ca. 720.000 Euro pro Anlage sowie mindestens 450.000 Euro an jährlichen Personalkosten angegeben; das BVerfG hat diesen Vortrag aber für zu einseitig erachtet, um auf seiner Grundlage eine Entscheidung über die Verfassungsmäßigkeit der Provider-Verpflichtungen aus der Vorratsdatenspeicherung zu treffen. ${ }^{350}$ In einem weiteren Verfahren vor dem VG Berlin wurden vom klagenden Provider einmalige Kosten zur Einrichtung der Speichersysteme für VoIP, Email und Internetzugang i.H.v. 1 Mio. Euro sowie jährliche Bereithaltungskosten i.H.v. 3 Mio. Euro angegeben. ${ }^{351}$ Aber auch die Angaben zur Gesamtdimension der Providerbelastungen durch Branchenverbände lassen kein einheitliches Bild erkennen. So spricht der Verband eco von Kosten i.H.v. 330 Mio. Euro, die die Provider im Zuge der ersten, verfassungswidrigen Umsetzung der Vorratsdatenspeicherungs-Richtlinie zu tragen gehabt hätten. ${ }^{352}$ Der BITKOM

\footnotetext{
${ }^{348}$ S. zur Ausgestaltung anhand Geschwindigkeiten auch unten Rn. 379 ff.

${ }^{349}$ Unter Zugrundelegung verschiedener Studien kommen zu dieser Einschätzung auch van Eijk/Poort/Rutten, Communication \& Strategies, Vol. 77, 1. Quart. 2010, S. 35, 42.

${ }^{350}$ BVerfG MMR 2009, 606, $607 \mathrm{f}$.

${ }^{351}$ VG Berlin ZUM-RD 2009, 483, 484.

${ }^{352}$ S. die Pressemitteilung vom 26.04.2012, abrufbar unter: http://www.eco.de/2012/pressemeldungen/ecoeinfuehrung-der-vorratsdatenspeicherung-verursacht-mehr-wirtschaftlichen-schaden-als-bruesselerstrafzahlungen.html.
} 
hatte hingegen 2008 im Vorfeld der Umsetzung von Kosten für Technik i.H.v. lediglich 75 Mio. Euro und jährlichen Betriebskosten „in zweistelliger Millionenhöhe“ gesprochen. ${ }^{353}$

152 Für das französische Three-Strikes-Modell und die dort eingerichtete Behörde HADOPI wird für das Jahr 2013 voraussichtlich ein Budget von 8 Mio. Euro zur Verfügung gestellt. ${ }^{354}$ Darüber hinaus wird davon gesprochen, dass die Provider mit entsprechenden Infrastruktur- und Überwachungsmaßnahmen mit nach französischen Schätzungen 70 Mio. Euro einmalig und 10-20 Mio. Euro Betriebskosten jährlich belastet seien. ${ }^{355}$

153 Für den US-amerikanischen Markt kommt eine Studie des US Social Science Research Council zu dem Ergebnis, dass trotz erheblicher Anstrengungen, illegales Filesharing zu unterbinden und entsprechende Webseiten zu schließen, nur kurzfristige Effekte erzielt werden können und die (vermutlichen) Zahlen illegaler Downloads bald wieder fast dieselbe Höhe erreichten. ${ }^{356}$ Ob dies auch für die Erfahrungen mit dem französischen HADOPI-Gesetz (Three-Strikes-Modell) zutrifft, ist derzeit umstritten. ${ }^{357}$ Eine amerikanische Studie stellte fest, dass seit dem Inkrafttreten des HADOPI-Gesetzes die Musikverkäufe über iTunes gestiegen waren, und sah darin einen Zusammenhang. ${ }^{358}$ Gleichzeitig ist aber einer von der HADOPI-Behörde stammenden Untersuchung, die im Januar 2011 veröffentlicht wurde, zu entnehmen, dass mehr als die Hälfte der Internetnutzer sich von HADOPI nicht in ihrem Verhalten beeinflussen lässt und immerhin 29\% aller Nutzer illegaler Content-Quellen erst innerhalb der letzten sechs Monate mit dieser Nutzung begonnen haben. ${ }^{359}$

154 Dem stehen die Kosten der Erhebung einer Abgabe bzw. der Kulturflatrate gegenüber, die mit den Kosten für die Erhebung der Geräteabgabe verglichen werden können. Würde man den Verwertungsgesellschaften die Erhebung und Verteilung der Abgaben überantworten, liegt es nahe, sich an den administrativen Kosten dieser Organisationen zu orientieren. So schlugen bei der GEMA bspw. im Jahr 2011 Aufwendungen in Höhe von ca. 123 Mio. Euro zu Buche, was knapp 15\% der Einnahmen entsprach. ${ }^{360}$ Bei der VG Wort betrug der Aufwendungsanteil im gleichen Jahr 7,46\% der Einnahmen, ${ }^{361}$ bei der VG Bild Kunst 5,48\%. ${ }^{362}$ Allerdings können diese Sätze nicht eins-zu-eins auf die Erhe-

\footnotetext{
${ }^{353}$ S. die Pressemitteilung vom 22.10.2008, abrufbar unter: http://www.bitkom.org/de/presse/56204_54288.aspx.

${ }^{354}$ S. den Anhang zum Entwurf des Haushaltsplans, S. 68, abrufbar unter: http://www.performancepublique.budget.gouv.fr/farandole/2013/pap/pdf/PAP2013_BG_Medias_livre_industries_culturelles.pdf; im Jahr 2012 betrug das Budget allerdings noch 12 Mio. Euro, s. https://netzpolitik.org/2012/hadopi-bilanz-nachzwei-jahren-24-millionen-euro-14-falle-bei-der-staatsanwaltschaft-keine-verurteilung/.

${ }^{355}$ Grassmuck, 2009, S. 22.

${ }^{356}$ US Social Science Research Council, Media piracy in emerging economies, Chapter 1, Rethinking Piracy, 2011, S. 30.

${ }^{357}$ Skeptisch etwa Hargreaves, Digital Opportunity, 2011, S. 78, unter Verweis auf HADOPI, Hadopi, cultural assets and internet use: practices and perceptions of French internet users, 2011, S. 27, 64; Ueberfeldt, in: Stiftung der Hessischen Rechtsanwaltschaft, Kulturflatrate, 2013, 115, 136 f.; s. aber auch den jüngsten Report der Überwachungsbehörde zu HADOPI oben Rn. $110 \mathrm{f}$.

${ }^{358}$ Danaher et al., 2012, S. 18 ff.; diese Studie wurde aber ebenfalls wieder in Frage gestellt, da es sich bei den vermeintlichen Zusammenhängen vielmehr um Zufälle und bei den Steigerungen der Verkaufszahlen um Auswirkungen des Weihnachtsgeschäfts und des Verkaufsstarts neuer Apple-Produkte handele, s. http://www.lemonde.fr/technologies/article/2012/01/24/hadopi-source-de-la-croissance-ditunes_1633919_651865.html.

${ }^{359}$ S. HADOPI, cultural assets and internet use: practices and perceptions of French internet users, 2011, S. 27, 64.

${ }^{360}$ GEMA, Geschäftsbericht 2011, S. 33.

${ }^{361}$ VG WORT, Geschäftsbericht 2011, S. 10.

${ }^{362}$ VG Bild-Kunst, Geschäftsbericht 2011, S. 5.
} 
bung einer Kulturflatrate übertragen werden, da die Einziehung der Gebühren weitgehend automatisiert und im Gefolge der Providergebühren erfolgen kann, mithin Außendienstmitarbeiter etc. zur Kontrolle nicht erforderlich sind. ${ }^{363}$

\section{Zusammenfassung}

Welche Effekte überwiegen und wie hoch sie in den verschiedenen Branchen veranschlagt werden können, ist nach wie vor weitgehend offen und lässt sich schwer einschätzen. Festgehalten werden kann aber, dass offensichtlich Substitutionseffekte nicht in einem Verhältnis eins-zu-eins vorliegen, mit anderen Worten nicht jeder getauschter Inhalt tatsächlich einem sonst durchgeführten Kauf entspricht. Zudem bestätigen fast alle Studien, dass auch die Promotionseffekte eine erhebliche Rolle spielen, also auch positive Effekte eintreten.

156 Selbst bei aller Vorsicht und unter Zugrundelegung von "worst case“ Szenarien deutet die ökonomische Tendenz offenbar zumindest auf eine Gleichgewichtigkeit der Effekte hin, so dass keine eindeutige Aussage zugunsten einer verschärften Rechtsverfolgung gemacht werden kann. Darauf weisen auch entsprechende ökonomische Wohlfahrtsuntersuchungen hinsichtlich verschiedener Strategien (Verschärfung der Rechtsverfolgung und Erhöhung von Strafen etc.; Erhebung von Abgaben; Subventionierung von Käufen) hin. Hier kamen etwa Chen/Png zu dem Ergebnis, dass jedenfalls eine Verschärfung des Sanktionensystems aus gesamtwirtschaftlicher Sicht den anderen Strategien unterlegen war. ${ }^{364}$ In gleicher Weise betont die oben referierte Studie von Huygen et al. im Auftrag der niederländischen Regierung, dass eine Verschärfung der Sanktionen eine suboptimale Strategie ist. ${ }^{365}$ Auch ökonomische Protagonisten eines starken urheberrechtlichen Schutzes verkennen nicht die Bedeutung von Geräteabgaben als mögliche Alternative. ${ }^{366}$

157 Angesichts einer derartigen weitgehend offenen Tatsachenlage, die sogar eher für die Abkehr vom traditionellen Sanktionensystem spricht, ist aus juristischer, insbesondere verfassungsrechtlicher Sicht die Reichweite der Einschätzungsprärogative bzw. des Prognosespielraums des Gesetzgebers angesprochen, die für die Frage der rechtlichen Grenzen eines Kulturflatratemodells eine erhebliche Rolle spielt:

\section{Rechtliche Grenzen eines Flatratemodells}

Die Einführung neuer Schranken für Verwertungsrechte und die Erweiterung bestehender Schranken wirkt sich auf die grundrechtlich geschützte Rechtsposition der Urheber und Rechteinhaber aus, da sie ihre Ausschließlichkeitsrechte nicht mehr unmittelbar wahrnehmen können und stattdessen Eingriffe gegen Kompensation dulden müssen. Daher liegt es auf der Hand, dass solche Schranken sowohl den nationalen verfassungsrechtlichen als auch europäischen primär sowie sekundärrechtlichen Vorgaben standhalten müssen.

\footnotetext{
${ }^{363}$ Grassmuck, Erwiderung auf das Musikindustrie-Positionspapier zur Kulturflatrate, 10.2.2010, Frage 4.

${ }^{364}$ Chen/Png, Information Systems Research, Vol. 14, No. 1, March 2003, S. 107, 118; ebenso: Bayaan, 2004, S. 17.

${ }^{365}$ Huygen et al., 2009, S. 121.

${ }^{366}$ S. Liebowitz/Watt, Journal of Economic Surveys, Vol. 20, No. 4, 2006, 513, 534 f.
} 


\section{Grenzen einer Erweiterung von Schranken}

\section{a) Verfassungsrechtliche Grenzen}

(1) Verfassungsrechtliche Prüfung und

\section{Einschätzungsprärogative des Gesetzgebers}

159 Eine Änderung der gesetzlichen Schrankenregelungen im UrhG müsste sich in verfassungsrechtlicher Hinsicht an den Grundrechten messen lassen, insbesondere hinsichtlich einer verhältnismäßigen Ausgestaltung der Regelungen. Unbeschadet einer abschließenden Prüfung der Verhältnismäßigkeit im engeren Sinne, d.h. der Angemessenheit etwaiger Grundrechtseingriffe für die Grundrechtsträger, muss das vom Gesetzgeber gewählte Mittel einen legitimen Zweck verfolgen und zur Zweckerreichung geeignet sein. Ein Gesetz ist geeignet, wenn mit seiner Hilfe der erstrebte Erfolg zumindest gefördert werden kann. Darüber hinaus muss das gewählte Mittel auch erforderlich sein, d.h. es darf - bei mindestens gleicher Wirksamkeit - kein grundrechtsschonenderes Mittel geben. ${ }^{367}$ Mit der Prüfung der Geeignetheit, der Erforderlichkeit und Angemessenheit wird zwangsläufig die Frage aufgeworfen, inwieweit das BVerfG befugt ist, die Beurteilungen und Entscheidungen des Gesetzgebers zu überprüfen und ggf. durch abweichende Bewertungen zu ersetzen.

160 Das BVerfG gesteht dabei dem Gesetzgeber einen Gestaltungsspielraum zu, auch Einschätzungsprärogative oder Prognosespielraum genannt, den das Gericht nur beschränkt überprüft: „Der Gesetzgeber hat bei der Entscheidung, ob er eine bestimmte Aufgabe in Angriff nehmen will und wie sie

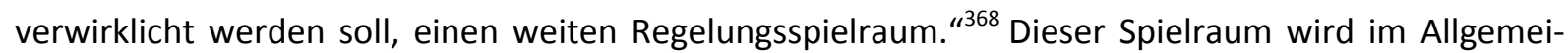
nen mit dem Demokratieprinzip begründet. ${ }^{369}$ Eine allgemeine, umfassende Befugnis des BVerfG, die sich auch auf die eigentlichen politischen Gestaltungsspielräume erstrecken würde, würde dieses Prinzip und die Gewaltenteilung zwischen den Staatsorganen unterlaufen. ${ }^{370}$

161 Darüber hinaus hat das BVerfG auch ausdrücklich die Befugnis des Gesetzgebers festgehalten, bei unklarer Folgenabschätzung mit den ihm zur Verfügung stehenden Mitteln zu experimentieren, d.h. „zeitlich und örtlich begrenzte Versuche“ durchzuführen. ${ }^{371}$ Dem Gesetzgeber komme dabei „erhebliche Gestaltungsfreiheit“ zu, „denn solche Versuche dienen der Aufgabe, Erfahrungen zu gewin-

\footnotetext{
${ }^{367}$ BVerfGE 30, 292, 316; 63, 88, 115; 67, 157, 173, 176; BVerfG NJW 1994, 1577, 1579.

${ }^{368}$ BVerfGE 37, 1, 20 - Stabilisierungsfonds; s. auch BVerfGE 62, 1, 50 unter Verweis auf BVerfGE 50, 290, 333; BVerfGE 71, 66, 77; 71, 206, 215; 73, 301, 315; 77, 84, 106: „weiter Gestaltungsraum“, „große Gestaltungsfreiheit“; ferner W. Meyer, in: v. Münch/Kunig, GG, 6. Aufl. 2012, Art. 93 Rn. 13.

${ }^{369}$ Daneben gibt es noch weitere umstrittene Ansätze; s. dazu Cremer, Freiheitsgrundrechte, 2003, S. 298 ff.; Heun, Funktionell-rechtliche Schranken der Verfassungsgerichtsbarkeit, 1992, S. 38 ff.; Schlaich/Korioth, Das Bundesverfassungsgericht, Rn. 519 ff. mwNachw.

${ }^{370}$ Raabe, in: Grabenwarter, Allgemeinheit der Grundrechte und Vielfalt der Gesellschaft, 1994, S. 86 f.; Cremer, Freiheitsgrundrechte, 2003, S. 298 ff.; Borowski, Grundrechte als Prinzipien, 2. Aufl. 2007, S. 159; Schenke, NJW 1979, 1321, 1323 f.; wobei es in der staatsrechtlichen Literatur in Reichweite und Begründung abweichende Meinungen gibt: s. dazu: Heun, Funktionell-rechtliche Schranken der Verfassungsgerichtsbarkeit, 1992, S. 38 ff.; Schlaich/Korioth, Das Bundesverfassungsgericht, Rn. 519 ff. mwNachw.

${ }^{371}$ BVerfGE 57, 295, 324 - Drittes Rundfunkurteil, dort in Bezug auf die Einführung privater Rundfunkangebote; s. zum „experimentierenden Gesetzgeber“ auch Bethge, in: Maunz/Schmidt-Bleibtreu/Klein/Bethge, BVerfGG, 38. EL 2012, Vorbemerkung Rn. 177, mwNachw; ders., Rundfunkfreiheit und öffentlich-rechtlicher Organisationsvorbehalt, 1987, S. 54 f.
} 
nen“. ${ }^{372}$ Das BVerfG versteht in diesem Rahmen die Gesetzgebung ausdrücklich auch als Prozess von „trial and error". ${ }^{373}$

162 Doch stehen diese Befugnisse dem Gesetzgeber selbstverständlich nur im Rahmen der verfassungsrechtlichen Grenzen zu. Dabei bezeichnet das BVerfG den Gesetzgeber insofern als den „Erstinterpreten des Grundgesetzes“. ${ }^{374}$ Damit wird zwar bereits angedeutet, dass das BVerfG ggf. als „Zweitinterpret" tätig werden kann. Allerdings muss zugleich berücksichtigt werden, dass dem Gesetzgeber nicht ex post vorgeworfen werden kann, dass ein Gesetz sich als verfassungswidrig erwiesen hat, wenn dies ex ante im Zeitpunkt der Beurteilung durch den Gesetzgeber noch nicht erkennbar war. ${ }^{375}$ Das BVerfG ist daher bei der Prüfung der Verfassungskonformität eines unter Nutzung des Prognosespielraums erlassenen Gesetzes daran gebunden, die gleiche Position einzunehmen, die auch der Gesetzgeber bei Erlass des Gesetzes innehatte; für den Fall, dass sich die vom Gesetzgeber ex ante getroffenen Prognosen ex post tatsächlich als unzutreffend erweisen, hat das BVerfG in seiner Entscheidung zur Neutralität der Bundesanstalt für Arbeit während eines Arbeitskampfes eine klare Aussage getroffen:

„Eine Einschätzung, von der der Gesetzgeber bei einer arbeitskampfrelevanten Regelung in zunächst unbedenklicher Weise ausgeht, kann sich im nachhinein als unzutreffend erweisen. Ursprünglich plausible Annahmen können durch die nachfolgende Entwicklung widerlegt, wohlbegründete Erwartungen hinsichtlich der komplexen Wirkungszusammenhänge enttäuscht werden. So kann sich trotz einer zunächst verfassungsrechtlich zulässigen Regelung eine nachhaltige Störung der Funktionsfähigkeit der Tarifautonomie einstellen. Eine solche Entwicklung ist in dem Maße korrekturbedürftig, in dem sich zeigt, daß strukturelle Ungleichgewichte auftreten, die ein ausgewogenes Aushandeln der Arbeits- und Wirtschaftsbedingungen nicht mehr zulassen und die in dem der Rechtsprechung gezogenen Rahmen nicht ausgeglichen werden können. Der Gesetzgeber ist dann verpflichtet, Maßnahmen zum Schutz der Koalitionsfreiheit zu treffen [...]. ${ }^{\text {1376 }}$

164 Damit ist die Möglichkeit einer "Sonderform der Unbegründetheitsentscheidung" angesprochen, die die beschränkte Kontrolldichte des BVerfG bei Prognoseentscheidungen des Gesetzgebers kompensieren kann. Haben sich die Rahmenbedingungen geändert, von denen der Gesetzgeber bei Erlass des Gesetzes nach sorgfältiger Sachverhaltsaufklärung berechtigterweise ausgehen durfte, kann das BVerfG den Gesetzgeber dazu anhalten, „die weitere Anwendung des vom Gericht für verfassungsrechtlich unbedenklich erklärten Gesetzes in der Praxis zu beobachten und es erforderlichenfalls so zu ändern, dass es auch weiterhin den Anforderungen der Verfassung genügt" ${ }^{377}$ Dadurch wird letztlich die Ausgangsüberlegung noch einmal unterstrichen, dass dem Gesetzgeber ein Prognosespiel-

\footnotetext{
${ }^{372}$ BVerfGE 57, 295, 324 - Drittes Rundfunkurteil, unter Verweis auf BVerfGE 54, 173, 202; dazu Stettner, NVwZ 1989, 806.

${ }^{373}$ Stettner, NVwZ 1989, 806, $806 \mathrm{f}$.

${ }^{374}$ BVerfGE 101, 158, 236.

${ }^{375}$ Neumann, RdA 2007, 71, 74; Stettner, NVwZ 1989, 806, 812.

${ }^{376}$ BVerfGE 92, 365, 397, unter Verweis auf BVerfGE 25, 1, 13; 49, 89, 130; 50, 290, 335; s. auch zur ex ante Perspektive des BVerfG Stettner, NVwZ 1989, 806, 808.

${ }^{377}$ Hömig, in: Maunz/Schmidt-Bleibtreu/Klein/Bethge, BVerfGG, 38. EL 2012, § 95 Rn. 71 mwNachw.; s. für Beispiele aus der verfassungsrechtlichen Rspr. BVerfGE 16, 147, 188 - Werkfernverkehr; 49, 89, 130, 132 Kalkar; 50, 290, 335 - Mitbestimmung; 73, 118, 169, 180, 182 - Niedersächsisches Landesrundfunkgesetz.
} 
raum zur Verfügung steht. Der dem Gesetzgeber auferlegten Beobachtungspflichtkommt verfassungsrechtliche Verbindlichkeit zu. ${ }^{378}$

Die eingeschränkte Überprüfbarkeit der Einschätzungsprärogative des Gesetzgebers wird allerdings in zweierlei Hinsicht konkretisiert: Zum einen muss notwendigerweise ein Fall vorliegen, in dem eine Prognose erforderlich ist, das heißt eine Sachlage, die ungeklärt ist oder in der eine Folgenabschätzung schwer zu treffen ist. Das BVerfG darf mindestens überprüfen, ob der Gesetzgeber alle seine Möglichkeiten ausgeschöpft hat, sich ein hinreichend sicheres Urteil zu bilden. ${ }^{379}$ „Intuition [...] darf nicht erreichbares, intersubjektiv vermittelbares Faktenwissen ersetzen“, jedenfalls nicht, soweit es sich vermeiden lässt. ${ }^{380}$ Zum anderen ist noch nichts über die Kontrolldichte ausgesagt, die das BVerfG als Maßstab anlegt. In der bereits zitierten Arbeitskampf-Entscheidung hat das BVerfG eine klare Rollenverteilung vorgezeichnet. Den Hintergrund der Entscheidung bildete die Koalitionsfreiheit nach Art. 9 Abs. 3 GG, die vom Gesetzgeber verlangt, den Einsatz von Arbeitskampfmitteln durch gesetzliche Rahmenbedingungen zu konturieren, „die sichern, daß Sinn und Zweck dieses Freiheitsrechts sowie seine Einbettung in die verfassungsrechtliche Ordnung gewahrt bleiben “381. Den Handlungsspielraum, der dem Gesetzgeber dabei verbleibt, beschrieb das BVerfG wie folgt:

„Bei der Beurteilung der Frage, ob die Regelung den Gewerkschaften die Fähigkeit nimmt, einen wirksamen Arbeitskampf zu führen, ist von der Einschätzung durch den Gesetzgeber auszugehen. Die Kampfstärke einer Arbeitnehmerkoalition hängt von einer im einzelnen kaum überschaubaren Fülle von Faktoren ab, die in ihren Wirkungen schwer abschätzbar sind. Nicht ohne weiteres erkennbar sind zudem die Möglichkeiten, die einer Gewerkschaft zu Gebote stehen, sich durch besondere Arten der Kampfführung an veränderte Umstände anzupassen. In einer solchen Lage trifft den Gesetzgeber die politische Verantwortung für eine zutreffende Erfassung und Bewertung der maßgebenden Faktoren. Das BVerfG kann sich nicht durch eine eigene Einschätzung an seine Stelle setzen. Die Grenze der Verfassungswidrigkeit ist erst dann überschritten, wenn sich deutlich erkennbar abzeichnet, daß eine Fehleinschätzung vorgelegen hat oder die angegriffene Maßnahme von vornherein darauf hinauslief, ein vorhandenes Gleichgewicht der Kräfte zu stören oder ein Ungleichgewicht zu verstärken. “382

167 Das BVerfG räumt sich selbst somit lediglich die Kompetenz ein, Entscheidungen zu korrigieren, die "deutlich erkennbar" verfassungswidrig sind (Evidenzkontrolle ${ }^{383}$ ) bzw. „,von vornherein“ auf ein nicht vertretbares Ergebnis hinauslaufen (Vertretbarkeitsprüfung ${ }^{384}$ ). Diese zwei Intensitätsstufen der Prüfung hat das BVerfG schließlich um eine dritte ergänzt für Fälle, in denen besonders gewichtige Grundrechte schwerwiegend eingeschränkt werden. In diesen Fällen - aber auch nur dann - gesteht

\footnotetext{
${ }^{378}$ Papier, in: Maunz/Dürig, GG, 68. EL 2013, Art. 14 Rn. 323; Mayer, Die Nachbesserungspflicht des Gesetzgebers, 1996, S. 34, 39 ff., 149 ff.; Heckmann, Geltungskraft und Geltungsverlust von Rechtsnormen, 1997, S. 83 f.; Hömig, in: Maunz/Schmidt-Bleibtreu/Klein/Bethge, BVerfGG, 38. EL 2012, § 95 Rn. 71; für die Pflicht des experimentierenden Gesetzgebers, nach der Experimentierphase wieder die „Normalität der dirigierenden Verfassung“ zu berücksichtigen, Bethge, in: Maunz/Schmidt-Bleibtreu/Klein/Bethge, BVerfGG, 38. EL 2012, Vorbemerkung Rn. 177; ferner ders., NVwZ 1997, 1, 4.

379 BVerfGE 77, 170, 215; 88, 203, 262; BVerfG NJW 1994, 1577, 1579.

${ }^{380}$ Stettner, NVwZ 1989, 806, 807.

${ }^{381}$ BVerfGE 92, 365, 395.

${ }^{382}$ BVerfGE 92, 365, 396.

${ }^{383}$ S. BVerfGE 36, 1, 17 - Grundvertrag; BVerfGE 37, 1, 20 - Stabilisierungsfonds; BVerfGE 40, 196, 223 - Güterkraftverkehrsgesetz.

${ }^{384}$ S. BVerfGE 25, 1, 12 f., 17 - Mühlengesetz; BVerfGE 30, 250, 263 - Absicherungsgesetz; BVerfGE 39, 210, 225 f. - Mühlenstrukturgesetz; BVerfGE 88, 203, 262; BVerfG NJW 1994, 1577, 1586.
} 
es sich einen intensiven Prüfungsmaßstab zu, in dem es eigene Wertungen treffen und an die Stelle derjenigen des Gesetzgebers stellen kann. ${ }^{385}$ Der Übergang zwischen den Intensitätsstufen ist flieBend und nicht immer eindeutig zu bestimmen, insbesondere zwischen der Evidenzkontrolle und der Vertretbarkeitsprüfung. ${ }^{386}$ Als maßgeblich erachtet das BVerfG den jeweiligen Sachbereich und die Dichte bzw. das Fehlen einer verfassungsrechtlich vorgegebenen Regelung, ${ }^{387}$ aber auch die Bedeutung der betroffenen Rechtsgüter. ${ }^{388}$

168 Im Sachbereich des Urheberrechts hat das BVerfG dem Gesetzgeber in der Vergangenheit ebenfalls einen Prognosespielraum eingeräumt, bei dessen Bestimmung die genannten Unsicherheitsaspekte in vollem Umfang zum Tragen kamen, namentlich bei der Festlegung eines angemessenen Vergütungssatzes im Rahmen von § 54 Abs. 4 UrhG a.F.: ${ }^{389}$

„Ähnlich wie bei der Ausgestaltung von Gebührenregelungen kommt dem Gesetzgeber auch in diesem Bereich ein weiter Entscheidungsspielraum zu (BVerfGE 20, 257 (270); 50, 217 (226 und 233)) [...]. Da der Werkkonsum ein eher immaterieller Vorgang ist, lassen sich kaum Maßstäbe für die Angemessenheit finden. Es kommt hinzu, daß das vom Gesetzgeber zulässigerweise gewählte Vergütungsprinzip notwendig alle Unsicherheiten enthält, die Prognoseentscheidungen anhaften. Das gilt insbesondere für die Frage, wann der Markt mit Audio- und Videogeräten gesättigt sein wird und in welchem Umfang der Kassettenabsatz bei steigender Versorgung der Haushalte mit diesen Geräten anwachsen wird. Arbeitszeitregelungen (vermehrte Freizeit) können ebenso eine Rolle spielen wie konjunkturelle Einflüsse und technische Neuentwicklungen, welche die Geräte möglicherweise noch weiter verbilligen. Die Beurteilung der Entwicklung war dem Gesetzgeber schließlich dadurch erschwert, daß die Abgabe unter Umständen ihrerseits den Absatz wegen der Verteuerung vor allem bei Leerkassetten negativ beeinflussen kann."

170 Das BVerfG hat sich in diesem Fall auf eine Evidenzkontrolle beschränkt: „Bei dieser Fülle widerstreitender Gesichtspunkte kann die Regelung nicht beanstandet werden. ${ }^{\text {“390 }}$ Dabei hat es mitberücksichtigt, dass der Gesetzgeber die Bundesregierung ersucht hat, alle drei Jahre über die Entwicklung des Vergütungsaufkommens Bericht zu erstatten, und sich zudem vorbehalten hat, im Falle einer Veränderung der maßgeblichen Rahmenbedingungen eine Anpassung der Vergütung vorzunehmen. ${ }^{391}$ Ähnlich hat das BVerfG in Bezug auf die Betreibervergütung für Fotokopien nach $\S 54$ Abs. 2 UrhG a.F. entschieden. Dabei hat das Gericht erneut betont, dass dem Gesetzgeber bei komplexen Sachverhalten das Recht zusteht, sich zunächst mit „gröberen Typisierungen und Generalisierungen“ zu begnügen, „um binnen angemessener Zeit Erfahrungen zu sammeln“ ${ }^{392}$ Auch hier ist die Anweisung des Gesetzgebers an die Bundesregierung, die zukünftigen Entwicklungen zu beobachten, wertend berücksichtigt worden.

\footnotetext{
${ }^{385}$ S. BVerfGE 7, 377, 406 ff. - Apotheken; BVerfGE 11, 30, 45 - Kassenärzte; BVerfGE 17, 269, 276 ff. - Arzneimittelgesetz; BVerfGE 39, 1, 51 ff. - Fristenregelung; BVerfGE 45, 187, 238 - Lebenslange Freiheitsstrafe; überblicksartig zu den drei Stufen BVerfGE 50, 290, 332 - Mitbestimmung.

${ }^{386}$ So zu Recht Schlaich/Korioth, Das Bundesverfassungsgericht, 9. Aufl. 2012, Rn. 536.

${ }^{387}$ Schlaich/Korioth, Das Bundesverfassungsgericht, 9. Aufl. 2012, Rn. 530.

${ }^{388}$ BVerfGE 50, 290, 332 f. - Mitbestimmung.

${ }^{389}$ BVerfGE 79, 1, 26 f. - Urheberrechtliche Vergütung.

390 BVerfGE 79, 1, 28 - Urheberrechtliche Vergütung.

391 BVerfGE 79, 1, 29 - Urheberrechtliche Vergütung.

392 BVerfG NJW 1997, 247, 248 - Kopierladen I, unter Verweis auf BVerfGE 75, 108, 162 - Künstlersozialabgabe.
} 
171 Insbesondere bei in die Zukunft gerichteten Prognoseentscheidungen, wie sie die Einführung einer urheberrechtlichen Schrankenregelung darstellt, belässt das BVerfG dem Gesetzgeber einen gewissen Spielraum, der erst dann überschritten wird, wenn die Erwägungen des Gesetzgebers so offensichtlich unrichtig sind, dass sie vernünftigerweise keine Grundlage für die gesetzgeberischen Maßnahmen darstellen können. Berücksichtigung finden müssen aber auch die Bedeutung der betroffenen Rechtsgüter sowie die Eingriffsintensität, die von der konkreten Ausgestaltung der gesetzlichen Schrankenregelung abhängt.

(2) Beeinträchtigung des Eigentums gem. Art. 14 GG

172 In erster Linie kommt ein Eingriff in das Eigentumsrecht gem. Art. 14 Abs. 1 GG der Rechteinhaber in Betracht.

\section{(a) Schutzbereich und Eingriff}

173 Unstreitig genießt das Urheberrecht bzw. die aus inm folgenden Verwertungsrechte den Schutz des Eigentums nach Art. 14 Abs. $1 \mathrm{GG}^{393}$ da es - neben dem Schutz der persönlichkeitsrechtlichen Interessen ${ }^{394}$ - dem Urheber die wirtschaftliche Nutzbarkeit und damit die vermögenswerten Ergebnisse seiner Werke zuordnet, ${ }^{395}$ sodass der Schutzbereich des Art. 14 GG eröffnet ist.

174 Ein Eigentumseingriff kann in dem vollständigen Entzug der Eigentumsposition oder in der Einschränkung der Nutzung und Verwendung des Eigentums liegen. Während die Enteignung nur unter den Bedingungen des Art. 14 Abs. 3 GG zulässig ist, werden Inhalts- und Schrankenbestimmungen hauptsächlich am Verhältnismäßigkeitsprinzip gemessen. Eine Enteignung gem. Art. 14 Abs. 3 GG scheidet hier als Maßstab von vornherein aus, da die urheberrechtlichen Schranken generell und abstrakt lediglich den Inhalt des enteignungsfähigen Eigentums festlegen, ${ }^{396}$ nicht aber eine bereits bestehende Rechtsposition entziehen - was für die bestehende Schranke auch allgemein anerkannt ist. ${ }^{397}$ Vielmehr wird durch die Ausdehnung der urheberrechtlichen Schranken der Umfang des urheberrechtlichen Eigentums für die Zukunft neu definiert. Die in die Zukunft gerichtete generelle und abstrakte Festlegung des Eigentums an sich ist zwar keine Beeinträchtigung des Grundrechts, allerdings muss sich der Gesetzgeber bei der gesetzlichen Ausgestaltung an die verfassungsrechtlichen Grenzen halten. ${ }^{398}$

\section{(b) Rechtfertigung}

175 Das Eigentum nach Art. 14 Abs. 1 GG wird nicht schrankenlos per se vom Grundgesetz geschützt. Vielmehr steht es dem Gesetzgeber im Rahmen der Verhältnismäßigkeit frei, den Inhalt des Eigentums auszugestalten, so dass es nicht das Eigentum an sich gibt, sondern nur bestimmte Ausprägungen. ${ }^{399} \mathrm{Ob}$ daraus folgt, dass die Verfassung nicht bestimmte Geschäftsmodelle schützen würde, et-

\footnotetext{
${ }^{393}$ St. Rspr. des BVerfG: BVerfGE 31, 229, 238 ff.; BVerfGE 79, 29, 40; BVerfG NJW 2011, 288, 290 ; statt vieler ferner: Dreier, in: Dreier/Schulze, UrhG, 4. Aufl. 2013, Einl. Rn. 39 ff.; Depenheuer, in: v. Mangoldt/Klein/Starck, GG, 6. Aufl. 2010, Art. 14 GG Rn. 148; s. auch Papier, in: Maunz/Dürig, GG, 68. EL 2013, Art. 14 Rn. 197 f.; Paulus/Wesche, ZGE 2010, 385, 389 mwNachw.

${ }^{394}$ S. dazu unten Rn. $188 \mathrm{ff}$.

395 So bereits BVerfG NJW 1971, 2163; bestätigend BVerfG GRUR Int. 2012, 567, 568.

${ }^{396}$ Zwengel, Kulturflatrates, 2012, S. 125; für das Modell einer Kulturflatrate auch Blanke-Roeser, in: Stiftung der Hessischen Rechtsanwaltschaft, Kulturflatrate, 2013, 29, 45; a.A. aber Fechner, 1999, S. 239.

397 Götting, in: Loewenheim, Handbuch des Urheberrechts, 2. Aufl. 2010, § 3 Rn. 4; Melichar, in: Schricker/Loewenheim, UrhR, 4. Aufl. 2010, Vor §§ 44a ff. UrhG Rn. 8.

${ }^{398}$ Wieland, in: Dreier, GG, 3. Aufl. 2013, Art. 14 Rn. 90.

${ }^{399}$ Grundlegend BVerfGE 58, 300, 330 - Naßauskiesung; BVerfG NJW 1992, 1303, 1305 f.; BVerfG NJW 1988, 1371.
} 
wa hinsichtlich des Verkaufs und der Verwertung einzelner Musikstücke etc. ${ }^{400}$ ist unerheblich; in Rede steht allein die Frage, ob die von Art. 14 Abs. 1 GG grundsätzlich verliehenen Ausschließlichkeitsrechte vom Gesetzgeber so ausgestaltet werden dürfen, dass der Eigentümer (Urheber) unter bestimmten Bedingungen die Nutzung seines Eigentums gegen angemessene Vergütung zu dulden hat, weil andere, ebenfalls grundrechtliche geschützte Interessen dies rechtfertigen.

176 Das BVerfG hat spezifisch für das Urheberrecht den Gesetzgeber für ermächtigt, aber auch verpflichtet gehalten, auf der einen Seite den grundlegenden Gehalt der Eigentumsgarantie - namentlich den verfassungsrechtlich garantierten Anspruch auf angemessene Verwertung der schöpferischen Leistung - zu wahren, andererseits aber auch einen Ausgleich und ein ausgewogenes Verhältnis mit den schutzwürdigen Interessen der Allgemeinheit herzustellen. ${ }^{401}$ In der grundlegenden „Schulbuch“-Entscheidung hat das BVerfG in Bezug auf die Schranken des $§ 46$ UrhG insofern ausgeführt:

„Bei der verfassungsrechtlichen Beurteilung dieser gesetzlichen Schranke ist davon auszugehen, daß der Gesetzgeber nicht nur die Individualbelange zu sichern hat, sondern ihm auch aufgetragen ist, den individuellen Berechtigungen und Befugnissen die im Interesse des Gemeinwohls erforderlichen Grenzen zu ziehen; er muß den Bereich des einzelnen und die Belange der Allgemeinheit in einen gerechten Ausgleich bringen. ${ }^{4402}$

178 Bezüglich der konkreten Ausgestaltung der Schranke räumte das BVerfG dem Gesetzgeber einen "verhältnismäßig weiten Entscheidungsraum" ein, da es sich hierbei um eine gesetzliche Ausformung der Eigentumsfreiheit gem. Art. 14 Abs. 1 S. 2 GG handelt. ${ }^{403}$ Das BVerfG trägt damit auch der Sozialbindung des Eigentums nach Art. 14 Abs. 2 GG Rechnung. ${ }^{404}$ Dies hat das Gericht auch in anderen Entscheidungen bekräftigt. ${ }^{405}$ Dabei muss aber auch das Institut Eigentum in seiner Funktion als freiheits- und existenzsicherndes Grundrecht gewahrt bleiben. Insbesondere muss die gesetzliche Ausgestaltung dem Verhältnismäßigkeitsgrundsatz genügen.

\section{(i) Legitimes und geeignetes Mittel}

179 Die Kulturflatrate muss dazu geeignet sein, einen legitimen Zweck zu erreichen, oder zumindest zu fördern. Nach dem eben Gesagten ist hier der legitime Zweck der Ausgleich der Rechte der Urheber und der Interessen der Nutzer, sowie das Sichern einer angemessenen Vergütung für die Urheber. ${ }^{406}$

\footnotetext{
${ }^{400}$ In diese Richtung aber argumentierend Roßnagel et al., Gutachten 2009, S. 12 f. - die Frage, ob Geschäftsmodelle durch die Verfassung geschützt werden, verdeckt das eigentliche Problem, wie weit Ausschließlichkeitsrechte gewährt werden, was jedem Geschäftsmodell vorgelagert ist; anders offenbar auch Schwartmann/Hentsch, ZUM 2012, 759, 769.

${ }^{401}$ BVerfG GRUR 1972, 481, 484; s. auch BVerfG NJW 1979, 2029, 2030; BVerfG NJW 1971, 2163, 2164; BVerfG GRUR Int. 2012, 567, 568; s. auch den für das Urheberrecht zuständige Berichterstatter im BVerfG Paulus, in: FS Papier, 2013, S. 561, 564 f.; Grzeszick, ZUM 2007, 344, 350.

402 BVerfGE 31, 229, 241 f. - Schulbuch.

${ }^{403}$ BVerfGE 79, 29, 40.

${ }^{404}$ Grzeszick, ZUM 2007, 344, 349; Götting, in: Loewenheim, Handbuch des Urheberrechts, 2. Aufl. 2010, § 3
} Rn. 3.

405 BVerfGE 21, 72, 83; BVerfGE 31, 229, 241; BVerfG GRUR 1972, 481, 483; Paulus, in: FS Papier, 2013, S. 561, 564 f.; Grzeszick, ZUM 2007, 344, 350 f.; Götting, in: Loewenheim, Handbuch des Urheberrechts, 2. Aufl. 2010, $\S 3 \mathrm{Rn} .3$.

${ }^{406}$ Blanke-Roeser, in: Stiftung der Hessischen Rechtsanwaltschaft, Kulturflatrate, 2013, 29, 46, nennt insofern nicht unzutreffend die „Neugestaltung des Kulturbetriebs“ und die „kulturelle Teilhabe der Allgemeinheit" als legitime Zwecke. Gerade durch ersteren Begriff werden konkrete Zwecksetzungen jedoch nicht präzise abge- 
Vor diesem Hintergrund ist die Einführung einer „Kulturflatrate“ als Abgabe für die Duldung bestimmter Nutzungshandlungen ein legitimes Mittel, da sie weitgehend ineffiziente und mit Gefährdungen der Grundrechtspositionen Dritter einhergehende Rechtsverfolgungsmaßnahmen ersetzen soll. ${ }^{407}$ Dies gilt auch im Rahmen der durch die vom BVerfG eingeschränkten Einschätzungsprärogative des Gesetzgebers, da ein gesteigertes öffentliches Interesse an der Lösung der dargestellten, durch die Möglichkeiten des Internet verschärften urheberrechtlichen Konflikte unterstellt werden kann: Insbesondere ist sie auch geeignet, die sonst eintretenden Einbußen der Urheber zu kompensieren. ${ }^{408}$ Schließlich kann auch aus Unschärfen in der Erfassung der Häufigkeit von Verwertungshandlungen noch nicht auf die Ungeeignetheit der Abgabe geschlossen werden; denn auch hier hat die verfassungsrechtliche Rechtsprechung das Bedürfnis nach einer gewissen Pauschalierung anerkannt. ${ }^{409}$ Zudem dürften die Methoden der Erfassung noch genauer sein als herkömmliche Erhebungen im Bereich der Geräte- und Leermedienabgaben, ${ }^{410}$ so dass insgesamt die Abgabe ein geeignetes Mittel darstellt.

\section{(ii) Erforderlichkeit}

180 Die Kulturflatrate-Regelung muss ebenso erforderlich, also das mildeste noch effektive Mittel sein. ${ }^{411}$ Dabei kann der Einführung einer Kulturflatrate als Schranke(n) mit Vergütungspflicht nicht entgegengehalten werden, dass sie angesichts von Streaming-Diensten nicht erforderlich sei, da der erleichterte, nur mit einer pauschalen Abgabe bezahlte Zugang zu Inhalten auch hier vom freien Markt ermöglicht würde. ${ }^{412}$ Denn eine solche Sichtweise würde die Kulturflatrate auf einen Zugang zu Inhalten mit pauschaler Vergütung reduzieren, die noch nicht einmal alle bei Streaming-Angeboten umfasst wären. Die beabsichtigte Kompensation für Umsatz- bzw. Einkommensverluste der Rechteinhaber würde damit ausgeblendet, ebenso die beabsichtigte Vermeidung der negativen Auswirkungen intensiver (oder potentiell noch weiter verschärfter) Rechtsverfolgungsmaßnahmen im Hinblick auf Persönlichkeitsrechte und Datenschutz der Nutzer sowie die Vermeidung der dadurch bedingten "chilling effects" in der Kommunikation. Mit anderen Worten bzw. nochmals betont stellt sich eine Kulturflatrate zwar als eine "second best"-Lösung im Vergleich zu üblichen Eigentumsrechten, jedoch angesichts struktureller Verfolgungsdefizite und erheblicher Nebenwirkungen auf Rechtspositionen Dritter (Datenschutz etc.) als effektivere Abhilfemaßnahme dar.

181 In Betracht käme als milderes Mittel, die Rechteinhaber auf DRM-Systeme als Selbstschutz zu verweisen und diese Systeme selbst - wie derzeit - einem strengen Schutz zu unterstellen. ${ }^{43}$ Die Erfahrungen haben indes gezeigt, dass, anders als in der Software- und Spielbranche, DRM-Systeme

bildet. Beide Begriffe dienen eher als Zusammenfassung der Gründe und Ansatzpunkte einer Kulturflatrate als urheberrechtliche Schranke, wie sie oben diskutiert werden.

${ }^{407}$ Zutr. und eingehend Roßnagel et al., Gutachten, 2009, S. 16; Zwengel, Kulturflatrates, 2012, S. $131 \mathrm{f}$.

${ }^{408}$ Zwengel, Kulturflatrates, 2012, S. 133 f.; Hennemann, Urheberrechtsdurchsetzung und Internet, 2011, S. 320 f.

${ }^{409}$ Auf europäischer Ebene EuGH, Urt. v. 21.10.2010 - C-467/08, GRUR 2011, 50, 54 f. Rn. 55 ff. - Padawan (im Folgenden verkürzt zitiert); aus nationaler Sicht BVerfG GRUR 1997, 123, 123 f.; BVerfG NJW 1992, 1303, 1304; vgl. ferner BGH GRUR 1988, 782, 783.

${ }^{410}$ Ausführlich dazu unten Rn. $427 \mathrm{ff}$.

${ }^{411}$ Starck, in: v. Mangoldt/Klein/Starck, GG, 6. Aufl. 2010, Art. 1 Abs. 3 Rn. 278; BVerfGE 17, 269, 279 f.; 30 , 292, 316; 39, 156, 165; 63, 88, 115 ff.; 102, 197, 217 f.; 104, 337, 347 f.; 120, 274, 319 f.

412 So aber Schwartmann/Hentsch, ZUM 2012, 759, 768; ähnlich Elbel, K\&R 2013, 439, 442; i.E. wie hier dagegen Klimanek, in: Stiftung der Hessischen Rechtsanwaltschaft, Kulturflatrate, 2013, 75, 87.

${ }^{413}$ Gerade Letzteres wäre als gesetzgeberische Maßnahme durchaus eine Alternative zur Einführung einer Urheberrechtsschranke, was die grundsätzliche Kritik von Blanke-Roeser, in: Stiftung der Hessischen Rechtsanwaltschaft, Kulturflatrate, 2013, 29, 48, am Vergleich mit DRM-Systemen zumindest relativiert. 
nicht geeignet waren, einen adäquaten Schutz der Rechteinhaber sicherzustellen, zudem ihre Akzeptanz bei den Nutzern sehr eingeschränkt zu sein scheint, und sie von der Industrie selbst wieder zurückgezogen wurden, so dass der Schluss gerechtfertigt ist, dass nur die Abgabe (derzeit) das mildeste Mittel darstellt. ${ }^{414}$

182 Zumal gewisse mit der Kulturflatrate (mit-)verfolgte Ziele - bspw. der Schutz der Grundrechte Dritter bei Rechtsverfolgungsmaßnahmen, die Entkriminalisierung der Bevölkerung, Entlastung der Rechtspflege oder die Schaffung einer höheren Verfügbarkeit kultureller Güter - mit DRM-Systemen gerade nicht erreicht werden können. ${ }^{415}$ Vielmehr kann eine Abgabe gerade dort Abhilfe schaffen, wo DRMSysteme umgangen werden, indem trotzdem eine Vergütung sichergestellt wird; der Verweis auf DRM-Systeme als individuelle Rechtsdurchsetzung vermag daher nicht ohne weiteres die Rechteinhaber vor Verlusten zu schützen. Allerdings ist durchaus fraglich und bedarf weiterer empirischer Untersuchungen, welche Faktoren dazu führen, dass DRM-Systeme in bestimmten Marktsektoren offensichtlich erfolgreich eingesetzt werden können, in anderen nicht. Entsprechendes gilt für abgestufte Rechtsverfolgungsmaßnahmen (HADOPI, Three-Strikes), zu denen kaum empirische Daten existieren und die oben genannten, möglicherweise auch mit einer Kulturflatrate verfolgten Ziele nicht erreichen können. ${ }^{416}$ Zudem versprechen die tradierten Rechtsverfolgungsmaßnahmen keine vollständige Abhilfe, sondern erinnern eher an ein Hase-und-Igel-Spiel. Ferner ist hier zu betonen, dass die Erfolgswahrscheinlichkeit von verschärften Rechtsverfolgungsmaßnahmen, etwa nach dem Vorbild des französischen Gesetzes (Loi HADOPI), bislang empirisch ungeklärt ist; man könnte umgekehrt auch für eine Verschärfung der Rechtsverfolgungsmaßnahmen plädieren. ${ }^{417}$ Maßgeblich ist daher, ob der Einschätzungs- und Prognosespielraum des Gesetzgebers bei der Wertung der vorliegenden Fakten eingehalten wird; hier ist aber - wie dargelegt - keineswegs deutlich, ob weitere Verschärfungen von Rechtsverfolgungsmaßnahmen ein gleich effektives Mittel darstellen. Erst recht werden die Fragen der Gefährdung von Persönlichkeitsrechten Dritter, bedingt durch die ständige Auswertung und Überwachung des Datenverkehrs, dabei nicht berücksichtigt.

183 Darüber hinaus wird geltend gemacht, dass kein Bedarf mehr für eine Kulturflatrate wegen zurückgehender Abmahnungen bestünde und die vorgeschlagenen Regelungen im Wesentlichen den stark rückläufigen Bereich der Tauschbörsen beträfen. Zudem dürften die Vorratsdaten nach § 113b TKG a.F. nicht zur Verfolgung von Urheberrechtsverletzungen herangezogen werden. ${ }^{418}$ Daran verwundert bereits die von denselben Autoren wenig später erhobene Forderung nach verstärkter Rechtsverfolgung gegenüber Nutzern, die zwar nicht mehr Tauschbörsen betreffen mag, wohl aber andere Wege, um Inhalte zu tauschen; das eigentliche Problem, die kritische Balance zwischen Rechtsverfolgung, Kompensation der Urheber und Wahrung des Datenschutzrechts etc., wird damit nur verschoben, aber nicht gelöst. Zudem hat das BVerfG in der Vorratsdatenspeicherung gerade

\footnotetext{
${ }^{414}$ So jedenfalls Roßnagel et al., Gutachten, 2009, S. 18; ähnlich Blanke-Roeser, in: Stiftung der Hessischen Rechtsanwaltschaft, Kulturflatrate, 2013, 29, 49; Klimanek, in: Stiftung der Hessischen Rechtsanwaltschaft, Kulturflatrate, 2013, 75, 89.

${ }^{415}$ Zwengel, Kulturflatrates, 2012, S. 135.

${ }^{416}$ Zwengel, Kulturflatrates, 2012, S. 137; Blanke-Roeser, in: Stiftung der Hessischen Rechtsanwaltschaft, Kulturflatrate, 2013, 29, 50.

${ }^{417}$ So denn auch konsequent Schwartmann/Hentsch, ZUM 2012, 759, 769 ff., die dies sogar auf eine verfassungsrechtlich abgeleitete Schutzpflicht des Staates gründen wollen.

${ }^{418}$ So Schwartmann/Hentsch, ZUM 2012, 759, 768.
} 
nicht die privatrechtliche Rechtsverfolgung pauschal als unzulässig bewertet. ${ }^{419}$ Schließlich ändern diese Verschiebungen zwischen den Filesharingaktivitäten nichts daran, dass eine Kompensation der Urheber über eine Kulturflatrate mindestens genauso erfolgversprechend wie verstärkte Rechtsverfolgungen erfolgen kann, so dass die Einschätzungsprärogative des Gesetzgebers nicht überschritten wäre. Bedenkt man, dass die bisherigen Rechtsverfolgungsmaßnahmen nicht die gewünschten Ergebnisse erzielt haben, ist mehr als fraglich, ob eine noch weitere Verschärfung vorzugswürdig gegenüber einer Abgabenlösung wäre oder ob eine Abgabe nicht vielmehr mehr Einnahmen und damit Kompensationen für Rechtseinbußen erzielen kann.

\section{(iii) Angemessenheit bzw. Verhältnismäßigkeit im engeren Sinne}

184 Fraglich ist allerdings, ob die Einführung einer neuen Schranke verbunden mit einer Abgabe auch verhältnismäßig im engeren Sinne ist. Verhältnismäßig im engeren Sinne ist eine Maßnahme dann, wenn die Einschränkungen des Grundrechts zu den Vorteilen, die der Allgemeinheit aus ihr erwachsen, ein angemessenes und vernünftiges Verhältnis besteht. ${ }^{420}$ Ein Eingriff in das Urheberrecht ist daher eher gerechtfertigt, wenn (aber auch nur soweit ${ }^{421}$ ) Allgemeininteressen betroffen sind, dagegen in der Regel nicht bei der Begünstigung von Individualinteressen. ${ }^{422} \mathrm{Im}$ Zusammenhang mit der Einschränkung des Urheberrechts als spezieller Ausformung des Eigentumsgrundrechts nach Art. 14 GG hat das BVerfG zudem eine Differenzierung vorgegeben: „Eingriffe in das Verfügungsrecht sind eher mit Gemeinwohlgründen zu rechtfertigen als eine Beschränkung des Verwertungsrechts. Diese kann wegen der Intensität des Eingriffs nur durch ein gesteigertes öffentliches Interesse gerechtfertigt werden. ${ }^{4423}$ Im Rahmen der Abwägung der verschiedenen Belange fallen zugunsten einer Abgabenlösung Interessen der Allgemeinheit an einfach zugänglichen Kulturgütern und Informationen, ${ }^{424}$ sowie vor allem die bereits dargelegten datenschutzrechtlichen Probleme ins Gewicht, die mit einer flächendeckenden Rechtsverfolgung verbunden sind, namentlich die de facto erforderliche Vorratsdatenspeicherung für zivile Zwecke, um die nötigen Identitätsdaten für Auskunftsansprüche zur Verfügung zu stellen, und die damit einhergehenden Gefährdungslagen für die informationelle Selbstbestimmung. ${ }^{425}$ Aber auch die Belastungen der Justiz spielen als Allgemeinbelang eine Rolle, wenn es um die Verwendung knapper Ressourcen geht. ${ }^{426}$ Dass Abgabenlösungen als Ersatz für Rechtsverfolgungen und Entlastungen des Justizsystems sowie Entkriminalisierungen ${ }^{427}$ eingesetzt werden, kann

\footnotetext{
${ }^{419}$ Ausdrücklich BVerfG NJW 2010, 833, 851: „Eine kategorische Trennung von ,Staatsaufgaben“ und ,privaten Aufgaben' mit der Folge der grundsätzlichen Unzulässigkeit einer Indienstnahme für Gemeinwohlzwecke von Privaten auf deren Kosten lässt sich der Verfassung nicht entnehmen“

${ }^{420}$ Starck, in: v. Mangoldt/Klein/Starck, GG, 6. Aufl. 2010, Art. 1 Abs. 3 Rn. 278; BVerfGE 90, 145, 185; 16, 194, 201 f.; 30, 292, 315; 68, 193, 219 f.; 83, 1, 19; 101, 331, 347.

${ }^{421}$ BVerfGE 79, 29, 40.

422 Insoweit zutr. Schwartmann/Hentsch, ZUM 2012, 759, 762

${ }^{423}$ BVerfGE 79, 29, 40, unter Verweis auf BVerfGE 31, 229 243; 49, 382, 400.

${ }^{424}$ Ausführlich Zwengel, Kulturflatrates, 2012, S. 149 ff., mit Verweis auf BVerfG GRUR 1972, 481; GRUR 1980, 44, 47; GRUR 1990, 438, 441; sowie Hennemann, Urheberrechtsdurchsetzung und Internet, 2011, S 314 f., 321 ; Runge, GRUR Int. 2007, 130, 135; Grzeszick, ZUM 2007, 344, 351; Honnefelder/Leenen, in: Depenheuer/Peifer, Geistiges Eigentum: Schutzrecht oder Ausbeutungstitel?, 2008, 47, 49; Hohagen, Die Freiheit der Vervielfältigung zum eigenen Gebrauch, 2004, S. 33 ff.; Badura, in: Ohly/Klippel, Geistiges Eigentum und Gemeinfreiheit, $2007,45,47 \mathrm{f}$.

425 In diese Richtung wohl auch: Zwengel, Kulturflatrates, 2012, S. 152 f.; i.E. auch Blanke-Roeser, in: Stiftung der Hessischen Rechtsanwaltschaft, Kulturflatrate, 2013, 29, 53 f.; krit. Hennemann, Urheberrechtsdurchsetzung und Internet, 2011, S. 325 f.

${ }^{426}$ Eingehend Roßnagel et al., Gutachten, 2009, S. 19 f.; krit., aber ohne nähere Begründung Klimanek, in: Stiftung der Hessischen Rechtsanwaltschaft, Kulturflatrate, 2013, 75, 93.

${ }^{427}$ Dies zu Recht in die Abwägung mit einbeziehend Zwengel, Kulturflatrates, 2012, S. 153 unter Bezug auf Lessig, Free Culture, 2004, S. 202; Fisher, 2004, S. 243; krit. aber: Hennemann, Urheberrechtsdurchsetzung und
} 
etwa auch an dem Beispiel von Quellensteuern für Zinseinkünfte demonstriert werden, die letztlich auf die punktgenaue Besteuerung zugunsten einer flächendeckenden Erfassung verzichten. Schließlich würde mit einer Abgabe auch eine finanzielle Kompensation derjenigen Rechteinhaber erreicht, die sich kostspielige Rechtsverfolgungsmaßnahmen nicht leisten können, so dass auch aus sozialstaatlichen Erwägungen diese Belange für eine Abgabe sprächen. Zwar wird dem entgegengehalten, dass auch eine Kulturflatrate eine Rechtsdurchsetzung der Vergütungsansprüche gegenüber dem Nutzer erfordere. ${ }^{428}$ Indes wird damit übersehen, dass die Abgabenlösung gerade bei den Internetanschlüssen ansetzt, mithin an der "Quelle“ und sich hier kaum Durchsetzungsprobleme stellen dürften, da die Nutzer sonst keine Möglichkeit der Internetverbindung hätten. ${ }^{429}$

185 Für die Schranke zugunsten nutzergenerierter Inhalte kann zudem ein allgemeines Interesse an der Förderung der Kreativität angeführt werden, was sich nicht zuletzt in der weitgezogenen Schranke der Kunstfreiheit Art. 5 Abs. 3 GG niederschlägt. ${ }^{430}$ Die Kunstfreiheit genießt einen hohen Stellenwert im Grundrechtsgefüge des Grundgesetzes. Zwar ist (mit Ausnahme der Menschenwürde als absoluter Höchstwert) die Bestimmung einer Rangordnung unter den Grundrechten unmöglich. ${ }^{431}$ Daran ändert prinzipiell auch nichts, dass die Kunstfreiheit anders als andere Grundrechte keinem einfachen Gesetzesvorbehalt unterworfen, sondern grundsätzlich schrankenlos gewährleistet wird. ${ }^{432}$ Doch kann sie ausschließlich zugunsten anderer Verfassungsgüter eingeschränkt werden. ${ }^{433}$ Neben der Abwehrfunktion besitzt die Kunstfreiheit auch eine objektiv-rechtliche Komponente, ${ }^{434}$ die Teil der Staatszielbestimmung des „Kulturstaats“ ist. ${ }^{435}$ Diese hat zwar mangels ausdrücklicher Festschreibung im GG keine trennscharfen Konturen, doch speist sie sich nicht zuletzt auch aus der Kunstfreiheit gem. Art. 5 Abs. 3 S. 1 GG als „Zentrum“ aller kulturellen Grundrechte. ${ }^{436}$ Die genannten Zusammenhänge hat das BVerfG im Hinblick auf eine etwaige Kunstförderungspflicht grundlegend in seiner Schallplatten-Entscheidung beschrieben, wo es über die Grundrechtsnorm des Art. 5 Abs. 3 S. 1 GG schreibt: „Als objektive Wertentscheidung für die Freiheit der Kunst stellt sie dem modernen Staat, der sich i.S. einer Staatszielbestimmung auch als Kulturstaat versteht, zugleich die Aufgabe, ein freiheitliches Kunstleben zu erhalten und zu fördern. ${ }^{4337}$ Daraus wird auch in der Literatur eine Förderungspflicht des Staates abgeleitet. ${ }^{438}$ Richtig ist aber auch, dass sich daraus keinesfalls konkrete Handlungsaufträge ableiten lassen, da es aufgrund der Komplexität des Kunstlebens und dessen gesellschaftlichen wie künstlerisch-persönlichen Wirkungsdimensionen eher um die Förderung von

Internet, 2011, S. 327; i.E. unklar Blanke-Roeser, in: Stiftung der Hessischen Rechtsanwaltschaft, Kulturflatrate, 2013, 29, $52 \mathrm{f}$.

${ }^{428}$ Schwartmann/Hentsch, ZUM 2012, 759, 768.

${ }^{429}$ Undifferenziert und ohne Bezug zu einem konkreten Abgabenmodell Schwartmann/Hentsch, ZUM 2012, 759, 768.

${ }^{430}$ Dies räumen selbst Schwartmann/Hentsch, ZUM 2012, 759, 765 ein.

${ }^{431}$ BVerfGE 35, 202, 225; Tettinger, JZ 2004, 1144, 1145; Isensee, AöR 131 (2006), 173, 191.

432 Bethge, in: Maunz/Schmidt-Bleibtreu/Klein/Bethge, BVerfGG, 38. EL 2012, § 90 Rn. 311.

${ }^{433}$ Für die Kunstfreiheit statt vieler nur BVerfGE 67, 213, 228 - Anachronistischer Zug.

${ }^{434}$ BVerfGE 30, 173, 188 ff.; 36, 321, 331; Scholz, in: Maunz/Dürig, GG, 68. EL 2013, Art. 5 Abs. 3 Rn. 16; Knies, Schranken der Kunstfreiheit als verfassungsrechtliches Problem, 1967, S. 177 ff., 195 ff.; Erbel, Inhalt und Auswirkungen der verfassungsrechtlichen Kunstfreiheitsgarantie, 1966, S. 99 ff., 102 ff.; Palm, Öffentliche Kunstförderung zwischen Kunstfreiheitsgarantie und Kulturstaat, 1997, S. 144 f.

435 BVerfGE 35, 79, 114; 36, 321, 331; Scholz, in: Maunz/Dürig, GG, 68. EL 2013, Art. 5 Abs. 3 Rn. 7 f.

${ }^{436}$ Palm, Öffentliche Kunstförderung zwischen Kunstfreiheitsgarantie und Kulturstaat, 1997, S. 129, 145.

${ }^{437}$ BVerfGE 36, 321, 331.

${ }^{438}$ Scholz, in: Maunz/Dürig, GG, 68. EL 2013, Art. 5 Abs. 3 Rn. 21; Ridder, Freiheit der Kunst nach dem Grundgesetz, 1963, S. 21; Ott, Kunst und Staat, 1968, S. 165; Palm, Öffentliche Kunstförderung zwischen Kunstfreiheitsgarantie und Kulturstaat, 1997, S. 177 f. mwNachw. 
allgemeinen Strukturen geht. ${ }^{439}$ Das bedeutet letztlich - gerade vor dem Hintergrund seiner Einschätzungsprärogative ${ }^{440}$ - dass dem Gesetzgeber die Entscheidung darüber zusteht, wo er korrigierend tätig wird, wenn sich bereichsspezifische, strukturelle Defizite zeigen. Im Falle der urheberrechtlichen Schranken ist insofern zu bedenken, dass das BVerfG ein „Interesse der Allgemeinheit an einem ungehinderten Zugang zu urheberrechtlich geschützten Werken" festgestellt hat. ${ }^{441}$ Dies liege daran, dass ein geschütztes Werk ab dem Moment seiner Publikation nicht mehr nur dem Urheber zur Verfügung stehe, „es tritt zugleich in den sozialen Raum und kann damit zu einem eigenständigen, das kulturelle und geistige Bild der Zeit mitbestimmenden Faktor werden. ${ }^{\prime 442}$ Auf diese Weise rechtfertigte das BVerfG die urheberrechtliche Schranke gem. § 46 UrhG zugunsten der Vervielfältigung, Verbreitung und öffentlichen Zugänglichmachung geschützter Werke als Elemente von Kirchen-, Schulund Unterrichtssammlungen. Die Argumentation kann entsprechend für die hier in Rede stehende Schranke herangezogen werden. Für verfassungswidrig erachtete das BVerfG bei § 46 UrhG lediglich die ursprünglich vorgesehene Vergütungsfreiheit. ${ }^{443}$ Dies ist in diesem Fall aber unproblematisch: Zwar kann der Urheber auch hier nicht mehr selbst seine Verwertungsrechte eigenständig geltend machen, insbesondere durch Lizenzierungen; doch steht dem im Interesse der Förderung der Kreativität bzw. neuer künstlerischer Werke ein Vergütungsanspruch gegenüber.

186 Schließlich ist behauptet worden, dass eine Kulturflatrate den Urhebern und Verwertern deren ausschließliche Nutzungsrechte gänzlich nehmen würde, im Gegensatz zur bereits existierenden Leermedien- bzw. Geräteabgabe, die die Abgabe lediglich für Zweitnutzungen im Rahmen der Störerhaftung als einen pauschalen Schadensersatzanspruch gewähre. ${ }^{444}$ Dieses im Wesentlichen auf Zweitnutzungen abstellende Argument setzt aber implizit voraus, dass der Private selbst bereits ein Werkstück erworben hätte; dies ist indes für die Privatkopie keineswegs erforderlich, die etwa auch bei Internetsendungen bzw. Internetradio eingreift, ebenso wie bei Kopien, die ein Privater von einem Werkstück eines Dritten anfertigt. ${ }^{445}$ Allenfalls für die Erweiterung der Schranke auf Uploads, also das Recht zur öffentlichen Zugänglichmachung, kann dieses Argument herangezogen werden. Aber auch hier gilt, dass es der Gestaltungsspielraum des Gesetzgebers erlaubt, gegen Vergütungen entsprechende Schranken aus Interessen der Allgemeinheit zu ziehen; zudem werden die ausschließlichen Verwertungsrechte keineswegs vollständig den Urhebern genommen, etwa nicht bei kommerziellem Gebrauch.

\footnotetext{
${ }^{439}$ S. Palm, Öffentliche Kunstförderung zwischen Kunstfreiheitsgarantie und Kulturstaat, 1997, S. 121.

${ }^{440}$ S. dazu oben Rn. $159 \mathrm{ff}$.

${ }^{441}$ BVerfG NJW 1971, 2163, 2164.

${ }^{442}$ BVerfG NJW 1971, 2163, 2164.

${ }^{443}$ BVerfG NJW 1971, 2163, $2164 \mathrm{f}$.

${ }^{444}$ So Schwartmann/Hentsch, ZUM 2012, 759, 768; dem folgend Klimanek, in: Stiftung der Hessischen Rechtsanwaltschaft, Kulturflatrate, 2013, 75, 87; ebenso Blanke-Roeser, in: Stiftung der Hessischen Rechtsanwaltschaft, Kulturflatrate, 2013, 29, 55.

445 BGH GRUR 1997, 459, 462 - CB-Infobank I; BGH GRUR 1997, 464, 466 - CB-Infobank II; Lüft, in: Wandtke/Bullinger, UrhR, 3. Aufl. 2009, § 53 UrhG Rn. 15; Dreier, in: Dreier/Schulze, UrhG, 4. Aufl. 2013, §53 UrhG Rn. 11; nach der Entscheidung des BGH zu Online-Videorecordern ist dem Begriff des Herstellers i.S.v. $§ 53$ Abs. 1S. 1 UrhG eine technische Sichtweise zugrunde zu legen, nach der dies auch derjenige ist, der die Kopie mit technischen Mitteln erstellt, die ihm von dritter Seite (hier dem Anbieter des Online-Videorecorders) zur Verfügung gestellt werden, was implizit bedeutet, dass die Vorlage der Kopie nicht zuvor vom Hersteller erworben worden sein muss, BGH GRUR 2009, 845, 846 - Internet-Videorecorder; s. dazu Wiebe, in: Spindler/Schuster, Recht der elektronischen Medien, 2. Aufl. 2011, §53 UrhG Rn. 4.
} 


\section{(c) Ergebnis}

187 Es kann daher festgehalten werden, dass eine Regelung wie die hier vorgeschlagene als Inhalts- und Schrankenbestimmung zwar in das Eigentumsgrundrecht Art. 14 Abs. 1 GG eingreift, allerdings aufgrund der gegenüberstehenden Allgemeininteressen und nicht zuletzt aufgrund der Einschätzungsprärogative des Gesetzgebers gerechtfertigt ist.

\section{(3) Persönlichkeitsrecht}

188 Da das Urheberrecht nach kontinentaleuropäischem Verständnis aber - wie bereits erwähnt - nicht nur ein wirtschaftlichen Zwecken dienendes Ausschließlichkeitsrecht ist, sondern auch gerade Ausdruck der persönlichen Kreativität ist, stellt es eine besondere Erscheinungsform des allgemeinen Persönlichkeitsrechts nach Art. 2 Abs. 1 GG dar. ${ }^{446}$ Das Urheberpersönlichkeitsrecht ist nach h.M. nicht identisch mit dem allgemeinen Persönlichkeitsrecht, sondern ist spezieller als dieses, ${ }^{447}$ wenngleich beide aus Art. 2 Abs. 1 i.V.m. Art. 1 Abs. 1 GG entspringen. ${ }^{448}$ Für den Inhaber bedeutet das Urheberpersönlichkeitsrecht zunächst das ausschließliche Recht,

„darüber zu bestimmen, ob, wann und in welcher Form sein Werk der Öffentlichkeit zugänglich gemacht werden soll [...]. Bei Werken, die unter Urheberrechtsschutz stehen, entspringt das sog. Veröffentlichungsrecht des Verfassers, soweit es nicht bereits aus dem allgemeinen Persönlichkeitsrecht erwächst, auch ohne ausdrückliche gesetzliche Regelung aus den persönlichkeitsrechtlichen Bestandteilen des Urheberrechts. ${ }^{4449}$

Das Veröffentlichungsrecht ist in § 12 UrhG kodifiziert, in Verbindung mit dem Recht auf Rückruf wegen gewandelter Überzeugung in $\S 42$ UrhG. Ersteres bliebe im Rahmen einer Kulturflatrate unberührt, da diese nur bereits veröffentlichte Werke umfassen würde. Letzteres bezieht sich nur auf vertragliche Nutzungsrechte, findet aber nicht auf gesetzliche Lizenzen - was die Kulturflatrate wäre - Anwendung. ${ }^{450}$ Der Schwerpunkt des Urheberpersönlichkeitsrechts liegt indes auf dem Recht auf Anerkennung der Urheberschaft nach § 13 UrhG, d.h. dem Recht des Urhebers darauf, dass er im Zusammenhang mit seinem Werk auch als Urheber genannt wird. ${ }^{451}$ Dies betont auch die Rechtsprechung:

„Das Namensnennungsrecht ist Ausfluß und besondere Erscheinungsform des Rechts auf Anerkennung der Urheberschaft im Sinne des $\S 13$ Satz 1 UrhG. Dieses Recht umfaßt die Anerkennung der Rechtsposition als Werkschöpfer und deren Dokumentation in der Außenwelt [...]. Es gehört zu den wesentlichen urheberpersönlichkeitsrechtlichen Berechtigungen, die ihre

\footnotetext{
${ }^{446}$ So bereits BVerfG NJW 1954, 1404, 1405 - Leserbrief; Ahlberg, in: Möhring/Nicolini, UrhG, 2. Aufl. 2000, Einl. Rn. 14; s. ausf. dazu Bullinger, in: Wandtke/Bullinger, UrhR, 3. Aufl. 2009, Vor § 12 ff. UrhG Rn. 16 ff. ${ }^{447}$ BGHZ 13, 334, 338 f. - Leserbrief; 15, 249, 257 ff. - Cosima Wagner; Bullinger, in: Wandtke/Bullinger, UrhR, 3. Aufl. 2009, Vor §§ 12 ff. UrhG Rn. 16; Schulze, in: Dreier/Schulze, UrhG, 4. Aufl. 2013, Vor § 12 UrhG Rn. 5; Dietz/Peukert, in: Loewenheim, Handbuch des Urheberrechts, 2. Aufl. 2010, § 15 Rn. 8 mwNachw; a.A. Wronka, UFITA 69 (1973), 71, 73; ein Überblick zum Streitstand bei Osenberg, Die Unverzichtbarkeit des Urheberpersönlichkeitsrechts, 1985, S. $10 \mathrm{ff}$.

${ }^{448}$ BGHZ 15, 249, 257 f. - Cosima Wagner; Bullinger, in: Wandtke/Bullinger, UrhR, 3. Aufl. 2009, Vor $\S 12 \mathrm{ff}$. UrhG Rn. 16.

449 BGHZ 15, 249, 258 - Cosima Wagner.

${ }^{450}$ Statt Vieler: Dreier, in: Dreier/Schulze, UrhG, 4. Aufl. 2013, § 42 Rn. 11.

${ }^{451}$ BGH GRUR 1995, 671, 672; Dietz/Peukert, in: Loewenheim, Handbuch des Urheberrechts, 2. Aufl. 2010, § 16 Rn. 66.
} 
Grundlage in den geistigen und persönlichen Beziehungen des Urhebers zu seinem Werk haben $[\ldots]^{\prime \prime} .{ }^{452}$

192 Ergänzt werden diese Ausprägungen des Urheberpersönlichkeitsrechts durch das Entstellungsverbot, das insbesondere in $\S \S 14,39$ UrhG zum Ausdruck kommt und sich „gegen Entstellungen sich gegen eine Beeinträchtigung der geistigen und persönlichen Urheberinteressen auch durch Form und Art der Werkwiedergabe und -nutzung richtet", nicht nur durch Eingriff in eine etwaige Werksubstanz. ${ }^{453}$

193 Jedoch kommt es nach der hier verfolgten Variante einer "Kulturflatrate“ nicht auf einen Eingriff in Persönlichkeitsrechte an, da dieser nur vorläge, wenn eine Rechtsverfolgung hinsichtlich der Entstellung von Werken nicht möglich wäre - genau dies ist aber mit der Erweiterung der Privatkopieschranken nicht beabsichtigt. ${ }^{454}$ Daran ändert auch nicht die Erweiterung der Bearbeitungsschranken bzw. -rechte etwas, da diese sich nur auf die Frage des Ausschließlichkeitsrechts beziehen, mithin auf die Modalität der Vergütungspflichtigkeit; das Recht, gegen Entstellungen vorzugehen, als eigentliche Ausprägung des Persönlichkeitsrechts soll davon unberührt bleiben. ${ }^{455}$ Schließlich wird der Urheber keineswegs dazu gezwungen, sein Werk zu veröffentlichen, oder der Entscheidung darüber beraubt, in welcher Weise er dies tun möchte; die vorgeschlagenen Schranken berühren diese Rechte nicht. ${ }^{456}$

194 Doch selbst wenn man einen Eingriff annehmen sollte, so ist dieser gerechtfertigt. Denn auch das Persönlichkeitsrecht wird durch kollidierende Grundrechte eingeschränkt, etwa durch die Informations- und Kommunikationsfreiheit Dritter, ${ }^{457}$ ebenso durch die allgemeinen Gesetze. ${ }^{458}$ Dementsprechend kann zunächst auf die gleichen Erwägungen wie zu Art. 14 GG zurückgegriffen werden.

\section{(4) Eingriff in Berufsfreiheit}

195 Die Einführung einer Kulturflatrate würde aller Wahrscheinlichkeit nach zudem dazu führen, dass kommerzielle Download-Portale (Musik, Film, E-Book etc.) Umsatzeinbußen erleiden würden, was einen Eingriff in deren Berufsfreiheit nach Art. 12 GG darstellen könnte. ${ }^{459}$ Problematisch ist hierbei, dass die Maßnahme nicht final auf kommerzielle Downloadportale gerichtet ist, sondern diese nur indirekt, bzw. mittelbar trifft. Diese sog. mittelbaren (oder auch nicht-imperativen) Grundrechtseingriffe werden im Rahmen der Berufsfreiheit in der Rechtsprechung des BVerfG besonders restriktiv

\footnotetext{
452 BGH GRUR 1995, 671, 672, unter Verweis auf RGZ 110, 393, 397 - Innenausstattung Riviera, sowie BGH GRUR 1963, 40, 42.

453 BGH GRUR 1982, 107, 109 - Kirchen-Innenraumgestaltung; s. auch BGHZ 150, 32, 41 f. - Unikatrahmen mwNachw; der Schutz des Urhebers kann bspw. dazu führen, dass die Verwendung eines Musikstücks als Klingelton auch ohne Veränderung des Werkes nach § 14 UrhG unzulässig ist, s. BGH NJW 2009, 774, 775 Rn. 14; s. ferner Bullinger, in: Wandtke/Bullinger, UrhR, 3. Aufl. 2009, Vor §§ 12 ff. UrhG Rn. 4; Dietz/Peukert, in: Loewenheim, Handbuch des Urheberrechts, 2. Aufl. 2010, § 16 Rn. 86.

${ }^{454}$ Dies verkennen offensichtlich Schwartmann/Hentsch, ZUM 2012, 759, 768 f., die nur pauschal die Kulturflatrate ohne Rücksicht auf die konkrete Ausgestaltung kritisieren.

455 S. oben Rn. $62 \mathrm{ff}$.

${ }^{456}$ Dies verkennt offensichtlich Elbel, K\&R 2013, 439, 442.

${ }^{457}$ Hierzu allgemein im Hinblick auf Internet-Sachverhalte Spindler, 69. DJT 2012, F 29, F $40 \mathrm{f}$.

${ }^{458}$ BVerfG NJW 1999, 1322, 1324; BVerfG NJW 2000, 1021, 1023; BVerfG NJW 2001, 594, 595; Di Fabio, in: Maunz/Dürig, GG, 68. EL 2013, Art. 2 Rn. 133.

${ }^{459}$ Ein Eingriff in Art. 14 GG kommt hier kaum in Betracht, da es um von Art. 14 GG nicht geschützte Zukunftsaussichten geht und auch der Schutz des eingerichteten Gewerbebetriebs nicht weiter geht als Art. 12 GG, zutr. für die Kulturflatrate Roßnagel et al., Gutachten, 2009, S. 22 unter Verweis auf BVerfGE 51, 193, 221 f.; zur bislang vom BVerfG noch nicht ausdrücklich anerkannten verfassungsrechtlichen Schutzfähigkeit des eingerichteten und ausgeübten Gewerbetriebes s. BVerfG NJW 1992, 1878, 1879; BVerfG NJW 2002, 2621, 2625 mwNachw; BVerfG NVwZ 2009, 1426, 1428 mwNachw; s. dazu auch Axer, in: Epping/Hillgruber, BeckOK GG, Art. 14 Rn. 51 ff.; Papier, in: Maunz/Dürig, GG, 68. EL 2013, Art. 14 Rn. 95 ff.
} 
gehandhabt. ${ }^{460}$ Ein Eingriff in Art. $12 \mathrm{GG}$ liegt demnach nur vor, wenn eine „objektiv berufsregelnde Tendenz", also ein besonders enger Zusammenhang mit der Berufsausübung besteht. ${ }^{461} \mathrm{Ob}$ ein solch enger Zusammenhang besteht oder nicht ist einzelfallabhängig und richtet sich stark nach der - nicht kritiklos gebliebenen ${ }^{462}$ - Rechtsprechung des BVerfG. So hat es beispielsweise hinsichtlich der Geräteabgaben eine objektiv berufsregelnde Tendenz verneint, mit dem Argument, dass hier nur interessenausgleichende Normen des Privatrechts vorlägen. ${ }^{463}$ Zwar ist die dort angesprochene und untersuchte Konstellation zwischen Urhebern, Nutzern und Geräteherstellern eine andere, doch auch die Kulturflatrate dient dem Ausgleich privatrechtlicher Interessen, so dass nach der Rechtsprechung des BVerfG eher kein Eingriff in die Berufsfreiheit der Download-Portalbetreiber vorliegt. ${ }^{464}$

Die Gegenauffassung, die die besonders enge Auslegung durch das BVerfG kritisiert, befürwortet demgegenüber, den allgemeinen Begriff des faktischen bzw. mittelbaren Eingriffes auch im Rahmen von Art. $12 \mathrm{GG}$ zu verwenden. ${ }^{465}$ Legt man diesen Maßstab an, so entscheidet über die Frage ob ein Eingriff vorliegt oder nicht, eine Gesamtschau aus Erheblichkeit, Finalität und Unmittelbarkeit der Maßnahme. ${ }^{466}$ Eine Kulturflatrate würde keine finale Wirkung haben und nur gemittelt über potentielle Kunden auf den Anbieter wirken. Ebenso sprechen die zu erwartenden Unterschiede zwischen kommerziell angebotenen digitalen Inhalten und den über Private (aufgrund der Schranken) zum Tausch angebotenen Files, insbesondere hinsichtlich ihrer Sicherheit gegen eine erhebliche Beeinträchtigung. Zumal bei kommerziellen Download-Portalen schon allein aufgrund der vertraglichen Beziehungen die Kunden entsprechende Gewährleistungsansprüche haben. Zudem können Download-Geschwindigkeiten und zusätzliche Angebote die kommerziellen Download-Portale von den privaten Anbietern hinreichend unterscheiden. ${ }^{467}$ Doch hängt die Beurteilung letztlich von den tatsächlichen Entwicklungen ab. Nähme man trotz allem eine erhebliche Beeinträchtigung an, müsste man weiter untersuchen, ob es sich um eine Regelung der Berufsausübung oder der Berufswahl handelt. ${ }^{468}$ So wäre nur dann, wenn durch die Einführung weitreichender Schranken und Abgaben der Betrieb eines kommerziellen Download-Portals unmöglich würde, die Berufswahl selbst beeinträchtigt. Ansonsten würde nur in den Geschäftsumfang der Download-Portale, nicht aber in ihre Existenz eingegriffen, ${ }^{469}$ so dass nur eine Berufsausübungsregelung vorläge, die ihrerseits bereits mit Allgemeinwohlbelangen gerechtfertigt werden kann, was wiederum auf die gleichen Erwägungen wie für

\footnotetext{
${ }^{460}$ Manssen, in: v. Mangoldt/Klein/Starck, GG, 6. Aufl. 2010, Art. 12 Abs. 1 Rn. 74; Kämmerer, in: von Münch/Kunig, GG, 6. Aufl. 2012, Art. 12 Rn. 46.

${ }^{461}$ BVerfGE 13, 181, 185; 52, 42, 54; 61, 291, 308; 70, 191, 214; Manssen, in: v. Mangoldt/Klein/Starck, GG, 6. Aufl. 2010, Art. 12 Abs. 1 Rn. 74; Kämmerer, in: von Münch/Kunig, GG, 6. Aufl. 2012, Art. 12 Rn. 46.

${ }^{462}$ Manssen, in: v. Mangoldt/Klein/Starck, GG, 6. Aufl. 2010, Art. 12 Abs. 1 Rn. 75; Kämmerer, in: von Münch/Kunig, GG, 6. Aufl. 2012, Art. 12 Rn. 46; Cremer, DÖV 2003, 921, 928;

${ }^{463}$ BVerfGE 31, 255, 265.

${ }^{464}$ So auch Zwengel, Kulturflatrates, 2012, S. 195 f.

${ }^{465}$ Manssen, in: v. Mangoldt/Klein/Starck, GG, 6. Aufl. 2010, Art. 12 Abs. 1 Rn. 75; Cremer, DÖV 2003, 921, 928; Gallwass, Faktische Beeinträchtigungen, 1970, S. 63 ff., 166; Papier, Der Staat 11 (1972), 483, 494; dagegen Kämmerer, in: von Münch/Kunig, GG, 6. Aufl. 2012, Art. 12 Rn. 46.

${ }^{466}$ Manssen, in: v. Mangoldt/Klein/Starck, GG, 6. Aufl. 2010, Art. 12 Abs. 1 Rn. 79; Sodan, DÖv 1987, 858, 863

f.; Ramsauer, Faktische Beeinträchtigungen des Eigentums, 1980, S. 36.

${ }^{467}$ Darauf stellen im Wesentlichen Roßnagel et al., Gutachten, 2009, S. 22 ab; ebenso: Zwengel, Kulturflatrates, 2012, S. 195 f.
}

${ }^{469}$ BVerfGE 24, 236, 251; BVerfGE 34, 252, 256. 
Art. 14 GG gestützt werden kann. ${ }^{470}$ Dementsprechend wird zu Recht darauf hingewiesen, dass es sich hier um vergleichsweise schwache Grundrechtspositionen handelt. ${ }^{471}$

197 Allerdings hängt diese Einordnung entscheidend von der getroffenen Annahme ab, dass die Qualität der angebotenen Inhalte von denjenigen der Download-Portale divergiert und auch sonstige Dienste von den Kunden honoriert werden. Problematisch erscheint hier, von der derzeitigen Situation der angebotenen privaten Inhalte im Netz auszugehen, ${ }^{472}$ denkbar wäre etwa, dass "gute“ Inhalte sich bei Einführung einer weitgehenden Schrankenregelung wesentlich öfter im Netz fänden, so dass die Substitutionsmöglichkeiten wesentlich größer wären.

\section{(5) Eingriff in Grundrechte der Provider}

198 Die Abgabenlösung einer Kulturflatrate könnte zudem auch in die Grundrechte der am Filesharing beteiligten Provider eingreifen, da diese die Messung des Nutzungsumfangs geschützter Werke ermöglichen und die entsprechenden Vergütungen einziehen und an Verwertungsgesellschaften etc. abführen müssten. ${ }^{473}$ Dies wäre zwangsläufig einerseits mit organisatorischen Maßnahmen (hauptsächlich personeller Natur) sowie mit Kosten (Anschaffung, Aufrüstung und Wartung von Geräten, Personalkosten etc.) verbunden, die in die Grundrechte der Berufsfreiheit (Art. $12 \mathrm{GG}$ ) sowie des Eigentums (Art. 14 GG, insbesondere hinsichtlich des Rechts am eingerichteten und ausgeübten Gewerbebetrieb) der Provider eingreifen würden. Die Grenzen der Zumutbarkeit derartiger Pflichten hat das VG Berlin im ähnlich gelagerten Fall der Vorratsdatenspeicherung skizziert: ${ }^{474}$

199 Konkret ging es darum, dass $\S 110$ Abs. 1 Nr. 1 TKG die Betreiber von TK-Anlagen, mit der öffentlich zugängliche TK-Dienste erbracht werden, dazu verpflichtet, „ab dem Zeitpunkt der Betriebsaufnahme auf eigene Kosten technische Einrichtungen zur Umsetzung gesetzlich vorgesehener Maßnahmen zur Überwachung der Telekommunikation vorzuhalten und organisatorische Vorkehrungen für deren unverzügliche Umsetzung zu treffen“. Eine Entschädigung ist im Gesetz hierfür nicht vorgesehen. ${ }^{475}$ Das VG Berlin legte das Verfahren dem BVerfG gem. Art. 100 Abs. 1 GG vor, da es das Fehlen einer Entschädigungsregelung für eine unverhältnismäßige Beschränkung des Art. 12 Abs. $1 \mathrm{GG}$ und Art. 14 Abs. 1 GG hielt. Die Vorlage durch das VG wurde vom BVerfG allerdings für unzulässig erachtet, da es an einer substantiierten Darlegung fehlte, weshalb das VG die fragliche gesetzliche Regelung für verfassungswidrig hielt. Das VG Berlin habe versäumt, insbesondere die konkrete finanzielle Belastung des klagenden TK-Unternehmens darzulegen, sodass die Prüfung einer Verletzung von Grundrechten ohne weitere Feststellungen nicht möglich sei. ${ }^{476}$

200 Das VG hatte zwar die Übertragung der organisatorischen Aufgabenlast auf die TK-Anbieter für zumutbar, mithin verhältnismäßig erachtet, da die Provider sachkundig seien, weshalb eine Übertragung im Lichte der zu schützenden Rechtsgüter sinnvoll erscheine, solange der normale Betriebsablauf „nicht zentral beeinflusst“ werde. ${ }^{477}$ Doch könne den Anbietern nicht die Kosten hierfür aufge-

\footnotetext{
${ }^{470}$ So Roßnagel et al., Gutachten, 2009, S. 22; Klimanek, in: Stiftung der Hessischen Rechtsanwaltschaft, Kulturflatrate, 2013, 75, 98; schon einen Eingriff verneinend, da nur eine unerhebliche Erschwerung vorläge: Zwengel, Kulturflatrates, 2012, S. 195 f.

471 Paulus, in: FS Papier, 2013, S. 561, 567.

${ }^{472}$ So aber Roßnagel et al., Gutachten, 2009, S. 22.

${ }^{473}$ S. dazu unten Rn. $393 \mathrm{ff}$.

${ }^{474}$ VG Berlin MMR 2008, 851 ff.; daran anschließend auch VG Berlin MMR 2009, 355 ff.

475 Lediglich das Justizvergütungs- und Entschädigungsgesetz (JVEG) sieht eine minimale Kostenübernahme durch den Staat vor, s. zu den Details BeckRS 2009, 34592.

${ }^{476}$ BVerfG MMR 2009, 606, $607 \mathrm{f}$.

${ }^{477}$ VG Berlin MMR 2008, 851, 852.
} 
bürdet werden, da es an einem Zurechnungszusammenhang fehle, der eine Kostentragung rechtfertigen könne, obwohl sie genuin hoheitliche Aufgaben übernehmen sollten, für die aufgrund ihres Allgemeinbezugs das verfassungsrechtliche Prinzip der Steuerstaatlichkeit gelte. ${ }^{478}$

201 Im Hinblick auf eine Kulturflatrate liegen die Parallelen nahe: Anders aber als im Fall der Vorratsdatenspeicherung, die mit originär staatlichen Aufgaben der Strafverfolgung verknüpft ist, betrifft eine Abgabenerhebung durch Provider nur die (mittelbare) Durchsetzung von Urheber- und Leistungsschutzrechten. Das BVerfG hat einer kategorischen Trennung von staatlichen und privaten Aufgaben „mit der Folge der grundsätzlichen Unzulässigkeit einer Indienstnahme für Gemeinwohlzwecke von Privaten auf deren Kosten" im Urteil zur Vorratsdatenspeicherung aber ohnehin eine klare Absage erteilt: „Vielmehr hat der Gesetzgeber einen weiten Gestaltungsspielraum, welche Pflichten (er) zur Sicherstellung von Gemeinwohlbelangen der Privaten im Rahmen ihrer Berufstätigkeit auferlegt" ${ }^{479}$ Somit ist im Rahmen einer Kulturflatrate nur noch entscheidend, dass die auferlegten Kostenlasten nicht unverhältnismäßig hoch ausfallen. ${ }^{480}$

202 Das VG Berlin hat schließlich noch einen Verstoß gegen den Gleichheitsgrundsatz gem. Art. 3 Abs. 1 GG angemahnt, da kleinere Anbieter bei gleicher Kostenlast wirtschaftlich härter getroffen würden als größere. ${ }^{481}$ Indes dürften alle diese Fragen keine ins Gewicht fallende Rolle spielen, da die AccessProvider die entstehenden Kosten auf die Nutzer überwälzen können - nicht anders als etwa die Hersteller, Händler und Importeure von Geräten, die unter die Geräteabgabe fallen.

(6) Ungleichbehandlung der Internetteilnehmer

203 Da die Kulturflatrate eine Abgabe enthält, die auf alle Internetanschlüsse erhoben würde, nimmt sie keinerlei Rücksicht darauf, ob ein Internetteilnehmer tatsächlich von den neuen Schranken Gebrauch macht, sondern „besteuert“ auch diejenigen, die das Netz z.B. lediglich zum Meinungsaustausch oder für nicht urheberrechtlich relevante Zwecke nützen. In einer solchen pauschalen Abgabe könnte eine Ungleichbehandlung und Einschränkung der allgemeinen Handlungsfreiheit nach Art. 2 Abs. 1 GG erblickt werden.

204 Roßnagel et al. haben jedoch zu Recht darauf hingewiesen, dass bereits bei bestehenden Abgaben wie der Rundfunkgebühr oder den Geräte- und Leermedienabgaben eine gewisse Pauschalierung in Anbetracht der damit verbundenen Vorteile hingenommen wird. ${ }^{482}$ Für den Rundfunkbereich etwa hat das BVerfG stets betont, dass der Zweck der Förderung des öffentlich-rechtlichen Rundfunks eine gleiche Belastung der Rundfunkteilnehmer rechtfertigt, auch wenn diese nicht das Programm des öffentlich-rechtlichen Rundfunks nutzen. ${ }^{483}$ Eine Pauschalierung liegt auch dem neuen rundfunkrechtlichen Finanzierungsmodell zugrunde, das ab 2013 im Zuge des 15. RundfunkänderungsStaatsvertrags gilt. Der dort neu eingeführte sog. Rundfunkbeitrag wird - anders als die Rundfunkgebühr genannte Abgabe im bisherigen, an Empfangsgeräte gekoppelten Modell - künftig gem. § $2 \mathrm{Abs.}$ 1 RBStV $^{484}$ für jede Wohnung sowie gem. $\S 5$ Abs. 1 RBStV für jede Betriebsstätte zu entrichten sein.

\footnotetext{
${ }^{478}$ VG Berlin MMR 2008, 851, $853 \mathrm{f}$.

479 BVerfG MMR 2010, 356, 368 Rn. 301 - Vorratsdatenspeicherung, unter Verweis auf BVerfGE 109, $64,85$.

${ }^{480}$ Im Ergebnis die Inanspruchnahme ebenso für verhältnismäßig haltend: Zwengel, Kulturflatrates, 2012, S. $187 \mathrm{f}$.

${ }^{481}$ VG Berlin MMR 2008, 851, 854.

${ }^{482}$ Roßnagel et al., Gutachten, 2009, S. 23 f.

${ }^{483}$ BVerfGE 90, 60, 91; BVerfG NJW 2000, 649, 649.

${ }^{484}$ Rundfunkbeitragsstaatsvertrag in der Fassung des 15. Staatsvertrags zur Änderung rundfunkrechtlicher Staatsverträge v. 15.12.2010/17.12.2010/21.12.2010.
} 
Die Zahlungsverpflichtung gilt unabhängig davon, ob in der jeweiligen Raumeinheit tatsächlich Rundfunk-Empfangsgeräte genutzt werden. Für den Rundfunkbeitrag wird aus finanzverfassungsrechtlicher Sicht vorgetragen, es handele sich dabei um einen echten Beitrag, der „im Gegensatz zur Steuer Korrelat einer individuell zurechenbaren (Gegen-)Leistung ist, die andererseits aber im Gegensatz zur Gebühr lediglich angeboten, nicht aber individuell in Anspruch genommen werden muss", solange ein individual- oder gruppennütziger Sondervorteil der finanziellen Belastung existiere. ${ }^{485}$ Auch wenn die Kulturflatrate als private Zahlungspflicht nicht der Finanzverfassung unterfällt und die Rundfunkabgabe besondere Aufgaben im Rahmen der Rundfunkfinanzierung erfüllt, lassen sich in gleichheitsrechtlicher Hinsicht die Aussagen des BVerfG auf die Kulturflatrate unproblematisch entsprechend anwenden. Die Gemeinnützigkeit liegt hierbei in der Förderung kultureller Vielfalt durch die Zugänglichkeit urheberrechtlich geschützter Werke. ${ }^{486}$ Auch in finanzverfassungsrechtlicher Hinsicht besteht Vergleichbarkeit, da die Inanspruchnahme der aus der urheberrechtlichen Schranke erwachsenden Befugnisse lediglich optional ist, aber bereits für die bloße Möglichkeit eine Zahlungspflicht besteht.

205 Aber auch im Bereich der traditionellen Geräteabgabe müssen heute Nutzer etwa von Speichermedien für den typischen urheberrechtlich relevanten Gebrauch die Abgabe entrichten, auch wenn sie selbst das Speichermedium zu völlig anderen Zwecken einsetzen. ${ }^{487}$ Abgesehen von dieser grundsätzlichen Rechtfertigung können durch entsprechende Differenzierungen nach Geschwindigkeit der Zugänge, die etwa höher ausfallen bei Nutzung digitaler Inhalte, oder durch prozentuale Erhebung eine gewisse Anpassung an das typische Nutzungsverhalten erzielt werden. ${ }^{488}$

\section{b) Internationale und europarechtliche Grenzen}

206 Während das deutsche Verfassungsrecht einer Kulturflatrate und neuen Schranken keine unüberwindlichen Hindernisse aufstellt, ist die Rechtslage im Hinblick auf das europäische Sekundärrecht anders:

\section{(1) InfoSoc-Richtlinie}

207 Entscheidend für die Einführung neuer Schranken ist der von der InfoSoc-RL gesetzte rechtliche Rahmen für Schranken in Mitgliedstaaten. Nach Art. 5 Abs. 1 bis Abs. 3 InfoSoc-RL können die Mitgliedstaaten nur in den vorgesehenen Fällen bzw. eingeräumten Möglichkeiten Schranken für die jeweiligen Verwertungsrechte vorsehen. ${ }^{489}$ Zusätzlich muss für jede Schranke der nach Art. 5 Abs. 5 InfoSoc-RL aus dem TRIPS-Abkommen ${ }^{490}$ und Art. 9 Abs. 2 der Revidierten Berner Übereinkunft (RBÜ) übernommene Dreistufen-Test eingehalten werden:

"It shall be a matter for legislation in the countries of the Union to permit the reproduction of such works in certain special cases, provided that such reproduction does not conflict with a

\footnotetext{
485 Bosman, K\&R 2012, 5, 9; ebenso Gall/Schneider, in: Hahn/Vesting, Rundfunkrecht, 3. Aufl. 2012, Vorbemerkung RBStV Rn. 37 mwNachw; Kritik an der konkreten Umsetzung des rundfunkrechtlichen Beitragsmodells unter Gleichheitsgesichtspunkten bei Geuer, MMR-Aktuell 2012, 335995.

${ }^{486}$ Roßnagel et al., Gutachten, 2009, S. 24; Klimanek, in: Stiftung der Hessischen Rechtsanwaltschaft, Kulturflatrate, 2013, 75, 99; krit. Blanke-Roeser, in: Stiftung der Hessischen Rechtsanwaltschaft, Kulturflatrate, 2013, 29, 59; Hennemann, Urheberrechtsdurchsetzung und Internet, 2011, S. 333.

${ }^{487}$ So auch schon zur Geräteabgabe das BVerfGE 31, 255; ferner Zwengel, Kulturflatrates, 2012, S. 194; dies übersieht etwa Blanke-Roeser, in: Stiftung der Hessischen Rechtsanwaltschaft, Kulturflatrate, 2013, $29,58 \mathrm{ff}$. gänzlich.

${ }^{488}$ S. oben Rn. 150 sowie unten Rn. 379 ff.; Roßnagel et al., Gutachten, 2009, S. 23.

489 Spindler, GRUR 2002, 105, $110 \mathrm{ff}$.

490 Übereinkommen über handelsbezogene Aspekte der Rechte des geistigen Eigentums (TRIPS), BGBI. II 1994, S. 1730 .
} 
normal exploitation of the work and does not unreasonably prejudice the legitimate interests of the author." ${ }^{491}$

\section{(a) Zulässige Schranken}

209 Für die private Vervielfältigung erlaubt Art. 5 Abs. 2 lit. b InfoSoc-RL den Mitgliedstaaten eine Schranke, wie sie der deutschen Privatkopieregelung in § 53 Abs. 2 UrhG entspricht - verknüpft mit der Bedingung, „dass die Rechteinhaber einen gerechten Ausgleich erhalten“. Demgemäß stünde die InfoSoc-Richtlinie auch einer entsprechenden Ausdehnung der Privatkopieschranke für den Download, also die Vervielfältigung, nicht entgegen - die Richtlinie beschränkt die Privatkopieschranke auch nicht wie $\S 53$ Abs. 1 UrhG auf rechtmäßige Vorlagen, von denen die Kopie angefertigt wird. ${ }^{492}$

210 Im Bereich des Rechts auf öffentliches Zugänglichmachen, Art. 3 Abs. 2 lit. a InfoSoc-RL, sieht Art. 5 Abs. 3 InfoSoc-RL dagegen einen exklusiven Schrankenkatalog vor, über den die Mitgliedstaaten nicht hinausgehen dürfen. Dieser Katalog enthält aber keinerlei Ausnahme für das private Zugänglichmachen. Auch die Erwägungsgründe lassen nicht erkennen, dass es sich hier um ein Versehen des Richtliniengebers handeln sollte. Die nationale Einführung einer entsprechenden Schranke würde daher de lege lata gegen die InfoSoc-RL verstoßen. ${ }^{493}$

211 Zwar wird von einigen Stimmen die Auffassung vertreten, dass es sich bei einer Kulturflatrate lediglich um eine zwangsweise kollektive Ausübung von Verwertungsrechten, mithin nicht um eine Schranke, sondern um eine Regelung der Ausübung der Rechte handele. ${ }^{494}$ Dies steht jedoch weder mit Wortlaut und Systematik der InfoSoc-RL noch mit dem Charakter der Kulturflatrate in Einklang: ${ }^{495}$ Schon vom Ansatz her kann eine kollektive Ausübung von Verwertungsrechten nur bedeuten, dass anstelle der Rechteinhaber eine kollektive Organisation tritt, die aber keineswegs gezwungen wäre, in die Nutzung von Rechten einzuwilligen. Mit anderen Worten wäre eine kollektive Ausübung von Rechten nicht automatisch mit einer Schranke der freien privaten Nutzung von Urheberrechten verbunden; es handelt sich vielmehr um die Übertragung einer Aktivlegitimation, ohne dass sich hieraus per se bestimmte Schranken ergäben. Ferner steht diese Auffassung nicht im Einklang mit Art. 5 Abs. 2 lit. a InfoSoc-RL: Denn die Schranke für private Vervielfältigungen wäre nach dieser Auffassung letztlich überflüssig, da es sich ebenfalls nur um die kollektive Rechtsdurchsetzung handeln würde, verknüpft mit einer Abgabe. Indes kann nicht wegdiskutiert werden, dass der Richtliniengeber die

\footnotetext{
${ }^{491}$ Revidierte Berner Übereinkunft zum Schutz von Werken der Literatur und Kunst 9. September 1886, in der Fassung vom 28. September 1979, abrufbar unter: http://www.wipo.int/export/sites/www/treaties/en/ip/berne/pdf/trtdocs_wo001.pdf.

${ }^{492}$ Art. 5 Abs. 2 lit. b InfoSoc-RL gestattet Ausnahmen in Bezug auf Vervielfältigungen durch Privatpersonen unterschiedslos und ohne weitere Einschränkungen „,auf beliebigen Trägern“. Damit war die engere Erlaubnis der Privatkopie in $\S 53$ Abs. 1 UrhG zwar zulässig i.S.d. InfoSoc-RL, aber von dieser nicht zwingend gefordert, s. Aschenbrenner, ZUM 2005, 145, 152; das Fehlen einer ausdrücklichen Klausel ist umso aussagekräftiger, als für die zwingenden Ausnahme gem. Art. 5 Abs. 1 InfoSoc-RL zwischenzeitlich ein solcher Passus vorgesehen war, dann aber im Gesetzgebungsverfahren wieder verworfen wurde, s. Walter/Lewinsky, European Copyright Law, 2010, InfoSocD. Rn. 11.0.43, 11.5.4 f.

493 Dies räumen auch Grassmuck, ZUM 2005, 104, 108 und Roßnagel et al., Gutachten, 2009, S. 25 ein; ebenso: Zwengel, Kulturflatrates, 2012, S. 273 f.; Blanke-Roeser, in: Stiftung der Hessischen Rechtsanwaltschaft, Kulturflatrate, 2013, 29, 62; Chatziathanasiou/Hartmann, in: Stiftung der Hessischen Rechtsanwaltschaft, Kulturflatrate, 2013, 153, 166, die angesichts der bestehenden europarechtlichen Hindernisse für die Schaffung einer „Experimentierklausel“ auf EU-Ebene für (ggf. zeitlich begrenzte) Modellprojekte einzelner Mitgliedstaaten plädieren.

${ }^{494}$ So im Wesentlichen Bernault/Lebois, 2006, S. 55 f. unter Berufung auf v. Lewinski, 2004, S. 7.

495 Wie hier, wenn auch ohne nähere Begründung Klimanek, in: Stiftung der Hessischen Rechtsanwaltschaft, Kulturflatrate, 2013, 75, 100; unentschieden demgegenüber Roßnagel et al., Gutachten, 2009, S. 25 f.
} 
Schranke nach Art. 5 Abs. 2 lit. a InfoSoc-RL ausdrücklich als Beschränkung des Verwertungsrechts eingeordnet hat, mithin auch die Mitgliedstaaten daran gebunden sind - dies gerade verknüpft mit einem Anspruch auf gerechten Ausgleich. Für die hier vertretene Auffassung spricht auch, dass etwa Art. 9 der Kabelweitersende-Richtlinie ${ }^{496}$ ausdrücklich eine kollektive Wahrnehmung der Rechte regelt - und damit verdeutlicht, dass eine solche Interpretation sich in der InfoSoc-RL hätte niederschlagen müssen. Es ist nach diesseitiger Auffassung höchst zweifelhaft, dass Art. 5 Abs. 3 InfoSoc-RL so interpretiert werden kann, dass er kollektive Zwangslizenzen auf mitgliedstaatlicher Ebene erlauben würde, da sonst die gesamte Systematik der Schrankensetzung und damit verknüpfter Vergütungsansprüche ausgehebelt würde. Denn dann würde es genügen, eine Vergütungspflicht verbunden mit der kollektiven Rechtewahrnehmung vorzusehen, um die gleichen Wirkungen wie jede Schranke zu erreichen - womit der abschließende Katalog des Art. 5 Abs. 3 InfoSoc-RL praktisch hinfällig wäre.

212 Schließlich ist zu berücksichtigen, dass Art. 6 InfoSoc-RL, der den Schutz von DRM-Maßnahmen regelt, keinerlei Schranke für die private Vervielfältigung vorsieht. Mit anderen Worten darf bei einem DRM-geschützten Werk nicht der technische Schutz umgegangen werden, um eine private Kopie herzustellen. Will man eine flächendeckende, auch DRM-geschützte Werke erfassende Schranke einführen, müsste daher auf europäischer Ebene auch diese Vorgabe geändert werden. ${ }^{497}$

\section{(b) Dreistufen-Test}

213 Neben dem exklusiven Katalog des Art. 5 Abs. 2, 3 InfoSoc-RL, aber auch aufgrund internationaler völkerrechtlicher Vorgaben, insbesondere Art. 9 Abs. 2 RBÜ, ${ }^{498}$ ist zudem gem. Art. 5 Abs. 5 InfoSocRL der Dreistufen-Test einzuhalten: Demnach dürfen die Schranken

„(...) nur in bestimmten Sonderfällen angewandt werden, in denen die normale Verwertung des Werks oder des sonstigen Schutzgegenstands nicht beeinträchtigt wird und die berechtigten Interessen des Rechtsinhabers nicht ungebührlich verletzt werden."

\section{(i) Die erste Stufe: Beschränkung auf bestimmte Sonderfälle}

215 Für die erste Stufe - die Beschränkung auf bestimmte Sonderfälle - besteht weitgehend Einigkeit, dass eine allgemeine Schranke zugunsten privater Aktivitäten dieses Erfordernis klar bestimmter, umgrenzter Fälle einhält. ${ }^{499}$ Denn nur der private Gebrauch wird erfasst, die betroffenen Verwertungsrechte können klar benannt werden.

\section{(ii) Die zweite Stufe: Die normale Verwertung}

216 Wesentliche größere Probleme stellen sich hinsichtlich der zweiten Stufe, die eine normale Verwertung des Werkes gewährleisten soll. Damit stellt die zweite Stufe letztlich darauf ab, ob der

\footnotetext{
${ }^{496}$ Richtlinie 93/83/EWG des Rates vom 27. September 1993 zur Koordinierung bestimmter urheber- und leistungsschutzrechtlicher Vorschriften betreffend Satellitenrundfunk Kabelweiterleitung, ABI. Nr. L 248 v. 6.10.1993, S. 15.

${ }^{497}$ Zutr. Roßnagel et al., Gutachten, 2009, S. 29; s. ebenf. Grassmuck, ZUM 2005, 104 ff., allerdings beide bezogen auf das nationale Recht, § 95b UrhG.

${ }^{498}$ S. ferner Art. 13 TRIPS, Art. 10 WCT sowie 16 Abs. 2 WPPT.

499 Senftleben, CR 2003, 914, 916; Runge, GRUR Int. 2007, 130, 134; Bernault/Lebois, 2006, S. 33 f.; Roßnagel et al., Gutachten, 2009, S. 27; im Ergebnis ebenso: Zwengel, Kulturflatrates, 2012, S. 231 f.; Klimanek, in: Stiftung der Hessischen Rechtsanwaltschaft, Kulturflatrate, 2013, 75, 102; Chatziathanasiou/Hartmann, in: Stiftung der Hessischen Rechtsanwaltschaft, Kulturflatrate, 2013, 153, 166; a.A. Blanke-Roeser, in: Stiftung der Hessischen Rechtsanwaltschaft, Kulturflatrate, 2013, 29, 63; Hennemann, Urheberrechtsdurchsetzung und Internet, 2011, S. 338.
} 
Rechteinhaber durch eine neue Schranke seiner Einnahmequellen auf den bisherigen Märkten beraubt wird, die allerdings ein ganz erhebliches Gewicht innerhalb der Gesamtverwertung aufweisen müssen. ${ }^{500}$ Dies würde gerade in den Fällen nahe liegen, in denen eine von einer Schrankenregelung gedeckte Nutzungsart in Konflikt bzw. - aus ökonomischer Perspektive betrachtet - in Wettbewerb mit einer solchermaßen gewichtigen Nutzungsart gerät, die nicht von einer Schrankenregelung gedeckt und somit allein dem Rechteinhaber vorbehalten ist. ${ }^{501}$ Nun scheint es auf der Hand zu liegen, dass neue Schranken, die das Filesharing legalisieren, zu einem Rückgang der kommerziell verkauften Werke führen werden, da sich die Content-Distribution über Download- und Streaming-Kanäle inzwischen zu einem Geschäftsmodell entwickelt hat, das nicht zuletzt davon lebt, dass der Content nicht anderweitig digital und vor allem nicht kostenlos verfügbar ist. ${ }^{502}$ Dem wird jedoch entgegengehalten, dass trotz des Verbots illegaler Kopien bereits erhebliche Verkaufsverluste eingetreten seien, zudem die kostenpflichtige Downloads nur 25\% des Gesamtumsatzes im Bereich der Musikindustrie ausmachten. Schließlich wird darauf verwiesen, dass eine Kontrolle der Downloadvorgänge aufgrund des massenhaften Austauschs unmöglich sei. ${ }^{503}$ Andere wiederum verweisen darauf, dass die Rechteinhaber die Möglichkeit haben, ihre Rechte durch den Einsatz von DRM-Systemen durchzusetzen, und daher eine Konkurrenz unterschiedlicher Rechte (zum einen durch die Kulturflatrate, zum anderen durch DRM-Systeme) vorläge. ${ }^{504}$ Noch weiter geht die Auffassung, die überhaupt eine "normale“ Verwertung verneint, wenn diese mit massenhaften Kontrollen und Beeinträchtigungen des freien Internetverkehrs verbunden sind. ${ }^{505}$

217 Diese Argumente vermögen allerdings nur zum Teil zu überzeugen: Zum einen kann die Situation massenhafter Rechtsverletzungen und der Probleme ihrer Durchsetzung nicht ohne weiteres dazu herangezogen werden, eine normale Verwertung zu verneinen bzw. davon auszugehen, dass bei bereits bestehenden Märkten und (eingeschränkten) Rechtsdurchsetzungsmöglichkeiten keine normale Verwertung möglich ist. Denn genauso gut spräche dieses Argument für eine weitere Verschärfung und Verbesserung der Rechtsdurchsetzungsmaßnahmen, um eben wieder eine normale Verwertung zu ermöglichen. Dass ferner derzeit der Downloadanteil nur ein Viertel des gesamten Umsatzes einer bestimmten Branche ausmacht, stellt auf den Status quo ab und bezieht nicht das Potential zukünftiger Marktentwicklungen ein, insbesondere die zu erwartende Substitution von klassischen Medienträgern hin zu Download- und Streamingangeboten - was ja gerade für den Rückgang privater Tauschangebote ins Feld geführt wird.

218 Dennoch weist der Vergleich mit dem Umsatzrückgang trotz privater Rechtsverfolgungsmaßnahmen auf den zentralen Punkt hin: Denn eine "normale Verwertung“ ist vom Telos her auf die Erzielung entsprechender Einnahmen und Vergütungen für den Urheber bzw. Rechteinhaber ausgerichtet. Ist aber das private Rechtsverfolgungssystem nicht mehr oder nur unter erheblicher Gefährdung der Rechte Dritter - hier des informationellen Selbstbestimmungsrechts - in der Lage, die Vergütungen sicherzustellen, kann die zweite Stufe des Dreistufen-Tests nicht zu einer Schlechterstellung der Ur-

\footnotetext{
${ }^{500}$ Entscheidung des WTO Panels vom 15.6.2000, WT/DS160/R, Rn. 6.180; ebenso Senftleben, GRUR Int. 2004, 200, 209; ausf. ders., Copyright, Limitations and the Three-Step Test, 2004, S. 189 ff.; s. auch Runge, GRUR Int. 2007, 130, 134; so wohl auch Knights, 2001, Rn. 16.

${ }^{501}$ Entscheidung des WTO Panels vom 15.6.2000, WT/DS160/R, Rn. 6.181; Runge, GRUR Int. 2007, $130,134$.

502 Ebenso Zwengel, Kulturflatrates, 2012, S. 242; Meschede, 2007, S. 158; siehe dazu aber auch: Aguiar/Martens, 2013, S. 16 f., die zu dem Ergebnis kommen, dass illegales Filesharing und legales Streaming kaum Auswirkung auf den Markt für Download-Titel hat.

${ }^{503}$ Bernault/Lebois, 2006, S. 35; dem weitgehend folgend Roßnagel et al., Gutachten, 2009, S. 27 f.

${ }^{504}$ Runge, GRUR Int. 2007, 130, 134, der aber letztlich eine Vereinbarkeit mit dem Dreistufen-Test verneint.

${ }^{505}$ So Aigrain, 2012, S. 73.
} 
heber bzw. Rechteinhaber führen. Wenn daher durch die Einführung einer Kulturflatrate erheblich mehr Vergütungen für die Urheber zu erwarten sind und dadurch gar Umsatzrückgänge kompensiert werden, kann eine solche Abgabe nicht die normale Verwertung behindern - im Gegenteil: Sie würde sie sogar wieder ermöglichen. ${ }^{506}$ Dies gilt auch im Hinblick auf den Einsatz von DRM-Systemen, die zum einen nicht durch die Kulturflatrate tangiert würden, dementsprechend nach wie vor zur individuellen Rechtsdurchsetzung eingesetzt werden könnten, ${ }^{507}$ zum anderen aber selbst darunter leiden, dass sie offenbar von den Nutzern kaum akzeptiert und oftmals durchbrochen werden. Daher kann auch hier nicht eindeutig davon ausgegangen werden, dass eine "normale Verwertung" nur durch individuelle Rechtsdurchsetzung ermöglicht würde. Daher kann die Abgabenlösung in Gestalt einer Kulturflatrate auch nicht auf eine Erleichterung der Transaktionen bzw. der Rechteeinholung durch die Nutzer reduziert werden: Schon für die Privatkopie stimmt diese Aussage nur bedingt, wie oben dargelegt wurde, da auch die Durchsetzungs- bzw. Rechtsverfolgungsprobleme eine erhebliche Rolle spielten. ${ }^{508}$

219 Schließlich ist auch fraglich, ob eine neue Schrankenregelung tatsächlich zu einer unmöglichen Verwertung in Gestalt der bisherigen Nutzungsarten, wie Download- oder Streaming-Portalen, führen würde; denn schon im Rahmen des bestehenden Filesharing konnten sich diese Portale durchsetzen bzw. am Markt etablieren. Zudem erscheint es durchaus möglich, dass zusätzliche Qualitätsangebote, z.B. von Schadsoftware freie Inhalte, besserer Zugang und einfach zu erreichendes Angebot eine hinreichende Differenzierung ermöglichen könnten. ${ }^{509}$ Allerdings ist bereits oben ${ }^{510}$ darauf hingewiesen worden, dass sich die ökonomischen Auswirkungen auf die neuen Geschäftsmodelle nur schwer auf der Grundlage der derzeitigen Datenbasis einschätzen lassen. Denn die bislang vorliegenden Untersuchungen zeigen ein unterschiedliches Bild, indem offenbar Nutzer einerseits bereit sind, Streaming-Portale bei entsprechend niedrigen Preisen zu nutzen, andererseits verbesserten Angeboten, einfacherem Zugang etc. keine große Bedeutung beigemessen wird. ${ }^{511}$

220 Ausschlaggebend ist daher auch hier - wie im Rahmen des Verfassungsrechts - die dem Gesetzgeber eingeräumte Einschätzungsprärogative, die auch im Bereich des Dreistufen-Tests Anwendung finden muss, ${ }^{512}$ wenn die zukünftige Entwicklung einer „normalen“ Verwertung nicht vollständig und nicht sicher prognostiziert werden kann. Aus rechtlicher Sicht ist in diesem Rahmen zudem darauf hinzuweisen, dass auch ein primär urheberrechtlich angelegter Dreistufen-Test die verfassungsrechtlichen

\footnotetext{
${ }^{506}$ Im Grundsatz ebenso Geiger/Griffiths/Hilty, GRUR Int. 2008, 822, 823 f.: „Demgegenüber begünstigen Ausnahmen und Beschränkungen unter gewissen Umständen die Interessen der Kreativen. Dies gilt insbesondere für Rechtsordnungen, in denen die Anwendung von Ausnahmen und Beschränkungen an die Zahlung einer angemessenen Vergütung geknüpft ist, an welcher der Kreative zwingend zu beteiligen ist“; ebenso Grassmuck, ZUM 2005, 104, 108; Zwengel, Kulturflatrates, 2012, S. 243, der zusätzlich noch darauf hinweist, dass die eigentlichen Urheber häufig nur mittelbar an den in Rede stehenden Verwertungsmöglichkeiten profitieren. Hauptakteure seien hier die Plattenfirmen u.ä. Intermediäre; wie hier auch Chatziathanasiou/Hartmann, in: Stiftung der Hessischen Rechtsanwaltschaft, Kulturflatrate, 2013, 153, 167.

${ }^{507}$ Darauf stellt maßgeblich Runge, GRUR Int. 2007, 130, 134 ab.

${ }^{508}$ S. dazu oben Rn. 13 ff.; nur zum Teil daher berechtigt Runge, GRUR Int. 2007, 130, 135 f., der die Privatkopie nur durch die Probleme bei der Rechteeinräumung gerechtfertigt sieht.

${ }^{509}$ Dagegen aber: Zwengel, Kulturflatrates, 2012, S. 242.

${ }^{510}$ S. oben Rn. $145 \mathrm{ff}$.

${ }^{511}$ S. dazu oben Rn. 96 ff. bzw. die Studien von Huygen et al., 2009, S. 81, 118.

512 Ähnlich Senftleben, GRUR Int. 2004, 200, 210 f.; dies verkennt Blanke-Roeser, in: Stiftung der Hessischen Rechtsanwaltschaft, Kulturflatrate, 2013, 29, 63, der zunächst die Unmöglichkeit einer sicheren Prognose anerkennt, sodann aber nicht konsequent argumentiert; wie hier hingegen Chatziathanasiou/Hartmann, in: Stiftung der Hessischen Rechtsanwaltschaft, Kulturflatrate, 2013, 153, 168 f., die grds. für eine innovative Regulierung plädieren, die nicht am starren Leitbild überkommener Geschäftsmodelle festhält.
} 
Wertungen (sei es des nationalen oder des europäischen Verfassungsrechts) zu berücksichtigen hat, wozu auch die gegenläufigen Interessen der Nutzer, insbesondere hinsichtlich ihres informationellen Selbstbestimmungsrechts, zählen. ${ }^{513}$

\section{(iii) Die dritte Stufe: Verhältnismäßigkeit}

221 Hinsichtlich der dritten Stufe kommt es auf die Wahrung der Verhältnismäßigkeit zwischen Interessen der Urheber einerseits und Interessen der Allgemeinheit andererseits an, insbesondere dass durch Vergütungssysteme die Eingriffe in die Urheberrechte ausgeglichen werden können. ${ }^{514}$ Dies verweist im Wesentlichen auf die gleichen Aspekte, wie sie im nationalen Recht im Rahmen der Verhältnismäßigkeit greifen, ${ }^{515}$ wobei wiederum die Gefährdungslagen für die informationelle Selbstbestimmung und die Sicherung der Vergütung des Urhebers im Vordergrund stehen. ${ }^{516}$ Insbesondere dürften angesichts der oben dargelegten empirischen Untersuchungen die Befürchtungen, dass eine Kulturflatrate zu dem Gegenteil des (angeblichen) Zwecks führen würde, nämlich eine weite Verbreitung von Inhalten zu erreichen, da Anreize für DRM-Systeme bestünden, ${ }^{517}$ weitgehend der Boden entzogen sein; denn wie dargelegt, sind die Gesamteffekte des Filesharing keineswegs eindeutig, DRM-Systeme haben trotz der weiten Verbreitung des Filesharing eher ab- als zugenommen. Warum bei einer Kulturflatrate das Gegenteil eintreten sollte, ist nicht recht ersichtlich. ${ }^{518}$

\section{(2) Enforcement-Richtlinie}

222 In Betracht könnte schließlich hinsichtlich der Rechtedurchsetzung und die Inpflichtnahme durch Abgaben die Kontrolle anhand der Enforcement-Richtlinie kommen. Indes lässt sich dieser Richtlinie keine Vorgabe für die Festlegung von Schranken entnehmen; anders formuliert setzt diese Richtlinie bereits voraus, dass ein bestimmtes Verhalten vom materiellen Urheberrecht als illegal qualifiziert wird, trifft aber keine Aussagen, welche Handlungen überhaupt als urheberrechtswidrig einzustufen sind.

\section{(3) Internationale Abkommen}

223 Den Vorgaben der InfoSoc-Richtlinie entsprechen die internationalen Abkommen, die allesamt Schranken durch die nationalen Urheberrechte zulassen. Zu beachten sind hier die Revidierte Berner Übereinkunft (RBÜ), ${ }^{519}$ das TRIPS-Abkommen, ${ }^{520}$ das WIPO Copyright Treaty (WCT), ${ }^{521}$ sowie das In-

\footnotetext{
${ }^{513}$ S. etwa deutlich der EuGH in seiner SABAM ./. Netlog-Entscheidung, EuGH, Urteil v. 16. 2. 2012 - Rs. C360/10, Rn. 48 (SABAM ./. Netlog NV), dazu Spindler, JZ 2012, 311, 313; Wiebe, WRP 2012, 1336.

${ }^{514}$ Senftleben, Copyright, Limitations and the Three-Step Test, 2004, S. 213, $216 \mathrm{ff}$., der exemplarisch verweist auf BVerfGE 31, 229, 244 f. - Schulbuchprivileg, und zudem betont, dass an dieser Stelle nicht nur Rechte oder Rechtsgüter, sondern Interessen in einen angemessenen Ausgleich gebracht werden müssen; s. auch Bernault/Lebois, 2006, S. 36.

${ }^{515}$ Zutr. Roßnagel et al., Gutachten, 2009, S. 27 f.

${ }^{516}$ Gem. der Entscheidung des WTO Panels vom 15.6.2000, WT/DS160/R, Rn. 6.229 ist die Grenze zur Unverhältnismäßigkeit an folgendem Punkt erreicht: „In our view, prejudice to the legitimate interests of right holders reaches an unreasonable level if an exception or limitation causes or has the potential to cause an unreasonable loss of income to the copyright owner", wodurch freilich auch keine präzisere Aussage gewonnen ist, da die Frage der Unverhältnismäßigkeit lediglich von den allgemeinen „Interessen“ auf die konkrete „Vergütung" verlagert wird; so zu Recht auch Knights, Limitations and Exceptions Under the „Three-Step-Test" and in National Legislation, WIPO/CR/MOW/01/2, 2001, Rn. 16, abrufbar unter: http://www.wipo.int/edocs/mdocs/copyright/en/wipo_cr_mow_01/wipo_cr_mow_01_2.pdf.

517 So aber Runge, GRUR Int. 2007, 130, 136.

518 Ebenso die Verhältnismäßigkeit bejahend: Zwengel, Kulturflatrates, 2012, S. 250.

${ }^{519}$ Berner Übereinkunft zum Schutz von Werken der Literatur und Kunst vom 9. September 1886 (BGBI. II S. 1071), zuletzt geändert durch Änderungsbeschluss vom 2. 10. 1979 (BGBI. 1985 II S. 81).
} 
ternationale Abkommen über den Schutz der ausübenden Künstler, der Hersteller von Tonträgern und der Sendeunternehmen (Rom-Abkommen), ${ }^{522}$ und das WIPO Performances and Phonograms Treaty (WPPT). ${ }^{523}$

\section{(a) Die Revidierte Berner Übereinkunft}

224 In der Revidierten Berner Übereinkunft sind von einer Kulturflatrate vor allem die Art. 9 Abs. 1 RBÜ (Vervielfältigungsrecht), $11^{\text {bis }}$ Abs. 1 Nr. 1 RBÜ (öffentliche Wiedergabe von literarischen und künstlerischen Werken), Art. 14 Abs. 1 Nr. 2 Alt. 2, 14 ${ }^{\text {bis }}$ Abs. 1 RBÜ (Verfilmungen von literarischen und künstlerischen Werken) und Art. 11 Abs. 1 Nr. 2, $11^{\text {ter }}$ Abs. 1 Nr. 2 RBÜ (öffentliche Wiedergabe von dramatisch und/oder musikalischen Werken, Art. $11^{\text {ter }}$ Abs. 1 Nr. 2 RBÜ (die öffentliche Wiedergabe von literarischen Vorträgen), sowie als Auffangnorm Art. 8 WCT betroffen. ${ }^{524}$ Beschränkungen sind im Bereich des Art. 9 Abs. 1 RBÜ (Vervielfältigungsrecht) gem. Art. 9 Abs. 2 RBÜ, sowie für Art. $11^{\text {bis }}$ Abs. 1 Nr. 1 RBÜ (öffentliche Wiedergabe von literarischen und künstlerischen Werken) gem. Art. $11^{\text {bis }}$ Abs. 2 S. 1 RBÜ möglich. Für die übrigen Rechte existieren keine geschriebenen Ausnahmen. Es sind allerdings ungeschrieben sog. „kleine Ausnahmen“ anerkannt, die dann vorliegen, wenn der Urheber nicht oder nur unwesentlich in seiner wirtschaftlichen Auswertung gestört wird. ${ }^{525}$ Damit sollen somit nur sog. de-minimis-Ausnahmen erfasst werden; doch soll diese Voraussetzung regelmäßig vorliegen, wenn der Dreistufen-Test erfüllt ist, ${ }^{526}$ ausnahmsweise dann nicht, wenn hauptsächlich normative und nicht wirtschaftliche Gesichtspunkte zur Bejahung des Dreistufen-Tests geführt haben. ${ }^{527} \mathrm{Hier}$ kann dazu auf die obigen Ausführungen verwiesen werden, denn durch die Kulturflatrate kann unter Umständen überhaupt erst die wirtschaftliche Ausbeute der ansonsten „schwarz" gehandelten Kulturgüter erfolgen, ${ }^{528}$ so dass eine „,kleine Ausnahme“ vorliegt. ${ }^{529}$

\section{(b) Rom-Abkommen, TRIPS, WCT, WPPT}

225 Das Rom-Abkommen (RA) ist nur hinsichtlich Vervielfältigungen i.S.v. Art. 10 RA einschlägig. Die Rechte aus Art. 7 RA werden dagegen nicht berührt. ${ }^{530}$ Eine Beschränkung der Rechte ist aber gem. Art. 15 Abs. 1 lit. a RA für private Benützung möglich. Eine derartige private Benützung würde aber

\footnotetext{
520 Übereinkommen über handelsbezogene Aspekte der Rechte des geistigen Eigentums vom 15. April 1994 (BGBI. II S. 1730), zuletzt geändert durch Beschluss des Rates vom 6. 12. 2005 (ABI. 2007 Nr. L 311 S. 35).

${ }^{521}$ WIPO-Urheberrechtsvertrag (WCT) vom 20. Dezember 1996 (BGBI. 2003 II S. 755).

522 Internationales Abkommen über den Schutz der ausübenden Künstler, der Hersteller von Tonträgern und der Sendeunternehmen (Rom-Abkommen) vom 26. Oktober 1961 (BGBI. Teil II/1965, S. 1244 ff.).

${ }^{523}$ WIPO-Vertrag über Darbietungen und Tonträger (WPPT) vom 20. Dezember 1996 (BGBI. 2003 II S. 754, 770);

s. dazu ausführlich Zwengel, Kulturflatrates, 2012, S. 197 ff.; ferner Runge, GRUR Int. 2007, 130.

${ }^{524}$ Zwengel, Kulturflatrates, 2012, S. 200 ff.; es soll mit Zwengel sowie Runge, GRUR Int. 2007, 130, 132 davon ausgegangen werden, dass „öffentliche Übertragung“ i.S.d. RBÜ auch nicht-lineare („on Demand“) Dienste erfasst, ferner Ricketson/Ginsburg, International Copyright and Neighbouring Rights, 2. Aufl. 2006, Rn. 12.49; dies wird u.a. bezweifelt von v. Ungern-Sternberg, in: Schricker/Loewenheim, UrhR, 4. Aufl. 2010, § $19 a$ Rn. 11. ${ }^{525}$ Zwengel, Kulturflatrates, 2012, S. 220; World Trade Organisation, WT/DS160/R, Tz. 6.81-6.82, Ricketson/Ginsburg, International Copyright and Neighbouring Rights, 2. Aufl. 2006, Rn. 13.113 f.

${ }^{526}$ World Trade Organisation, WT/DS160/R, Rn. 6.82, 6.93; Zwengel, Kulturflatrates, 2012, S. 253; dagegen aber: Ricketson/Ginsburg, International Copyright and Neighbouring Rights, 2. Aufl. 2006, Rn. 13.113 f.; He, IIC 2009, 274, $293 \mathrm{f}$.

${ }^{527}$ Ricketson/Ginsburg, International Copyright and Neighbouring Rights, 2. Aufl. 2006, Rn. 13.113 f.; He, IIC 2009, 274, $293 \mathrm{f}$.

528 S. oben Rn. $216 \mathrm{ff}$.

${ }^{529}$ So im Ergebnis auch: Zwengel, Kulturflatrates, 2012, S. $253 \mathrm{f}$.

${ }^{530}$ Zwengel, Kulturflatrates, 2012, S. 203 f.
} 
im Falle einer Kulturflatrate, die gerade nicht die kommerzielle Verwendung erfasst, vorliegen. ${ }^{531}$ Das TRIPS-Abkommen verweist hinsichtlich der zu beachtenden Rechte auf die RBÜ (Art. 9 Abs. 1 S. 1 TRIPS), statuiert aber zusätzlich in Art. 14 Abs. 1 S. 1 Alt. 2 und Abs. 2 TRIPS eigene zu beachtende Rechte für ausübende Künstler. ${ }^{532}$ Für die Beschränkung der Rechte, die aus der Verweisung auf die RBÜ resultieren, gelten auch deren Regelungen. Für die in Art. 14 Abs. 1 S. 1 Alt. 2 und Abs. 2 TRIPS garantierten Rechte verweist das TRIPS dagegen auf das Rom-Abkommen, so dass auch hier eine Beschränkung i.S.e. Kulturflatrate möglich ist. ${ }^{533}$ Der WPPT, wäre von einer Kulturflatrate in den Art. 7, 10, 11 und 14 berührt. ${ }^{534}$ Gleichzeitig gestattet er aber die Beschränkung dieser Rechte in Art. 16 Abs. 1 WPPT. ${ }^{535}$

\section{Vorgaben für Abgabenlösungen}

226 Neben den rechtlichen Grenzen für die Erweiterung der Schranken selbst gilt es ferner verfassungsund europarechtliche Vorgaben für die Ausgestaltung von Vergütungs- und Abgabenlösungen zu berücksichtigen. Denn diese können maßgeblich werden für die Berechnung der Abgabenhöhe selbst, ebenso aber auch für die Verteilung:

\section{a) Europarechtliche Vorgaben}

\section{(1) InfoSoc-RL, insbesondere die Padawan-Entscheidung des EuGH}

227 Wiederum kommt vor allem die InfoSoc-RL als erster rechtlicher Eckstein in Betracht, hier vor allem die in Art. 5 Abs. 2 lit. b enthaltene Verknüpfung der Schranke zugunsten privater Vervielfältigung mit einem gerechten Ausgleich für den Rechteinhaber.

228 Von nicht zu unterschätzender Bedeutung sind hier die jüngsten Entscheidungen des EuGH, insbesondere die sog. Padawan-Entscheidung vom 21.10.2010: ${ }^{536}$

(a) Die Rechtsprechung des EuGH zu Abgaben

(i) Padawan

229 Zentral für die Entscheidung des Falls war die Auslegung des Begriffs des „gerechten Ausgleichs“ in Art. 5 Abs. 2 lit. b InfoSoc-RL. Zwar qualifiziert der EuGH zu Recht diesen Begriff als autonom europarechtlich zu interpretierenden Topos, ${ }^{537}$ doch räumt er den Mitgliedstaaten ausdrücklich die Befugnis ein, den gerechten Ausgleich näher auszugestalten, insbesondere dessen Erhebung und Höhe. ${ }^{538}$ Allerdings ist diese Freiheit in der Ausgestaltung nicht grenzenlos; vielmehr sieht der EuGH in Erwägungsgrund 35 der InfoSoc-RL eine Konkretisierung des Begriffs „gerechter Ausgleich“, der den Mitgliedstaaten Schranken bei der Ausgestaltung setzt.

230 Demnach muss der "gerechte Ausgleich“ auf der Grundlage des Schadens berechnet werden, der dem Urheber durch die Privatkopie entsteht. ${ }^{539}$ In diesem Rahmen erkennt der EuGH ausdrücklich

\footnotetext{
${ }^{531}$ Ebenso Zwengel, Kulturflatrates, 2012, S. 221 f.; Runge, GRUR Int. 2007, 130, 133; a.A Ricketson/Ginsburg, International Copyright and Neighbouring Rights, 2. Aufl. 2006, Rn. 19.12.

${ }^{532}$ Ausführlich dazu Zwengel, Kulturflatrates, 2012, S. 206 f.

${ }^{533}$ Zwengel, Kulturflatrates, 2012, S. 223; v. Lewinsky, International copyright law and policy, 2008, Tz. 10.100.

${ }^{534}$ Zwengel, Kulturflatrates, 2012, S. 208.

${ }^{535}$ So auch Zwengel, Kulturflatrates, 2012, S. 223.

${ }^{536}$ EuGH GRUR 2011, 50 - Padawan.

${ }^{537}$ Zuvor bereits EuGH, Urt. v. 6.2.2003 - Rs. C-245/00, Slg. 2003, I-1251 Rn. 24 - SENA/NOS = GRUR 2003, 325, $326 \mathrm{f}$.

${ }^{538}$ EuGH GRUR 2011, 50, 53 Rn. 37 - Padawan.

${ }^{539}$ EuGH GRUR 2011, 50, 53 f. Rn. 39 ff., 50 - Padawan.
} 
die Schwierigkeit der tatsächlichen Durchsetzung von Vergütungs- bzw. „Schadensersatzansprüchen“ gegenüber Privaten als Grund an, Abgaben für Privatkopien einzuführen und mit diesen diejenigen zu belasten, die Vervielfältigungsgeräte oder Dienstleistungen Privaten zur Verfügung stellen, da diese als vorbereitende Handlungen qualifiziert werden und die Abgaben an Private weiterbelastet werden können. ${ }^{540}$ Deutlich und für die hier zu konkretisierende Abgabenhöhe von zentraler Bedeutung ist jedoch der Bezug, den der EuGH mit dem "Schaden“ des Urhebers aufgrund der Vervielfältigungen durch Private herstellt. Dieser muss demnach die Grundlage jeder Berechnung darstellen. ${ }^{541}$

231 Diese Rechtfertigung für die Abgabe stellt aber nach Auffassung des EuGH gleichzeitig auch deren Beschränkung dar: Denn die in Frage stehenden Geräte, Medien oder Dienstleistungen müssen zur Anfertigung von Privatkopien genutzt werden können, so dass keine Abgaben erheben werden dürfen für Geräte, Medien etc., die eindeutig nicht zu Zwecken der Anfertigung von Privatkopien erworben werden. ${ }^{542}$

232 Der EuGH ist sich jedoch auf Grundlage des Erwägungsgrundes 35 der InfoSoc-RL, der nur von „possible harm" spricht, der Schwierigkeiten bewusst, die die Feststellung einer solchen Erwerbsabsicht in der Praxis begegnet - einen Nachweis des tatsächlichen Schadens des Urhebers bzw. dass tatsächlich Kopien durch Private angefertigt werden, verlangt er nicht, ${ }^{543}$ sondern stellt auf eine tatsächliche Vermutung ab, dass die Geräte, Medien etc. zu diesen Zwecken in vollem Umfang benützt werden, sofern sie von Privaten erworben wurden. ${ }^{544}$ Eine ähnliche Vermutung hatte der EuGH auch bereits im Zusammenhang mit einer Entscheidung zu Art. 3 Abs. 1 InfoSoc-RL aufgestellt, indem dort davon ausgegangen worden ist, dass eine „öffentliche Wiedergabe“ i.S.d. Richtlinie bereits bei der Verbreitung eines Fernsehsignals mittels in Hotelzimmern aufgestellter Fernsehapparate - unabhängig vom tatsächlichen Einschalten der Geräte während des Aufenthalts der Gäste - anzunehmen sei. $^{545}$

\section{(ii) Opus Supplies}

233 In der Rechtssache Opus hatte der EuGH Gelegenheit, seinen Ansatz weiter auszubauen: ${ }^{546}$ Der Fall betraf einen in Deutschland ansässigen Anbieter, der Speichermedien in die Niederlande verkaufte, ohne dort oder in Deutschland Abgaben auf die Medien zu entrichten. Der EuGH rekurrierte im Wesentlichen auf seine im Padawan-Urteil statuierten Grundsätze, indem wesentliches Prinzip für Art. 5 Abs. 2 lit. b die "fair compensation“ des Urhebers auf der Grundlage seines erlittenen Schadens sein müsse. ${ }^{547}$ Das Gericht bestätigt nochmals ausdrücklich die Schwierigkeiten, von den Privaten eine Kompensation zu erhalten, so dass auch Hersteller etc. von den Mitgliedstaaten in die Pflicht genommen werden können. ${ }^{548}$ Der EuGH nimmt aber auch die Mitgliedstaaten, die eine Privatkopieschranke geschaffen haben, dafür in die Pflicht, dass sie ein effektives Abgabensystem schaffen, ${ }^{549}$ so

\footnotetext{
${ }^{540}$ EuGH GRUR 2011, 50, 54 Rn. 46, 48, 50 - Padawan.

${ }^{541}$ Ebenso: Riesenhuber, GRUR 2013, 582, $583 \mathrm{f}$.

542 EuGH GRUR 2011, 50, 54 f. Rn. 53, 59 - Padawan; unter Verweis auf Rn. 59 des Padawan-Urteils des EuGH lässt das BVerfG eine Differenzierung des BGH zwischen privater und gewerblicher Benutzung der Geräte ausdrücklich zu, BVerfG GRUR 2011, 225, 226 f. Rn. 26.

${ }^{543}$ EuGH GRUR 2011, 50, 54 Rn. 54 - Padawan; s. dazu auch: Riesenhuber, GRUR 2013, 582, 584.

${ }^{544}$ EuGH GRUR 2011, 50, 54 Rn. 55 - Padawan.

${ }^{545}$ EuGH, Urt. v. 7.12.2006 - Rs. C-306/05, Slg. 2006, I-1519 Rn. 43, 48 ff. = MMR 2007, 164, 165 f.

${ }^{546}$ EuGH, Urt. v. 16.6.2011 - Rs. C-462/09 Stichting de Thuiskopie ./. Opus Supplies Deutschland, GRUR 2011, 909 (im Folgenden verkürzt zitiert).

${ }^{547}$ EuGH GRUR 2011, 909, 910 Rn. 24.

${ }^{548}$ EUGH GRUR 2011, 909, 910 Rn. 29.

${ }^{549}$ EuGH GRUR 2011, 909, 911 Rn. 36, 39.
} 
dass auch im Ausland ansässige Händler bzw. Hersteller in die Abgabepflicht eingebunden werden können. ${ }^{550}$

\section{(iii) VEWA}

234 Schließlich bekräftigte der EuGH indirekt seinen Ansatz aus der Rechtssache Padawan in einer Entscheidung, die die Kalkulation von Abgaben für den Verleih von Werken in Belgien betraf. ${ }^{551}$ Auch wenn die Verleih-Richtlinie nur von einer "compensation“ spricht, stellt der EuGH auch hier auf den für den Urheber entstehenden Schaden („,harm“) ab, der die Grundlage der Berechnungen bilden müsse. ${ }^{552}$ Allerdings betont das Gericht auch den Ermessensspielraum der Mitgliedstaaten bei der Festlegung von Kriterien für die Vergütung der Urheber, der gerade bei der Verleihung von Werken durch nicht-kommerzielle Institutionen die Berücksichtigung von kulturpolitischen Maßnahmen zulasse. $^{553}$

\section{(b) Folgerungen}

235 Diese vom EuGH betonte Konnexität von Schaden des Urhebers und Abgabe sowie der Notwendigkeit der Typisierung führt zu der Frage, ob im Rahmen einer "fair compensation“ auch etwa kompensierende Effekte des Filesharing berücksichtigt werden können, insbesondere der empirisch offenbar abgesicherte sog. Promotionseffekt, der zu vermehrten Käufen gerade von Filesharern führt, ebenso wie der vermehrte Besuch etwa von Musikkonzerten, aber auch anderen Konsum.

236 Allerdings erscheint es zweifelhaft, ob eine derartige, eher volkswirtschaftlich ausgerichtete Perspektive in die Auslegung des Begriffs "gerechter Ausgleich“ Eingang finden kann. Denn Ausgangspunkt für den EuGH ist - wie oben dargelegt - der "Schaden“ bzw. Schadensersatzanspruch des Urhebers im Sinne des Erwägungsgrundes 35 der InfoSoc-RL, für den die Abgabe nur ein Substitut darstellt. Zwar kann man bereits an dieser Stelle einwenden, dass die englische Sprachfassung der Richtlinie hier nur von "harm“ statt von damage spricht, so dass eine rein abstrakte "Schadens"berechnung genügen könnte. ${ }^{554}$ Indes sind die anderen Sprachfassungen der Richtlinie auch nicht eindeutig: So wird der Begriff des „préjudice“ (französisch), des „daño" (spanisch) oder des „pregiudizio“ (italienisch), des „nadeel“ (niederländisch) etc. verwandt. Ob daher tatsächlich daraus der Schluss gezogen werden kann, dass völlig abstrakte Berechnungen im Sinne einer Interessenabwägung genügen, erscheint zweifelhaft.

237 Andererseits kann eine konkrete Schadensberechnung etwa im Sinne der §§ 249 ff. BGB bzw. § 97 UrhG nicht zur Grundlage einer Abgabe gemacht werden - dies schließt schon der typisierende Charakter einer Abgabe von vornherein aus, sie wäre völlig sinnentleert, da sie gerade der Vereinfachung durch Pauschalierung dienen soll. Dies wird auch vom EuGH betont, wenn er zwar einerseits auf die "tatsächliche Nutzung" der Geräte und auf die Gegenleistung für den dem Urheber entstandenen Schaden abstellt, ${ }^{555}$ andererseits aber anerkennt, dass gerade kein konkreter Nachweis eines Schadens zu führen ist, ${ }^{556}$ da sonst dem Urheber Steine statt Brot angesichts der Berechnungs- und Durchsetzungsprobleme gegeben werden. Stattdessen spricht nach dem EuGH gar eine Vermutung

\footnotetext{
${ }^{550}$ EuGH GRUR 2011, 909, 911 Rn. 41.

${ }^{551}$ EuGH, Urt. v. 30.6.2011 - Rs. C-271/10 Vereniging van Educatieve en Wetenschappelijke Auteurs (VEWA) ./. Belgische Staat, GRUR 2011, 913 (im Folgenden verkürzt zitiert).

552 EuGH GRUR 2011, 913, 913 f. Rn. 28.

${ }^{553}$ EuGH GRUR 2011, 913, 914 Rn. 35 f.

${ }^{554}$ So Dreier, ZUM 2011, 281, 284.

555 EuGH GRUR 2011, 50, 53 Rn. 39 f., 42 - Padwan.

${ }^{556}$ EuGH GRUR 2011, 50, 54 Rn. 54 - Padawan.
} 
dafür, dass die Vervielfältigungsfunktionen „vollständig“ ausgeschöpft werden, solange das Gerät dazu nur in der Lage ist. ${ }^{557}$ Daraus ist zu Recht der Schluss gezogen worden, dass die InfoSoc-RL demnach einer Pauschalierung im Rahmen einer Abgabenlösung nicht entgegensteht. ${ }^{558}$

238 Allerdings wird damit auch eine Grenze bei der Bestimmung der Vergütungshöhe eingezogen: Denn wenn auch bei abstrakter Sichtweise der Schaden der Urheber Grundlage zu sein hat, kann darüber bei der Vergütungshöhe nicht hinausgegangen werden - sie darf aber auch grundsätzlich nicht unterschritten werden. Zwar dürfte sich der Schaden etwa von Musikverlegern in einer gesamten Branche schwer exakt berechnen lassen, erst recht wenn kaum belegt werden kann, wie Vervielfältigungen bzw. Filesharing sich kausal auf einen Umsatz- bzw. Erlösrückgang wirklich auswirken; ${ }^{559}$ dennoch ist es nicht auszuschließen, dass z.B. über statistische Berechnungen Anhaltspunkte für mögliche Schäden bestimmt werden. Daher ist nach wie vor eine abstrahierende Sichtweise zulässig (und für Abgaben auch geboten), wobei die Grenze aber in tatsächlich möglichen Schäden liegt, die typisiert werden können.

239 Eines der wesentlichen Kriterien im deutschen Recht zur Berechnung der Vergütung ist der Gerätetyp. Dies schlägt sich auch in der Praxis der geschlossenen Gesamtverträgen zwischen der ZPÜ als Zusammenschluss der Verwertungsgesellschaften und den (Verbänden der) Geräteherstellern ${ }^{560}$ oder den von den Verwertungsgesellschaften festgesetzten Tarifen nieder, ${ }^{561}$ die auf die typischen Nutzungswerte bei den typischen Endabnehmern von bestimmten Geräte rekurrieren. ${ }^{562}$ Dagegen stellt der EuGH unmittelbar nicht darauf ab, welche Geräte zum Einsatz kommen oder welche Vervielfältigungsintensität ihnen inne wohnt. Andererseits hebt das Gericht ausdrücklich auf Geräte, Medien und Anlagen zur Vervielfältigung $a b,{ }^{563}$ so dass daraus der Schluss gezogen werden könnte, dass auch hier eine typisierende Betrachtung anhand des Gerätes zulässig ist. ${ }^{564}$ Aber auch hier gilt, dass an den Gerätetyp nur solange angeknüpft werden kann, wie sein statistisch in Annäherungswerten zu ermittelnder Beitrag zu privaten Vervielfältigungen reicht - was offenbar auch in der Praxis geschieht.

240 Bezogen auf eine Kulturflatrate müsste demnach bestimmt werden, wie viele Vervielfältigungsvorgänge typischerweise über einen privaten Internetanschluss getätigt werden - was nur mit einem prozentualen Anteil am Gesamtvolumen eines Internetanschlusses bestimmt werden kann.

241 Die deutsche Regelung zur Bestimmung der Vergütungshöhe rekurriert indes nicht nur auf pauschalierende Schadensfaktoren wie den Gerätetyp (und die daraus resultierende Intensität einer Vervielfältigung) und die Nutzungshäufigkeit, sondern bezieht auch Kriterien wie die wirtschaftliche

\footnotetext{
${ }^{557}$ EuGH GRUR 2011, 50, 54 Rn. 55 - Padawan.

${ }^{558}$ Dreier, ZUM 2011, 281, 286.

559 Bspw. sieht Vitorino, Recommendations, S. 19 f. die Schäden durch Privatkopien als grundsätzlich geringer als weithin angenommen, da er auf die Anzahl der Kopien schaut, die tatsächlich trotzdem lizenziert worden wären, wenn es die Privatkopieschranke nicht gäbe; er bezieht somit eine Art Substitutionsrate (dazu s. oben Rn. 97 ff.; sowie zur Einbeziehung in die Berechnungsmodelle s. unten Rn. 326 ff.) in seine Schadensberechnung mit ein.

${ }^{560}$ S. etwa den „Gemeinsamen Tarif der ZPÜ und VG Wort / VG Bild-Kunst über die Vergütung für PCs und zum Einbau bestimmter Brenner", Bundesanzeiger, 6. Mai 2010, Nr. 69, S. 1634.

${ }^{561}$ S. etwa den „Gemeinsamen Tarif der VG Wort und der VG Bild-Kunst, Bundesanzeiger, 23. Dezember 2008, Nr. 195, S. 4691.

562 S. dazu Dreier, ZUM 2011, 281, 285, mit Verweis auf die Schiedsstelle, ZUM-RD 2011, 46, 52.

563 EuGH GRUR 2011, 50, 54 Rn. 52 - Padawan.

${ }^{564}$ So Dreier, ZUM 2011, 281, 286, 289 f.
} 
Zumutbarkeit mit ein, die eher einer Interessenabwägung zwischen Herstellern und Urhebern zuzuordnen sind. Diese finden ihren Niederschlag in der sog. (variablen ${ }^{565}$ ) Kappungsgrenze des § 54a Abs. 4 UrhG im Hinblick auf das Verhältnis von Geräteabgabe und Preis des Gerätes bzw. Speichermediums. Eine solche Kappung einer Abgabe wäre auch für eine „Kulturflatrate“ im Verhältnis zu den Gebühren für einen Internetzugang denkbar. Ob diese Kappung indes mit den vom EuGH aufgestellten Grundsätzen vereinbar ist, die sich allein auf eine - abstrakte - Schadensberechnung stützen, erscheint zweifelhaft. ${ }^{566}$ Zwar eröffnet die InfoSoc-RL insbesondere in Erwägungsgrund 31 eine Interessenabwägung zwischen Urhebern und Nutzern, doch bezieht sich der EuGH auch angesichts dieses Erwägungsgrundes allein auf den "Schaden“ des Urhebers, ${ }^{567}$ so dass eine Kappung im Hinblick auf die wirtschaftliche Zumutbarkeit bzw. den Preis nicht mehr vom Schadensbegriff gedeckt wäre. ${ }^{568}$ Allerdings dürfte der EuGH hier über das Ziel hinausgeschossen sein, indem er die eindeutige in der Richtlinie angelegte Abwägung zwischen Nutzern (und damit indirekt auch den Herstellern etc., die die Abgabe an die Nutzer weiterreichen) und Rechteinhabern einseitig zugunsten des Schadens der Urheber ausblendet. Auch wenn daher der EuGH offenbar eindeutig gegen eine Einbeziehung wirtschaftlicher Interessen von Nutzern (und Herstellern ${ }^{569}$ ) Stellung bezieht, spricht die Richtlinie eine andere Sprache - so dass zu hoffen ist, dass der EuGH seine Ausführungen revidieren bzw. richtigstellen kann. Andernfalls muss die Richtlinie geändert werden, um breiteren Raum für eine Interessenabwägung zu finden.

242 In diesem Rahmen ist auch zu berücksichtigen, dass gerade der Dreistufen-Test, der für die Zulässigkeit einer Schranke maßgeblich heranzuziehen ist (und vom EuGH nicht näher erwähnt wird) durchaus die Möglichkeit eröffnet, keine 1:1 Vergütung anhand ersparter Lizenzen zu bestimmen. So hebt Senftleben zu Recht hervor: ${ }^{570}$

„Dass im Wege derartiger Pauschalzahlungen etwa in Deutschland jemals eine Vergütungshöhe erreicht worden wäre, die im Verdacht stehen könnte, auch nur ein schwacher Abglanz des leuchtenden Vorbilds zu sein, auf das Ricketson [der eine Vergütungshöhe gefordert hatte, die dem Satz ohne eine Schranke entsprach] verweist, steht wohl außer Frage. Es ist allerdings zu berücksichtigen, dass eine volle Entschädigung im Dreistufentest auch gar nicht angelegt ist. In funktionaler Hinsicht dient die Zahlung einer angemessenen Vergütung nicht dazu, die entgangene Lizenzgebühr auszugleichen. Es soll lediglich insoweit Ersatz geleistet werden, wie eine Beschränkung die Schwelle einer zulässigen Verletzung berechtigter Interessen überschreitet. Die Vergütungszahlung dient somit als Mittel zur Reduzierung einer ungebührlichen Verletzung auf ein den Umständen nach angemessenes Maß. Das Konzept für die Zahlung einer angemessenen Vergütung, das dem Dreistufentest zu Grunde liegt, ist folglich ein fließender Übergang von Konstellationen, in denen keine Vergütungszahlungen notwendig sind, weil die Beschränkung keine ungebührliche Interessenverletzung mit sich bringt, bis hin zu Fällen, in denen große Beträge angemessen erscheinen, weil von der Beschränkung einschneidende Auswirkungen auf die berechtigten Interessen des Urhebers ausgehen."

244 Auf gleicher Linie liegen die Erläuterungen der EU-Kommission zur InfoSoc-Richtlinie:

\footnotetext{
${ }^{565}$ Eine absolute Kappungsgrenze wäre mit dem EuGH-Urteil schwerlich vereinbar, zutr. Jani/Ebbinghaus, GRUR-Prax 2011, 71, 72; Dreier, ZUM 2011, 281, 287.

${ }^{566}$ S. Dreier, ZUM 2011, 281, 287; Riesenhuber, GRUR 2013, 582, $589 \mathrm{f}$.

${ }^{567}$ EuGH GRUR 2011, 50, 53 Rn. 42 f.

568 So denn auch Jani/Ebbinghaus, GRUR-Prax 2011, 71, 72.

${ }^{569}$ Generell kritisch zur Einbeziehung der Interessen der Hersteller: Riesenhuber, GRUR 2013, 582, 589.

${ }^{570}$ Senftleben, GRUR Int. 2004, 200, 211.
} 
'The term 'fair compensation' is not identical to the term 'equitable remuneration' as it is used in Articles 4(4) and 8(2) of the EC rental and lending rights directive. While the notion of 'equitable remuneration' is based on the assumption that authors are entitled to remuneration for every act of usage of their protected works, fair compensation is, inter alia, linked to the possible harm that derives from acts of private copying (cf. recital 35 of the Directive). Article $5(2)(b)$ therefore requires that any payment to rightholders must be compensatory in nature." ${ }^{1571}$

"This is explained further in recital 35. The payment of any compensation should take into account: (i) the 'possible harm to the rightsholders'; (ii) whether rightsholders 'have already received payment'; and (iii) that no obligation for payment arises where there is minimal harm to rightsholders. Fair compensation is for the harm that could result from the act of private copying itself ('harm to the rightsholders resulting from the act in question'). ${ }^{\text {“572 }}$

247 Dies gilt auch für einen mehr schadensrechtlichen Ansatz, der zwar denkbar, aber mit vielen Unsicherheiten hinsichtlich der Auffassung des EuGH behaftet ist: Demnach wäre denkbar, dass angesichts der nur schwierig darzulegenden Kausalität von Vervielfältigungs- oder anderen Verwertungsvorgängen zu „Schäden“ der Urheber auch im Sinne einer Vorteilsanrechnung, wie sie aus den $\S \S 249$ ff. BGB bekannt ist, die nicht minder schwer zu belegenden bzw. kausalen Promotionseffekte angerechnet werden könnten, da sie entsprechende Umsatzausfälle bei dem Verkauf von Tonträgern oder Downloadportalen kompensieren könnten. So wie Schäden hier typisierend berechnet werden müssen, könnte dann auch daran gedacht werden, die offenbar vorhandenen positiven Effekte ebenfalls typisierend einzubeziehen. Wie bereits dargelegt, verlässt eine solche Vorteilsanrechnung, die eher gesamtwirtschaftlich argumentiert, den Boden der traditionellen Schadensberechnung und kann daher hier nur als mögliche Argumentation angedeutet werden.

\section{(c) Zusammenfassung}

248 Festzuhalten ist daher, dass die EuGH-Rechtsprechung zur InfoSoc-RL im Wesentlichen an dem "Schaden“ des Urhebers anknüpft, der durch Verwertungsvorgänge entsteht, hier allerdings eine Typisierung zulässt. Ob eine Berücksichtigung von gegenläufigen Effekten etwa im Sinne einer gesamtwirtschaftlichen Interessenabwägung oder einer Art Vorteilsanrechnung möglich ist, erscheint fraglich.

\section{(2) Datenschutz-Richtlinien}

249 Die Erfassung der Nutzung bzw. des Downloads etc. von Werken (s. Verteilungsschlüssel etc.) bedarf der Erhebung von Daten - daher sind auch die datenschutzrechtliche Vorgaben von großer Bedeutung, insbesondere die Grundsätze der Datensparsamkeit und der Zweckbindung. Generell gilt hier, dass diejenigen Lösungen vorzugswürdig sind, die möglichst wenig personenbezogene Daten zur Messung der Nutzungsvorgänge der urheberrechtlich relevanten Inhalte erfordern. ${ }^{573}$ Dem steht andererseits das Interesse der Urheber gegenüber, die konkreten Eingriffe in ihre Rechte vergütungsmäßig zu erfassen - entsprechend den oben dargelegten europarechtlichen Grundsätzen der InfoSoc-RL bzw. Padawan-Entscheidung des EuGH.

\footnotetext{
${ }^{571}$ EU Kommission, Background Document „Fair Compensation For Acts of Private Copying”, 14.02.2008, S. 4, abrufbar unter: http://ec.europa.eu/internal_market/copyright/docs/levy_reform/background_en.pdf.

${ }^{572}$ EU Kommission, Background Document „Fair Compensation For Acts of Private Copying”, 14.02.2008, S. 4 Fn. 7, abrufbar unter: http://ec.europa.eu/internal_market/copyright/docs/levy_reform/background_en.pdf. ${ }^{573}$ Zutr. Roßnagel et al., Gutachten, 2009, S. 29 f.
} 
250 Von Bedeutung ist hier der derzeit rechtspolitisch intensiv diskutierte Vorschlag einer europäischen Datenschutz-Grund-VO. ${ }^{574}$ Dieser enthält allerdings in Fortführung der bereits existierenden europäischen Ansätze in Form der Datenschutz-Richtlinie sowie der ePrivacy-Richtlinie ${ }^{575}$ die bereits bezeichneten Grundsätze der Zweckbindung und Datensparsamkeit. Detailliertere Vorgaben für die Erfassung von Down- und Uploadvorgängen enthalten allerdings de lege lata weder die DatenschutzRL noch die ePrivacy-Richtlinie, de lege ferenda auch nicht der neue Vorschlag - allerdings erlaubt der neue Vorschlag delegierte Rechtsakte der Kommission, die demnach auch für die Erhebung von Daten für die Berechnung und Verteilung einer Abgabe Vorgaben machen könnte. Aus Datenschutzgründen sollten die Nutzer zumindest pseudonymisiert, möglichst aber nur die verteilungsrelevanten Daten erhoben werden, also etwa die Urheber von Werken, Titel etc. ${ }^{576}$ Ferner spielt das Datenschutzrecht im Rahmen der Vergütungsverteilung eine Rolle, wenn es um die Anforderungen an eine Datenschutzerklärung und Einwilligung gegenüber Nutzern geht, die im Rahmen von statistischen Erhebungen entsprechende Plug-ins in ihren Browsern oder Software auf ihren Rechnern installieren, die die Erhebung nutzungsrelevanter Daten erlaubt. ${ }^{577}$

\section{b) Verfassungsrechtliche Vorgaben}

\section{(1) Eingriff in Urheber- und Verwertungsrecht}

251 In Bezug auf Pauschalabgaben für Urheber zur Kompensation von Einnahmeeinbußen aufgrund urheberrechtlicher Schrankenregelungen hat sich das BVerfG im Jahr 2010 in zwei Entscheidungen geäußert. ${ }^{578}$ Gegenstand beider Entscheidungen waren unter anderem die Fragen, ob die Geräteabgaben nach der bis zum 31.12.2007 geltenden Fassung des § 54a UrhG auch auf gewisse Gerätetypen anwendbar waren, die ausschließlich in Verbindung mit anderen Geräten zur Vervielfältigung geeignet sind und, darüber hinaus, ob auch an Urheber digitaler Werkvorlagen eine angemessene Vergütung nach dieser Regelung zu entrichten war. ${ }^{579}$

252 Neben der Bezugnahme auf europarechtliche Vorgaben und mögliche Auslegungen der Urheberrechtsrichtlinie ${ }^{580}$ hebt das Gericht erneut die grundsätzliche Zuordnung der vermögenswerten Ergebnisse der schöpferischen Leistung an den Urheber und dessen Verfügungsfreiheit über seine Werke im Rahmen der Eigentumsgarantie aus Art. 14 Abs. $1 \mathrm{GG}$ hervor. ${ }^{581}$ Aufgrund der technischen Entwicklung entstehende Schutzlücken seien zu schließen und eine Auslegung des § 54a UrhG a.F. habe im Lichte des Art. 14 Abs. 1 GG dahingehend zu erfolgen, dass der Gesetzgeber den verfassungsrechtlich garantierten Verwertungsanspruch für Fälle sichern wollte, in denen der Werknutzer

\footnotetext{
${ }^{574}$ Vorschlag für eine Verordnung des Europäischen Parlaments und des Rates zum Schutz natürlicher Personen bei der Verarbeitung personenbezogener Daten und zum freien Datenverkehr (Datenschutz-

Grundverordnung), KOM(2012) 11 endg., abrufbar unter: http://eurlex.europa.eu/LexUriServ/LexUriServ.do?uri=COM:2012:0011:FIN:DE:PDF.

${ }^{575}$ Richtlinie 2009/136/EG des Europäischen Parlaments und des Rates vom 25. November 2009 zur Änderung der Richtlinie 2002/22/EG über den Universaldienst und Nutzerrechte bei elektronischen Kommunikationsnetzen und -diensten, der Richtlinie 2002/58/EG über die Verarbeitung personenbezogener Daten und den Schutz der Privatsphäre in der elektronischen Kommunikation und der Verordnung (EG) Nr. 2006/2004 über die Zusammenarbeit im Verbraucherschutz, ABI. Nr. L 337 v. 18.12.2009, S. 11.

${ }^{576}$ Zur möglichen Erhebung der Daten s. unten Rn. 393 ff.

577 Dazu unten Rn. $457 \mathrm{ff}$.

578 BVerfG NJW 2011, 288 - Drucker und Plotter; s. zu dieser Entscheidung auch die zusammenfassende Darstellung bei Arlt, MMR-Aktuell 2011, 313015; BVerfG MMR 2011, 749.

${ }^{579}$ BVerfG NJW 2011, 288 - Drucker und Plotter; BVerfG MMR 2011, 749; Arlt, MMR 2011, 749, 750.

${ }^{580}$ BVerfG MMR 2011, 749, 750; BVerfG 2011, 288, $289 \mathrm{f.}$

${ }^{581}$ BVerfG NJW 2011, 288, 290, unter Verweis auf BVerfGE 31, 229, 240 f.; BVerfGE 79, 1, 25.
} 
nicht belangt werden könne. ${ }^{582}$ Das BVerfG beanstandet in diesem Zusammenhang die vorhergegangene Entscheidung des $\mathrm{BGH}^{583} \S 54 \mathrm{a}$ UrhG a.F. neben den analogen nicht auch auf digitale Werkvorlagen angewandt zu haben.

253 Allerdings haben sich diese Auslegungsprobleme mit der neuen Fassung des $\S 54$ UrhG erübrigt: Es unterfallen nun sämtliche Geräte der Abgabepflicht, die „allein oder in Verbindung mit anderen Geräten, Speichermedien oder Zubehör zur Vornahme (von) Vervielfältigungen benutzt" werden. Zudem verlangt § 54 UrhG n.F. lediglich, dass nach der Art des Werkes eine Vervielfältigung nach der Schrankenbestimmung des $\S 53$ Abs. 1 bis 3 UrhG zu erwarten ist, unterscheidet aber nicht wie noch die alte Rechtslage zwischen Geräten, die Vervielfältigungen im Wege der Ablichtung oder eines ähnlichen Verfahrens ( $\$$ 54a UrhG a.F.) ${ }^{584}$ oder im Wege der Bild- und Tonaufzeichnung ( $\$ 54$ UrhG a.F. $)^{585}$ vornehmen. ${ }^{586}$ Somit ist nunmehr eine Unterscheidung zwischen Geräten nach § 54 UrhG a.F. und § 54a UrhG a.F. hinfällig. Jetzt sind unabhängig vom Vervielfältigungsverfahren und Herkunft der Kopiervorlage auch digitale Werkevorlagen und alle - auch nur als Teil einer Funktionseinheit - zur Vervielfältigung i.S.d. $\S 53$ Abs. 1 bis 3 UrhG geeigneten Geräte von der Abgaberegelung des $\S 54$ UrhG n.F. erfasst. ${ }^{587}$

254 Trotz der mittlerweile erfolgten Novellierung des Urhebergesetzes bleiben die genannten und vorhergegangenen Entscheidungen des BVerfG von Bedeutung: Auch wenn dem Gesetzgeber gem. Art. 14 Abs. 1 S. 2 GG die Aufgabe der Festlegung des Inhalts und der Schranken des Urheberrechts unter Beachtung der sozialen Bedeutung des Eigentums zukommt, muss er dabei doch in jedem Fall den aufgezeigten vermögenswerten Kern der schöpferischen Leistung des Urhebers wahren. ${ }^{588}$ Von diesen verfassungsrechtlichen Vorgaben dürfte auch bei der Anpassung des Urhebergesetzes an eine Kulturflatrate nicht abgewichen werden.

\footnotetext{
${ }^{582}$ BVerfG NJW 2011, 288, 290.

583 BGH NJW 2008, 751.

${ }^{584}$ Bis zum 31.12.2007 lautete Abs. 1 des § 54a UrhG:

Ist nach der Art eines Werkes zu erwarten, daß es nach $\S 53$ Abs. 1 bis 3 durch Ablichtung eines Werkstücks oder in einem Verfahren vergleichbarer Wirkung vervielfältigt wird, so hat der Urheber des Werkes gegen den Hersteller von Geräten, die zur Vornahme solcher Vervielfältigungen bestimmt sind, Anspruch auf Zahlung einer angemessenen Vergütung für die durch die Veräußerung oder sonstiges Inverkehrbringen der Geräte geschaffene Möglichkeit, solche Vervielfältigungen vorzunehmen.

${ }^{585}$ Bis zum 31.12.2007 lautete Abs. 1 des $§ 54$ UrhG:

Ist nach der Art eines Werkes zu erwarten, daß es durch Aufnahme von Funksendungen auf Bild-oder Tonträger oder durch Übertragungen von einem Bild-oder Tonträger auf einen anderen nach § 53 Abs. 1 oder 2 vervielfältigt wird, so hat der Urheber des Werkes gegen den Hersteller

1. von Geräten und

2. von Bild- oder Tonträgern,

die erkennbar zur Vornahme solcher Vervielfältigungen bestimmt sind, Anspruch auf Zahlung einer angemessenen Vergütung für die durch die Veräußerung der Geräte sowie der Bild-oder Tonträger geschaffene Möglichkeit, solche Vervielfältigungen vorzunehmen. [2] Neben dem Hersteller haftet als Gesamtschuldner, wer die Geräte oder die Bild- oder Tonträger in den Geltungsbereich dieses Gesetzes gewerblich einführt oder wiedereinführt oder wer mit ihnen handelt. [3] Der Händler haftet nicht, wenn er im Kalenderhalbjahr Bild-oder Tonträger von weniger als 6000 Stunden Spieldauer und weniger als 100 Geräte bezieht.

${ }^{586}$ S. ausf. zur alten Rechtslage Loewenheim, in: Loewenhiem, Handbuch des Urheberrechts, 2. Aufl. $2010, \S 86$ Rn. $18 \mathrm{ff}$.

${ }^{587}$ S. ausf. dazu Dreier, in: Dreier/Schulze, UrhG, 4. Aufl. 2013, § 54 UrhG Rn. 6 ff.; Loewenheim, in: Loewenhiem, Handbuch des Urheberrechts, 2. Aufl. 2010, § 86 Rn. 11 ff; s. auch Lüft, GRUR-Prax 2011, 313646; Arlt, MMR 2011, 749, 750.

${ }^{588}$ Dazu grundlegend BVerfGE 31, 229, $240 \mathrm{f.}$
} 


\section{(2) Datenschutz-Grundrechte}

255 Ähnlich dem europäischen Recht müssen auch nach deutschem Verfassungsrecht die Grundrechte auf informationelle Selbstbestimmung ${ }^{589}$ und Vertrauen in die Integrität von IT-Systemen ${ }^{590}$ bei der Erhebung von Daten zur Bestimmung der Vergütungen beachtet werden. Wiederum gelten die Grundsätze der Datensparsamkeit, der möglichst anonymen, zumindest pseudonymen Erhebung der Daten. Darüber hinaus greift angesichts einiger Methoden, die direkt den Datenverkehr durch Deep Packet Inspection ${ }^{591}$ überwachen, das Fernmeldegeheimnis nach Art. $10 \mathrm{GG}$ ein.

256 In den Schutzbereich des Fernmeldegeheimnisses fallen der Inhalt, aber auch die Umstände jeder Individualkommunikation, die unkörperlich übermittelt wird, ${ }^{592}$ was zu einer besonderen Weite des Schutzbereiches führt. ${ }^{593}$ Insbesondere schützt das Fernmeldegeheimnis nicht nur Sprach- oder Textkommunikation, sondern jede Datenübertragung, ${ }^{594}$ da das Grundrecht entwicklungsoffen ausgestaltet ist und ,jede Übermittlung von Informationen mit Hilfe der verfügbaren Kommunikationstechniken ${ }^{4595}$ umfasst, grundsätzlich also auch den Internetverkehr. ${ }^{596}$ Allerdings wird gefordert, dass der Kommunikationsvorgang hinreichend individualisiert wurde ${ }^{597}$ Angesichts dessen, dass im Internet die Grenzen zwischen Individual- und Massenkommunikation verschwimmen, muss diese Wertung normativ und kann nicht technisch erfolgen, ${ }^{598}$ sodass der Schutzbereich dann nicht eröffnet ist, wenn ein Kommunikationsvorgang öffentlich zugänglich ist. ${ }^{599}$ So fallen beispielsweise geschlossene Benutzergruppen, der E-Mail Verkehr oder auch geschützte Chaträume in den Schutzbereich des Art. $10 \mathrm{GG}{ }^{600}$ Ein Indiz für Individualverkehr soll sein, ob für eine Überwachung Zugangshindernisse zu überwinden sind, ebenso soll er dann vorliegen, wenn kein Zweifel am individuellen Charakter der Kommunikation besteht. ${ }^{601}$ Kein Individualverkehr soll bestehen, wenn sich eine grundrechtsgebundene Stelle unter denselben technischen oder ökonomischen Voraussetzungen in die Kommunikation einschalten kann wie jeder Dritte, ${ }^{602}$ so dass etwa nicht besonders geschützte P2P-Netzwerke oder Filehoster wie Rapidshare (solange kein besonderes Zugangshindernis wie eine Passwortsperre besteht) nicht dem Schutzbereich von Art. $10 \mathrm{GG}$ unterfallen. ${ }^{603}$ Gegen eine solche Annahme spricht aber die oben erwähnte Entwicklung hin zu einer Vermischung von Massen- und Individualkommuni-

\footnotetext{
${ }^{589}$ BVerfGE 65, 1, 43 - Volkszählung.

${ }^{590}$ BVerfGE 120, 274, 302.

${ }^{591}$ S. unten Rn. 439 ff.; sowie zur Deep Packet Inspection allgemein Bedner, CR 2010, 339.

592 BVerfGE 115, 166, 183; Durner, in: Maunz/Dürig, GG, 68. EL 2013, Art. 10 Rn. 81.

${ }^{593}$ Durner, in: Maunz/Dürig, GG, 68. EL 2013, Art. 10 Rn. 82; Löwer, in: v. Münch/Kunig, GG, 6. Aufl. 2012, Art. 10 Rn. 19.

${ }^{594}$ Durner, in: Maunz/Dürig, GG, 68. EL 2013, Art. 10 Rn. 82; Hermes, in: Dreier, GG, 3. Aufl. 2013, Art. 10 Rn. 41.

${ }^{595}$ BVerfGE 115, 166, 182.

${ }^{596}$ Durner, in: Maunz/Dürig, GG, 68. EL 2013, Art. 10 Rn. 47; Löwer, in: v. Münch/Kunig, GG, 6. Aufl. 2012, Art. 10 Rn. 19.

${ }^{597}$ Böckenförde, JZ 2008, 925, 936; Hermes, in: Dreier, GG, 3. Aufl. 2013, Art. 10 Rn. 39.

598 Durner, in: Maunz/Dürig, GG, 68. EL 2013, Art. 10 Rn. 94; Gusy, in: v. Mangoldt/Klein/Starck, GG, 6. Aufl. 2010, Art. 10 Rn. 42; Hermes, in: Dreier, GG, 3. Aufl. 2013, Art. 10 Rn. 40.

${ }^{599}$ Durner, in: Maunz/Dürig, GG, 68. EL 2013, Art. 10 Rn. 93 Hermes, in: Dreier, GG, 3. Aufl. 2013, Art. 10 Rn. 39.

${ }^{600}$ Durner, in: Maunz/Dürig, GG, 68. EL 2013, Art. 10 Rn. 94; Löwer, in: v. Münch/Kunig, GG, 6. Aufl. 2012, Art. 10 Rn. 20; Hermes, in: Dreier, GG, 3. Aufl. 2013, Art. 10 Rn. 39.

${ }^{601}$ Durner, in: Maunz/Dürig, GG, 68. EL, 2012, Art. 10 Rn. 94.

${ }^{602}$ Gusy, in: v. Mangoldt/Klein/Starck, GG, 6. Aufl. 2010, Art. 10 Rn. 44.

${ }^{603}$ Beck/Kreißig, NStZ 2007, 304, 307; Zombik, ZUM 2006, 450, 454; Czychowski, MMR 2004, 514, 518; davon zu unterscheiden ist die Frage, ob es sich um einen Eingriff in die Fernmeldefreiheit handelt, wenn bei dem Access-Provider die Identifikation einer Person anhand einer IP-Adresse verlangt wird.
} 
kation, ${ }^{604}$ und der Schutzzweck des Art. $10 \mathrm{GG}$. Hiernach soll das Fernmeldegeheimnis gerade die Vertraulichkeit der Information schützen und sie gegen eine unbefugte Kenntniserlangung durch Dritte abschirmen. ${ }^{605}$ Die Vertraulichkeit wäre bei einer Analyse des Internettraffic aber nicht mehr gegeben, da sich erst nach dieser herausstellen würde, ob deren Inhalt Individualkommunikation ist oder nicht, es würde somit erst nachdem der Kommunikationsanhalt zur Kenntnis genommen wurde über dessen Grundrechtsschutz entschieden ${ }^{606}$ Daher muss bereits bei der Möglichkeit individueller Kommunikation der Schutzbereich des Art. $10 \mathrm{GG}$ eröffnet sein. ${ }^{607}$ Wollte man also mit Techniken wie der Deep Packet Inspection arbeiten und den Internetverkehr überwachen, so ließe es sich kaum vermeiden, auch solche Daten zu durchleuchten, die dem Schutz des Fernmeldegeheimnisses unterfallen, womit der Schutzbereich von Art. 10 GG eröffnet ist.

257 In dessen Schutzbereich greift der Staat ein, wenn er selber Kenntnis vom Inhalt der Telekommunikation nimmt, ${ }^{608}$ aber auch dann, wenn er sich Privater als Hilfspersonen zur Erledigung staatlicher Aufgaben bedient. ${ }^{609}$ Beides ist hier zunächst nicht einschlägig, denn weder nimmt der Staat selber Kenntnis, noch benutzen die Telekommunikationsanbieter die durch die DPI gewonnenen Daten für staatliche Aufgaben. Allerdings begründet Art. $10 \mathrm{GG}$ auch Schutzpflichten. Der Gesetzgeber muss ein hinreichendes Schutzniveau durch Regulierung der privaten Telekommunikationsanbieter sicherstellen. Dazu gehört insbesondere auch die gesetzliche Normierung und Begrenzung von Ausnahmetatbeständen. Üblicherweise liegt der Schutzpflicht aber eine Konstellation zugrunde bei der die Grundrechtsgefährdung ursprünglich von dem anderen Privatrechtssubjekt ausgeht, nicht aber von der gesetzlichen Anordnung. Der Gesetzgeber begäbe sich somit, indem er Private dazu verpflichtet die Daten zu erheben und weiterzugeben, in den Grenzbereich von Eingriff und Schutzpflichtverletzung. ${ }^{610}$ Während eine Erlaubnis zur Weitergabe an Behörden eher noch dem Eingriffsbereich zugeordnet werden kann, ${ }^{611}$ ist dies hier aufgrund einer anderen Gefährdungslage anders zu beurteilen. Denn der Gesetzgeber nimmt zu keinem Zeitpunkt Kenntnis von den Daten und hat ohne entsprechende Rechtsgrundlage auch keine rechtliche Möglichkeit dazu. Indem der Gesetzgeber also die privaten Anbieter dazu verpflichtet eine Deep Packet Inspection vorzunehmen und die erhobenen Daten zu übermitteln nimmt er Einfluss auf das Schutzniveau des Telekommunikationsgeheimnisses im Privatrecht, so dass eine Schutzpflichtenkonstellation vorliegt.

258 Zwar hat der Gesetzgeber auch bei der Ausgestaltung von Schutzpflichten einen weiten Spielraum und das Bundesverfassungsgericht übt nur eine eingeschränkte Kontrolle aus, ${ }^{612}$ doch gibt es auch

\footnotetext{
${ }^{604}$ Hermes, in: Dreier, GG, 3. Aufl. 2013, Art. 10 Rn. 39; Löwer, in: v. Münch/Kunig, GG, 6. Aufl. 2012, Art. 10 Rn. 20.

${ }^{605}$ BVerfGE 115, 166, 183; Hermes, in: Dreier, GG, 3. Aufl. 2013, Art. 10 Rn. 40; Löwer, in: v. Münch/Kunig, GG, 6. Aufl. 2012, Art. 10 Rn. 24.

${ }^{606}$ Hermes, in: Dreier, GG, 3. Aufl. 2013, Art. 10 Rn. 40; ähnlich Löwer, in: v. Münch/Kunig, GG, 6. Aufl. 2012, Art. 10 Rn. 20; Gusy, in: v. Mangoldt/Klein/Starck, GG, 6. Aufl. 2010, Art. 10 Rn. 44; anders dagegen Stern, Das Staatsrecht der Bundesrepublik Deutschland, Band IV/1, 2006, S. 228, der insoweit auf den einzelnen Kommunikationsvorgang abstellen will.

${ }^{607}$ Hermes, in: Dreier, GG, 3. Aufl. 2013, Art. 10 Rn. 40; so auch Gusy, in: v. Mangoldt/Klein/Starck, GG, 6. Aufl. 2012, Art. 10 Rn. 44; Stettner, in: Merten/Papier, Handbuch der Grundrechte, Bd. IV, 2011, § 92 Rn. 33; wohl auch: Löwer, in: v. Münch/Kunig, GG, 6. Aufl. 2012, Art. 10 Rn. 20.

${ }^{608}$ Gusy, in: v. Mangoldt/Klein/Starck, GG, 6. Aufl. 2012, Art. 10 Rn. 58.

${ }^{609}$ BVerfGE 125, 260, 311 Rn. 193.

${ }^{610}$ Hermes, in: Dreier, GG, 3. Aufl. 2013, Art. 10 Rn. 53.

${ }^{611}$ Hermes, in: Dreier, GG, 3. Aufl. 2013, Art. 10 Rn. 53 unter Berufung auf BVerfGE 125, 260, 312 Rn. 194.

${ }^{612}$ Durner, in: Maunz/Dürig, GG, 68. EL 2013, Art. 10 Rn. 115; zur Einschätzungsprärogative allgemein s. oben Rn. $159 \mathrm{ff}$.
} 
hier eine Grenze bei besonders intensiven Beeinträchtigungen ${ }^{613}$ In Anbetracht der Menge an erhobenen Daten, die für einen gewissen Abrechnungszeitraum gespeichert werden müssen, könnten daher Bedenken bestehen, ob damit nicht diese Grenze überschritten ist. Ähnlich wie bei der Vorratsdatenspeicherung, die vom BVerfG für verfassungswidrig erklärt wurde. ${ }^{614}$ Hierzu bestehen allerdings - abgesehen von der Speicherung der Daten zu privatrechtlichen Zwecken - entscheidende Unterschiede. Zunächst wäre eine Speicherung der urheberrechtlich relevanten Vorgänge vom Umfang her geringer. Entscheidender ist allerdings, dass die Speicherung nicht anlasslos geschehen würde, sondern zu einem konkreten Zweck und in konkretem Umfang, so dass die Speicherung nicht geeignet wäre, ein „diffus bedrohliches Gefühl des Beobachtetseins hervorzurufen, das eine unbefangene Wahrnehmung der Grundrechte in vielen Bereichen beeinträchtigen kann". ${ }^{615}$ Dennoch ist der hohe Rang, den Art. $10 \mathrm{GG}$ unter den Grundrechten einnimmt zu beachten, sowie, dass der Mitschnitt oder die Kenntnisnahme von Kommunikationsinhalten das Telekommunikationsgeheimnis stark beeinträchtigen. ${ }^{616}$ Da die Regelungen zur Kulturflatrate dem Ausgleich von Grundrechten dienen, müssen bei der Beurteilung ob der Gesetzgeber seinen Gestaltungsspielraum überschritten hat, auch die kollidierenden Rechtsgüter betrachtet werden. ${ }^{617}$ Während bei Maßnahmen der Strafverfolgung oder Gefahrenabwehr - wie sie für die Vorratsdatenspeicherung in Rede stehen - auf hochrangige Gemeinwohlbelange zurückgegriffen werden kann, ${ }^{618}$ greift bei der Kulturflatrate vor allem Art. 14 Abs. 1 GG zugunsten der Rechteinhaber auf der gegenüberliegenden Seite. Dies könnte gegen die Zulässigkeit einer Deep Packet Inspection auch zu privatrechtlichen Zwecken sprechen.

259 Andererseits könnte man sich an den Forderungen des Bundesverfassungsgerichts zu einer verfassungsgemäßen verdachtsunabhängigen Vorratsdatenspeicherung orientieren. ${ }^{619}$ Eine entsprechende Regelung sollte sich beispielsweise durch hohe Datenschutzstandards, eine strikte Zweckbindung und Transparenz auszeichnen und darüber hinaus sicherstellen, dass keine Nutzerzuordnung geschehen kann, die Daten also soweit wie möglich (bis auf eine evtl. Geo-Zuordnung) anonymisiert werden. ${ }^{620}$ Die Intensität der Deep Packet Inspection müsste so gering wie zur Identifzierung der Daten gerade erforderlich ausfallen. Ebenso müssten effektive Rechtsschutzmöglichkeiten geschaffen werden, um ggf. Verstöße verfolgen zu können. ${ }^{621}$

260 Doch selbst wenn diese Voraussetzungen erfüllt sind, bleiben aufgrund der Tiefe der Erfassung des Internettraffic auf der einen Seite und dem dagegen stehenden Grundrecht auf Eigentum der Urheber, verfassungsrechtlich Zweifel bestehen; denn eine komplette Sichtung der Internetkommunikation stellt einen der schwerwiegendsten vorstellbaren Eingriffe in das Fernmeldegeheimnis dar. ${ }^{622}$ Daher wird in den meisten Fällen die staatliche Anordnung einer eine flächendeckende Anwendung

\footnotetext{
${ }^{613}$ Durner, in: Maunz/Dürig, GG, 68. EL 2013, Art. 10 Rn. 115; s. auch Callies, in: Merten/Papier, Handbuch der Grundrechte, Band 2, 2006, § 44 Rn. 6.

${ }^{614}$ BVerfGE 125, 260.

${ }^{615}$ BVerfGE 125, 260, 320.

${ }^{616}$ Gusy, in: v. Mangoldt/Klein/Starck, GG, 6. Aufl. 2012, Art. 10 Rn. 72; Löwer, in: v. Münch/Kunig, GG, 6. Aufl. 2012, Art. 10 Rn. 28.

${ }^{617}$ Gusy, in: v. Mangoldt/Klein/Starck, GG, 6. Aufl. 2012, Art. 10 Rn. 72; Dumer, in: Maunz/Dürig, GG, 68. EL 2013, Art. 10 Rn. 149.

${ }^{618}$ Löwer, in: v. Münch/Kunig, GG, 6. Aufl. 2012, Art. 10 Rn. 56, 62; Gusy, in v. Mangoldt/Klein/Starck, GG, 6. Aufl. 2010, Art. 10 Rn. $81 \mathrm{ff}$.

${ }^{619}$ BVerfGE 125, 260, $325 \mathrm{ff}$.

${ }^{620}$ Dazu s. auch unten Rn. $377 \mathrm{ff}$.

${ }^{621}$ Löwer, in: v. Münch/Kunig, GG, 6. Aufl. 2012, Art. 10 Rn. 62.

${ }^{622}$ Dumer, in: Maunz/Dürig, GG, 68. EL 2013, Art. 10 Rn. 147; Arndt, DÖV 1996, 459, 460, der (bzgl. Funksignalen) die globale Erfassung als verfassungswidrig ansieht; krit. auch Bendner, CR 2010, 339, 344.
} 
von Deep Packet Inspection zur Sicherung privater Rechte den Gestaltungsspielraum des Gesetzgebers überschreiten.

\section{Transitorische Probleme: Eingriffe in bestehende Verträge und Rechte?}

261 Bei Einführung einer Kulturflatrate muss auch das Schicksal bestehender Verträge und übertragener Rechte geklärt werden. Allerdings hat das BVerfG schon für Art. 14 GG frühzeitig festgehalten, dass das Grundrecht kein „versteinertes“ Eigentumsrecht kennt, sondern der Gesetzgeber vielmehr frei ist, das Eigentum neu auszugestalten ${ }^{623}$ - wobei natürlich auch Übergangslösungen und Kompensationen zu bedenken sind , um „einen schonenden Übergang vom alten ins neue Recht“ zu gewährleisten. ${ }^{624}$ Daher kann auch eine Übergangsfrist der Schrankenregelung für ältere Werke in Betracht kommen - allerdings kann angesichts des 70 Jahre über den Tod des Urhebers hinausdauernden Schutzes eine solche Übergangslösung nicht so ausgestaltet werden, dass die Schranken nur für neue Werke gälten, da der „Kulturflatrate“ sonst nur ein äußerst schmaler Bereich verbliebe, und zudem die Nutzer stets mit der Frage konfrontiert wären, von wann ein Werk stammt und ob es noch unter eine Übergangslösung fiele. Daher spricht vieles für nur kurze Übergangszeiten, um bestehende Verträge anzupassen.

\section{International-privatrechtliche Probleme}

262 Schließlich gilt es, auf ein grundsätzliches Problem hinzuweisen, das sich bei einer Erweiterung der Schranken auf den privaten Upload stellt. Da das Internet von vornherein einen globalen Charakter aufweist, kann ein aus Deutschland öffentlich zugänglich gemachter Inhalt in jedem Staat der Welt abgerufen werden. Tatsächlich haben einige empirische Studien ergeben, dass etwa aus den USA zahlreiche Inhalte gerade während der deutschen Schulferien aus Deutschland abgerufen werden. ${ }^{625}$ Damit ist aber von vornherein die Frage angesprochen, welche Rechtsordnung darüber bestimmt, ob Schranken zugunsten eines Verwertungsvorgangs eingreifen. Hier ist zwischen dem Download bzw. der Vervielfältigung einerseits und dem Upload bzw. dem Recht auf Öffentliches Zugänglichmachen zu unterscheiden:

263 Für die Vervielfältigung kommt es darauf an, wo die Kopie entstanden ist, da dies der eigentliche urheberrechtliche Verwertungsvorgang ist. ${ }^{626}$ Demnach ist es für den Download weitgehend anerkannt, dass die Rechtsordnung des Landes, in dem das Vervielfältigungsstück angefertigt wird, berufen ist, um über mögliche Ansprüche, aber auch über die Schranken der Verwertungsrechte zu entscheiden. ${ }^{627}$

264 Für das Recht zum öffentlichen Zugänglichmachen bzw. den Upload ist die Rechtslage dagegen diffiziler: Hier besteht der eigentliche Verwertungsvorgang darin, dass der Zugang für jedermann eröffnet wird, so dass fraglich ist, ob der Akt des Zugänglichmachens nur auf den Ort bezogen ist, an dem das Werk „ins Netz“ gestellt wird, ${ }^{628}$ oder ob nicht vielmehr der Ort der Abrufbarkeit entschei-

\footnotetext{
${ }^{623}$ BVerfGE 31, 275, $284 \mathrm{f}$.

${ }^{624}$ BVerfGE 53, 336, 351, unter Verweis auf BVerfGE 43, 242, 288; 71, 137, 144 f.; 71, 230, 252; 95, 64, $86 \mathrm{ff}$.; Papier, in: Maunz/Dürig, GG, 68. EL 2013, Art. 14 Rn. 327.

${ }^{625}$ Oberholzer-Gee/Strumpf, 2007, S. 14 ff.

${ }^{626}$ BGH GRUR 1965, 323, 325 - Cavalleria rusticana.

${ }^{627}$ Dreier, in: Dreier/Schulze, 4. Aufl. 2013, Vorb. §§ 120 ff. Rn. 33; Katzenberger, in: Schricker/Loewenheim, UrhR, 4. Aufl. 2010, Vor §§ 120 ff. Rn. 136, 145; Nordemann-Schiffel, in: Fromm/Nordemann, UrhR, 10. Aufl. 2008, Vor $\S \S 120$ ff. Rn. 67; Spindler, in: Leible, Die Bedrohung des internationalen Privatrechts im Zeitalter der neuen Medien, 2003, S. 155, 163 ff.; zur Beurteilung wann eine Vervielfältigung im Inland hergestellt wird: BGH GRUR 1965, 323, 325 - Cavalleria rusticana; BGHSt 49, 93, 97.

${ }^{628}$ Dieselhorst, ZUM 1998, 293, 299 f.; Koch, CR 1999, 121, 123.
} 
dend ist (modifizierte Bogsch-Theorie ${ }^{629}$ ) - der dann allerdings aufgrund der Globalität des Internet in jedem Staat liegen kann, aus dem das Angebot abgerufen werden kann. In den letzten Jahren zeichnet sich allgemein das Vordringen der letzteren Auffassung ab - auch wenn bislang eine höchstrichterliche Entscheidung aussteht. ${ }^{630}$

265 Übertragen auf eine Kulturflatrate mit entsprechenden Schranken bedeutet dies, dass ein deutscher Nutzer zwar beim Download aufgrund der eingreifenden Schranke vor entsprechender Inanspruchnahme geschützt ist, nicht aber was den Upload angeht. Hier kann bei einem Abruf aus einem anderen Staat, der keinerlei vergleichbare Schranken wie eine Kulturflatrate dem Nutzer eine entsprechende Rechtsverfolgung drohen - so dass sich der Nutzer nur durch die Einrichtung von Geolokalisierungsdiensten und -sperren dagegen schützen kann. Diese aus internationalem Privatrecht resultierenden Probleme werden leider von vielen Studien völlig verkannt. ${ }^{631}$ Nur mit dem Einsatz von Geolokalisierungen würde zudem dem Argument begegnet werden können, dass Nutzer in Deutschland für die Nutzung in anderen Ländern mitbezahlen müssten. ${ }^{632}$

266 Diese Geolokalisierungen sind inzwischen vor allem aus dem Bereich der Glücksspiele im Internet bekannt und erlauben weitgehend präzise Ortslokalisierungen bis auf einige Kilometer, in Kombination mit anderen Methoden, wie z.B. der Handyortung sogar bis auf wenige Meter. ${ }^{633}$ Allerdings lassen sich diese Maßnahmen ihrerseits durch den Einsatz von Anonymisierungsdiensten, Virtual Private Networks (VPN) oder Proxyservern, die am gewünschten Ort aufgestellt werden, umgehen, sofern

${ }^{629}$ Grundlegend bereits Katzenberger, GRUR Int. 1983, 895, 916 f.; die Bogsch-Theorie wurde von Arpad Bogsch, ehem. Generaldirektor der WIPO, im Zusammenhang mit dem Senderecht beim Satellitenfernsehen entwickelt, s. dazu Kur, WRP 2011, 971, 977; krit. Schwarz/Reber, in: Loewenheim, Handbuch des Urheberrechts, 2. Aufl. 2010, § 21 Rn. 100 ff. mwNachw.

${ }^{630}$ S. v. Welser, in: Wandtke/Bullinger, UrhR, 3. Aufl. 2009, Vor §§ 120 ff. UrhG Rn. 19; Katzenberger, in: Schricker/Loewenheim, UrhR, 4. Aufl. 2010, vor §§ 120 ff. UrhG Rn. 145; Hoeren, in: Hoeren/Sieber, Multimedia-

Recht, 32. EL 2012, Teil 7.8 Rn. 23; Harte-Bavendamm/Wiebe, in: Kilian/Heussen, Computerrechts-Handbuch, 32. EL 2012, Urheberrecht Rn. 141; aus der Rspr. s. dazu LG Hamburg, Entsch. v. 26.09.2008 - 308 $0248 / 07=$ BeckRS 2008, 23065, das § 19a UrhG in einem Fall anwendete, in dem ein Unternehmen mit Sitz in den USA Thumbnails von urheberrechtlich geschützten Bildern über das Internet öffentlich zugänglich gemacht hatte; s. auch schon Spindler, IPRax 2003, 412, 418 ff. mwNachw., u.a. mit dem vermittelnden Vorschlag, das Recht desjenigen Ortes zur Anwendung gelangen zu lassen, an dem derjenige seinen Sitz hat, der die Inhalte auf dem speichernden Server beherrscht und steuert; ferner Dreyer, in: Dreyer/Kotthoff/Meckel, UrhR, 2. Aufl. 2009, § 19a UrhG Rn. 31, die zusätzlich zur Rechtsordnung des Landes, in die Bereitstellung des Inhalts erfolgt, auch diejenige des Landes anwenden möchte, in dem das Werk geschaffen wurde.

${ }^{631}$ So auch Aigrain, 2012, S. 129 f., der die kollisionsrechtliche Frage leider völlig verkennt.

${ }^{632}$ Darauf stellt etwa unter anderem auch Runge, GRUR Int. 2007, 130, 135 ab.

${ }^{633}$ Dazu Hoeren, ZfWG 2008, 311, 312 f.; die genannten Zahlen stützen sich allerdings auf die Angaben entsprechender Geolokalisierungs-Diensteanbieter, so behauptet die Firma BigChampagne bspw., eine Verlässlichkeit von „well over 99 percent“, s. BigChampagne Online Media Measurement, Monitoring and Identifying P2P Media, S. 2; krit. zur technischen Realisierbarkeit hinreichend präziser Geolokalisierung im glücksspielrechtlichen Kontext OVG Lüneburg NVwZ 2009, 1241, 1243; ebenso VG Berlin, Beschl. v. 21.02.2012 - 35 L $376.11=$ BeckRS 2012, 48575; a.A. VG Düsseldorf, Urt. v. 12.07.2011 - 27 K 5009/08 = BeckRS 2011, 53037, das die zur Verfügung stehenden Methoden glücksspielrechtlich für ausreichend hält; ebenso OVG Münster, Beschl. v. 13.07.2010 - 13 B 676/10 = BeckRS 2010, 51049; wNachw zur Rspr. bei Winkelmüller/Kessler, GewArch 2009, 181, 182; s. ferner Blaufus/Freyer/Trinks, DStR 2011, 2269, 2276, die den Einsatz der Geolokalisierung auch im Rahmen der Umsatzbesteuerung elektronischer Dienstleistungen anregen, um Fälle abwickeln zu können, in denen diese an Kunden in Drittländern außerhalb der EU erbracht werden; s. ferner für weitere Anwendungsgebiete und Dienste Hoeren, MMR 2007, 3, $3 \mathrm{f}$. 
nicht weitere Ortungsdienste (wie Handyortung) damit verknüpft werden. ${ }^{634}$ Auch die Verwendung sog. „deep links" kann u.U. eine Geolokalisierung, verbunden mit einer Abrufsperre, ins Leere laufen lassen. Erforderlich ist dafür lediglich, dass ein bestimmter, urheberrechtlich geschützter Inhalt unmittelbar über seine eigene Internet-Adresse (d.h. über den „deep link“) abgerufen wird, statt über die Webseite, in die sie eingebettet ist und deren Abruf die Geolokalisierung auslösen würde. Dies ist möglich, wenn die Datei nicht auf demselben Server gespeichert ist wie die Webseite, was gerade bei Multimedia-Inhalten häufig vorkommt. ${ }^{635}$ Verhindern lässt sich eine solche Umgehung allerdings mit verhältnismäßig geringem Aufwand, indem beim Zugriff auf die Datei eine „Session-ID“ mitgesendet wird, sodass jeder Zugriff außerhalb der jeweiligen „Sitzung“ verweigert wird, oder aber indem der Datei ein Dateiname zugewiesen wird, der immer nur für einen Abruf gültig ist. ${ }^{636} \mathrm{Da}$ indes Geolokalisierungen die Erhebung personenbezogener Daten erfordern, indem die Herkunft der IP-Adressen und die Anfragen ermittelt werden, sind wiederum zumindest Pseudonymisierungen unumgänglich. Die Datenschutzbeauftragten des Bundes und der Länder halten bei IP-Adressen nach dem Internetprotokoll IPv6 eine Verwendung nur der ersten 4 Bytes für die Geolokalisierung für ausreichend. ${ }^{637}$

267 Aber auch hier ist zu berücksichtigen, dass dem Nutzer oftmals die Möglichkeit fehlt, derartige Sperren durchzuführen und durchzusetzen, etwa gegenüber Filehostern wie Rapidshare, die dann selbst eine Geolokalisierung und entsprechende Maßnahmen vorsehen müssten. Dies ließe sich allenfalls mit einem ggf. gesetzlich verankerten Anspruch darauf, dass derartige Möglichkeiten für die Nutzer bereitgestellt werden, beheben. Andernfalls müsste eine Änderung bzw. von der derzeitig vorherrschenden Auffassung abweichende Kodifizierung des Internationalen Urheberkollisionsrecht erfolgen, indem eine reine auf den Server bzw. heraufladenden Nutzer bezogene Anknüpfung gesetzlich verankert würde.

268 Eine weitere Lösung könnte darin bestehen, die Schranken nur auf solche Plattformen anzuwenden, zu denen unmittelbar ein deutscher Access-Provider Zugang gewährt, also in Gestalt von Tauschplattformen etc. angesiedelt bei einem deutschen Access-Provider. ${ }^{638}$ Da die Einwahl von außerhalb Deutschlands hier eher unwahrscheinlich ist, wäre eine Beschränkung auf deutsche Teilnehmer wahrscheinlich. Zudem könnte der Zugang zu einer solchen Plattform von der Abfrage abhängig gemacht werden, wo sich ein Teilnehmer befindet. Die Schranken müssten dann aber auf derartige Plattformen beschränkt werden und würden nicht andere Up- und Downloadvorgänge im Netz umfassen.

\footnotetext{
${ }^{634}$ S. ausf. dazu Prill, Webradio-Streamripping: Eine neue Form der Musikpiraterie?, erscheint demnächst, S. 44 ff. mwNachw zur Technik; s. auch Hoeren, ZfWG 208, 311, 311 f.; ders., MMR 2007, 3, 6; zu Anonymisierungsdiensten s. Rau/Behrens, K\&R 2009, $766 \mathrm{ff}$.

${ }^{635}$ S. zur Funktionsweise von „deep links“ Prill, Webradio-Streamripping: Eine neue Form der Musikpiraterie?, erscheint demnächst, S. $38 \mathrm{f}$.

${ }^{636}$ Prill, Webradio-Streamripping: Eine neue Form der Musikpiraterie?, erscheint demnächst, S. 38 f.

${ }^{637}$ S. Entschließung der 82. Konferenz der Datenschutzbeauftragten des Bundes und der Länder am 28./29. September 2011 in München, S. 2, abrufbar unter:

http://www.bfdi.bund.de/SharedDocs/Publikationen/Entschliessungssammlung/DSBundLaender/82DSK_IPv6.p df;jsessionid=0E81AF686CF133FA9272C5BFF4342070.1_cid354?_blob=publicationFile; s. aber auch die Differenzierung nach dem Lokalisierungszweck bei Kühn, DuD 2009, 747, 751.

${ }^{638}$ S. das Bsp. bei Renner/Renner, Digital ist besser, 2011, S. 145 f.
} 


\section{Mögliche Einnahme- und Verteilungsschlüssel}

Einer der wesentlichen Kritikpunkte, die immer wieder gegen eine Kulturflatrate ins Feld geführt wurden, bezieht sich zum einen auf die unklare Höhe der Abgabe, zum anderen auf die - so die Kritik - unpräzisen Verteilungsschlüssel, indem eine derartige Abgabe im Gießkannenprinzip an alle Urheber ausgeschüttet würde und damit besonders Künstler, die aufwendige oder hochwertige Werke schaffen, gegebenenfalls benachteiligt würden. ${ }^{639}$ Einigkeit herrscht jedenfalls auf allen Seiten der Diskussion um eine Kulturflatrate, dass ein solches System eine gerechte Einnahmenverteilung und damit eine Entlohnung der Kreativen gewährleisten müsse:

"The amount and distribution of this contribution must respect a number of principles regarding: the revenues of individual creators, the financing of the production of new works, the equitable character of the distribution in relation to activities conducted over the Internet, and the impact on the welfare of all." ${ }^{640}$

271 Neben der reinen "Zahlungs"-Seite muss zudem beleuchtet werden, in welchem Verfahren Tarife festgelegt werden können, wer Verhandlungspartner sein kann, ebenso welche Streitschlichtungsformen in Betracht kommen können.

\section{A. Die Einnahmenseite}

\section{Rechtliche Rahmenbedingungen}

272 Maßgeblich ist nach dem geltenden UrhWahrnG ${ }^{641}$ der geldwerte Vorteil, der durch die Verwertung geschützter Werke erzielt wird ( $\$ 13$ Abs. 3 S. 1 UrhWahrnG). ${ }^{642}$ Dabei wird meist auf eine Höhe von $10 \%$ an den durch die Verwertung erzielten Bruttoerlösen abgestellt, ${ }^{643}$ ohne dass dies eine starre Obergrenze bilden würde. ${ }^{644}$ Daraus darf allerdings nicht der Rückschluss gezogen werden, dass es etwa nur auf den Verkaufspreis eines Produktes ankäme ${ }^{645}-$ und damit auch für Internetanschlüsse nicht auf den monatlichen Beitrag. Denn entscheidend ist das Ausmaß der Verwertung der Werke, da der Preis eines Produktes nur wenig darüber aussagt, wie die urheberrechtlich relevante Nutzung beschaffen ist. ${ }^{646}$ Die Grenze des § 54a Abs. 4 UrhG wurde vom Gesetzgeber im Wesentlichen zur

\footnotetext{
${ }^{639}$ S. dazu Hennemann, Urheberrechtsdurchsetzung und Internet, 2011, S. 345; Runge, GRUR Int. 2007, 130, 135; Bundesverband Musikindustrie, Positionspapier zur Kulturflatrate v. 25.1.2010; Börsenverein des Deutschen Buchhandels, Fragenkatalog zur Kulturflatrate, 2009; Arbeitskreis Urheberrecht der SPD-

Bundestagsfraktion, Zwölf Thesen für ein faires und zeitgemäßes Urheberrecht, 2012; ein Überblick an Pro- und Contra Argumenten bei Petschulat, Kulturflatrate, 2010, S. 6 f.

${ }^{640}$ Aigrain, 2012, S. 76.

${ }^{641}$ Urheberrechtswahrnehmungsgesetz vom 9. September 1965 (BGBI. I S. 1294), das zuletzt durch Artikel 2 des Gesetzes vom 26. Oktober 2007 (BGBI. I S. 2513) geändert worden ist.

${ }^{642}$ Dazu Gerlach, in: Wandtke/Bullinger, UrhR, 3. Aufl. 2009, § 13 UrhWahrnG Rn. 7 ff.; Reinbothe, in: Schricker/Loewenheim, UrhR, 4. Aufl. 2010, § 13 UrhWahrnG Rn. 6 ff., jeweils mwNachw.

${ }^{643}$ Schulze, in: Dreier/Schulze, Kommentar zum UrhG, 4. Aufl. 2013, § 13 UrhWG Rn. 16 und 17.

${ }^{644}$ BGH GRUR 2004, 669, 671 - Mehrkanaldienste; Schiedsstelle, ZUM 2005, 90, 92; Schiedsstelle, ZUM 2007, 77, 81; Nordemann, in: Fromm/Nordemann, UrhR, 10. Aufl. 2008, § 13 UrhWahrnG Rn. 4; Gerlach, in: Wandtke/Bullinger, UrhR, 3. Aufl. 2009, § 13 UrhWahrnG Rn. 7.

${ }^{645}$ Insoweit zutr. Becker/Müller, in: FS Pfennig, 2012, S. 373, 378.

${ }^{646}$ Begr RegE BT-Drs. 10/837, S. 10 f., 19.
} 
Erhaltung der Wettbewerbsfähigkeit gegenüber Staaten, die keine Geräteabgabe kennen, eingefügt. ${ }^{647}$ Selbst diese Grenze ist nach der oben diskutierten Padawan-Entscheidung des EuGH erheblichen Zweifeln ausgesetzt, ${ }^{648}$ da sie nicht im Verhältnis zur urheberrechtlich relevanten Nutzung eines Werkes infolge von Schranken steht. ${ }^{649}$

273 Damit - und vor allem mit den oben dargelegten Leitlinien des EuGH zur Auslegung des „gerechten Ausgleichs" für den Urheber im Rahmen der InfoSoc-RL - sind die Grenzen und Grundlagen de lege lata einer Abgabe vorgezeichnet. Wie dargelegt, existieren allerdings etliche "Stellschrauben“, von denen die Höhe einer Vergütung entscheidend abhängt. Schon aus europarechtlicher Sicht seien diese hier kurz in Erinnerung gerufen: Zum einen die Frage einer Typisierung des Schadens und der Kausalität zwischen Filesharing und Verlusten der Rechteinhaber insbesondere, zum anderen das Problem, ob eine Art Vorteilsanrechnung existiert, mithin der Begriff des gerechten Ausgleichs auf die gesamte Branche und nicht nur auf einen einzelnen Sektor (z.B. Tonträgerverkauf) bezogen werden kann, so dass z.B. die sog. 360-Grad-Verträge der Musikindustrie berücksichtigt werden könnten.

\section{Die Geräteabgabe als Vorbild? Volumenbasierte Abgaben ohne Substitutionsrate}

\section{a) Bisherige Berechnungen der Abgaben}

274 Die Schiedsstelle beim Deutschen Patent- und Markenamt (DPMA) legt derzeit den durchschnittlichen Endgerätepreis abzüglich der in ihm enthaltenen (alten) Urheberrechtsabgaben und der Umsatzsteuer als Kalkulationsbasis zugrunde, da dieser die einzige objektiv und neutral feststellbare Größe sei. ${ }^{650}$ Dieser wird dann multipliziert mit dem Anteil der urheberrechtlich relevanten Nutzungen des jeweiligen Gerätes, der auf empirische Messungen ${ }^{651}$ zurückgeht. $^{652}$ Dafür wird das Verhältnis der Nutzung von Vervielfältigungsfunktionen gegenüber der Nutzung der sonstigen Funktionen der Geräte herausgearbeitet. ${ }^{63}$ Dabei wird die tatsächliche Dauer, für die ein Gerät zur Vervielfältigung eingesetzt wird, ins Verhältnis zu der gesamten Nutzungsdauer gesetzt. ${ }^{654}$ Anschließend wird ermittelt, welchen Anteil urheberrechtlich relevante Vervielfältigungen daran haben. ${ }^{655}$ Hierfür zieht die Schiedsstelle nach § 14 Abs. 5a UrhWahrnG empirische Untersuchungen heran, derzeit von GfK Panel Services Deutschland und TNS Infratest GmbH. ${ }^{656}$

\footnotetext{
${ }^{647}$ Begr RegE BT-Drs. 16/1828, S. 30.

${ }^{648}$ S. dazu oben Rn. $229 \mathrm{ff}$.

${ }^{649}$ Zutr. Dreier, ZUM 2011, 281, 286 f.; Spindler, in: FS Pfennig, 2012, S. 387, 399, maßgeblich ist hier die Erwägung des EuGH, dass im Rahmen der InfoSoc-Richtlinie dem Urheber seine Nachteile ausgeglichen werden müssen; mit Kritik an der Regelung des § 54a UrhG auch Loewenheim, in: Schricker/Loewenheim, UrhR, 4. Aufl. 2010, § 54a UrhG Rn. 10.

${ }^{650}$ Schiedsstelle, ZUM-RD 2011, 46, 55 f.; Schiedsstelle, ZUM-RD 2010, 575, 584 f.

${ }^{651}$ Die genaue Methode wird aus den Einigungsvorschlägen nicht ganz ersichtlich, zumindest in Teilen muss mit Umfragen gearbeitet worden sein, Schiedsstelle, ZUM-RD 2011, 46, 49; Schiedsstelle, Einigungsvorschlag v. 15.2.2012 - Sch-Urh 37/08, S. 26 f. liegt dagegen eine empirische Untersuchung mittels Tagebuchbefragung zugrunde, S. 14.

${ }^{652}$ Schiedsstelle, ZUM-RD 2010, 575, 577 beruft sich für CD- und DVD-Rohlinge auf eine unveröffentlichte Studie der GfK aus dem Juni 2009 über die Vervielfältigung vergütungspflichtiger Inhalte; Schiedsstelle, ZUM-RD 2011, 46, 51 zitiert eine unveröffentlichte Studien für diverse Rekorder-Geräte der TNS Infratest GmbH vom 22.12.2009 und der GfK vom 7.5.2010; sowie Schiedsstelle, Einigungsvorschlag v. 15.2.2012 - Sch-Urh 37/08 eine Studie der TNS Infratest GmbH v. 24.1.2012 zur Erfassung von urheberrechtlich relevanten Nutzungen privat genutzter PCs.

${ }^{653}$ Schiedsstelle, ZUM-RD 2011, 46, $50 \mathrm{f}$.

${ }^{654}$ Schiedsstelle, Einigungsvorschlag v. 15.2.2012 - Sch-Urh 37/08, S. 32 f.

${ }^{655}$ Schiedsstelle, Einigungsvorschlag v. 15.2.2012 - Sch-Urh 37/08, S. 36 ff.

${ }^{656}$ Schiedsstelle, ZUM-RD 2011, $46 \mathrm{ff}$.
} 
275 Dabei kommt es auf die Zahl der Brenn- oder Aufnahmevorgänge an, nicht auf die Speichergröße oder Zahl der kopierten Titel. Vervielfältigungen privater Inhalte (nicht Kopien nach $\S 53$ Abs. 1 UrhG), Kopien von mit DRM geschützten Werken und die erste Kopie bei kostenpflichtigen Downloads bleiben unberücksichtigt. ${ }^{657}$

276 Nicht mit in die Rechnung einbezogen werden rechtswidrige Vervielfältigungen, bspw. Kopien, die unter Umgehung eines Kopierschutzes ( $\S 95 a$ UrhG) erstellt wurden, oder deren Vorlage aus einer offensichtlich rechtswidrigen Quelle ( $\$ 53$ Abs. 1 UrhG) stammt. ${ }^{658}$ Die Abgabe stelle einen Ausgleich für die gesetzliche Lizenz des $\S 53$ UrhG und keine Schadensnorm dar, so dass Handlungen die nicht unter $\S 53$ UrhG fallen, nicht berücksichtigt werden können. ${ }^{659}$ Allerdings darf die Vergütung gem. § 54a Abs. 4 UrhG den Hersteller nicht unzumutbar beeinträchtigen. In Anbetracht dessen, dass eine durchschnittliche urheberrechtliche Vergütung von $10 \%$ der Bruttoeinnahmen gezahlt werde, sei dann entsprechend eine Abwägung, einerseits der wirtschaftlichen Interessen des Herstellers und andererseits der Höhe der urheberrechtlichen Nutzungen, vorzunehmen. ${ }^{660}$ Allerdings soll selbst eine erhebliche urheberrechtliche Nutzung nicht zu einer Abgabe von mehr als 18\% des Endverkaufspreises führen. ${ }^{661}$ Für Geräteabgaben sei dies aber noch zu modifizieren, da dort aufgrund der generell höheren Kosten und selteneren Anschaffung die Gefahr bestünde, dass Verbraucher auf ausländische Angebote auswichen, so dass dort die Kappungsgrenze im Höchstfall 13,5\% des bereinigten Endverkaufspreises betrage.

277 So werde bspw. ein PC zu 8,14\% für Vervielfältigungen eingesetzt. Von diesen 8,14\% wird dann die Prozentzahl der urheberrechtlich relevanten Nutzungen ermittelt $(23,7 \%)$, letztlich mit dem durchschnittlichen Endgerätepreis (559 Euro) multipliziert. Es ergibt sich also folgende Formel: ${ }^{662}$

Dauer der Vervielfältigung $\times$ Anteil der urheberrechtlich relevanten Nutzungen $\mathrm{x}$ durchschnittlicher Endgerätepreis = Angemessene Vergütung

(Am Beispiel eines PC also: 8,14\% x 23,7\% x $559=10,78$ ) - eine Kappung erfolgt nicht, da die dafür notwendige kritische Masse von 13,5\% nicht erreicht wird.

279 Vor diesem Hintergrund hat die Zentralstelle für private Überspielungsrechte (ZPÜ) für Hersteller/Importeure, die nicht Mitglied eines Gesamtvertrages sind, Tarife aufgestellt, die etwa MP3Player, ${ }^{663}$ externe Brenner, ${ }^{664}$ Festplatten, ${ }^{665}$ und Rohlinge ${ }^{666}$ erfassen; leider kann aus den veröffentlichen Angaben nicht immer vollständig nachvollzogen werden, wie und auf welcher methodischen Grundlage die Tarife ermittelt werden.

\footnotetext{
${ }^{657}$ Schiedsstelle, ZUM-RD 2011, 46, 51 ff.; Schiedsstelle, ZUM-RD 2010, 575, 579 ff.; dies entspricht den Forderungen des Mediators Vitorino im Rahmen der EU-Konsultation zu Vergütungsabgaben für die Privatkopie: Vitorino, Recommendations, S. $6 \mathrm{f}$.

${ }^{658}$ Schiedsstelle, ZUM-RD 2011, 46, 52; Schiedsstelle, Einigungsvorschlag v. 15.2.2012 - Sch-Urh 37/08, S. 40 f.

${ }^{659}$ Schiedsstelle, Einigungsvorschlag v. 15.2.2012 - Sch-Urh 37/08, S. 38 f.

${ }^{660}$ Schiedsstelle, ZUM-RD 2010, 575, 585.

${ }^{661}$ Schiedsstelle, ZUM-RD 2011, 46, 57; Schiedsstelle, ZUM-RD 2010, 575, 585.

662 Schiedsstelle, Einigungsvorschlag v. 15.2.2012 - Sch-Urh 37/08, S. 44.

${ }^{663}$ https://www.gema.de/fileadmin/user_upload/Musiknutzer/Tarife/Tarife_sonstige/Tarif_MP3-_und_MP4Player_ab_2010.pdf.

${ }^{664}$ https://www.gema.de/fileadmin/user_upload/Musiknutzer/Tarife/Tarife_sonstige/Tarif_externe_CD_und_DVD-Brenner_ab_2010.pdf.

$\overline{665}$ https://www.gema.de/fileadmin/user_upload/Musiknutzer/Tarife/Tarife_sonstige/ Tarif_Festplatten_ab_2008.pdf.

${ }^{666}$ https://www.gema.de/fileadmin/user_upload/Musiknutzer/Tarife/Tarife_sonstige/Tarif_Rohlinge.pdf.
} 
280 Allerdings hat die Schiedsstelle im Schlichtungsvorschlag für Leermedien (CD-/DVD-Rohlinge usw.) angenommen, dass bei einer urheberrechtlich relevanten Nutzung von

- unter 25\% die Kappungsgrenze $12 \%$ des Endverkaufspreises

- bei 25\% - 50\% die Kappungsgrenze 14\% des Endverkaufspreises

- bei 50\% - 75\% die Kappungsgrenze 16\% des Endverkaufspreises

- und bei $75 \%$ und mehr die Kappungsgrenze $18 \%$ des Endverkaufspreises

beträgt. ${ }^{667}$ Von einer der Geräteabgabe entsprechenden Grenze von 13,5\% sieht die Schiedsstelle bei Leermedien ab, da sie davon ausgeht, dass Leermedien trotz der höheren Abgabe nicht im Ausland erworben werden. ${ }^{668}$ Analog auf eine Kulturflatrate angewandt, wäre abhängig von der urheberrechtlich relevanten Nutzung somit maximal eine Abgabe i.H.v. 18\% des durchschnittlichen Marktpreises eines Internetanschlusses möglich.

281 Zu beachten ist aber, dass, sofern man sich im Rahmen einer Kulturflatrate für eine $\S 54 a$ Abs. 4 UrhG entsprechende Regelung und die damit verbundene Begrenzung einer Abgabe auf Internetanschlüsse auf ein wirtschaftlich angemessenes Verhältnis zum Preisniveau entscheidet, sich eine gegenüber der Geräteabgabe unterschiedliche Situation ergibt: Denn Internetanschlüsse können nicht wie Geräte ohne weiteres aus dem Ausland bezogen werden, um die dort günstigeren Preise in Anspruch nehmen zu können. Daher scheint der Grundgedanke des § 54a Abs. 4 UrhG, nämlich die Vermeidung von Wettbewerbsnachteilen für den Verkauf von Geräten in Deutschland gegenüber dem Kauf in anderen Staaten, ${ }^{669}$ bei Internetzugängen nicht zu verfangen.

282 Zudem ist fraglich, ob die Kappung bei Leermedien ohne weiteres auf eine Internetflatrate übertragen werden kann, da die Internetzugangsgebühr weder vom Volumen noch der Zeit abhängig ist, während die Preise für Leermedien üblicherweise anhand der Speicherkapazitäten berechnet werden.

283 Sollte dennoch eine $\S 54$ a Abs. 4 UrhG entsprechende Regelung für eine Kulturflatrate geschaffen werden, sind keine Gründe ersichtlich, warum ein auf die Kulturflatrate weitergedachtes Schiedsstellenmodell nicht auch für eine Kappungsgrenze die urheberrechtlich relevante Nutzung des Internetanschlusses berücksichtigen sollte. Auch wenn, wie dargestellt, Leermedien nicht absolut vergleichbar mit einem Internetanschluss sind, werden doch zu Darstellungszwecken bei den nachfolgenden Berechnungen die dafür entwickelten Grundsätze angewandt.

\section{b) Lizenzanallogiemodell}

284 Demgegenüber stellen die Verwertungsgesellschaften in ihren Verfahren vor der Schiedsstelle und in den anhängigen Rechtsstreitigkeiten um die angemessene Vergütung für CD- und DVD-Rohlinge, ${ }^{670}$ diverse Rekorder-Geräte, ${ }^{671}$ sowie Desktop-PCs und Notebooks ${ }^{672} \mathrm{im}$ Wesentlichen auf die schon

\footnotetext{
${ }^{667}$ Schiedsstelle, ZUM-RD 2010, 575, 586.

${ }^{668}$ Schiedsstelle, ZUM-RD 2010, 575, 586.

${ }^{669}$ RegE eines Zweiten Gesetzes zur Regelung des Urheberrechts in der Informationsgesellschaft v. 15.6.2008, BT-Drs. 16/1828, S. 30.

${ }^{670}$ Schiedsstelle, ZUM-RD 2010, 575.

${ }^{671}$ Schiedsstelle, ZUM-RD 2011, 46.

${ }^{672}$ Schiedsstelle, Einigungsvorschlag v. 15.2.2012 - Sch-Urh 37/08.
} 
unter dem alten Abgabenrecht übliche Lizenzanalogie ${ }^{673}$ zu den Erstverwertungen ab, indem sie darauf verweisen, dass für die Abgabenhöhe der Substitutionseffekt gegenüber einem normalen Lizenzerwerb maßgeblich ist, anders ausgedrückt, der durch die Schranke ersetzte Kauf der Verwertungsrechte. Dabei muss aber beachtet werden, dass im Rahmen der Lizenzanalogie streng genommen nicht der Schaden betrachtet wird, den die Rechteinhaber erlitten haben, sondern - entsprechend der dreifachen Schadensberechnung nach $\S 97 \mathrm{UrhG}^{674}$ - die gegenüberliegende Perspektive eingenommen wird: Demnach ist dasjenige entscheidend, was der Verbraucher durch die private Vervielfältigung an geldwertem Vorteil erlangt. ${ }^{675}$ Als Orientierungsmaßstab für die Höhe dieses Werts können dabei die Erlöse der Rechteinhaber aus der Primärverwertung verwendet werden, also der Anteil am Endverkaufspreis, der an die Rechteinhaber bei einem gewöhnlichen Medienkauf abfallen würde. ${ }^{676}$ Damit sind in diesem Modell für jede Mediengattung zwei Werte von Bedeutung, einerseits der Endverkaufspreis und andererseits der Anteil daran, der an die Rechteinhaber abgeführt wird.

\section{(1) Ausgangspunkt: Endverkaufspreis}

285 Stellt man auf den Endverkaufspreis ab, muss man festlegen, ob das digitale oder physische Medium als Grundlage der Rechnung dienen soll. Denn der durchschnittliche Ladenpreis eines Musikalbums, das man herunterlädt, und der eines Musikalbums, das man gepresst auf CD erwirbt, können unterschiedlich sein. Man könnte hier entweder komplett auf den physischen oder digitalen Markt abstellen, oder aber den Markt entsprechend den tatsächlichen Verhältnissen aufteilen und so beide Medien in die Berechnung einfließen lassen. Letztere Möglichkeit erscheint zunächst vorzugswürdig, da sich in ihr die realen Substitutionsverhältnisse am ehesten widerspiegeln. So hätte höchstwahrscheinlich nicht jeder, der ein Album aufgrund der Kulturflatrate herunterlädt, dieses in einem Downloadshop, sondern möglicherweise auch auf CD gekauft. Dieser Gedanke passt allerdings nicht zu dem Modell der Lizenzanalogie, denn bei dieser ist, wie oben ausgeführt, nicht die Schadenssituation, die durch den Download entsteht entscheidend, sondern der tatsächliche wirtschaftliche Vorteil des Kopierenden. Dies ist aber bei der Kulturflatrate in jedem Fall die digitale Datei, so dass als Berechnungsgrundlage nur digitale Medien (Musik-, Filmdownload, E-Book) in Frage kommen.

Insbesondere bei Musikkäufen stellt sich die Frage, ob man auf den Kauf eines Songs oder den Kauf eines Albums abstellen sollte. Bei der Geräteabgabe wird auf ganze CDs, also i.d.R. Alben abgestellt, da in aller Regel einzelne Songs auf physischen Datenträgern nicht separat erworben werden können (abgesehen von besonders ausgekoppelten Singles). Dementsprechend wird aufgrund der pauschalen Größe der Speichermedien deren Volumen in Spielstunden umgerechnet, sodass eine Anlehnung an den Preis eines Musikalbums erfolgen kann. ${ }^{677}$ Bei einer rein digitalen Kulturflatrate kann man allerdings jeden Song einzeln erwerben. So läge es näher, das Volumen mit Hilfe der Kompressionsrate direkt umzurechnen, damit man - entsprechend der Möglichkeit, einzelne Songs zu kaufen/herunterzuladen - das Volumen nicht zunächst in Spielstunden umrechnen muss, sondern direkt die Anzahl der Lieder berechnen kann. Eine Rechnung pro Song führt allerdings auch zu einem letztlich höheren Endpreis, da die Rabattierung, die beim Kauf eines ganzen Albums stattfindet, beim Kauf eines einzelnen Songs entfällt.

\footnotetext{
${ }^{673}$ BT-Drs. 10/837, S. 19.

${ }^{674}$ S. nur statt aller: Wild, in: Schricker/Loewenheim, UrhR, 4. Aufl. 2010, § 97 Rn. 145; Dreier, in: Dreier/Schulze, UrhG, 4. Aufl. 2013, §97 Rn. 58; v. Wolff, in: Wandtke/Bullinger, UrhR, 3. Aufl. 2009, § 97 Rn. 58.

${ }^{675}$ Müller, ZUM 2007, 777, 781.

${ }^{676}$ Becker/Müller, in: FS Pfennig, 2012, S. 373, 381.

${ }^{677}$ Becker/Müller, in: FS Pfennig, 2012, S. 373, 381.
} 
287 Entsprechendes gilt bezüglich Filmdownloads, nur dass sich hier der Unterschied zu einer Spielstunden-Berechnung in Grenzen hält, da sich hier in der Regel auch nur ein Film auf einer DVD befindet.

288 Als Durchschnittspreise sollen bei der folgenden Berechnung zugrunde gelegt werden für einen Musikdownload: 9,99 Euro/Album bzw. 0,99 Euro/Lied, ${ }^{678}$ für einen Filmdownload: 7,71 Euro, ${ }^{679}$ sowie für ein E-Book: 8,07 Euro. ${ }^{680}$

\section{(2) Abzug der Vertriebsstufen}

289 Die Lizenzanalogie stellt aber nicht auf den Endverkaufspreis im Laden ab, sondern auf einen Lizenzerwerb direkt beim Rechteinhaber. Der reine Verkaufspreis kann daher nicht als Grundlage genommen werden. Denn in ihm sind nicht nur die Entgelte enthalten, die an die Rechteinhaber gehen, sondern auch solche, die an Handel und Vertrieb abfließen. Um also zu dem tatsächlichen Wert einer Lizenz zu gelangen, müssen die Kosten der Vertriebsstufen abgezogen werden. ${ }^{681}$ Dies gilt schon bei der Bestimmung der jetzigen Geräteabgabe; denn Berechtigte aus der Abgabe sind nur die Urheber und Inhaber von Leistungsschutzrechten, da nur Eingriffe in ihre Verwertungsrechte abgegolten werden. Der Handel bzw. Vertrieb ist zwar mittelbar ebenfalls betroffen; doch kann er mangels einer eigenen urheberrechtlichen Rechtsstellung nicht an entsprechenden Abgaben partizipieren.

290 Vorliegend können die entsprechenden Werte mangels ausreichender Daten nur grob geschätzt werden. Konkretere Berechnungen wären erst möglich, sobald genaue Zahlen aus der Industrie vorlägen. Genau wie bei den durchschnittlichen Verkaufspreisen müssen auch hier die unkörperlichen Vertriebswege zugrunde gelegt werden, da sich gerade die Vertriebskosten digitaler und physischer Werke erheblich voneinander unterscheiden. Dies, entsprechend auf den deutschen Markt angewandt, führt zu Folgendem:

291 Für den digitalen Musikmarkt ist eine pauschale Bewertung schwierig, da es aufgrund der zahlreichen Angebote auch eine Vielzahl an Kostenmodellen gibt. Ein Problem, das alle Online-Distributoren betrifft, besteht darin, dass es wenig Datenmaterial dazu gibt, welchen Anteil die Beteiligten am Umsatz genau erhalten. Denn die jeweiligen Distributoren treffen offenbar individuelle Vereinbarungen mit den Plattenfirmen und auch mit der GEMA, die der Öffentlichkeit nicht ohne weiteres zugänglich sind. Zweckmäßig erscheint es, dabei auf das Modell des Marktführers iTunes (in den USA zwischen $66 \%$ (NPD-Group ${ }^{682}$ ) und 76\% (Billboard ${ }^{683}$ ) Marktanteil) abzustellen, für dessen Erlöse es zumindest einige Schätzungen gibt. ${ }^{684}$ So sollen bei dem Vertrieb über den iTunes Store durchschnittlich ca. 30\% des Erlöses an Apple gehen. Darin enthalten sind sowohl MwSt. als auch die Abgaben an die GEMA, die im Jahr 2006 nach Tarif VR-OD2 15\%, nach dem heutigen Tarif VR-OD7 10,25\% betragen. ${ }^{685}$ Die

\footnotetext{
${ }^{678}$ Mangels genauer statistischer Werte soll hier der übliche Preis eines Albums/Lieds im iTunes Store zugrunde gelegt werden.

${ }^{679}$ BVV/GfK, Der deutsche Videomarkt 2011, S. 50.

${ }^{680}$ Dieser Preis umfasst allerdings nicht Schul- und Fachbücher, GfK Verbraucherpanel Media*Scope Buch, Durchschnittspreis von E-Books in Deutschland im Jahr 2010 und 2011.

${ }^{681}$ Becker/Müller, in: FS Pfennig, 2012, S. 373, 383.

682 The Wall Street Journal v. 16.12.2010, Amazon Can't Dent iTunes, abrufbar unter: http://online.wsj.com/article/SB10001424052748704073804576023913889536374.html.

${ }^{683}$ The Wall Street Journal v. 16.12.2010, Amazon Can't Dent iTunes, abrufbar unter: http://online.wsj.com/article/SB10001424052748704073804576023913889536374.html.

${ }^{684}$ Z.B. Schwetter/Volz, Preiszusammensetzung bei Hardware und Software Tonträgern, 2004; Erdmann/Stanek, Der iTunes Store für Musiker, 2. Aufl. 2006, S. 18.

${ }^{685}$ Erdmann/Stanek, Der iTunes Store für Musiker, 2. Aufl. 2006, S. 18.
} 
Tarifangaben können, wie dargelegt, aufgrund möglicher vertraglicher Vereinbarungen nur als Anhaltspunkt dienen. Es bleiben somit 70\% für die Plattenfirma und Künstler zzgl. des GEMA-Anteils i.H.v. 10,25\%, der letztlich auch an die Künstler ausgeschüttet wird.

292 Für den Download-Markt bei Filmen soll mangels anderer Angaben vorläufig von einem ähnlichen Anteil am Erlös wie bei Musik-Downloads ausgegangen werden, allerdings ohne den GEMA-Anteil i.H.v. 10,25\%, so dass ein Erlösanteil von ca. $70 \%$ zugrunde gelegt wird.

293 Für den E-Book-Markt kann eine geschätzte Pauschale von 50\% für Handel und Vertrieb abgezogen werden. ${ }^{686}$

\section{(3) Abschlag für Zweitverwertung?}

294 Hiervon wird bei der Geräteabgabe aufgrund der Zweitverwertung durch Private ein Abschlag, üblicherweise von $75 \%$, gemacht. ${ }^{677}$ Dies begründe sich in dem wertmäßigen Unterschied, der zwischen der Erstverwertung und der privaten Vervielfältigung, also zwischen Kopie und Original liege. Als Richtwert wird dabei der Unterschied in der Vergütung der Rechteinhaber zwischen erstmaliger und zweitmaliger Erstverwertung zugrunde gelegt, der $50 \%$ betrage. Die private Vervielfältigung stehe aber noch unter der zweitmaligen Erstverwertung, so dass sich eine nochmalige Halbierung ergebe und letztlich die Vergütung der Zweitverwertung nur noch 25\% der Vergütung der erstmaligen Erstverwertung betrage ${ }^{688}$ Daraus ergibt sich dann für Speichermedien, dass die Spielstunden, die das Speichermedium (oder der PC etc.) erlaubt, mit dem Speicherplatz verglichen werden, einschließlich der möglichen Komprimierungen, den etwa eine CD oder eine DVD benötigt. ${ }^{689}$

295 Allerdings ist zweifelhaft, ob ein entsprechender Abschlag auch bei einer Kulturflatrate anzuwenden ist. ${ }^{690}$ Der Hintergrund für den Abschlag im Rahmen der Geräteabgabe ist der Wertunterschied zwischen Original und Kopie. Während dies bei analogen Geräten aufgrund der mit jedem Kopiervorgang einhergehenden Qualitätsverluste einleuchtend und selbst bei der körperlichen (allerdings verlustfrei möglichen) Kopie einer CD aufgrund der Haltbarkeit, der Verpackung, des Booklet oder selbst des sentimentalen Werts noch nachvollziehbar ist, so ist eine unkörperliche digitale Kopie $100 \%$ identisch mit dem Original und es tritt kein Wertverlust ein. Damit entfällt aber auch die Rechtfertigung dafür, dass ein Abzug vorgenommen wird. Folglich ist bei der Kulturflatrate von einem Abschlag für die Zweitverwertung Abstand zu nehmen.

\section{(4) Transposition auf Internettraffic}

296 Auf der Basis des Modells der Verwertungsgesellschaften für die Geräteabgabe wäre die Spieldauer in Speicherplatz maßgeblich, transponiert auf Down- und Uploadvorgänge, mithin die Spieldauer umgerechnet in Datenvolumen, das verbraucht wurde, da dies wiederum den urheberrechtlichen Verwertungsvorgängen der Vervielfältigung und der öffentlichen Zugänglichmachung entspräche. Bei der Geräte- und Leermedienabgabe wird die Vergütung pro Spielstunde errechnet, um eine einheitliche, zwischen Werk und Gerät bzw. Leermedium vergleichbare Einheit zu erhalten. Bei einer Kulturflatrate könnte diese Umrechnung dagegen entbehrlich sein. Denn anders als bislang beim physi-

\footnotetext{
${ }^{686}$ Gerundet von 48,5\%, die sich aus Vertriebskosten und Buchhandelsrabatt ergeben, PricewaterhouseCoopers, E-Books in Deutschland - Der Beginn einer neuen Gutenberg-Ära?, 2010, S. 27.

${ }^{687}$ Becker/Müller, in: FS Pfennig, 2012, S. 373, 384.

${ }^{688}$ Becker/Müller, in: FS Pfennig, 2012, S. 373, 384.

${ }^{689}$ Näher zum Berechnungsmodell der Verwertungsgesellschaften, auch im Hinblick auf die Unterschiede zwischen Audio- und Audiovisuellen Werken Becker/Müller, in: FS Pfennig, 2012, S. 373, $380 \mathrm{ff}$.

${ }^{690}$ Dies wäre der Fall, wenn man der Meinung von Vitorino, Recommendations, S. 19 f. folgt, der aber nicht den wertmäßigen Unterschied, sondern die Substitution als Maßstab nimmt.
} 
schen Vertrieb kann jedes digitale Werk einzeln erworben werden und hat einen eigenen Preis. Eine Umrechnung in Spielstunden wäre damit ein rechnerischer Umweg und zudem der digitalen Welt, in der jede Datei in (Mega- bzw. Giga-)Byte gemessen werden kann, als Einheit fremd, zumal auch EBooks nicht in Spielstunden gemessen werden können. Stattdessen genügt es, die durchschnittliche Größe einer Audio-, Video oder E-Book-Datei festzulegen. Selbst wenn man davon ausginge, dass bspw. auf dem Musiksektor weniger einzelne Songs, sondern viel mehr ganze Musikalben getauscht werden, so kann man eine durchschnittliche Album-Dateigröße festlegen und einen durchschnittlichen Albumpreis. Daher sollte das Lizenzanalogiemodell dahingehend modifiziert werden, dass nicht erst das Volumen in Spielstunden umgerechnet werden muss. Es genügt, eine durchschnittliche Dateigröße für jeden Medientyp festzulegen und damit die durchschnittliche Anzahl an Werken im anteiligen Internettraffic zu errechnen.

Allerdings stehen für den Internettraffic kaum verlässliche Zahlen zur Verfügung. So gibt der Verband der Anbieter von Telekommunikations- und Mehrwertdiensten e.V. (VATM) für 2012 lediglich ein prognostiziertes durchschnittliches Datenvolumen von 12,5 GB pro Internetanschluss an, ${ }^{691}$ ohne dass dieses zwischen gewerblichen oder privaten Anschlüssen differenzieren würde. Allerdings nennt das Statistische Bundesamt in einer Studie von 2010 die Zahl von rund 30 Mio. Internetanschlüssen in privaten Haushalten in Deutschland. ${ }^{692}$ Davon betrug laut der Studie des Statistischen Bundesamtes im Jahr 2010 der Anteil der Breitbandanschlüsse 91\%, ${ }^{693}$ also 27,3 Mio. Diese Zahl entspricht in etwa der des VATM (26,3 Mio. $)^{694}$, weswegen naheliegt, dass beide Studien Internetanschlüsse privater Haushalte erfassen. Es sollte bei den nachfolgenden Berechnungen auch bei anderen Modellen diese Zahl zugrunde gelegt werden, da über einen nicht breitbandigen Anschluss Filesharing allenfalls in einem sehr geringen Umfang möglich ist. Allerdings wird in diesen Studien nicht weiter definiert, ab welcher Übertragungsgeschwindigkeit von einem Breitbandanschluss ausgegangen werden kann, es mangelt insoweit an einer Alternative, so dass auf die in den Studien verwandte Definition, ab welcher Geschwindigkeit ein Breitbandanschluss vorliegt, vertraut werden muss. ${ }^{695}$

Bei Einführung einer Abgabe müssten hier aber in jedem Fall zusätzliche empirische Erhebungen durchgeführt werden, um zu aktuelleren, genaueren und gesicherten Zahlen zu kommen.

299 Dies gilt erst recht für den urheberrechtlich relevanten Anteil am Datenvolumen über Internetanschlüsse. Hier liegen - soweit ersichtlich - keinerlei empirische Daten vor, so dass diese erst ermittelt werden müssten. Selbst für die momentan im Streit stehenden Geräteabgaben bei Speichermedien oder PCs sind kaum genauere empirisch gesicherte Daten - soweit ersichtlich - verfügbar. Daher können im Folgenden nur als Näherungswert die von den Verwertungsgesellschaften für die Speichermedien zugrunde gelegten Zahlen $(23,7 \%$ - der Einfachheit halber soll nachfolgend von $25 \%$ ausgegangen werden) herangezogen werden, in der Annahme, dass die Nutzung von Speichermedien in ähnlich urheberrechtlich relevantem Umfang erfolgt wie von Internetanschlüssen. Auch hier ist aber nochmals ein ausdrückliches Caveat angebracht, da nur mit derartigen Näherungslösungen und Annahmen gearbeitet werden kann.

\footnotetext{
${ }^{691}$ Dialog Consult GmbH/VATM, 14. Marktstudie zum Telekommunikationsmarkt Deutschland 2012, S. 17, für 2011 werden 11,3 GB genannt.

${ }^{692}$ Czajka, Statistisches Bundesamt, Wirtschaft und Statistik, August 2011, 709, 710.

${ }^{693}$ Czajka, Statistisches Bundesamt, Wirtschaft und Statistik, August 2011, 709, 710.

${ }^{694}$ Anzahl der „stationären Breitbandanschlüsse“ für das Jahr 2010; für das Jahr 2011 werden 27,3 Mio. angegeben: Dialog Consult GmbH/VATM, 14. Marktstudie zum Telekommunikationsmarkt Deutschland 2012, S. 13 ${ }^{695}$ Unklar ist ferner, ob nur Festnetzanschlüsse oder die neuen LTE-Verfahren im Mobilfunk berücksichtigt wurden.
} 
(5) Beachtung unterschiedlicher Kompressionsraten

300 Ein weiteres Problem, das sich stellt, sind die unterschiedlichen Datenvolumina, die ein Musikstück, ein Video oder ein E-Book belegt. Im Folgenden wurde mit durchschnittlichen Kompressionsraten gerechnet, so dass ein Musikstück mit $5 \mathrm{MB}$, ein Film mit $700 \mathrm{MB}$ und ein Buch mit $2 \mathrm{MB}$ angesetzt wurde. ${ }^{696}$ Dem würde eine Spielstunden-Umrechnung von $100 \mathrm{MB}$ pro Spielstunde an Musik, bzw. 466,67 MB pro Spielstunde an Film entsprechen. Um bestimmen zu können, wie viel Prozent des Datenvolumens auf die jeweilige Medienkategorie abfällt, wurde die DCN-Studie des Jahres 2011 zugrunde gelegt, ${ }^{697}$ in der aufgeschlüsselt ist, wie viel Musik, Filme und E-Books im Jahr 2010 heruntergeladen wurden, ${ }^{698}$ so dass sich ein Verhältnis von $12,6 \%$ (Musik) zu 87,3\% (Film) zu 0,1\% (E-Book) ergibt.

(6) Formel und Berechnung

301 Will man trotz dieser erheblichen Unsicherheiten an der Formel der Lizenzanalogie festhalten, ergäbe sich für eine Kulturflatrate für jeden Industriezweig folgende Formel:

Zahl der Breitbandanschlüsse $x$ durchschnittliches Volumen des Datenverkehrs pro Monat $x$ prozentualer Anteil der urheberrechtlich relevanten Nutzungen $x$ prozentualer Anteil des getauschten Mediums am Datenvolumen / MB pro Werk bzw. Werksammlung (Album-, bzw. Songdownload, Filmdownload, E-Book) x durchschnittlicher Ladenpreis abzgl. Vertriebsstufen

303 Setzt man einen restriktiven Maßstab mit einem urheberrechtlich relevanten Anteil von $25 \%$ an, so ergibt sich für Deutschland folgende Berechnung (getrennt nach Industrien):

(a) „Reines“ Lizenzanalogiemodell

304 Zunächst könnte man ein „reines" Lizenzanalogiemodell bilden, welches mit Spielstunden und einem Zweitverwertungsabzug rechnet.

\footnotetext{
${ }^{696}$ Allerdings muss beachtet werden, dass sich in Zukunft die Datenvolumina anders darstellen werden, da etwa im Videobereich die Tendenz zu immer höher auflösenden Inhalten verläuft, so dass dort trotz der Weiterentwicklung von Komprimierungstechnologien mit steigenden Dateigrößen zu rechnen ist. ${ }^{697}$ GfK, DCN-Studie 2011, Presseversion, S. 14.

${ }^{698}$ Dabei wurden entsprechend den Kompressionsraten angelegt: 5 MB für ein Musikstück, 100 MB für ein Musikalbum und aufgrund höherer Kompressionsrate (m.a.W. geringerer Qualität) $250 \mathrm{MB}$ für ein durchschnittliches 10-Stunden-Hörbuch. Für einen Film wurden 700 MB und 466,67 MB für eine Fernseh-/TV-SerienFolge angerechnet.
} 
305 Für die Musikindustrie:

\begin{tabular}{|l|l|l|l|l|l|}
\hline $\begin{array}{l}\text { Volumen } \\
\text { pro Nutzer } \\
\text { in MB } \\
\text { monatlich }\end{array}$ & $\begin{array}{l}\text { Zahl der Breitbandan- } \\
\text { schlüsse }\end{array}$ & $\begin{array}{l}\text { Volumen pro } \\
\text { Monat in MB }\end{array}$ & $\begin{array}{l}\text { Urheberrechtlich } \\
\text { relevanter Anteil }\end{array}$ & $\begin{array}{l}\text { Anteil von Musik- } \\
\text { downloads am } \\
\text { Gesamtvolumen }\end{array}$ & $\begin{array}{l}\text { Spielstunde } \\
\text { in MB }\end{array}$ \\
\hline 12.500 & 27.300 .000 & 341.250 .000 .000 & $25 \%$ & $12,6 \%$ & 100 \\
\hline
\end{tabular}

\begin{tabular}{|c|c|c|c|c|c|}
\hline $\begin{array}{l}\text { Spielstunden } \\
\text { insg. }\end{array}$ & $\begin{array}{l}\text { Durchschnittli- } \\
\text { cher Endver- } \\
\text { kaufspreis eines } \\
\text { Albums }\end{array}$ & $\begin{array}{l}\text { Anteil der } \\
\text { Rechteinhaber } \\
\text { am Erlös }\end{array}$ & $\begin{array}{l}\text { Abschlag für } \\
\text { Zweitverwer- } \\
\text { tung }\end{array}$ & Preis monatlich & $\begin{array}{l}\text { Preis umgelegt } \\
\text { auf Anschluss } \\
\text { monatlich }\end{array}$ \\
\hline 107.493 .750 & $9,99 €$ & $80,25 \%$ & $75 \%$ & $215.443 .676,60 €$ & $7,89 €$ \\
\hline
\end{tabular}

Für die Filmindustrie:

\begin{tabular}{|l|l|l|l|l|l|}
\hline $\begin{array}{l}\text { Volumen } \\
\text { pro Nutzer } \\
\text { in MB }\end{array}$ & Zahl der Breitbandan- \\
monatlich & $\begin{array}{l}\text { Schlüsse } \\
\text { Solumen pro } \\
\text { Monat in MB }\end{array}$ & $\begin{array}{l}\text { Urheberrechtlich } \\
\text { relevanter Anteil }\end{array}$ & $\begin{array}{l}\text { Anteil von Film- } \\
\text { downloads am } \\
\text { Gesamtvolumen }\end{array}$ & $\begin{array}{l}\text { Spielstunde } \\
\text { in MB }\end{array}$ \\
\hline 12.500 & 27.300 .000 & 341.250 .000 .000 & $25 \%$ & $87,3 \%$ & 466,67 \\
\hline
\end{tabular}

\begin{tabular}{|c|c|c|c|c|c|}
\hline $\begin{array}{l}\text { Spielstunden } \\
\text { insg. }\end{array}$ & $\begin{array}{l}\text { Durchschnittlicher } \\
\text { Endverkaufspreis: } \\
7,71 € \text { für } 90 \text { min.; } \\
\text { somit durchschnitt- } \\
\text { licher Endverkaufs- } \\
\text { preis für } 1 \text { Spiel- } \\
\text { stunde: }\end{array}$ & $\begin{array}{l}\text { Anteil der } \\
\text { Rechteinha- } \\
\text { ber am Erlös }\end{array}$ & $\begin{array}{l}\text { Abschlag für } \\
\text { Zweitverwertung }\end{array}$ & Preis monatlich & $\begin{array}{l}\text { Preis um- } \\
\text { gelegt auf } \\
\text { Anschluss } \\
\text { monatlich }\end{array}$ \\
\hline $159.594 .172,54$ & $5,14 €$ & $70 \%$ & $75 \%$ & $143.554 .958,20 €$ & $5,26 €$ \\
\hline
\end{tabular}

307 Für die Buchindustrie:

\begin{tabular}{|l|l|l|l|l|l|}
\hline $\begin{array}{l}\text { Volumen pro } \\
\begin{array}{l}\text { Nutzer in MB } \\
\text { monatlich }\end{array}\end{array}$ & $\begin{array}{l}\text { Zahl der Breitbandan- } \\
\text { schlüsse }\end{array}$ & $\begin{array}{l}\text { Volumen pro } \\
\text { Monat in MB }\end{array}$ & $\begin{array}{l}\text { Urheberrechtlich } \\
\text { relevanter Anteil }\end{array}$ & $\begin{array}{l}\text { Anteil von Buch- } \\
\text { downloads am Ge- } \\
\text { samtvolumen }\end{array}$ & $\begin{array}{l}\text { MB } \\
\text { pro } \\
\text { Buch }\end{array}$ \\
\hline 12.500 & 27.300 .000 & 341.250 .000 .000 & $25 \%$ & $0,10 \%$ & 2 \\
\hline
\end{tabular}

\begin{tabular}{|c|c|c|c|c|c|}
\hline $\begin{array}{l}\text { Summe Bü- } \\
\text { cher }\end{array}$ & $\begin{array}{l}\text { Durchschnittlicher } \\
\text { Endverkaufspreis }\end{array}$ & $\begin{array}{l}\text { Anteil der Recht- } \\
\text { einhaber am } \\
\text { Erlös }\end{array}$ & $\begin{array}{l}\text { Abschlag für } \\
\text { Zweitverwer- } \\
\text { tung }\end{array}$ & Preis monatlich & $\begin{array}{l}\text { Preis um- } \\
\text { gelegt auf } \\
\text { Anschluss } \\
\text { monatlich }\end{array}$ \\
\hline 42.656 .250 & $8,07 €$ & $50 \%$ & $75 \%$ & $43.029 .492,1875 €$ & $1,58 €$ \\
\hline
\end{tabular}

308 Mithin ergäbe sich ein Abgabenpreis von 14,73 Euro pro Anschluss monatlich, ohne Anwendung einer Kappung.

309 Würde man dagegen keinen Abschlag für die Zweitverwertung abziehen, so käme man auf einen Preis von 58,91 Euro pro Anschluss monatlich. 


\section{(b) Modifiziertes Lizenzanalogiemodell}

310 Passt man das Lizenzanalogiemodell dagegen an die Gegebenheiten der Kulturflatrate an, rechnet man also nicht mit Spielstunden und nicht mit einem Abschlag für die Zweitverwertung, ergibt sich folgende Berechnung.

\section{Für die Musikindustrie:}

\begin{tabular}{|r|l|l|l|l|}
\hline $\begin{array}{l}\text { Volumen pro } \\
\begin{array}{l}\text { Nutzer in MB } \\
\text { monatlich }\end{array}\end{array}$ & $\begin{array}{l}\text { Zahl der Breitbandan- } \\
\text { schlüsse }\end{array}$ & $\begin{array}{l}\text { Volumen pro Mo- } \\
\text { nat in MB }\end{array}$ & $\begin{array}{l}\text { Urheberrechtlich } \\
\text { relevanter Anteil }\end{array}$ & $\begin{array}{l}\text { Anteil von Musikdown- } \\
\text { loads am Gesamtvolu- } \\
\text { men }\end{array}$ \\
\hline 12.500 & 27.300 .000 & 341.250 .000 .000 & $25 \%$ & $12,6 \%$ \\
\hline
\end{tabular}

\begin{tabular}{|l|l|l|l|l|r|}
\hline $\begin{array}{l}\text { Durchschnittliche } \\
\text { Größe einer Mu- } \\
\text { sikdatei in MB }\end{array}$ & $\begin{array}{l}\text { Musikdownloads } \\
\text { in Stück }\end{array}$ & $\begin{array}{l}\text { Durchschnittli- } \\
\text { cher Endver- } \\
\text { kaufspreis eines } \\
\text { Songs }\end{array}$ & $\begin{array}{l}\text { Anteil der } \\
\text { Rechteinha- } \\
\text { ber am Erlös }\end{array}$ & Preis monatlich & $\begin{array}{l}\text { Preis um- } \\
\text { gelegt auf } \\
\text { Anschluss } \\
\text { monatlich }\end{array}$ \\
\hline 5 & 2.149 .875 .000 & $0,99 €$ & $80,25 \%$ & $1.708 .021 .940,63 €$ & $\mathbf{6 2 , 5 6 €}$ \\
\hline
\end{tabular}

312 Für die Filmindustrie:

\begin{tabular}{|r|r|r|r|l|l|}
\hline $\begin{array}{l}\text { Volumen } \\
\text { pro Nut- } \\
\text { zer in MB } \\
\text { monatlich }\end{array}$ & $\begin{array}{l}\text { Zahl der Breitband- } \\
\text { anschlüsse }\end{array}$ & $\begin{array}{l}\text { Volumen pro Mo- } \\
\text { nat in MB }\end{array}$ & $\begin{array}{l}\text { Urheberrechtlich } \\
\text { relevanter Anteil }\end{array}$ & $\begin{array}{l}\text { Anteil von Film- } \\
\text { downloads am } \\
\text { Gesamtvolumen }\end{array}$ & $\begin{array}{l}\text { Durchschnittliche } \\
\text { Größe eines Films } \\
\text { in MB }\end{array}$ \\
\hline 12.500 & 27.300 .000 & 341.250 .000 .000 & $25 \%$ & $87,3 \%$ & 700 \\
\hline
\end{tabular}

\begin{tabular}{|c|c|c|c|c|}
\hline $\begin{array}{l}\text { Filmdownloads in } \\
\text { Stück }\end{array}$ & $\begin{array}{l}\text { Durchschnittlicher Endver- } \\
\text { kaufspreis eines Films }\end{array}$ & \begin{tabular}{|l} 
Anteil der \\
Rechteinhaber \\
am Erlös
\end{tabular} & Preis monatlich & $\begin{array}{l}\text { Preis umgelegt auf } \\
\text { Anschluss monat- } \\
\text { lich }\end{array}$ \\
\hline 106.396 .875 & $7,71 €$ & $70 \%$ & $574.223 .934,38 €$ & $21,03 €$ \\
\hline
\end{tabular}

313 Für die Buchindustrie:

\begin{tabular}{|r|l|l|l|l|l|}
\hline $\begin{array}{l}\text { Volumen pro } \\
\begin{array}{l}\text { Nutzer in MB } \\
\text { monatlich }\end{array}\end{array}$ & $\begin{array}{l}\text { Zahl der Breitbandan- } \\
\text { schlüsse }\end{array}$ & $\begin{array}{l}\text { Volumen pro } \\
\text { Monat in MB }\end{array}$ & $\begin{array}{l}\text { Urheberrechtlich } \\
\text { relevanter Anteil }\end{array}$ & $\begin{array}{l}\text { Anteil von Buch- } \\
\text { downloads am Ge- } \\
\text { samtvolumen }\end{array}$ & $\begin{array}{l}\text { MB } \\
\text { pro } \\
\text { Buch }\end{array}$ \\
\hline 12.500 & 27.300 .000 & 341.250 .000 .000 & $25 \%$ & $0,10 \%$ & 2 \\
\hline
\end{tabular}

\begin{tabular}{|c|r|r|r|r|}
\hline $\begin{array}{l}\text { Summe Bü- } \\
\text { cher }\end{array}$ & $\begin{array}{l}\text { Durchschnittlicher Endver- } \\
\text { kaufspreis }\end{array}$ & $\begin{array}{l}\text { Anteil der Rechtein- } \\
\text { haber am Erlös }\end{array}$ & Preis monatlich & $\begin{array}{l}\text { Preis umgelegt } \\
\text { auf Anschluss } \\
\text { monatlich }\end{array}$ \\
\hline 42.656 .250 & $8,07 €$ & $50 \%$ & $172.117 .968,75 €$ & $\mathbf{6 , 3 0 €}$ \\
\hline
\end{tabular}

314 Mithin ergäbe sich aus einer rein auf das monatliche Downloadvolumen basierenden Berechnung ein Abgabenpreis von 89,89 Euro pro Anschluss monatlich, ohne Anwendung einer Kappung.

315 Würde man keinen Abzug für die Vertriebsstufen vornehmen, so ergäbe sich ein Preis von 120,62 Euro pro Anschluss pro Monat. 
316 Würde man, wie für die Geräteabgabe, einen Abschlag für die Zweitverwertung vornehmen, aber keinen für die Vertriebsstufen, so ergäbe sich ein Preis 30,16 Euro pro Anschluss pro Monat.

317 Würde man, wie für die Geräteabgabe, einen Abschlag für die Zweitverwertung i.H.v. 75\% vornehmen und den Abzug für die Vertriebsstufen einbeziehen, so ergäbe sich ein Preis von 22,48 Euro pro Anschluss pro Monat.

\section{(7) Zusammenfassung}

318 Würde man das Lizenzanalogiemodell, welches die Verwertungsgesellschaften zur Berechnung der Geräteabgabe verwenden, eins zu eins auf die Kulturflatrate übertragen, ergäbe sich ein monatlicher Preis von 14,73 Euro pro Anschluss pro Monat. Rechnete man aus diesem Modell den Abschlag für die Zweitverwertung heraus, ergäbe sich ein monatlicher Preis von 58,91 Euro pro Anschluss.

319 Nähme man einen geringeren urheberrechtlich relevanten Anteil am Datenstrom an, so ergäbe sich bei einem Anteil von 15\% ein monatlicher Preis von 8,85 Euro mit Zweitverwertungsabschlag und 35,34 Euro ohne Zweitverwertungsabschlag. Setzte man den Anteil urheberrechtlich relevanter Tauschvorgänge besonders niedrig bei $5 \%$ an, so ergäbe sich ein monatlicher Preis pro Anschluss von 2,95 Euro mit und 11,78 Euro ohne Zweitverwertungsabschlag.

320 Würde man dieses Modell allerdings an die Besonderheiten der Kulturflatrate anpassen, also keine Umrechnung in Spielstunden vornehmen, ergäbe sich ein Preis von 89,89 Euro monatlich pro Anschluss. Würde man dagegen, wie für die Geräteabgabe, einen Abschlag für die Zweitverwertung i.H.v. 75\% abziehen und den Abzug für die Vertriebsstufen einbeziehen, so ergäbe sich ein Preis von 22,48 Euro pro Anschluss pro Monat.

321 Nähme man auch hier einen geringeren urheberrechtlich relevanten Anteil (15\%) an, so ergäbe sich ein Preis von 53,94 Euro ohne, bzw. 13,49 Euro mit Zweitverwertungsabschlag und für einen besonders niedrigen Anteil von 5\% ohne Zweitverwertungsabschlag 17,98 Euro, bzw. mit Abschlag 4,50 Euro. $^{699}$

\section{c) Kappungsgrenzen-Modell}

322 Ginge man hingegen von einer Kappungsgrenze aus, ergäbe sich:

Durchschnittlicher Marktpreis des Internetzugangs pro Monat(abzüglich USt. i.H.v. 19\%) $x$ prozentualer Anteil der monatlichen urheberrechtlich relevanten Vervielfältigungen, insgesamt begrenzt auf 14\% des Endverkaufspreises aufgrund der unterstellten urheberrechtlich relevanten Nutzung von 25\% (sofern man eine § 54a Abs. 4 UrhG entsprechende Regelung für die Kulturflatrate schafft).

324 Legt man dieses Modell zugrunde, wären bei einem durchschnittlichen Flatratepreis für einen Internetanschluss von ca. 25 Euro monatlich 5,25 Euro monatlich oder, bei Schaffung einer $\$ 54 a$ Abs. 4 UrhG entsprechenden Begrenzungsregelung, maximal 3,50 Euro monatlich möglich.

\footnotetext{
${ }^{699}$ Für die detaillierten Rechnungen s. unten Rn. 558 ff.
} 
325 Allerdings ist - wie oben dargelegt - mehr als fraglich, ob die Kappung ohne weiteres von Leermedien auf eine Internetflatrate übertragen werden kann, da die Internetzugangsgebühr weder vom Volumen noch der Zeit abhängig ist, während die Preise für Leermedien üblicherweise anhand der Speicherkapazitäten berechnet werden. Zudem scheint der Grundgedanke des § 54a Abs. 4 UrhG, nämlich die Vermeidung von Wettbewerbsnachteilen für den Verkauf von Leermedien in Deutschland gegenüber dem Kauf in anderen Staaten, bei Internetzugängen nicht zu verfangen, da hier eine alternative Verbindung über das Ausland schwer möglich bzw. kaum vorzufinden sein wird, somit keine Wettbewerbsnachteile zu erwarten sind.

\section{Auf tatsächlicher Substitution basiertes Gegenmodell (Fisher)700}

326 Während das Modell der Verwertungsgesellschaften im Wesentlichen auf der Annahme beruht, dass die urheberrechtlich relevanten Verwertungsvorgänge der Privatvervielfältigung tatsächlich als Substitution von Lizenzen dienen, also Kaufvorgänge ersetzen, und damit eine Kausalität postuliert wird, setzt das für den US-amerikanischen Musikmarkt entwickelte Modell von Fisher an der empirisch weitgehend belegten Tatsache an, ${ }^{701}$ dass Filesharing nicht im Verhältnis 1:1 Kaufvorgänge substituiert, sondern vielmehr wesentlich mehr Vervielfältigungsvorgänge stattfinden, als tatsächlich Inhalte gekauft worden wären. Mit anderen Worten ist der Substitutionseffekt wesentlich geringer. Im Sinne einer Art Differenzhypothese wird danach gefragt, welcher Zustand eingetreten wäre, wenn es das schädigende Ereignis (hier: Filesharing) nicht gegeben hätte ${ }^{702}$ - dann aber wäre es nicht zu gleich hohen Verkaufszahlen entsprechender Inhalte gekommen, wie sie tatsächlich vervielfältigt und getauscht wurden.

327 Akzeptiert man diese zentrale Annahme auch aus rechtlicher Sicht, indem man die Differenzhypothese im Rahmen einer Schadensberechnung zugrunde legt, ergeben sich andere Ausgangswerte und damit auch Abgabenhöhen - ohne überhaupt auf eine Interessenabwägung zwischen Urhebern und Nutzern eingehen zu müssen:

\section{a) Ausgangspunkt: Veränderungen der Erlössituation}

328 Fisher wählt als Ausgangspunkt die Erlöse der Musikindustrie für das Jahr 2000, bevor die technologischen Änderungen ebenso wie die Einbrüche in den Verkaufszahlen eintraten. ${ }^{703}$ Allerdings zeigt bereits diese Einschränkung die grundsätzliche Problematik bei der Wahl einer Bezugsgröße; denn die Einführung der CD etwa führte zu erheblichen Substitutionseffekten und damit zu einem Boom, der erst Anfang der neunziger Jahre abebbte, so dass oft der Vorwurf erhoben wurde, dass die Zahlen verzerrt im positiven Sinne zugunsten der Musikindustrie seien. ${ }^{704}$ Derartige Effekte müssten z.B. eliminiert werden, um zu einer verlässlichen Größe zu gelangen. Dies ließe sich allerdings durch eine gleitende Durchschnittsberechnung erreichen.

329 Der Einfachheit halber werden hier im Folgenden die Erlöse der deutschen Musikindustrie der letzten fünf Jahre zugrunde gelegt, mithin 2007 bis 2011, so dass sich für diesen Zeitraum ein gleitender Mittelwert von 1.563,8 Mio. Euro ergibt. Dieser gemittelte Bruttoerlös lässt sich in die unterschiedlichen Vertriebswege aufteilen. So wurden 171,2 Mio. Euro über Digitalverkäufe erzielt und 1.392,6

\footnotetext{
${ }^{700}$ Grundlegend für den folgenden Abschnitt Fisher, 2004, S. 199 ff.

${ }^{701} \mathrm{~S}$. dazu oben die erwähnten Studien unter Rn. $80 \mathrm{ff}$.

702 Zur Differenzhypothese im Schadensrecht s. Oetker, in: Münchener Kommentar zum BGB, Bd. 2, 6. Aufl. 2012, § 249 Rn. 18 ff. mwNachw.

${ }^{703}$ Fisher, 2004, S. 210, unter Bezug auf die yearend statistics der RIAA, abrufbar unter: http://www.icce.rug.nl/ soundscapes/VOLUME02/Trends_and_shifts_Appendix.shtml.

704 Das Problem der Bezugsgröße differenziert dargestellt bei Tschmuck, Ursachenanalyse, 2009.
} 
Mio. Euro durch den Verkauf physischer Tonträger. Dabei soll für physische Tonträger hier nur die CD entscheidend sein, die mit $88,1 \%$ den größten Anteil am Verkauf physischer Tonträger hat. ${ }^{705}$ Diese Aufteilung ist für den nächsten Schritt wichtig, in dem die Anteile für Mehrwertsteuer, Handel und Vertrieb abgezogen werden, da diese keine Einbußen für die Musikindustrie darstellen und folglich nicht ersetzt werden müssen. Dazu addieren sich noch 168,8 Mio. Euro für GVLLeistungsschutzrechte, so dass sich letztlich eine Summe von 1.732,6 Mio. Euro ergibt.

330 Hinzu gerechnet werden müssen ferner die Verluste, die sich für die Künstler aus einem möglichen Rückgang der Einnahmen der GEMA aus der Wahrnehmung von Aufführungs-, Vorführungs-, Sende-, und Wiedergaberechten ergeben, insbesondere durch einen Wegfall von Radiosendern, allerdings erst auf einer späteren Stufe, da hier die Substitutionsrate mit $5 \%$ deutlich niedriger angesetzt werden muss. ${ }^{706}$ Die GEMA weist in ihren Jahresberichten nur den Gesamtanteil des Rundfunks aus, ohne aufzuschlüsseln, was davon auf Fernsehen und was auf Radio zurückfällt. ${ }^{707}$ Dieser Gesamtanteil betrug im Jahr 2011 261,322 Mio. Euro.

331 S. dazu auch folgendes Diagramm:

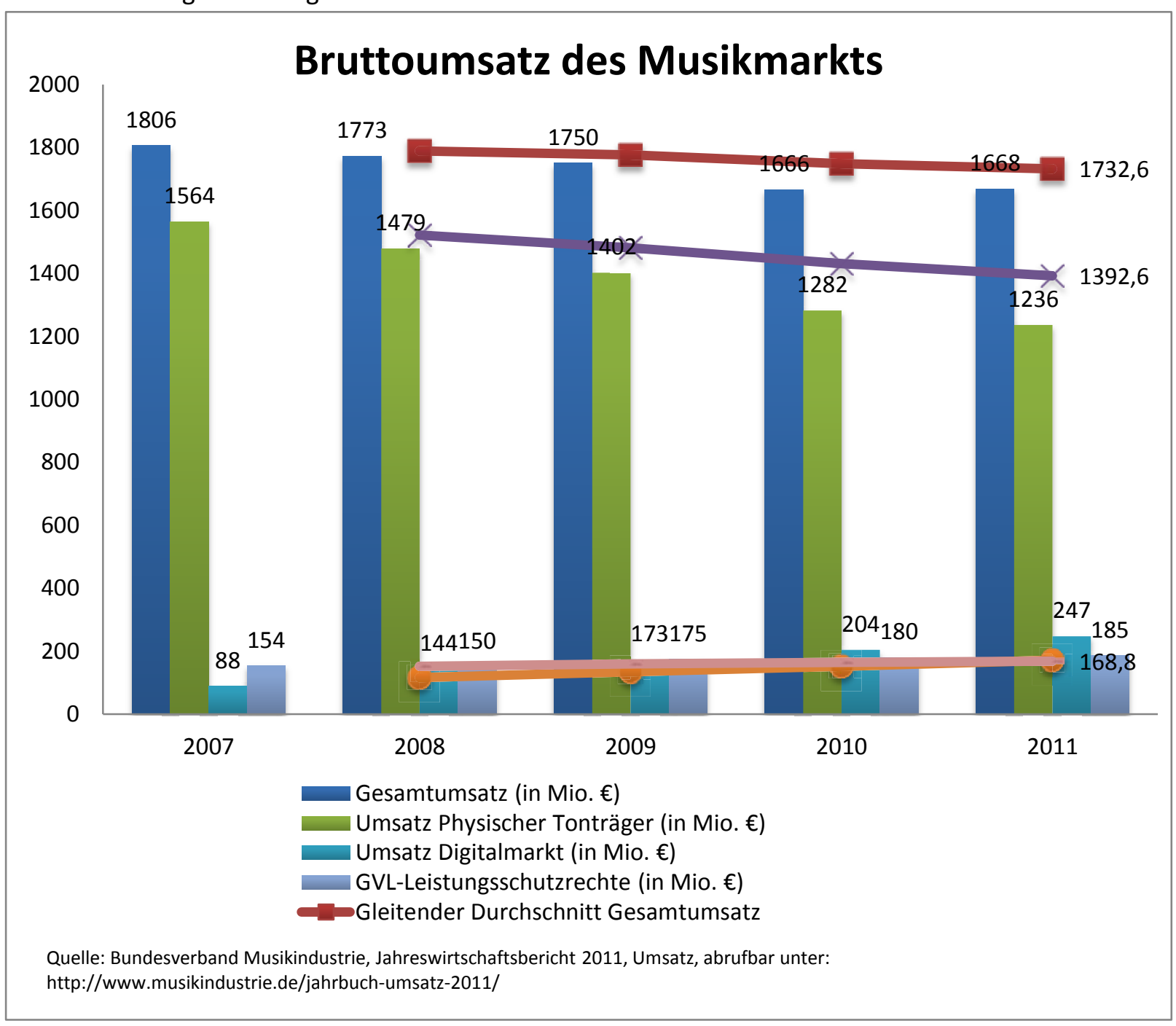

\footnotetext{
${ }^{705}$ Bundesverband Musikindustrie, Jahreswirtschaftsbericht 2011, Absatz.

${ }^{706}$ So für den amerikanischen Markt Fisher, 2004, S. 211.

707 GEMA, Geschäftsbericht 2011, S. 49.
} 
332 Für den Filmmarkt sind die Ausgaben der Verbraucher in den letzten Jahren insgesamt gestiegen, während die Erlöse aus der Vermietung gefallen sind. So beträgt der gleitende Mittelwert der Jahre 2007-2011 des von den Kunden bezahlten Bruttopreises für Filmkäufe 1.367,8 Mio. Euro. Für gemietete Filme beträgt er dagegen 268,4 Mio. Euro. Insgesamt haben Kunden 1.636,2 Mio. Euro für gemietete oder gekaufte Filme ausgegeben. Davon umfasst sind sowohl physisch abgesetzte Datenträger wie BluRays oder DVDs, als auch digitale Angebote. Nicht mit einberechnet werden von Fisher die Erlöse aus Kinovorführungen. ${ }^{708}$ Hinzu rechnet er dagegen den Pay-TV-Markt. ${ }^{709}$ Dieser hatte in Deutschland zwischen 2007 und 2011 einen Umsatz im gleitenden Mittelwert von 1.206 Mio. Euro. ${ }^{710}$

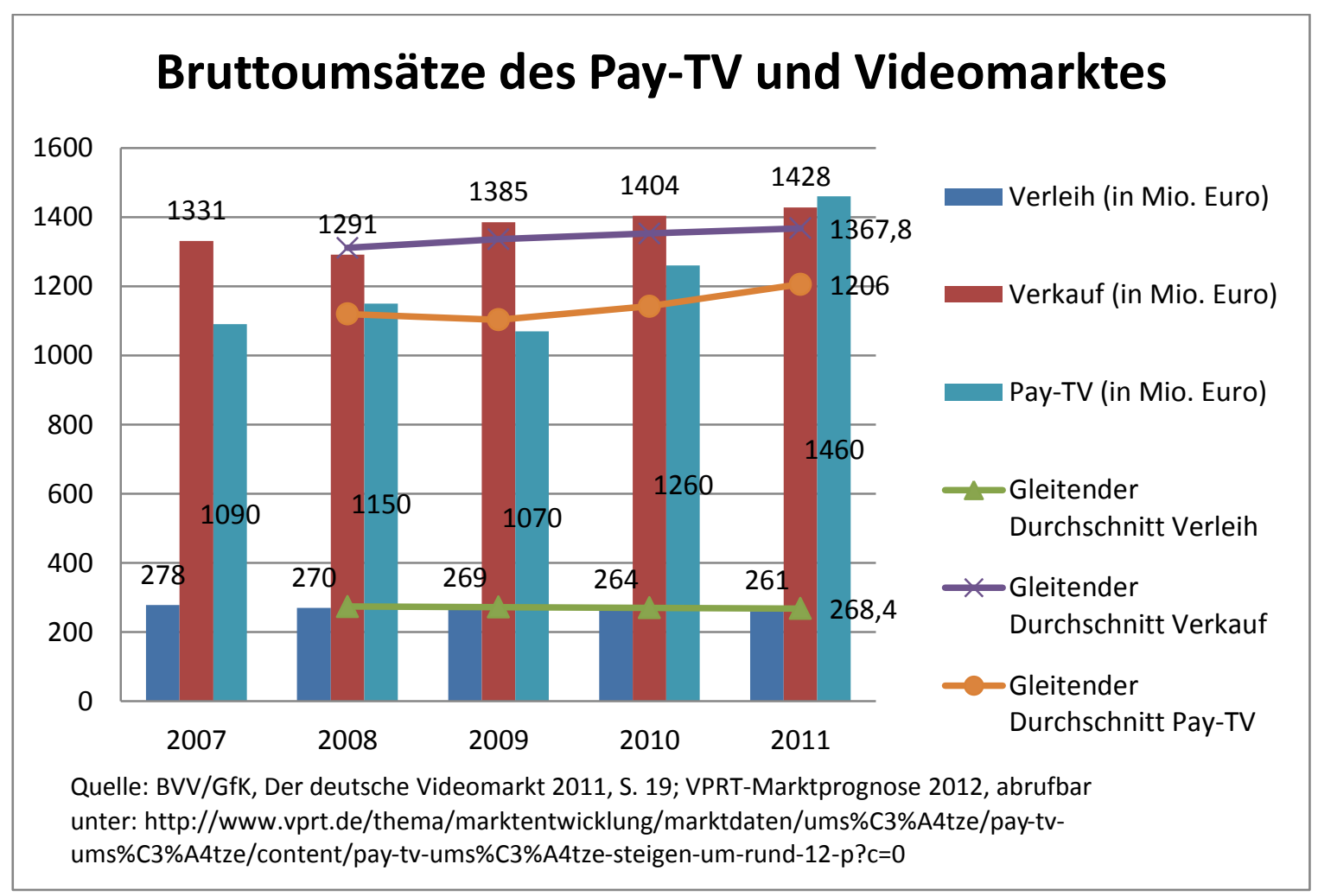

\footnotetext{
${ }^{708}$ Fisher, 2004, S. 212 ff.

${ }^{709}$ Fisher, 2004, S. 212.

${ }^{710}$ VPRT-Marktprognose 2012.
} 
333 Der Büchermarkt hatte in den Jahren 2007-2011 einen Umsatz im gleitenden Mittelwert von 9.643 Mio. Euro. ${ }^{711}$

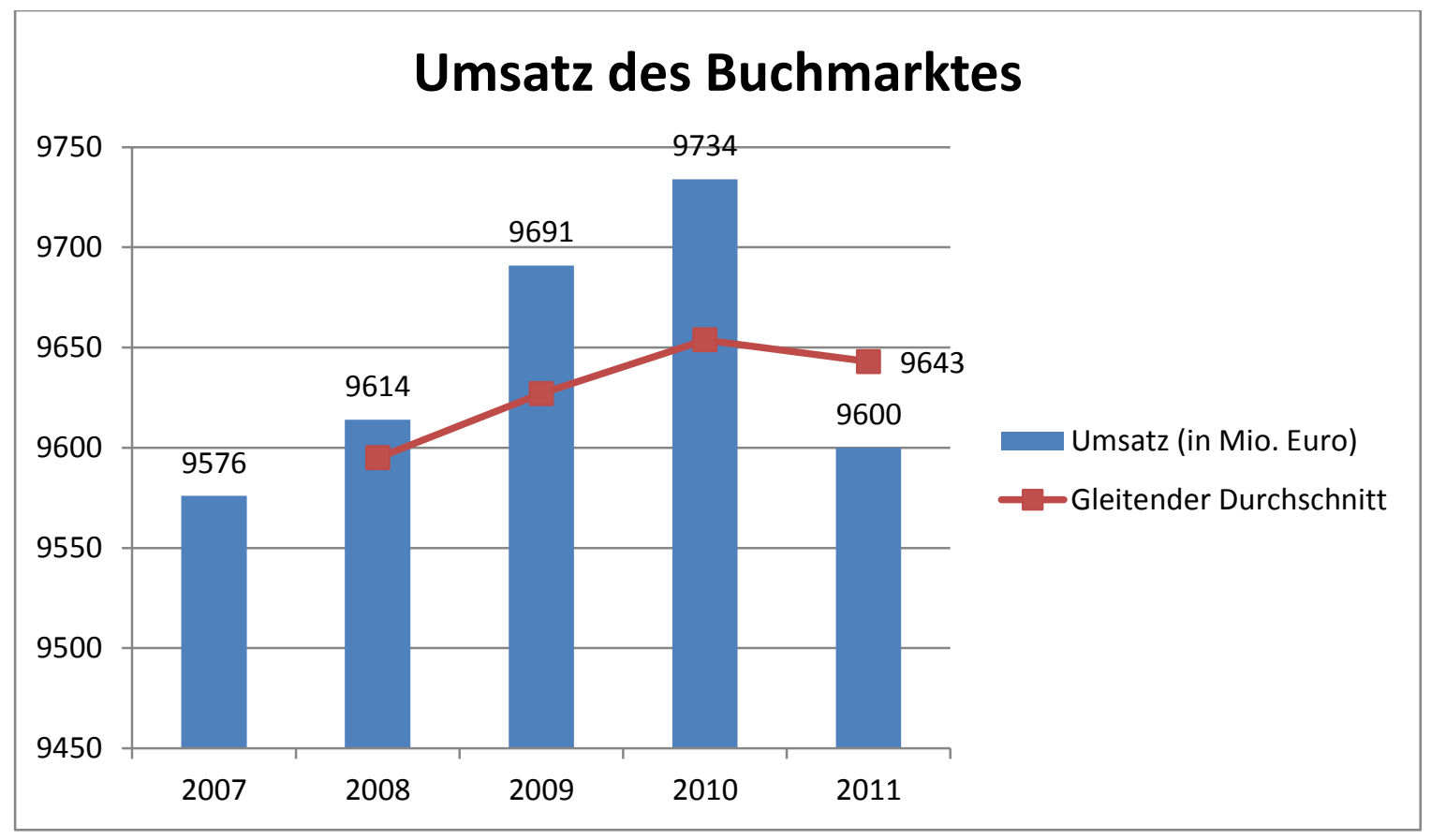

\section{b) Einfluss der Substitutionsrate}

334 Im nächsten Schritt zieht Fisher die Substitutionsrate heran, also den durch Filesharing tatsächlich ausgelösten Verzicht auf vergleichbare Kauftransaktionen. Fisher selbst geht hier von einer Rate von $20 \%$ des Angebots aus. ${ }^{712}$ Für die fehlenden Erträge der Musikindustrie aus dem Rundfunk setzt Fisher den substituierenden Anteil bei $5 \%$ an, ${ }^{713}$ was auch hier als grobe Merkzahl angesetzt werden soll. Dieser betrug im Jahr 2011 261,322 Mio. Euro. Davon 5\% sind 13,0661 Mio. Euro.

335 Für die allgemeine Substitutionsrate bezieht er sich auf Studien von Netanel, ${ }^{714}$ dieser sich wiederum auf Liebowitz. ${ }^{715}$ Liebowitz hat seinerzeit für den Musikmarkt analysiert, wie sich das Kaufverhalten von Personen bzgl. CD-Käufen von 1999 bis 2003 entwickelt hat. Dabei kam er zu dem Ergebnis, dass durchschnittlich (gerundet) jede Person im Jahr 1999 (dem Jahr, in dessen Herbst Napster erschien) 4,97 CDs und im Jahr 2002 4,09 CDs gekauft hat, also 0,88 CDs weniger, woraus sich ergebe, dass Filesharing zu einem Einbruch des Musikmarktes um 20-25\% geführt habe. ${ }^{716}$ Wie oben bereits dargelegt, wird die Methode zur Ermittlung dieses Wertes teilweise kritisch gesehen, insbesondere da Liebowitzs mikroökonomisches Modell Besonderheiten des Medienmarktes außer Acht lasse. ${ }^{717} 2008$ wurde der Wert durch Liebowitz selbst relativiert. ${ }^{718}$ In einer Studie von 99 US-amerikanischen Städten über einen Zeitraum von 1999 bis 2003 verglich er nicht nur die Anzahl gekaufter Musik-CDs pro Person von 1999 mit denen von 2003 (2,9 zu 2,44, also ein Rückgang um 16\%), sondern stellte zusätz-

\footnotetext{
${ }^{711}$ PricewaterhouseCoopers, German Entertainment and Media Outlook: 2011-2015; Börsenverein des Deutschen Buchhandels, Umsatz- und Preisentwicklung, 2011; anders dagegen der BVV/GfK, Der deutsche Videomarkt 2011, S. 16, der 2011 einen Jahresumsatz i.H.v. 4.151 Mio. Euro (!) angibt.

712 Fisher, 2004, S. 210 f., unter Verweis auf Netanel, Harvard JOLT Vol. 17, 2003, 1, 11.

${ }^{713}$ Fisher, 2004, S. $211 \mathrm{f}$.

${ }^{714}$ Netanel, Harvard JOLT Vol. 17, 2003, 1, 48.

715 Liebowitz, 2003, S. 30.

${ }^{716}$ Liebowitz, 2003, S. 29.

${ }^{717}$ S. oben Rn. 81 ff. sowie Tschmuck, Economics, 2010, S. 7.

718 Liebowitz, Management Science, Vol. 54, 2008, 852, $858 \mathrm{f}$.
} 
lich die These auf, dass die CD-Verkäufe ohne P2P-Technologien jährlich um 0,47-5,75\%, durchschnittlich um 3,63\% gestiegen wären. ${ }^{719}$ So seien für ein hypothetisches Jahr 2003 ohne Filesharing nicht 2,9 Käufe pro Person in Rechnung zu stellen, sondern 3,74 und folglich ein Unterschied von 1,3 CDs pro Person, oder ein Rückgang der Käufe um 35\%.

Dies bestätigt, was oben im Rahmen der empirischen Studien gezeigt wurde: Die Aussagen über die Substitutionsrate sind durchaus unterschiedlich, ${ }^{720}$ ohne dass ein Konsens ersichtlich ist. Teilweise werden sie auch mit dem Promotionseffekt verrechnet. ${ }^{721}$ An Liebowitz' Ansatz zur Bestimmung der Substitutionsrate (von 2008) wird u.a. kritisiert, dass er als Proxy-Variable für die tatsächliche Verbreitung von Filesharing die Verbreitung von Internet-Anschlüssen nimmt, ${ }^{722}$ sowie dass seine Datengrundlagen unvollkommen verbunden seien. ${ }^{723}$

337 Allerdings sind auch andere Studien nicht frei von Kritik an ihren jeweiligen methodischen Ansätzen zur Ermittlung der Substitutionsrate. ${ }^{724}$ Huygen et al. haben treffend bemerkt, dass ein negativer Effekt von Filesharing auf Musikverkäufe nach der derzeitigen Forschungslage weder bestätigt noch ausgeschlossen werden kann. ${ }^{725}$ Für eine Kulturflatrate sind diese Zahlen ferner problematisch, da bislang die Substitutionsraten weitgehend für den Musikmarkt erstellt wurden, selten dagegen für andere Märkte, wie bspw. den Film- oder Büchermarkt. ${ }^{726}$ Auffällig ist jedenfalls, das unabhängig von der Methode der größte Teil der Studien auf eine Substitutionsrate zwischen $10 \%$ und $30 \%$ kommt. $^{727}$ Im "worst case"-Szenario kann hier also mit einer Rate von $30 \%$ gerechnet werden. ${ }^{728}$ Aber auch wenn man bezüglich dieser Annahmen Skepsis äußern wollte, könnten die von der Musikindustrie selbst vorgebrachten Schäden aufgrund von "Internetpiraterie“ herangezogen und in Bezug zu den früheren Umsätzen gesetzt werden, so dass sich dann Substitutionsraten von 1/3 bzw. 33\% ergäben.

338 Die Substitutionsrate ist damit allerdings nicht für alle Zeit festgelegt. Fisher selbst betont hier, ${ }^{729}$ dass nur grob geschätzt werden könne, zu welchen Umsatzeinbußen die Einführung einer Kulturflatrate in Zukunft tatsächlich führen würde, wenn erst einmal das Filesharing legalisiert wäre. Da sich das Konsumverhalten mit der Zeit an die neuen Möglichkeiten anpassen werde, müsse dieses stetig beobachtet werden, um den Wert der Substitutionsrate an die neuen Verhältnisse anzupassen. ${ }^{730} \mathrm{Es}$ ist daher sehr wohl möglich, dass diese Rate in Zukunft wesentlich höher ausfällt, mit der Folge, dass

\footnotetext{
${ }^{719}$ Liebowitz, Management Science, Vol. 54, 2008, 852, 858.

${ }^{720}$ Einen Überblick über das Meinungsspektrum bietet Tschmuck, Economics, 2010.

${ }^{721}$ S. oben Rn. $79 \mathrm{ff}$.

${ }^{722}$ Tschmuck, Economics, 2010, S. 8.

${ }^{723}$ Tschmuck, Economics, 2010, S. 16.

${ }^{724}$ S. dazu oben Rn. 81 ff. sowie ausführlich Tschmuck, Economics, 2010, S. 7, 16 f., 23 f., 29 f., 34 ff.; sowie Martens/Herfert/Karbe, 2012, S. 19 ff.; ebenso Huygen et al., S. 102.

${ }^{725}$ Huygen et al., 2009, S. 98.

${ }^{726}$ S. dazu oben Rn. 96 ff.; so kommt die TERA-Studie unter Berufung auf die Datengrundlage der Erhebung IPSOS, Digital \& Physical Piracy in GB, 2007, zu dem Ergebnis, dass die Substitutionsrate für Kauf-DVDs $10 \%$ beträgt, für Leih-DVDs dagegen $0 \%$.

${ }^{727}$ So z.B. Martens/Herfert/Karbe, 2012, S. 21 (30\%); Rob/Waldfogel, 2004, S. 1, 3 (20\%); Peitz/Waelbroeck, 2004, S. 15 (10\%); Zentner, Journal of Law and Economics, Vol. 49, 2006, 63, 87 (7,8\%); Hong, 2011, S. 32 (20\%); s. dazu auch den Überblick in der TERA-Studie (s. oben Rn. 96 ff.), S. 57 sowie Tschmuck, Creativity, 2012, S. 188 f.

${ }^{728}$ Ähnlich Tschmuck, Creativity, 2012, S. 189, der einen Wert zwischen 20\% und 30\% für eine pessimistische Schätzung hält.

${ }^{729}$ Fisher, 2004, S. 210.

${ }^{730}$ Fisher, 2004, S. 210.
} 
auch eine Abgabe wesentlich höher wäre. Eine verlässliche Prognose lässt sich hier kaum treffen - so dass wiederum auf die Einschätzungsprärogative des Gesetzgebers zu verweisen ist.

\section{c) Abzug für Vertriebsstufen}

339 Fisher zieht zusätzlich die Anteile der Erlöse ab, die nicht auf die Rechteinhaber, sondern auf Händler, Vertrieb etc. entfallen. ${ }^{731}$ Vorliegend können die entsprechenden Werte mangels ausreichender Daten nur grob geschätzt werden. Konkretere Berechnungen wären erst möglich, sobald genaue Zahlen aus der Industrie vorlägen. Im Gegensatz zu der Berechnung im Rahmen des Lizenzanalogiemodells, genügt es hier nicht, nur den Digitalmarkt zu betrachten. Fisher stellt nicht wie die Verwertungsgesellschaften auf den Vermögenszuwachs beim Kopierenden ab, sondern geht von der entgegengesetzten Ausgangslage aus, dem verursachten Schaden. Daher kann hier nicht darauf abgestellt werden, dass der Kopierende einen Vermögenszuwachs in Höhe des Wertes einer digitalen Lizenz erlangt, sondern es müssen auch die physischen Märkte beachtet werden, insbesondere da sich teilweise erhebliche Unterschiede zwischen den Kosten für Vertrieb und Handel im digitalen Markt einerseits und im physischen Markt andererseits ergeben. Dies, entsprechend auf den deutschen Markt angewandt, führt zu Folgendem:

340 Für physische Tonträger auf dem deutschen Musikmarkt wurde errechnet, dass im Durchschnitt vom Bruttopreis einer CD 16\% auf die MwSt., 22\% auf den Handel, 18,6\% auf den Vertrieb, 5,2\% auf die Herstellung der CD, 1\% auf GEMA-Administrationskosten, 20,1\% auf die Plattenfirma, 2,5\% auf den Verlag und (insg.) 14,6\% auf den Künstler entfallen. ${ }^{732}$ Dabei sind für die Kulturflatrate nur die letzten drei Werte entscheidend (also insg. 37,2\% des Gesamtumsatzes), so dass sich für Künstler und Plattenfirmen ein Erlös aus dem Verkauf physischer Tonträger in Höhe von 518,0472 Mio. Euro ergibt.

341 Für den digitalen Musikmarkt soll, wie oben zum Lizenzmodell ausgeführt, ein Vertriebsstufenanteil i.H.v. $80,25 \%$ zugrunde gelegt werden. ${ }^{733}$ Damit sind 137,388 Mio. Euro für den digitalen Musikerwerb zu veranschlagen. Addiert mit dem Betrag der GVL-Leistungsschutzrechte i.H.v. 168,8 Mio. Euro, beläuft sich der zu ersetzende Erlös somit auf 824,2352 Mio. Euro.

342 Die Zahlen für den Filmmarkt sind noch gröber geschätzt. Die Erlöskette im Videovertrieb lässt sich vereinfacht so beschreiben, dass der Produzent stellvertretend für die Filmschaffenden die Rechte für die Videoverwertung an den Videoverwerter abtritt. ${ }^{734}$ Dieser Videoverwerter übernimmt Produktion und Vertrieb der Videos und verkauft diese anschließend an den Einzelhandel. Von diesem Erlös erhält der Produzent einen gewissen Prozentsatz, den dieser wiederum an die an der Filmproduktion Beteiligten ausschüttet. Letztlich bedeutend ist damit nur dieser Prozentsatz, da der Rest sozusagen nur die Unkosten sind, die aus der physischen Verwertung entstehen. Von dem ermittelten Brutto-Durchschnittspreis muss somit zunächst die MwSt. i.H.v. 19\% abgezogen werden. Ferner müssen weitere $30 \%$ abgezogen werden, um auf den Handelsabgabepreis (HAP) zu kommen. Von diesem Handelsabgabepreis sollen geschätzt 20\%, also $10 \%$ insgesamt, auf den Videolizenzgeber entfallen, ${ }^{735}$ während andere Quellen von insgesamt ca. $30 \%$ ausgehen. ${ }^{736}$ Allerdings sei hier noch

\footnotetext{
${ }^{731}$ Fisher, 2004, S. 210 f.

${ }^{732}$ Bundesverband Musikindustrie, Jahreswirtschaftsbericht 2009, Kap. 3, Umsatz, S. 17.

${ }^{733}$ S. oben Rn. $235 \mathrm{ff}$.

${ }^{734}$ S. dazu Wirtz, Medien- und Internetmanagement, 4. Aufl. 2004, S. $256 \mathrm{ff}$.

735 Pense, Erlösverteilung - Erlösprognose bei der Film- und Fernsehproduktion, 19.4.2010, S. 6 (die Berechnung steht unter der Prämisse, dass der Lizenzgeber der Produzent ist).

${ }^{736}$ Wegmann, in: FS Matschke, 2008, S. 149, 168; was näher an dem Wert liegt, den auch Fisher, 2004, S. 212 angenommen hat (36\%).
} 
einmal darauf hingewiesen, dass dies bloße Schätzungen sind, solange keine genauen Daten vorliegen. Zugunsten der Rechteinhaber und i.S.e. „worst case“-Rechnung, soll hier von einem Anteil von $30 \%$ ausgegangen werden. Aus dem Verkauf von DVDs sind somit 410,34 Mio. Euro zu ersetzen.

343 Für Leih-DVDs ist dies schwieriger zu ermitteln, da dort nicht ohne weiteres vom Nettoerlös der Videothek auf den Erlösanteil des Produzenten geschlossen werden kann. Dazu wäre der Handelsabgabepreis, den die Videotheken an den Videoverwerter zahlen, erforderlich. Für einen reellen Wert müssten diesbezüglich genauere Daten erhoben werden. Der Einfachheit halber, sozusagen als Platzhalter, soll hier, analog zum Videoverkauf, ein Satz von $30 \%$ veranschlagt werden, so dass nach Abzug des Vertriebsanteils ein Betrag von 80,52 Mio. Euro verbleibt.

344 Der Verkauf- und Leihmarkt müsste für genaue Werte wiederum in einen digitalen und einen physischen Markt aufgeteilt werden. Angesichts dessen, dass der digitale Videomarkt insgesamt aber nur $1 \%$ des gesamten Videomarktes ausmacht ${ }^{737}$ und die Höhe der Vertriebskosten allenfalls grob geschätzt werden könnten, soll dieser Unterschied hier vernachlässigt werden.

345 Die Quote des Vertriebsanteils im Pay-TV-Sektor ist ebenso schwierig zu bestimmen. Fisher geht hierbei für den amerikanischen Markt von einer Quote von ca. $85 \%$ aus. ${ }^{738}$ Solange kein anderer Wert zur Verfügung steht, soll dieser hier zugrunde gelegt werden. Damit ergibt sich nach Abzug des Vertriebsanteils für den deutschen Markt ein Wert von 180,9 Mio. Euro.

346 Für den Buchmarkt kann eine geschätzte Pauschale von 65\% für Handel, Vertrieb und Buchdruck abgezogen werden, ${ }^{739}$ so dass letztlich ein Betrag von 3.375,05 Mio. Euro verbleibt.

\section{d) Administrative Kosten}

347 Zu diesen Beträgen sind dann die administrativen Kosten hinzuzufügen, für die Fisher für die USA ca. $20 \%$ veranschlagt. ${ }^{740}$ Für das Jahr 2004 gelangt Fisher damit insgesamt zu einer Abgabe in der Größenordnung von 5-6 US-Dollar - eine Bezugsgröße, die später oft aufgegriffen wurde. ${ }^{741}$

348 Für deutsche Verhältnisse könnte zu Vergleichszwecken auf die Kosten für die Erhebung der Geräteabgabe zurückgegriffen werden, die allerdings in den Geschäftsberichten der Verwertungsgesellschaften nicht gesondert ausgewiesen werden - vorbehaltlich dessen, dass die Einziehung und Verteilung anderen Organisationen überantwortet würde. Indes dürfte der administrative Aufwand sich nicht wesentlich anders gestalten. So schlugen bei der GEMA bspw. im Jahr 2011 Aufwendungen in Höhe von ca. 123 Mio. Euro zu Buche, was knapp 15\% der Einnahmen entsprach. ${ }^{742}$ Bei der VG Wort betrug der Aufwendungsanteil im gleichen Jahr 7,46\% der Einnahmen, ${ }^{743}$ bei der VG Bild Kunst $5,48 \% .{ }^{744}$ Allerdings können diese Sätze nicht eins-zu-eins auf die Erhebung einer Kulturflatrate über-

\footnotetext{
${ }^{737}$ BVV/GfK, Der deutsche Videomarkt 2011, S. 22.

${ }^{738}$ Fisher, 2004, S. 212; ähnlich Wegmann, in: FS Matschke, 2008, S. 149, 168.

${ }^{739}$ Wolter, Buchkalkulation - Was verdienen Autor und Verlag an Büchern, 21.6.2012; ein ähnliches Ergebnis ergibt sich aus PriceWaterhouseCoopers, E-Books in Deutschland, 2010, S. 27, die sich auf eine Modellrechnung des kaufmännischen Geschäftsführers des S. Fischer Verlags beziehen: Der Buchhandelsrabatt wird dort auf $50 \%$ des Nettoladenpreises festgelegt, sowie für Lager und Vertrieb ein Wert i.H.v. 32\% des Nettoverlagserlöses zugrunde gelegt, was umgerechnet in etwa den hier angenommenen $15 \%$ des Ladenpreises entspricht.

${ }^{740}$ Fisher, 2004, S. 214.

${ }^{741}$ Etwa Aigrain, Sharing, Culture and the Economy in the Internet Age, 2012, S. 89, oder die französische Initiative L'Alliance Public Artistes in ihrem FAQ (abrufbar unter: http://www.lalliance.org/pages/4_1.html).

742 GEMA, Geschäftsbericht 2011, S. 33.

${ }^{743}$ VG WORT, Geschäftsbericht 2011, S. 10.

${ }^{744}$ VG Bild-Kunst, Geschäftsbericht 2011, S. 5.
} 
tragen werden, da die Einziehung bei einer Kulturflatrate weitgehend automatisiert und im Gefolge der Providergebühren erfolgen könnte, mithin eine kostspielige Organisation mit Außendienstmitarbeitern etc. zur Kontrolle von vornherein nicht erforderlich wäre. ${ }^{75}$ Dennoch soll ähnlich wie bei der Substitutionsrate von einem "worst case“ ausgegangen und die 15\%-Rate der GEMA als Maßstab herangezogen werden, zumal es anfangs wahrscheinlich eines Einrichtungsaufwandes bedürfte, der kompensiert werden müsste, insbesondere wenn man die Hilfe der Provider in Anspruch nähme.

\section{e) Zusammenfassung}

349 Insgesamt resultiert daraus als Formel für eine Abgabe:

Umsatz der Industrie (bereinigt um Vertriebsstufen) multipliziert mit der Substitutionsrate zuzüglich der administrativen Kosten der Kulturflatrate geteilt durch die Zahl der privaten Internetnutzer/privaten Internetanschlüsse

350 Legt man die deutschen Zahlen für einen „worst case“ zugrunde, ergibt sich daraus Folgendes:

\begin{tabular}{|l|l|}
\hline $\begin{array}{l}\text { Umsatzerlöse pro Jahr (gleitender Mittelwert aus fünf } \\
\text { Jahren) }\end{array}$ & $14.217,8$ Mio. $€$ \\
\hline Umsatz, bereinigt um die Vertriebsstufen & $4.871,0452$ Mio. $€$ \\
\hline Substitutionsrate „worst case & $30 \%$ \\
\hline Administrative Kosten der Kulturflatrate & $15 \%$ \\
\hline Zahl der privaten Internetnutzer & Ca. 52,79 Mio. \\
\hline Zahl der privaten Breitbandanschlüsse & Ca. 27,3 Mio. $^{747}$ \\
\hline
\end{tabular}

\section{Mithin ergäbe sich für die Musikindustrie:}

\begin{tabular}{|l|l|}
\hline Gesamtumsatz durchschnittlich (2007-2011) & $1.732,6$ Mio. $€^{748}$ \\
\hline Umsatz physischer Markt & $1.392,6$ Mio. $€$ \\
\hline $\begin{array}{l}\text { Umsatz physisch, bereinigt um Vertriebskosten i.H.v. } \\
72,8 \%\end{array}$ & 518,0472 Mio. $€$ \\
\hline Umsatz digitaler Markt & 171,2 Mio. $€$ \\
\hline $\begin{array}{l}\text { Umsatz digital, bereinigt um Vertriebskosten i.H.v. } \\
\text { 19,75\% }\end{array}$ & 137,388 Mio. $€$ \\
\hline Zzgl. Einnahmen aus GVL-Rechten & 168,8 Mio. $€$ \\
\hline $\begin{array}{l}\text { Umsatzerlöse der Musikindustrie (netto) bereinigt } \\
\text { um Vertriebskosten }\end{array}$ & 824,2352 Mio. $€$ \\
\hline Multipliziert mit der Substitutionsrate von 30\% & 247,27056 Mio. $€$ \\
\hline $\begin{array}{l}\text { Zuzüglich der Einkünfte aus Rundfunk (multipliziert } \\
\text { mit einer Substitutionsrate von 5\%) }\end{array}$ & $246,620535+13,0661=260,33666$ Mio. € \\
\hline Pro Internetnutzer & $4,93 €$ jährlich \\
\hline $\begin{array}{l}\text { Pro Anschluss (s. oben Zahl der privaten Breitband- } \\
\text { anschlüsse) }\end{array}$ & $9,54 €$ jährlich \\
\hline
\end{tabular}

745 Grassmuck, Erwiderung auf das Musikindustrie-Positionspapier zur Kulturflatrate, 10.2.2010.

${ }^{746}$ Die zur Verfügung stehenden Studien sind hier nicht absolut deckungsgleich, weswegen ein Mittelwert gebildet wurde: AGOF e.V. Studie Oktober 2012, S. 3 nennt 51,77 Mio. Internetnutzer ab 14 Jahre, INITIATIVE D21 (N)Onliner Atlas 2012 nennt 53,2 Mio. Internetnutzer ab 14 Jahren und ARD/ZDF kommen in ihrer Onlinestudie 1998-2012 zu einem Ergebnis von 53,4 Mio. Internetnutzern ab 14 Jahren.

${ }^{747}$ Czajka, Statistisches Bundesamt, Wirtschaft und Statistik, August 2011, 709, 710; das entspricht etwa der Zahl, die sich aus den Angaben bei DIALOG CONSULT/VATM, Analyse Telekommunikationsmarkt Deutschland 2012, 2012, S. 17 ergibt: Bei 12,5 GB monatlich und einem jährlichen Gesamtdatenvolumen aller Anschlüsse kommt man auf 29,3 Mio. Internetanschlüsse.

${ }^{748}$ Gleitender Mittelwert aus 5 Jahren, 2007-2011, entnommen Bundesverband Musikindustrie, Jahreswirtschaftsbericht 2011. 
352 Für die anderen Industrien ergäben sich ansatzweise folgende Zahlen - dabei ist allerdings nochmals ausdrücklich auf die bislang nur spärlich vorhandenen empirischen Ergebnisse hinzuweisen, insbesondere hinsichtlich der Substitutionsraten; alternativ wird im Folgenden mit der "worst case“Substitutionsrate von $30 \%$ gerechnet:

353 Für die Filmindustrie ergäbe sich:

\begin{tabular}{|l|l|}
\hline Gesamtumsatz durchschnittlich (2007-2011): & $2.842,2$ Mio. $€^{749}$ \\
\hline Umsatz DVD-Verkauf und Verleih & $1.636,2$ Mio. $€$ \\
\hline $\begin{array}{l}\text { Umsatz DVD-Verkauf und Verleih, bereinigt um Ver- } \\
\text { triebskosten i.H.v. 70\% }\end{array}$ & 490,86 Mio. $€$ \\
\hline Umsatz Pay-TV & 1.206 Mio. $€$ \\
\hline $\begin{array}{l}\text { Umsatz Pay-TV, bereinigt um Vertriebskosten i.H.v. } \\
85 \%\end{array}$ & 180,9 Mio. $€$ \\
\hline $\begin{array}{l}\text { Umsatzerlöse der Filmindustrie (netto), bereinigt um } \\
\text { Vertriebskosten }\end{array}$ & 671,76 Mio. $€$ \\
\hline Multipliziert mit der Substitutionsrate von 30\% & 201,528 Mio. $€$ \\
\hline Pro Internetnutzer & $3,82 €$ jährlich \\
\hline Pro Anschluss & $7,38 €$ jährlich \\
\hline
\end{tabular}

\section{Für die Buchindustrie:}

\begin{tabular}{|l|l|}
\hline Gesamtumsatz (2007-2011) & 9.643 Mio. $€^{750}$ \\
\hline $\begin{array}{l}\text { Umsatz Buchindustrie bereinigt um Vertriebskosten } \\
\text { i.H.v. 65\% }\end{array}$ & $3.375,05$ Mio. $€$ \\
\hline Multipliziert mit der Substitutionsrate von 30\% & $1.012,515$ Mio. € \\
\hline Pro Internetnutzer & $19,19 €$ jährlich \\
\hline Pro Anschluss & $37,09 €$ jährlich \\
\hline
\end{tabular}

\begin{tabular}{|l|l|}
\hline $\begin{array}{l}\text { Für die anfallenden Kosten durch die Administration } \\
\text { einer Kulturflatrate: Höhe des einzunehmenden Be- } \\
\text { trags }\end{array}$ & $259,686635+201,528+1.012,515=1.473,73$ Mio. $€$ \\
\hline $\begin{array}{l}\text { Verwaltungskosten i.H.v. 15\% des einzunehmenden } \\
\text { Betrags }\end{array}$ & 221,05944525 Mio. $€$ \\
\hline Pro Internetnutzer & $4,19 €$ jährlich \\
\hline Pro Anschluss & $8,10 €$ jährlich \\
\hline
\end{tabular}

355 Bezieht man alle drei Branchen sowie die Administrationskosten mit ein, ergibt sich eine Zahl von 32,12 Euro jährlich und 2,68 Euro monatlich pro Internetnutzer. Pro Anschluss läge der Betrag bei 62,11 Euro jährlich und monatlich bei 5,18 Euro.

356 Diese Beträge würden sich noch einmal verringern, sofern man von niedrigeren Substitutionsraten und Verwaltungskosten ausgeht, wobei die Substitutionsrate gegenüber den Verwaltungskosten einen wesentlich höheren Einfluss auf den Endbetrag hat. Setzt man die Substitutionsrate bspw. bei einem Wert von $20 \%$ an und die Verwaltungskosten bei 7,5\%, so ergibt sich ein monatlicher Betrag von 1,68 Euro pro Nutzer oder 3,24 Euro pro Anschluss. Setzt man beide Werte besonders niedrig an

\footnotetext{
${ }^{749}$ Gleitender Mittelwert aus 5 Jahren, 2007-2011, entnommen BVV/GfK, Der deutsche Videomarkt 2011, S. 14.

${ }^{750}$ Gleitender Mittelwert aus 5 Jahren, 2007-2011, entnommen von 2007-2010 aus PricewaterhouseCoopers, German Entertainment and Media Outlook: 2011-2015; für 2011 aus Börsenverein des Deutschen Buchhandels, Umsatz- und Preisentwicklung, 2011.
} 
(10\% Substitutionsrate, bzw. 5\% Verwaltungskosten), so müssten Nutzer 0,83 Euro oder Anschlussinhaber 1,60 Euro monatlich zahlen. ${ }^{751}$

357 Allerdings ist gegenüber dem von Fisher (und auch nachfolgenden Vorschlägen) zugrunde gelegten Umsatzeinbußen zu Recht darauf hingewiesen worden, dass damit diejenigen, die das Internet als eigenen (und ausschließlichen) kreativen Raum nutzen, leer ausgehen, da sie eben nicht den klassischen Vertriebsweg über Verleger bzw. Intermediäre suchen. Anders gewendet würde eine Vergütungskalkulation, die sich nur auf die Einbußen der Rechteinhaber in klassischen Vertriebssituationen bezieht, letztlich sogar zu einer zu geringen Vergütung der Kreativen führen können, da ihre Werke nicht in die Berechnung der Höhe der Abgabe einflössen (wohl aber bei der Verteilung). ${ }^{752}$

\section{Mischmodell: Volumenbasiert mit Substitutionsrate}

358 Ein Mischmodell könnte schließlich darin bestehen, dass man zwar das Lizenzanalogiemodell zugrunde legt, mithin vom durchschnittlichen Volumen eines Internetanschlusses ausgeht, diesen aber anders als im Modell der Verwertungsgesellschaften nicht 1:1 übernimmt bzw. fingierte entgangene Transaktionen annimmt, sondern eine Substitutionsrate ${ }^{753}$ von max. $30 \%$ einbezieht.

Mithin ergäbe sich folgende Formel:

Zahl der privaten Breitbandanschlüsse $x$ durchschnittliches Volumen des Datenverkehrs pro Monat $x$ prozentualer Anteil der urheberrechtlich relevanten Nutzungen $x$ prozentualer Anteil des getauschten Mediums am Datenvolumen / MB pro Werk bzw. Werksammlung (Album-, bzw. Songdownload, Filmdownload, E-Book) x durchschnittlicher Ladenpreis abzgl. Vertriebsstufen $\times$ Substitutionsrate.

361 Nimmt man die oben angegebenen Zahlen, ergibt sich folgende Berechnung:

\section{Für die Musikindustrie:}

\begin{tabular}{|l|l|l|l|l|l|}
\hline $\begin{array}{l}\text { Volumen } \\
\text { pro Nut- } \\
\text { zer in MB } \\
\text { monatlich }\end{array}$ & $\begin{array}{l}\text { Zahl der Breitband- } \\
\text { anschlüsse }\end{array}$ & $\begin{array}{l}\text { Volumen pro Mo- } \\
\text { nat in MB }\end{array}$ & $\begin{array}{l}\text { Urheberrecht- } \\
\text { lich relevanter } \\
\text { Anteil }\end{array}$ & $\begin{array}{l}\text { Anteil von Mu- } \\
\text { sikdownloads am } \\
\text { Gesamtvolumen }\end{array}$ & $\begin{array}{l}\text { Durchschnittliche } \\
\text { Größe einer Mu- } \\
\text { sikdatei in MB }\end{array}$ \\
\hline 12.500 & 27.300 .000 & 341.250 .000 .000 & $25 \%$ & $12,6 \%$ & 5 \\
\hline
\end{tabular}

\begin{tabular}{|c|c|l|l|l|l|}
\hline $\begin{array}{l}\text { Musikdownloads } \\
\text { in Stück }\end{array}$ & $\begin{array}{l}\text { Preis pro } \\
\text { Album }\end{array}$ & $\begin{array}{l}\text { Anteil der } \\
\text { Rechteinhaber } \\
\text { am Erlös }\end{array}$ & Preis monatlich & $\begin{array}{l}\text { Substitutions- } \\
\text { rate }\end{array}$ & $\begin{array}{l}\text { Preis umgelegt auf } \\
\text { Anschlussinhaber } \\
\text { monatlich }\end{array}$ \\
\hline 2.149 .875 .000 & $0,99 €$ & $80,25 \%$ & $1.708 .021 .940,63 €$ & $30 \%$ & $\mathbf{1 8 , 7 7} €$ \\
\hline
\end{tabular}

\footnotetext{
${ }^{751}$ Für detaillierte Berechnungen, sowie weitere Werte und Kombinationsmöglichkeiten, s. unten Rn. $616 \mathrm{ff}$.

752 So Aigrain, 2012, S. 89: „In other words, we would neither reward nor finance those who build the heart of the digital cultural commons".

${ }^{753}$ Eine ähnliche Überlegung findet sich zur Vergütungsabgabe für die Privatkopie bei Vitorino, Recommendations, S. 19 f., der bei der Auslegung des Wortes „harm“ (s. oben Rn. 236) darauf abstellen will, wie viele Vergütungspflichtige Kopien tatsächlich hergestellt worden wären, wenn es nicht die Privatkopieschranke gebe; wobei er später aber noch weiter geht und es für gerechtfertigt hält, auf den Wert den eine Kopie für den Nutzer hat abzustellen (S. 20).
} 
363 Für die Filmindustrie:

\begin{tabular}{|r|r|r|r|r|r|}
\hline $\begin{array}{l}\text { Volumen } \\
\text { pro Nut- } \\
\text { zer in MB } \\
\text { monatlich }\end{array}$ & $\begin{array}{l}\text { Zahl der Breitband- } \\
\text { anschlüsse }\end{array}$ & $\begin{array}{l}\text { Volumen pro Mo- } \\
\text { nat in MB }\end{array}$ & $\begin{array}{l}\text { Urheberrecht- } \\
\text { lich relevanter } \\
\text { Anteil }\end{array}$ & $\begin{array}{l}\text { Anteil von Film- } \\
\text { downloads am } \\
\text { Gesamtvolumen }\end{array}$ & $\begin{array}{l}\text { Durchschnittliche } \\
\text { Größe eines Films }\end{array}$ \\
\hline 12.500 & 27.300 .000 & 341.250 .000 .000 & $25 \%$ & $87,3 \%$ & 700 \\
\hline
\end{tabular}

\begin{tabular}{|c|c|c|c|c|c|}
\hline $\begin{array}{l}\text { Heruntergeladene } \\
\text { Filme in Stück }\end{array}$ & \begin{tabular}{|l} 
Preis \\
pro \\
Film \\
\end{tabular} & \begin{tabular}{|l} 
Anteil der \\
Rechtein- \\
haber am \\
Erlös \\
\end{tabular} & Preis monatlich & $\begin{array}{l}\text { Substitutionsra- } \\
\text { te }\end{array}$ & $\begin{array}{l}\text { Preis umgelegt } \\
\text { auf Anschluss- } \\
\text { inhaber monat- } \\
\text { lich } \\
\end{array}$ \\
\hline 106.396 .875 & $7,71 €$ & $70 \%$ & $574.223 .934,38 €$ & $30 \%$ & $6,31 €$ \\
\hline
\end{tabular}

364 Für die Buchindustrie:

\begin{tabular}{|c|r|r|r|l|l|}
\hline $\begin{array}{l}\text { Volumen pro } \\
\text { Nutzer in MB } \\
\text { monatlich }\end{array}$ & $\begin{array}{l}\text { Zahl der Breitbandan- } \\
\text { schlüsse }\end{array}$ & $\begin{array}{l}\text { Volumen pro } \\
\text { Monat in MB }\end{array}$ & $\begin{array}{l}\text { Urheberrechtlich } \\
\text { relevanter Anteil }\end{array}$ & $\begin{array}{l}\text { Anteil von Buch- } \\
\text { downloads am Ge- } \\
\text { samtvolumen }\end{array}$ & $\begin{array}{l}\text { MB } \\
\text { pro } \\
\text { Buch }\end{array}$ \\
\hline 12.500 & 27.300 .000 & 341.250 .000 .000 & $25 \%$ & $0,10 \%$ & 2 \\
\hline
\end{tabular}

\begin{tabular}{|c|l|l|l|r|l|}
\hline $\begin{array}{l}\text { Anzahl Bü- } \\
\text { cher }\end{array}$ & $\begin{array}{l}\text { Preis } \\
\text { pro E- } \\
\text { Book }\end{array}$ & $\begin{array}{l}\text { Anteil der Ver- } \\
\text { triebskosten }\end{array}$ & Preis monatlich & Substitutionsrate & $\begin{array}{l}\text { Preis umgelegt auf } \\
\text { Anschlussinhaber } \\
\text { monatlich }\end{array}$ \\
\hline 42.656 .250 & $8,08 €$ & $50 \%$ & $172.117 .968,75,00 €$ & $30 \%$ & $\mathbf{1 , 8 9 €}$ \\
\hline
\end{tabular}

365 Mithin ergäbe sich nach dem Mischmodell insgesamt ein monatlicher Abgabenpreis von 26,97 Euro pro Anschluss.

366 Ginge man von einer geringeren Substitutionsrate und einem geringeren urheberrechtlich relevanten Anteil am Datenvolumen aus, so ergäbe dies einen monatlichen Betrag von 10,79 Euro pro Anschluss. Bei einer besonders niedrig angesetzten Substitutionsrate und einem besonders niedrigen urheberrechtlich relevanten Anteil bliebe ein Betrag von 1,80 Euro pro Anschluss pro Monat.

\section{Zusammenfassung}

367 Fasst man die Ergebnisse zusammen, so sind vier Berechnungen einer Kulturflatrate möglich - die indes alle auf Annahmen und nur schwach verlässlichen empirischen Daten beruhen. Legt man die restriktivsten Werte ${ }^{754}$ zugrunde (urheberrechtlich relevanter Anteil von 25\%, Substitutionsrate von $30 \%$ und Verwaltungsaufwand i.H.v. 15\% der eingenommenen Beträge), ergeben sich folgende Zahlen:

- Das Modell einer Abgabe basierend auf getauschten Datenvolumen (Verwertungsgesellschaften) und einer 1:1 Substitution (Lizenzanalogie) mit dem (Näherungs-)Wert von $\mathbf{8 9 , 8 9}$ Euro pro Anschluss pro Monat

- Schließlich das Modell der Kappungsgrenze, bei dem ein maximaler monatlicher Preis von 3,50 Euro pro Internetanschluss möglich wäre.

\footnotetext{
${ }^{754}$ Für die ausführlichen Berechnungen und weitere Kombinationsmöglichkeiten, s. unten Rn. $558 \mathrm{ff}$.
} 
- Das Modell einer Abgabe basierend auf Umsatzeinbußen (Fisher), das eine Substitutionsrate einbezieht und zu einem monatlichen Preis pro Anschluss von 5,18 Euro gelangt.

- Als letztes kommt ein Mischmodell in Betracht, welches auf der Berechnung des Lizenzanalogiemodells beruht, aber zusätzlich Fishers Substitutionsrate berücksichtigt. Danach ergibt sich ein Monatspreis von 26,97 Euro.

368 Geht man dagegen von geringeren Zahlen aus (urheberrechtlich relevanter Anteil von 15\%, Substitutionsrate von $20 \%$ und Verwaltungsaufwand i.H.v. $7.5 \%$ ), so kommt es zu folgenden Ergebnissen:

- Für das Modell der (modifizierten) Lizenzanalogie: 53,94 Euro pro Anschluss pro Monat

- Das Modell der Kappungsgrenze bleibt unverändert bei 3,50 Euro

- Geht man vom Substitutionsmodell aus, ergibt sich ein Betrag von monatlich 3,24 Euro pro Anschluss

- Schließlich für das Mischmodell ein Betrag von monatlich 10,79 Euro pro Anschluss.

369 Legt man sehr niedrige Ausgangswerte zugrunde (urheberrechtlich relevanter Anteil von 5\%, Substitutionsrate von $10 \%$, sowie Verwaltungsaufwand i.H.v. 5\%), ergeben sich Werte von:

- 17,98 Euro pro Anschluss pro Monat nach dem modifizierten Lizenzanalogiemodell

- Weiterhin unveränderten 3,50 Euro für das Kappungsgrenzenmodell

- Nach dem Substitutionsmodell i.H.v. 1,60 Euro pro Anschluss pro Monat

- Letztlich für das Mischmodell 1,80 Euro pro Anschluss pro Monat.

370 Betrachtet man die verschiedenen Modelle, fällt zunächst für das Modell der Lizenzanalogie auf, dass es im höchsten Fall zu einem Abgabenerlös von insgesamt 29.447.964.000 Euro jährlich käme. Die gesamte (!) Industrie (Musik, Film, Buch) erwirtschaftet aber einen Umsatz (nicht Gewinn) von 14.217.800.000 Euro jährlich, in dem sämtliche Einkünfte enthalten sind, auch Vertriebskosten etc. Dies zeigt deutlich, dass eine vollständige Lizenzanalogie kaum geeignet sein dürfte, die Abgabe sinnvoll zu strukturieren bzw. zu berechnen, da sie zu einer Überkompensation führen würde.

371 Eine Einbeziehung einer Substitutionsrate in die Berechnungen ist daher erforderlich, erst recht wenn man in den Schadensbegriff auch eintretende Vorteile einbezieht (im Sinne eines Vorteilsausgleichs). Auch erscheint es schwierig, angesichts der vielen und sich schnell entwickelnden Dateiformate und Kompressionstechniken eine einheitliche Größe zu finden, um die benötigten Datenvolumina zu berechnen.

372 Nach dem Modell der Kappungsgrenze ergibt sich ein monatlicher Betrag pro Anschluss von maximal 3,50 Euro. Allerdings weist dieses Modell Begründungs- und Berechnungsdefizite auf: Der Anknüpfungspunkt des durchschnittlichen Preises einer Internetflatrate führt zwar zu einem für den Nutzer angemessenen Betrag, berücksichtigt dabei aber die Interessen der Urheber nur unzureichend. Dies gilt erst recht im Hinblick auf die europarechtlichen Vorgaben, da deren wirtschaftliche Verhältnisse in keiner Weise in die Formel einbezogen werden, so dass er ungeeignet erscheint, um einen ange- 
messenen Ausgleich zwischen den Interessen der Kulturschaffenden und der Nutzer herzustellen. Schließlich dürfte der Grundgedanke des § 54a UrhG, nämlich die Vermeidung von Wettbewerbsnachteilen gegenüber ausländischen Angeboten, kaum einschlägig sein.

373 Das Fisher-Modell dagegen geht von dem tatsächlichen Umsatzrückgang der Industrien aus. Die Vergleichsweise umfangreichen und vielfältigen Datenerhebungen, die zur präziseren Berechnung nötig wären, könnten durchaus durchgeführt werden. Die Substitutionsrate, die in periodischen Abständen neu zu ermitteln wäre, bietet dabei ein flexibles Anpassungskriterium. Es müsste allerdings überlegt werden, wie man auch diejenigen Kulturschaffenden in die Rechnung einbeziehen kann, die nicht in den klassischen Vertriebswegen arbeiten, etwa Künstler, die primär (oder ausschließlich) ohne Verwerter arbeiten, indem sie auf eigenen Internet-Plattformen ihre Werke allein zum Download anbieten. Denn diese Kulturschaffenden würden aufgrund des Ansatzes bei den Industrieumsätzen bei der Bemessung der Höhe der Abgabe nicht berücksichtigt werden (wohl aber bei der Verteilung).

374 Das Mischmodell wiederum bezieht zwar die Substitutionsrate mit ein, kann aber dennoch die Kritik am unrealistisch hohen Preis des reinen Lizenzanalogiemodells und auch die Bedenken hinsichtlich der Berechnung des Datenvolumens nicht völlig ausräumen. Letzterem wiederum könnte durch eine genaue technische Erfassung der Dateitypen und -größen entgegengesteuert werden.

Insgesamt erscheint daher im Rahmen der Einschätzungsprärogative eine Wahl zwischen dem Fisher-Modell und dem Mischmodell auf der Basis der Lizenzanalogie als empfehlenswert.

\section{B. Ausgestaltungsmöglichkeiten einer Flatrate}

377 Vor diesem Hintergrund gilt es weitere Ausdifferenzierungen einer Kulturflatrate zu diskutieren, insbesondere was der mögliche Bezugspunkt für eine Vergütung sein kann. Während in den USA Abgaben im Zusammenhang mit einer Kulturflatrate nicht nur auf Internetzugänge, sondern auch auf Produkte, Geräte, Leermedien und Web-Services diskutiert werden, ${ }^{755}$ steht in Europa angesichts der bereits bestehenden Geräteabgaben nur der Internetzugang als Anknüpfungspunkt für eine Abgabe im Zentrum der Diskussion. ${ }^{756}$ Dabei ist gerade unter Datenschutzgesichtspunkten eine individuelle Ausgestaltung anhand der konkret herunter- oder heraufgeladenen Daten keine zu diskutierende Alternative; denn dies würde eine konkrete Zuordnung aller Nutzungsvorgänge zu jedem individuellen Nutzer bedingen und damit gerade zum unerwünschten „gläsernen Nutzer" führen. Dies schließt keineswegs aus, dass auf der Verteilungsseite eine weitgehende Anknüpfung an die konkreten Nutzungen einzelner Werkstücke durchgeführt wird, wie noch zu zeigen sein wird. ${ }^{757}$

\footnotetext{
755 Netanel, Harvard JOLT, Vol. 17, 2003, 1, 43 f., der auch eine Ausweitung in Erwägung zieht auf alle zukünftigen „,consumer products and services whose value is substantially enhanced [...] by P2P file sharing [...] As technology evolves"; Fisher, 2004, S. 218 f.

${ }^{756}$ S. nur Bernault/Lebois, 2006, S. 66; Roßnagel et. al., Gutachten, 2009, S. 23, 30; Initiative privatkopie.net u.a., Kompensation ohne Kontrolle, 2004, S. 8; ferner mit weiteren Nachweisen aus der außereuropäischen Diskussion: Zwengel, Kulturflatrates, 2012, S. 99 Fn. 489.

${ }^{757}$ S. unten Rn. $427 \mathrm{ff}$.
} 
378 Während für das Modell, das maßgeblich auf eine allgemeine Substitutionsrate abstellt (Fisher), kein eindeutiger Bezugspunkt besteht, da sowohl Datenvolumina als auch Geschwindigkeiten etc. als Anknüpfungspunkt herangezogen werden könnten, würde ein Modell, das den Verwertungsgesellschaftenansatz folgt, klar auf die Datenvolumina abstellen:

\section{Anknüpfung an Breitbandanschlüsse}

379 Zunächst kann in einer typisierenden Betrachtungsweise (ähnlich den Geräteabgaben) darauf abgestellt werden, mit welchen Anschlussformen und damit vor allem welchen Geschwindigkeiten Inhalte getauscht und heruntergeladen werden können. Dabei ist allerdings zu beachten, dass Kompressionstechnologien die Frage der Geschwindigkeiten erheblich verändern können, so dass ein entsprechender Abgabentatbestand quasi jährlich neu den Kreis der einzubeziehenden Anschlussarten zu berücksichtigen hätte.

Maßgeblich ist jedenfalls, dass Komprimierungstechniken ebenso wie Änderungen in der Übertragungstechnologie stets Auswirkungen auf den Bezugspunkt der Abgabe haben können, so dass zwar keine vollständige Technologieneutralität erreicht werden kann, aber eine stets wiederkehrende Prüfung erforderlich ist.

381 Eine andere Frage bezieht sich auf den bereits diskutierten Einwand, dass bei einer Abgabe auf Internetzugangsanschlüsse Nutzer unter Umständen für etwas zahlen müssen, was sie gar nicht nutzen wollen. Diesem Einwand könnte im Rahmen einer Abgabe dadurch Rechnung getragen werden, dass die Höhe der Abgabe unter Berücksichtigung der Geschwindigkeiten oder des Datenvolumens ausgestaltet wird, da anzunehmen ist, dass digitaler Content häufig mehr Datenvolumen beansprucht als etwa reine Meinungsäußerungen, Texte etc., ebenso dass Nutzer, die tauschen wollen, keine langsamen Anschlüsse verwenden werden. ${ }^{758}$ Andere Vorschläge sehen einen prozentualen Aufschlag auf die Internetgebühren vor, so dass für schnellere und teurere Zugänge auch mehr Abgaben zu entrichten sind. ${ }^{759}$

382 Ein erster Einwand bezieht sich auf die damit eintretende Doppelbelastung von Nutzern, die eines der Download- bzw. Streaming-Modelle, wie etwa T-Entertain, benutzen. Gerade solche Angebote, die auch Fernsehen und Rundfunk als internetgestützten Dienst anbieten (Triple-Play), benötigen hohe Geschwindigkeiten ebenso wie ein hohes Datenvolumen. Im Ergebnis müsste ein Nutzer solcher Geschäftsmodelle daher sowohl eine hohe Abgabe in Gestalt der Kulturflatrate bezahlen, da er hohe Geschwindigkeiten benötigt, als auch gleichzeitig Gebühren für das gesamte Angebot. Wie schon oben ausgeführt, ist daher nicht auszuschließen, dass erhebliche negative Effekte für die Preisgestaltung bzw. ökonomische Überlebensfähigkeit solcher Modelle eintreten - was aber mangels tragfähiger Daten ohne weitere Felduntersuchungen bzw. empirische Erhebungen nicht im Rahmen dieses Gutachtens eingeschätzt werden kann. Denkbar wäre jedenfalls, dass Nutzer, die entsprechende Verträge nachweisen können, in den Genuss eines Abschlags auf ihre Abgabe kommen könnten. Angesichts der auch bei diesen Diensten weitgehend automatisierten Verwaltung ist es möglich, dass sich die zusätzlichen Verwaltungskosten noch im Rahmen der Verhältnismäßigkeit bewegen würden; indes wären auch hier zusätzliche Informationen seitens der entsprechenden Anbieter über geschätzte Höhen des administrativen Aufwandes etc. erforderlich.

\footnotetext{
${ }^{758}$ Roßnagel et al., Gutachten, 2009, S. 23.

${ }^{759}$ Fisher, 2004, S. 221.
} 
383 Zwar sind nicht alle getauschten Inhalte gleichermaßen relevant für Datenvolumen und/oder Anschlussgeschwindigkeiten; so werden E-Books und Texte kaum derartige Datenmengen oder Geschwindigkeiten beanspruchen wie komplexe Videofiles oder gar BluRay-Inhalte. Dennoch kann und muss im Rahmen einer Abgabe mit einer Typisierung gearbeitet werden, indem der erste Anschein dafür spricht, dass Filesharer höhere Geschwindigkeiten und Datenvolumina nachfragen - es dürfte eher unwahrscheinlich sein, dass nur E-Books getauscht werden. Schließlich würde eine genau Erfassung des Verhaltens der jeweiligen Nutzer eine lückenlose Kontrolle ihrer Inhalte erfordern, was schon verfassungs- und datenschutzrechtlich größten Zweifeln begegnet, da sie eine permanente Deep Packet Inspection erfordern würde. ${ }^{760}$ Aber auch hier wäre eine Feinsteuerung durch empirische Erhebungen in Gestalt von Umfragen möglich.

384 Ähnlich der Abgabe auf USB-Sticks, wo zwei Kapazitätskategorien bis 4GB und ab 4GB gebildet werden ${ }^{761}$ sollte auch eine Abgabe aus einer Kulturflatrate nicht jede denkbare Bandbreite erfassen. Denn derzeit lässt sich allenfalls mit dem angesprochenen Anschein arbeiten, wonach Nutzer schnellerer Anschlüsse vermutlich in höherem Maße urheberrechtlich relevant agieren als Nutzer mit langsameren Anschlüssen. Es lässt sich aber zumindest derzeit, wie dargestellt, anhand der vorhandenen empirischen Studien nicht sagen, mit welchem MBit-Anstieg ein Filesharing-Anstieg verbunden sein könnte. Ohnehin ist wohl nur schwer möglich und zu aufwendig, empirische Nachweise für jeden einzelnen Anschlusstypus zu liefern. Begründbar wäre daher allenfalls eine kategorische Aufteilung etwa in langsame, mittlere und schnelle Anschlüsse. Die Kategorisierung kann zudem zukünftig dabei helfen, schnell auf technische Entwicklungen zu reagieren, indem sich die mittlere Kategorie immer an dem Wert der am häufigsten genutzten Geschwindigkeit zum jeweiligen Zeitpunkt orientiert. Die dafür relevanten Daten werden von der Bundesnetzagentur (BNetzA) zur Verfügung gestellt. ${ }^{762}$

385 Ließe man bei der vorgeschlagenen Aufteilung nun die Abgabe proportional ansteigen, müsste man die Durchschnittsgeschwindigkeiten der jeweiligen Kategorien zueinander ins Verhältnis setzen. Die derzeit am häufigsten gewählte Anschlussgeschwindigkeit - und damit die mittlere Geschwindigkeit - stellen mit 46,3\% die Angebote von über 2 bis unter $10 \mathrm{MBit} / \mathrm{s}$ (Abgabe Kat. 2). ${ }^{763}$ Breitbandanschlüsse von $144 \mathrm{Kbit} / \mathrm{s}$ bis $2 \mathrm{MBit} / \mathrm{s}$ repräsentieren die langsame (Abgabe Kat. 1) und Anschlüsse ab $10 \mathrm{MBit} / \mathrm{s}$ die schnelle Kategorie (Abgabe Kat. 3). ${ }^{764}$ Kategorie 1 hat damit einen Durchschnittsgeschwindigkeitswert von rund 1,1 MBit/s, Kategorie 2 von rund $6 \mathrm{MBit} / \mathrm{s}$ und Kategorie 3 von 55 $\mathrm{MBit} / \mathrm{s} .{ }^{765}$ Würde man jetzt eine proportional an die Geschwindigkeit angelegte Abgabe einführen, würden Nutzer der schnellen Kategorie etwa eine rund 9 mal höhere Abgabe zahlen als Nutzer der mittleren Kategorie. Es liegen allerdings, wie aufgezeigt, keinerlei empirische Nachweise vor, dass ein Anstieg der Geschwindigkeit proportional zu mehr Filesharing führt. Das erscheint sogar unrealistisch, da ab einem gewissen Punkt das Maximum dessen erreicht ist, was ein privater Nutzer überhaupt noch an urheberrechtlich relevantem Material abspeichern kann bzw. wird, da zumindest ein Teil der Nutzer die Filesharingaktivitäten reduzieren wird, sobald das heruntergeladene Material nicht mehr konsumiert werden kann.

\footnotetext{
${ }^{760}$ S. dazu unten Rn. $439 \mathrm{ff}$.

761 ZPÜ, Tarif USB-Sticks und Speicherkarten ab 07.2012, S. 1, abrufbar unter: http://www.zpue.de/fileadmin/user_upload/pdf/Tarif_USB-Sticks_und_SpK_ab_07_2012.pdf.

762 BNetzA, Jahresbericht 2011, S. 76.

763 BNetzA, Jahresbericht 2011, S. 76.

764 BNetzA, Jahresbericht 2011, S. 76.

${ }^{765}$ BNetzA, Jahresbericht 2011, S. 76.
} 
386 Ähnliche Gedanken scheinen auch grundlegend für die Tarife der ZPÜ für die Abgabe auf USB-Sticks gewesen zu sein. ${ }^{766}$ Denn USB-Sticks ab 4GB werden nur mit einer rund $60 \%$ höheren Abgabe belegt als Sticks unter $4 \mathrm{~GB},{ }^{767}$ obwohl bereits Kapazitäten von $128 \mathrm{~GB}$ und mehr erreicht werden. Mangels Daten zu dem ungefähren durchschnittlichen Anstieg des Filesharing von Anschlusskategorie zu Anschlusskategorie soll sich nachfolgend für die Kulturflatrate zur Veranschaulichung daran orientiert werden; allerdings auch an dieser Stelle mit dem ausdrücklichen Hinweis auf den Bedarf weiterer empirischer Untersuchungen. Legt man die oben in den Berechnungen verwandten Werte zugrunde, ergeben sich für die vier Modelle folgende Preise (je pro Anschluss, pro Monat): ${ }^{768}$

- für das modifizierte Lizenzanalogiemodell eine beispielhafte Preisverteilung von 34,17 Euro (Kat. 1) zu 85,43 Euro (Kat. 2) zu 136,69 Euro (Kat. 3).

- Das Kappungsgrenzenmodell führt zu Preisen von 1,33 Euro (Kat. 1), 3,33 Euro (Kat. 2) und 5,32 Euro (Kat. 3)

- Nach dem Substitutionsmodell verteilt sich die Kostenlast wie folgt: 1,97 Euro (Kat. 1) zu 4,92 Euro (Kat. 2) zu 7,88 Euro (Kat. 3).

- Das Mischmodell führt entsprechend zu Werten von 10,25 Euro (Kat. 1) über 25,63 Euro (Kat. 2) bis hin zu 41,01 Euro (Kat. 3).

387 Würde sich der Gesetzgeber für diesen Weg entscheiden, wäre dies auch noch von der ihm zugestandenen Einschätzungsprärogative gedeckt. ${ }^{769}$

\section{Personenbezogene Anknüpfung? (Vermeidung der Zahlung mehrerer Internetan- schlüisse)}

388 Aus der Tatsache, dass die Abgabe als Kompensation für die urheberrechtlichen Verwertungsvorgänge eingeführt werden soll, resultiert auch bereits, dass es nur um Abgaben auf Anschlüsse gehen kann, über die die entsprechenden Vorgänge realisiert werden - nicht aber auf Personen. Mit anderen Worten kommt es auf die Zahl der urheberrelevanten Vorgänge über einen Anschluss an, nicht aber auf die Zahl der Personen, die über einen Internetanschluss mit dem Netz verbunden sind. Auch bei einer hohen Zahl von Personen etwa über ein hauseigenes WLAN wird damit die Übertragungskapazität eines solchen Anschlusses nicht erhöht - was aber allein maßgeblich ist für die Bestimmung der Abgabe als Kompensation für den Urheber. ${ }^{770}$

\section{Vergütungsschuldner}

389 Als Vergütungsschuldner kommen im Wesentlichen die Intermediäre in Betracht, hier vor allem die Internetprovider. Zwar könnten theoretisch entsprechend den Entscheidungen zur (Mit-) Haftung für illegales Filesharing auch die Softwareanbieter herangezogen werden, die ihrerseits die Gebühren auf die Nutzer abwälzen könnten. Allerdings sprechen hiergegen erhebliche praktische Probleme, da

\footnotetext{
${ }^{766}$ ZPÜ, Tarif USB-Sticks und Speicherkarten ab 07.2012, S. 1, abrufbar unter: http://www.zpue.de/fileadmin/user_upload/pdf/Tarif_USB-Sticks_und_SpK_ab_07_2012.pdf.

767 ZPÜ, Tarif USB-Sticks und Speicherkarten ab 07.2012, S. 1, abrufbar unter: http://www.zpue.de/fileadmin/user_upload/pdf/Tarif_USB-Sticks_und_SpK_ab_07_2012.pdf.

${ }^{768} \mathrm{Zu}$ den genauen Berechnungen und den Ergebnissen bei variierenden Ausgangswerten, s. unten Rn. $558 \mathrm{ff}$.

${ }^{769}$ S. oben Rn. $159 \mathrm{ff}$.

${ }^{770}$ Zu Fragen der Mischnutzung etwa von Privaten im Rahmen eines Arbeitsverhältnisses oder an Schulen, Universitäten etc. s. unten Rn. 484 ff.
} 
die entsprechende Software oder Dienste oftmals nicht im Inland angeboten werden, zudem es sich meist um kostenlose oder rein werbefinanzierte Produkte handelt. ${ }^{771}$

390 Demgegenüber bieten sich die Internet-Access-Provider als diejenigen „intermediären“ Vergütungsschuldner an, da nur über ihre Zugänge zum Netz die entsprechenden Down- und Uploadvorgänge vorgenommen werden können. ${ }^{772}$

\section{Verhältnis zu den traditionellen Geräteabgaben}

391 Angesichts der Tatsache, dass bereits jetzt schon für die Privatkopie Geräteabgaben sowie Abgaben auf Leermedien erhoben werden, liegt es auf der Hand, dass diese Abgabenmodelle sich überschneiden, da eine Kulturflatrate zwar nicht nur, aber eben auch die Privatkopie erfassen würde. Mithin kann eine Abgabe sowohl auf den Internetzugang als auch auf ein Speichermedium etc. den gleichen Vervielfältigungsvorgang mehrfach erfassen, so dass eine entsprechende Reduktion erforderlich wäre, sei es bei der Geräteabgabe, sei es bei der Kulturflatrate. Das Ausmaß an Überlappungen lässt sich nur durch empirische Erhebungen ermitteln, die den Einsatz von Speichermedien etc. ohne Zusammenhang mit dem Internet analysieren, mit anderen Worten also den Offline-Anteil der Vervielfältigungsvorgänge, z.B. die Kopie von CDs auf einen USB-Stick oder eine Festplatte, ohne dass irgendein Zusammenhang mit Downloadvorgängen besteht. Umgekehrt geht die Kulturflatrate auch einen Schritt weiter als die traditionelle Geräte- und Leermedienabgabe, da auch das öffentliche Zugänglichmachen sowie das Bearbeiten von den neuen Schranken erfasst wäre.

392 Sinnvoll wäre daher angesichts des umfassenderen Anwendungsbereichs der Kulturflatrate eine prozentuale Absenkung der Geräteabgabe, um aufgrund der Überschneidungen zwischen beiden Abgaben im Bereich der Privatkopien eine doppelte Erfassung zu verhindern.

\section{Die Verteilungsseite: Vergütungen an Kreative}

\section{Rechtliche Vorüberlegungen}

393 Anknüpfungspunkt für die Verteilung an Kreative ist zunächst das Ausmaß der Nutzung ihrer Werke. Dies gebietet indirekt auch das europäische Recht, wenn es für die Abgabenerhebung darauf abstellt, welcher Nachteil den Rechteinhabern entstanden ist. ${ }^{773}$ Auch aus verfassungsrechtlicher Sicht muss den Rechteinhabern eine angemessene Kompensation zur Verfügung gestellt werden. Dies schließt Verteilungsschlüssel aus, die überwiegend oder ausschließlich auf nicht auf die Nutzung bezogene Kriterien abstellen, sondern auf verteilungs- bzw. kulturpolitische Kriterien, da sie nicht dem Eingriff in das eigentumsähnliche Recht des Urhebers entsprächen. Ebenso wenig können schon aus diesen rechtlichen Gründen allein freiwillige Votings für Künstler die Verteilung nicht entscheiden, da es primär auf die tatsächliche Nutzung des Werkes ankommt.

394 Umgekehrt gebietet es die Kompensation der Urheber bzw. Rechteinhaber nicht, dass ausschließlich nutzungsbezogene Kriterien Eingang in die Verteilung finden. So können auch kulturpolitische Bezugspunkte, wie etwa die Förderung der E- oder Kirchenmusik (wie bei der GEMA) Berücksichtigung finden. ${ }^{774}$ Hier schlägt sich letztlich zum einen die Befugnis des Gesetzgebers zur inhaltlichen Ausge-

\footnotetext{
${ }^{771}$ Bernault/Lebois, 2006, S. $37 \mathrm{f}$.

772 Bernault/Lebois, 2006, S. 38 f.; zusätzlich entsprechen sie auch der Forderung von Vitorino, Recommendations, S. 14 f., dass pauschale Abgaben nicht bei Geräteherstellern oder Importeuren, sondern beim Händler, bzw. demjenigen, der dem Endverbraucher das Produkt letztlich verschafft, erhoben werden sollen.

${ }^{773}$ S. oben Rn. 229 ff.; EuGH GRUR 2011, 50 - Padawan.

${ }^{774}$ Roßnagel et al., Gutachten, 2009, S. 30.
} 
staltung des Eigentums, aber auch dessen Sozialbindung nieder, so dass auch kultur- und sozialpolitische Belange in die Verteilung Eingang finden können, ${ }^{775}$ solange der Kern des Eigentums unangetastet bleibt und aus europarechtlicher Sicht ein „gerechter Ausgleich“ hergestellt wird.

395 Schließlich scheiden auch die Vertriebsstufen (Händler etc.) aus der Verteilung der Abgabeneinnahmen aus - wie schon bereits derzeit im Rahmen der Geräteabgabe, da sie nicht Rechteinhaber im urheberrechtlichen Sinne sind und die Abgabe nur der Kompensation des Eingriffs in die Verwertungsrechte dient.

Die einfachste Form der Verteilung bestünde in der „gießkannenartigen“ Verteilung des Gebührenaufkommens an alle Urheber bzw. Rechteinhaber. Sofern indes technische Verfahren oder empirische Grundlagen zur Verfügung stehen, die die Verteilung präziser anhand der Nutzung der Werke zu bestimmen vermögen und unter wirtschaftlichen Gesichtspunkten zumutbar sind, sind solche "Gießkannen"-Verfahren in der Regel rechtlich nicht zulässig, da sie den jeweiligen Rechteinhaber nicht angemessen entschädigen. ${ }^{776} \S 54$ a Abs. 1 S. 1 UrhG und $\S 7$ S. 1 UrhWahrnG formulieren den Grundsatz, dass sich die Vergütungshöhe an der tatsächlichen Werknutzung zu orientieren hat und im Falle der kollektiven Rechtewahrnehmung durch Verwertungsgesellschaften diese die Ausschüttung nicht nach „willkürlichen“ Kriterien vornehmen dürfen. Nur wenn eine gleichmäßige Verteilung auf alle Berechtigten nicht willkürlich wäre, d.h. nicht gegen den verfassungsrechtlichen Gleichheitsgrundsatz verstieße, ${ }^{777}$ wäre sie zulässig. Eine Pauschalierung, wie sie die gleichmäßige Verteilung der Ausschüttungssumme zweifellos darstellen würde, ließe sich zwar grundsätzlich durch sachliche Gründe rechtfertigen. ${ }^{778}$ Doch dürften sich angesichts der Tatsache, dass sich durch empirische Ermittlungen die tatsächlichen Ausmaße der Werknutzung zumindest einzelner Werkkategorien mit einem vertretbaren Aufwand ermitteln und somit die Pauschalierungen vermindern lassen, überzeugende Gründe für eine gleichmäßige Verteilung wohl kaum finden lassen. ${ }^{779}$

397 Paradigmatisch ist ein derzeit vor dem OLG München anhängiger Streit um die korrekte Verteilung von Einnahmen durch die VG Wort:

398 Hier entschied erstinstanzlich das LG München I, dass die VG Wort nicht in der in ihren Verteilungsplänen vorgesehenen Art und Weise an Verleger Gebührenanteile ausschütten dürfe, da damit gegen das Willkürverbot gem. § 7 UrhWahrnG verstoßen werde. Zugrunde lag der Fall, dass ein Autor im Jahr 1984 in einem Wahrnehmungsvertrag alle seine bestehenden und zukünftigen Rechte an seinen Werken an die VG Wort abgetreten hat, diese jedoch in ihren Verteilungsplänen pauschale Zahlungen an Verleger (mithin auch an den des betreffenden Autors) vorsieht. ${ }^{780}$ Das LG München I hat

\footnotetext{
${ }^{775}$ So hinsichtlich § 8 UrhWahrnG: Gerlach, in: Wandtke/Bullinger, UrhR, 3. Aufl., 2009, § 8 UrhWahrnG; ebenso die Einflussmöglichkeit von Art. 14 GG bejahend, aber abl. zur konkreten Ausgestaltung von $\S 8$ UrhWahrnG: Augenstein, Rechtliche Grundlagen des Verteilungsplans urheberrechtlicher Verwertungsgesellschaften, 2004, S. $127,142$.

${ }^{776}$ BVerfGE 79, 1, 18 - Urheberrechtliche Vergütung; BVerfG ZUM 1997, 555, 555 f. - Bandübernahmeverträge.

777 So Reinbothe, in: Schricker/Loewenheim, UrhR, 4. Aufl. 2010, § 7 UrhWahrnG Rn. 3.

778 BVerfG ZUM 1997, 555 - Bandübernahmeverträge; BGH GRUR 1988, 782, 783 - GEMA-Wertungsverfahren.

${ }^{779}$ S. dazu als Maßstab nur das kürzlich ergangene Urteil des LG München I MMR 2012, 618 ff., das die Verteilungspraxis der VG Wort als willkürlich einstufte, da diese pauschal Vergütungen an die Verlage ausschüttete, ohne zu ermitteln, ob diesen im Einzelfall tatsächlich Rechte an einem Werk zustanden.

${ }^{780} \S 3$ Nr. 1 des Verteilungsplans Wissenschaft der VG Wort, Fassung 21. Mai 2011, lautet: „Die Verteilungssummen bestehen zur gleichen Hälfte aus einem Urheber- und einem Verlagsanteil. Beide Teile werden den Berechtigten gegenüber gesondert abgerechnet und verteilt“; aus $§ 3$ Nr. 2 und 3 des Verteilungsplans können
} 
darin einen Verstoß gegen das Willkürverbot gesehen, da diese Pauschalierung den Urheber unangemessen benachteilige. Aufgrund des Vorausabtritts seiner Rechte an die VG Wort konnte er an den Verleger keine Rechte mehr abtreten, die diesen dazu berechtigen könnten, an den Ausschüttungen der VG Wort zu partizipieren. ${ }^{781}$ Das Gericht führt weiter aus: „Deshalb wäre das Verteilungssystem für den KI. offensichtlich ungerecht und er würde mit seinem Werk die Abtretungen anderer Autoren finanzieren, die ihrerseits zu Unrecht begünstigt wären, soweit sie nach altem Recht alle Rechte an die Verleger abgetreten haben, bevor die Werke bei der Bekl. gemeldet wurden“. ${ }^{782}$

399 Pauschalierungen seien zwar zu einem gewissen Grad bei der kollektiven Rechtewahrnehmung unvermeidbar, doch sei eine genaue Erfassung der Rechte - d.h. einschließlich der Rechtslage bzgl. etwaiger Abtretungen - nach dem heutigen Stand der Datenverarbeitung der VG Wort ohne unverhältnismäßigen organisatorischen Mehraufwand möglich. ${ }^{783}$ Neben der pauschalen Beteiligung der Verleger an den Ausschüttungen beurteilte das LG München I auch die in den Verteilungsplänen der VG Wort vorgesehene pauschale Beteiligung bestimmter Berufsverbände für willkürlich i.S.v. § 7 UrhWahrnG. ${ }^{784}$ Die VG Wort hätte demnach den „Bezug zum Tatsächlichen“ etwaiger Pauschalierungen durch repräsentative Stichproben herstellen und darlegen müssen, „dass die tatsächlich an die Berufsverbände erfolgten Zahlungen in einem angemessenen Verhältnis zu den Vergütungsansprüchen stehen“. ${ }^{785}$ Da dies unterblieben sei, seien die vorgesehenen Pauschalierungen willkürlich.

400 Gegen die Ansicht des LG wird allerdings eingewandt, dass es gerade willkürlich sei, auf die dingliche Rechtslage und mithin das Prioritätsprinzip abzustellen. ${ }^{786}$ In vielen Fällen sei es zufällig, wer in welcher Reihenfolge die Rechte abgetreten habe, so dass dies kein verlässlicher Anknüpfungspunkt dafür sei, an wen die Einnahmen ausgeschüttet werden dürfen. ${ }^{787}$ Stattdessen sei bei einer kollidierenden Vorauseinräumung auf den Verlagsvertrag abzustellen. ${ }^{788}$ Häufig werde auch auf den Verteilungsplan im Verlagsvertrag ausdrücklich Bezug genommen. ${ }^{789}$ Zwar sei der Verlagsvertrag grundsätzlich nur relativ im Verhältnis zwischen Verlag und Autor von Bedeutung, allerdings gehe es bei dem Streit um den Verteilungsplan auch nur vordergründig um das Verhältnis Autor/Verwertungsgesellschaft, vielmehr gehe es um die Legitimation Autor/Verlag. ${ }^{790}$ Weiterhin sei die Verteilungsautonomie der Verwertungsgesellschaften zu beachten. Eine Grenze befinde sich erst dort, wo eine Quote festgelegt

sich Änderungen dahingehend ergeben, dass dem Verlag im ungünstigsten Fall 38\% der Ausschüttungssumme zustehen.

${ }^{781}$ LG München I MMR 2012, 618, 619; krit. zur Geltung des Prioritätsprinzips im Rahmen der Ausschüttung durch Verwertungsgesellschaften Riesenhuber, ZUM 2012, 746, $749 \mathrm{f}$.

782 LG München I MMR 2012, 618, 619; diametral entgegengesetzt: Riesenhuber, ZUM 2012, 746, 750, demzufolge es gerade willkürlich sei, die Verteilung nach dem Prioritätsprinzip vorzunehmen, da es zufällig wäre, wer und wann die Rechte an die Verwertungsgesellschaft abgetreten hätte.

783 LG München I MMR 2012, 618, 620.

${ }^{784} \S 12$ Nr. 1 des Verteilungsplans Wissenschaft der VG Wort, Fassung 21. Mai 2011, lautet: „Nach Ablauf von 4 Jahren ab dem Jahr, für das Rückstellungen gemäß § 1 Abs. 3 gebildet wurden, werden die für noch nicht wahrnehmungsberechtigte Urheber zurückgestellten Mittel an diejenigen Urheberorganisationen ausgeschüttet, denen Berechtigte ihre Ansprüche übertragen haben und welche die VG WORT entsprechend freistellen"; gem. § 12 Nr. 2 sind diesbezüglich derzeit der Deutsche Hochschulverband, die Gesellschaft Deutscher Chemiker sowie die Deutsche Physikalische Gesellschaft anerkannt.

785 LG München I MMR 2012, 618, 620.

${ }^{786}$ Riesenhuber, ZUM 2012, 746, 750.

${ }^{787}$ Riesenhuber, ZUM 2012, 746, 753.

${ }^{788}$ Riesenhuber, ZUM 2012, 746, $751 \mathrm{f.}$

${ }^{789}$ Riesenhuber, ZUM 2012, 746, 751.

${ }^{790}$ Riesenhuber, ZUM 2012, 746, 753. 
werde, die den Grundsatz missachte, dass die Rechte und deren Erlöse primär dem Urheber zugeordnet werden soll. ${ }^{791}$

401 Für allgemein zu beachtende Grundsätze der Verteilungsmöglichkeiten hat dieser konkrete Streit allerdings nur bedingt Aussagekraft, da es hier um die Verteilung zwischen zwei Parteien mit einem sehr speziellen rechtlichen Verhältnis geht. In den Fällen, in denen es um die Verteilung zwischen bspw. zwei Urhebern geht, die nur über die Mitgliedschaft in der Verwertungsgesellschaft und kein anderweitiges Vertragsverhältnis verbunden sind, hat diese Diskussion wenig Bedeutung, da hier kein schuldrechtliches Korrektiv der dinglichen Rechtslage greifen kann. Zudem beruft sich diese Ansicht zur Begründung der Pauschalierung auf das wirtschaftliche Gebot der Verhältnismäßigkeit, ${ }^{792}$ also dass Pauschalierungen im Interesse eines möglichst geringen Verwaltungsaufwandes hinzunehmen sind. ${ }^{793}$ Andersherum ausgedrückt, dürfen dann keine Pauschalierungen erfolgen, wenn eine Differenzierung ohne unverhältnismäßig viel Verwaltungsaufwand möglich ist, was letztlich mit der Meinung des LG München I übereinstimmt. ${ }^{794}$

402 Da eine möglichst auf die konkrete Nutzung der jeweiligen Werke bezogene Verteilung erforderlich ist, lohnt auch hier ein Blick auf die Praxis der Verwertungsgesellschaften zur Verteilung der aus den Geräteabgaben erzielten Einnahmen:

\section{2. Überblick über die Verteilung der Einnahmen bei Verwertungsgesellschaften}

\section{a) GEMA795}

\section{(1) Überblick}

403 Gem. § 7 UrhWahrnG sind die Einnahmen aus der Tätigkeit der GEMA nach festen Regeln aufzuteilen und die Grundsätze des Verteilungsplans in die Satzung der GEMA aufzunehmen. Für die von der GEMA festgelegten unterschiedlichen Nutzungsbereiche existieren aktuell drei verschiedene Verteilungspläne: $:^{796}$

- A: Das Aufführungs- und Senderecht ${ }^{797}$

- B: Das Mechanische Vervielfältigungsrecht ${ }^{798}$

- C: Der Nutzungsbereich Online ${ }^{799}$

Die Verteilungssumme wird um die in den Verteilungsplänen vorgesehenen Abzüge vermindert und ergibt dann die Nettoverteilsumme. ${ }^{800}$ So sind etwa im Bereich des Verteilungsplanes A von den Gesamteinnahmen die Kosten für die Tätigkeit der GEMA abzuziehen (Aufwendungsersatz nach § 670

\footnotetext{
${ }^{791}$ Riesenhuber, ZUM 2012, 746, 756.

${ }^{792}$ Riesenhuber, ZUM 2012, 746, 756.

793 So schon BGH GRUR 1988, 782, 783.

794 LG München I MMR 2012, 618, 620.

${ }^{795}$ S. zum Verteilungsplan A im Aufführungs- und Senderecht Müller, in: Kreile/Becker/Riesenhuber, Recht und Praxis der GEMA, 2. Aufl. 2008, Kap. 11.1, S. 402 ff., für Verteilungsplan B (sog. mechanische Vervielfältigungsrecht) s. Kap. 11.2, S. 531 ff., für den vorläufigen Verteilungsplan C (Nutzungsbereich Online) s. Kap. 11.3, S. 573 ff.; s. auch die Übersicht der Verteilungspläne bei Brandhorst, virtuos, März 2010, S. 55.

${ }^{796}$ Die Verteilungspläne sind einsehbar im GEMA-Jahrbuch 2011/2012, S. 291 ff.

797 GEMA-Jahrbuch 2011/2012, S. $291 \mathrm{ff}$.

798 GEMA-Jahrbuch 2011/2012, S. $330 \mathrm{ff}$.

799 GEMA-Jahrbuch 2011/2012, S. 345 ff.

${ }^{800}$ Müller, in: Kreile/Becker/Riesenhuber, Recht und Praxis der GEMA, 2. Aufl. 2008, Kap. 11.1, Rn. 206.
} 
BGB). ${ }^{801}$ Von dieser Verteilungssumme werden dann noch weitere Kosten für die Unterstützungskasse für Angestellte sowie für soziale und kulturelle Zwecke ${ }^{802}$ abgezogen, woraus sich dann in diesem Bereich die Nettoverteilungssumme ergibt. ${ }^{803}$ Verteilungsplan B sieht hingegen für die Bestimmung des Aufwandes der GEMA eine Kommission gegenüber den Berechtigten i.H.v. bis zu $25 \%$ vor ( $\S 1$ Abs. 1 Verteilungsplan B); es erfolgt kein Abzug für soziale und kulturelle Zwecke, es sei denn, es handelt sich um Zinserträge, Aufnahme- sowie Verwaltungsgebühren, Konventionalstrafen und andere unteilbare Beträge (§ 1 Abs. 2 Verteilungsplan B). ${ }^{804}$

405 Die jeweiligen Verteilungspläne sehen dann zur Konkretisierung der Verteilung der Nettoverteilsummen eine Vielzahl unterschiedlicher Abrechnungssparten vor. ${ }^{805}$ Sparte $\mathrm{R}$ behandelt etwa die Ausschüttung für die Sendung von Werken im Hörfunk. ${ }^{806}$

(2) Verteilungsarten

406 Die Verteilung erfolgt je nach Sparte entweder nach der sog. individuellen Verteilung, der kollektiven Verrechnung oder der analogen Verrechnung: ${ }^{807}$

- Bei der individuellen Verteilung werden die Einnahmen nach Abzug der Kosten direkt an den/die Berechtigten verteilt (sog. Nettoeinzelverrechnung). ${ }^{808}$

- Bei der analogen Verrechnung erfolgt die Abrechnung entsprechend einer bereits in einer anderen Sparte vorgenommen Verteilung ohne eigenständige Auswertung. ${ }^{809}$

- Bei der kollektiven Verrechnung werden zunächst sämtliche Einnahmen einer Nutzungsart gesammelt und dann eine Verteilung anhand bestimmter Kriterien vorgenommen. ${ }^{810}$

407 Neben der Anzahl der Aufführungen oder Sendeminuten pro Jahr sowie dem Anteil des Berechtigten an dem abzurechnenden Werk ${ }^{811}$ ergibt sich die Höhe der Ausschüttung auf Basis entweder von Minuten- oder Punktwerten. ${ }^{812}$ Wird in einer Sparte der Punktwert bei der Abrechnung berücksichtigt, ${ }^{813}$ legt die GEMA für jedes abzurechnende Musikstück zuvor einen Punktwert für diese Sparte

\footnotetext{
${ }^{801}$ Müller, in: Kreile/Becker/Riesenhuber, Recht und Praxis der GEMA, 2. Aufl. 2008, Kap. 11.1, Rn. 2 ff., s. auch Rn. 5 für den verminderten Kostensatz bei Einnahmen durch ausländische Verwertungsgesellschaften.

${ }^{802}$ Für die Unterstützungskasse für Angestellte ( $§ 1 \mathrm{Abs}$. 3 Verteilungsplan A) und für soziale und kulturelle Zwecke (§ 1 Abs. 4a Verteilungsplan A), s. dazu Müller, in: Kreile/Becker/Riesenhuber, Recht und Praxis der GEMA, 2. Aufl. 2008, Kap. 11.1, Rn. 7 ff.

${ }^{803}$ Müller, in: Kreile/Becker/Riesenhuber, Recht und Praxis der GEMA, 2. Aufl. 2008, Kap. 11.1, Rn. $6 \mathrm{ff}$.

${ }^{804}$ Müller, in: Kreile/Becker/Riesenhuber, Recht und Praxis der GEMA, 2. Aufl. 2008, Kap. 11.2, Rn. $4 \mathrm{ff}$.

${ }^{805}$ S. dazu den Überblick über die Sparten bei Brandhorst, virtuos, März 2010, S. 56; s. auch Müller, in: Kreile/Becker/Riesenhuber, Recht und Praxis der GEMA, 2. Aufl. 2008, Kap. 11.1, Rn. 207 ff.

${ }^{806}$ S. dazu Brandhorst, virtuos, August 2011, S. 49 ff.

${ }^{807}$ Brandhorst, virtuos, März 2010, S. 55 f.

${ }^{808}$ Brandhorst, virtuos, März 2010, S. 55, 56.

${ }^{809}$ S. dazu Brandhorst, virtuos, März 2010, S. 55, 57.

${ }^{810}$ Brandhorst, virtuos, März 2010, S. 55, 56.

${ }^{811}$ Die Anteile werden i.R.d. Verteilungsplanes A in Zwölfteln berechnet, s. § 4 Verteilungsplan A: Brandhorst, virtuos, Mai 2010, S. 52; i.R.d. Verteilungsplanes B hingegen in Prozent, s. § 3 Verteilungsplan B.

812 Brandhorst, virtuos, März 2010, S. 55, 56; Bei Punktwerten ergibt sich bspw. für die Sparte E folgende Formel zur Berechnung der Ausschüttung: Anteile $x$ Punkbewertung $\times$ Punktwert $x$ Anzahl Aufführungen, Brandhorst, virtuos, Mai 2010, S. 52; für die Sparte R hingegen Sendedauer x Punktbewertung x Senderkoeffizient x Minutenwert x Anteil, Brandhorst, virtuos, August 2011, S. 49.

${ }^{813}$ So etwa bin den Sparten E, U und M; s. dazu Brandhorst, virtuos, Mai 2010, S. 52; Brandhorst, virtuos, Mai 2010, S. $52 \mathrm{ff}$.
} 
fest, der sich an der Größe der Besetzung, der Spieldauer und der Art des Werkes orientiert. ${ }^{814}$ Jedem Punkt wird bei der Abrechnung ein Geldbetrag zugewiesen, wodurch eine Abrechnung losgelöst vom individuellen Inkasso erfolgt und das sog. Solidarprinzip der GEMA zum Tragen kommt: Der einheitliche Abrechnungsbetrag pro Punkt führt dazu, dass Berechtigte mit hohem Inkasso (etwa aus großen Veranstaltungshäusern mit hohen Eintrittsgeldern) Berechtigte mit geringerem Inkasso (etwa aus kleinen Veranstaltungshäusern mit geringen Eintrittsgeldern) fördern. ${ }^{815}$

\section{(a) Verfahren zur Hochrechnung von Informationen zur Abrechnung: Das sog. PRO-Verfahren}

In der Sparte U (Live-Veranstaltungen von Unterhaltungs- und Tanzmusik) ${ }^{816}$ fehlten zumindest noch vor einigen Jahren ca. 3/4 bis 6/7 der Informationen über tatsächliche Programme von Veranstaltungen. ${ }^{817} \mathrm{Um}$ anhand der vorhandenen Informationen eine Hochrechnung der Zahl der Aufführungen eines Werkes vorzunehmen, wird von der GEMA seit dem 1.1.1998 bislang noch das sog. PROverfahren genutzt. ${ }^{818}$ Der BGH erläutert dieses Verfahren wie folgt: ${ }^{819}$

„Zunächst werden die in den verwertbaren Programmen angegebenen Aufführungen eines Werkes (genauer: einer Werkversion) gezählt. In einem zweiten Schritt werden die Aufführungszahlen der nicht durch Programme belegten Werkaufführungen hinzugerechnet. Deren Anzahl wird mit Hilfe des so genannten PRO-Faktors ermittelt. Anders als das frühere lineare Hochrechnungsverfahren berücksichtigt das PRO-Verfahren nicht allein die Zahl der durch Programme belegten Aufführungen, sondern auch weitere, ebenfalls den Programmen entnommene Umstände wie die Verteilung der Aufführungsorte auf die Verwaltungsbezirke der Bekl. (GEMA-Bezirke) und die Verteilung der Aufführungszeiten auf die Kalendermonate.

Im Einzelnen wird der so genannte PRO-Faktor wie folgt bestimmt:

\footnotetext{
${ }^{814}$ Die Punktwerte reichen dabei von 12 bis 2400 Punkte; zu den Punktwerten bei E-Musik s. Brandhorst, virtuos, Mai 2010, S. 52, $53 \mathrm{ff}$.

${ }^{815}$ Brandhorst, virtuos, Mai 2010, S. 52, 55; Brandhorst, virtuos, März 2010, S. 55, 57; Müller, in: Kreile/Becker/Riesenhuber, Recht und Praxis der GEMA, 2. Aufl. 2008, Kap. 11.1, Rn. 213.

${ }^{816}$ S. dazu Müller, in: Kreile/Becker/Riesenhuber, Recht und Praxis der GEMA, 2. Aufl. 2008, Kap. 11.1, Rn. 223, wonach in dieser Sparte „(...) Einnahmen aus der Vergabe des Aufführungsrechts nach $\S 19$ Abs. 2 UrhG abgerechnet (werden), die für die einzelnen Veranstaltungen der Unterhaltungsmusik erzielt worden sind. "Veranstaltungen definiert die GEMA als „(...) alle öffentlichen Einzelereignisse, die aus einem bestimmten Anlass stattfinden - dazu zählen zum Beispiel auch Live-Konzerte. Von Veranstaltungen zu unterscheiden sind ständige, zum alltäglichen Geschehen gehörende Musikwiedergaben, also beispielsweise die Musikuntermalung in Geschäften oder Gaststätten“, https://www.gema.de/musiknutzer/lizenzieren/meine-lizenz/veranstalter-vonevents-konzerten-und-theaterauffuehrungen/veranstaltungen.html.

${ }^{817}$ BGH GRUR 2005, 757, 758; LG Berlin ZUM-RD 2000, 340; Müller, in: Kreile/Becker/Riesenhuber, Recht und Praxis der GEMA, 2. Aufl. 2008, Kap. 11.1, Rn. 173; nach eigenen Angaben der GEMA beläuft sich aber der Anteil der vorhandenen Programme nunmehr auf einen erhöhten Anteil von 45\%, s. dazu GEMA, Präsentation INKA, 2012, Folie Nr. 4.

${ }^{818}$ BGH GRUR 2005, 757, 758; Müller, in: Kreile/Becker/Riesenhuber, Recht und Praxis der GEMA, 2. Aufl. 2008, Kap. 11.1, Rn. 173 ff. mwNachw.; zuvor wurde die Hochrechnung linear anhand der vorliegenden Informationen vorgenommen; ab dem Geschäftsjahr 2013 wird dann das PRO-Verfahren durch das sog. InkA-Modell (Abkrz. für „Inkassobezogene Abrechnung im Bereich U-Musik“) ersetzt, s. dazu unten und unter https://www.gema.de/nl/062012/mitgliedernews/inka.html.

${ }^{819}$ Der BGH überprüfte in dieser Entscheidung nicht abstrakt das PRO-Verfahren oder dessen rechtskonforme Aufnahme in die Satzung der GEMA, sondern beschäftigte sich vielmehr mit der Frage, ob ein Urheber - der Kläger - durch die Anwendung des PRO-Verfahrens im Jahr 1998 unbillig benachteiligt wurde. Dies wurde vom Gericht jedoch verneint, BGH GRUR 2005, 757, 759 ff.; zur Verteilgerechtigkeit des PRO-Verfahrens s. bereits LG Berlin ZUM-RD 2000, 340.
} 
Aus der Anzahl der Aufführungsorte und der Anzahl der Aufführungszeiten wird zunächst ein Gewichtungsfaktor (Matrix-Kennzahl) gebildet, der mindestens ein (ein Monat in einem GEMABezirk) und maximal 144 betragen kann (zwölf Monate in zwölf GEMA-Bezirken). Dabei geht die Bekl. auch nach Schließung ihrer Bezirksdirektionen in Düsseldorf und Köln von zwölf Regionen aus. Die Anzahl der Aufführungen einer Werkversion wird mit ihrer jeweiligen MatrixKennzahl multipliziert. Diese Hochrechnung wird anschließend durch einen Normierungsfaktor ausgeglichen, da die Anzahl der Aufführungen infolge der Gewichtung rein rechnerisch ansteigt. So wird gegenwärtig entsprechend dem rechnerischen Anstieg der Aufführungszahl auf das 59-fache die zuvor ermittelte Aufführungszahl durch 59 geteilt. Das wechselnde Verhältnis der durch Programme belegten Aufführungen zu den nicht belegten Aufführungen (derzeit 1/7 zu 6/7) wird dadurch berücksichtigt, dass die gewichtete Hochrechnung nur auf die nicht durch Programme belegten Aufführungen angewandt wird. Die Multiplikation der Matrix-Kennzahl mit dem Normierungsfaktor sowie mit dem Anteil der nicht durch Programme belegten Aufführungen ergibt nach Hinzurechnung des Anteils der durch Programme belegten Aufführungen den PRO-Faktor. Die Zahl aller Aufführungen eines Werkes wird durch Multiplikation der Anzahl der durch Programme belegten Aufführungen mit dem PRO-Faktor ermittelt. ${ }^{\text {(820 }}$

412 Ab 2013 kommt bei der Einnahmenverteilung der GEMA das PRO-Verfahren zur Ermittlung der Aufführungszahlen nicht mehr zum Einsatz. ${ }^{821}$ Stattdessen wird dann eine „inkassobezogene Abrechnung im Bereich der U-Musik“ (INKA) vorgenommen. ${ }^{822}$ Hierbei erfolgt eine inkassoorientierte Zuordnung der gemeldeten Veranstaltung in zwölf Segmente. ${ }^{823}$ Die Bandbreite der Segmente reicht dabei von Inkassi von bis zu 50 Euro bis über 10.000 Euro, sowie einem Auffangtatbestandssegment für Fälle, wo eine Inkassozuordnung nicht möglich ist. ${ }^{824}$ Bis 500 Euro Inkasso erfolgt eine vereinfachte kollektive Verrechnung, bei der jedem aufgeführten Werk einer Veranstaltung abhängig vom Inkassosegment ein bestimmter Punktwert zugeordnet wird. ${ }^{825}$ Jede Veranstaltung wird gesondert im jeweiligen Segment abgerechnet. ${ }^{826}$ Für den Anteil der Veranstaltungen, für die keine Programmmeldung zu Verfügung steht, erfolgt eine lineare Hochrechnung der Aufführungszahlen der Werke unter Berücksichtigung der Programmabdeckungsquote des jeweiligen Segments der Veranstaltung. ${ }^{827}$ In den Segmenten ab 500,01 Euro kommt es hingegen zu einer Nettoeinzelverrechnung des Inkassos der Veranstaltung. ${ }^{828}$ Liegen keine Angaben über das Programm einer Veranstaltung aus einem dieser Inkassosegmente vor, wird das daraus resultierende überschüssige Inkasso gesondert für jedes Segment auf die Veranstaltungen mit Programmnachweis aufgeschlagen und mitverrechnet. ${ }^{829}$

\footnotetext{
${ }^{820}$ BGH GRUR 2005, 757, 758.

${ }^{821}$ GEMA, Präsentation INKA, 2012, Folie Nr. 20.

${ }^{822}$ GEMA, Tagesordnung Mitgliederversammlung 2012, S. 44 ff., insb. 57 ff.; GEMA, INKA FAQs, 2012.

${ }^{823}$ GEMA, Tagesordnung Mitgliederversammlung 2012, S. 58; ferner

https://www.gema.de/nl/062012/mitgliedernews/inka.html.

${ }^{824}$ S. dazu die Übersicht auf https://www.gema.de/nl/062012/mitgliedernews/inka.html.

${ }^{825}$ GEMA, Tagesordnung Mitgliederversammlung 2012, S. 58.

${ }^{826}$ GEMA, Tagesordnung Mitgliederversammlung 2012, S. 58.

${ }^{827}$ GEMA, Tagesordnung Mitgliederversammlung 2012, S. 58.

${ }^{828}$ GEMA, Tagesordnung Mitgliederversammlung 2012, S. 58.

${ }^{829}$ GEMA, Tagesordnung Mitgliederversammlung 2012, S. 58; s. auch GEMA, Präsentation INKA, 2012, Folie Nr. 10, wonach die Verteilung der Einnahmen aus nicht durch Programme belegte Veranstaltungen unter 500 Euro per linearer Hochrechnung, ab 500 Euro hingegen per linearer inkassobezogener Hochrechnung erfolgt.
} 


\section{(b) Abrechnung im Hör- und Fernsehfunk}

413 In der Sparte R (Hörfunk) und FS (Fernsehrundfunk) erfolgt eine Aufteilung der Einnahmen aufgrund der unterschiedlichen betroffenen Verwertungsrechte zu 2/3 zugunsten des Senderechts und zu 1/3 zugunsten des mechanischen Vervielfältigungsrechts und Herstellungsrechts. ${ }^{830}$ Dementsprechend greifen bei der Verteilung dieser Einnahmen sowohl Verteilungsplan A als auch Verteilungsplan B ein. $^{831}$

414 Für die Abrechnung wird die Sendedauer eines einzelnen Werkes grundsätzlich anhand von exakten Angaben zur Spieldauer auf Basis von Sendeanmeldungen der Sendeanstalten vorgenommen. ${ }^{832} \mathrm{Al}-$ lerdings müssen Sendeanstalten unter einem gewissen Inkassobetrag keine Sendeanmeldung vornehmen; ${ }^{833}$ vielmehr wird auf diesen - als unverhältnismäßig angesehen - Aufwand verzichtet und vermutet, dass das Programm der kleineren Sender dem der größeren Sendeanstalten entspricht. ${ }^{834}$ Allerdings ist ein Nachweis durch die Berechtigten der GEMA-Ausschüttung möglich, welcher eine höhere Sendedauer ihrer Werke belegt. ${ }^{835}$ Zudem wird in Sparte R ebenfalls eine Bewertung der einzelnen Musikstücke anhand der gleichen Kriterien wie im Aufführungsrecht vorgenommen, allerdings nicht auf einer Punkteskala von 12 bis 2400 , sondern von Faktor 1 bis $2 \frac{1 / 2 .}{{ }^{836}}$ Zudem ist der Senderkoeffizient $\mathrm{t}^{837}$ und der für das jeweilige Jahr geltende Minutenwert ${ }^{838}$ von Relevanz. Insgesamt ergibt sich somit folgende Formel zur Berechnung der Ausschüttung im Hörfunk: Sendedauer x Punktebewertung $x$ Senderkoeffizient $x$ Minutenwert x Anteil.

\section{(3) Abrechnung im Nutzungsbereich Online}

415 Der Verteilungsplan C regelt den Nutzungsbereich Online. ${ }^{839}$ Ähnlich wie im Rundfunk können von der Nutzung von Werken im Internet unterschiedliche Rechte der Urheber betroffen sein, in diesem Fall abhängig davon, ob das Werk auf einem Server gespeichert wird (§ 16 Abs. 1 UrhG), öffentlich zugänglich gemacht ( $\S 19 a$ UrhG) und der Werkkonsum einen Download erfordert (ebenfalls $\S 16$ Abs. 1 UrhG).$^{840}$ Dementsprechend sieht Verteilungsplan $C$ je nach konkreter Nutzung Sparten vor, die eine Zuordnung zu den unterschiedlichen Nutzungsrechten ermöglichen; wobei sich die Aufteilung an dem jeweiligen Schwerpunkt der einzelnen Nutzung orientiert und ein Rückgriff auf die Grundsätze der Verteilungspläne A und B erfolgt. ${ }^{841} \mathrm{Im}$ Bereich Online findet dann grundsätzlich eine Nettoeinzelverrechnung statt. ${ }^{842}$ Einnahmen aus Internetradioangeboten werden aufgrund des geringen Inkassos ohne Programmabrechnung vorgenommen und mit den Einnahmen der Sparte $R$ abgerechnet, was somit in der Sparte R zu einer Sendeminutenerhöhung führt. ${ }^{843}$

\footnotetext{
${ }^{830} \S 1$ Abs. 1 S. 2 Verteilungsplan A, GEMA-Jahrbuch 2011/2012, S. 291 ff.

${ }^{831}$ S. dazu Müller, in: Kreile/Becker/Riesenhuber, Recht und Praxis der GEMA, 2. Aufl. 2008, Kap. 11.1, Rn. 18a.

${ }^{832}$ Müller, in: Kreile/Becker/Riesenhuber, Recht und Praxis der GEMA, 2. Aufl. 2008, Kap. 11.1, Rn. 177 f.; Brandhorst, virtuos, August 2011, S. 49.

${ }^{833}$ Müller, in: Kreile/Becker/Riesenhuber, Recht und Praxis der GEMA, 2. Aufl. 2008, Kap. 11.1, Rn. 186.

${ }^{834}$ Brandhorst, virtuos, August 2011, S. 49.

${ }^{835}$ Brandhorst, virtuos, August 2011, S. 49.

${ }^{836}$ GEMA-Jahrbuch 2011/2012, S. 313 ff.; Brandhorst, virtuos, August 2011, S. 49.

${ }^{837}$ S. dazu Müller, in: Kreile/Becker/Riesenhuber, Recht und Praxis der GEMA, 2. Aufl. 2008, Kap. 11.1, Rn. 179

ff.; Brandhorst, virtuos, August 2011, S. 49, 50.

${ }^{838}$ Für das Jahr 2010 betrug der Wert 2,7581 Euro, Brandhorst, virtuos, August 2011, S. 49, 50.

${ }^{839}$ GEMA-Jahrbuch 2011/2012, S. 345 ff.

${ }^{840}$ Müller, in: Kreile/Becker/Riesenhuber, Recht und Praxis der GEMA, 2. Aufl. 2008, Kap. 11.3, Rn. $1 \mathrm{f}$.

${ }^{841}$ Müller, in: Kreile/Becker/Riesenhuber, Recht und Praxis der GEMA, 2. Aufl. 2008, Kap. 11.3, Rn. 3, 7.

${ }^{842}$ Müller, in: Kreile/Becker/Riesenhuber, Recht und Praxis der GEMA, 2. Aufl. 2008, Kap. 11.3, Rn. 8.

${ }^{843}$ Müller, in: Kreile/Becker/Riesenhuber, Recht und Praxis der GEMA, 2. Aufl. 2008, Kap. 11.3, Rn. 10.
} 
416 Am Beispiel YouTube ergibt sich mit GEMA-relevanten Werken per Abruf eine Verteilung nach dem Verteilungsplan $C$ in den Sparten MOD und VOD. ${ }^{844}$ Diese Einnahmen werden zu 66,67\% im Rahmen des Verteilungsplans A und zu 33,33\% im Rahmen des Verteilungsplans B mit abgerechnet; sofern es sich nicht um Streaming-Angebote sondern um Downloadangebote per Abruf handelt (Sparten MODVR und VOD-VR), erfolgt eine Abrechnung zu 66,67\% nach Verteilungsplan B und zu 33,33\% in Verteilungsplan $A^{845}$

417 Grundsätzlich wird, wie dargestellt, im Bereich Online eine Nettoeinzelverrechnung durchgeführt, was also in diesem Fall dazu führt, dass nach den Abzügen die Einnahmen vom jeweiligen OnDemand-Anbieter auf die Berechtigten verteilt werden, deren Werke im eingereichten Programm vorkommen. ${ }^{846}$ Der Verteilungsplan sieht allerdings folgende Ausnahme von diesem Grundsatz vor:

„Eine Nettoeinzelverrechnung wird nicht durchgeführt, soweit für Einnahmen aus OnlineNutzungen keine Programme erhältlich sind oder die Kosten für eine Verteilung im Wege der Nettoeinzelverrechnung außer Verhältnis zu den Einnahmen stünden.

In solchen Fällen erfolgt die Verteilung in den Nutzungsbereichen Music-on-Demand und Ruftonmelodien als Zuschlag in den Sparten des jeweiligen Nutzungsbereichs. Die Erträge im Nutzungsbereich Internetradio werden zugunsten der Sparten des Tonrundfunks gemäß den Verteilungsplänen A und B verrechnet. In den Nutzungsbereichen Internet-TV, Websites und Cinema-/Video-on-Demand werden die Erträge zugunsten der Sparten des Fernsehrundfunks gemäß den Verteilungsplänen $A$ und $B$ verrechnet. Für das Filmherstellungsrecht erfolgt die Verteilung in den genannten Fällen zugunsten der mechanischen Vervielfältigungsrechte der Sparte des Fernsehrundfunks gemäß dem Verteilungsplan B. ${ }^{\text {} 847}$

420 Das bedeutet also u.a., dass - sofern kein Programm von YouTube zur Erfassung vorhanden ist - die erzielten Einnahmen prozentual in den jeweiligen Sparten der Verteilungspläne A und B hinzuaddiert und entsprechend den dort geltenden Grundsätzen für nicht werk- und nutzungsbezogene verteilt werden. $^{848}$

(4) Geräteabgaben

421 Die von der GEMA aus den Geräteabgaben gem. $\S \S 54 \mathrm{ff}$. UrhG ${ }^{849}$ erzielten Einnahmen werden zu den anderen Abrechnungssparten hinzuaddiert - bei Bildtonträgern im Audiobereich zu 95\% dem Rundfunkbereich und zu 5\% den Lizenzeinnahmen aus der Tonträgerindustrie. ${ }^{850}$ Es wird also angenommen, dass die privat vervielfältigten Titel zu 95\% denen aus dem Rundfunk entsprechen, wobei nicht ersichtlich wird, auf welcher Grundlage diese Verteilung zustande gekommen ist. Im Rundfunkbzw. Tonträgerbereich wird der Betrag entsprechend den dort geltenden Regeln mitverteilt. ${ }^{851}$ Somit

\footnotetext{
${ }^{844}$ GEMA-Jahrbuch 2011/2012, S. 345 ff.

${ }^{845}$ GEMA-Jahrbuch 2011/2012, S. 347.

${ }^{846}$ Müller, in: Kreile/Becker/Riesenhuber, Recht und Praxis der GEMA, 2. Aufl. 2008, Kap. 11.1, Rn. 319.

${ }^{847}$ GEMA-Jahrbuch 2011/2012, S. 345 f.

${ }^{848}$ Zum Zuschlag in Verteilungsplan A s. GEMA-Jahrbuch 2011/2012, S. 295 f.

${ }^{849}$ S. dazu Staudt, in: Kreile/Becker/Riesenhuber, Recht und Praxis der GEMA, 2. Aufl. 2008, Kap. 10, Rn. $221 \mathrm{ff}$.

${ }^{850}$ Abschnitt VI Ziffer 8 des GEMA-Verteilungsplans; vgl. auch die Parallelregelung in Abschnitt IV Ziffer 8 für die Industrie.

${ }^{851}$ Kreile, GRUR Int. 1992, 24, 36.
} 
kommt es also in den betroffenen Sparten zu einem Zuschlag für jeden Berechtigten bzw. zu einer Erhöhung des Minutenwertes. ${ }^{852}$

\section{b) VG WORT}

422 Die Geräte- und Speichermedienvergütung nach § 54 UrhG stellt die Haupteinnahmequelle der VG WORT dar. ${ }^{853}$ Allerdings konnte die VG WORT ebenso wie die GEMA im audiovisuellen Bereich ${ }^{854}$ für Vervielfältigungsgeräte und Speichermedien seit 2008 so gut wie keine Einnahmen erzielen. ${ }^{855}$ Das liegt zum einen an fehlenden Einigungen mit der Industrie über die mit der tatsächlichen Nutzung der Gerätetypen zusammenhängenden Abgabe ${ }^{856}$ (wie es das Urheberrecht seit 2008 vorsieht), ${ }^{857}$ zum anderen an fehlenden Einigungen zwischen den beteiligten Verwertungsgesellschaften über die Aufteilung der Abgaben. ${ }^{858}$ Die VG WORT und die GEMA kritisieren gleichermaßen in diesem Zusammenhang die mit dem Zweiten Korb der Urheberrechtsreform geschaffenen Bestimmungen hinsichtlich der Geräteabgaben. ${ }^{859}$

423 Die Einnahmen aus diesen Abgaben werden dann aber grundsätzlich anhand der von der VG WORT durchgeführten Ermittlungen hinsichtlich der Kopieranteile in den einzelnen Sparten mitverteilt. ${ }^{860}$ Für die Sparte Hör- und Fernsehrundfunk etwa sind die Minutenzahl der Sendung, die Bewertung der Kategorie des Werkes und die Einstufung der Sendeanstalten ausschlaggebend. ${ }^{861}$ In der Sparte der Bibliothekstantiemen werden hingegen $20 \%$ der Einnahmen zu gleichen Teilen an die Berechtigten verteilt; ${ }^{862}$ die Verteilung der restlichen $80 \%$ erfolgt mittels hochgerechneter Ausleihstatistiken, ${ }^{863}$ die stichprobenartig jedes Jahr von 12 nach bestimmten Kriterien ausgewählten Bibliotheken übermittelt werden. ${ }^{864}$ Die Ergebnisse der Stichproben der letzten drei Jahre werden dann addiert und gedrittelt. Der so errechnete Mittelwert ist die Grundlage der Auszahlung. ${ }^{865}$.

\section{c) VG Bild-Kunst}

424 Auch die VG Bild-Kunst erzielt einen Großteil ihrer Einnahmen aus den Geräte- und Betreiberabgaben nach $\S \S 54 \mathrm{ff}$. UrhG. ${ }^{866}$ Die Einnahmen aus Fotokopie-Geräteabgaben werden auf Basis eines Vertei-

\footnotetext{
${ }^{852}$ Kreile, GRUR Int. 1992, 24, 36.

${ }^{853}$ VG WORT, Geschäftsbericht 2011, S. 2, 8 f.

${ }^{854}$ Der andere Bereich neben dem audiovisuellen ist der der Reprographie.

${ }^{855}$ VG WORT, Geschäftsbericht 2011, S. 3; GEMA-Jahrbuch 2011/2012, S. 39.

${ }^{856}$ VG WORT, Geschäftsbericht 2011, S. 3; GEMA-Jahrbuch 2011/2012, S. 39.

${ }^{857}$ S. eing. Staudt, in: Kreile/Becker/Riesenhuber, Recht und Praxis der GEMA, 2. Aufl. 2008, Kap. 10, Rn. 228.

${ }^{858}$ VG WORT, Geschäftsbericht 2011, S. 3; VG Bild-Kunst: „Es bedarf erheblicher Anstrengung, einen Wertevergleich zwischen einem Bild, einem Song und einem Film zu entwickeln; in einem weiteren Schritt gilt es dann, im Film die Ansprüche von Drehbuch, Regie, Produzent, Darstellern, Filmmusik usw. anteilig zu definieren. Nachdem Untersuchungen über die tatsächliche Nutzung von Werken vorliegen, gehen wir davon aus, dass es noch in 2012 zu einem Abschluss kommen wird und dann wenigstens die bei der ZPÜ noch vorhandenen Rückstellungen verteilt werden können.", Geschäftsbericht 2011, S. 1.

${ }^{859}$ VG WORT, Geschäftsbericht 2011, S. 3; GEMA-Jahrbuch 2011/2012, S. 39.

${ }^{860}$ VG WORT, Verteilungsplan, S. 8 ff., $\S \S 23$ ff., $41 \mathrm{ff}$.

${ }^{861}$ VG WORT, Verteilungsplan, S. 12, §§ $25 \mathrm{ff}$; bzw. 13 f., $\S \S 31 \mathrm{ff}$.

${ }^{862}$ VG WORT, Verteilungsplan, S. 8, § 10 Abs. 1 a).

${ }^{863}$ VG WORT, Verteilungsplan, S. 8, § 10 Abs. 1 b).

${ }^{864}$ Zusatzvereinbarung zum Vertrag über die Abgeltung urheberrechtlicher Ansprüche nach § 27 des Urhebergesetzes für die Jahre 2010 bis 2014, abrufbar unter:

http://www.bibliotheksverband.de/fileadmin/user_upload/DBV/vereinbarungen/Vertrag_27Abs2UrhG_unter schrieben_20110814.pdf.

${ }^{865}$ VG WORT, Auszahlungen Belletristik und Kinderbücher.

${ }^{866}$ VG Bild-Kunst, Geschäftsbericht 2011, S. 2 f.
} 
lungsbeschlusses nach festen Quoten auf die einzelnen Berufsgruppen verteilt. ${ }^{867}$ Die „Geräteabgaben Fotokopie“ werden zu 5\% dem Bereich „Fotokopieren in Schulen“ und zu 95\% der „Betreiberabgabe" zugeführt. ${ }^{868}$ Bei der Berechnung der Anteile für das Fotokopieren aus Büchern werden zudem zuvor festgelegte Multiplikatoren berücksichtigt. ${ }^{869}$ Der Verteilungsbeschluss basiert an dieser Stelle auf Empfehlungen von Berufsgruppenversammlungen. ${ }^{870}$ Die Geräte- und Leerkassettenabgaben im Bereich Film werden zwischen den an dem Werk Beteiligten nach festen Prozentsätzen verteilt. ${ }^{871}$ Zudem wird für jeden Film ein Punktewert gebildet, der sich aus der Multiplikation des Zeitfaktors, der Ausstrahlungsdauer, der Senderwerte und dem Werkfaktor ergibt. ${ }^{872}$

425 Die weitere Verteilung von Einnahmen etwa aus dem Bereich des Senderechts ergibt sich aus der Bewertung des Senders, der Anzahl der Ausstrahlungen und der Dauer der Ausstrahlung. ${ }^{873}$ Besondere Regeln gelten allerdings für aktuelle Berichterstattungen. ${ }^{874}$

426 Die Verteilung der Bibliothekstantiemen richtet sich hingegen nach der Ausleihfrequenz, dem Multiplikator der Werkart und des Empfangsberechtigten, dem Zeitraum für Bewertungen von Altauflagen sowie den Mindest- und Höchstgrenzen der Verteilung. ${ }^{875}$ Die erforderlichen Daten werden ebenso wie die bei der VG WORT ermittelt. ${ }^{876}$

\section{Erfassung nach Nutzungsintensität: Folgen für die Kulturflatrate}

427 Wie die oben dargelegten Verteilungsschlüssel der Verwertungsgesellschaften und die rechtlichen Vorüberlegungen gezeigt haben, ist die Verteilung nach Nutzungsintensität von Werken ein entscheidender Schlüssel, den auch eine Kulturflatrate bzw. Abgabe zu berücksichtigen hätte. So wie etwa von der GEMA unterschiedliche Werkkategorien festgelegt werden, müssten auch für die Kulturflatrate zunächst bestimmte Klassen gebildet werden, z.B. Musik, Film oder E-Book (oder sonstige digitale Inhalte). In einem zweiten Schritt müsste die Nutzungsintensität und -häufigkeit ermittelt werden, insbesondere wie häufig ein Werk heruntergeladen, angeboten oder bearbeitet wurde. ${ }^{877}$

428 Entgegen der oft zu hörenden Kritik an der Kulturflatrate, dass diese nicht geeignet sei, erfolgreiche Urheber angemessen zu entlohnen und die Verteilung problematisch sei, sind gerade die Systeme, die die Internetnutzung erfassen, weit mehr und präziser in der Lage, die Beliebtheit und den Nutzungsgrad von Werken zu erfassen. Hier ist vorweg anhand der oben dargestellten Verteilungspläne daran zu erinnern, dass auch im Bereich der Geräteabgabe und selbst bei unmittelbar einzuholenden Lizenzen im Rahmen der derzeitigen Modelle der Verwertungsgesellschaften oftmals wenig verlässli-

\footnotetext{
${ }^{867}$ VG Bild-Kunst, Verteilungsplan, S. $8 \mathrm{f}$.

${ }^{868}$ VG Bild-Kunst, Verteilungsplan, S. 9.

${ }^{869}$ VG Bild-Kunst, Verteilungsplan, S. 9.

${ }^{870}$ VG Bild-Kunst, Verteilungsplan, S. 8.

${ }^{871}$ VG Bild-Kunst, Verteilungsplan, S. 19.

${ }^{872}$ VG Bild-Kunst, Verteilungsplan, S. 20.

${ }^{873}$ VG Bild-Kunst, Verteilungsplan, S. 4.

${ }^{874}$ Hier sind 10 Werke je Sendung vergütungsfrei, VG Bild-Kunst, Verteilungsplan, S. 2 f.

${ }^{875}$ VG Bild-Kunst, Verteilungsplan, S. 6 f.

${ }^{876}$ Zusatzvereinbarung zum Vertrag über die Abgeltung urheberrechtlicher Ansprüche nach § 27 des Urhebergesetzes für die Jahre 2010 bis 2014, abrufbar unter:

http://www.bibliotheksverband.de/fileadmin/user_upload/DBV/vereinbarungen/Vertrag_27Abs2UrhG_unter schrieben_20110814.pdf; hinsichtlich des konkreten Verteilungsvorgangs kann aufgrund des gleichen Datenerhebungsverfahrens davon ausgegangen werden, dass die VG Bild-Kunst ebenso verfährt; für Details s. oben Rn. $422 \mathrm{ff}$.

${ }^{877}$ S. auch Roßnagel et al., Gutachten, 2009, S. 30.
} 
che Zahlen vorliegen, die zudem oft auf Hochrechnungen etc. basieren. ${ }^{878}$ Wie zu zeigen sein wird, können dagegen verschiedene, in Kombination anzuwendende Systeme einen größeren Präzisionsgrad bei der Verteilung der Vergütungen erreichen - ohne dass dazu notwendigerweise die Identität der jeweiligen Nutzer (bzw. ihre IP-Adresse) genutzt werden müsste:

\section{a) Messverfahren}

429 Um möglichst genau die Nutzungsintensitäten zu erfassen, muss gewährleistet sein:

- Dass Werke erfasst sind und identifiziert werden können

- Die Tausch- bzw. Download- und Uploadvorgänge quantitativ erfasst werden können

- Gleichzeitig ist ein höchstmöglicher Datenschutz zu gewährleisten, möglichst Anonymität

- Missbräuche müssen möglichst ausgeschaltet werden

(1) Zentrale Datenbank

430 Datenbanken zum Abgleich von Down- und Uploadvorgängen mit registrierten Werken stellen das Herzstück einer punktgenauen Verteilung der Vergütungen an die Kreativen dar. Ohne einen wie auch immer gearteten Abgleich zwischen Files, die im Datenverkehr oder auf PCs von Nutzern vorzufinden sind, kann eine genaue Ermittlung der Nutzung von Werken nicht erfolgen. ${ }^{879}$

431 Dies ist indes kein Novum; selbst im Bereich traditioneller Medien bedienen sich Sendehäuser etc. entsprechender Datenbanken, anhand derer die Rückmeldungen erfolgen. Aber auch sonst existieren zahlreiche Datenbanken wie Gracenote ${ }^{880}$ oder Audible Magic, ${ }^{881}$ die Audiofingerprints abgleichen; in gleicher Weise stützen sich Anwendungen wie Shazam auf vergleichbare Datenbanken mit Audiostrukturen. ${ }^{882}$ Parallel dazu finden sich auch freie Datenbanken wie freedb.org. ${ }^{883}$ Zahlreiche dieser Datenbanken werden in Media Playern eingebettet, etwa durch Funktion wie „Autotagger" im Rahmen des weit verbreiteten (und kostenlosen) Musikplayers Winamp. ${ }^{884}$ Dies gilt ebenso für zahlreiche andere Tools, die dem Nutzer helfen, seine Musiksammlungen mit weiteren Daten aus dem Internet zu vervollständigen.

432 Mit Hilfe dieser Audio-Fingerprints können filigrane Abgleiche durchgeführt werden, indem kurze Teile eines Musikstücks mit den in der Datenbank hinterlegten Charakteristika verglichen werden, so dass schon allein durch die sog. Hüllkurve (also das typische gesamte Audiosignal) eine sehr zuverlässige Identifizierung des jeweiligen Stückes möglich ist. Eine solche Technik scheint auch nicht auf Musik beschränkt zu sein, auch wenn etwa für Videodateien eine wohl komplexere Analyse erforderlich ist - wie aber die Suchfunktionen bei Videoportalen wie YouTube zeigen, scheint auch dies nicht

\footnotetext{
${ }^{878}$ S. oben Rn. 274 ff.; dies wird auch angemerkt bei BigChampagne Online Media Measurement, Monitoring and Identifying P2P Media, S. 2, unter Verweis auf die schon immer auf Hochrechnungen basierende Messung beim traditionellen Rundfunk, die aber schon lange nicht mehr in Frage gestellt werde.

${ }^{879}$ Aigrain, 2012, S. 149 f.

${ }^{880} \mathrm{http}: / /$ www.gracenote.com: Nach den dortigen Angaben werden 130 Millionen Musiksongs gespeichert.

${ }^{881}$ http://audiblemagic.com/.

${ }^{882}$ Zur Funktionsweise von Shazam s. Wang, Communications of the ACM, Vol. 49, No. 8, 2006, 44, $46 \mathrm{ff}$.; Shazam greift inzwischen auf eine Datenbank mit mehr als 20 Mio. Musiktiteln zu, s. http://netzwertig.com/2012/09/18/shazam-will-second-screen-erobern-der-naechste-gigant-entsteht/. 883 http://www.freedb.org/.

${ }^{884}$ S. einen Überblick zu den Funktionen, zu denen auch der Auto-Tagger gehört, unter http://www.winamp.com/help/Player_Features.
} 
ausgeschlossen zu sein. ${ }^{885}$ Auch wird der Einsatz solcher Software im Musikbereich durch britische Verwertungsgesellschaften (PRS for Music) berichtet, um genauere Informationen über die Häufigkeit der Sendung bestimmter Musikstücke zu erlangen. ${ }^{886}$ Allerdings bedingt die Verwendung dieser „Fingerprints“, dass die Hüllkurven durch kurzfristige Analyse des Files abgeglichen werden müssen was zeitaufwendiger ist als der unmittelbare Vergleich bereits ohne Analyse vorliegender Metadaten.

433 Dem Vorbild etwa von Gracenote und Audible Magic folgend, würde eine solche Datenbank zahlreiche Meta-Informationen enthalten, z.B. über Komponist, Interpret, Rechteinhaber (Verlag) etc. $^{887}$ - was sich problemlos auch auf andere Werke übertragen ließe, wie etwa Filme, wobei hier die Daten angesichts der zahlreichen involvierten Kreativen wesentlich umfangreicher wären. Die Eintragung der entsprechenden Dateien könnte ohne weiteres den Kreativen oder den Rechteinhabern überlassen bleiben, da sie ein Eigeninteresse haben, an der Vergütung zu partizipieren ${ }^{888}-$ nicht anders als heute teilweise auch bei den Verwertungsgesellschaften, wie etwa der VG Wort, bei deren Online-Datenbanksystem der Autor seine Werke anmelden muss. Über einen „unique identifier“ wie etwa den bereits gebräuchlichen DOI-Standard (Digital Object Identifier ${ }^{889}$ ) oder die an die konkrete Aufnahme gekoppelten ISRC-Codes ${ }^{890}$ könnten dann die Werke zugeordnet werden.

434 Generell werden auch auf internationaler Ebene derartige Datenbanken für die Vereinfachung und Automatisierung der Lizenzierung dringend empfohlen, auch unter Beteiligung des Staates, da die Rechteeinräumung gerade im Downloadbereich oftmals als extrem umständlich und damit als Innovationshindernis empfunden wird. ${ }^{891}$ Demgemäß könnte eine derartige Datenbank gleichzeitig für kommerzielle Anwender die nötigen Lizenzinformationen bzw. Angebote für eine automatisierte Rechtevergabe enthalten, so dass sie mehrere Vorteile in sich vereinigen würde.

435 Für alle Methoden, ob Fingerprinting oder Metadaten, gilt aber, dass es nicht darauf ankommt, zu ermitteln, wer die Daten herunterlädt - es genügt, die heruntergeladene Datei zu analysieren, so dass es nicht der Erhebung personenbezogener Daten bedarf. ${ }^{892}$

\footnotetext{
${ }^{885}$ S. dazu bspw. das Projekt „Digitale Wasserzeichen“ des Fraunhofer SIT: https://www.sit.fraunhofer.de/de/angebote/projekte/wasserzeichen/.

${ }^{886}$ Leeb, Der Wert künstlerischer Arbeit, 2009, S. 155.

${ }^{887}$ S. die Produktbeschreibung von Audible Magic, abrufbar unter:

http://www.audiblemagic.com/technology.php.

${ }^{888}$ S. auch Aigrain, 2012, S. 150; auf diesem Weg werden werden schon jetzt die kommerziellen Datenbanken wie die von Audible Magic gespeist, s. dazu die Selbstbeschreibung: „Record labels, artists, movie and television studios from around the world register content directly with Audible Magic for content they want protected. A full complement of metadata and business usage rules are provided on lookup matches", s. http://www.audiblemagic.com/technology.php.

${ }^{889}$ Zur Funktionsweise von DOI s. die Beschreibung des Börsenvereins des Deutschen Buchhandels e.V., abrufbar unter: http://www.boersenverein.de/de/181906; zu Einsatzmöglichkeiten in der Praxis s. ferner Langston/Tyler, Internet and Higher Education, Vol. 7, 2004, 51, $54 \mathrm{ff}$.

${ }^{890}$ S. zu ISRC-Codes die Beschreibung auf der Internetseite des Bundesverbands Musikindustrie e.V.: http://www.musikindustrie.de/isrc/; dazu auch: BigChampagne Online Media Measurement, Monitoring and Identifying P2P Media, S. 3.

${ }^{891}$ So für UK bzw. die EU Hargreaves, Digital Opportunity, 2011, S. 29 ff.

${ }^{892}$ Dies wird auch betont in BigChampagne Online Media Measurement, Monitoring and Identifying P2P Media,
} S. 3. 
(2) Passive Datenerhebung

436 Die Erfassung von Werken ist aber nur der erste Schritt, um die Nutzung von Werken quantitativ zu erfassen. Daneben bedarf es der Messung der jeweiligen Werknutzung bzw. der Down- und Uploadvorgänge:

437 Die erste erfolgversprechende - und soweit ersichtlich auch schon angewandte Methode - bestünde in der sog. passiven Erhebung (passive measurement), die die in P2P-Netzwerken bereitgestellten und getauschten Dateien erfasst. Da alle an ein P2P-Netzwerk angeschlossenen Nutzer für jeden im Netzwerk befindlichen Teilnehmer Dateien zum Download zur Verfügung stellen, können diese Angebote durchsucht und damit auch die Zahl bzw. die jeweiligen Musikstücke erhoben werden, wiederum durch die Audio-Fingerprinting-Methode oder auch durch Metatags bzw. ID3v-TagInformationen, die häufig den Musikstücken beigefügt sind. ${ }^{893}$

438 Derartige Verfahren macht sich offenbar die Musikindustrie schon seit geraumer Zeit zunutze - und nicht zur Rechtsverfolgung, sondern um Erkenntnisse über die Musikgewohnheiten der Nutzer zu gewinnen. Schon vor einem Jahrzehnt begann das Unternehmen BigChampagne im Rahmen von Napster die Häufigkeit der Nutzung bestimmter Musikstücke auszuwerten. Später erstreckten sich die angewandten Methoden auch auf P2P-Netzwerke ohne zentralen Server sowie andere BitTorrent-Portale etc. Die Musikindustrie ebenso wie einzelne Musiker nutzen offenbar diese Dienste, um zu ermitteln, welche Musikstücke beim Publikum besonders Anklang finden. ${ }^{894}$ Ein Nachteil dieser Methode liegt darin, dass sich das Nutzerverhalten hinsichtlich der eingesetzten Technologien ständig verändert und somit u.U. die einmal in die Beobachtung aufgenommenen P2P-Netzwerke mit der Zeit nicht mehr repräsentative Zahlen widerspiegeln könnten. ${ }^{895}$ Fraglich ist auch, ob mit diesen Methoden die immer beliebteren Filehosting-Provider bzw. die dortigen Up- und Downloadvorgänge wirkungsvoll erfasst werden können. Der Vorteil liegt wiederum darin, dass für diese Form der Messung keine offizielle Kooperation mit Providern erforderlich ist, ${ }^{896}$ was den finanziellen Gesamtaufwand der Umstellung auf ein Messsystem verringern würde.

(3) Messung auf Providerebene

439 Ferner könnten Provider den Datenverkehr mit Hilfe der oben dargestellten Methoden darauf untersuchen, ob und welche Dateien herauf- und heruntergeladen werden. In einem gewissen Sinne wären erste Schritte auch im Rahmen einer Ausdifferenzierung der Netzkapazitäten damit verbunden, wie die Debatte um die Netzneutralität zeigt; demnach sind Provider offenbar durchaus in der Lage, nach der Art von Daten zu differenzieren, sofern keine oder keine hinreichende Verschlüsselung eingesetzt wird. ${ }^{897}$ Dann läge der Schritt nicht fern, sie bezüglich der getauschten Files in die Pflicht zu nehmen, diese näher zu analysieren.

440 Gängig sind zwei verschiedene Methoden: Das sog. Flow monitoring und die Deep Packet Inspection (DPI) ${ }^{898}$ Für beide Methoden gilt allerdings, dass sie Probleme hinsichtlich der zu überprüfenden Masse an Daten aufwerfen, so dass sie letztlich nur stichprobenartig angewandt werden können, nicht aber flächendeckend. Praktisch unmöglich ist dagegen die Überprüfung von Daten, die ver-

\footnotetext{
${ }^{893}$ S. dazu http://de.wikipedia.org/wiki/ID3-Tag.

${ }^{894}$ S. dazu http://de.wikipedia.org/wiki/BigChampagne.

895 Oberholzer-Gee/Strumpf, 2010, S. 12.

${ }^{896}$ So BigChampagne Online Media Measurement, Monitoring and Identifying P2P Media, S. 7.

${ }^{897}$ Im Zusammenhang mit der Diskussion um die Netzneutralität wird die „Deep Packet Inspection“Technologie angesprochen, Görisch, EuZW 2012, 494, 495; Frevert, MMR 2012, 510 f.; dazu sogleich.

${ }^{898}$ Oberholzer-Gee/Strumpf, 2010, S. 11.
} 
schlüsselt sind ${ }^{899}$ was anscheinend zunehmend gerade im Filesharing-Bereich auftritt - allerdings nicht zuletzt durch die Rechtsverfolgungsmaßnahmen bedingt, die bei einer Kulturflatrate teilweise entfallen würden.

441 Das „Flow monitoring" untersucht dabei Datenpakete auf der Router-Ebene, indem die HeaderInformationen und die Ports untersucht werden, weist aber aufgrund der eher "flachen" Untersuchungsdichte ein hohe Fehlerquote auf. ${ }^{900}$

442 Größere Genauigkeit erlaubt dagegen die „Deep Packet Inspection“-Technologie, die sich nicht nur auf den Packet Header verlässt und eine Identifizierung von Dateien aus dem Datenstrom ermöglicht, dabei allerdings auch hohe technologische Anforderungen stellt. ${ }^{901}$ In der Praxis wird durch das Unternehmen Sandvine das Filesharing auf diese Art und Weise untersucht, mit dem interessanten Ergebnis, dass bis 2008 ca. 61\% des Datenverkehr-Upstreams (und ca. 22\% des Downstreams) auf Filesharing zurückzuführen waren. ${ }^{902} \mathrm{DPI}$ arbeitet dabei auf den Levels 4 bis 7 des OSI-Modells. ${ }^{903} \mathrm{DPI}-$ Systeme analysieren das gesamte Datenpaket und filtern dessen Inhalt nach vordefinierten Regeln und Suchbegriffen. Genauer gesagt, werden alle Daten innerhalb eines Datenpaketes (packet payload) mit einer Datenbank abgeglichen. Darüber hinaus kann dieser statische Abgleich um statistische oder historische Algorithmen ergänzt werden. ${ }^{904}$ Bei der simpleren Analyse von HeaderInformationen werden nur wenige Daten abgeglichen. Die dazu benötigten Felder innerhalb des Datenpaketes sind zudem durch Protokoll-Standards genauestens definiert und beschränkt. Eine Suche im erheblich größeren und nicht definierten Bereich des Datenpaketes ist aufgrund dessen erheblich aufwendiger und somit teurer. Durch die Vielzahl an möglichen Suchbegriffen stellt dies auch einen wesentlich größeren Aufwand an Rechenleistung dar. ${ }^{905}$ Wie allerdings bereits dargelegt, begegnet jedenfalls in Deutschland ein DPI-Verfahren erheblichen Bedenken im Hinblick auf Art. 10 GG. ${ }^{906}$

443 Zudem dürfte dies einen Quantensprung hinsichtlich der Kosten und der erforderlichen Ressourcen darstellen, da etwa Fingerprintanalysen auch bei schnellen Rechnern umfangreiche Kapazitäten er-

\footnotetext{
${ }^{899}$ Bedner, CR 2010, 339, 342.

900 Shalunov/Teitelbaum, TCP Use and Performance on Internet2, ACM SIGCOMM Internet Measurement Workshop, 2001, S. $1 \mathrm{ff}$.

901 Bedner, CR 2010, 339 ff., insb. 341 f.; Spies, MMR 2008, XII.

902 Sandvine, 2008 Global Broadband Phenomena, S. 3; s. auch Ferguson, Trends and Statistivs in Peer-to-Peer, Präsentation d. CasheLogic Workshop on Technical and legal aspects of peer-to-peer television, 2006, S. 4, der noch 50-65\% des Downstreams und 75-90\% des Upstreams P2P zuweist; aktuelle Zahlen sind dagegen weitaus geringer (39,6\% im Upstream und 6,4\% im Downstream für Amerika; bzw. 51,4\% und 14,9\% für Europa) - was vermutlich nicht zuletzt durch den starken Anstieg von sog. „Real-Life-Entertainments“ verursacht wurde, Sandvine, Global Internet Phenomena Report, 2013, S. 5, 12.

${ }^{903}$ Grundlegend zum OSI-Modell Day/Zimmermann, Proceedings of the IEEE, Vol. 71, 1983, $1334 \mathrm{ff}$.

${ }^{904}$ Anderson, Deep packet inspection meets 'net neutrality, CALEA', 2007; Allot Communications, Digging deeper into deep packet inspection (DPI), 2007; Deep Packet Inspection and Internet Censorship: International Convergence on an 'Integrated Technology of Control', 2009, S. 4, abrufbar unter: http://advocacy.globalvoicesonline.org/wp-content/uploads/2009/06/deeppacketinspectionandinternetcensorship2.pdf.
}

\section{${ }^{905}$ Porter, The Perils of Deep Packet Inspection, 2010. \\ ${ }^{906}$ S. dazu oben Rn. 255 ff.; wie hier auch Runge, GRUR Int. 2007, 130, 135.}


fordern. ${ }^{907}$ Eine Beschränkung auf Stichproben wäre daher auf jeden Fall erforderlich. Zudem können diese Verfahren verschlüsselte Dateien nicht erfassen. Allerdings wären derartige Methoden im Gegensatz zur passiven Durchsuchung von P2P-Netzwerken keineswegs darauf beschränkt, sondern würden auch bei Filesharing-Hostern wie Rapidshare greifen, da spätestens beim Up- und Downloadvorgang die Files analysiert werden können. Eine Erfassung der Nutzer bzw. ihrer IP-Adressen wäre, abgesehen von einer groben Geolokalisierung, um ausländische Files und Nutzungen auszuschließen, nicht erforderlich. ${ }^{908}$

\section{(4) Messung auf Nutzerebene}

444 Aber auch die Messung auf Nutzerebene ist möglich: Eine Variante bestünde darin, dass die eingesetzte P2P-Software selbst die entsprechenden Daten analysiert und an eine zentrale Meldestelle weitergibt - wiederum ohne dass die Identität des Nutzers dadurch offenbart werden müsste. Allerdings impliziert dies, dass die Entwickler der P2P-Software entsprechende Tools vorsehen - was gerade bei Open Source-Entwicklungen schwierig sein dürfte. ${ }^{909}$

445 Erfolgversprechender dürfte es sein, derartige Analysetools auf freiwilliger Basis bei den Nutzern einzusetzen - vergleichbar den Set-Top-Boxen, die im Bereich TV und Rundfunk stichprobenartig aufgrund der Kooperation mit Zuschauern bzw. Nutzern verwandt werden, sog. Sampling. ${ }^{910}$

446 In Deutschland kommen insbesondere das „TC Score“- und das „TC UMX“-Verfahren zum Einsatz. Bei der „TC Score“-Technik misst ein Gerät im Haushalt der freiwilligen Teilnehmer das An-, Ab- und Umschalten von Geräten sowie weitere Ereignisse, wie etwa die Nutzung des Teletextes. ${ }^{911}$ Durch ein Anmeldeverfahren vor der Konsumierung von Fernsehinhalten können Fernsehnutzungen auf die Sekunde genau einzelnen Nutzern bzw. Alters- und Geschlechtergruppen zugeordnet werden. ${ }^{912}$ Das Messgerät wird dann über einen Festnetz- oder GSM/UMTS-Anschluss mit der Zentrale verbunden und die ermittelten Daten übertragen. ${ }^{913}$ Nach Angaben der Arbeitsgemeinschaft Fernsehforschung (AGF) können mit dieser von der Gesellschaft für Konsumforschung (GfK) entwickelten Methode sämtliche klassische Empfangswege Kabel, Terrestrik, Satellit (jeweils analog und digital) sowie analoge Videorecorder, aber auch die Nutzung zeitversetzten Sehens über digitale Aufzeichnungsgeräte wie Festplatten- und DVD-Recorder erfasst werden. Zudem sei die Integration weiterer Messmodule durch offene Schnittstellen, die auch den Einsatz der Technologien Dritter erlaubt, jederzeit möglich, um etwa die TV-Nutzung mit einer TV-Karte am PC, IPTV, Nutzung an MHP-Boxen oder auch mobiles Fernsehen (DVB-H, Handy TV) erfassen zu können. ${ }^{914}$

447 Hinzu kommt die "TC UMX“-Technik, eine Art Audio-Fingerprinting-Verfahren, das etwa dort eingesetzt wird, wo die „TC Score“-Messtechnik technisch nicht realisierbar ist. ${ }^{915}$ Hierbei werden

\footnotetext{
${ }^{907}$ So auch BigChampagne Online Media Measurement, Monitoring and Identifying P2P Media, S. 7; s. zum Spannungsfeld zwischen Datenminimierung und Erzielung möglichst hoher Trefferquoten bei ContentIdentification-Technologien Fitzner, Von Digital-Rights-Management zu Content Identification, 2011, S. 148.

${ }^{908}$ S. dazu Rn. 262 ff.; s. auch BigChampagne Online Media Measurement, Monitoring and Identifying P2P Media, S. 6.

909 Darauf weist zu Recht Bauer, Dipl. Arbeit 2011, S. 80 f. hin; diesen Vorschlag aber ausdrücklich in Erwägung ziehend BigChampagne Online Media Measurement, Monitoring and Identifying P2P Media, S. 5 f.

${ }^{910}$ Fisher, 2004, S. 226 ff.; leicht modifiziert im Ansatz aber ähnlich Aigrain, 2012, S. 146 f., 152 f.

${ }^{911}$ GfK, Pressemitteilung vom 3.7.2009, S. $1 \mathrm{f}$.

${ }^{912}$ GfK, Pressemitteilung vom 3.7.2009, S. 2.

913 http://www.agf.de/fsforschung/messtechnik/tcscore/.

914 http://www.agf.de/fsforschung/messtechnik/tcscore.

${ }^{915}$ http://www.agf.de/fsforschung/messtechnik/umx.
} 
lediglich die Audiosignale am Fernseher erfasst und komprimiert, an eine zentrale Datenbank übermittelt und dort mit Tonmustern der Radio- und TV-Sender verglichen, um so anhand der Übereinstimmungen das Nutzungsverhalten der Teilnehmer festzustellen bzw. auszuwerten. ${ }^{916}$

448 Abbildungen 1 und 2 verdeutlichen diese heute in Deutschland angewandten Verfahren:

MESSUNG IM HAUSHALT (JE NACH HAUSHALTSAUSSTATTUNG) TC score

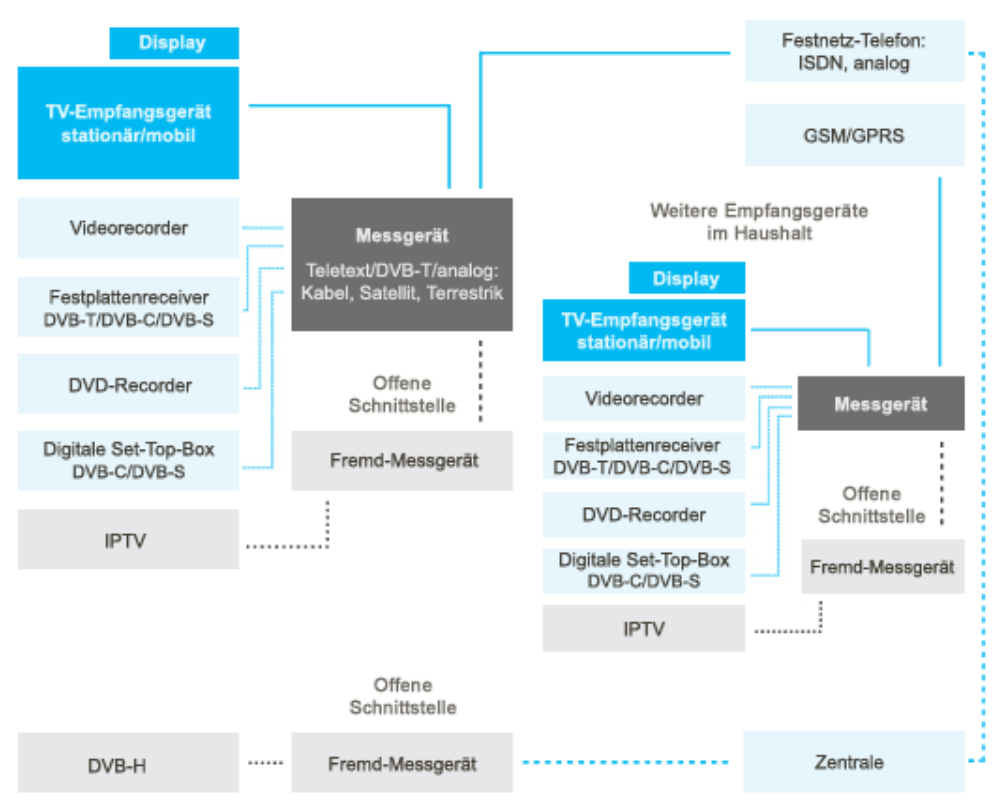

DVB-C, $-\mathrm{S},-\mathrm{T}=$ Digital Video Broadcasting, Cable, Satellite, Terrestrial

Abbildung 1: Messtechnik „TC Score ${ }^{4917}$

${ }^{916}$ http://www.agf.de/fsforschung/messtechnik/umx.

${ }^{917}$ Quelle: http://www.agf.de/fsforschung/messtechnik/tcscore/. 


\section{DAS UMX-AUDIOMATCHINGVERFAHREN}
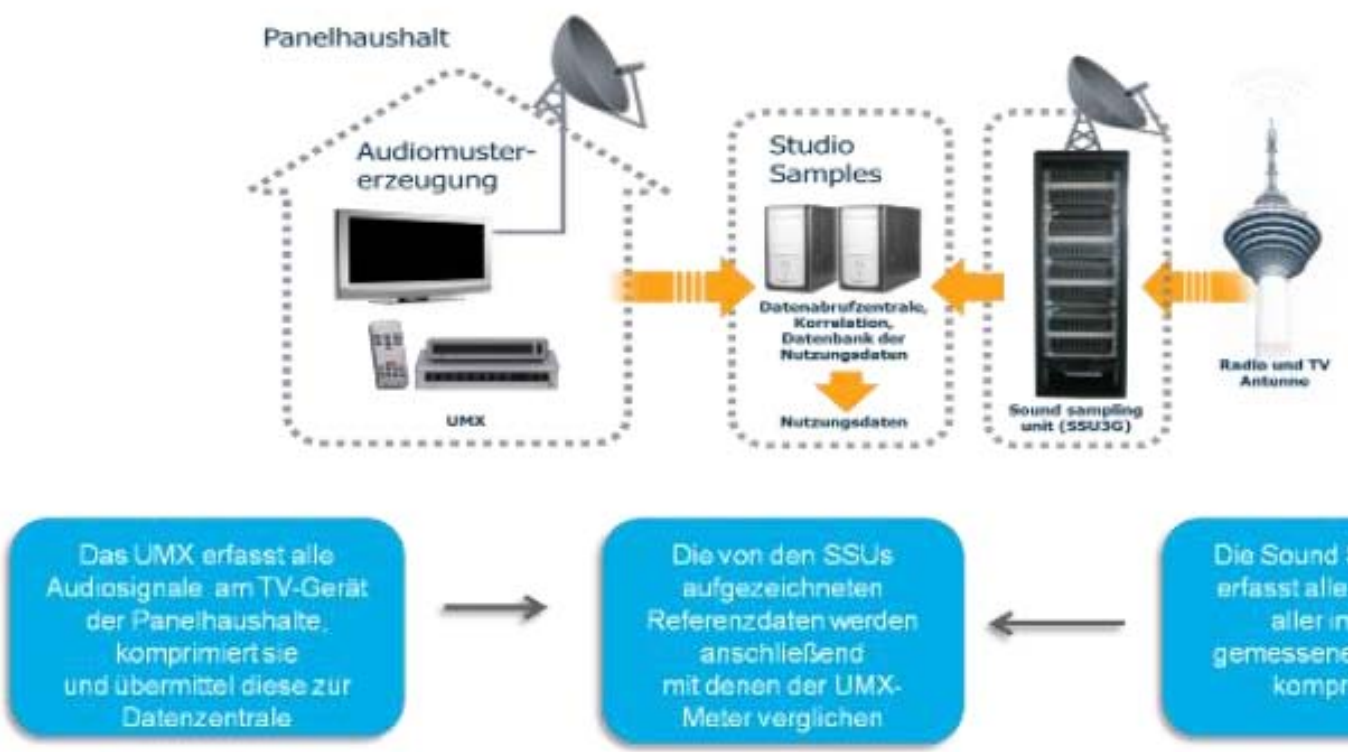

Die Sound Sampling Unit erfasstalie Audiosignale aller im System

gemessenen Sender und komprimiertsio

\section{Aus den _positiven" Matches werden - ggt unter

Abbildung 2: Messtechnik „TC UMX“918

449 In technischer Hinsicht könnten diese Funktionen im Rahmen einer Kulturflatrate entweder Log-Tools oder Plug-ins in Browsern übernehmen. Finanzielle Anreize zugunsten der Nutzer könnten hier zudem eingreifen, so dass statistisch gesehen repräsentative Werte ermittelt werden könnten. Wiederum wäre eine Identifizierung der jeweiligen Nutzer nicht erforderlich; eine Anonymisierung und Aggregation der Daten wäre vollkommen ausreichend. Gleichzeitig könnte auch durch entsprechende Kontrollen sowie durch Aufklärung der Nutzer die Einhaltung des Datenschutzes gewährleistet werden. ${ }^{919}$

\section{b) Andere Werknutzungen}

450 Wesentlich schwieriger zu erfassen wäre die Zahl der Werknutzungen durch Bearbeitung (Mashups, Remixes etc.). Zwar ließe sich die Zahl der mit Originalmusik verbundenen Videos z.B. bei YouTube erfassen. Ein solches System setzt YouTube mit dem Programm VideoID ein, indem Rechteinhaber ihre Musik, aber auch Filme, bei YouTube registrieren lassen können. YouTube kann dann mit AudioFingerprinting auf der Basis von Audible Magic Musik identifizieren, die in von Nutzern hochgeladenen Filmen verwandt wird; aber offensichtlich ist es auch möglich, Filme entsprechend zu identifizieren. ${ }^{920}$

\footnotetext{
${ }^{918}$ Quelle: http://www.agf.de/fsforschung/messtechnik/umx.

${ }^{919}$ So auch BigChampagne Online Media Measurement, Monitoring and Identifying P2P Media, S. 9, die zudem eine Ergänzung der nutzerbasierten Messung durch Audiofingerprints oder ähnliche Identifizierungsmethoden vorschlagen.

${ }^{920}$ Zur Funktionsweise s. http://www.youtube.com/t/video_id_about; s. auch Fitzner, Von Digital-RightsManagement zu Content Identification, 2011, S. 152 ff. mwNachw.
} 


\section{Overview of VideolD System}

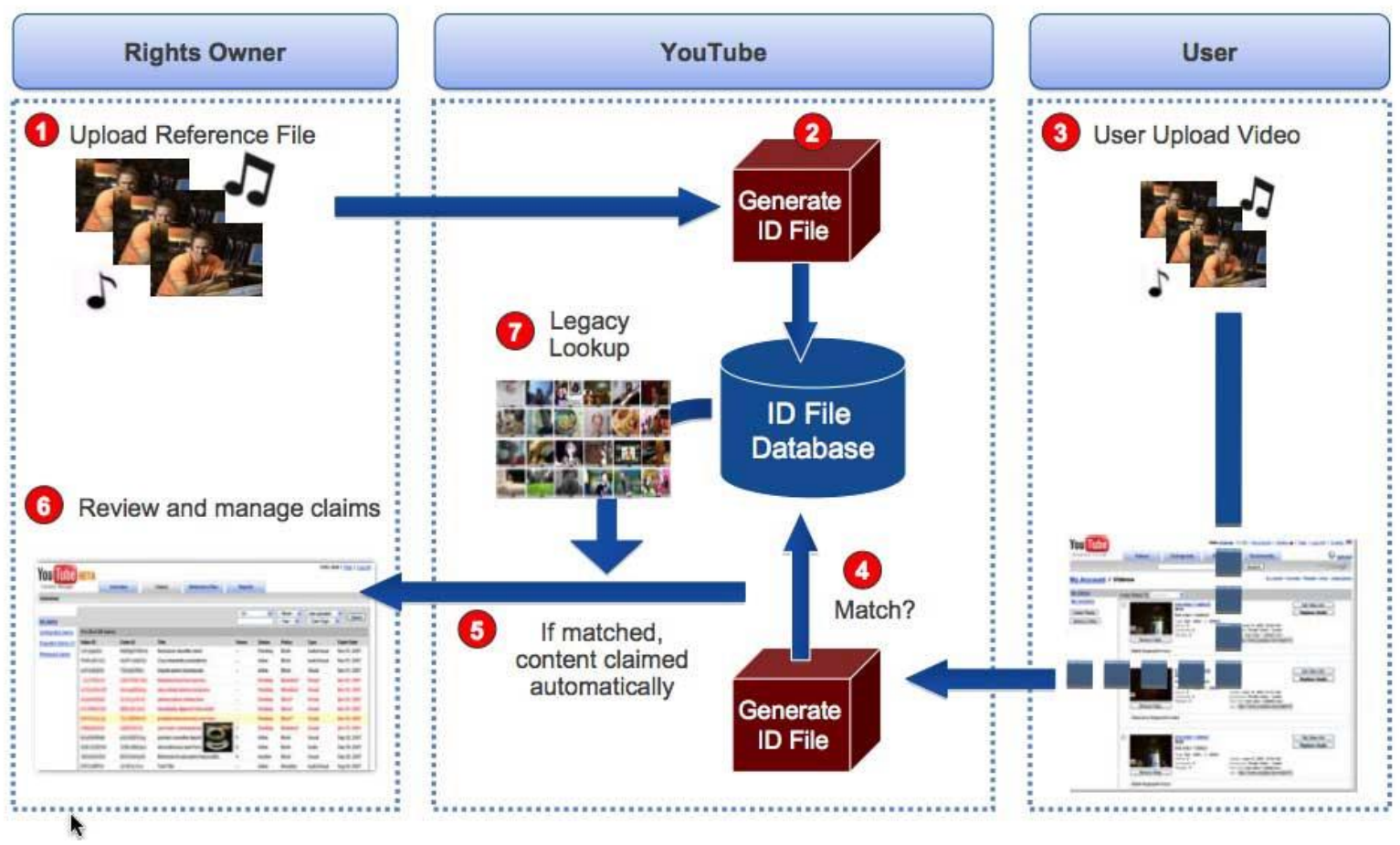

Abbildung 3: Funktionsweise von Video-ID bei YouTube ${ }^{921}$

451 Schwer zu beurteilen ist allerdings die Frage, in welchem Ausmaße Bearbeitungen mit Hilfe dieser Software erfasst werden können, die eben Musik oder Filme verändern, da hier unter Umständen kein ausreichender „digitaler Fingerabdruck“ aufgrund der Verarbeitung zur Verfügung steht. Die Erkennung von Tonfetzen oder Filmfetzen dürfte sich wesentlich aufwendiger gestalten, hängt allerdings von den technischen Möglichkeiten ab, was näherer Untersuchung bedürfte, aber den Rahmen dieses Gutachtens sprengen würde. Indes dürfte es hier auch mit den Methoden des Sampling, also der nutzerbasierten freiwilligen Angabe, möglich sein, zu ermitteln, inwiefern und wie häufig bestimmte Werkstücke für eine Bearbeitung genutzt werden. Zudem ist zu vermuten - und könnte gegebenenfalls empirisch belegt bzw. untersucht werden - dass die meisten Mashups bzw. Remixes im Rahmen von YouTube eine Werkart unberührt lassen, insbesondere Musik, indem oftmals eigene Filme mit entsprechender Musik unterlegt werden.

452 Im Bereich der Textformate sind dagegen Bearbeitungen schwer zu erkennen, wie die umfangreiche Diskussion um Plagiate in der Wissenschaft belegt; auch entsprechende Plagiatssoftware ist nicht immer in der Lage, Bearbeitungen zu erkennen. ${ }^{922}$ Umgekehrt kann mit Hilfe solcher, ggf. zu modifizierender Software für zahlreiche Fälle eine Nutzung und Bearbeitung des ursprünglichen Werkes erkannt werden, so dass für die Zwecke der Verteilung der Vergütung eine recht hohe Präzision er-

\footnotetext{
${ }^{921}$ Quelle: http://www.mr-gadget.de/drm/2008-12-02/youtube-videoid-system-wie-google-die-simpsonsvideos-entfernt.

922 Beispiele für Software zur Identifizierung von Plagiaten sind etwa Tunitin (http://turnitin.com/de/home), Plagaware (http://www.plagaware.de/), Copyscape Premium (http://www.copyscape.com/signup.php?pro=1) und CatchltFirst (http://www.catchitfirst.com/); s. dazu auch http://www.faz.net/aktuell/feuilleton/forschungund-lehre/plagiatssoftware-an-universitaeten-software-oder-lektuere-11855656.html.
} 
reicht werden könnte - eine 100\%ige Präzision wird auch im Bereich der Geräteabgaben keineswegs verlangt, in dem mit wesentlich gröberen Annahmen und Zahlen gearbeitet wird. ${ }^{923}$

\section{c) Missbrauchsrisiken}

453 Von Kritikern einer Kulturflatrate wird häufig eingewandt, dass die Verwendung von Metadaten (in Metatags) ebenso wie die Registrierung in zentralen Datenbanken Missbrauchshandlungen provozieren können, mit denen die jeweiligen Täter versuchen, sich größere als die ihnen zustehenden Vergütungsanteile zu sichern. ${ }^{924}$ Hier können mehrere Möglichkeiten unterschieden werden: ${ }^{925}$

454 In Betracht kommt zunächst die Registrierung von Werken unter einem falschen Namen bei der zentralen Datenbank, um an dem Erfolg populärer Werke teilzuhaben. Zum einen ließe sich aber dieses Problem durch entsprechende Identifizierungen vermeiden, etwa mit Hilfe der neuen De-Mail oder des elektronischen Personalausweises, zum anderen sind auch bei den gegenwärtigen Meldesystemen diese Missbräuche nicht ausgeschlossen, werden allerdings dann aufgedeckt, wenn der wahre Urheber sein Werk anmeldet; ${ }^{926}$ bei Missbräuchen könnten Vertragsstrafen oder andere Sanktionen verhängt werden, die mit Hilfe der Identifizierung eingetrieben werden können, ggf. verbunden mit der Zahlung von Kautionen, falls dies als erforderlich angesehen wird. Wenn Urheber (bzw. Rechteinhaber) nur gegen Gebühr und/oder Kaution eine Registrierung ihrer Werke erreichen könnten, wäre zum einen eine wirksame Sanktion im Falle des Missbrauchs gewährleistet (Verfall der Kaution), zum anderen eine Seriositätsschranke aufgebaut, die beliebige Registrierungen verhindern könnte.

455 Die Manipulation von Metadaten eines Inhalts ist dagegen weniger missbrauchsanfällig, da diese zwar verändert werden können, nicht aber dazu führen, dass dann die Suche nach diesem Inhalt automatisch zu entsprechenden Zuweisungen führt. Die Suche geht dann schlicht ins Leere. Bestehende Manipulationen könnten zudem über ein Beschwerdesystem registriert werden. Schließlich ist bei einer (zusätzlichen) Anwendung des Audio-Fingerprinting auch diese Form des Missbrauchs ausgeschlossen. ${ }^{927}$ Da Content-Identification-Systeme inzwischen aufgrund der Sensibilität rein kryptografischer Hash-Werte, bei denen selbst die geringste Veränderung an der Datei zu einem anderen Hash-Wert führt, stattdessen sog. Perceptual Hash Functions verwenden, kommt es für die Erkennbarkeit auf Meta-Daten nicht an. Dabei wird der Hash-Wert allein aus den Charakteristika des Werkes erstellt, die für den Rezipienten sinnlich wahrnehmbar sind, während bspw. Kompressionsrate, Codecs, Bitrates etc. ohne Auswirkungen auf den Hash-Wert geändert werden können. ${ }^{928}$

456 Möglich bleibt dagegen eine Manipulation dergestalt, dass automatisiert ständig Downloadvorgänge eines bestimmten Inhalts vorgenommen werden, ohne dass tatsächlich eine Nutzung bei einem physischen Nutzer damit verbunden ist; vielmehr handelt es sich um Programmschleifen, die dem Überwachungssystem suggerieren würden, dass ein bestimmter Inhalt äußerst beliebt ist - obwohl es sich

\footnotetext{
${ }^{923}$ S. oben Rn. $274 \mathrm{ff}$.

${ }^{924}$ S. dazu Börsenverein des Deutschen Buchhandels, Fragenkatalog zur Kulturflatrate, 2009; einen Überblick an Pro- und Contra Argumenten bei Petschulat, Kulturflatrate, 2010, S. $6 \mathrm{ff}$.

925 S. auch Aigrain, 2012, S. 148 f.

${ }^{926}$ Zutr. Bauer, Dipl. Arbeit, 2011 S. 85.

927 Dazu auch Bauer, Dipl. Arbeit, 2011, S. 86 f.; s. auch Fitzner, Von Digital-Rights-Management zu Content Identification, 2011, S. 148 f. zur Treffsicherheit in der Praxis verwendeter Content-Identification-Technologien.

${ }^{928}$ S. ausf. zur Technik m. zahlr. wNachw. Fitzner, Von Digital-Rights-Management zu Content Identification, 2011, S. 146 ff., dort insb. auch Fn. 611.
} 
nur um ständig simulierte Down- und Uploadvorgänge handelt. ${ }^{929}$ Derartige Missbräuche ließen sich nur durch die Beobachtung von Anomalien im Daten-Traffic vermeiden. Zudem könnte die kombinierte Anwendung mehrerer Messmethoden solche Manipulationen eher aufdecken, etwa durch gleichzeitige Verwendung (und Gegenkontrolle) durch freiwilliges Sampling (bzw. Umfragen oder auch Votings für bestimmte Künstler). ${ }^{930}$

\section{Progressionsverteilungsmodelle (Abflachung bei Stars etc.) - Förderungen}

457 Sofern die Kompensation der Rechteinhaber für die Nutzung ihrer Werke im Vordergrund steht, ist es zulässig, dass ein Teil der Abgabe zur Förderung kulturpolitischer Vorhaben genützt wird. ${ }^{931}$

Vorbilder für einen derartigen Einbehalt finden sich bei zahlreichen in- und ausländischen Verwertungsgesellschaften. In Österreich etwa dient dazu der Fonds für soziale und kulturelle Einrichtungen (SKE-Fonds ${ }^{932}$ ) bei den Leermedien als Fördereinrichtung der österreichischen Verwertungsgesellschaft AustroMechana, die gem. § 13 Abs. 2 des österreichischen VerwGesG ${ }^{933}$ dazu verpflichtet ist, 50\% der Einnahmen aus der Leerkassettenvergütung an eigene soziale und kulturelle Einrichtungen abzuführen. ${ }^{934}$ Durch den Fonds werden zeitgenössische Künstler gefördert, die über die AustroMechana Tantiemen erhalten, bspw. durch die Zurverfügungstellung von Tonstudios oder Weiterbildungsmöglichkeiten. ${ }^{935}$

459 In Deutschland bestimmt § 7 S. 2 UrhWahrnG, dass die Verteilungspläne der Verwertungsgesellschaften „dem Grundsatz entsprechen [sollen], dass kulturell bedeutende Werke und Leistungen zu fördern sind“. Dementsprechend sieht z.B. die GEMA in $\S 1 \mathrm{Nr}$. 4a ihres Verteilungsplans vor, dass jeweils $10 \%$ der Verteilungssumme für soziale und kulturelle Zwecke bereitgestellt werden. Dies entsprach im Jahr 2010 einer Summe von 44,9 Mio. Euro, von denen 7,3 Mio. Euro in die Sozialkasse gingen, 2,6 Mio. Euro in die Alterssicherung der Mitglieder eingespeist wurden und der Rest den Wertungs- und Schätzungsverfahren der GEMA zugutekamen. ${ }^{936}$ Diese Wertungsverfahren dienen dazu, bestimmte kulturell bedeutsame Werkkategorien im Verhältnis zu anderen finanziell besonders zu fördern. ${ }^{937}$ Die Filmverwertungsgesellschaft VFF wiederum hat, der AustroMechana vergleichbar, durch $\S 3$ ihres Verteilungsplans einen Förderungsfonds eingerichtet, in den $4 \%$ der Ausschüttungssumme fließen.

\footnotetext{
${ }^{929}$ Bauer, Dipl. Arbeit, 2011, S. 86 f.

${ }^{930}$ S. zu dem Modell der Kulturwertmark, die auf Votings beruht, unten Rn. $501 \mathrm{ff}$.

${ }^{931}$ S. dazu EU, Background Document, 'Fair Compensation for Acts of Private Copying', 2008, S. 9 f., abrufbar unter: http://ec.europa.eu/internal_market/copyright/docs/levy_reform/background_en.pdf, insb. dort die Übersicht über die Verwendung der Pauschalabgaben innerhalb der EU für kulturelle und soziale Zwecke im Jahr 2004.

932 Zum Hintergrund des SKE-Fonds s. http://www.ske-fonds.at/show_content.php?sid=1; die Richtlinien des SKE-Fonds sind abrufbar unter: http://www.ske-fonds.at/rte/upload/download/richtlinien_ske.pdf.

933 Öst. Bundesgesetz über die Verwertungsgesellschaften (Verwertungsgesellschaftengesetz 2006 - VerwGesG 2006), BGBI. I Nr. 9/2006, zuletzt geändert durch Bundesgesetz vom 19.07.2010, BGBI. I Nr. 50/2010.

${ }^{934} \S 13$ Abs. 2 des öst. VerwGesG lautet: „Verwertungsgesellschaften, die Ansprüche auf Leerkassettenvergütung geltend machen, haben sozialen und kulturellen Zwecken dienende Einrichtungen zu schaffen und diesen $50 \%$ der Gesamteinnahmen aus dieser Vergütung abzüglich der darauf entfallenden Verwaltungskosten zuzuführen. Die Verpflichtung zur Schaffung sozialer Einrichtungen gilt jedoch nicht für Verwertungsgesellschaften, deren Bezugsberechtigte ausschließlich Rundfunkunternehmer sind“.

935 S. den Überblick unter http://www.ske-fonds.at/show_content.php?hid=2.

${ }^{936}$ GEMA-Jahrbuch 2011/2012, S. 49.

${ }^{937}$ Melichar, in: Loewenheim, Handbuch des Urheberrechts, 2. Aufl. 2010, § 47 Rn. 37; näher zum Wertungsverfahren Vogel, GRUR 1993, 513, 524; zu den kulturellen und sozialen Aufgaben der Verwertungsgesellschaften in Deutschland und Österreich s. Leeb, Der Wert künstlerischer Arbeit, 2009, S. 89 ff.
} 
460 Aber auch generelle Erwägungen sprechen für eine Abflachung gegenüber einem rein linearproportionalen Modell, das die Vergütung allein anhand der Popularität messen würde. Akzeptiert man auch kulturpolitische Gründe im Rahmen des Urheber(abgaben)rechts, so müssen Werke, die zum ersten Mal publiziert werden, überproportional gegenüber ihrer Popularität honoriert werden, um die kulturelle Vielfalt zu fördern und zu erhalten. Vergleichbare Modelle sind bereits auch in anderen Ländern diskutiert worden, ${ }^{938}$ wobei allerdings auch radikale Vorschläge zu verzeichnen sind, etwa der Einführung einer "Quadrat-Wurzel“-Methode, ${ }^{939}$ die zu einer übermäßigen Abflachung der Vergütung bei populären Werken führen würde.

461 Verfassungsrechtlich ist damit zwar ein Eingriff in Art. 14 GG für den Urheber eines populären Werkes verbunden, doch kann dieser gerechtfertigt und verhältnismäßig auf der Grundlage des kulturpolitischen Auftrag des Staates sein, ${ }^{940}$ sofern der Urheber des populären Werkes nach wie vor einen zwar nicht mehr linearen, aber doch ausreichend hohen Anteil an der Vergütung erhält (degressives Modell). Vergleichbare Erwägungen liegen bereits jetzt den Verteilungsmodellen auch der Verwertungsgesellschaften zugrunde.

\section{Empfänger der Vergütungen}

462 Schließlich müssen auch die Berechtigten bzw. Empfänger der Vergütungen bestimmt werden. Je nachdem, welche Rechte betroffen sind, müssen nicht nur Urheber und Rechteinhaber einbezogen werden, sondern auch Inhaber von Leistungsschutzrechten, was bei Bildern, Tonträgern, in Zukunft unter Umständen auch Presseverlagen eine Rolle spielen kann.

463 Die Verteilung zwischen den jeweiligen Gruppen kann hier nach dem Vorbild der Leermedienabgabe erfolgen, die zwischen den Verwertungsgesellschaften ausgehandelt wird, etwa hinsichtlich Bildern (VG Bild-Kunst), Texten (VG Wort) oder Musik (GEMA), wie oben dargelegt. ${ }^{941}$

464 Da sich diese Verwertungsgesellschaften (zusammen mit anderen) gemeinsam der ZPÜ als Einzugsstelle bedienen, liegen bei dieser die Einnahmen zunächst gebündelt vor und werden nach einem von den Verwertungsgesellschaften untereinander ausgehandelten Verteilungsschlüssel zur weiteren Ausschüttung an diese weitergeleitet. Aus dem Bereich Audio gehen die eingenommenen Beträge zu je $42 \%$ an die GEMA und die GVL sowie zu 16\% an die VG Wort. Die Einnahmen aus dem Bereich Video gehen zu je 21\% an die GEMA und die GVL, zu 8\% an die VG Wort sowie zu 50\% an die VG Bild-Kunst und die Filmverwertungsgesellschaften. ${ }^{942}$ Gerade die Verteilung im Bereich Video hat sich in der Vergangenheit allerdings als problematisch erwiesen, nicht zuletzt weil die Rechtewahr-

\footnotetext{
${ }^{938}$ Aigrain, 2012, S. $96 \mathrm{f}$.

${ }^{939}$ Stallmann, Freedom - or Copyright?, 2009.

940 Die Verwirklichung des Kulturstaats ist für die Bundesrepublik Deutschland dem Grundgesetz zufolge sowohl Staatszielbestimmung als auch Verfassungsauftrag, BVerfGE 35, 79, 114; 36, 321, 331; Scholz, in: Maunz/Dürig, GG, 68. EL 2013, Art. 5 Abs. 3 Rn. 8; zur Konkretisierung dieser Zielvorgaben s. auch Steiner, in: Isensee/Kirchhof, Handbuch des Staatsrechts, Bd. IV, 3. Aufl. 2006, § 86 Rn. 11, demzufolge es sich bei der Kulturpflege um eine „kulturpolitisch zu konkretisierende Gestaltungsaufgabe“ handele; ferner Butzer, in: Isensee/Kirchhof, Handbuch des Staatsrechts, Bd. IV, 3. Aufl. 2006, § 74 Rn. 35, der dem Staat im Bereich der Kulturförderung „Sicherstellungsaufgaben“ zuschreibt; beispielhaft sei die Vielfaltsicherung gem. § 25 Abs. 3 des Rundfunk-Staatsvertrags der Länder genannt („Im Rahmen des Zulassungsverfahrens soll die Landesmedienanstalt darauf hinwirken, dass an dem Veranstalter auch Interessenten mit kulturellen Programmbeiträgen beteiligt werden."), hierzu Rossen-Stadtfeld, in: Hahn/Vesting, Rundfunkrecht, 3. Aufl. 2012, § 25 RStV Rn. 30, 60. ${ }^{941}$ S. oben Rn. $403 \mathrm{ff}$.

${ }^{942}$ Kreile, GRUR Int. 1992, 24, $34 \mathrm{f}$.
} 
nehmung dort auf fünf Verwertungsgesellschaften verteilt ist (GÜFA, VFF, VGF, GWFF und VG BildKunst). ${ }^{943}$

In diesen Rahmen sind auch Bagatellschwellen einzuführen, da nicht jede auch nur minimale Nutzung eine Vergütung auslösen kann, da die Kosten zur Erfassung und Vergütung dann diejenigen der tatsächlichen Inanspruchnahme übersteigen. ${ }^{944}$

\section{E. Einziehung und Verteilung: Verwertungsgesellschaften und andere Lösungen}

Von der Frage, wer die Verteilung übernimmt, zu trennen ist der Problemkreis, wer die relevanten Verteilungsschlüssel ermittelt, mit anderen Worten: Wer die Daten erhebt, die als Bezugsgröße für die Bestimmung des Verteilungsschlüssels dienen. Vorgeschlagen wird etwa die Einrichtung einer unabhängigen Stelle, die die aggregierten Daten bzw. Schlüssel an die Verwertungsgesellschaften übermittelt, so dass eine Trennung von Verteilung und Datenerhebung stattfindet. ${ }^{945}$

467 Da die Kulturflatrate den bereits eingeführten Abgaben auf Geräte und Leermedien entspricht, liegt es nahe, ihre Erhebung und Einziehung denjenigen Organisationen zu überantworten, die bereits Erfahrung mit kollektiven Abgaben haben, namentlich den Verwertungsgesellschaften. Auch käme die Überantwortung dieser Aufgabe an die ZPÜ in Betracht. ${ }^{946}$ Für die Zuweisung dieser Kompetenzen an Verwertungsgesellschaften soll sprechen, dass sie keine eigenen wirtschaftlichen Interessen verfolgen und deswegen Garant für das Bemühen um eine gerechte Verteilung der Gebühren seien. ${ }^{947}$

Allerdings ebbt die Diskussion über die Rolle und die innere Ausgestaltung der Verwertungsgesellschaften in der öffentlichen Wahrnehmung nicht ab, vor allem induziert durch z.T. offensives Vorgehen der GEMA in verschiedenen Fällen, was etwa in Vorwürfen, die GEMA sei der "Mafia“ ähnlich, gipfelte. ${ }^{948}$ Aber nicht nur auf nationaler Ebene hält die Kritik an, sondern auch im Ausland. So fordert etwa der Hargreaves-Report, dass die Verwertungsgesellschaften transparenter zu strukturieren seien und verpflichtet werden sollten, Codes of Conduct einzuhalten. ${ }^{949}$ Auch der Vorschlag der Europäischen Kommission für eine Richtlinie des Europäischen Parlaments und des Rates über kollektive Wahrnehmung von Urheber- und verwandten Schutzrechten und die Vergabe von Mehrgebietslizenzen für die Online-Nutzung von Rechten an Musikwerken im Binnenmarkt ${ }^{950}$ enthält in Kapitel I (Art. 4-20) etliche Vorgaben für Minimalgrundsätze der inneren Organisation, insbesondere der Organe sowie der Rechte und Pflichten der Mitglieder, und der Transparenz der Verwertungsgesellschaften.

\footnotetext{
${ }^{943}$ Kreile, GRUR Int. 1992, 24, 34 ff.; s. dazu auch den Überblick über die Verwertungsgesellschaften, welche über eine Erlaubnis nach $\S 1$ UrhWahrnG verfügen: http://www.dpma.de/docs/dpma/aufgaben/listederverwertungsgesellschaften2.pdf.

944 Ebenso Aigrain, 2012, S. 97.

945 So Aigrain, 2008, S. 98: „observatoire“.

${ }^{946}$ So Spitz/Dittrich, Schöne neue kreative Welt, 2010.

947 So Roßnagel et al., Gutachten, 2009, S. 62.

${ }^{948}$ Hoeren hatte in einem Interview gesagt: „Man darf den Einfluss von bestimmten Unternehmen nicht unterschätzen, und man muss außerdem wissen, dass es damals [in den 30er Jahren] innerhalb der Gema ,mafiöse' Strukturen gab, die die Gema noch heute beherrschen“, s. http://www.brandeins.de/magazin/digitalewirtschaft/mafioese-strukturen.html. Bezüglich dieser Aussage hat Hoeren auf Verlangen der GEMA eine Unterlassungserklärung abgegeben, s. dazu http://blog.beck.de/2012/08/07/ist-die-gema-mafioes.

${ }^{949}$ Hargreaves, Digital Opportunity, 2011, S. 36.

${ }^{950}$ Vorschlag vom 11.07.2012, COM(2012) 372 final 2012/0180 (COD), Text abrufbar unter: http://ec.europa.eu/internal_market/copyright/docs/management/com-2012-3722_de.pdf; krit. zum Vorschlag Ventroni, MMR 2012, $565 \mathrm{ff}$.
} 
469 Daher hängt die Überantwortung an die Verwertungsgesellschaften in entscheidendem Maße von deren innerer "Corporate Governance" ab. Generell wird eine "demokratische" Organisation gefordert, die sowohl der Beteiligung über Internetverfahren offen steht als auch konsequente Entscheidungen gerade in Vergütungsfragen ermöglicht. ${ }^{951}$ Eine kritische Überprüfung würde allerdings den hier gesetzten Rahmen sprengen und muss einer weiteren Untersuchung vorbehalten bleiben.

470 Will man nicht den Weg einer inneren Strukturreform der Verwertungsgesellschaften gehen, kommt nur die Schaffung einer neuen Organisation in Betracht, etwa einer staatsfernen ${ }^{952}$ bzw. unabhängigen Stiftung, die die Verteilung der Erlöse aus einer Abgabe übernehmen soll. ${ }^{953}$ Ohne hier auf Einzelheiten einzugehen, würde eine solche Stiftung indes keine anderen Funktionen als eine herkömmliche Verwertungsgesellschaft wahrnehmen. Vorzugswürdig ist daher eher eine Reform des geltenden Rechts der Verwertungsgesellschaften, insbesondere ihrer "Corporate Governance“, als die Schaffung neuer, ihrerseits mit zahlreichen strukturellen Fragen behafteten Organisationen. ${ }^{954}$

\footnotetext{
${ }^{951}$ Aigrain, 2012, S. 139 f.

${ }^{952} \mathrm{Zu}$ den verfassungsrechtlichen Problemen, die eine staatliche Einzugsstelle verursachen würde, wie sie bspw. Fisher, 2004, S. 205 ff. vorschlägt s. ausführlich: Zwengel, Kulturflatrates, 2012, S. 166 ff., 182.

${ }^{953}$ So der Vorschlag im Rahmen des Kulturwertmarkmodells des Chaos-Computer-Clubs, s. CCC, Ein Vorschlag zur Güte - die Kulturwertmark.

${ }^{954}$ Ebenso Grassmuck, 2011, S. 55 f.
} 


\section{Weitere Folgefragen}

\section{A. Beschränkung auf nicht-kommerzielle Tätigkeiten}

471 Die auf private Tätigkeiten begrenzte Schranke einer Kulturflatrate wirft naturgemäß die Frage auf, wie kommerzielle Anbieter und Nutzer von den privaten sinnvoll abgegrenzt und diese Einschränkungen in der Praxis überwacht werden können, ohne dass im Übermaß in die datenschutzrechtlichen Belange der Nutzer eingegriffen würde.

\section{Prüfung der privaten Tätigkeit}

472 Das Problem der "Gewerblichkeit" bzw. der Privatheit ist für das Urheberrecht nicht neu. Allen voran setzt schon die bestehende Schranke des $\S 53$ Abs. 1 UrhG voraus, dass die Vervielfältigungen nur zum privaten Gebrauch angefertigt werden. Ebenso grenzt umgekehrt § 101 Abs. 1 UrhG den Auskunftsanspruch auf solche Verstöße ein, die in einem gewerblichen Ausmaß begangen werden. Daher kann grundsätzlich an die dort entwickelten Kriterien angeknüpft werden, ohne dass sich für eine Ausdehnung der Schranken materiell-rechtlich Besonderheiten ergäben. Darüber hinaus bieten die "Creative Commons" (CC) Musterlizenzverträge Urhebern die Möglichkeit, online verfügbar gemachte Werke auf die nicht-kommerzielle Nutzung durch Dritte zu beschränken. ${ }^{955}$

473 Privat ist ein Gebrauch im Rahmen des $\S 53$ Abs. 1 UrhG dann, wenn dieser in der Privatsphäre zur Befriedigung rein persönlicher Bedürfnisse durch die eigene Person oder durch eine durch persönliches Band verbundene Person stattfindet. ${ }^{956}$ Die angefertigten Vervielfältigungsstücke dürfen dann nach dem ausdrücklichen Wortlaut des Regelungstextes keinen unmittelbaren und mittelbaren Erwerbszwecken dienen. Ähnlich verhält es sich bei dem Begriff des "gewerblichen Ausmaßes" aus $\S 101$ Abs. 1 UrhG, der auf die EU-Richtlinie 2004/48/EG zurück geht und Verletzten einen Auskunftsanspruch nur in Fällen gewährt, wo die Rechtsverletzungen „zwecks Erlangung eines unmittelbaren oder mittelbaren wirtschaftlichen oder kommerziellen Vorteils vorgenommen werden", was Handlungen von gutgläubigen Endverbrauchern ausschließen soll. ${ }^{957}$

474 Allerdings können die Tatbestände der genannten Regelungen im Rahmen der Einführung einer Kulturflatrate, die auch den Upload erfassen will, nicht ohne Modifikationen übernommen werden. Bislang knüpfen sowohl § 53 Abs. 1 als auch § 101 Abs. 1 UrhG an quantitative Voraussetzungen an: Das Privatkopieprivileg greift nur in Fällen, wo „einzelne Vervielfältigungen eines Werkes “958 angefer-

\footnotetext{
${ }^{955}$ S. grds. zu den CC und den dort zur Verfügung gestellten Vertragsbausteinen - zu denen auch die Beschränkung auf nicht kommerzielle Nutzungen gehört - unter: http://de.creativecommons.org/was-ist-cc/; zum Hintergrund der CC s. Paul, in: Hoeren/Sieber, Multimedia-Recht, 32. EL 2012, Teil 7.4 Rn. $121 \mathrm{ff}$.

956 So bereits BGH GRUR 1978, 474, 475 zum damals gleichbedeutenden Begriff des „persönlichen Gebrauchs“; s. dazu Loewenheim, Handbuch des Urheberrechts, 2. Aufl. 2010, § 31 Rn. 26 Fn. 1; s. weiter Wiebe, in: Spindler/Schuster, Recht der elektronischen Medien, 2. Aufl. 2011, § 53 UrhG Rn. 3; Dreier, in: Dreier/Schulze, UrhG, 4. Aufl. 2013, § 53 UrhG Rn. 7.

957 S. Erwägungsgrund 14 der Richtlinie 2004/48/EG des Europäischen Parlaments und des Rates vom 29. April 2004 zur Durchsetzung der Rechte des geistigen Eigentums, ABI. Nr. L 195 v. 2.6.2004, S. 16; s. auch Dreier, in: Dreier/Schulze, UrhG, 4. Aufl. 2013, § 101 UrhG Rn. 6; Wiebe, in: Spindler/Schuster, Recht der elektronischen Medien, 2. Aufl. 2011, § 101 UrhG Rn. 4.

${ }^{958}$ Nach derzeitiger Rspr. liegen noch „einzelne Vervielfältigungsstücke“ vor, wenn die Zahl sieben nicht überschritten wird, s. dazu Wiebe, in: Spindler/Schuster, Recht der elektronischen Medien, 2. Aufl. 2011, §52 UrhG Rn. 3 mwNachw.
} 
tigt werden; im Rahmen des Auskunftsanspruchs kann sich das "gewerbliche Ausmaß" aus der Anzahl der Rechtsverletzungen ergeben. Privatkopien sind also nur zulässig bzw. Auskunftsansprüche ausgeschlossen, wenn keine große Anzahl von Musikstücken, Filmen oder anderen Werken betroffen ist. Ein „privater" Betrieb einer Tauschbörse ist daher nach geltendem Recht nicht mit dem Begriff des „privaten“ Charakters vereinbar.

475 Entscheidet sich ein Urheber dazu, seine Werke auf die nicht-kommerzielle Weiternutzung mittels der „Creative Commons“-Musterlizenzverträge zu beschränken, formuliert die inzwischen dritte Version dieser Verträge die Rechteeinräumung wie folgt:

„Die Rechteeinräumung gemäß Abschnitt 3 gilt nur für Handlungen, die nicht vorrangig auf einen geschäftlichen Vorteil oder eine geldwerte Vergütung gerichtet sind (,nichtkommerzielle Nutzung', ,Non-commercial-Option'). Wird Ihnen in Zusammenhang mit dem Schutzgegenstand dieser Lizenz ein anderer Schutzgegenstand überlassen, ohne dass eine vertragliche Verpflichtung hierzu besteht (etwa im Wege von File-Sharing), so wird dies nicht als auf geschäftlichen Vorteil oder geldwerte Vergütung gerichtet angesehen, wenn in Verbindung mit dem Austausch der Schutzgegenstände tatsächlich keine Zahlung oder geldwerte Vergütung geleistet wird. ${ }^{4959}$

477 Die Formulierung "geschäftlicher Vorteil“ in Kombination mit der der "geldwerten Vergütung“ ermöglicht auch in diesem Bereich die Erfassung von sowohl unmittelbaren als auch mittelbaren Einnahmen. Gleichzeitig wird aber auch eine kommerzielle Nutzung auf Vorgänge beschränkt, die vorranging auf diese Einnahmenerzielung gerichtet ist. Damit wird der Anwendungsbereich für nichtkommerzielle Nutzungen nicht ausgehöhlt, da zumindest eine Zwecksetzung durch den Verwender der fremden Werke stattzufinden hat, die vor allem anderen die Erzielung von Einnahmen zum Gegenstand haben muss. Demgemäß bleiben Sachverhalte noch vertragskonform, bei denen die Einnahmen nur zufällig oder einem anderen Zweck untergeordnet als Nebenprodukt realisiert werden. Zudem wird Filesharing ausdrücklich gestattet, solange dieses unentgeltlich stattfindet. Der Empfänger beim Filesharingvorgang wird dann ebenfalls CC-Vertragspartner des Rechteinhabers, solange die Rechteeinräumung unter die auflösende Bedingung gestellt wird, dass der verteilende Lizenznehmer als Bote des Rechteinhabers die Lizenzbedingungen weitergibt. ${ }^{960}$

478 Maßgeblich muss daher auch im Rahmen einer Kulturflatrate sein, ob mit den Down- und Uploads die Erzielung von Einnahmen beabsichtigt ist, sei es unmittelbar durch Zahlungen an den Nutzer oder mittelbar durch die Erzielung etwa von Werbeeinnahmen oder den Handel mit persönlichen Daten der angeschlossenen Nutzer. ${ }^{961}$ Bei der weiteren Bestimmung der mittelbaren Einnahmen kann auf die Ausführungen zu den „Creative Commons“-Musterlizenzverträgen und auf die zu § 53 Abs. 1 S. 1 UrhG entwickelten Grundsätze bezüglich der „mittelbaren Erwerbszwecke“ zurückgegriffen werden. Auch im Rahmen einer Kulturflatrate sollte davon abgesehen werden, die Schranke praktisch leerlaufen zu lassen, indem Sachverhalte als kommerziell qualifiziert würden, bei denen irgendwann sehr indirekt Einnahmen erzielt werden könnten. ${ }^{962}$ Entscheidend muss auch bei einer Kulturflatrate sein, ob hinter dem Werktausch im Internet ein langfristiges wirtschaftliches Interesse steht (mittelbarer

\footnotetext{
${ }^{959}$ http://creativecommons.org/licenses/by-nc/3.0/de/legalcode.

${ }^{960}$ CC-Lizenzvertrag Ver. 3.0, Ziff. 4 Lit. a, http://creativecommons.org/licenses/by-nc/3.0/de/legalcode; s. auch Paul, in: Hoeren/Sieber, Multimedia-Recht, 32. EL 2012, Teil 7.4 Rn. 128, 130.

961 Zum Handel mit Daten von Nutzern als Form der Entgeltlichkeit jüngst Bräutigam, MMR 2012, 635, 636.

962 Dreier, in: Dreier/Schulze, UrhG, 4. Aufl. 2012, § 53 UrhG Rn. 10.
} 
Erwerbszweck), oder nur zufällig bzw. mit einer erheblichen Latenz wirtschaftliche Vorteile bei den handelnden Personen eintreten (kein mittelbarer Erwerbszweck). ${ }^{963}$ Lädt also etwa jemand ein mit urheberrechtlich relevantem Inhalt versehendes Video bei YouTube hoch, wäre grundsätzlich das Tatbestandsmerkmal der unmittelbaren Einnahmen zu verneinen, da die Person für den Upload weder ein direktes Entgelt erhält noch auf Basis der Anzahl der „Klicks“ des Angebotes Gelder ausgeschüttet werden. Deutlicher noch wäre dies bei Videos etc. auf sozialen Netzwerkplattformen wie Facebook. Werden aber etwa die Videos oder deren Beschreibungen mit Hinweisen auf eigene Internetpräsenzen versehen, könnten durchaus mittelbare Einnahmen generiert werden, was die Berufung auf eine Kulturflatrateschranke ausschließen würde. Hilfreich bei der Abgrenzung - und damit bei der praktischen Handhabbarkeit - wäre wie bei den "Creative Commons"-Musterlizenzverträgen eine Beschränkung des Tatbestandsmerkmales des wirtschaftlichen Vorteils auf Sachverhalte, in denen die Erzielung von Einnahmen den vorrangigen Zweck einnimmt bzw. der kommerzielle Charakter der Handlung als tragendes Motiv erkennbar ist.

479 Entgegen $\S 53$ Abs. 2 und Abs. 3 UrhG kann aber in jedem Fall bei einer Kulturflatrate von Schrankenbestimmungen zum sonstigen eigenen Gebrauch abgesehen werden, die auch bei juristischen Personen anwendbar wären. Ein Bedarf für eine an den sonstigen eigenen Gebrauch angelegte Privilegierung für juristische Personen ist nicht ersichtlich. Die Privilegierungen einer Kulturflatrate sollten ausschließlich Privatpersonen erfassen. Daher kann eine Kulturflatrateschranke ebenso wie der private Gebrauch nach § 53 Abs. 1 S. 1 UrhG auf natürliche Personen beschränkt bleiben. ${ }^{964}$

Das Verhalten Dritter hätte keinerlei Auswirkungen auf die durch eine Kulturflatrate Privilegierten: Wenn jemand unter Einhaltung der Voraussetzungen einer Kulturflatrateschranke geschützte Inhalte rechtmäßig vervielfältigt und öffentlich zugänglich gemacht hat, bleibt das auch selbst dann rechtmäßig, wenn ein Dritter die Inhalte zu Erwerbszwecken nutzt. Die Frage ist hierbei aber, ab welchem Punkt der Dritte die Verantwortung für Inhalte anderer übernehmen muss. Die Bestimmung dessen sollte in diesen Fällen nach den Grundsätzen der Haftung für fremde Inhalte erfolgen. Dabei ist zu untersuchen, ob sich jemand die Informationen eines anderen zu eigen macht. ${ }^{965}$ Das wiederum ist der Fall, wenn nach der wertenden Betrachtung eines objektiven Dritten der Anbieter für die Information die Verantwortung tragen will. ${ }^{966}$ In ähnlicher Weise hat der EuGH versucht, die nicht mehr „neutrale“ Unterstützung eines Providers aus dem Anwendungsbereich der Haftungsprivilegierungen der Art. $10 \mathrm{E}$-Commerce-R $\mathrm{L}^{967}$ herauszunehmen, insbesondere bei flankierenden Angeboten für die speichernden Nutzer. Demnach muss der Provider sich weitgehend auf eine technische neutrale

\footnotetext{
963 Grübler, in: Ahlberg/Götting, BeckOK UrhR, Stand 15.9.2012, § 53 Rn. 10; Dreier, in: Dreier/Schulze, UrhG, 4. Aufl. 2012, § 53 UrhG Rn. 10.

${ }^{964}$ Zur Beschränkung auf natürliche Personen im Falle des § 53 Abs. 1 S. 1 UrhG s. Dreier, in: Dreier/Schulze, UrhG, 4. Aufl. 2013, § 53 UrhG Rn. 7; Lüft, in: Wandtke/Bullinger, UrhG, 3. Aufl. 2009, §53 UrhG Rn. 17; Grübler, in: Ahlberg/Götting, BeckOK UrhR, Stand 15.9.2012, §53 Rn. 7.

${ }^{965}$ S. dazu Wolff, in: Hoeren/Sieber, Multimedia-Recht, 32. EL 2012, Teil 11 Rn. 76; Hoffmann, in: Spindler/Schuster, Recht der elektronischen Medien, 2. Aufl. 2011, § 7 TMG Rn. 17.

${ }^{966}$ Grundlegend BGH NJW-RR 2010, 1276, 1278 - marions-kochbuch.de; Hoffmann, in: Spindler/Schuster, Recht der elektronischen Medien, § 7 TMG Rn. 17.

${ }^{967}$ Richtlinie 2000/31/EG des Europäischen Parlaments und des Rates vom 8. Juni 2000 über bestimmte rechtliche Aspekte der Dienste der Informationsgesellschaft, insbesondere des elektronischen Geschäftsverkehrs, im Binnenmarkt („Richtlinie über den elektronischen Geschäftsverkehr“), ABI. Nr. L 178 v. 17.7.2000, S. 1.
} 
Handlung beschränken. ${ }^{968}$ Wann allerdings welche Tätigkeiten der Provider noch als neutral einzustufen sind, ist derzeit noch im Fluss und lässt sich schwerlich prognostizieren.

481 Hat sich der Dritte die fremden Inhalte zu eigen gemacht, müsste er sich selbst auf die Kulturflatrateschranke berufen können oder eine Lizenzierung nachweisen, um sich nicht rechtswidrig zu verhalten. Das wäre, wie aufgezeigt, bei juristischen Personen und beabsichtigten Einnahmen ausgeschlossen. Zu denken wäre hier etwa an Anbieter, die ihr Geschäftsmodell auf der Verbreitung von nutzerbasierten Inhalten aufbauen. Als prominentestes Beispiel kann hier der anhängige Rechtsstreit über die Stellung von YouTube genannt werden. ${ }^{969}$ Angebote, bei denen nicht mehr die technische Speicherung und öffentliche Zugänglichmachung fremder Inhalte im Vordergrund stehen, sondern bei denen die fremden Inhalte selbst zur Einkünfteerzielung benutzt werden, wären entsprechend der derzeitigen Rechtslage auch zukünftig nur zulässig, wenn für zu eigen gemachte Inhalte die entsprechenden Lizenzen der Rechteinhaber für die von Betreibern durchgeführten Verwertungshandlungen vorliegen würden. ${ }^{970}$ Zu betonen ist allerdings nochmals, dass die Kriterien für diese Abgrenzungsfragen nach wie vor der Diskussion harren und hier nicht weiter vertieft werden können, da etwa die werbefinanzierte Bereitstellung von Plattformen zur Darbietung fremder Inhalte noch nicht allein als sich zu eigen Machen oder nicht-neutrale Handlungen qualifiziert wird.

482 Fraglich bleibt dabei aber, wie mit den Plattformen zu verfahren wäre, welche zwar selbst keine Werke zugänglich und sich auch keine fremden Inhalte zu eigen machen, aber daran adäquat kausal mitwirken, indem sie Hyperlinks zu von Nutzern hochgeladenen Werken sammeln und systematisieren und damit das Auffinden der Werke und das Filesharing erleichtern. Nach derzeitigem Recht haften diese Unternehmen nur akzessorisch als Störer, sofern die verlinkten Inhalte rechtswidrig sind; es sei denn auf den Plattformen sind die hinter dem Link stehenden Inhalte direkt mittels des sog. Framings zu eigen gemacht worden, was genau wie bei Plattformen wie YouTube ohne Lizenz einen eigenen und direkten Eingriff in die Rechte der Urheber durch den Anbieter zur Folge hat und einer Lizenzierung bedarf. ${ }^{971}$ Sobald die öffentliche Zugänglichmachung durch die Internetnutzer im Rahmen einer Kulturflatrate zulässig wäre, würden auch diese linksammelnden und verbreitungserleichternden Plattformen nicht mehr akzessorisch als Störer auf Unterlassung in Anspruch genommen werden können. Gleichzeitig würden aber derartige Plattformen von den nunmehr legal verfügbaren Inhalten finanziell profitieren, da sie die Seiten mit den Linksammlungen mit Werbung anreichern könnten. Derartige Angebote würden in Konkurrenz mit bisherigen Geschäftsmodellen wie Spotify oder T-Entertain treten. Allerdings ist auch hier keineswegs ausgemacht, ob derartige Plattformen noch als „neutral“ bewertet werden können.

483 Insbesondere Angebote, die etwa hochladende Nutzer finanziell für hochgeladene und verlinkte Inhalte entlohnen würden, könnten demnach nach wie vor akzessorisch als Störer in Anspruch genommen werden. Selbst dürften die Anbieter erst recht keine Werke zugänglich machen. Es verbleibt damit also nur ein recht geringes Geschäftsfeld, das sich immer auf dem schmalen Grat zwischen „zu

\footnotetext{
968 Grundlegend EuGH MMR 2011, 596, 602 Rn. 113 - L'Oréal SA u.a./eBay International AG u.a., unter Bezugnahme auf EuGH MMR 2010, 315, 320 Rn. 114, 120 - Google France und Google; dazu Spindler, MMR 2011, 703, 704 f.; s. auch Spindler, JZ 2012, 311, 312; ferner Wiebe, WRP 2012, 1182, 1185 ff.; sowie die Besprechungen von Nordemann, GRUR 2011, 977 ff., und Meyerdierks, ZD 2012, 29 ff.

969 LG Hamburg MMR 2012, 404.

${ }^{970}$ Zu der Frage, wann sich YouTube Inhalte zu eigen macht s. Wolff, in: Hoeren/Sieber, Multimedia-Recht, 32. EL 2012, Teil 11 Rn. 76.

${ }^{971}$ S. ausf. dazu Dreier, in: Dreier/Schulze, UrhG, 4. Aufl. 2013, § 19a UrhG Rn. 6, m. Nachw. aus der Rspr.; s. auch Ott, ZUM 2008, $556 \mathrm{ff}$.
} 
eigen machen“ und „nicht zu eigen machen“ fremder Inhalte bewegen würde. Zudem würde die dadurch ggf. erhöhte Werknutzung über eine Kulturflatrateabgabe kompensiert werden, womit zumindest die Rechteinhaber schadlos gestellt würden.

\section{Behandlung von Resellern und Routern (Internet-Cafés, Schulen etc.)}

Probleme können in diesem Rahmen vor allem gemischte Tätigkeiten hervorrufen, insbesondere private Up- und Downloads am Arbeitsplatz oder in Universitäten oder Schulen. Denn offenbar entfalten Angehörige solcher Institutionen und Organisationen in signifikanter Weise private Internetaktivitäten auch über solche Netze, was statistisch belegt ist. 28\% der Berufstätigen nutzen das Internet am Arbeitsplatz einmal täglich zu privaten Zwecken, 11\% mehrmals die Woche, 5 Prozent mehrmals im Monat, 6\% einmal im Monat oder seltener. Damit nutzen 50\% derjenigen, die beruflich über einen Internetanschluss verfügen, diesen zumindest auch privat. ${ }^{972}$

Gerade für solche Netze, die von Internet-Cafés, Schulen, Universitäten oder Organisationen, ebenso von Arbeitgebern, betrieben werden und die die Schnittstelle zum Internet bieten, ist fraglich, ob und inwieweit sie unter eine Abgabe fielen. Anknüpfungspunkt wäre hier wiederum der Anschluss mit seinen Kapazitäten als solcher; indes liegt es auf der Hand, dass Arbeitgeber, Universitäten, Schulen etc. selbst nicht in vollem Umfang zur Abgabe herangezogen werden können, da sie selbst nicht von den Schranken profitieren. Andererseits würde ein erheblicher Anteil der privaten Nutzung nicht von der Abgabe erfasst, wenn man diese Institutionen völlig außer Acht ließe im Rahmen der Berechnungen.

486 Die Problematik der gemischten gewerblichen bzw. nicht-privaten und privaten Nutzung ist indes keineswegs neu, sondern taucht in anderem Gewande im Gefolge der bereits erwähnten PadawanEntscheidung des EuGH wieder auf, der die Abgabe strikt auf den Anteil des privaten Gebrauchs beschränkt. ${ }^{973}$ Das Gericht betont aber nur, dass nur die „eindeutige“ nicht-private Nutzung von der Vergütungspflicht nach Art. 5 Abs. 2 lit. b InfoSoc-RL ausgenommen sei, ${ }^{974}$ die gemischten Nutzungen dagegen der Abgabe unterfallen können - jedenfalls dem Grunde nach. Dabei kommt es noch nicht einmal auf die tatsächliche Nutzung an, sondern nur auf die Möglichkeit der privaten Nutzung. Allerdings formuliert der EuGH dies als eine Art Beweislastregel, indem er von einer Vermutung spricht:

„Wenn dagegen die fraglichen Anlagen natürlichen Personen zu privaten Zwecken überlassen worden sind, ist es nicht erforderlich nachzuweisen, dass diese mit Hilfe dieser Geräte tatsächlich Privatkopien angefertigt und somit dem Urheber des geschützten Werks tatsächlich einen Nachteil zugefügt haben. Bei diesen natürlichen Personen wird nämlich rechtmäßig vermutet, dass sie diese Überlassung vollständig ausschöpfen, d.h., es wird davon ausgegangen, dass sie sämtliche mit diesen Anlagen verbundenen Funktionen, einschließlich der Vervielfältigungsfunktion, nutzen. Daraus folgt, dass allein die technische Fähigkeit dieser Anlagen oder dieser Geräte, Kopien zu fertigen, ausreicht, um die Anwendung der Abgabe für Privatkopien zu rechtfertigen, sofern diese Anlagen oder Geräte natürlichen Personen als private Nutzer überlassen worden sind. ${ }^{\prime 975}$

488 Diese Vermutung greift aber nur bei der Überlassung an private Personen ein und bezieht sich auch nur auf die vollständige Ausnutzung der Gerätschaften zu Zwecken der privaten Vervielfältigung.

\footnotetext{
${ }^{972}$ S. BITKOM, Studie Netzgesellschaft, 2011, S. 50 f.

${ }^{973}$ S. oben Rn. $229 \mathrm{ff}$.

${ }^{974}$ EuGH GRUR 2011, 50, 54 Rn. 53 - Padawan.

${ }^{975}$ EuGH GRUR 2011, 50, 54 Rn. 54 ff. - Padawan.
} 
Damit steht andererseits dem Hersteller, Händler etc. im Prinzip der Gegenbeweis offen, dass der Abnehmer trotz an sich möglicher gemischter Nutzung nur gewerbliche Vervielfältigungen vornimmt, ebenso, dass Vervielfältigungen nur außerhalb der nach § 53 Abs. 2 - 7 UrhG privilegierten Nutzungen vorgenommen werden, mithin keine Abgabepflicht besteht.

489 Fraglich ist allerdings in diesem Zusammenhang, wer die Beweislast für fehlende private Vervielfältigungen trägt, was in der Praxis angesichts immer wieder möglicher, wenn auch eventuell nur geringfügiger privater Vervielfältigungen ${ }^{976}$ von großer Bedeutung ist. So soll dem EuGH-Urteil zu entnehmen sein, dass Unternehmen noch nicht einmal die Darlegungs- und Beweislast tragen sollen, da diese nur für Privatnutzer eingreife. ${ }^{977}$ Indes trifft der EuGH gerade zu gemischten Nutzungen keinerlei Aussage; vielmehr bezieht sich die Vermutungsregel nur auf die Ausnützung der Geräte, wenn sie an Private geliefert werden. Zudem spricht sich der EuGH nur bei „eindeutiger" gewerblicher Nutzung gegen eine Vergütungspflicht aus. Daher ist es den Mitgliedstaaten nach wie vor überlassen, welche Beweislastregeln sie bei einer gemischten Nutzung verwenden.

490 Für eine Kulturflatrate würde dies bedeuten, dass eine Organisation bzw. Institution den Beweis antreten könnte, dass sie durch Anweisungen und z.B. erforderliche Freigaben für Programme (durch den Admin etc.) dafür Sorge trägt, dass keine privaten Aktivitäten über die Internetanschlüsse der Institution durchgeführt werden. Allerdings besagt das Bestehen eines solchen Verbots noch nichts darüber, ob es auch tatsächlich umgesetzt wird, so dass es zu keinen privaten Vervielfältigungen mehr kommt. In solchen Fällen wird das Unternehmen zumindest darlegen müssen, welche Compliance-Maßnahmen es ergriffen hat, damit etwa private Nutzungen am Arbeitsplatz unterbleiben.

491 Schließlich könnte auch daran gedacht werden, dass Arbeitgeber bei erlaubter privater Nutzung der Arbeitnehmer die Abgabe an diese weiterbelasten; die entsprechenden arbeitsrechtlichen Fragen können hier jedoch nicht behandelt werden.

492 Auch für gemischte Nutzungszwecke erlaubt es die EuGH-Entscheidung daher, auf Pauschalierungen zurückzugreifen, insbesondere auf die Nutzungsintensität und Nutzungszwecke. Letztlich wird es hier auf empirische bzw. Stichprobenerhebungen ankommen, um zu ermitteln, ob und inwieweit etwa Internetanschlüsse in Unternehmen, Universitäten etc. zu privaten Vervielfältigungen herangezogen werden. ${ }^{978}$ In diesem Maße wären dann diese Organisationen ebenso zur Abgabe heranzuziehen wie rein private Internetanschlüsse.

\section{Feststellung des privaten Charakters}

493 Diese Fragen deuten bereits auf ein weiteres Problem hin, nämlich die Überprüfung und Feststellung des privaten Charakters von Internetaktivitäten bzw. -anschlüssen in der Praxis. Allein auf das Datenvolumen kann hier nicht abgestellt werden, da angesichts des Up- und Downloads von Videodateien, gestreamten Inhalten etc. auch bei privaten Anschlüssen teilweise massiver Datentraffic erzeugt werden kann, erst recht, wenn Private Tauschbörsen betreiben. Für die meisten Fälle des Uploading wird indes schon durch die Gestaltung des Angebots ersichtlich sein, ob es sich noch um einen privaten Charakter handelt. Zudem scheidet bei Anschlüssen von juristischen Personen sowie Handelsgesellschaften oder Kaufleuten von vornherein der private Charakter aus; bei natürlichen Personen

\footnotetext{
${ }^{976}$ Erwägungsgrund 35 der InfoSoc-RL; hier kann allerdings auch fraglich sein, ob überhaupt noch eine Vergütungspflicht besteht, da der EuGH für geringfügige Vervielfältigungen offenbar überhaupt keine Abgabepflicht annimmt, EuGH GRUR 2011, 50, 53 f. Rn. 39, 46 - Padawan; darauf weist auch Frank, CR 2011, 1, 5 hin.

977 So Niemann, CR 2011, 69, 75.

${ }^{978}$ So zu Recht Dreier, ZUM 2011, 281, 289.
} 
(Kaufleuten, OHGs etc.) wird das Gegenteil entsprechend der Padawan-Entscheidung des EuGH von diesen dargelegt und bewiesen werden müssen. In allen anderen noch verbleibenden Fällen muss wie bislang auch - mit Indizien gearbeitet werden, um die kommerzielle Absicht festzustellen.

\section{B. Festlegung der Tarife}

494 Die Einziehung und Verteilung einer Kulturflatrate bzw. Abgabe lässt die Frage offen, in welchem Verfahren die Höhe der Abgabe unter Berücksichtigung der oben dargelegten Eckwerte bestimmt wird. Zwar wird quasi durch die nötigen empirischen Erhebungen die mögliche Bandbreite einer Abgabe rechtlich vorgegeben; doch bleibt in concreto ein gewisser Spielraum, der zu der Frage führt, wer in welchem Verfahren die Abgabe festlegen kann:

\section{Vorbild §§ 54a ff. UrhG?}

495 Es liegt auf der Hand, auf das in $\S \S 54 a \mathrm{ff}$. UrhG geregelte Verfahren der Verhandlungen über Abgaben zurückzugreifen. Allerdings sind die Erfahrungen mit der reinen Verhandlungslösung bislang eher ernüchternd, da die Verfahren erhebliche Zeit in Anspruch nehmen und bislang nur wenige Vereinbarungen vorliegen. ${ }^{979}$

496 Zudem könnten gerade für eine Kulturflatrate erhebliche Probleme bestehen, wer als Verhandlungspartner sinnvollerweise in Betracht käme. Zwar stellt sich die Lage im Grundsatz nicht anders als bei der Geräteabgabe dar: Auf Seiten der Rechteinhaber könnten nach wie vor die Verwertungsgesellschaften auftreten, ggf. auch (neue) Urhebervereinigungen, wie dies der Fall im Rahmen der Vergütungsregeln nach $\S 32 a$ UrhG ist. ${ }^{980}$ Indes ist fraglich, wer Ihnen auf Seiten der Nutzer gegenüberstünde: Ähnlich der Geräteabgabe kämen hier die Internetprovider (Access-Provider) in Betracht, die zwar nicht selbst die Abgabe schulden, aber mittelbare Vergütungsschuldner sind, indem sie für ihre Nutzer die Abgabe erheben müssen - dies entspricht der Rechtslage bei der Geräteabgabe, da auch hier die Hersteller bzw. Importeure letztlich nicht die Vergütungsschuldner sind, aber zur Vereinfachung als mittelbare Vergütungsschuldner herangezogen werden. Denkbar ist allerdings auch, dass besondere, gegebenenfalls neu zu gründende Verbände oder der Bundesverband für Verbraucherschutz für die Nutzer in den Verhandlungen auftreten. ${ }^{981}$

497 Hinsichtlich der langwierigen Verfahrensdauern kämen Beschleunigungsmaßnahmen prozessualer Art in Betracht, etwa die Verkürzung des Rechtswegs auf eine Tatsachen- sowie eine Revisionsinstanz, Fristen zur Einreichung und Erarbeitung empirischer Grundlagen etc., deren nähere Behandlung indes den Rahmen dieses Gutachtens sprengen würde und letztlich auf eine allgemeine Problematik der Abgabenbestimmung bezogen ist, die hier nicht näher zu vertiefen ist. Eine andere Alternative bestünde in der Rückkehr zur alten staatlich verordneten Abgabe; ${ }^{982}$ die Kriterien zur Bestimmung der Abgabe müssten sich hier an den oben dargelegten Eckwerten orientieren, das Verfahren

\footnotetext{
979 Müller, ZUM 2008, 377, 377 ff.: bei Ausbleiben einer gütlichen Einigung mindestens fünf Jahre; dies kommt auch in BGH GRUR 2000, 872, 873, deutlich zum Ausdruck; s. zur Verfahrensdauer vor der Schlichtungsstelle ferner Gerlach, in: Wandtke/Bullinger, UrhR, 3. Aufl. 2009, § 11 UrhWahrnG Rn. 11; Reinbothe, in: Schricker/Loewenheim, UrhR, 4. Aufl. 2010, § 14a UrhWahrnG Rn. 1, § 14c UrhWahrnG Rn. 5; Nordemann, in: Fromm/Nordemann, UrhR, 10. Aufl. 2008, §§ 14-16 UrhWahrnG Rn. 8.

${ }^{980}$ S. dazu Spindler, ZUM 2012, 921, 922 f.

${ }^{981}$ S. dazu Grassmuck, 2011.

${ }^{982}$ S. zur Reform der Abgabe im Zweiten Korb Hoeren, MMR 2007, 615, 619; ferner Krüger, GRUR 2005, 206, 208 f.; jüngst auch Verweyen, GRUR 2012, 875 ff., zugl. Anm. zu BGH GRUR 2012, 705 ff. - PC als Bild- und Tonaufzeichnungsgerät.
} 
könnte als eine untergesetzliche Verordnung, ggf. auch mit Anhörung des Parlaments und der Beteiligten ausgestaltet werden.

\section{Streitschlichtung (UrhWahrnG)}

498 Legt man das Modell der §§ 54a ff. UrhG zugrunde, liegt es nahe, das im UrhWahrnG geregelte Verfahren zur Streitschlichtung zu übernehmen. Insbesondere empirische Erhebungen und Berechnungen sind quasi das „tägliche Brot“ der Schiedsstelle, so dass auf deren Erfahrungen zurückgegriffen werden könnte. 


\section{Auswirkungen auf die (Urheber-) Persönlichkeitsrechte}

499 Eine Kulturflatrate und die damit einhergehenden Schranken betreffen Eingriffe in Verwertungsrechte, lassen aber den Schutz des Urheberpersönlichkeitsrechts unberührt. Hier kann der Urheber (nicht automatisch auch der Rechteinhaber als Verwerter, da er keine Persönlichkeitsrechte an dem Werk hat) nach wie vor seine Ansprüche gegen Nutzer durchsetzen, wenn sein Werk entstellt wurde oder er nicht namentlich als dessen Urheber genannt wird. ${ }^{983}$ Diese Ansprüche sind auch weitgehend verfassungsrechtlich geboten, da der Urheberschutz sich nicht im Eigentumsschutz erschöpft, sondern auch den Schutz der Persönlichkeit und damit auch das Werk als Ausfluss der Persönlichkeit umfasst. ${ }^{984}$

500 Allerdings darf nicht verkannt werden, dass der fortbestehende - verfassungsrechtlich gebotene Schutz der Urheber bedingt, dass die Rechte auch durchgesetzt werden können. Eine Rechtsdurchsetzung wiederum aber erfordert entweder die Identifizierung von Rechtsverletzern oder aber zumindest die Inanspruchnahme der Intermediäre, also der Provider und Diensteanbieter etwa im Rahmen der Störerhaftung. Wenn Nutzer völlig anonymisiert würden und damit jegliche Identifizierung entfiele, wäre eine Rechtsverfolgung ihnen gegenüber nicht mehr möglich. In diesem Fall könnten nur noch die Intermediäre in Anspruch genommen werden, was zum einen zu Problemen im Bereich der Störerhaftung führen würde, zum anderen zu einer Neujustierung der Haftungsprivilegierung der Intermediäre führen müsste, da sonst keinerlei Schadensersatzansprüche seitens der Geschädigten mehr durchsetzbar wären. Anders formuliert vermag eine Kulturflatrate nur die Probleme der Rechtsverfolgung im Rahmen der Verwertungsrechte zu lösen - eine völlige Aufhebung der Enforcementprobleme ist damit nicht verbunden. ${ }^{985}$

\footnotetext{
${ }^{983}$ S. zu den Ausprägungen des Urheberpersönlichkeitsrechts oben Rn. 62 ff.

${ }^{984}$ S. oben Rn. $188 \mathrm{ff}$.

985 Eingehend zu dem Problem der anonymen Kommunikation im Netz Spindler, 69. DJT 2012, F 12, F 25, F 33, sowie ZUM 2013, erscheint demnächst.
} 



\section{Alternative Modelle}

\section{A. Freiwillige Vergabe von Vergütungen}

\section{Die Kulturwertmark}

501 Quasi als Gegenentwurf zu einer Kulturflatrate stellt der Chaos-Computer-Club das Modell einer Kulturwertmark vor. Gegenüber der Abgabenlösung unterscheidet sich dieses Modell nicht hinsichtlich der einzuführenden Schranken, ${ }^{986}$ die sich ebenfalls auf den privaten Down- und Upload beziehen sollen, ebenso wenig hinsichtlich der Ansätze zur Berechnung, wohl aber hinsichtlich der Verteilung der zu erhebenden Abgabe. Statt einer auf Down- und Uploadvorgänge bezogenen Auswertung sollen die Nutzer eine Art Mikrowährung erhalten, die Kulturwertmark, die sie dann an diejenigen Urheber verteilen bzw. zahlen können, die ihnen gefallen haben. ${ }^{987}$

502 Diese freiwillige Vergabe von „Kulturwertmarken“ je nach dem, welchen Wert man persönlich einem Inhalt beimisst, stößt indes auf zahlreiche Probleme: Denn nicht der tatsächliche Konsum würde damit erfasst, sondern nur die subjektive Wertschätzung eines bestimmten Inhaltes, obwohl etwa durchaus andere „minderwertige“ Werke ebenso oft genutzt würden. Selbst wenn die subjektive Wertschätzung gegenüber einem Werk sich proportional zur Nutzungshäufigkeit verhielte, könnte es passieren, dass die Zahlung an den Künstler dennoch unterbliebe, aus Gründen, die mit dem Werk als solchem nichts zu tun haben, wohl aber bspw. mit dem persönlichen Image des Künstlers. Des Weiteren bestünden Missbrauchsmöglichkeiten, da jeder Nutzer sich ohne nennenswerten Aufwand selbst als Urheber (bspw. eines Textes oder Fotos) anmelden und sich selbst den gesamten zu verteilenden Betrag zuweisen könnte, und sei es auf Umwegen über einen Mittelsmann. ${ }^{988}$

503 Aber auch aus rechtlicher Sicht ergeben sich erhebliche Bedenken gegen eine von tatsächlichen Verwertungsvorgängen losgelösten Verteilung der Erlöse: Denn nicht zuletzt aus verfassungsrechtlichen Gründen (Art. 14 GG) muss die Vergütung der Urheber an der tatsächlichen Werknutzung orientiert sein, wie u.a. in §§ 54a Abs. 1 UrhG, 7 UrhWahrnG zum Ausdruck kommt. Der Vorschlag des CCC wendet sich von diesem Grundsatz zwar bewusst ab, da diesem zufolge die tatsächliche Nutzung zu einer Konzentration der Zahlung auf einige wenige Superstars führe (sog. Pareto-Effekt), die im Sinne einer kulturellen Vielfalt nicht wünschenswert sei. ${ }^{989}$ Doch kann dies allenfalls zu einer Abflachung der Vergütung bei Superstars etc. führen, nicht aber zu einer Aufgabe der Verknüpfung zwischen Häufigkeit von Verwertungsvorgängen und Vergütung.

504 Zudem wird zu Recht eingewandt, dass die Erfahrungen mit dem freiwilligen Vergütungssystem "flattr" belegen, dass sich auch hier Aufmerksamkeit und Zahlungen der Nutzer auf wenige Urheber konzentrieren. ${ }^{990}$ Auch kann eine auf subjektiven Einschätzungen (bzw. Umfragen etc.) basierende Verteilung der Ressourcen darauf hinauslaufen, dass in verzerrter Weise zu häufig diejenigen Werke genannt würden, die im Gedächtnis haften geblieben sind, nicht aber unbedingt alle benutzten Wer-

\footnotetext{
${ }^{986}$ Allerdings sollen zusätzlich die Schutzfristen verkürzt werden, s. CCC, Ein Vorschlag zur Güte - die Kulturwertmark, S. 5.

${ }^{987}$ S. CCC, Ein Vorschlag zur Güte - die Kulturwertmark, S. 4.

${ }^{988}$ Grassmuck, 2011, S. 38.

${ }^{989}$ S. CCC, Ein Vorschlag zur Güte - die Kulturwertmark, S. 11.

${ }^{990}$ Grassmuck, 2011, S. 35.
} 
ke. ${ }^{991}$ So ist die Kritik nachvollziehbar, dass die Kulturwertmark "keinen Anspruch auf angemessene Vergütung" biete, sondern lediglich „die Hoffnung, dass sich durch individuelle Belohnungsentscheidungen ein faires Verhältnis zwischen Popularität und Ertrag“ einstelle. ${ }^{992}$

505 Die nötige Konnexität zwischen Verwertungsvorgängen und Vergütung fehlt aber ebenso bei anderen Modellen, die etwa auf ein „global sponsorship“ abstellen wollen, indem die Nutzer über die Zuordnung der Vergütungen zu bestimmten Künstlern entscheiden, ${ }^{993}$ ohne dass es darauf ankäme, wie oft tatsächlich die entsprechenden Werke herunter- bzw. heraufgeladen würden.

\section{Auktions- und Vorfinanzierungsmodelle mit Freigabe von Urheberrechten (Crowdfunding; Micropayment)}

506 Die gleichen Bedenken des Auseinanderfallens von tatsächlichen Nutzungshandlungen und freiwilliger Vergabe von Vergütungen richten sich auch gegen andere Modelle, die auf eine Art Vorfinanzierungsmodell, dem Crowdfunding-Gedanken folgend, hinauslaufen, wie etwa die im Rahmen des Blur Workshop on Power at Play in Digital Art and Culture am Banff Center for the Arts und anschließend durch James Love ${ }^{994}$ sowie Alain Toner verfeinerten Modelle. ${ }^{995}$ Nach diesen Modellen sollten die Nutzer zwar eine monatliche Abgabe entrichten, doch würden sie darüber entscheiden, welcher Organisation sie die Abgaben zugestehen wollten, die sich ihrerseits mit verschiedenen Vergütungs- bzw. Vergabepolitiken um die Gunst der Nutzer bemühen.

507 Auch hier kann ein solches Modell nicht den rechtlichen Vorgaben genügen, die prinzipiell an die konkreten Nutzungen der jeweiligen Werke anknüpfen (müssen). An der Messung und Hochrechnung bzw. Schätzung der jeweiligen Nutzungsintensität führt rechtlich gesehen kein Weg vorbei. ${ }^{996}$ Ebenso wenig ist einsichtig, warum die Zwischenschaltung neuer Intermediäre einen Fortschritt gegenüber einer Vergabe über (reformierte) Verwertungsgesellschaften erbringen sollte.

\section{B. Opt-out-Modelle}

508 Ein weiteres, ohne gesetzliche Schranke auskommendes Modell sieht vor, dass Provider ihren Kunden von vornherein im Rahmen der Anschlussgebühr eine Flatrate anbieten, aus der die Nutzer herausoptieren können (Opt-out). In diesem Rahmen müssten die Provider dann allerdings zwangsweise Lizenzen der Rechteinhaber einwerben.

509 Als Variante ist daher vorgeschlagen worden, dass die Rechteinhaber verpflichtet sind, den Providern entsprechende Lizenzen anzubieten, wobei bei fehlender Einigung eine staatliche Stelle die Vergütung etc. festsetzen würde, ${ }^{997}$ vergleichbar etwa den Konflikten um die Einspeisung von Rundfunkprogrammen in Kabelnetze etc.

510 Ob derartige Angebote allerdings „konkurrenzfähig“ gegenüber den „kostenlosen“ Angeboten der Filesharing-Gemeinde sind, ist eine offene Frage. Zudem ist fraglich, welcher Vorteil gegenüber bestehenden Download- bzw. Musikflatrate-Angeboten besteht. Zudem würde die Attraktivität eines solchen Systems bedingen, dass die Rechtsverfolgungsmaßnahmen gegenüber illegalen Nutzern (mangels Schranke) fortgesetzt würden.

\footnotetext{
${ }^{991}$ Aigrain, 2012, S. 80.

${ }^{992}$ Grassmuck, 2011, S. 36.

993 So etwa das Modell von Muguet, Le mécénat global, septembre 2008.version 0.3.

${ }^{994}$ Love, Artists want to be paid: The Blur/Banff proposal, 2002.

${ }^{995}$ Toner, Blur Banff proposal. P2P Foundation, January 2008.

${ }^{996}$ S. oben Rn. $227 \mathrm{ff}$.

997 So das Bsp. bei Renner/Renner, Digital ist besser, 2011, S. 145.
} 


\section{Marktlösungen}

511 Anstelle einer Abgabenlösung verbunden mit Schranken könnte auf Marktlösungen vertraut werden, die sich im Hinblick auf den trotz Rechtsverfolgungsmaßnahmen bestehenden Druck durch das Filesharing entwickeln. In Betracht kommen hier etwa die Fortentwicklung von Kollektivlizenzen für beliebte Videoplattformen wie YouTube oder von Streaming-Portalen, schließlich auch das Vertrauen auf die weitere vertikale Integration von Download-Portalen mit Hardwareangeboten wie von iTunes bzw. Apple, aber auch anderen wie neuerdings Google Music.

512 Allen Varianten ist gemeinsam, dass auf eine gesetzliche Schranke mitsamt den einzutreibenden Abgaben verzichtet würde; aber auch, dass sie Rechtsverfolgungsmaßnahmen nicht verzichtbar machen, sondern lediglich das Niveau in dem Ausmaß verringern, in dem Nutzer auf Filesharing zugunsten von Streaming-Angeboten verzichten. Die Vorteile liegen umgekehrt darin, dass in der Regel Marktmechanismen eher in der Lage sind, die Bedürfnisse von Nutzern effizient (bei knappen Ressourcen) zu befriedigen und unnötige Kosten vermeiden. Zudem würde der Auf- bzw. Ausbau von Organisationen vermieden, die die Abgaben eintreiben und verteilen müssen.

\section{Beibehaltung der autonom vereinbarten Kollektivlizenzen (YouTube)}

513 Einer der angeführten Marktmechanismen könnte in dem Ausbau von Kollektivlizenzen für viel genutzte Download- und Upload-Portale wie YouTube bestehen, ggf. unter Einschluss von Lizenzen für die Bearbeitung von Werken.

514 Allerdings würde dies nur einen gewissen Sektor von Up- und Downloadvorgängen überhaupt betreffen, wenngleich offenbar eine zunehmende Zahl von Nutzern, insbesondere Jugendliche, YouTube als Quelle für ihre Musikdownloads nutzen. Wie dargelegt, würde dies an den übrigen Rechtsverfolgungsmaßnahmen nichts ändern.

515 Abgesehen von diesen Einschränkungen darf aber auch nicht verkannt werden, dass es keinerlei Abschlusszwang der Rechteinhaber gegenüber YouTube oder anderen Download- oder StreamingPortalen gibt, so dass keineswegs gewährleistet wäre, dass Nutzer Zugang zu allen Inhalten hätten. Nur wenn der Markt aufgrund seiner Nachfrage tatsächlich für einen entsprechenden Druck auf Streaming- bzw. Portale wie YouTube sorgt, kann von einem entsprechenden Grad an Zugang zu Inhalten ausgegangen werden - was letztlich nur schwer einzuschätzen ist.

516 Darüber hinaus würde aus Sicht der Nutzer nur eine klare und transparente Lizenzierungspraxis für die nötige Rechtssicherheit sorgen, da dem Nutzer oftmals nicht ersichtlich ist, welche Rechte tatsächlich von einem Streaming- oder sonstigen Portal erworben wurden. Eine potentielle Grauzone bliebe daher auch hier bestehen.

\section{Entwicklung von vertikal integrierten Systemen (Apple/iTunes) sowie Streaming-Angeboten}

517 Wie oben bereits dargelegt, würde sich die Einführung einer Kulturflatrate als Schranke auch auf die Attraktivität bestehender Streaming-Angebote wie Spotify oder integrierter Download- und Hardwareportale wie iTunes etc. auswirken. Gerade diese beiden erfolgreichen am Markt herausgebildeten Lösungen zeigen, dass Unternehmen, auch und gerade neu eintretende Unternehmen in der Lage sind, auf bestehende Marktunvollkommenheiten zu reagieren.

518 Allerdings ist derzeit nicht absehbar, wie etwa gerade Streaming-Angebote wie Spotify dazu führen können, dass für Nutzer attraktive Alternativen zum Filesharing dauerhaft angeboten werden. Glei- 
ches gilt für iTunes und vertikal integrierte, korrespondierende Hardwareangebote; je nachdem, wie sich hier Marktstrukturen entwickeln, ob sich enge Oligopole herausbilden etc., können für die Nutzer derartige Angebote auch wieder unattraktiv werden und damit als Substitutionsmöglichkeiten ausfallen. 


\section{Zusammenfassung}

519 Seit mehr als zehn Jahren ist ein weiterhin steigender Austausch von auch urheberrechtlich geschützten Inhalten über das Netz ebenso zu verzeichnen wie erhebliche Schwierigkeiten, die jeweiligen Rechte effektiv durchzusetzen. Zahlreiche Anstrengungen zur Rechtsverfolgung haben sich in der Vergangenheit zwar als kurzfristig erfolgreich erwiesen, mittelfristig aber auch zu „Abmahnwellen" sowie entsprechenden Reaktionen im Netz durch neue technische Verfahren geführt. Gleichzeitig verstärkt sich die Unsicherheit über die Verantwortlichkeit Dritter, insbesondere von Anschlussinhabern, Eltern, Arbeitgebern oder Universitäten, für Rechtsverletzungen, die über ihre Anschlüsse oder unter ihrer Aufsicht begangen werden. Technische Kopierschutzsysteme haben aufgrund fehlender Akzeptanz der Nutzer nur in einigen Teilbereichen Abhilfe schaffen können, zumal sie auch schnell wieder umgegangen werden konnten. Andererseits haben sich in jüngster Zeit Musikabobzw. Streamingdienste mit Flatrates entwickelt, wie Spotify oder Simfy, die offenbar zu einer Abnahme der Filesharingaktivitäten geführt haben. Ferner ist eine erhebliche Zunahme nutzergenerierter Inhalte etwa auf YouTube zu beobachten, die ihrerseits die Frage der Verwertung von Urheberrechten (Bearbeitungsrecht) aufwerfen.

520 Die Lage entspricht damit in frappierender Weise den Erfahrungen vor Einführung der Geräteabgaben im Jahre 1966 - so dass eine analoge Lösung des Dilemmas zwischen effektiver Kompensation der Rechteinhaber und Wahrung der Freiheiten der Nutzer in Gestalt einer Internetabgabe („Kulturflatrate") nahe liegt, die auch Gefährdungen der Rechte auf informationelle Selbstbestimmung vermeidet.

\section{A. Ausgestaltung der Kulturflatrate}

521 Eine solche „Kulturflatrate“ sollte zum einen die Einführung bzw. Erweiterung von Schranken, zum anderen eine neue Abgabe auf Internetanschlüsse enthalten. Die Schranken wären auf die nichtkommerzielle oder auf die private Nutzung bezogen, sowohl hinsichtlich des Downloads bzw. der Vervielfältigung ( $§ 16$ UrhG, § 53 Abs. 1 UrhG) sowie des öffentlichen Zugänglichmachens bzw. des Uploads (§ 19a UrhG); kommerzielle Verwertungsvorgänge wären nach wie vor rechtswidrig. DRMSysteme sollten in diesem Rahmen nicht erfasst werden, um die individuelle Rechtsdurchsetzung zu ermöglichen, zumal die Abgabe dem Ausgleich einer ineffektiven Rechtsdurchsetzung gilt. Aus diesem Grund werden auch Software und Spiele aus den erfassten Werkkategorien ausgenommen.

522 In diesem Rahmen wäre die Privatkopieschranke des §53 Abs. 1 UrhG zu modifizieren, indem die Einschränkung auf nicht offensichtlich rechtswidrige Werkvorlagen entfiele. Darüber hinaus müsste $\S 53$ Abs. 6 UrhG dahingehend modifiziert werden, dass davon nur die Abs. 2 und 3 des $\S 53$ UrhG erfasst werden, nicht aber Abs. 1. §53 Abs. 6 UrhG untersagt die Verbreitung - also auch die öffentliche Zugänglichmachung nach $\S 19 a U^{\prime}{ } G^{998}$ - von Vervielfältigungsstücken, die zum privaten oder sonstigen eigenen Gebrauch erstellt wurden. Ohne Modifikation ergäbe sich eine Situation, in der nur Originale per Filesharing verbreitet werden dürften, nicht aber rechtmäßig erstellte Privatkopien. Eine Beibehaltung des Erfordernisses, dass keine Privatkopien von offensichtlich rechtswidrigen Vorlagen erstellt werden dürfen, würde dazu führen, dass Nutzer beurteilen (können) müssten, ob ein

\footnotetext{
${ }^{998}$ S. dazu Lüft, in: Wandtke/Bullinger, UrhR, 3. Aufl. 2009, § 53 UrhG Rn. 44.
} 
Upload bzw. ein öffentliches Zugänglichmachen eines Werkes privaten oder kommerziellen Zwecken dient. Dies dürfte noch größere Probleme aufwerfen als derzeit die Einschätzung der offensichtlichen Rechtswidrigkeit.

523 Zudem müsste zur Erfassung der Angebotsseite (Upload) eine neue Schranke zugunsten privater Nutzer hinsichtlich des Rechts auf öffentliche Zugänglichmachung (§ 19a UrhG) eingeführt werden. Schließlich wäre im Bereich der Schranken eine Ausnahme für private Bearbeitungen im Rahmen von $\S 23$ UrhG einzuführen. Die jeweiligen Beschränkungen auf private Nutzer stellen sicher, dass sich gewerbliche Anbieter nicht auf die Schranken berufen können.

524 Die Schranken wären allein auf veröffentlichte digitale Werke bezogen; damit wird erreicht, dass der Urheber nach wie vor als Ausfluss seines Persönlichkeitsrecht das Recht beibehält, über die Veröffentlichung seines Werkes zu entscheiden.

525 Unberührt bliebe von den Schranken zudem das Urheberpersönlichkeitsrecht, indem ein Urheber sein Werk zurufen kann oder sich gegen Entstellungen wehren kann.

526 Auf der Abgabenseite wäre eine allgemeine Abgabe auf jeden Internetanschluss einzuführen, die in Abhängigkeit entweder vom Datenvolumen über den Anschluss oder von den Geschwindigkeiten des Anschlusses zu staffeln wäre.

527 Zwar wäre es auch denkbar, nur die Privatkopieschranke des § 53 Abs. 1 UrhG wieder auf Downloads jedweder Vorlage zu erweitern, nicht dagegen die Uploadvorgänge, vergleichbar der Rechtslage in den Niederlanden. ${ }^{999}$ Hierzu bedürfte es auch, im Gegensatz zu der Einführung einer Schranke für Uploadvorgänge, keiner Änderung europäischen Rechts, da die InfoSoc-RL hinsichtlich der Privatkopie keine weiteren Vorgaben enthält, was die Rechtmäßigkeit der Vorlage, von der die Vervielfältigung angefertigt wird, angeht. ${ }^{1000}$ Allerdings werden etliche Filesharing-Programme damit nicht erfasst, da sie - wie z.B. viele BitTorrent-Sharingprogramme - gleichzeitig einen Download und einen Upload vornehmen, und sei es auch nur im Hinblick auf einzelne kleinere Fragmente. Privilegiert wären nur solche Download-Vorgänge, die ohne jeden Upload ihrerseits auskommen, wie etwa das Herunterladen von Files von Filesharing-Hostern wie z.B. Rapidshare.

528 Denkbar wäre es ferner, neben der Erweiterung der Privatkopieschranke zwar keine Schranke für das Uploading ( $§ 19 a$ UrhG) einzuführen, dafür aber die Strafbarkeit auf kommerzielle Aktivitäten zu beschränken (als besonders qualifizierten Tatbestand). Allerdings würde damit die zivilrechtliche Rechtsverfolgung weiterhin möglich sein - eine weitere Einschränkung in diesem Bereich käme einer Schranke gleich, da der Gesetzgeber dann zwar ein Recht einräumen, aber die Verfolgung nicht ermöglichen würde, was den Wirkungen einer Schranke entspräche.

\section{B. Rechtliche und ökonomische Beurteilung}

529 Für die rechtliche und ökonomische Beurteilung ist neben anderen Aspekten von maßgeblicher Bedeutung, welche Auswirkungen das Filesharing auf die verschiedenen Bereiche hatte, sei es auf die jeweiligen Verwerter, sei es auf Anreize für Künstler, neue Werke zu produzieren.

\footnotetext{
${ }^{999}$ S. dazu Huygen et al., 2009, S. 51 f.

${ }^{1000}$ So verlangt die InfoSoc-Richtlinie weder in ihren Erwägungsgründen noch in Art. 5 Abs. 2 b), dass die Vorlage rechtmäßig sein muss; allerdings hat der Österreichische Oberste Gerichtshof u.a. diese Frage dem EuGH vorgelegt, Vorabentscheidungsersuchen v. 29.6.2012 - Az. C-314/12 - UPC Telekabel Wien GmbH gegen Constantin Film Verleih GmbH.
} 


\section{1. Ökonomische Beurteilung}

$530 \mathrm{Zu}$ betonen ist zunächst, dass die empirische Basis zur Beurteilung von positiven und negativen Effekten des Filesharing sowie einer Kulturflatrate bislang noch nicht völlig gesichert ist, erst recht nicht für neuere über das Internet getauschte Inhalte wie Filme oder E-Books. Zu beobachten ist eine Verlagerung der Erlöse vom Verkauf physischer Medien auf digitale Inhalte, aber auch vermehrt auf sog. „Live Acts“, was allerdings nicht für jede Branche gleichermaßen gilt. Am intensivsten wurde bislang die Musikindustrie empirisch untersucht: Geht man von den Studien aus, die im Sinne eines "worst case"-Szenarios eine Substitution des Kaufs von Musik durch Filesharing annehmen, gelangt man zu einer Substitutionsrate von maximal 30\%. Mit anderen Worten führt nicht jeder getauschte Inhalt dazu, dass an dessen Stelle der Kauf des Inhalts getreten wäre. Für die anderen Branchen liegen bislang nur wenige oder gar keine Zahlen für mögliche Substitutionsraten vor. Anstelle dieser „worst-case“-Szenarien können natürlich auch andere Parameter verwandt werden, die von geringeren Substitutionsraten ausgehen.

531 Im Hinblick auf die Anreizwirkungen für Kreative liegen nur wenige Studien vor, wiederum für den Musikmarkt. Offenbar hat nach diesen Studien das Filesharing aber keine signifikanten Auswirkungen auf das Erscheinen neuer Musik bzw. Werke, was seinerseits an einer Vielzahl von Faktoren liegen kann. Für andere Branchen fehlen entsprechende Studien.

532 Die Berücksichtigung dieser Umstände, insbesondere der Substitutionsrate, ist von essentieller Bedeutung für die Berechnung der Abgabenhöhe.

\section{Rechtliche Beurteilung}

\section{a) Deutsches Verfassungsrecht}

533 Aus rechtlicher Sicht ist eine Kulturflatrate kein unverhältnismäßiger Eingriff in die Eigentumsrechte der Urheber, da sie für eine angemessene Kompensation der Urheber anstelle einer nicht immer effektiven Rechtsdurchsetzung sorgt. Maßgeblich ist in diesem Rahmen der Prognose- und Einschätzungsspielraum des Gesetzgebers hinsichtlich der Auswirkungen auf die Urheber und Verwerter, der die oben genannten gegenläufigen Effekte berücksichtigen kann. Hinzu kommt, dass mit einer Abgaben- sowie Schrankenlösung auch datenschutzrechtliche Probleme vermieden werden können.

534 Das (Urheber-) Persönlichkeitsrecht steht einer Kulturflatrate ebenfalls nicht entgegen, da die Schranken gerade nicht die dem Persönlichkeitsrecht entstammenden Ansprüche berühren, sondern vielmehr entsprechende Ansprüche weiterhin bestehen bleiben. Allerdings muss es auch bei den Möglichkeiten einer entsprechenden Rechtsverfolgung bleiben, insbesondere bei Auskunftsansprüchen und der Möglichkeit der Identifizierung von Rechtsverletzern, da sonst die Persönlichkeitsrechte schutzlos blieben.

535 Eingriffe in die Grundrechte der Provider als mittelbare Schuldner der Vergütungsansprüche belasten diese nicht unverhältnismäßig, da sie keine Aufgaben für den Staat durchführen, sondern selbst in die Verwertungskette eingeschaltet sind, was schon die bestehenden Auskunftsansprüche belegen, zudem die Kosten an ihre Kunden weitergeben können.

536 Die Persönlichkeitsrechte bzw. Rechte auf informationelle Selbstbestimmung der Nutzer können ebenfalls im Rahmen einer Kulturflatrate gewahrt werden, sofern die Messung der Nutzungsintensität von Werken sich auf freiwillige Befragungen (empirische Erhebungen) und anonymisierte Messungen beschränkt. 
537 Schließlich resultiert keine Ungleichbehandlung der Internetnutzer im Hinblick auf diejenigen, die kein Filesharing betreiben. Zum einen berücksichtigt eine Staffelung nach Geschwindigkeiten und/oder Volumen die unterschiedliche Nutzung des Internet, zum anderen ist eine (minimale) Heranziehung der nicht-tauschenden Nutzer gerechtfertigt, wenn es auch ihren Interessen dient, insbesondere der Vermeidung datenschutzrechtlicher Gefährdungen. Problematisch ist allerdings eine Doppelbelastung derjenigen, die Triple-Play-Modelle verwenden und dadurch per se höhere Geschwindigkeiten benötigen; hier ist über entsprechende Abschläge nachzudenken.

\section{b) Europarecht}

538 Im Gegensatz zum Verfassungsrecht setzt die bestehende europarechtliche Rechtslage in Gestalt der Richtlinie über Urheberrechte in der Informationsgesellschaft - sog. InfoSoc-RL - der Einführung einer Kulturflatrate schwer zu überwindende Grenzen. Während die Erweiterung der Privatkopie im Ermessen der Mitgliedstaaten steht, sieht die Richtlinie für Schranken im Bereich des Rechts auf öffentliches Zugänglichmachen (Upload) einen abschließenden Katalog vor. Zwar wird vereinzelt vertreten, dass eine Kulturflatrate mit entsprechenden Einschränkungen für die Verwertungsrechte beim Upload nur als eine Regelung der Ausübung der Verwertungsrechte zu interpretieren sei; doch stößt eine solche Auslegung auf erhebliche systematische und teleologische Bedenken hinsichtlich der Richtlinie und hat sich bislang nicht durchsetzen können. Eine Änderung der InfoSoc-RL wäre daher im Sinne einer Erweiterung der Schranken für das Recht auf öffentliche Zugänglichmachung erforderlich (Art. 5 Abs. 3 der Richtlinie).

539 Demgegenüber bestehen keine Bedenken hinsichtlich der Einführung einer Schranke für die Bearbeitungsrechte, so dass nutzergenerierte Inhalte im wesentlich größeren Maße als bislang ermöglicht würden.

540 Unabhängig von dem abschließenden Katalog der Schranken in Art. 5 Abs. 3 InfoSoc-RL würden entsprechende Schranken im Rahmen der wiederum maßgeblichen Einschätzungsprärogative des Gesetzgebers dem Dreistufen-Test standhalten. Der Urheber würde mit einer Abgabe im Rahmen der Kulturflatrate letztlich mindestens gleich, wenn nicht sogar besser gestellt als im Rahmen einer individuellen Rechtsdurchsetzung, da angesichts der zeit- und kostenintensiven individuellen Rechtsdurchsetzung im Internet die Kulturflatrate Verluste kompensieren könnte, die bei derzeitiger Rechtslage oder selbst durch eine verschärfte Rechtsdurchsetzung nicht oder nur schwer zu ersetzen sind. Zudem stände dem Rechteinhaber nach wie vor der Einsatz von DRM-Systemen offen. Ob Einbußen von aktuellen Streaming-Diensten wie Spotify, iTunes oder T-Entertain dem entgegenstehen oder diese Geschäftsmodelle nach wie vor neben einer Kulturflatrate möglich sind, lässt sich derzeit kaum abschätzen, bewegt sich aber im Rahmen der Einschätzungsprärogative des Gesetzgebers.

541 Hinsichtlich der Abgabenerhebung sowohl für den Adressatenkreis als auch für ihre Höhe enthält die InfoSoc-RL ebenfalls Vorgaben, die durch die Rechtsprechung des EuGH vor allem in der PadawanEntscheidung präzisiert worden sind. Demnach müssen die Nachteile bzw. Schäden der Urheber, die durch die Privatkopie entstehen, angemessen kompensiert werden. Ein gewisser Bezug zum Individualschaden des Urhebers ist daher durch die Richtlinie vorgegeben, wenngleich Pauschalierungen und Typisierungen möglich sind. Zudem sind aufgrund des Schadensbezugs aber auch Anrechnungen von gleichzeitig entstehenden Vorteilen möglich, so dass für die Berücksichtigung einer Substitutionsrate Spielraum besteht. 


\section{c) Internationales Urheberrecht (Kollisionsrecht)}

542 Schließlich resultiert aus den kollisionsrechtlichen Vorgaben des Urheberrechts ein praktisches Anwendungsproblem für eine Kulturflatrate: Während für die Schranke der Privatkopie bzw. des Downloads diejenige Rechtsordnung Anwendung findet, in deren Land die Kopie erstellt wird, wird weithin für den Upload, also das Recht auf öffentliches Zugänglichmachen jeder mögliche Abrufort des Inhalts als Anknüpfungspunkt gewählt. Dies bedeutet, dass auch bei einer Schranke in Deutschland bzw. der EU zugunsten des Uploads ein anderes Land diesen Vorgang anders behandeln könnte - so dass hieraus de facto ein Zwang zum territorial beschränkten Zugriff auf die Inhalte resultiert, der in praxi aber mittels Geolocation-Tools erreicht werden könnte, auch ohne dass ein konkreter Anschlussinhaber identifiziert werden müsste.

543 Andere denkbare Modelle wären die Identifizierung der abrufenden Nutzer auf Tauschbörsen in Deutschland oder die Ansiedlung von solchen Tauschbörsen bei Access-Providern oder anderen Dienstleistern mit Sitz in Deutschland mit entsprechenden Anmelderoutinen. Zwar könnten damit wahrscheinlich die meisten ausländischen Nutzer von dem Abruf ausgeschlossen werden; doch darf nicht verkannt werden, dass die Staatsbürgerschaft rechtlich gesehen nichts mit dem Abrufort eines Inhaltes zu tun hat, auf den es aber maßgeblich für das Internationale Urheberrecht ankommt. Mit anderen Worten kann sich ein deutscher Staatsbürger auch bei einer solchen nationalen Tauschbörse aus dem Ausland anmelden - und dennoch wäre das ausländische Recht anwendbar, da es lediglich auf den Abrufort ankommt. ${ }^{1001}$ Diese Fälle müssten dann durch eine zusätzliche Angabe des Ortes, von dem aus der Nutzer die Inhalte abruft, ausgeschlossen werden. Das Ziel der Anonymität wäre zudem erheblich beeinträchtigt; zumindest bedürfte es entsprechender datenschutzrechtlicher Vorkehrungen.

\section{Berechnung und Verteilung}

\section{Höhe der Abgabe}

544 Vor diesem Hintergrund kann die Bandbreite einer möglichen Abgabe bestimmt werden, wobei verschiedene Modelle der Berechnung in Betracht kommen:

- Geht man von dem modifizierten Modell der Lizenzanalogie aus, das bei der Geräteabgabe Verwendung findet, kommt es auf das durchschnittliche Volumen an, das pro Internetanschluss genutzt wird, auf dessen Basis dann die urheberrechtlich relevanten Vervielfältigungsvorgänge geschätzt werden. Ein Problem stellt bei der Anknüpfung an das Volumen die Tatsache dar, dass nicht nur die Dateigrößen zwischen Film, Buch und Musik variieren, sondern es zusätzlich innerhalb der Gattungen durch die Verwendung verschiedener Kompressionsraten zu unterschiedlichen Dateigrößen kommt. Dem kann man dadurch abhelfen, dass für die verschiedenen Medienarten durchschnittliche Kompressionsraten festlegt werden. Zudem kann der Datenverkehr anhand empirischer Studien aufgeschlüsselt und den verschiedenen Medienkategorien zugeteilt werden. ${ }^{1002}$ Je nachdem, ob man eine Substitutionsrate berücksichtigt, ergibt sich folgendes Tableau:

${ }^{1001}$ S. oben Rn. $262 \mathrm{ff}$.

1002 S. dazu ausführlich die Berechnungen für das VG-Modell Rn. 274 ff. 


\begin{tabular}{|l|l|l|l|}
\hline $\begin{array}{l}\text { Preis umgelegt auf pri- } \\
\text { vate Breitbandanschlüs- } \\
\text { se, monatlich, ohne } \\
\begin{array}{l}\text { Substitutionsrate, ohne } \\
\text { Zweitverwertungsab- } \\
\text { schlag }\end{array}\end{array}$ & $\begin{array}{l}\text { Preis umgelegt auf pri- } \\
\text { vate Breitbandanschlüs- } \\
\text { se, ohne Substitutionsra- } \\
\text { te, mit Zweitverwer- } \\
\text { tungsabschlag }\end{array}$ & $\begin{array}{l}\text { Preis umgelegt auf pri- } \\
\text { vate Breitbandanschlüs- } \\
\text { se, monatlich mit Substi- } \\
\text { tutionsrate, ohne Zweit- } \\
\text { verwertungsabschlag }\end{array}$ & $\begin{array}{l}\text { Preis umgelegt auf } \\
\text { private Breitbandan- } \\
\text { schlüsse, monatlich mit } \\
\text { Substitutionsrate, mit } \\
\text { Zweitverwertungsab- } \\
\text { schlag }\end{array}$ \\
\hline \multicolumn{2}{|c|}{$22,47 €$} & $26,97 €$ & $6,74 €$ \\
\hline
\end{tabular}

Realistisch erscheint hier ein Preis von 6,74 Euro ${ }^{1003}$, möglicherweise auch von 22,47 Euro, wobei aber beachtet werden muss, dass der in diese Rechnung eingezogene Zweitverwertungsabschlag bei einer Kulturflatrate kritisch zu sehen ist. ${ }^{1004}$ Setzt man nur eine Substitutionsrate an, ergibt sich ein Preis i.H.v. 26,97 Euro, doch ist auch dieser Preis zu hoch. ${ }^{1005}$ Zum Vergleich: 2011 betrug der durchschnittliche monatliche Preis für eine $16 \mathrm{MBit} / \mathrm{s}$ DSL-Flatrate inkl. Telefon-Flatrate 28,16 Euro. ${ }^{1006}$ Eine Abgabe nach dem reinen Lizenzanalogiemodell i.H.v. 89,89 Euro würde nicht berücksichtigen, dass Substitutionen nicht in einem Verhältnis 1:1 erfolgen; zudem würde sie zu einer unrealistischen Abgabe führen, die fast dem Doppelten (!) der derzeitigen Erlöse der gesamten (!) Verwerterindustrie und somit einer Überkompensation entspräche.

- Demgegenüber würde das Modell einer Kappungsgrenze anhand des Gerätepreises, übertragen auf eine Kulturflatrate anhand der monatlichen Internetzugangsgebühr eine wesentliche geringere Rate vorsehen. Bei einer urheberrechtlich relevanten Nutzung von $25 \%$ würde sich hier eine Kappung bei 14\% der Zugangsgebühr ergeben, so dass sich bei einer durchschnittlichen Flatrate von 25 Euro eine maximale Abgabe von 3,50 Euro ergäbe. Allerdings bestehen aufgrund der Padawan-Entscheidung des EuGH erhebliche Bedenken, ob die im deutschen Urheberrecht vorgesehene Kappung europarechtlich zulässig ist. Zudem erscheint eine Anknüpfung an die monatliche Internetzugangsgebühr ungeeignet, um einen gerechten Ausgleich zwischen Urhebern und Nutzern herzustellen, da urheberrechtliche Parameter keine Berücksichtigung finden.

- Legt man dagegen das von Fisher entwickelte Modell zugrunde, dass die Umsätze der jeweiligen Verwerter bzw. Urheber, bereinigt um die Vertriebskosten und Gewinnspannen der Zwischenhandelsstufen, als Ausgangspunkt nimmt, ferner die Substitutionsraten, gelangt man zu einer monatlichen Abgabe von ca. 5 Euro. Allerdings ist anzumerken, dass es sich hier nur um grobe Schätzungen handelt, da aus einigen Branchen verlässliche Zahlen fehlen, ebenso empirische Erhebungen über Substitutionsraten.

\footnotetext{
${ }^{1003}$ Nach einer Umfrage von The American Assembly, Copy Culture in the US and Germany, 2013, S. 58, wären 74\% der Befragten, die eine monatliche Pauschalabgabe favorisieren, bereit einen Betrag von maximal 9,99 Euro zu bezahlen.

${ }^{1004}$ S. dazu oben Rn. 294 f.

1005 Und würde vermutlich an Akzeptanzproblemen in der Bevölkerung leiden: Nach der Umfrage von The American Assembly, Copy Culture in the US and Germany, 2013, S. 58, wären nur 15\% der Befragten, die ohnehin eine monatliche Pauschalabgabe favorisieren, bereit einen Betrag von 25 Euro oder mehr zu bezahlen.

${ }^{1006}$ Verivox, DSL und Kabel-Internet: Preisentwicklung im Jahr 2011, abrufbar unter: www.verivox.de/presse/dsl-und-kabel-internet-preisentwicklung-im-jahr-2011-82490.aspx.
} 
545 Im Rahmen der Einschätzungsprärogative des Gesetzgebers erscheint eine Abgabe in Höhe zwischen 5 Euro und 22,47 Euro rechtlich zulässig.

\section{Vergütungsschuldner}

546 Eine Abgabe sollte an einen Breitbandanschluss anknüpfen, möglichst gestaffelt nach Volumen und/oder Geschwindigkeit, um unterschiedliches Nutzungsverhalten zu erfassen.

547 Vergütungsschuldner sind die Internet-Access-Provider, die diese Abgabe an ihre Kunden weiterbelasten - vergleichbar der Geräteabgabe.

548 Die Geräteabgaben wären bei Einführung einer solchen Abgabe entsprechend zu kürzen, um urheberrechtlich relevante Vorgänge, etwa die Vervielfältigung im Rahmen eines Downloadvorgangs, nicht doppelt zu erfassen.

\section{Verteilung}

549 Die Verteilung der Abgabe sollte entsprechend den Vorbildern der derzeitigen Verwertungsgesellschaften, ggf. mit Modifikationen, anhand der Nutzungshäufigkeit der jeweiligen Werke vorgenommen werden. Dabei können in einem gewissen Maße auch soziale oder kulturpolitische Erwägungen berücksichtigt werden, z.B. durch Progressionsvorbehalte, solange der eigentliche Anknüpfungspunkt die Nutzungsintensität des jeweiligen Werkes bleibt.

550 Die Nutzungshäufigkeit kann datenschutzrechtlich neutral auf mehrfache Weise erfolgen, etwa durch:

- Plug-ins in Browsern (auf freiwilliger und anonymisierter Basis) bei den Nutzern

- Durch Monitoring von Filesharingnetzwerken oder Sharehostern und Audiofingerprinting

- Durch Umfragen bzw. Erhebungen

551 Voraussetzung ist allerdings, dass die Werke in einer Datenbank registriert werden (einschließlich Audio- und Videofingerprint) - was aber bereits für zahlreiche Werke der verschiedenen Branchen der Fall ist. Eine Messung auf diese Weise ist teilweise präziser als bei der derzeitigen Geräteabgabe und sollte, bei Wahrung der statistischen Repräsentativität, auch Nischenprodukte bis zu einer gewissen Bagatellschwelle erfassen. Gleichwohl müssen etwaige Ungenauigkeiten oder Pauschalierungen, die sich aus den Messmethoden ergeben, dabei zugunsten des Datenschutzes hingenommen werden, da sie sich nur durch eine komplette Filterung des Datenverkehrs beseitigen ließen.

552 Missbräuche müssen durch eine Kombination der oben erwähnten Ermittlungssysteme vermieden werden, teilweise auch durch Kontrollen auf Anomalien im Datenverkehr, etwa bestimmte häufige Abfragen von Werken durch wenige IP-Adressen oder gehäuftes Auftreten zu einem eng umgrenzten Zeitpunkt.

\section{Erhebung und Festlegung der Tarife}

553 Die Abgaben können über die Internetprovider von den bestehenden Verwertungsgesellschaften erhoben werden. Zwar bedarf die Struktur der Verwertungsgesellschaften evtl. der Reform, doch beziehen sich diese Fragen auf allgemeine Probleme der "Corporate Governance“ der Verwertungs- 
gesellschaften und sollten umfassend im Rahmen des EU-Vorschlags zum Collective rights management ${ }^{1007}$ aufbereitet werden.

554 Die Festlegung der Tarife sollte entsprechend §§ 54a ff. UrhG in Verhandlungen zwischen den Verwertungsgesellschaften und neu zu schaffenden Nutzerverbänden, ggf. auch bestehenden Verbraucherschutzvereinigungen erfolgen. Eine Streitschlichtung sollte anhand der Vorgaben des UrhWahrnG erfolgen.

\section{Alternative Modelle und Auswirkungen auf Geschäftsmodelle}

555 Alternative Modelle wie die Kulturwertmark sind nicht in der Lage, die Urheber entsprechend der Nutzungsintensität ihrer Werke zu entgelten und sind europarechtlich daher fragwürdig.

556 Welche Auswirkungen eine Kulturflatrate allerdings für Streaming-Dienste und kommerzielle Download-Portale hätte, kann nur schwer abgeschätzt werden, insbesondere ob sich diese Modelle durch verbesserte Qualität, Zugang und ein breiteres sofort verfügbares Angebot genügend von den „Angeboten“ durch Filesharing abheben könnten. Die vorliegenden empirischen Studien bzw. Umfragen zeigen zwar zum einen, dass Nutzer bereit sind, bei geringem Entgelt solche Dienste zu nutzen, wie man an den schon heute etablierten Streaming- und Downloadportalen sieht; andererseits zeigen die Studien zum Teil auch, dass die Kriterien der Qualität und Verfügbarkeit offenbar kaum entscheidungserheblich sind für die Nutzer. Dem Gesetzgeber stünde hier jedoch ein Prognosespielraum bzw. eine Einschätzungsprärogative sowie eine mögliche Befristung und Überprüfung einer solchen Abgabe zur Verfügung.

\footnotetext{
${ }^{1007}$ Europäische Kommission, Vorschlag für eine Richtlinie des Europäischen Parlaments und des Rates über kollektive Wahrnehmung von Mehrgebietslizenzen für die Online-Nutzung von Rechten an Musikwerken im Binnenmarkt vom 11.7.2012, COM(2012) 372 final.
} 


\section{Anhang}

\section{A. Übersicht über vorliegende empirische Studien aus Hargreaves-Report}

557 Die vom Hargreaves-Report ausgewerteten Studien geben einen interessanten, da vergleichenden Aufschluss zur deutschen Situation: ${ }^{1008}$

1. Music

\begin{tabular}{|c|c|}
\hline $\begin{array}{l}2010 \text { - Harris Interactive/BPI Digital Music Sur- } \\
\text { vey }^{1009}-5,000+\text { surveyed, aged 16-54 }\end{array}$ & $\begin{array}{l}29 \text { per cent engaged in unauthorised music down- } \\
\text { loading. } \\
\text { Films, TV programmes, software (non-gaming) and } \\
\text { video games respectively were the next most popu- } \\
\text { lar downloads. } \\
76 \text { per cent of all music obtained online was unli- } \\
\text { censed. }\end{array}$ \\
\hline $\begin{array}{l}2010 \text { - Nielsen, The Hyper-Fragmented World of } \\
\text { Music survey, on behalf of Midem }{ }^{1010}-26,644 \\
\text { respondents across } 53 \text { markets }\end{array}$ & $\begin{array}{l}35 \text { per cent worldwide admitted to downloading } \\
\text { music without paying for it (potentially illegally). }\end{array}$ \\
\hline $\begin{array}{l}2010 \text { - Music Matters/Synovate/MidemNet Global } \\
\text { Survey }{ }^{1011} \text { of } 8,500 \text {, aged } 18+\text { in } 13 \text { countries }\end{array}$ & $\begin{array}{l}\text { UK - } 13 \text { per cent admitted to file sharing (not clear } \\
\text { whether this is in response to the same question as } \\
\text { below). } \\
\text { USA - } 15 \text { per cent downloaded a song from the } \\
\text { internet without paying for it. } \\
\text { Globally }-29 \text { per cent. } \\
\text { China (the highest) }-68 \text { per cent . } \\
\text { S Korea (second highest) - } 60 \text { per cent. } \\
\text { Spain (third highest) }-46 \text { per cent. }\end{array}$ \\
\hline 2010 - BPI, Digital Music Nation & 65 per cent of music downloads are illegal. ${ }^{1012}$ \\
\hline $\begin{array}{l}2011 \text { - International Federation of the Phonograph- } \\
\text { ic Industry report does not aggregate data but } \\
\text { quotes the } 2010 \text { Nielsen survey }\end{array}$ & $\begin{array}{l}23 \text { per cent across the top five EU markets (of ac- } \\
\text { tive internet users) admitted to downloading with- } \\
\text { out paying. } \\
45 \text { per cent in Brazil. } \\
44 \text { per cent in Spain. }\end{array}$ \\
\hline $\begin{array}{l}2009 \text { - International Federation of the Phonograph- } \\
\text { ic Industry report } \\
\text { Collating studies from } 16 \text { countries over four years }\end{array}$ & $\begin{array}{l}2010 \text { - } 29.8 \text { million frequent users of file sharing } \\
\text { services in the top five EU markets. } \\
2009 \text { - } 95 \text { per cent of music downloads are unau- } \\
\text { thorized. } \\
2008 \text { - over } 40 \text { billion unauthorised files shared - } \\
\text { meaning that globally around } 95 \text { per cent of music } \\
\text { tracks are downloaded without payment. } \\
16 \text { per cent of internet users in Europe regularly } \\
\text { swap music on P2P networks (Jupiter Research). }\end{array}$ \\
\hline
\end{tabular}

${ }^{1008}$ Quelle: Tabelle aus Hargreaves, Digital Opportunity, 2011, S. 70 ff. Allerdings sind die Quellenangaben nicht frei von Widersprüchen oder nicht auffindbar, s. dazu die einzelnen Anmerkungen.

${ }^{1009} \mathrm{http}: / /$ www.bpi.co.uk/assets/files/Digital\%20Music\%20Nation\%202010.pdf.

${ }^{1010} \mathrm{http}: / /$ www.nielsen.com/us/en/insights/reports-downloads/2011/hyper-fragmented-world-of-music.html.

${ }^{1011} \mathrm{http}: / /$ www.midem.com/RM/RM_Midem_v2/pdf/industry-

insight/library/music_consumption_around_the_world.pdf.

${ }_{1012}$ Die genaue Quelle ist hier nicht klar. Es kann nur vermutet werden, dass Harris Interactive/BPI Digital Music Survey (s. Fn. 1009) gemeint ist; wobei hierin die angegebene Prozentzahl von 65\% nicht zu finden ist.

${ }^{1013}$ http://www.ifpi.org/content/library/DMR2009-real.pdf. 


\begin{tabular}{|c|c|}
\hline $\begin{array}{l}2007 \text { \& } 2009 \text { - Brindley \& Walker, The Leading } \\
\text { Question/Music Ally Speakerbox survey of } 1000 \\
\text { music fans (aged 14-64) }{ }^{1014}\end{array}$ & $\begin{array}{l}\text { Overall - per month: } \\
2007-22 \text { per cent file share (potentially illegally). } \\
2009-17 \text { per cent file-share. } \\
14-18 \text { year olds: } \\
2007-42 \text { per cent file share. } \\
2009-26 \text { per cent file share. }\end{array}$ \\
\hline $\begin{array}{l}\text { 2002-2008 - Sandvine Intelligent Broadband Net- } \\
\text { works, Global Internet Phenomena Reports - deep } \\
\text { packet inspection of payloads on computer net- } \\
\text { works }\end{array}$ & $\begin{array}{l}\text { File sharing accounted for between } 40 \text { and } 60 \text { per } \\
\text { cent of all bandwidth. }\end{array}$ \\
\hline $\begin{array}{l}2006 \text { - Birgitte Andersen and Marion Frenz, The } \\
\text { Impact of Music Downloads and P2P File-Sharing } \\
\text { on the Purchase of Music: A Study for Industry } \\
\text { Canada, }{ }^{1016} \text { Decima Research survey, Survey of } \\
2,100 \text { Canadian people, quota based random sam- } \\
\text { ple to represent Canadian population as a whole. }\end{array}$ & $\begin{array}{l}29 \text { per cent download through P2P networks. } \\
29.2 \text { per cent rip from CDs. } \\
20.5 \text { per cent used friends to copy MP3s. } \\
8.5 \text { per cent downloaded from free sites. }\end{array}$ \\
\hline
\end{tabular}

2. Feature films/TV programmes

\begin{tabular}{|l|l|}
\hline 2010 - Harris Interactive & $\begin{array}{l}14 \text { per cent of internet users download films \& TV } \\
\text { programmes from illegal P2P services. }\end{array}$ \\
\hline $2008-09$ - Wiggin Entertainment Media Rese- & Watch pirate DVDs of movies: \\
$\operatorname{arch}^{1017}$ & $2008-29$ per cent \\
& $2009-29$ per cent. \\
& File sharing unauthorised films/programmes: \\
& $2008-21$ per cent \\
& $2009-21$ per cent. \\
\hline
\end{tabular}

\section{Games/software}

\begin{tabular}{|l|l|}
\hline 2008-09 - Wiggin Entertainment Media Research & File sharing unauthorised games/software: \\
& $2008-14$ per cent. \\
& $2009-16$ per cent. \\
\hline 2008 - Nielsen, Video Gamers in Europe, Piracy and & Europe: \\
$\begin{array}{l}\text { Digital Downloading for the Interactive Software } \\
\text { Federation of Europe, Survey of 6,000 active gam- } \\
\text { ers }\end{array}$ & $\begin{array}{l}2007-40 \text { per cent owned at least one pirate/ } \\
\text { copied game. } \\
2008-35 \text { per cent owned at least one pirate/ } \\
\\
\text { copied game (14 per cent in UK). }\end{array}$ \\
\hline
\end{tabular}

\section{Business Software}

\begin{tabular}{|l|l|}
\hline $\begin{array}{l}\text { 2008-09 - British Software Alliance \& International } \\
\text { Data Corporation }\end{array}$ & $\begin{array}{l}27 \text { per cent of software installed in the UK in that } \\
\text { year was illegal. }\end{array}$ \\
\hline
\end{tabular}

5. Books

\begin{tabular}{|l|l|}
\hline Jan 2010 - Atttibutor (anti-piracy business) & $\begin{array}{l}10 \text { per cent of the total United States book sales were } \\
\text { pirated. }\end{array}$ \\
\hline
\end{tabular}

\footnotetext{
${ }^{1014}$ Die Angaben konnten nicht überprüft werden, da die Quelle nicht im Volltext verfügbar war.

1015 Die Angaben konnten nicht überprüft werden, da die Quelle nicht im Volltext verfügbar war.

${ }^{1016}$ http://www.ic.gc.ca/eic/site/ippd-

dppi.nsf/vwapj/IndustryCanadaPaperMay4_2007_en.pdf/\$FILE/IndustryCanadaPaperMay4_2007_en.pdf.

${ }^{1017}$ Die Angaben konnten nicht überprüft werden, da die Quelle nicht im Volltext verfügbar war.

${ }^{1018}$ Die Angaben konnten nicht überprüft werden, da die Quelle nicht im Volltext verfügbar war. 1019
}

http://www.bsa.org/sitecore/shell/Controls/Rich\%20Text\%20Editor/ /media/Files/idc_studies/bsa_idc_united _kingdom_final\%20pdf.ashx. 
6. Unauthorised Content Generally

\begin{tabular}{|l|l|}
\hline $\begin{array}{l}\text { April 2011- } \text { eBizMBA Rank Fifteen Most Popular } \\
\text { Torrent Websites }\end{array}$ & $\begin{array}{l}\text { Over } 45 \text { million estimated unique monthly visitors } \\
\text { worldwide on } 15 \text { most popular sites. }\end{array}$ \\
\hline $\begin{array}{l}\text { Jan } 2011 \text { - USA, MarkMonitor, Traffic Report: } \\
\text { Online pirating and counterfeiting }\end{array}$ & $\begin{array}{l}1022 \\
\text { digital piracy \& traffic generated by these sites was } \\
\text { over } 146 \text { million visits per day or } 53 \text { billion per year. }\end{array}$ \\
\hline $\begin{array}{l}2010 \text { - Tera Consultants/Business Action to Stop } \\
\text { Counterfeiting and Piracy }\end{array}$ & $\begin{array}{l}778 \text { million digital piracy copyright infringements per } \\
\text { year. }\end{array}$ \\
\hline $2008-09-$ IPOQUE $^{1024}$ & $\begin{array}{l}34 \text { to } 70 \text { per cent of global internet traffic taken up } \\
\text { with file sharing depending on region. }\end{array}$ \\
\hline $\begin{array}{l}\text { 2008 - Forrester Research survey of 1,176 } \\
\text { consumers }\end{array}$ & $\begin{array}{l}11.6 \text { per cent of respondents admitted to } \\
\text { engaging in illegal file sharing. Scaled up } \\
\text { to } 16.3 \text { per cent because of under reporting concern. } \\
\text { This equates to } 6.7 \text { million people. }\end{array}$ \\
\hline
\end{tabular}

${ }^{1020}$ Die Angaben konnten nicht überprüft werden, da die Quelle nicht im Volltext verfügbar war.

${ }^{1021}$ http://www.ebizmba.com/articles/torrent-websites.

1022 https://www.markmonitor.com/download/report/MarkMonitor_-_Traffic_Report_110111.pdf.

${ }^{1023}$ http://www.droit-technologie.org/upload/dossier/doc/219-1.pdf.

${ }^{1024}$ Die Angaben konnten nicht überprüft werden, da die Quelle nicht im Volltext verfügbar war.

${ }^{1025}$ Die Angaben konnten nicht überprüft werden, da die Quelle nicht im Volltext verfügbar war. 


\section{B. Berechnungstabellen}

\section{Lizenzanalogiemodell}

Formel: Zahl der Breitbandanschlüsse $x$ durchschnittliches Volumen des Datenverkehrs pro Monat x prozentualer Anteil der urheberrechtlich relevanten Nutzungen x prozentualer Anteil des getauschten Mediums am Datenvolumen / MB pro Werk bzw. Werksammlung (Album-, bzw. Songdownload, Filmdownload, E-Book) x durchschnittlicher Ladenpreis abzgl. Vertriebsstufen

\section{a) „Reines“ Lizenzanalogiemodel}

(1) Urheberrechtlich relevanter Anteil von $25 \%$

(a) Ohne Abschlag für Zweitverwertung

559 Für die Musikindustrie:

\begin{tabular}{|l|l|l|l|l|l|}
\hline $\begin{array}{l}\text { Volumen } \\
\text { pro Nutzer } \\
\text { in MB } \\
\text { monatlich }\end{array}$ & $\begin{array}{l}\text { Zahl der Breitbandan- } \\
\text { schlüsse }\end{array}$ & $\begin{array}{l}\text { Volumen pro Mo- } \\
\text { nat in MB }\end{array}$ & $\begin{array}{l}\text { Urheberrechtlich } \\
\text { relevanter Anteil }\end{array}$ & $\begin{array}{l}\text { Anteil von Musik- } \\
\text { downloads am } \\
\text { Gesamtvolumen }\end{array}$ & $\begin{array}{l}\text { Spielstunde } \\
\text { in MB }\end{array}$ \\
\hline 12.500 & 27.300 .000 & 341.250 .000 .000 & $25 \%$ & $12,6 \%$ & 100 \\
\hline
\end{tabular}

\begin{tabular}{|l|l|l|l|l|}
\hline & $\begin{array}{l}\text { Durchschnittlicher End- } \\
\text { verkaufspreis eines Al- } \\
\text { bums }\end{array}$ & $\begin{array}{l}\text { Anteil der Rechtein- } \\
\text { haber am Erlös }\end{array}$ & Preis monatlich & $\begin{array}{l}\text { Preis umge- } \\
\text { legt auf An- } \\
\text { schluss mo- } \\
\text { natlich }\end{array}$ \\
\hline 107.493 .750 & $9,99 €$ & $80,25 \%$ & $861.774 .706,41 €$ & $\mathbf{3 1 , 5 7 €}$ \\
\hline
\end{tabular}

\section{Für die Filmindustrie:}

\begin{tabular}{|l|l|l|l|l|l|}
\hline $\begin{array}{l}\text { Volumen } \\
\text { pro Nutzer } \\
\text { in MB mo- } \\
\text { natlich }\end{array}$ & $\begin{array}{l}\text { Zahl der Breitband- } \\
\text { anschlüsse }\end{array}$ & $\begin{array}{l}\text { Volumen pro Mo- } \\
\text { nat in MB }\end{array}$ & $\begin{array}{l}\text { Urheberrechtlich } \\
\text { relevanter Anteil }\end{array}$ & $\begin{array}{l}\text { Anteil von Film- } \\
\text { downloads am } \\
\text { Gesamtvolumen }\end{array}$ & $\begin{array}{l}\text { Spielstunde } \\
\text { in MB }\end{array}$ \\
\hline 12.500 & 27.300 .000 & 341.250 .000 .000 & $25 \%$ & $87,3 \%$ & 466,67 \\
\hline
\end{tabular}

\begin{tabular}{|l|l|l|l|l|}
\hline & $\begin{array}{l}\text { Durchschnittlicher Endver- } \\
\text { kaufspreis: 7,71 } € \text { für 90 } \\
\text { min.; somit durchschnittli- } \\
\text { cher Endverkaufspreis für 1 } \\
\text { Spielstunde: }\end{array}$ & $\begin{array}{l}\text { Anteil der Recht- } \\
\text { einhaber am Erlös }\end{array}$ & Preis monatlich & $\begin{array}{l}\text { Preis umge- } \\
\text { legt auf An- } \\
\text { schluss mo- } \\
\text { natlich }\end{array}$ \\
\hline Spielstunden insg. & $\begin{array}{l}\text { 70\% } \\
159.594 .172,54\end{array}$ & $5,14 €$ & $574.219 .832,81 €$ & $\mathbf{2 1 , 0 3 €}$ \\
\hline
\end{tabular}


561 Für die Buchindustrie:

\begin{tabular}{|l|l|l|l|l|l|}
\hline $\begin{array}{l}\text { Volumen pro } \\
\begin{array}{l}\text { Nutzer in MB } \\
\text { monatlich }\end{array}\end{array}$ & $\begin{array}{l}\text { Zahl der Breitbandan- } \\
\text { schlüsse }\end{array}$ & $\begin{array}{l}\text { Volumen pro } \\
\text { Monat in MB }\end{array}$ & $\begin{array}{l}\text { Urheberrechtlich } \\
\text { relevanter Anteil }\end{array}$ & $\begin{array}{l}\text { Anteil von Buch- } \\
\text { downloads am Ge- } \\
\text { samtvolumen }\end{array}$ & $\begin{array}{l}\text { MB } \\
\text { pro } \\
\text { Buch }\end{array}$ \\
\hline 12.500 & 27.300 .000 & 341.250 .000 .000 & $25 \%$ & $0,10 \%$ & 2 \\
\hline
\end{tabular}

\begin{tabular}{|l|l|l|l|l|}
\hline & $\begin{array}{l}\text { Durchschnittlicher Endver- } \\
\text { kaufspreis }\end{array}$ & $\begin{array}{l}\text { Anteil der Rechtein- } \\
\text { haber am Erlös }\end{array}$ & Preis monatlich & $\begin{array}{l}\text { Preis umgelegt } \\
\text { auf Anschluss } \\
\text { monatlich }\end{array}$ \\
\hline 42.656 .250 & $8,07 €$ & $50 \%$ & $172.117 .968,75 €$ & $\mathbf{6 , 3 0} €$ \\
\hline
\end{tabular}

562 Insgesamt:

\begin{tabular}{|l|l|l|l|}
\hline Musikindustrie & Filmindustrie & Buchindustrie & Gesamt \\
\hline $31,57 €$ & $21,03 €$ & $6,30 €$ & $\mathbf{5 8 , 9 1 €}$ \\
\hline
\end{tabular}

563 Gestaffelt nach Breitbandgeschwindigkeiten:

\begin{tabular}{|l|l|l|}
\hline $\begin{array}{l}\text { Kategorie } 1 \text { (bis einschließlich } 2 \\
\text { MBit/s) }\end{array}$ & Kategorie 2 (2-10 MBit/s) & Kategorie 3 (10 MBit/s und mehr) \\
\hline $22,39 €$ & $55,98 €$ & $89,57 €$ \\
\hline
\end{tabular}

(b) Mit Abschlag für Zweitverwertung

564 Für die Musikindustrie:

\begin{tabular}{|l|l|l|l|l|l|}
\hline $\begin{array}{l}\text { Volumen } \\
\text { pro Nutzer } \\
\text { in MB } \\
\text { monatlich }\end{array}$ & $\begin{array}{l}\text { Zahl der Breitbandan- } \\
\text { schlüsse }\end{array}$ & $\begin{array}{l}\text { Volumen pro Mo- } \\
\text { nat in MB }\end{array}$ & $\begin{array}{l}\text { Urheberrechtlich } \\
\text { relevanter Anteil }\end{array}$ & $\begin{array}{l}\text { Anteil von Musik- } \\
\text { downloads am } \\
\text { Gesamtvolumen }\end{array}$ & $\begin{array}{l}\text { Spielstunde } \\
\text { in MB }\end{array}$ \\
\hline 12.500 & 27.300 .000 & 341.250 .000 .000 & $25 \%$ & $12,6 \%$ & 100 \\
\hline
\end{tabular}

\begin{tabular}{|c|c|c|c|c|c|}
\hline $\begin{array}{l}\text { Spielstunden } \\
\text { insg. }\end{array}$ & $\begin{array}{l}\text { Durchschnittlicher } \\
\text { Endverkaufspreis } \\
\text { eines Albums }\end{array}$ & $\begin{array}{l}\text { Anteil der } \\
\text { Rechteinhaber } \\
\text { am Erlös }\end{array}$ & $\begin{array}{l}\text { Abschlag für } \\
\text { Zweitverwertung }\end{array}$ & Preis monatlich & $\begin{array}{l}\text { Preis um- } \\
\text { gelegt auf } \\
\text { Anschluss } \\
\text { monatlich }\end{array}$ \\
\hline 107.493 .750 & $9,99 €$ & $80,25 \%$ & $75 \%$ & $215.443 .676,60 €$ & $7,89 €$ \\
\hline
\end{tabular}


565 Für die Filmindustrie:

\begin{tabular}{|l|l|l|l|l|l|}
\hline $\begin{array}{l}\text { Volumen } \\
\text { pro Nutzer } \\
\text { in MB mo- } \\
\text { natlich }\end{array}$ & $\begin{array}{l}\text { Zahl der Breitband- } \\
\text { anschlüsse }\end{array}$ & $\begin{array}{l}\text { Volumen pro Mo- } \\
\text { nat in MB }\end{array}$ & $\begin{array}{l}\text { Urheberrechtlich } \\
\text { relevanter Anteil }\end{array}$ & $\begin{array}{l}\text { Anteil von Film- } \\
\text { downloads am } \\
\text { Gesamtvolumen }\end{array}$ & $\begin{array}{l}\text { Spielstunde } \\
\text { in MB }\end{array}$ \\
\hline 12.500 & 27.300 .000 & 341.250 .000 .000 & $25 \%$ & $87,3 \%$ & 466,67 \\
\hline
\end{tabular}

\begin{tabular}{|c|c|c|c|c|c|}
\hline Spielstunden insg. & $\begin{array}{l}\text { Durchschnittlicher } \\
\text { Endverkaufspreis: } \\
7,71 € \text { für } 90 \text { min.; } \\
\text { somit durchschnittli- } \\
\text { cher Endverkaufs- } \\
\text { preis für } 1 \text { Spiel- } \\
\text { stunde: }\end{array}$ & $\begin{array}{l}\text { Anteil der } \\
\text { Rechtein- } \\
\text { haber am } \\
\text { Erlös }\end{array}$ & $\begin{array}{l}\text { Abschlag für } \\
\text { Zweitverwertung }\end{array}$ & Preis monatlich & $\begin{array}{l}\text { Preis um- } \\
\text { gelegt auf } \\
\text { Anschluss } \\
\text { monatlich }\end{array}$ \\
\hline $159.594 .172,54$ & $5,14 €$ & $70 \%$ & $75 \%$ & $143.554 .958,20 €$ & $5,26 €$ \\
\hline
\end{tabular}

\section{Für die Buchindustrie:}

\begin{tabular}{|l|l|l|l|l|l|}
\hline $\begin{array}{l}\text { Volumen pro } \\
\text { Nutzer in MB } \\
\text { monatlich }\end{array}$ & $\begin{array}{l}\text { Zahl der Breitbandan- } \\
\text { schlüsse }\end{array}$ & $\begin{array}{l}\text { Volumen pro } \\
\text { Monat in MB }\end{array}$ & $\begin{array}{l}\text { Urheberrechtlich } \\
\text { relevanter Anteil }\end{array}$ & $\begin{array}{l}\text { Anteil von Buch- } \\
\text { downloads am Ge- } \\
\text { samtvolumen }\end{array}$ & $\begin{array}{l}\text { MB } \\
\text { pro } \\
\text { Buch }\end{array}$ \\
\hline 12.500 & 27.300 .000 & 341.250 .000 .000 & $25 \%$ & $0,10 \%$ & 2 \\
\hline
\end{tabular}

\begin{tabular}{|l|l|l|l|l|l|}
\hline $\begin{array}{l}\text { Summe } \\
\text { Bücher }\end{array}$ & $\begin{array}{l}\text { Durchschnittlicher } \\
\text { Endverkaufspreis }\end{array}$ & $\begin{array}{l}\text { Anteil der Recht- } \\
\text { einhaber am } \\
\text { Erlös }\end{array}$ & $\begin{array}{l}\text { Abschlag für } \\
\text { Zweitverwertung }\end{array}$ & Preis monatlich & $\begin{array}{l}\text { Preis umge- } \\
\text { legt auf } \\
\text { Anschluss } \\
\text { monatlich }\end{array}$ \\
\hline 42.656 .250 & $8,07 €$ & $50 \%$ & $75 \%$ & $43.029 .492,19 €$ & $\mathbf{1 , 5 8} €$ \\
\hline
\end{tabular}

\section{Insgesamt:}

\begin{tabular}{|l|l|l|l|}
\hline Musikindustrie & Filmindustrie & Buchindustrie & Gesamt \\
\hline $7,89 €$ & $5,26 €$ & $1,58 €$ & $\mathbf{1 4 , 7 3 €}$ \\
\hline
\end{tabular}

\section{Gestaffelt nach Breitbandgeschwindigkeiten:}

\begin{tabular}{|l|l|l|}
\hline $\begin{array}{l}\text { Kategorie } \mathbf{1} \text { (bis einschließlich } \mathbf{2} \\
\text { MBit/s) }\end{array}$ & Kategorie 2 (2-10 MBit/s) & Kategorie 3 (10 MBit/s und mehr) \\
\hline $5,60 €$ & $14,00 €$ & $22,39 €$ \\
\hline
\end{tabular}


(2) Urheberrechtlich relevanter Anteil von 15\%:

(a) Ohne Abschlag für Zweitverwertung

569 Für die Musikindustrie:

\begin{tabular}{|l|l|l|l|l|l|}
\hline $\begin{array}{l}\text { Volumen } \\
\text { pro Nutzer } \\
\text { in MB mo- } \\
\text { natlich }\end{array}$ & $\begin{array}{l}\text { Zahl der Breitband- } \\
\text { anschlüsse }\end{array}$ & $\begin{array}{l}\text { Volumen pro Mo- } \\
\text { nat in MB }\end{array}$ & $\begin{array}{l}\text { Urheberrechtlich } \\
\text { relevanter Anteil }\end{array}$ & $\begin{array}{l}\text { Anteil von Musik- } \\
\text { downloads am } \\
\text { Gesamtvolumen }\end{array}$ & $\begin{array}{l}\text { Spielstunde } \\
\text { in MB }\end{array}$ \\
\hline 12.500 & 27.300 .000 & 341.250 .000 .000 & $15 \%$ & $12,60 \%$ & 100 \\
\hline
\end{tabular}

\begin{tabular}{|l|l|l|l|l|}
\hline & $\begin{array}{l}\text { Durchschnittlicher End- } \\
\text { verkaufspreis eines Al- } \\
\text { bums }\end{array}$ & $\begin{array}{l}\text { Anteil der Rechtein- } \\
\text { haber am Erlös }\end{array}$ & Preis monatlich & $\begin{array}{l}\text { Preis umge- } \\
\text { legt auf An- } \\
\text { schluss mo- } \\
\text { natlich }\end{array}$ \\
\hline 64.496 .250 & $9,99 €$ & $80,25 \%$ & $517.064 .823,84 €$ & $\mathbf{1 8 , 9 4 €}$ \\
\hline
\end{tabular}

570 Für die Filmindustrie:

\begin{tabular}{|l|l|l|l|l|l|}
\hline $\begin{array}{l}\text { Volumen } \\
\text { pro Nutzer } \\
\text { in MB mo- } \\
\text { natlich }\end{array}$ & $\begin{array}{l}\text { Zahl der Breitband- } \\
\text { anschlüsse }\end{array}$ & $\begin{array}{l}\text { Volumen pro Mo- } \\
\text { nat in MB }\end{array}$ & $\begin{array}{l}\text { Urheberrechtlich } \\
\text { relevanter Anteil }\end{array}$ & $\begin{array}{l}\text { Anteil von Film- } \\
\text { downloads am } \\
\text { Gesamtvolumen }\end{array}$ & $\begin{array}{l}\text { Spielstunde } \\
\text { in MB }\end{array}$ \\
\hline 12.500 & 27.300 .000 & 341.250 .000 .000 & $15 \%$ & $87,30 \%$ & 466,67 \\
\hline
\end{tabular}

\begin{tabular}{|l|l|l|l|l|}
\hline & $\begin{array}{l}\text { Durchschnittlicher Endverkaufspreis: } \\
7,71 € \text { für } 90 \text { min.; somit durch- } \\
\text { Schnittlicher Endverkaufspreis für 1 } \\
\text { Spielstunden } \\
\text { Spielstunde: }\end{array}$ & $\begin{array}{l}\text { Anteil der } \\
\text { Rechteinhaber } \\
\text { am Erlös }\end{array}$ & Preis monatlich & $\begin{array}{l}\text { Preis umgelegt } \\
\text { auf Anschluss } \\
\text { monatlich }\end{array}$ \\
\hline $95.756 .503,52$ & $5,14 €$ & $70 \%$ & $344.531 .899,68 €$ & $\mathbf{1 2 , 6 2} €$ \\
\hline
\end{tabular}

571 Für die Buchindustrie:

\begin{tabular}{|l|l|l|l|l|l|}
\hline $\begin{array}{l}\text { Volumen pro } \\
\begin{array}{l}\text { Nutzer in MB } \\
\text { monatlich }\end{array}\end{array}$ & $\begin{array}{l}\text { Zahl der Breitbandan- } \\
\text { schlüsse }\end{array}$ & $\begin{array}{l}\text { Volumen pro } \\
\text { Monat in MB }\end{array}$ & $\begin{array}{l}\text { Urheberrechtlich } \\
\text { relevanter Anteil }\end{array}$ & $\begin{array}{l}\text { Anteil von Buch- } \\
\text { downloads am Ge- } \\
\text { samtvolumen }\end{array}$ & $\begin{array}{l}\text { MB } \\
\text { pro } \\
\text { Buch }\end{array}$ \\
\hline 12.500 & 27.300 .000 & 341.250 .000 .000 & $15 \%$ & $0,10 \%$ & 2 \\
\hline
\end{tabular}

\begin{tabular}{|l|l|l|l|l|}
\hline $\begin{array}{l}\text { Summe Bü- } \\
\text { cher }\end{array}$ & $\begin{array}{l}\text { Durchschnittlicher Endver- } \\
\text { kaufspreis }\end{array}$ & $\begin{array}{l}\text { Anteil der Rechtein- } \\
\text { haber am Erlös }\end{array}$ & Preis monatlich & $\begin{array}{l}\text { Preis umgelegt auf } \\
\text { Anschluss monatlich }\end{array}$ \\
\hline 25.593 .750 & $8,07 €$ & $50 \%$ & $103.270 .781,25 €$ & $\mathbf{3 , 7 8} €$ \\
\hline
\end{tabular}

\section{Insgesamt:}

\begin{tabular}{|l|l|l|l|}
\hline Musikindustrie & Filmindustrie & Buchindustrie & Gesamt \\
\hline $18,94 €$ & $12,62 €$ & $3,78 €$ & $\mathbf{3 5 , 3 4} €$ \\
\hline
\end{tabular}


573 Gestaffelt nach Breitbandgeschwindigkeiten:

\begin{tabular}{|l|l|l|}
\hline $\begin{array}{l}\text { Kategorie } \mathbf{1} \text { (bis einschließlich 2 } \\
\text { MBit/s) }\end{array}$ & Kategorie 2 (2-10 MBit/s) & Kategorie 3 (10 MBit/s und mehr) \\
\hline $13,44 €$ & $33,59 €$ & $53,74 €$ \\
\hline
\end{tabular}

(b) Mit Abschlag für Zweitverwertung

\section{Für die Musikindustrie:}

\begin{tabular}{|l|l|l|l|l|l|}
\hline $\begin{array}{l}\text { Volumen } \\
\text { pro Nutzer } \\
\text { in MB mo- } \\
\text { natlich }\end{array}$ & $\begin{array}{l}\text { Zahl der Breitband- } \\
\text { anschlüsse }\end{array}$ & $\begin{array}{l}\text { Volumen pro Mo- } \\
\text { nat in MB }\end{array}$ & $\begin{array}{l}\text { Urheberrechtlich } \\
\text { relevanter Anteil }\end{array}$ & $\begin{array}{l}\text { Anteil von Musik- } \\
\text { downloads am } \\
\text { Gesamtvolumen }\end{array}$ & $\begin{array}{l}\text { Spielstunde } \\
\text { in MB }\end{array}$ \\
\hline 12.500 & 27.300 .000 & 341.250 .000 .000 & $15 \%$ & $12,60 \%$ & 100 \\
\hline
\end{tabular}

\begin{tabular}{|l|l|l|l|l|l|}
\hline & $\begin{array}{l}\text { Durchschnittlicher } \\
\text { Spielstunden } \\
\text { Endverkaufspreis } \\
\text { eines Albums }\end{array}$ & $\begin{array}{l}\text { Anteil der } \\
\text { Rechteinhaber } \\
\text { am Erlös }\end{array}$ & $\begin{array}{l}\text { Abschlag für } \\
\text { Zweitverwertung }\end{array}$ & Preis monatlich & $\begin{array}{l}\text { Preis um- } \\
\text { gelegt auf } \\
\text { Anschluss } \\
\text { monatlich }\end{array}$ \\
\hline 64.496 .250 & $9,99 €$ & $80,25 \%$ & $75 \%$ & $129.266 .205,96 €$ & $\mathbf{4 , 7 4} €$ \\
\hline
\end{tabular}

\section{Für die Filmindustrie:}

\begin{tabular}{|l|l|l|l|l|l|}
\hline $\begin{array}{l}\text { Volumen } \\
\text { pro Nutzer } \\
\text { in MB } \\
\text { monatlich }\end{array}$ & $\begin{array}{l}\text { Zahl der Breitbandan- } \\
\text { schlüsse }\end{array}$ & $\begin{array}{l}\text { Volumen pro } \\
\text { Monat in MB }\end{array}$ & $\begin{array}{l}\text { Urheberrechtlich } \\
\text { relevanter Anteil }\end{array}$ & $\begin{array}{l}\text { Anteil von Film- } \\
\text { downloads am } \\
\text { Gesamtvolumen }\end{array}$ & $\begin{array}{l}\text { Spielstunde } \\
\text { in MB }\end{array}$ \\
\hline 12.500 & 27.300 .000 & 341.250 .000 .000 & $15 \%$ & $87,30 \%$ & 466,67 \\
\hline
\end{tabular}

\begin{tabular}{|c|c|c|c|c|c|}
\hline $\begin{array}{l}\text { Spielstunden } \\
\text { insg. }\end{array}$ & $\begin{array}{l}\text { Durchschnittlicher } \\
\text { Endverkaufspreis: } 7,71 \\
€ \text { für } 90 \text { min.; somit } \\
\text { durchschnittlicher } \\
\text { Endverkaufspreis für } 1 \\
\text { Spielstunde: }\end{array}$ & $\begin{array}{l}\text { Anteil der Recht- } \\
\text { einhaber am } \\
\text { Erlös }\end{array}$ & $\begin{array}{l}\text { Abschlag für } \\
\text { Zweitverwer- } \\
\text { tung }\end{array}$ & Preis monatlich & $\begin{array}{l}\text { Preis umge- } \\
\text { legt auf } \\
\text { Anschluss } \\
\text { monatlich }\end{array}$ \\
\hline $95.756 .503,52$ & $5,14 €$ & $70 \%$ & $75 \%$ & $86.132 .974,92 €$ & $3,16 €$ \\
\hline
\end{tabular}


576 Für die Buchindustrie:

\begin{tabular}{|l|l|l|l|l|l|}
\hline $\begin{array}{l}\text { Volumen pro } \\
\text { Nutzer in MB } \\
\text { monatlich }\end{array}$ & $\begin{array}{l}\text { Zahl der Breitbandan- } \\
\text { schlüsse }\end{array}$ & $\begin{array}{l}\text { Volumen pro } \\
\text { Monat in MB }\end{array}$ & $\begin{array}{l}\text { Urheberrechtlich } \\
\text { relevanter Anteil }\end{array}$ & $\begin{array}{l}\text { Anteil von Buch- } \\
\text { downloads am Ge- } \\
\text { samtvolumen }\end{array}$ & $\begin{array}{l}\text { MB } \\
\text { pro } \\
\text { Buch }\end{array}$ \\
\hline 12.500 & 27.300 .000 & 341.250 .000 .000 & $15 \%$ & $0,10 \%$ & 2 \\
\hline
\end{tabular}

\begin{tabular}{|l|l|l|l|l|l|}
\hline & & Anteil der Recht- & & & $\begin{array}{l}\text { Preis umge- } \\
\text { legt auf } \\
\text { Summe }\end{array}$ \\
Bücher & $\begin{array}{l}\text { Durchschnittlicher } \\
\text { Endverkaufspreis }\end{array}$ & $\begin{array}{l}\text { einhaber am } \\
\text { Erlös }\end{array}$ & $\begin{array}{l}\text { Abschlag für } \\
\text { Zweitverwertung }\end{array}$ & Preis monatlich & monatlich \\
\hline 25.593 .750 & $8,07 €$ & $50 \%$ & $75 \%$ & $25.817 .695,31 €$ & $\mathbf{0 , 9 5} €$ \\
\hline
\end{tabular}

577 Insgesamt:

\begin{tabular}{|l|l|l|l|}
\hline Musikindustrie & Filmindustrie & Buchindustrie & Gesamt \\
\hline $4,74 €$ & $3,16 €$ & $0,95 €$ & $\mathbf{8 , 8 5} €$ \\
\hline
\end{tabular}

\section{Gestaffelt nach Breitbandgeschwindigkeiten:}

\begin{tabular}{|l|l|l|}
\hline $\begin{array}{l}\text { Kategorie } 1 \text { (bis einschließlich 2 } \\
\text { MBit/s) }\end{array}$ & Kategorie 2 (2-10 MBit/s) & Kategorie 3 (10 MBit/s und mehr) \\
\hline $3,36 €$ & $8,40 €$ & $13,44 €$ \\
\hline
\end{tabular}


(3) Urheberrechtlich relevanter Anteil von 5\%

(a) Ohne Abschlag für Zweitverwertung

\section{Für die Musikindustrie:}

\begin{tabular}{|l|l|l|l|l|l|}
\hline $\begin{array}{l}\text { Volumen } \\
\text { pro Nutzer } \\
\text { in MB } \\
\text { monatlich }\end{array}$ & $\begin{array}{l}\text { Zahl der Breitbandan- } \\
\text { schlüsse }\end{array}$ & $\begin{array}{l}\text { Volumen pro Mo- } \\
\text { nat in MB }\end{array}$ & $\begin{array}{l}\text { Urheberrechtlich } \\
\text { relevanter Anteil }\end{array}$ & $\begin{array}{l}\text { Anteil von Musik- } \\
\text { downloads am } \\
\text { Gesamtvolumen }\end{array}$ & $\begin{array}{l}\text { Spielstunde } \\
\text { in MB }\end{array}$ \\
\hline 12.500 & 27.300 .000 & 341.250 .000 .000 & $5 \%$ & $12,60 \%$ & 100 \\
\hline
\end{tabular}

\begin{tabular}{|l|l|l|l|l|}
\hline & $\begin{array}{l}\text { Durchschnittlicher End- } \\
\text { verkaufspreis eines Al- } \\
\text { bums }\end{array}$ & $\begin{array}{l}\text { Anteil der Rechtein- } \\
\text { haber am Erlös }\end{array}$ & Preis monatlich & $\begin{array}{l}\text { Preis umge- } \\
\text { legt auf An- } \\
\text { schluss mo- } \\
\text { natlich }\end{array}$ \\
\hline 21.498 .750 & $9,99 €$ & $80,25 \%$ & $172.354 .941,28 €$ & $\mathbf{6 , 3 1} €$ \\
\hline
\end{tabular}

580 Für die Filmindustrie:

\begin{tabular}{|l|l|l|l|l|l|}
\hline $\begin{array}{l}\text { Volumen } \\
\text { pro Nutzer } \\
\text { in MB } \\
\text { monatlich }\end{array}$ & $\begin{array}{l}\text { Zahl der Breitbandan- } \\
\text { schlüsse }\end{array}$ & $\begin{array}{l}\text { Volumen pro } \\
\text { Monat in MB }\end{array}$ & $\begin{array}{l}\text { Urheberrechtlich } \\
\text { relevanter Anteil }\end{array}$ & $\begin{array}{l}\text { Anteil von Film- } \\
\text { downloads am } \\
\text { Gesamtvolumen }\end{array}$ & $\begin{array}{l}\text { Spielstunde } \\
\text { in MB }\end{array}$ \\
\hline 12.500 & 27.300 .000 & 341.250 .000 .000 & $5 \%$ & $87,30 \%$ & 466,67 \\
\hline
\end{tabular}

\begin{tabular}{|l|l|l|l|l|}
\hline & $\begin{array}{l}\text { Durchschnittlicher Endver- } \\
\text { kaufspreis: 7,71 } € \text { für 90 min.; } \\
\text { somit durchschnittlicher End- } \\
\text { verkaufspreis für 1 Spielstun- } \\
\text { Se: }\end{array}$ & $\begin{array}{l}\text { Anteil der Recht- } \\
\text { einhaber am Erlös }\end{array}$ & Preis monatlich & $\begin{array}{l}\text { Preis umge- } \\
\text { legt auf An- } \\
\text { schluss mo- } \\
\text { natlich }\end{array}$ \\
\hline $31.918 .834,51$ & $5,14 €$ & $70 \%$ & $114.843 .966,57 €$ & $\mathbf{4 , 2 1} €$ \\
\hline
\end{tabular}

\section{Für die Buchindustrie:}

\begin{tabular}{|l|l|l|l|l|l|}
\hline $\begin{array}{l}\text { Volumen pro } \\
\begin{array}{l}\text { Nutzer in MB } \\
\text { monatlich }\end{array}\end{array}$ & $\begin{array}{l}\text { Zahl der Breitbandan- } \\
\text { schlüsse }\end{array}$ & $\begin{array}{l}\text { Volumen pro } \\
\text { Monat in MB }\end{array}$ & $\begin{array}{l}\text { Urheberrechtlich } \\
\text { relevanter Anteil }\end{array}$ & $\begin{array}{l}\text { Anteil von Buch- } \\
\text { downloads am Ge- } \\
\text { samtvolumen }\end{array}$ & $\begin{array}{l}\text { MB } \\
\text { pro } \\
\text { Buch }\end{array}$ \\
\hline 12.500 & 27.300 .000 & 341.250 .000 .000 & $5 \%$ & $0,10 \%$ & 2 \\
\hline
\end{tabular}

\begin{tabular}{|l|l|l|l|l|}
\hline & $\begin{array}{l}\text { Durchschnittlicher Endver- } \\
\text { Summe Bücher }\end{array}$ & $\begin{array}{l}\text { Anteil der Rechtein- } \\
\text { haber am Erlös }\end{array}$ & Preis monatlich & $\begin{array}{l}\text { Preis umgelegt } \\
\text { auf Anschluss } \\
\text { monatlich }\end{array}$ \\
\hline 8.531 .250 & $8,07 €$ & $50 \%$ & $34.423 .593,75 €$ & $\mathbf{1 , 2 6} €$ \\
\hline
\end{tabular}

Insgesamt:

\begin{tabular}{|l|l|l|l|}
\hline Musikindustrie & Filmindustrie & Buchindustrie & Gesamt \\
\hline $6,31 €$ & $4,21 €$ & $1,26 €$ & $\mathbf{1 1 , 7 8 €}$ \\
\hline
\end{tabular}


582 Gestaffelt nach Breitbandgeschwindigkeiten:

\begin{tabular}{|l|l|l|}
\hline $\begin{array}{l}\text { Kategorie } \mathbf{1} \text { (bis einschließlich } \mathbf{2} \\
\text { MBit/s) }\end{array}$ & Kategorie 2 (2-10 MBit/s) & Kategorie 3 (10 MBit/s und mehr) \\
\hline $4,48 €$ & $11,20 €$ & $17,91 €$ \\
\hline
\end{tabular}

(b) Mit Abschlag für Zweitverwertung

\section{Für die Musikindustrie:}

\begin{tabular}{|l|l|l|l|l|l|}
\hline $\begin{array}{l}\text { Volumen } \\
\text { pro Nutzer } \\
\text { in MB } \\
\text { monatlich }\end{array}$ & $\begin{array}{l}\text { Zahl der Breitbandan- } \\
\text { schlüsse }\end{array}$ & $\begin{array}{l}\text { Volumen pro Mo- } \\
\text { nat in MB }\end{array}$ & $\begin{array}{l}\text { Urheberrechtlich } \\
\text { relevanter Anteil }\end{array}$ & $\begin{array}{l}\text { Anteil von Musik- } \\
\text { downloads am } \\
\text { Gesamtvolumen }\end{array}$ & $\begin{array}{l}\text { Spielstunde } \\
\text { in MB }\end{array}$ \\
\hline 12.500 & 27.300 .000 & 341.250 .000 .000 & $5 \%$ & $12,60 \%$ & 100 \\
\hline
\end{tabular}

\begin{tabular}{|c|c|c|c|c|c|}
\hline $\begin{array}{l}\text { Spielstunden } \\
\text { insg. }\end{array}$ & $\begin{array}{l}\text { Durchschnittlicher } \\
\text { Endverkaufspreis } \\
\text { eines Albums }\end{array}$ & $\begin{array}{l}\text { Anteil der } \\
\text { Rechteinhaber } \\
\text { am Erlös }\end{array}$ & $\begin{array}{l}\text { Abschlag für } \\
\text { Zweitverwertung }\end{array}$ & Preis monatlich & \begin{tabular}{|l|} 
Preis um- \\
gelegt auf \\
Anschluss \\
monatlich \\
\end{tabular} \\
\hline 21.498 .750 & $9,99 €$ & $80,25 \%$ & $75 \%$ & $43.088 .735,32 €$ & $1,58 €$ \\
\hline
\end{tabular}

\section{Für die Filmindustrie:}

\begin{tabular}{|l|l|l|l|l|l|}
\hline $\begin{array}{l}\text { Volumen } \\
\text { pro Nutzer } \\
\text { in MB } \\
\text { monatlich }\end{array}$ & $\begin{array}{l}\text { Zahl der Breitbandan- } \\
\text { schlüsse }\end{array}$ & $\begin{array}{l}\text { Volumen pro } \\
\text { Monat in MB }\end{array}$ & $\begin{array}{l}\text { Urheberrechtlich } \\
\text { relevanter Anteil }\end{array}$ & $\begin{array}{l}\text { Anteil von Film- } \\
\text { downloads am } \\
\text { Gesamtvolumen }\end{array}$ & $\begin{array}{l}\text { Spielstunde } \\
\text { in MB }\end{array}$ \\
\hline 12.500 & 27.300 .000 & 341.250 .000 .000 & $5 \%$ & $87,30 \%$ & 466,67 \\
\hline
\end{tabular}

\begin{tabular}{|l|l|l|l|l|l|}
\hline & $\begin{array}{l}\text { Durchschnittlicher } \\
\text { Endverkaufspreis: 7,71 } \\
€ \text { für 90 min.; somit } \\
\text { durchschnittlicher }\end{array}$ & & & & \\
Spielstunden & $\begin{array}{l}\text { Endverkaufspreis für 1 } \\
\text { Spielstunde: }\end{array}$ & $\begin{array}{l}\text { Anteil der } \\
\text { Rechteinhaber } \\
\text { insg. }\end{array}$ & $\begin{array}{l}\text { am Erlös } \\
\text { Awschlag für } \\
\text { tung }\end{array}$ & Preitverwer- & $\begin{array}{l}\text { Preis um- } \\
\text { gelegt auf } \\
\text { Anschluss } \\
\text { monatlich }\end{array}$ \\
\hline $31.918 .834,51$ & $5,14 €$ & $70 \%$ & $28.710 .991,64 €$ & $\mathbf{1 , 0 5}$ \\
\hline
\end{tabular}


Für die Buchindustrie:

\begin{tabular}{|l|l|l|l|l|l|}
\hline $\begin{array}{l}\text { Volumen pro } \\
\text { Nutzer in MB } \\
\text { monatlich }\end{array}$ & $\begin{array}{l}\text { Zahl der Breitbandan- } \\
\text { schlüsse }\end{array}$ & $\begin{array}{l}\text { Volumen pro } \\
\text { Monat in MB }\end{array}$ & $\begin{array}{l}\text { Urheberrechtlich } \\
\text { relevanter Anteil }\end{array}$ & $\begin{array}{l}\text { Anteil von Buch- } \\
\text { downloads am Ge- } \\
\text { samtvolumen }\end{array}$ & $\begin{array}{l}\text { MB } \\
\text { pro } \\
\text { Buch }\end{array}$ \\
\hline 12.500 & 27.300 .000 & 341.250 .000 .000 & $5 \%$ & $0,10 \%$ & 2 \\
\hline
\end{tabular}

\begin{tabular}{|c|c|c|c|c|c|}
\hline $\begin{array}{l}\text { Summe } \\
\text { Bücher }\end{array}$ & $\begin{array}{l}\text { Durchschnittlicher } \\
\text { Endverkaufspreis }\end{array}$ & $\begin{array}{l}\text { Anteil der Recht- } \\
\text { einhaber am } \\
\text { Erlös }\end{array}$ & $\begin{array}{l}\text { Abschlag für } \\
\text { Zweitverwertung }\end{array}$ & Preis monatlich & $\begin{array}{l}\text { Preis umge- } \\
\text { legt auf } \\
\text { Anschluss } \\
\text { monatlich }\end{array}$ \\
\hline 8.531 .250 & $8,07 €$ & $50 \%$ & $75 \%$ & $8.605 .898,44 €$ & $0,32 €$ \\
\hline
\end{tabular}

585 Insgesamt:

\begin{tabular}{|l|l|l|l|}
\hline Musikindustrie & Filmindustrie & Buchindustrie & Gesamt \\
\hline $1,58 €$ & $1,05 €$ & $0,32 €$ & $\mathbf{2 , 9 5} €$ \\
\hline
\end{tabular}

586 Gestaffelt nach Breitbandgeschwindigkeiten:

\begin{tabular}{|l|l|l|}
\hline $\begin{array}{l}\text { Kategorie } 1 \text { (bis einschließlich } 2 \\
\text { MBit/s) }\end{array}$ & Kategorie 2 (2-10 MBit/s) & Kategorie 3 (10 MBit/s und mehr) \\
\hline $1,12 €$ & $2,80 €$ & $4,48 €$ \\
\hline
\end{tabular}




\section{b) Modifiziertes Lizenzanalogiemodell}

(1) Urheberrechtlich relevanter Anteil von 25\%

(a) Ohne Abschlag für Zweitverwertung

\section{Für die Musikindustrie:}

\begin{tabular}{|l|l|l|l|l|}
\hline $\begin{array}{l}\text { Volumen pro } \\
\begin{array}{l}\text { Nutzer in MB } \\
\text { monatlich }\end{array}\end{array}$ & $\begin{array}{l}\text { Zahl der Breitbandan- } \\
\text { schlüsse }\end{array}$ & $\begin{array}{l}\text { Volumen pro Mo- } \\
\text { nat in MB }\end{array}$ & $\begin{array}{l}\text { Urheberrechtlich } \\
\text { relevanter Anteil }\end{array}$ & $\begin{array}{l}\text { Anteil von Musikdown- } \\
\text { loads am Gesamtvolu- } \\
\text { men }\end{array}$ \\
\hline 12.500 & 27.300 .000 & 341.250 .000 .000 & $25 \%$ & $12,6 \%$ \\
\hline
\end{tabular}

\begin{tabular}{|l|l|l|l|l|l|}
\hline $\begin{array}{l}\text { Durchschnittliche } \\
\text { Größe einer Mu- } \\
\text { sikdatei in MB }\end{array}$ & $\begin{array}{l}\text { Musikdownloads } \\
\text { in Stück }\end{array}$ & $\begin{array}{l}\text { Durchschnittlicher } \\
\text { Endverkaufspreis } \\
\text { eines Songs }\end{array}$ & $\begin{array}{l}\text { Anteil der } \\
\text { Rechtein- } \\
\text { haber am } \\
\text { Erlös }\end{array}$ & Preis monatlich & $\begin{array}{l}\text { Preis um- } \\
\text { gelegt auf } \\
\text { Anschluss } \\
\text { monatlich }\end{array}$ \\
\hline 5 & 2.149 .875 .000 & $0,99 €$ & $80,25 \%$ & $1.708 .021 .940,63 €$ & $\mathbf{6 2 , 5 6 €}$ \\
\hline
\end{tabular}

588 Für die Filmindustrie:

\begin{tabular}{|l|l|l|l|l|l|}
\hline $\begin{array}{l}\text { Volumen } \\
\text { pro Nut- } \\
\text { zer in MB } \\
\text { monatlich }\end{array}$ & $\begin{array}{l}\text { Zahl der Breit- } \\
\text { bandanschlüsse }\end{array}$ & $\begin{array}{l}\text { Volumen pro Mo- } \\
\text { nat in MB }\end{array}$ & $\begin{array}{l}\text { Urheberrechtlich } \\
\text { relevanter Anteil }\end{array}$ & $\begin{array}{l}\text { Anteil von Film- } \\
\text { downloads am } \\
\text { Gesamtvolumen }\end{array}$ & $\begin{array}{l}\text { Durchschnittliche } \\
\text { Größe eines Films } \\
\text { in MB }\end{array}$ \\
\hline 12.500 & 27.300 .000 & 341.250 .000 .000 & $25 \%$ & $87,3 \%$ & 700 \\
\hline
\end{tabular}

\begin{tabular}{|l|l|l|l|l|}
\hline $\begin{array}{l}\text { Filmdownloads in } \\
\text { Stück }\end{array}$ & $\begin{array}{l}\text { Durchschnittlicher Endver- } \\
\text { kaufspreis eines Films }\end{array}$ & $\begin{array}{l}\text { Anteil der Recht- } \\
\text { einhaber am Erlös }\end{array}$ & Preis monatlich & $\begin{array}{l}\text { Preis umgelegt auf } \\
\text { Anschluss monat- } \\
\text { lich }\end{array}$ \\
\hline 106.396 .875 & $7,71 €$ & $70 \%$ & $574.223 .934,38 €$ & $\mathbf{2 1 , 0 3 €}$ \\
\hline
\end{tabular}

\section{Für die Buchindustrie:}

\begin{tabular}{|l|l|l|l|l|l|}
\hline $\begin{array}{l}\text { Volumen pro } \\
\begin{array}{l}\text { Nutzer in MB } \\
\text { monatlich }\end{array}\end{array}$ & $\begin{array}{l}\text { Zahl der Breitbandan- } \\
\text { schlüsse }\end{array}$ & $\begin{array}{l}\text { Volumen pro } \\
\text { Monat in MB }\end{array}$ & $\begin{array}{l}\text { Urheberrechtlich } \\
\text { relevanter Anteil }\end{array}$ & $\begin{array}{l}\text { Anteil von Buch- } \\
\text { downloads am Ge- } \\
\text { samtvolumen }\end{array}$ & $\begin{array}{l}\text { MB } \\
\text { pro } \\
\text { Buch }\end{array}$ \\
\hline 12.500 & 27.300 .000 & 341.250 .000 .000 & $25 \%$ & $0,10 \%$ & 2 \\
\hline
\end{tabular}

\begin{tabular}{|l|l|l|l|l|}
\hline $\begin{array}{l}\text { Summe Bü- } \\
\text { cher }\end{array}$ & $\begin{array}{l}\text { Durchschnittlicher Endver- } \\
\text { kaufspreis }\end{array}$ & $\begin{array}{l}\text { Anteil der Rechtein- } \\
\text { haber am Erlös }\end{array}$ & Preis monatlich & $\begin{array}{l}\text { Preis umgelegt } \\
\text { auf Anschluss } \\
\text { monatlich }\end{array}$ \\
\hline 42.656 .250 & $8,07 €$ & $50 \%$ & $172.117 .968,75 €$ & $6,30 €$ \\
\hline
\end{tabular}

\section{Insgesamt:}

\begin{tabular}{|l|l|l|l|}
\hline Musikindustrie & Filmindustrie & Buchindustrie & Gesamt \\
\hline $62,56 €$ & $21,03 €$ & $6,30 €$ & $\mathbf{8 9 , 8 9} €$ \\
\hline
\end{tabular}


591 Gestaffelt nach Breitbandgeschwindigkeiten:

\begin{tabular}{|l|l|l|}
\hline $\begin{array}{l}\text { Kategorie } 1 \text { (bis einschließlich } 2 \\
\text { MBit/s) }\end{array}$ & Kategorie 2 (2-10 MBit/s) & Kategorie 3 (10 MBit/s und mehr) \\
\hline $34,18 €$ & $85,44 €$ & $136,71 €$ \\
\hline
\end{tabular}

(b) Mit Abschlag für Zweitverwertung

592 Für die Musikindustrie:

\begin{tabular}{|l|l|l|}
\hline Preis monatlich & Abschlag i.H.v. 75\% & $\begin{array}{l}\text { Preis umgelegt auf Anschluss mo- } \\
\text { natlich }\end{array}$ \\
\hline $1.708 .021 .940,63 €$ & $427.005 .485,16 €$ & $15,64 €$ \\
\hline
\end{tabular}

593 Für die Filmindustrie:

\begin{tabular}{|l|l|l|}
\hline Preis monatlich & Abschlag i.H.v. 75\% & $\begin{array}{l}\text { Preis umgelegt auf Anschluss mo- } \\
\text { natlich }\end{array}$ \\
\hline $574.223 .934,38 €$ & $143.555 .983,60 €$ & $5,26 €$ \\
\hline
\end{tabular}

594 Für die Buchindustrie:

\begin{tabular}{|l|l|l|}
\hline Preis monatlich & Abschlag i.H.v. 75\% & $\begin{array}{l}\text { Preis umgelegt auf Anschluss mo- } \\
\text { natlich }\end{array}$ \\
\hline $172.117 .968,75 €$ & $43.029 .492,19 €$ & $1,58 €$ \\
\hline
\end{tabular}

\begin{tabular}{|l|l|l|l|}
\hline Musikindustrie & Filmindustrie & Buchindustrie & Gesamt \\
\hline $15,64 €$ & $5,26 €$ & $1,58 €$ & $\mathbf{2 2 , 4 8} €$ \\
\hline
\end{tabular}

595 Gestaffelt nach Breitbandgeschwindigkeiten:

\begin{tabular}{|l|l|l|}
\hline $\begin{array}{l}\text { Kategorie } \mathbf{1} \text { (bis einschließlich } \mathbf{2} \\
\mathbf{M B i t} / \mathbf{s})\end{array}$ & Kategorie 2 (2-10 MBit/s) & Kategorie $\mathbf{3}$ (10 MBit/s und mehr) \\
\hline $8,54 €$ & $21,36 €$ & $34,18 €$ \\
\hline
\end{tabular}


(2) Urheberrechtlich relevanter Anteil von 15\%:

(a) Ohne Abschlag für Zweitverwertung

596 Für die Musikindustrie:

\begin{tabular}{|l|l|l|l|l|}
\hline $\begin{array}{l}\text { Volumen } \\
\text { pro Nutzer } \\
\text { in MB } \\
\text { monatlich }\end{array}$ & $\begin{array}{l}\text { Zahl der Breitbandan- } \\
\text { schlüsse }\end{array}$ & $\begin{array}{l}\text { Volumen pro Monat in } \\
\text { MB }\end{array}$ & $\begin{array}{l}\text { Urheberrechtlich relevan- } \\
\text { ter Anteil }\end{array}$ & $\begin{array}{l}\text { Anteil von Musik- } \\
\text { downloads am } \\
\text { Gesamtvolumen }\end{array}$ \\
\hline 12.500 & 27.300 .000 & 341.250 .000 .000 & $15 \%$ & $12,60 \%$ \\
\hline
\end{tabular}

\begin{tabular}{|l|l|l|l|l|l|}
\hline $\begin{array}{l}\text { Durchschnittliche } \\
\text { Größe einer Mu- } \\
\text { sikdatei in MB }\end{array}$ & $\begin{array}{l}\text { Musikdownloads } \\
\text { in Stück }\end{array}$ & $\begin{array}{l}\text { Durchschnittlicher } \\
\text { Endverkaufspreis } \\
\text { eines Songs }\end{array}$ & $\begin{array}{l}\text { Anteil der } \\
\text { Rechtein- } \\
\text { haber am } \\
\text { Erlös }\end{array}$ & Preis monatlich & $\begin{array}{l}\text { Preis um- } \\
\text { gelegt auf } \\
\text { Anschluss } \\
\text { monatlich }\end{array}$ \\
\hline 5 & 1.289 .925 .000 & $0,99 €$ & $80,25 \%$ & $1.024 .813 .164,38 €$ & $\mathbf{3 7 , 5 4 €}$ \\
\hline
\end{tabular}

597 Für die Filmindustrie:

\begin{tabular}{|l|l|l|l|l|l|}
\hline $\begin{array}{l}\text { Volumen } \\
\text { pro Nut- } \\
\text { zer in MB } \\
\text { monatlich }\end{array}$ & $\begin{array}{l}\text { Zahl der Breit- } \\
\text { bandanschlüsse }\end{array}$ & $\begin{array}{l}\text { Volumen pro Mo- } \\
\text { nat in MB }\end{array}$ & $\begin{array}{l}\text { Urheberrechtlich } \\
\text { relevanter Anteil }\end{array}$ & $\begin{array}{l}\text { Anteil von Film- } \\
\text { downloads am } \\
\text { Gesamtvolumen }\end{array}$ & $\begin{array}{l}\text { Durchschnittliche } \\
\text { Größe eines Films } \\
\text { in MB }\end{array}$ \\
\hline 12.500 & 27.300 .000 & 341.250 .000 .000 & $15 \%$ & $87,3 \%$ & 700 \\
\hline
\end{tabular}

\begin{tabular}{|l|l|l|l|l|}
\hline $\begin{array}{l}\text { Filmdownloads in } \\
\text { Stück }\end{array}$ & $\begin{array}{l}\text { Durchschnittlicher Endver- } \\
\text { kaufspreis eines Films }\end{array}$ & $\begin{array}{l}\text { Anteil der Recht- } \\
\text { einhaber am Erlös }\end{array}$ & Preis monatlich & $\begin{array}{l}\text { Preis umgelegt } \\
\text { auf Anschluss } \\
\text { monatlich }\end{array}$ \\
\hline 63.838 .125 & $7,71 €$ & $70 \%$ & $344.534 .360,63 €$ & $\mathbf{1 2 , 6 2} €$ \\
\hline
\end{tabular}

\section{Für die Buchindustrie:}

\begin{tabular}{|l|l|l|l|l|l|}
\hline $\begin{array}{l}\text { Volumen pro } \\
\begin{array}{l}\text { Nutzer in MB } \\
\text { monatlich }\end{array}\end{array}$ & $\begin{array}{l}\text { Zahl der Breitbandan- } \\
\text { schlüsse }\end{array}$ & $\begin{array}{l}\text { Volumen pro } \\
\text { Monat in MB }\end{array}$ & $\begin{array}{l}\text { Urheberrechtlich } \\
\text { relevanter Anteil }\end{array}$ & $\begin{array}{l}\text { Anteil von Buch- } \\
\text { downloads am Ge- } \\
\text { samtvolumen }\end{array}$ & $\begin{array}{l}\text { MB } \\
\text { pro } \\
\text { Buch }\end{array}$ \\
\hline 12.500 & 27.300 .000 & 341.250 .000 .000 & $15 \%$ & $0,10 \%$ & 2 \\
\hline
\end{tabular}

\begin{tabular}{|l|l|l|l|l|}
\hline $\begin{array}{l}\text { Summe Bü- } \\
\text { cher }\end{array}$ & $\begin{array}{l}\text { Durchschnittlicher Endver- } \\
\text { kaufspreis }\end{array}$ & $\begin{array}{l}\text { Anteil der Rechtein- } \\
\text { haber am Erlös }\end{array}$ & Preis monatlich & $\begin{array}{l}\text { Preis umgelegt } \\
\text { auf Anschluss } \\
\text { monatlich }\end{array}$ \\
\hline 25.593 .750 & $8,07 €$ & $50 \%$ & $103.270 .781,25 €$ & $\mathbf{3 , 7 8} €$ \\
\hline
\end{tabular}

\section{Insgesamt:}

\begin{tabular}{|l|l|l|l|}
\hline Musikindustrie & Filmindustrie & Buchindustrie & Gesamt \\
\hline $37,54 €$ & $12,62 €$ & $3,78 €$ & $53,94 €$ \\
\hline
\end{tabular}


Gestaffelt nach Breitbandgeschwindigkeiten:

\begin{tabular}{|l|l|l|}
\hline $\begin{array}{l}\text { Kategorie } 1 \text { (bis einschließlich } 2 \\
\text { MBit/s) }\end{array}$ & Kategorie 2 (2-10 MBit/s) & Kategorie 3 (10 MBit/s und mehr) \\
\hline $20,51 €$ & $51,27 €$ & $82,03 €$ \\
\hline
\end{tabular}

(b) Mit Abschlag für Zweitverwertung

601 Für die Musikindustrie:

\begin{tabular}{|l|l|l|}
\hline Preis monatlich & Abschlag i.H.v. 75\% & $\begin{array}{l}\text { Preis umgelegt auf Anschluss mo- } \\
\text { natlich }\end{array}$ \\
\hline $1.024 .813 .164,375 €$ & $256.203 .291,09 €$ & $9,38 €$ \\
\hline
\end{tabular}

602 Für die Filmindustrie:

\begin{tabular}{|l|l|l|}
\hline Preis monatlich & Abschlag i.H.v. 75\% & $\begin{array}{l}\text { Preis umgelegt auf Anschluss mo- } \\
\text { natlich }\end{array}$ \\
\hline $344.534 .360,63 €$ & $86.133 .590,16 €$ & $3,16 €$ \\
\hline
\end{tabular}

603 Für die Buchindustrie:

\begin{tabular}{|l|l|l|}
\hline Preis monatlich & Abschlag i.H.v. 75\% & $\begin{array}{l}\text { Preis umgelegt auf Anschluss mo- } \\
\text { natlich }\end{array}$ \\
\hline $103.270 .781,25 €$ & $25.817 .695,31 €$ & $0,95 €$ \\
\hline
\end{tabular}

604

Insgesamt:

\begin{tabular}{|l|l|l|l|}
\hline Musikindustrie & Filmindustrie & Buchindustrie & Gesamt \\
\hline $9,38 €$ & $3,16 €$ & $0,95 €$ & $\mathbf{1 3 , 4 9}$ \\
\hline
\end{tabular}

605 Gestaffelt nach Breitbandgeschwindigkeiten:

\begin{tabular}{|l|l|l|}
\hline $\begin{array}{l}\text { Kategorie } \mathbf{1} \text { (bis einschließlich } \mathbf{2} \\
\text { MBit/s) }\end{array}$ & Kategorie 2 (2-10 MBit/s) & Kategorie 3 (10 MBit/s und mehr) \\
\hline $5,13 €$ & $12,82 €$ & $20,51 €$ \\
\hline
\end{tabular}


(3) Urheberrechtlich relevanter Anteil von 5\%:

(a) Ohne Abschlag für Zweitverwertung

Für die Musikindustrie:

\begin{tabular}{|l|l|l|l|l|}
\hline $\begin{array}{l}\text { Volumen } \\
\text { pro Nutzer } \\
\text { in MB }\end{array}$ & Zahl der Breitbandan- \\
monatlich & $\begin{array}{l}\text { Schlüsse } \\
\text { Solumen pro Monat in } \\
\text { MB }\end{array}$ & $\begin{array}{l}\text { Urheberrechtlich relevan- } \\
\text { ter Anteil }\end{array}$ & $\begin{array}{l}\text { Anteil von Musik- } \\
\text { downloads am } \\
\text { Gesamtvolumen }\end{array}$ \\
\hline
\end{tabular}

\begin{tabular}{|l|l|l|l|l|l|}
\hline $\begin{array}{l}\text { Durchschnittliche } \\
\text { Größe einer Mu- } \\
\text { sikdatei in MB }\end{array}$ & $\begin{array}{l}\text { Musikdownloads } \\
\text { in Stück }\end{array}$ & $\begin{array}{l}\text { Durchschnittlicher } \\
\text { Endverkaufspreis } \\
\text { eines Songs }\end{array}$ & $\begin{array}{l}\text { Anteil der } \\
\text { Rechteinha- } \\
\text { ber am Erlös }\end{array}$ & Preis monatlich & $\begin{array}{l}\text { Preis um- } \\
\text { gelegt auf } \\
\text { Anschluss } \\
\text { monatlich }\end{array}$ \\
\hline 5 & 429.975 .000 & $0,99 €$ & $80,25 \%$ & $341.604 .388,13 €$ & $\mathbf{1 2 , 5 1 €}$ \\
\hline
\end{tabular}

607 Für die Filmindustrie:

\begin{tabular}{|l|l|l|l|l|l|}
\hline $\begin{array}{l}\text { Volumen } \\
\text { pro Nut- } \\
\text { zer in MB } \\
\text { monatlich }\end{array}$ & $\begin{array}{l}\text { Zahl der Breit- } \\
\text { bandanschlüsse }\end{array}$ & $\begin{array}{l}\text { Volumen pro Mo- } \\
\text { nat in MB }\end{array}$ & $\begin{array}{l}\text { Urheberrechtlich } \\
\text { relevanter Anteil }\end{array}$ & $\begin{array}{l}\text { Anteil von Film- } \\
\text { downloads am } \\
\text { Gesamtvolumen }\end{array}$ & $\begin{array}{l}\text { Durchschnittliche } \\
\text { Größe eines Films } \\
\text { in MB }\end{array}$ \\
\hline 12.500 & 27.300 .000 & 341.250 .000 .000 & $5 \%$ & $87,3 \%$ & 700 \\
\hline
\end{tabular}

\begin{tabular}{|l|l|l|l|l|}
\hline $\begin{array}{l}\text { Filmdownloads in } \\
\text { Stück }\end{array}$ & $\begin{array}{l}\text { Durchschnittlicher Endver- } \\
\text { kaufspreis eines Films }\end{array}$ & $\begin{array}{l}\text { Anteil der Recht- } \\
\text { einhaber am Erlös }\end{array}$ & Preis monatlich & $\begin{array}{l}\text { Preis umgelegt auf } \\
\text { Anschluss monat- } \\
\text { lich }\end{array}$ \\
\hline 21.279 .375 & $7,71 €$ & $70 \%$ & $114.844 .786,88 €$ & $\mathbf{4 , 2 1 €}$ \\
\hline
\end{tabular}

Für die Buchindustrie:

\begin{tabular}{|l|l|l|l|l|l|}
\hline $\begin{array}{l}\text { Volumen pro } \\
\begin{array}{l}\text { Nutzer in MB } \\
\text { monatlich }\end{array}\end{array}$ & $\begin{array}{l}\text { Zahl der Breitbandan- } \\
\text { schlüsse }\end{array}$ & $\begin{array}{l}\text { Volumen pro } \\
\text { Monat in MB }\end{array}$ & $\begin{array}{l}\text { Urheberrechtlich } \\
\text { relevanter Anteil }\end{array}$ & $\begin{array}{l}\text { Anteil von Buch- } \\
\text { downloads am Ge- } \\
\text { samtvolumen }\end{array}$ & $\begin{array}{l}\text { MB } \\
\text { pro } \\
\text { Buch }\end{array}$ \\
\hline 12.500 & 27.300 .000 & 341.250 .000 .000 & $5 \%$ & $0,10 \%$ & 2 \\
\hline
\end{tabular}

\begin{tabular}{|l|l|l|l|l|}
\hline $\begin{array}{l}\text { Summe Bü- } \\
\text { cher }\end{array}$ & $\begin{array}{l}\text { Durchschnittlicher Endver- } \\
\text { kaufspreis }\end{array}$ & $\begin{array}{l}\text { Anteil der Rechtein- } \\
\text { haber am Erlös }\end{array}$ & Preis monatlich & $\begin{array}{l}\text { Preis umgelegt } \\
\text { auf Anschluss } \\
\text { monatlich }\end{array}$ \\
\hline 8.531 .250 & $8,07 €$ & $50 \%$ & $34.423 .593,75 €$ & $\mathbf{1 , 2 6} €$ \\
\hline
\end{tabular}

\begin{tabular}{|l|l|l|l|}
\hline Musikindustrie & Filmindustrie & Buchindustrie & Gesamt \\
\hline $12,51 €$ & $4,21 €$ & $1,26 €$ & $17,98 €$ \\
\hline
\end{tabular}


Gestaffelt nach Breitbandgeschwindigkeiten:

\begin{tabular}{|l|l|l|}
\hline $\begin{array}{l}\text { Kategorie } 1 \text { (bis einschließlich } 2 \\
\text { MBit/s) }\end{array}$ & Kategorie 2 (2-10 MBit/s) & Kategorie 3 (10 MBit/s und mehr) \\
\hline $6,84 €$ & $17,09 €$ & $27,34 €$ \\
\hline
\end{tabular}

(b) Mit Abschlag für Zweitverwertung

\section{Für die Musikindustrie:}

\begin{tabular}{|l|l|l|}
\hline Preis monatlich & Abschlag i.H.v. 75\% & $\begin{array}{l}\text { Preis umgelegt auf Anschluss mo- } \\
\text { natlich }\end{array}$ \\
\hline $341.604 .388,13 €$ & $85.401 .097,03 €$ & $3,13 €$ \\
\hline
\end{tabular}

\section{Für die Filmindustrie:}

\begin{tabular}{|l|l|l|}
\hline Preis monatlich & Abschlag i.H.v. 75\% & $\begin{array}{l}\text { Preis umgelegt auf Anschluss mo- } \\
\text { natlich }\end{array}$ \\
\hline $114.844 .786,88 €$ & $28.711 .196,72 €$ & $1,05 €$ \\
\hline
\end{tabular}

\section{Für die Buchindustrie:}

\begin{tabular}{|l|l|l|}
\hline Preis monatlich & Abschlag i.H.v. 75\% & $\begin{array}{l}\text { Preis umgelegt auf Anschluss mo- } \\
\text { natlich }\end{array}$ \\
\hline $34.423 .593,75 €$ & $8.605 .898,44 €$ & $0,32 €$ \\
\hline
\end{tabular}

614 Insgesamt:

\begin{tabular}{|l|l|l|l|}
\hline Musikindustrie & Filmindustrie & Buchindustrie & Gesamt \\
\hline $3,13 €$ & $1,05 €$ & $0,32 €$ & $\mathbf{4 , 5 0 €}$ \\
\hline
\end{tabular}

\section{Gestaffelt nach Breitbandgeschwindigkeiten:}

\begin{tabular}{|l|l|l|}
\hline $\begin{array}{l}\text { Kategorie } 1 \text { (bis einschließlich } 2 \\
\text { MBit/s) }\end{array}$ & Kategorie 2 (2-10 MBit/s) & Kategorie 3 (10 MBit/s und mehr) \\
\hline $1,71 €$ & $4,27 €$ & $6,84 €$ \\
\hline
\end{tabular}




\section{Auf tatsächlicher Substitution basiertes Gegenmodell (Fisher)}

Formel: Umsatz der Industrie (bereinigt um Vertriebsstufen) multipliziert mit der Substitutionsrate zuzüglich administrative Kosten der Kulturflatrate geteilt durch die Zahl der privaten Internetnutzer/privaten Internetanschlüsse

\section{a) Für eine Substitutionsrate von $30 \%$}

(1) Verwaltungskosten $15 \%$

\begin{tabular}{|l|l|}
\hline $\begin{array}{l}\text { Umsatzerlöse pro Jahr (gleitender Mittelwert aus fünf } \\
\text { Jahren) }\end{array}$ & $14.217,8$ Mio. $€$ \\
\hline Umsatz bereinigt um die Vertriebsstufen & $4.871,0452$ Mio. $€$ \\
\hline Substitutionsrate „worst case“ & $30 \%$ \\
\hline Administrative Kosten der Kulturflatrate & $15 \%$ \\
\hline Zahl der privaten Internetnutzer & Ca. 52,79 Mio. ${ }^{1026}$ \\
\hline Zahl der privaten Breitbandanschlüsse & Ca. 27,3 Mio. ${ }^{1027}$ \\
\hline
\end{tabular}

\section{Mithin ergäbe sich für die Musikindustrie:}

\begin{tabular}{|l|l|}
\hline Gesamtumsatz durchschnittlich (2007-2011) & $1.732,6$ Mio. $€^{1028}$ \\
\hline Umsatz physischer Markt & $1.392,6$ Mio. $€$ \\
\hline $\begin{array}{l}\text { Umsatz physisch, bereinigt um Vertriebskosten i.H.v. } \\
72,8 \%\end{array}$ & 518,0472 Mio. $€$ \\
\hline Umsatz digitaler Markt & 171,2 Mio. $€$ \\
\hline $\begin{array}{l}\text { Umsatz digital, bereinigt um Vertriebskosten i.H.v. } \\
19,75 \%\end{array}$ & 137,388 Mio. $€$ \\
\hline Zzgl. Einnahmen aus GVL-Rechten & 168,8 Mio. $€$ \\
\hline $\begin{array}{l}\text { Umsatzerlöse der Musikindustrie (netto), bereinigt } \\
\text { um Vertriebskosten }\end{array}$ & 824,2352 Mio. $€$ \\
\hline Multipliziert mit der Substitutionsrate von 30\% & 247,27056 Mio. € \\
\hline $\begin{array}{l}\text { Zuzüglich der Einkünfte aus Rundfunk (multipliziert } \\
\text { mit einer Substitutionsrate von 5\%) }\end{array}$ & $246,620535+13,0661=260,33666$ Mio. € \\
\hline Pro Internetnutzer & $4,93 €$ jährlich \\
\hline Pro Anschluss & $9,54 €$ jährlich \\
\hline
\end{tabular}

\footnotetext{
${ }^{1026}$ Die zur Verfügung stehenden Studien sind hier nicht absolut deckungsgleich, weswegen ein Mittelwert gebildet wurde: AGOF e.V. Studie Oktober 2012, S. 3 nennt 51,77 Mio. Internetnutzer ab 14 Jahre, INITIATIVE D21 (N)Onliner Atlas 2012 nennt 53,2 Mio. Internetnutzer ab 14 Jahren und ARD/ZDF kommen in ihrer Onlinestudie 1998-2012 zu einem Ergebnis von 53,4 Mio. Internetnutzern ab 14 Jahren.

${ }^{1027}$ Czajka, Statistisches Bundesamt, Wirtschaft und Statistik, August 2011, 709, 710; das entspricht etwa der Zahl, die sich aus den Angaben bei DIALOG CONSULT/VATM, Analyse Telekommunikationsmarkt Deutschland 2012, 2012, S. 17 ergibt: Bei 12,5 GB monatlich und einem jährlichen Gesamtdatenvolumen aller Anschlüsse kommt man auf 29,3 Mio. Internetanschlüsse.

${ }^{1028}$ Gleitender Mittelwert aus 5 Jahren, 2007-2011, entnommen Bundesverband Musikindustrie, Jahreswirtschaftsbericht 2011, abrufbar unter.
} 
618 Für die Filmindustrie ergäbe sich:

\begin{tabular}{|l|l|}
\hline Gesamtumsatz durchschnittlich (2007-2011): & $2.842,2$ Mio. $€^{1029}$ \\
\hline Umsatz DVD-Verkauf und Verleih & $1.636,2$ Mio. $€$ \\
\hline $\begin{array}{l}\text { Umsatz DVD-Verkauf und Verleih, bereinigt um Ver- } \\
\text { triebskosten i.H.v. 70\% }\end{array}$ & 490,86 Mio. $€$ \\
\hline Umsatz Pay-TV & 1.206 Mio. $€$ \\
\hline $\begin{array}{l}\text { Umsatz Pay-TV, bereinigt um Vertriebskosten i.H.v. } \\
85 \%\end{array}$ & 180,9 Mio. $€$ \\
\hline $\begin{array}{l}\text { Umsatzerlöse der Filmindustrie (netto), bereinigt um } \\
\text { Vertriebskosten }\end{array}$ & 671,76 Mio. $€$ \\
\hline Multipliziert mit der Substitutionsrate von 30\% & 201,528 Mio. $€$ \\
\hline Pro Internetnutzer & $3,82 €$ jährlich \\
\hline Pro Anschuss & $7,38 €$ jährlich \\
\hline
\end{tabular}

\section{Für die Buchindustrie:}

\begin{tabular}{|l|l|}
\hline Gesamtumsatz (2007-2011) & 9.643 Mio. $€^{1030}$ \\
\hline $\begin{array}{l}\text { Umsatz Buchindustrie bereinigt um Vertriebskosten } \\
\text { i.H.v. 65\% }\end{array}$ & $3.375,05$ Mio. $€$ \\
\hline Multipliziert mit der Substitutionsrate von 30\% & $1.012,515$ Mio. € \\
\hline Pro Internetnutzer & $19,19 €$ jährlich \\
\hline Pro Anschuss & $37,09 €$ jährlich \\
\hline
\end{tabular}

\section{Verwaltungskosten:}

\begin{tabular}{|l|l|}
\hline $\begin{array}{l}\text { Für die anfallenden Kosten durch die Administration } \\
\text { einer Kulturflatrate: Höhe des einzunehmenden Be- } \\
\text { trags }\end{array}$ & $259,686635+201,528+1.012,515=1.473,73$ Mio. $€$ \\
\hline $\begin{array}{l}\text { Verwaltungskosten i.H.v. 15\% des einzunehmenden } \\
\text { Betrags }\end{array}$ & 221,05944525 Mio. $€$ \\
\hline Pro Internetnutzer & $4,19 €$ jährlich \\
\hline Pro Anschluss & $8,10 €$ jährlich \\
\hline
\end{tabular}

\section{Insgesamt pro Nutzer:}

\begin{tabular}{|r|r|r|r|r|r|}
\hline Musikindustrie & Filmindustrie & Buchindustrie & Verwaltung & Gesamt/Jahr & Gesamt/Monat \\
\hline $4,93 €$ & $3,82 €$ & $19,19 €$ & $4,19 €$ & $\mathbf{3 2 , 1 2} €$ & $\mathbf{2 , 6 8} €$ \\
\hline
\end{tabular}

${ }^{1029}$ Gleitender Mittelwert aus 5 Jahren, 2007-2011, entnommen BVV/GfK, Der deutsche Videomarkt 2011, S.

14.

${ }^{1030}$ Gleitender Mittelwert aus 5 Jahren, 2007-2011, entnommen von 2007-2010 aus PricewaterhouseCoopers, German Entertainment and Media Outlook: 2011-2015; für 2011 aus Börsenverein des Deutschen Buchhandels, Umsatz- und Preisentwicklung, 2011. 
Insgesamt pro Anschluss:

\begin{tabular}{|r|r|r|r|r|r|}
\hline Musikindustrie & Filmindustrie & Buchindustrie & Verwaltung & Gesamt/Jahr & Gesamt/Monat \\
\hline $9,54 €$ & $7,38 €$ & $37,09 €$ & $8,10 €$ & $\mathbf{6 2 , 1 1} €$ & $\mathbf{5 , 1 8} €$ \\
\hline
\end{tabular}

Gestaffelt nach Breitbandgeschwindigkeiten (pro Monat/pro Anschluss):

\begin{tabular}{|r|r|r|}
\hline $\begin{array}{l}\text { Kategorie } 1 \text { (bis einschließlich 2 } \\
\text { MBit/s) }\end{array}$ & Kategorie 2 (2-10 MBit/s) & Kategorie 3 (10 MBit/s und mehr) \\
\hline $1,97 €$ & $4,92 €$ & $7,87 €$ \\
\hline
\end{tabular}

(2) Verwaltungskosten 7,5\%

623 Mithin ergäbe sich für die Musikindustrie:

\begin{tabular}{|l|l|}
\hline $\begin{array}{l}\text { Umsatzerlöse der Musikindustrie (netto) bereinigt } \\
\text { um Vertriebskosten }\end{array}$ & 824,2352 Mio. $€$ \\
\hline Multipliziert mit der Substitutionsrate von 30\% & 247,27056 Mio. $€$ \\
\hline $\begin{array}{l}\text { Zuzüglich der Einkünfte aus Rundfunk (multipliziert } \\
\text { mit einer Substitutionsrate von 5\%) }\end{array}$ & $246,620535+13,0661=260,33666$ Mio. € \\
\hline Pro Internetnutzer & $4,93 €$ jährlich \\
\hline Pro Anschluss & $9,54 €$ jährlich \\
\hline
\end{tabular}

\section{Mithin ergäbe sich für die Filmindustrie:}

\begin{tabular}{|l|l|}
\hline $\begin{array}{l}\text { Umsatzerlöse der Filmindustrie (netto), bereinigt um } \\
\text { Vertriebskosten }\end{array}$ & 671,76 Mio. $€$ \\
\hline Multipliziert mit der Substitutionsrate von 30\% & 201,528 Mio. $€$ \\
\hline Pro Internetnutzer & $3,52 €$ jährlich \\
\hline Pro Anschuss & $7,38 €$ jährlich \\
\hline
\end{tabular}

625 Mithin ergäbe sich für die Buchindustrie:

\begin{tabular}{|l|l|}
\hline $\begin{array}{l}\text { Umsatz Buchindustrie bereinigt um Vertriebskosten } \\
\text { i.H.v. } 65 \%\end{array}$ & $3.375,05$ Mio. $€$ \\
\hline Multipliziert mit der Substitutionsrate von 30\% & $1.012,515$ Mio. € \\
\hline Pro Internetnutzer & $19,19 €$ jährlich \\
\hline Pro Anschuss & $37,09 €$ jährlich \\
\hline
\end{tabular}

626 Mithin ergäbe sich für die Verwaltungskosten:

Für die anfallenden Kosten durch die Administration einer Kulturflatrate: Höhe des einzunehmenden Betrags

Verwaltungskosten i.H.v. 7,5\% des einzunehmenden Betrags

Pro Internetnutzer

Pro Anschluss
$259,686635+201,528+1012,515=1.473,73$ Mio. $€$

110,578.474.50 Mio. €

$2,09 €$ jährlich

$4,05 €$ jährlich 
Insgesamt pro Nutzer:

\begin{tabular}{|r|r|r|r|r|r|}
\hline Musikindustrie & Filmindustrie & Buchindustrie & Verwaltung & Gesamt/Jahr & Gesamt/Monat \\
\hline $4,93 €$ & $3,82 €$ & $19,19 €$ & $2,09 €$ & $\mathbf{3 0 , 0 2} €$ & $\mathbf{2 , 5 0} €$ \\
\hline
\end{tabular}

Insgesamt pro Anschluss:

\begin{tabular}{|r|r|r|r|r|r|}
\hline Musikindustrie & Filmindustrie & Buchindustrie & Verwaltung & Gesamt/Jahr & Gesamt/Monat \\
\hline $9,54 €$ & $7,38 €$ & $37,09 €$ & $4,05 €$ & $\mathbf{5 8 , 0 6} €$ & $\mathbf{4 , 8 4} €$ \\
\hline
\end{tabular}

629 Gestaffelt nach Breitbandgeschwindigkeiten (pro Monat/pro Anschluss):

\begin{tabular}{|r|r|r|}
\hline $\begin{array}{l}\text { Kategorie } 1 \text { (bis einschließlich } 2 \\
\text { MBit/s) }\end{array}$ & Kategorie 2 (210 MBit/s) & Kategorie 3 (10 MBit/s und mehr) \\
\hline $1,84 €$ & $4,60 €$ & $7,36 €$ \\
\hline
\end{tabular}

(3) Verwaltungskosten 5\%

630 Mithin ergäbe sich für die Musikindustrie:

\begin{tabular}{|l|l|}
\hline $\begin{array}{l}\text { Umsatzerlöse der Musikindustrie (netto) bereinigt } \\
\text { um Vertriebskosten }\end{array}$ & 824,2352 Mio. $€$ \\
\hline Multipliziert mit der Substitutionsrate von 30\% & 247,27056 Mio. $€$ \\
\hline $\begin{array}{l}\text { Zuzüglich der Einkünfte aus Rundfunk (multipliziert } \\
\text { mit einer Substitutionsrate von 5\%) }\end{array}$ & $246,620535+13,0661=260,33666$ Mio. $€$ \\
\hline Pro Internetnutzer & $4,93 €$ jährlich \\
\hline Pro Anschluss & $9,54 €$ jährlich \\
\hline
\end{tabular}

631 Mithin ergäbe sich für die Filmindustrie:

\begin{tabular}{|l|l|}
\hline $\begin{array}{l}\text { Umsatzerlöse der Filmindustrie (netto) bereinigt um } \\
\text { Vertriebskosten }\end{array}$ & 671,76 Mio. $€$ \\
\hline Multipliziert mit der Substitutionsrate von 30\% & 201,528 Mio. $€$ \\
\hline Pro Internetnutzer & $3,52 €$ jährlich \\
\hline Pro Anschuss & $7,38 €$ jährlich \\
\hline
\end{tabular}

\section{Mithin ergäbe sich für die Buchindustrie:}

\begin{tabular}{|l|l|}
\hline $\begin{array}{l}\text { Umsatz Buchindustrie bereinigt um Vertriebskosten } \\
\text { i.H.v. } 65 \%\end{array}$ & $3.375,05$ Mio. $€$ \\
\hline Multipliziert mit der Substitutionsrate von 30\% & $1.012,515$ Mio. $€$ \\
\hline Pro Internetnutzer & $19,19 €$ jährlich \\
\hline Pro Anschuss & $37,09 €$ jährlich \\
\hline
\end{tabular}




\section{Mithin ergäbe sich für die Verwaltungskosten:}

\begin{tabular}{|l|l|}
\hline $\begin{array}{l}\text { Für die anfallenden Kosten durch die Administration } \\
\text { einer Kulturflatrate: Höhe des einzunehmenden Be- } \\
\text { trags }\end{array}$ & $259,686635+201,528+1.012,515=1.473,73$ Mio. $€$ \\
\hline $\begin{array}{l}\text { Verwaltungskosten i.H.v. 7,5\% des einzunehmenden } \\
\text { Betrags }\end{array}$ & 73,718983 Mio. € \\
\hline Pro Internetnutzer & $1,40 €$ jährlich \\
\hline Pro Anschluss & $2,70 €$ jährlich \\
\hline
\end{tabular}

Insgesamt pro Nutzer:

\begin{tabular}{|r|r|r|r|r|r|}
\hline Musikindustrie & Filmindustrie & Buchindustrie & Verwaltung & Gesamt/Jahr & Gesamt/Monat \\
\hline $4,93 €$ & $3,82 €$ & $19,19 €$ & $1,40 €$ & $\mathbf{2 9 , 3 3} €$ & $\mathbf{2 , 4 4} €$ \\
\hline
\end{tabular}

635 Insgesamt pro Anschluss:

\begin{tabular}{|r|r|r|r|r|r|}
\hline Musikindustrie & Filmindustrie & Buchindustrie & Verwaltung & Gesamt/Jahr & Gesamt/Monat \\
\hline $9,54 €$ & $7,38 €$ & $37,09 €$ & $2,70 €$ & $\mathbf{5 6 , 7 1} €$ & $\mathbf{4 , 7 3} €$ \\
\hline
\end{tabular}

636 Gestaffelt nach Breitbandgeschwindigkeiten (pro Monat/pro Anschluss):

\begin{tabular}{|r|r|r|}
\hline $\begin{array}{l}\text { Kategorie } 1 \text { (bis einschließlich } 2 \\
\text { MBit/s) }\end{array}$ & Kategorie 2 (2-10 MBit/s) & Kategorie 3 (10 MBit/s und mehr) \\
\hline $1,71 €$ & $4,28 €$ & $6,84 €$ \\
\hline
\end{tabular}




\section{b) Für eine Substitutionsrate von $20 \%$}

(1) Verwaltungskosten $15 \%$

\begin{tabular}{|l|l|}
\hline $\begin{array}{l}\text { Umsatzerlöse pro Jahr (gleitender Mittelwert aus } 5 \\
\text { Jahren) }\end{array}$ & $14.217,8$ Mio. $€$ \\
\hline Umsatz bereinigt um die Vertriebsstufen & $4.871,0452$ Mio. $€$ \\
\hline Substitutionsrate „worst case“ & $20 \%$ \\
\hline Administrative Kosten der Kulturflatrate & $15 \%$ \\
\hline Zahl der privaten Internetnutzer & Ca. 52,79 Mio..$^{1031}$ \\
\hline Zahl der privaten Breitbandanschlüsse & Ca. 27,3 Mio. ${ }^{1032}$ \\
\hline
\end{tabular}

637 Für die Musikindustrie:

\begin{tabular}{|l|l|}
\hline Gesamtumsatz durchschnittlich (2007-2011) & $1.732,6$ Mio. $€^{1033}$ \\
\hline Umsatz physischer Markt & $1.392,6$ Mio. $€$ \\
\hline $\begin{array}{l}\text { Umsatz physisch, bereinigt um Vertriebskosten i.H.v. } \\
72,8 \%\end{array}$ & 518,0472 Mio. $€$ \\
\hline Umsatz digitaler Markt & 171,2 Mio. $€$ \\
\hline $\begin{array}{l}\text { Umsatz digital, bereinigt um Vertriebskosten i.H.v. } \\
19,75 \%\end{array}$ & 137,388 Mio. $€$ \\
\hline Zzgl. Einnahmen aus GVL-Rechten & 168,8 Mio. € \\
\hline $\begin{array}{l}\text { Umsatzerlöse der Musikindustrie (netto) bereinigt um } \\
\text { Vertriebskosten }\end{array}$ & 824,2352 Mio. e \\
\hline Multipliziert mit der Substitutionsrate von 20\% & 164,84704 Mio. € \\
\hline $\begin{array}{l}\text { Zuzüglich der Einkünfte aus Rundfunk (multipliziert } \\
\text { mit einer Substitutionsrate von 5\%) }\end{array}$ & $164,84704+13,0661=177,91314$ Mio. € \\
\hline Pro Internetnutzer & $3,37 €$ jährlich \\
\hline Pro Anschluss & $6,52 €$ jährlich \\
\hline
\end{tabular}

\section{Für die Filmindustrie:}

\begin{tabular}{|l|l|}
\hline Gesamtumsatz durchschnittlich (2007-2011): & $2.842,2$ Mio. $€^{1034}$ \\
\hline Umsatz DVD-Verkauf und Verleih & $1.636,2$ Mio. $€$ \\
\hline $\begin{array}{l}\text { Umsatz DVD-Verkauf und Verleih, bereinigt um Ver- } \\
\text { triebskosten i.H.v. 70\% }\end{array}$ & 490,86 Mio. $€$ \\
\hline Umsatz Pay-TV & 1.206 Mio. $€$ \\
\hline Umsatz Pay-TV, bereinigt um Vertriebskosten i.H.v. 85\% & 180,9 Mio. $€$ \\
\hline $\begin{array}{l}\text { Umsatzerlöse der Filmindustrie (netto) bereinigt um } \\
\text { Vertriebskosten }\end{array}$ & 671,76 Mio. $€$ \\
\hline Multipliziert mit der Substitutionsrate von 20\% & 134,352 Mio. $€$ \\
\hline Pro Internetnutzer & $2,55 €$ jährlich \\
\hline
\end{tabular}

${ }^{1031}$ Die zur Verfügung stehenden Studien sind hier nicht absolut deckungsgleich, weswegen ein Mittelwert gebildet wurde: AGOF e.V. Studie Oktober 2012, S. 3 nennt 51,77 Mio. Internetnutzer ab 14 Jahre, INITIATIVE D21 (N)Onliner Atlas 2012 nennt 53,2 Mio. Internetnutzer ab 14 Jahren und ARD/ZDF kommen in ihrer Onlinestudie 1998-2012 zu einem Ergebnis von 53,4 Mio. Internetnutzern ab 14 Jahren.

${ }^{1032}$ Czajka, Statistisches Bundesamt, Wirtschaft und Statistik, August 2011, 709, 710; das entspricht etwa der Zahl, die sich aus den Angaben bei DIALOG CONSULT/VATM, Analyse Telekommunikationsmarkt Deutschland 2012, 2012, S. 17 ergibt: Bei 12,5 GB monatlich und einem jährlichen Gesamtdatenvolumen aller Anschlüsse kommt man auf 29,3 Mio. Internetanschlüsse.

${ }^{1033}$ Gleitender Mittelwert aus 5 Jahren, 2007-2011, entnommen Bundesverband Musikindustrie, Jahreswirtschaftsbericht 2011.

${ }^{1034}$ Gleitender Mittelwert aus 5 Jahren, 2007-2011, entnommen BVV/GfK, Der deutsche Videomarkt 2011, S. 14. 
Für die Buchindustrie:

\begin{tabular}{|l|l|}
\hline Gesamtumsatz (2007-2011) & 9.643 Mio. $€^{1035}$ \\
\hline $\begin{array}{l}\text { Umsatz Buchindustrie bereinigt um Vertriebskosten } \\
\text { i.H.v. 65\% }\end{array}$ & $3.375,05$ Mio. $€$ \\
\hline Multipliziert mit der Substitutionsrate von 20\% & 675,010 Mio. $€$ \\
\hline Pro Internetnutzer & $12,79 €$ jährlich \\
\hline Pro Anschuss & $24,73 €$ jährlich \\
\hline
\end{tabular}

640 Mithin ergäbe sich für die Verwaltungskosten:

\begin{tabular}{|l|l|}
\hline $\begin{array}{l}\text { Für die anfallenden Kosten durch die Administration } \\
\text { einer Kulturflatrate: Höhe des einzunehmenden Be- } \\
\text { trags }\end{array}$ & $177,913.140+134,352+675,01=987,27514$ Mio. $€$ \\
\hline $\begin{array}{l}\text { Verwaltungskosten i.H.v. 15\% des einzunehmenden } \\
\text { Betrags }\end{array}$ & 148,091271 Mio. € \\
\hline Pro Internetnutzer & $2,81 €$ jährlich \\
\hline Pro Anschluss & $5,42 €$ jährlich \\
\hline
\end{tabular}

641 Insgesamt pro Nutzer:

\begin{tabular}{|r|r|r|r|r|r|}
\hline Musikindustrie & Filmindustrie & Buchindustrie & Verwaltung & Gesamt/Jahr & Gesamt/Monat \\
\hline $3,37 €$ & $2,55 €$ & $12,79 €$ & $2,81 €$ & $\mathbf{2 1 , 5 1}$ & $\mathbf{1 , 7 9} €$ \\
\hline
\end{tabular}

642 Insgesamt pro Anschluss:

\begin{tabular}{|r|r|r|r|r|r|}
\hline Musikindustrie & Filmindustrie & Buchindustrie & Verwaltung & Gesamt/Jahr & Gesamt/Monat \\
\hline $6,52 €$ & $4,92 €$ & $24,73 €$ & $5,42 €$ & $\mathbf{4 1 , 5 9} €$ & $\mathbf{3 , 4 7} €$ \\
\hline
\end{tabular}

643 Gestaffelt nach Breitbandgeschwindigkeiten (pro Monat/pro Anschluss):

\begin{tabular}{|r|r|r|}
\hline $\begin{array}{l}\text { Kategorie } 1 \text { (bis einschließlich } 2 \\
\text { MBit/s) }\end{array}$ & Kategorie 2 (2-10 MBit/s) & Kategorie 3 (10 MBit/s und mehr) \\
\hline $1,32 €$ & $3,29 €$ & $5,27 €$ \\
\hline
\end{tabular}

${ }^{1035}$ Gleitender Mittelwert aus 5 Jahren, 2007-2011, entnommen von 2007-2010 aus PricewaterhouseCoopers, German Entertainment and Media Outlook: 2011-2015; für 2011 aus Börsenverein des Deutschen Buchhandels, Umsatz- und Preisentwicklung, 2011. 
(2) Verwaltungskosten 7,5\%

644 Legt man die deutschen Zahlen zugrunde, ergibt sich daraus Folgendes:

\begin{tabular}{|l|l|}
\hline $\begin{array}{l}\text { Umsatzerlöse pro Jahr (gleitender Mittelwert aus 5 } \\
\text { Jahren) }\end{array}$ & $14.217,8$ Mio. $€$ \\
\hline Umsatz bereinigt um die Vertriebsstufen & $4.871,0452$ Mio. $€$ \\
\hline Substitutionsrate „worst case“ & $20 \%$ \\
\hline Administrative Kosten der Kulturflatrate & $7,5 \%$ \\
\hline Zahl der privaten Internetnutzer & Ca. 52,79 Mio. $^{1036}$ \\
\hline Zahl der privaten Breitbandanschlüsse & Ca. 27,3 Mio. $^{1037}$ \\
\hline
\end{tabular}

\section{Für die Musikindustrie:}

\begin{tabular}{|l|l|}
\hline $\begin{array}{l}\text { Umsatzerlöse der Musikindustrie (netto) bereinigt um } \\
\text { Vertriebskosten }\end{array}$ & 824,2352 Mio. $€$ \\
\hline Multipliziert mit der Substitutionsrate von 20\% & 164,84704 Mio. € \\
\hline $\begin{array}{l}\text { Zuzüglich der Einkünfte aus Rundfunk (multipliziert } \\
\text { mit einer Substitutionsrate von 5\%) }\end{array}$ & $164,84704+13,0661=177,91314$ Mio. € \\
\hline Pro Internetnutzer & $3,37 €$ jährlich \\
\hline Pro Anschluss & $6,52 €$ jährlich \\
\hline
\end{tabular}

\section{Für die Filmindustrie:}

\begin{tabular}{|l|l|}
\hline $\begin{array}{l}\text { Umsatzerlöse der Filmindustrie (netto) bereinigt um } \\
\text { Vertriebskosten }\end{array}$ & 671,76 Mio. $€$ \\
\hline Multipliziert mit der Substitutionsrate von 20\% & 134,352 Mio. $€$ \\
\hline Pro Internetnutzer & $2,55 €$ jährlich \\
\hline Pro Anschuss & $4,92 €$ jährlich \\
\hline
\end{tabular}

\section{Für die Buchindustrie:}

\begin{tabular}{|l|l|}
\hline $\begin{array}{l}\text { Umsatz Buchindustrie bereinigt um Vertriebskosten } \\
\text { i.H.v. } 65 \%\end{array}$ & $3.375,05$ Mio. $€$ \\
\hline Multipliziert mit der Substitutionsrate von $20 \%$ & 675,010 Mio. € \\
\hline Pro Internetnutzer & $12,79 €$ jährlich \\
\hline Pro Anschuss & $24,73 €$ jährlich \\
\hline
\end{tabular}

\footnotetext{
${ }^{1036}$ Die zur Verfügung stehenden Studien sind hier nicht absolut deckungsgleich, weswegen ein Mittelwert gebildet wurde: AGOF e.V. Studie Oktober 2012, S. 3 nennt 51,77 Mio. Internetnutzer ab 14 Jahre, INITIATIVE D21 (N)Onliner Atlas 2012 nennt 53,2 Mio. Internetnutzer ab 14 Jahren und ARD/ZDF kommen in ihrer Onlinestudie 1998-2012 zu einem Ergebnis von 53,4 Mio. Internetnutzern ab 14 Jahren.

${ }^{1037}$ Czajka, Statistisches Bundesamt, Wirtschaft und Statistik, August 2011, 709, 710; das entspricht etwa der Zahl, die sich aus den Angaben bei DIALOG CONSULT/VATM, Analyse Telekommunikationsmarkt Deutschland 2012, 2012, S. 17 ergibt: Bei 12,5 GB monatlich und einem jährlichen Gesamtdatenvolumen aller Anschlüsse kommt man auf 29,3 Mio. Internetanschlüsse.
} 
Mithin ergäbe sich für die Verwaltungskosten:

Für die anfallenden Kosten durch die Administration einer Kulturflatrate: Höhe des einzunehmenden Betrags

Verwaltungskosten i.H.v. 7,5\% des einzunehmenden Betrags

Pro Internetnutzer

Pro Anschluss

$177,913.140+134,352+675,01=987,27514$ Mio. $€$

74,0456355 Mio. €

$1,40 €$ jährlich

2,71 € jährlich

Insgesamt pro Nutzer:

\begin{tabular}{|r|r|r|r|r|r|}
\hline Musikindustrie & Filmindustrie & Buchindustrie & Verwaltung & Gesamt/Jahr & Gesamt/Monat \\
\hline $3,37 €$ & $2,55 €$ & $12,79 €$ & $1,40 €$ & $\mathbf{2 0 , 1 0} €$ & $\mathbf{1 , 6 8} €$ \\
\hline
\end{tabular}

Insgesamt pro Anschluss:

\begin{tabular}{|r|r|r|r|r|r|}
\hline Musikindustrie & Filmindustrie & Buchindustrie & Verwaltung & Gesamt/Jahr & Gesamt/Monat \\
\hline $6,52 €$ & $4,92 €$ & $24,73 €$ & $2,71 €$ & $38,88 €$ & $3,24 €$ \\
\hline
\end{tabular}

651 Gestaffelt nach Breitbandgeschwindigkeiten (pro Monat/pro Anschluss):

\begin{tabular}{|r|r|r|}
\hline $\begin{array}{l}\text { Kategorie 1 (bis einschließlich 2 } \\
\text { MBit/s) }\end{array}$ & Kategorie 2 (2-10 MBit/s) & Kategorie 3 (10 MBit/s und mehr) \\
\hline $1,23 €$ & $3,08 €$ & $4,93 €$ \\
\hline
\end{tabular}

(3) Verwaltungskosten 5\%

652 Für die Musikindustrie:

\begin{tabular}{|l|l|}
\hline $\begin{array}{l}\text { Umsatzerlöse der Musikindustrie (netto) bereinigt um } \\
\text { Vertriebskosten }\end{array}$ & 824,2352 Mio. $€$ \\
\hline Multipliziert mit der Substitutionsrate von 20\% & 164,84704 Mio. $€$ \\
\hline $\begin{array}{l}\text { Zuzüglich der Einkünfte aus Rundfunk (multipliziert } \\
\text { mit einer Substitutionsrate von 5\%) }\end{array}$ & $164,84704+13,0661=177,91314$ Mio. $€$ \\
\hline Pro Internetnutzer & $3,37 €$ jährlich \\
\hline Pro Anschluss & $6,52 €$ jährlich \\
\hline
\end{tabular}

\section{Für die Filmindustrie:}

\begin{tabular}{|l|l|}
\hline $\begin{array}{l}\text { Umsatzerlöse der Filmindustrie (netto) bereinigt um } \\
\text { Vertriebskosten }\end{array}$ & 671,76 Mio. $€$ \\
\hline Multipliziert mit der Substitutionsrate von 20\% & 134,352 Mio. $€$ \\
\hline Pro Internetnutzer & $2,55 €$ jährlich \\
\hline Pro Anschuss & $4,92 €$ jährlich \\
\hline
\end{tabular}


654 Für die Buchindustrie:

\begin{tabular}{|l|l|}
\hline $\begin{array}{l}\text { Umsatz Buchindustrie bereinigt um Vertriebskosten } \\
\text { i.H.v. } 65 \%\end{array}$ & $3.375,05$ Mio. $€$ \\
\hline Multipliziert mit der Substitutionsrate von 20\% & 675,010 Mio. $€$ \\
\hline Pro Internetnutzer & $12,79 €$ jährlich \\
\hline Pro Anschluss & $24,73 €$ jährlich \\
\hline
\end{tabular}

655 Mithin ergäbe sich für die Verwaltungskosten:

\begin{tabular}{|l|l|}
\hline $\begin{array}{l}\text { Für die anfallenden Kosten durch die Administration } \\
\text { einer Kulturflatrate: Höhe des einzunehmenden Be- } \\
\text { trags }\end{array}$ & $177,913.140+134,352+675,01=987,27514$ Mio. $€$ \\
\hline $\begin{array}{l}\text { Verwaltungskosten i.H.v. 5\% des einzunehmenden } \\
\text { Betrags }\end{array}$ & 49,363757 Mio. € \\
\hline Pro Internetnutzer & $0,94 €$ jährlich \\
\hline Pro Anschluss & $1,81 €$ jährlich \\
\hline
\end{tabular}

Insgesamt pro Nutzer:

\begin{tabular}{|r|r|r|r|r|r|}
\hline Musikindustrie & Filmindustrie & Buchindustrie & Verwaltung & Gesamt/Jahr & Gesamt/Monat \\
\hline $3,37 €$ & $2,55 €$ & $12,79 €$ & $0,94 €$ & $\mathbf{1 9 , 6 4} €$ & $\mathbf{1 , 6 4} €$ \\
\hline
\end{tabular}

Insgesamt pro Anschluss:

\begin{tabular}{|r|r|r|r|r|r|}
\hline Musikindustrie & Filmindustrie & Buchindustrie & Verwaltung & Gesamt/Jahr & Gesamt/Monat \\
\hline $6,52 €$ & $4,92 €$ & $24,73 €$ & $1,81 €$ & $\mathbf{3 7 , 9 7} €$ & $\mathbf{3 , 1 6} €$ \\
\hline
\end{tabular}

657 Gestaffelt nach Breitbandgeschwindigkeiten (pro Monat/pro Anschluss):

\begin{tabular}{|r|r|r|}
\hline $\begin{array}{l}\text { Kategorie } 1 \text { (bis einschließlich } 2 \\
\text { MBit/s) }\end{array}$ & Kategorie 2 (2-10 MBit/s) & Kategorie 3 (10 MBit/s und mehr) \\
\hline $1,20 €$ & $3,01 €$ & $4,81 €$ \\
\hline
\end{tabular}




\section{c) Für eine Substitutionsrate von $10 \%$}

(1) Verwaltungskosten 15\%

\begin{tabular}{|l|l|}
\hline $\begin{array}{l}\text { Umsatzerlöse pro Jahr (gleitender Mittelwert aus } 5 \\
\text { Jahren) }\end{array}$ & $14.217,8$ Mio. $€$ \\
\hline Umsatz bereinigt um die Vertriebsstufen & $4.871,0452$ Mio. $€$ \\
\hline Substitutionsrate & $10 \%$ \\
\hline Administrative Kosten der Kulturflatrate & $15 \%$ \\
\hline Zahl der privaten Internetnutzer & Ca. 52,79 Mio..$^{1038}$ \\
\hline Zahl der privaten Breitbandanschlüsse & Ca. 27,3 Mio. ${ }^{1039}$ \\
\hline
\end{tabular}

658 Mithin ergäbe sich für die Musikindustrie:

\begin{tabular}{|l|l|}
\hline Gesamtumsatz durchschnittlich (2007-2011) & $1.732,6$ Mio. $€^{1040}$ \\
\hline Umsatz physischer Markt & $1.392,6$ Mio. $€$ \\
\hline $\begin{array}{l}\text { Umsatz physisch, bereinigt um Vertriebskosten i.H.v. } \\
72,8 \%\end{array}$ & 518,0472 Mio. $€$ \\
\hline Umsatz digitaler Markt & 171,2 Mio. $€$ \\
\hline $\begin{array}{l}\text { Umsatz digital, bereinigt um Vertriebskosten i.H.v. } \\
19,75 \%\end{array}$ & 137,388 Mio. $€$ \\
\hline Zzgl. Einnahmen aus GVL-Rechten & 168,8 Mio. € \\
\hline $\begin{array}{l}\text { Umsatzerlöse der Musikindustrie (netto) bereinigt } \\
\text { um Vertriebskosten }\end{array}$ & 824,2352 Mio. € \\
\hline Multipliziert mit der Substitutionsrate von 10\% & 82,42352 Mio. € \\
\hline $\begin{array}{l}\text { Zuzüglich der Einkünfte aus Rundfunk (multipliziert } \\
\text { mit einer Substitutionsrate von 5\%) }\end{array}$ & $82,42352+13,0661=95,48962$ Mio. € \\
\hline Pro Internetnutzer & $1,81 €$ jährlich \\
\hline Pro Anschluss & $3,50 €$ jährlich \\
\hline
\end{tabular}

${ }^{1038}$ Die zur Verfügung stehenden Studien sind hier nicht absolut deckungsgleich, weswegen ein Mittelwert gebildet wurde: AGOF e.V. Studie Oktober 2012, S. 3 nennt 51,77 Mio. Internetnutzer ab 14 Jahre, INITIATIVE D21 (N)Onliner Atlas 2012 nennt 53,2 Mio. Internetnutzer ab 14 Jahren und ARD/ZDF kommen in ihrer Onlinestudie 1998-2012 zu einem Ergebnis von 53,4 Mio. Internetnutzern ab 14 Jahren.

${ }^{1039}$ Czajka, Statistisches Bundesamt, Wirtschaft und Statistik, August 2011, 709, 710; das entspricht etwa der Zahl, die sich aus den Angaben bei DIALOG CONSULT/VATM, Analyse Telekommunikationsmarkt Deutschland 2012, 2012, S. 17 ergibt: Bei 12,5 GB monatlich und einem jährlichen Gesamtdatenvolumen aller Anschlüsse kommt man auf 29,3 Mio. Internetanschlüsse.

${ }^{1040}$ Gleitender Mittelwert aus 5 Jahren, 2007-2011, entnommen Bundesverband Musikindustrie, Jahreswirtschaftsbericht 2011. 
Für die Filmindustrie:

\begin{tabular}{|l|l|}
\hline Gesamtumsatz durchschnittlich (2007-2011): & $2.842,2$ Mio. $€^{1041}$ \\
\hline Umsatz DVD-Verkauf und Verleih & $1.636,2$ Mio. $€$ \\
\hline $\begin{array}{l}\text { Umsatz DVD-Verkauf und Verleih, bereinigt um Ver- } \\
\text { triebskosten i.H.v. 70\% }\end{array}$ & 490,86 Mio. $€$ \\
\hline Umsatz Pay-TV & 1.206 Mio. $€$ \\
\hline Umsatz Pay-TV, bereinigt um Vertriebskosten i.H.v. 85\% & 180,9 Mio. $€$ \\
\hline $\begin{array}{l}\text { Umsatzerlöse der Filmindustrie (netto) bereinigt um } \\
\text { Vertriebskosten }\end{array}$ & 671,76 Mio. $€$ \\
\hline Multipliziert mit der Substitutionsrate von 10\% & 67,176 Mio. $€$ \\
\hline Pro Internetnutzer & $1,27 €$ jährlich \\
\hline Pro Anschuss & $2,46 €$ jährlich \\
\hline
\end{tabular}

Für die Buchindustrie:

\begin{tabular}{|l|l|}
\hline Gesamtumsatz (2007-2011) & 9.643 Mio. $€^{1042}$ \\
\hline $\begin{array}{l}\text { Umsatz Buchindustrie bereinigt um Vertriebskosten } \\
\text { i.H.v. 65\% }\end{array}$ & $3.375,05$ Mio. $€$ \\
\hline Multipliziert mit der Substitutionsrate von 10\% & 337,505 Mio. $€$ \\
\hline Pro Internetnutzer & $6,39 €$ jährlich \\
\hline Pro Anschuss & $12,36 €$ jährlich \\
\hline
\end{tabular}

\section{Mithin ergäbe sich für die Verwaltungskosten:}

\begin{tabular}{|l|l|}
\hline $\begin{array}{l}\text { Für die anfallenden Kosten durch die Administration } \\
\text { einer Kulturflatrate: Höhe des einzunehmenden Be- } \\
\text { trags }\end{array}$ & $95,48962+67,176+337,505=1.473,73$ Mio. $€$ \\
\hline $\begin{array}{l}\text { Verwaltungskosten i.H.v. 15\% des einzunehmenden } \\
\text { Betrags }\end{array}$ & 75,025593 Mio. € \\
\hline Pro Internetnutzer & $1,42 €$ jährlich \\
\hline Pro Anschluss & $2,75 €$ jährlich \\
\hline
\end{tabular}

Insgesamt pro Nutzer:

\begin{tabular}{|r|r|r|r|r|r|}
\hline Musikindustrie & Filmindustrie & Buchindustrie & Verwaltung & Gesamt/Jahr & Gesamt/Monat \\
\hline $1,81 €$ & $1,27 €$ & $6,39 €$ & $1,42 €$ & $\mathbf{1 0 , 9 0} €$ & $\mathbf{0 , 9 1} €$ \\
\hline
\end{tabular}

663 Insgesamt pro Anschluss:

\begin{tabular}{|r|r|r|r|r|r|}
\hline Musikindustrie & Filmindustrie & Buchindustrie & Verwaltung & Gesamt/Jahr & Gesamt/Monat \\
\hline $3,50 €$ & $2,46 €$ & $12,36 €$ & $2,75 €$ & $\mathbf{2 1 , 0 7} €$ & $\mathbf{1 , 7 6} €$ \\
\hline
\end{tabular}

${ }^{1041}$ Gleitender Mittelwert aus 5 Jahren, 2007-2011, entnommen BVV/GfK, Der deutsche Videomarkt 2011, S.

14.

1042 Gleitender Mittelwert aus 5 Jahren, 2007-2011, entnommen von 2007-2010 aus PricewaterhouseCoopers, German Entertainment and Media Outlook: 2011-2015; für 2011 aus Börsenverein des Deutschen Buchhandels, Umsatz- und Preisentwicklung, 2011. 
Gestaffelt nach Breitbandgeschwindigkeiten (pro Monat/pro Anschluss):

\begin{tabular}{|r|r|r|}
\hline $\begin{array}{l}\text { Kategorie 1 (bis einschließlich 2 } \\
\text { MBit/s) }\end{array}$ & Kategorie 2 (2-10 MBit/s) & Kategorie 3 (10 MBit/s und mehr) \\
\hline $0,67 €$ & $1,67 €$ & $2,67 €$ \\
\hline
\end{tabular}

(2) Verwaltungskosten 7,5\%

\begin{tabular}{|l|l|}
\hline $\begin{array}{l}\text { Umsatzerlöse pro Jahr (gleitender Mittelwert aus } 5 \\
\text { Jahren) }\end{array}$ & $14.217,8$ Mio. $€$ \\
\hline Umsatz bereinigt um die Vertriebsstufen & $4.871,0452$ Mio. $€$ \\
\hline Substitutionsrate & $10 \%$ \\
\hline Administrative Kosten der Kulturflatrate & $7,5 \%$ \\
\hline Zahl der privaten Internetnutzer & Ca. 52,79 Mio. ${ }^{1043}$ \\
\hline Zahl der privaten Breitbandanschlüsse & Ca. 27,3 Mio. ${ }^{1044}$ \\
\hline
\end{tabular}

\section{Mithin ergäbe sich für die Musikindustrie:}

\begin{tabular}{|l|l|}
\hline $\begin{array}{l}\text { Umsatzerlöse der Musikindustrie (netto) bereinigt } \\
\text { um Vertriebskosten }\end{array}$ & 824,2352 Mio. $€$ \\
\hline Multipliziert mit der Substitutionsrate von 10\% & 95,48962 Mio. $€$ \\
\hline Pro Internetnutzer & $1,81 €$ jährlich \\
\hline Pro Anschluss & $3,50 €$ jährlich \\
\hline
\end{tabular}

666 Für die Filmindustrie:

\begin{tabular}{|l|l|}
\hline $\begin{array}{l}\text { Umsatzerlöse der Filmindustrie (netto) bereinigt um } \\
\text { Vertriebskosten }\end{array}$ & 671,76 Mio. $€$ \\
\hline Multipliziert mit der Substitutionsrate von 10\% & 67,176 Mio. $€$ \\
\hline Pro Internetnutzer & $1,27 €$ jährlich \\
\hline Pro Anschuss & $2,46 €$ jährlich \\
\hline
\end{tabular}

\section{Für die Buchindustrie:}

\begin{tabular}{|l|l|}
\hline $\begin{array}{l}\text { Umsatz Buchindustrie bereinigt um Vertriebskosten } \\
\text { i.H.v. } 65 \%\end{array}$ & $3.375,05$ Mio. $€$ \\
\hline Multipliziert mit der Substitutionsrate von 10\% & 337,505 Mio. $€$ \\
\hline Pro Internetnutzer & $6,39 €$ jährlich \\
\hline Pro Anschuss & $12,36 €$ jährlich \\
\hline
\end{tabular}

${ }^{1043}$ Die zur Verfügung stehenden Studien sind hier nicht absolut deckungsgleich, weswegen ein Mittelwert gebildet wurde: AGOF e.V. Studie Oktober 2012, S. 3 nennt 51,77 Mio. Internetnutzer ab 14 Jahre, INITIATIVE D21 (N)Onliner Atlas 2012 nennt 53,2 Mio. Internetnutzer ab 14 Jahren und ARD/ZDF kommen in ihrer Onlinestudie 1998-2012 zu einem Ergebnis von 53,4 Mio. Internetnutzern ab 14 Jahren.

${ }^{1044}$ Czajka, Statistisches Bundesamt, Wirtschaft und Statistik, August 2011, 709, 710; das entspricht etwa der Zahl, die sich aus den Angaben bei DIALOG CONSULT/VATM, Analyse Telekommunikationsmarkt Deutschland 2012, 2012, S. 17 ergibt: Bei 12,5 GB monatlich und einem jährlichen Gesamtdatenvolumen aller Anschlüsse kommt man auf 29,3 Mio. Internetanschlüsse. 
Mithin ergäbe sich für die Verwaltungskosten:

Für die anfallenden Kosten durch die Administration einer Kulturflatrate: Höhe des einzunehmenden Betrags

Verwaltungskosten i.H.v. 7,5\% des einzunehmenden Betrags

Pro Internetnutzer

Pro Anschluss

$95,48962+67,176+337,505=1.473,73$ Mio. $€$

37,51279650 Mio. €

$0,71 €$ jährlich

$1,37 €$ jährlich

Insgesamt pro Nutzer:

\begin{tabular}{|r|r|r|r|r|r|}
\hline Musikindustrie & Filmindustrie & Buchindustrie & Verwaltung & Gesamt/Jahr & Gesamt/Monat \\
\hline $1,81 €$ & $1,27 €$ & $6,39 €$ & $0,71 €$ & $\mathbf{1 0 , 1 9} €$ & $\mathbf{0 , 8 5} €$ \\
\hline
\end{tabular}

670 Insgesamt pro Anschluss:

\begin{tabular}{|r|r|r|r|r|r|}
\hline Musikindustrie & Filmindustrie & Buchindustrie & Verwaltung & Gesamt/Jahr & Gesamt/Monat \\
\hline $3,50 €$ & $2,46 €$ & $12,36 €$ & $1,37 €$ & $\mathbf{1 9 , 7 0}$ & $\mathbf{1 , 6 4} €$ \\
\hline
\end{tabular}

671 Gestaffelt nach Breitbandgeschwindigkeiten (pro Monat/pro Anschluss):

\begin{tabular}{|r|r|r|}
\hline $\begin{array}{l}\text { Kategorie } 1 \text { (bis einschließlich } 2 \\
\text { MBit/s) }\end{array}$ & Kategorie 2 (2-10 MBit/s) & Kategorie 3 (10 MBit/s und mehr) \\
\hline $0,62 €$ & $1,56 €$ & $2,50 €$ \\
\hline
\end{tabular}

(3) Verwaltungskosten 5\%

672 Für die Musikindustrie:

\begin{tabular}{|l|l|}
\hline $\begin{array}{l}\text { Umsatzerlöse der Musikindustrie (netto) bereinigt um } \\
\text { Vertriebskosten }\end{array}$ & 824,2352 Mio. $€$ \\
\hline Multipliziert mit der Substitutionsrate von 10\% & 95,48962 Mio. € \\
\hline Pro Internetnutzer & $1,81 €$ jährlich \\
\hline Pro Anschluss & $3,50 €$ jährlich \\
\hline
\end{tabular}

\section{Für die Filmindustrie:}

\begin{tabular}{|l|l|}
\hline $\begin{array}{l}\text { Umsatzerlöse der Filmindustrie (netto) bereinigt um } \\
\text { Vertriebskosten }\end{array}$ & 671,76 Mio. $€$ \\
\hline Multipliziert mit der Substitutionsrate von 10\% & 67,176 Mio. $€$ \\
\hline Pro Internetnutzer & $1,27 €$ jährlich \\
\hline Pro Anschuss & $2,46 €$ jährlich \\
\hline
\end{tabular}


674 Für die Buchindustrie:

\begin{tabular}{|l|l|}
\hline $\begin{array}{l}\text { Umsatz Buchindustrie bereinigt um Vertriebskosten } \\
\text { i.H.v. } 65 \%\end{array}$ & $3.375,05$ Mio. $€$ \\
\hline Multipliziert mit der Substitutionsrate von 10\% & 337,505 Mio. $€$ \\
\hline Pro Internetnutzer & $6,39 €$ jährlich \\
\hline Pro Anschluss & $12,36 €$ jährlich \\
\hline
\end{tabular}

675 Mithin ergäbe sich für die Verwaltungskosten:

Für die anfallenden Kosten durch die Administration einer Kulturflatrate: Höhe des einzunehmenden Betrags

Verwaltungskosten i.H.v. 5\% des einzunehmenden Betrags

Pro Internetnutzer

Pro Anschluss

$95,48962+67,176+337,505=1.473,73$ Mio. $€$

25,008531 Mio. €

$0,47 €$ jährlich

$0,92 €$ jährlich

676 Insgesamt pro Nutzer:

\begin{tabular}{|r|r|r|r|r|r|}
\hline Musikindustrie & Filmindustrie & Buchindustrie & Verwaltung & Gesamt/Jahr & Gesamt/Monat \\
\hline $1,81 €$ & $1,27 €$ & $6,39 €$ & $0,47 €$ & $\mathbf{9 , 9 5} €$ & $\mathbf{0 , 8 3} €$ \\
\hline
\end{tabular}

677 Insgesamt pro Anschluss:

\begin{tabular}{|r|r|r|r|r|r|}
\hline Musikindustrie & Filmindustrie & Buchindustrie & Verwaltung & Gesamt/Jahr & Gesamt/Monat \\
\hline $3,50 €$ & $2,46 €$ & $12,36 €$ & $0,92 €$ & $\mathbf{1 9 , 2 4} €$ & $1,60 €$ \\
\hline
\end{tabular}

\section{Gestaffelt nach Breitbandgeschwindigkeiten (pro Monat/pro Anschluss):}

\begin{tabular}{|r|r|r|}
\hline $\begin{array}{l}\text { Kategorie 1 (bis einschließlich 2 } \\
\text { MBit/s) }\end{array}$ & Kategorie 2 (2-10 MBit/s) & Kategorie 3 (10 MBit/s und mehr) \\
\hline $0,61 €$ & $1,52 €$ & $2,44 €$ \\
\hline
\end{tabular}




\section{Mischmodell}

Formel: Zahl der privaten Breitbandanschlüsse $x$ durchschnittliches Volumen des Datenverkehrs pro Monat $x$ prozentualer Anteil der urheberrechtlich relevanten Nutzungen $x$ prozentualer Anteil des getauschten Mediums am Datenvolumen / MB pro Werk bzw. Werksammlung (Album-, bzw. Songdownload, Filmdownload, E-Book) $x$ durchschnittlicher Ladenpreis abzgl. Vertriebsstufen $x$ Substitutionsrate.

\section{a) Urheberrechtlicher Anteil von 25\%}

(1) Substitutionsrate von 30\%

680 Für die Musikindustrie:

\begin{tabular}{|l|l|l|l|l|l|}
\hline $\begin{array}{l}\text { Volumen } \\
\text { pro Nut- } \\
\text { zer in MB } \\
\text { monatlich }\end{array}$ & $\begin{array}{l}\text { Zahl der Breitband- } \\
\text { anschlüsse }\end{array}$ & $\begin{array}{l}\text { Volumen pro Mo- } \\
\text { nat in MB }\end{array}$ & $\begin{array}{l}\text { Urheberrechtlich } \\
\text { relevanter Anteil }\end{array}$ & $\begin{array}{l}\text { Anteil von Mu- } \\
\text { sikdownloads am } \\
\text { Gesamtvolumen }\end{array}$ & $\begin{array}{l}\text { Durchschnittliche } \\
\text { Größe einer Mu- } \\
\text { sikdatei in MB }\end{array}$ \\
\hline 12.500 & 27.300 .000 & 341.250 .000 .000 & $25 \%$ & $12,6 \%$ & 5 \\
\hline
\end{tabular}

\begin{tabular}{|l|l|l|l|l|l|}
\hline & $\begin{array}{l}\text { Anteil der } \\
\text { Rechtein- } \\
\text { haber am } \\
\text { in Stück }\end{array}$ & $\begin{array}{l}\text { Prös } \\
\text { Album }\end{array}$ & Preis monatlich & Substitutionsrate & $\begin{array}{l}\text { Preis umgelegt auf } \\
\text { Anschlussinhaber } \\
\text { monatlich }\end{array}$ \\
\hline 2.149 .875 .000 & $0,99 €$ & $80,25 \%$ & $1.708 .021 .940,63 €$ & $30 \%$ & $\mathbf{1 8 , 7 7 €}$ \\
\hline
\end{tabular}

\section{Für die Filmindustrie:}

\begin{tabular}{|l|l|l|l|l|l|}
\hline $\begin{array}{l}\text { Volumen } \\
\text { pro Nut- } \\
\text { zer in MB } \\
\text { monatlich }\end{array}$ & $\begin{array}{l}\text { Zahl der Breitband- } \\
\text { anschlüsse }\end{array}$ & $\begin{array}{l}\text { Volumen pro Mo- } \\
\text { nat in MB }\end{array}$ & $\begin{array}{l}\text { Urheberrechtlich } \\
\text { relevanter Anteil }\end{array}$ & $\begin{array}{l}\text { Anteil von Film- } \\
\text { downloads am } \\
\text { Gesamtvolumen }\end{array}$ & $\begin{array}{l}\text { Durchschnittliche } \\
\text { Größe eines Films }\end{array}$ \\
\hline 12.500 & 27.300 .000 & 341.250 .000 .000 & $25 \%$ & $87,3 \%$ & 700 \\
\hline
\end{tabular}

\begin{tabular}{|l|l|l|l|l|l|}
\hline $\begin{array}{l}\text { Heruntergeladene } \\
\text { Filme in Stück }\end{array}$ & $\begin{array}{l}\text { Preis pro } \\
\text { Film }\end{array}$ & $\begin{array}{l}\text { Anteil der } \\
\text { Rechteinha- } \\
\text { ber am Erlös }\end{array}$ & Preis monatlich & Substitutionsrate & $\begin{array}{l}\text { Preis umgelegt auf } \\
\text { Anschlussinhaber } \\
\text { monatlich }\end{array}$ \\
\hline 106.396 .875 & $7,71 €$ & $70 \%$ & $574.223 .934,38 €$ & $30 \%$ & $\mathbf{6 , 3 1 €}$ \\
\hline
\end{tabular}

\section{Für die Buchindustrie:}

\begin{tabular}{|l|l|l|l|l|l|}
\hline $\begin{array}{l}\text { Volumen pro } \\
\text { Nutzer in MB } \\
\text { monatlich }\end{array}$ & $\begin{array}{l}\text { Zahl der Breitbandan- } \\
\text { schlüsse }\end{array}$ & $\begin{array}{l}\text { Volumen pro } \\
\text { Monat in MB }\end{array}$ & $\begin{array}{l}\text { Urheberrechtlich } \\
\text { relevanter Anteil }\end{array}$ & $\begin{array}{l}\text { Anteil von Buch- } \\
\text { downloads am Ge- } \\
\text { samtvolumen }\end{array}$ & $\begin{array}{l}\text { MB } \\
\text { pro } \\
\text { Buch }\end{array}$ \\
\hline 12.500 & 27.300 .000 & 341.250 .000 .000 & $25 \%$ & $0,10 \%$ & 2 \\
\hline
\end{tabular}

\begin{tabular}{|l|l|l|l|l|l|}
\hline $\begin{array}{l}\text { Anzahl Bü- } \\
\text { cher }\end{array}$ & $\begin{array}{l}\text { Preis } \\
\text { pro E- } \\
\text { Book }\end{array}$ & $\begin{array}{l}\text { Anteil der Ver- } \\
\text { triebskosten }\end{array}$ & Preis monatlich & Substitutionsrate & $\begin{array}{l}\text { Preis umgelegt auf } \\
\text { Anschlussinhaber } \\
\text { monatlich }\end{array}$ \\
\hline 42.656 .250 & $8,07 €$ & $50 \%$ & $172.117 .968,75 €$ & $30 \%$ & $\mathbf{1 , 8 9} €$ \\
\hline
\end{tabular}


683 Insgesamt:

\begin{tabular}{|l|l|l|l|}
\hline Musikindustrie & Filmindustrie & Buchindustrie & Gesamt \\
\hline $18,77 €$ & $6,31 €$ & $1,89 €$ & $\mathbf{2 6 , 9 7} €$ \\
\hline
\end{tabular}

Gestaffelt nach Breitbandgeschwindigkeiten (pro Monat/pro Anschluss):

\begin{tabular}{|l|l|l|}
\hline $\begin{array}{l}\text { Kategorie } \mathbf{1} \text { (bis einschließlich } \mathbf{2} \\
\mathbf{M B i t} / \mathbf{s})\end{array}$ & Kategorie 2 (2-10 MBit/s) & Kategorie 3 (10 MBit/s und mehr) \\
\hline $10,25 €$ & $25,63 €$ & $41,01 €$ \\
\hline
\end{tabular}

(2) Substitutionsrate von $20 \%$

684 Für die Musikindustrie:

\begin{tabular}{|l|l|l|l|l|l|}
\hline $\begin{array}{l}\text { Volumen } \\
\text { pro Nut- } \\
\text { zer in MB } \\
\text { monatlich }\end{array}$ & $\begin{array}{l}\text { Zahl der Breitband- } \\
\text { anschlüsse }\end{array}$ & $\begin{array}{l}\text { Volumen pro Mo- } \\
\text { nat in MB }\end{array}$ & $\begin{array}{l}\text { Urheberrechtlich } \\
\text { relevanter Anteil }\end{array}$ & $\begin{array}{l}\text { Anteil von Mu- } \\
\text { sikdownloads am } \\
\text { Gesamtvolumen }\end{array}$ & $\begin{array}{l}\text { Durchschnittliche } \\
\text { Größe einer Mu- } \\
\text { sikdatei in MB }\end{array}$ \\
\hline 12.500 & 27.300 .000 & 341.250 .000 .000 & $25 \%$ & $12,6 \%$ & 5 \\
\hline
\end{tabular}

\begin{tabular}{|l|l|l|l|l|l|}
\hline $\begin{array}{l}\text { Musikdownloads } \\
\text { in Stück }\end{array}$ & $\begin{array}{l}\text { Preis pro } \\
\text { Album }\end{array}$ & $\begin{array}{l}\text { Anteil der } \\
\text { Rechteinhaber } \\
\text { am Erlös }\end{array}$ & Preis monatlich & Substitutionsrate & $\begin{array}{l}\text { Preis umgelegt auf } \\
\text { Anschlussinhaber } \\
\text { monatlich }\end{array}$ \\
\hline 2.149 .875 .000 & $0,99 €$ & $80,25 \%$ & $1.708 .021 .940,63 €$ & $20 \%$ & $\mathbf{1 2 , 5 1 €}$ \\
\hline
\end{tabular}

\section{Für die Filmindustrie:}

\begin{tabular}{|l|l|l|l|l|l|}
\hline $\begin{array}{l}\text { Volumen } \\
\text { pro Nut- } \\
\text { zer in MB } \\
\text { monatlich }\end{array}$ & $\begin{array}{l}\text { Zahl der Breit- } \\
\text { bandanschlüsse }\end{array}$ & $\begin{array}{l}\text { Volumen pro Mo- } \\
\text { nat in MB }\end{array}$ & $\begin{array}{l}\text { Urheberrechtlich } \\
\text { relevanter Anteil }\end{array}$ & $\begin{array}{l}\text { Anteil von Film- } \\
\text { downloads am } \\
\text { Gesamtvolumen }\end{array}$ & $\begin{array}{l}\text { Durchschnittliche } \\
\text { Größe eines Films }\end{array}$ \\
\hline 12.500 & 27.300 .000 & 341.250 .000 .000 & $25 \%$ & $87,3 \%$ & 700 \\
\hline
\end{tabular}

\begin{tabular}{|l|l|l|l|l|l|}
\hline $\begin{array}{l}\text { Heruntergeladene } \\
\text { Filme in Stück }\end{array}$ & $\begin{array}{l}\text { Preis } \\
\text { pro Film }\end{array}$ & $\begin{array}{l}\text { Anteil der } \\
\text { Rechteinha- } \\
\text { ber am Erlös }\end{array}$ & Preis monatlich & Substitutionsrate & $\begin{array}{l}\text { Preis umgelegt auf } \\
\text { Anschlussinhaber } \\
\text { monatlich }\end{array}$ \\
\hline 106.396 .875 & $7,71 €$ & $70 \%$ & $574.223 .934,38 €$ & $20 \%$ & $\mathbf{4 , 2 1 €}$ \\
\hline
\end{tabular}

686 Für die Buchindustrie:

\begin{tabular}{|l|l|l|l|l|l|}
\hline $\begin{array}{l}\text { Volumen pro } \\
\text { Nutzer in MB } \\
\text { monatlich }\end{array}$ & $\begin{array}{l}\text { Zahl der Breitbandan- } \\
\text { schlüsse }\end{array}$ & $\begin{array}{l}\text { Volumen pro } \\
\text { Monat in MB }\end{array}$ & $\begin{array}{l}\text { Urheberrechtlich } \\
\text { relevanter Anteil }\end{array}$ & $\begin{array}{l}\text { Anteil von Buch- } \\
\text { downloads am Ge- } \\
\text { samtvolumen }\end{array}$ & $\begin{array}{l}\text { MB } \\
\text { pro } \\
\text { Buch }\end{array}$ \\
\hline 12.500 & 27.300 .000 & 341.250 .000 .000 & $25 \%$ & $0,10 \%$ & 2 \\
\hline
\end{tabular}

\begin{tabular}{|l|l|l|l|l|l|}
\hline $\begin{array}{l}\text { Anzahl } \\
\text { Bücher }\end{array}$ & $\begin{array}{l}\text { Preis } \\
\text { pro E- } \\
\text { Book }\end{array}$ & $\begin{array}{l}\text { Anteil der Ver- } \\
\text { triebskosten }\end{array}$ & Preis monatlich & Substitutionsrate & $\begin{array}{l}\text { Preis umgelegt auf An- } \\
\text { schlussinhaber monatlich }\end{array}$ \\
\hline 42.656 .250 & $8,07 €$ & $50 \%$ & $172.117 .968,75 €$ & $20 \%$ & $\mathbf{1 , 2 6} €$ \\
\hline
\end{tabular}


687 Insgesamt:

\begin{tabular}{|l|l|l|l|}
\hline Musikindustrie & Filmindustrie & Buchindustrie & Gesamt \\
\hline $12,51 €$ & $4,21 €$ & $1,26 €$ & $\mathbf{1 7 , 9 8} €$ \\
\hline
\end{tabular}

688 Gestaffelt nach Breitbandgeschwindigkeiten (pro Monat/pro Anschluss):

\begin{tabular}{|l|l|l|}
\hline $\begin{array}{l}\text { Kategorie } \mathbf{1} \text { (bis einschließlich } \mathbf{2} \\
\text { MBit/s) }\end{array}$ & Kategorie 2 (2-10 MBit/s) & Kategorie 3 (10 MBit/s und mehr) \\
\hline $6,84 €$ & $17,09 €$ & $27,34 €$ \\
\hline
\end{tabular}

(3) Substitutionsrate von 10\%

689 Für die Musikindustrie:

\begin{tabular}{|l|l|l|l|l|l|}
\hline $\begin{array}{l}\text { Volumen } \\
\text { pro Nut- } \\
\text { zer in MB } \\
\text { monatlich }\end{array}$ & $\begin{array}{l}\text { Zahl der Breit- } \\
\text { bandanschlüsse }\end{array}$ & $\begin{array}{l}\text { Volumen pro Monat } \\
\text { in MB }\end{array}$ & $\begin{array}{l}\text { Urheberrechtlich } \\
\text { relevanter Anteil }\end{array}$ & $\begin{array}{l}\text { Anteil von Mu- } \\
\text { sikdownloads am } \\
\text { Gesamtvolumen }\end{array}$ & $\begin{array}{l}\text { Durchschnittliche } \\
\text { Größe einer Mu- } \\
\text { sikdatei in MB }\end{array}$ \\
\hline 12.500 & 27.300 .000 & 341.250 .000 .000 & $25 \%$ & $12,6 \%$ & 5 \\
\hline
\end{tabular}

\begin{tabular}{|l|l|l|l|l|l|}
\hline $\begin{array}{l}\text { Musikdownloads } \\
\text { in Stück }\end{array}$ & $\begin{array}{l}\text { Preis } \\
\text { pro } \\
\text { Album }\end{array}$ & $\begin{array}{l}\text { Anteil der } \\
\text { Rechteinha- } \\
\text { ber am Erlös }\end{array}$ & Preis monatlich & Substitutionsrate & $\begin{array}{l}\text { Preis umgelegt auf } \\
\text { Anschlussinhaber } \\
\text { monatlich }\end{array}$ \\
\hline 2.149 .875 .000 & $0,99 €$ & $80,25 \%$ & $1.708 .021 .940,63 €$ & $10 \%$ & $\mathbf{6 , 2 6} €$ \\
\hline
\end{tabular}

690 Für die Filmindustrie:

\begin{tabular}{|l|l|l|l|l|l|}
\hline $\begin{array}{l}\text { Volumen } \\
\text { pro Nutzer } \\
\text { in MB mo- } \\
\text { natlich }\end{array}$ & $\begin{array}{l}\text { Zahl der Breit- } \\
\text { bandanschlüsse }\end{array}$ & $\begin{array}{l}\text { Volumen pro Mo- } \\
\text { nat in MB }\end{array}$ & $\begin{array}{l}\text { Urheberrechtlich } \\
\text { relevanter Anteil }\end{array}$ & $\begin{array}{l}\text { Anteil von Film- } \\
\text { downloads am } \\
\text { Gesamtvolumen }\end{array}$ & $\begin{array}{l}\text { Durchschnittliche } \\
\text { Größe eines Films }\end{array}$ \\
\hline 12.500 & 27.300 .000 & 341.250 .000 .000 & $25 \%$ & $87,3 \%$ & 700 \\
\hline
\end{tabular}

\begin{tabular}{|l|l|l|l|l|l|}
\hline $\begin{array}{l}\text { Heruntergeladene } \\
\text { Filme in Stück }\end{array}$ & $\begin{array}{l}\text { Preis } \\
\text { pro } \\
\text { Film }\end{array}$ & $\begin{array}{l}\text { Anteil der } \\
\text { Rechteinhaber } \\
\text { am Erlös }\end{array}$ & Preis monatlich & Substitutionsrate & $\begin{array}{l}\text { Preis umgelegt auf } \\
\text { Anschlussinhaber } \\
\text { monatlich }\end{array}$ \\
\hline 106.396 .875 & $7,71 €$ & $70 \%$ & $574.223 .934,38 €$ & $10 \%$ & $\mathbf{2 , 1 0 €}$ \\
\hline
\end{tabular}

691 Für die Buchindustrie:

\begin{tabular}{|l|l|l|l|l|l|}
\hline $\begin{array}{l}\text { Volumen pro } \\
\text { Nutzer in MB } \\
\text { monatlich }\end{array}$ & $\begin{array}{l}\text { Zahl der Breitbandan- } \\
\text { schlüsse }\end{array}$ & $\begin{array}{l}\text { Volumen pro } \\
\text { Monat in MB }\end{array}$ & $\begin{array}{l}\text { Urheberrechtlich } \\
\text { relevanter Anteil }\end{array}$ & $\begin{array}{l}\text { Anteil von Buch- } \\
\text { downloads am Ge- } \\
\text { samtvolumen }\end{array}$ & $\begin{array}{l}\text { MB } \\
\text { pro } \\
\text { Buch }\end{array}$ \\
\hline 12.500 & 27.300 .000 & 341.250 .000 .000 & $25 \%$ & $0,10 \%$ & 2 \\
\hline
\end{tabular}

\begin{tabular}{|l|l|l|l|l|l|}
\hline $\begin{array}{l}\text { Anzahl } \\
\text { Bücher }\end{array}$ & $\begin{array}{l}\text { Preis } \\
\text { pro E- } \\
\text { Book }\end{array}$ & $\begin{array}{l}\text { Anteil der Ver- } \\
\text { triebskosten }\end{array}$ & Preis monatlich & Substitutionsrate & $\begin{array}{l}\text { Preis umgelegt auf An- } \\
\text { schlussinhaber monatlich }\end{array}$ \\
\hline 42.656 .250 & $8,07 €$ & $50 \%$ & $172.117 .968,75 €$ & $10 \%$ & $\mathbf{0 , 6 3} €$ \\
\hline
\end{tabular}


692 Insgesamt:

\begin{tabular}{|l|l|l|l|}
\hline Musikindustrie & Filmindustrie & Buchindustrie & Gesamt \\
\hline $6,26 €$ & $2,10 €$ & $0,63 €$ & $\mathbf{8 , 9 9} €$ \\
\hline
\end{tabular}

693 Gestaffelt nach Breitbandgeschwindigkeiten (pro Monat/pro Anschluss):

\begin{tabular}{|l|l|l|}
\hline $\begin{array}{l}\text { Kategorie } 1 \text { (bis einschließlich } 2 \\
\text { MBit/s) }\end{array}$ & Kategorie 2 (2-10 MBit/s) & Kategorie 3 (10 MBit/s und mehr) \\
\hline $3,42 €$ & $8,54 €$ & $13,67 €$ \\
\hline
\end{tabular}




\section{b) Urheberrechtlich relevanter Anteil von 15\%}

(1) Substitutionsrate von 30\%

694 Für die Musikindustrie:

\begin{tabular}{|l|l|l|l|l|l|}
\hline $\begin{array}{l}\text { Volumen } \\
\text { pro Nut- } \\
\text { zer in MB } \\
\text { monatlich }\end{array}$ & $\begin{array}{l}\text { Zahl der Breitband- } \\
\text { anschlüsse }\end{array}$ & $\begin{array}{l}\text { Volumen pro Mo- } \\
\text { nat in MB }\end{array}$ & $\begin{array}{l}\text { Urheberrechtlich } \\
\text { relevanter Anteil }\end{array}$ & $\begin{array}{l}\text { Anteil von Mu- } \\
\text { sikdownloads am } \\
\text { Gesamtvolumen }\end{array}$ & $\begin{array}{l}\text { Durchschnittliche } \\
\text { Größe einer Mu- } \\
\text { sikdatei in MB }\end{array}$ \\
\hline 12.500 & 27.300 .000 & 341.250 .000 .000 & $15 \%$ & $12,6 \%$ & 5 \\
\hline
\end{tabular}

\begin{tabular}{|l|l|l|l|l|l|}
\hline $\begin{array}{l}\text { Musikdownloads } \\
\text { in Stück }\end{array}$ & $\begin{array}{l}\text { Preis pro } \\
\text { Album }\end{array}$ & $\begin{array}{l}\text { Anteil der } \\
\text { Rechteinha- } \\
\text { ber am Erlös }\end{array}$ & Preis monatlich & Substitutionsrate & $\begin{array}{l}\text { Preis umgelegt auf } \\
\text { Anschlussinhaber } \\
\text { monatlich }\end{array}$ \\
\hline 1.289 .925 .000 & $0,99 €$ & $80,25 \%$ & $1.024 .813 .164,38 €$ & $30 \%$ & $\mathbf{1 1 , 2 6 €}$ \\
\hline
\end{tabular}

\section{Für die Filmindustrie:}

\begin{tabular}{|l|l|l|l|l|l|}
\hline $\begin{array}{l}\text { Volumen } \\
\text { pro Nut- } \\
\text { zer in MB } \\
\text { monatlich }\end{array}$ & $\begin{array}{l}\text { Zahl der Breitband- } \\
\text { anschlüsse }\end{array}$ & $\begin{array}{l}\text { Volumen pro Mo- } \\
\text { nat in MB }\end{array}$ & $\begin{array}{l}\text { Urheberrechtlich } \\
\text { relevanter Anteil }\end{array}$ & $\begin{array}{l}\text { Anteil von Film- } \\
\text { downloads am } \\
\text { Gesamtvolumen }\end{array}$ & $\begin{array}{l}\text { Durchschnittliche } \\
\text { Größe eines Films }\end{array}$ \\
\hline 12.500 & 27.300 .000 & 341.250 .000 .000 & $15 \%$ & $87,3 \%$ & 700 \\
\hline
\end{tabular}

\begin{tabular}{|l|l|l|l|l|l|}
\hline $\begin{array}{l}\text { Heruntergeladene } \\
\text { Filme in Stück }\end{array}$ & $\begin{array}{l}\text { Preis } \\
\text { pro } \\
\text { Film }\end{array}$ & $\begin{array}{l}\text { Anteil der } \\
\text { Rechteinhaber } \\
\text { am Erlös }\end{array}$ & Preis monatlich & Substitutionsrate & $\begin{array}{l}\text { Preis umgelegt auf } \\
\text { Anschlussinhaber } \\
\text { monatlich }\end{array}$ \\
\hline 63.838 .125 & $7,71 €$ & $70 \%$ & $344.534 .360,63 €$ & $30 \%$ & $\mathbf{3 , 7 9} €$ \\
\hline
\end{tabular}

696 Für die Buchindustrie:

\begin{tabular}{|l|l|l|l|l|l|}
\hline $\begin{array}{l}\text { Volumen pro } \\
\begin{array}{l}\text { Nutzer in MB } \\
\text { monatlich }\end{array}\end{array}$ & $\begin{array}{l}\text { Zahl der Breitbandan- } \\
\text { schlüsse }\end{array}$ & $\begin{array}{l}\text { Volumen pro } \\
\text { Monat in MB }\end{array}$ & $\begin{array}{l}\text { Urheberrechtlich } \\
\text { relevanter Anteil }\end{array}$ & $\begin{array}{l}\text { Anteil von Buch- } \\
\text { downloads am Ge- } \\
\text { samtvolumen }\end{array}$ & $\begin{array}{l}\text { MB } \\
\text { pro } \\
\text { Buch }\end{array}$ \\
\hline 12.500 & 27.300 .000 & 341.250 .000 .000 & $15 \%$ & $0,10 \%$ & 2 \\
\hline
\end{tabular}

\begin{tabular}{|l|l|l|l|l|l|}
\hline $\begin{array}{l}\text { Anzahl } \\
\text { Bücher }\end{array}$ & $\begin{array}{l}\text { Preis pro } \\
\text { E-Book }\end{array}$ & $\begin{array}{l}\text { Anteil der Ver- } \\
\text { triebskosten }\end{array}$ & Preis monatlich & Substitutionsrate & $\begin{array}{l}\text { Preis umgelegt auf An- } \\
\text { schlussinhaber monatlich }\end{array}$ \\
\hline $\mathbf{2 5 . 5 9 3 . 7 5 0}$ & $8,07 €$ & $50 \%$ & $103.398 .750 €$ & $30 \%$ & $\mathbf{1 , 1 3} €$ \\
\hline
\end{tabular}

\section{Insgesamt:}

\begin{tabular}{|l|l|l|l|}
\hline Musikindustrie & Filmindustrie & Buchindustrie & Gesamt \\
\hline $11,26 €$ & $3,79 €$ & $1,13 €$ & $\mathbf{1 6 , 1 8} €$ \\
\hline
\end{tabular}


698 Gestaffelt nach Breitbandgeschwindigkeiten (pro Monat/pro Anschluss):

\begin{tabular}{|l|l|l|}
\hline $\begin{array}{l}\text { Kategorie } \mathbf{1} \text { (bis einschließlich } \mathbf{2} \\
\mathbf{M B i t} / \mathbf{s})\end{array}$ & Kategorie 2 (2-10 MBit/s) & Kategorie $\mathbf{3}$ (10 MBit/s und mehr) \\
\hline $6,15 €$ & $15,38 €$ & $24,61 €$ \\
\hline
\end{tabular}

(2) Substitutionsrate von $20 \%$

699 Für die Musikindustrie:

\begin{tabular}{|l|l|l|l|l|l|}
\hline $\begin{array}{l}\text { Volumen } \\
\text { pro Nut- } \\
\text { zer in MB } \\
\text { monatlich }\end{array}$ & $\begin{array}{l}\text { Zahl der Breit- } \\
\text { bandanschlüsse }\end{array}$ & $\begin{array}{l}\text { Volumen pro Monat } \\
\text { in MB }\end{array}$ & $\begin{array}{l}\text { Urheberrechtlich } \\
\text { relevanter Anteil }\end{array}$ & $\begin{array}{l}\text { Anteil von Mu- } \\
\text { sikdownloads am } \\
\text { Gesamtvolumen }\end{array}$ & $\begin{array}{l}\text { Durchschnittliche } \\
\text { Größe einer Mu- } \\
\text { sikdatei in MB }\end{array}$ \\
\hline 12.500 & 27.300 .000 & 341.250 .000 .000 & $15 \%$ & $12,6 \%$ & 5 \\
\hline
\end{tabular}

\begin{tabular}{|l|l|l|l|l|l|}
\hline $\begin{array}{l}\text { Musikdownloads } \\
\text { in Stück }\end{array}$ & $\begin{array}{l}\text { Preis pro } \\
\text { Album }\end{array}$ & $\begin{array}{l}\text { Anteil der } \\
\text { Rechteinhaber } \\
\text { am Erlös }\end{array}$ & Preis monatlich & Substitutionsrate & $\begin{array}{l}\text { Preis umgelegt auf } \\
\text { Anschlussinhaber } \\
\text { monatlich }\end{array}$ \\
\hline 1.289 .925 .000 & $0,99 €$ & $80,25 \%$ & $1.024 .813 .164,38 €$ & $20 \%$ & $\mathbf{7 , 5 1} €$ \\
\hline
\end{tabular}

Für die Filmindustrie:

\begin{tabular}{|l|l|l|l|l|l|}
\hline $\begin{array}{l}\text { Volumen } \\
\text { pro Nut- } \\
\begin{array}{l}\text { zer in MB } \\
\text { monatlich }\end{array}\end{array}$ & $\begin{array}{l}\text { Zahl der Breit- } \\
\text { bandanschlüsse }\end{array}$ & $\begin{array}{l}\text { Volumen pro Mo- } \\
\text { nat in MB }\end{array}$ & $\begin{array}{l}\text { Urheberrechtlich } \\
\text { relevanter Anteil }\end{array}$ & $\begin{array}{l}\text { Anteil von Film- } \\
\text { downloads am } \\
\text { Gesamtvolumen }\end{array}$ & $\begin{array}{l}\text { Durchschnittliche } \\
\text { Größe eines Films }\end{array}$ \\
\hline 12.500 & 27.300 .000 & 341.250 .000 .000 & $15 \%$ & $87,3 \%$ & 700 \\
\hline
\end{tabular}

\begin{tabular}{|l|l|l|l|l|l|}
\hline $\begin{array}{l}\text { Heruntergeladene } \\
\text { Filme in Stück }\end{array}$ & $\begin{array}{l}\text { Preis } \\
\text { pro } \\
\text { Film }\end{array}$ & $\begin{array}{l}\text { Anteil der } \\
\text { Rechteinhaber } \\
\text { am Erlös }\end{array}$ & Preis monatlich & Substitutionsrate & $\begin{array}{l}\text { Preis umgelegt auf } \\
\text { Anschlussinhaber } \\
\text { monatlich }\end{array}$ \\
\hline 63.838 .125 & $7,71 €$ & $70 \%$ & $344.534 .360,63 €$ & $20 \%$ & $\mathbf{2 , 5 2} €$ \\
\hline
\end{tabular}

701 Für die Buchindustrie:

\begin{tabular}{|l|l|l|l|l|l|}
\hline $\begin{array}{l}\text { Volumen pro } \\
\begin{array}{l}\text { Nutzer in MB } \\
\text { monatlich }\end{array}\end{array}$ & $\begin{array}{l}\text { Zahl der Breitbandan- } \\
\text { schlüsse }\end{array}$ & $\begin{array}{l}\text { Volumen pro } \\
\text { Monat in MB }\end{array}$ & $\begin{array}{l}\text { Urheberrechtlich } \\
\text { relevanter Anteil }\end{array}$ & $\begin{array}{l}\text { Anteil von Buch- } \\
\text { downloads am Ge- } \\
\text { samtvolumen }\end{array}$ & $\begin{array}{l}\text { MB } \\
\text { pro } \\
\text { Buch }\end{array}$ \\
\hline 12.500 & 27.300 .000 & 341.250 .000 .000 & $15 \%$ & $0,10 \%$ & 2 \\
\hline
\end{tabular}

\begin{tabular}{|l|l|l|l|l|l|}
\hline $\begin{array}{l}\text { Anzahl } \\
\text { Bücher }\end{array}$ & $\begin{array}{l}\text { Preis pro } \\
\text { E-Book }\end{array}$ & $\begin{array}{l}\text { Anteil der Ver- } \\
\text { triebskosten }\end{array}$ & Preis monatlich & Substitutionsrate & $\begin{array}{l}\text { Preis umgelegt auf An- } \\
\text { schlussinhaber monatlich }\end{array}$ \\
\hline 25.593 .750 & $8,07 €$ & $50 \%$ & $103.398 .750 €$ & $20 \%$ & $\mathbf{0 , 7 6 €}$ \\
\hline
\end{tabular}

\begin{tabular}{|l|l|l|l|}
\hline Musikindustrie & Filmindustrie & Buchindustrie & Gesamt \\
\hline $7,51 €$ & $2,52 €$ & $0,76 €$ & $10,79 €$ \\
\hline
\end{tabular}


703 Gestaffelt nach Breitbandgeschwindigkeiten (pro Monat/pro Anschluss):

\begin{tabular}{|l|l|l|}
\hline $\begin{array}{l}\text { Kategorie } \mathbf{1} \text { (bis einschließlich } \mathbf{2} \\
\text { MBit/s) }\end{array}$ & Kategorie 2 (2-10 MBit/s) & Kategorie 3 (10 MBit/s und mehr) \\
\hline $4,10 €$ & $10,25 €$ & $16,41 €$ \\
\hline
\end{tabular}

(3) Substitutionsrate von 10\%

704 Für die Musikindustrie:

\begin{tabular}{|l|l|l|l|l|l|}
\hline $\begin{array}{l}\text { Volumen } \\
\text { pro Nut- } \\
\text { zer in MB } \\
\text { monatlich }\end{array}$ & $\begin{array}{l}\text { Zahl der Breitband- } \\
\text { anschlüsse }\end{array}$ & $\begin{array}{l}\text { Volumen pro Mo- } \\
\text { nat in MB }\end{array}$ & $\begin{array}{l}\text { Urheberrechtlich } \\
\text { relevanter Anteil }\end{array}$ & $\begin{array}{l}\text { Anteil von Mu- } \\
\text { sikdownloads am } \\
\text { Gesamtvolumen }\end{array}$ & $\begin{array}{l}\text { Durchschnittliche } \\
\text { Größe einer Mu- } \\
\text { sikdatei in MB }\end{array}$ \\
\hline 12.500 & 27.300 .000 & 341.250 .000 .000 & $15 \%$ & $12,6 \%$ & 5 \\
\hline
\end{tabular}

\begin{tabular}{|l|l|l|l|l|l|}
\hline $\begin{array}{l}\text { Musikdownloads } \\
\text { in Stück }\end{array}$ & $\begin{array}{l}\text { Preis } \\
\text { pro } \\
\text { Album }\end{array}$ & $\begin{array}{l}\text { Anteil der } \\
\text { Rechteinha- } \\
\text { ber am Erlös }\end{array}$ & Preis monatlich & Substitutionsrate & $\begin{array}{l}\text { Preis umgelegt auf } \\
\text { Anschlussinhaber } \\
\text { monatlich }\end{array}$ \\
\hline 1.289 .925 .000 & $0,99 €$ & $80,25 \%$ & $1.024 .813 .164,38 €$ & $10 \%$ & $\mathbf{3 , 7 5 €}$ \\
\hline
\end{tabular}

705 Für die Filmindustrie:

\begin{tabular}{|l|l|l|l|l|l|}
\hline $\begin{array}{l}\text { Volumen } \\
\text { pro Nut- } \\
\text { zer in MB } \\
\text { monatlich }\end{array}$ & $\begin{array}{l}\text { Zahl der Breitband- } \\
\text { anschlüsse }\end{array}$ & $\begin{array}{l}\text { Volumen pro Mo- } \\
\text { nat in MB }\end{array}$ & $\begin{array}{l}\text { Urheberrechtlich } \\
\text { relevanter Anteil }\end{array}$ & $\begin{array}{l}\text { Anteil von Film- } \\
\text { downloads am } \\
\text { Gesamtvolumen }\end{array}$ & $\begin{array}{l}\text { Durchschnittliche } \\
\text { Größe eines Films }\end{array}$ \\
\hline 12.500 & 27.300 .000 & 341.250 .000 .000 & $15 \%$ & $87,3 \%$ & 700 \\
\hline
\end{tabular}

\begin{tabular}{|l|l|l|l|l|l|}
\hline $\begin{array}{l}\text { Heruntergeladene } \\
\text { Filme in Stück }\end{array}$ & $\begin{array}{l}\text { Preis } \\
\text { pro } \\
\text { Film }\end{array}$ & $\begin{array}{l}\text { Anteil der } \\
\text { Rechteinhaber } \\
\text { am Erlös }\end{array}$ & Preis monatlich & Substitutionsrate & $\begin{array}{l}\text { Preis umgelegt auf } \\
\text { Anschlussinhaber } \\
\text { monatlich }\end{array}$ \\
\hline 63.838 .125 & $7,71 €$ & $70 \%$ & $344.534 .360,63 €$ & $10 \%$ & $\mathbf{1 , 2 6} €$ \\
\hline
\end{tabular}

706 Für die Buchindustrie:

\begin{tabular}{|l|l|l|l|l|l|}
\hline $\begin{array}{l}\text { Volumen pro } \\
\text { Nutzer in MB } \\
\text { monatlich }\end{array}$ & $\begin{array}{l}\text { Zahl der Breitbandan- } \\
\text { schlüsse }\end{array}$ & $\begin{array}{l}\text { Volumen pro } \\
\text { Monat in MB }\end{array}$ & $\begin{array}{l}\text { Urheberrechtlich } \\
\text { relevanter Anteil }\end{array}$ & $\begin{array}{l}\text { Anteil von Buch- } \\
\text { downloads am Ge- } \\
\text { samtvolumen }\end{array}$ & $\begin{array}{l}\text { MB } \\
\text { pro } \\
\text { Buch }\end{array}$ \\
\hline 12.500 & 27.300 .000 & 341.250 .000 .000 & $15 \%$ & $0,10 \%$ & 2 \\
\hline
\end{tabular}

\begin{tabular}{|l|l|l|l|l|l|}
\hline $\begin{array}{l}\text { Anzahl } \\
\text { Bücher }\end{array}$ & $\begin{array}{l}\text { Preis pro } \\
\text { E-Book }\end{array}$ & $\begin{array}{l}\text { Anteil der Ver- } \\
\text { triebskosten }\end{array}$ & Preis monatlich & Substitutionsrate & $\begin{array}{l}\text { Preis umgelegt auf An- } \\
\text { schlussinhaber monatlich }\end{array}$ \\
\hline 25.593 .750 & $8,07 €$ & $50 \%$ & $103.398 .750 €$ & $10 \%$ & $\mathbf{5 , 3 9} €$ \\
\hline
\end{tabular}

707 Insgesamt:

\begin{tabular}{|l|l|l|l|}
\hline Musikindustrie & Filmindustrie & Buchindustrie & Gesamt \\
\hline $3,75 €$ & $1,26 €$ & $5,39 €$ & $\mathbf{1 0 , 7 9} €$ \\
\hline
\end{tabular}


Gestaffelt nach Breitbandgeschwindigkeiten (pro Monat/pro Anschluss):

\begin{tabular}{|l|l|l|}
\hline $\begin{array}{l}\text { Kategorie } \mathbf{1} \text { (bis einschließlich } 2 \\
\text { MBit/s) }\end{array}$ & Kategorie 2 (2-10 MBit/s) & Kategorie 3 (10 MBit/s und mehr) \\
\hline $2,05 €$ & $5,13 €$ & $8,20 €$ \\
\hline
\end{tabular}

\section{c) Urheberrechtlich relevanter Anteil von 5\%}

(1) Substitutionsrate von 30\%

709 Für die Musikindustrie:

\begin{tabular}{|l|l|l|l|l|l|}
\hline $\begin{array}{l}\text { Volumen } \\
\text { pro Nut- } \\
\text { zer in MB } \\
\text { monatlich }\end{array}$ & $\begin{array}{l}\text { Zahl der Breitband- } \\
\text { anschlüsse }\end{array}$ & $\begin{array}{l}\text { Volumen pro Mo- } \\
\text { nat in MB }\end{array}$ & $\begin{array}{l}\text { Urheberrechtlich } \\
\text { relevanter Anteil }\end{array}$ & $\begin{array}{l}\text { Anteil von Mu- } \\
\text { sikdownloads am } \\
\text { Gesamtvolumen }\end{array}$ & $\begin{array}{l}\text { Durchschnittliche } \\
\text { Größe einer Mu- } \\
\text { sikdatei in MB }\end{array}$ \\
\hline 12.500 & 27.300 .000 & 341.250 .000 .000 & $5 \%$ & $12,6 \%$ & 5 \\
\hline
\end{tabular}

\begin{tabular}{|l|l|l|l|l|l|}
\hline $\begin{array}{l}\text { Musikdownloads } \\
\text { in Stück }\end{array}$ & $\begin{array}{l}\text { Preis pro } \\
\text { Album }\end{array}$ & $\begin{array}{l}\text { Anteil der } \\
\text { Rechteinhaber } \\
\text { am Erlös }\end{array}$ & Preis monatlich & Substitutionsrate & $\begin{array}{l}\text { Preis umgelegt auf } \\
\text { Anschlussinhaber } \\
\text { monatlich }\end{array}$ \\
\hline 429.975 .000 & $0,99 €$ & $80,25 \%$ & $341.604 .388,13 €$ & $30 \%$ & $\mathbf{3 , 7 5} €$ \\
\hline
\end{tabular}

710 Für die Filmindustrie:

\begin{tabular}{|l|l|l|l|l|l|}
\hline $\begin{array}{l}\text { Volumen } \\
\text { pro Nut- } \\
\text { zer in MB } \\
\text { monatlich }\end{array}$ & $\begin{array}{l}\text { Zahl der Breit- } \\
\text { bandanschlüsse }\end{array}$ & $\begin{array}{l}\text { Volumen pro Mo- } \\
\text { nat in MB }\end{array}$ & $\begin{array}{l}\text { Urheberrechtlich } \\
\text { relevanter Anteil }\end{array}$ & $\begin{array}{l}\text { Anteil von Film- } \\
\text { downloads am } \\
\text { Gesamtvolumen }\end{array}$ & $\begin{array}{l}\text { Durchschnittliche } \\
\text { Größe eines Films }\end{array}$ \\
\hline 12.500 & 27.300 .000 & 341.250 .000 .000 & $5 \%$ & $87,3 \%$ & 700 \\
\hline
\end{tabular}

\begin{tabular}{|l|l|l|l|l|l|}
\hline $\begin{array}{l}\text { Heruntergeladene } \\
\text { Filme in Stück }\end{array}$ & $\begin{array}{l}\text { Preis } \\
\text { pro } \\
\text { Film }\end{array}$ & $\begin{array}{l}\text { Anteil der } \\
\text { Rechteinhaber } \\
\text { am Erlös }\end{array}$ & Preis monatlich & Substitutionsrate & $\begin{array}{l}\text { Preis umgelegt auf } \\
\text { Anschlussinhaber } \\
\text { monatlich }\end{array}$ \\
\hline $\mathbf{2 1 . 2 7 9 . 3 7 5}$ & $7,71 €$ & $70 \%$ & $114.844 .786,88 €$ & $30 \%$ & $\mathbf{1 , 2 6} €$ \\
\hline
\end{tabular}

\section{Für die Buchindustrie:}

\begin{tabular}{|l|l|l|l|l|l|}
\hline $\begin{array}{l}\text { Volumen pro } \\
\text { Nutzer in MB } \\
\text { monatlich }\end{array}$ & $\begin{array}{l}\text { Zahl der Breitbandan- } \\
\text { schlüsse }\end{array}$ & $\begin{array}{l}\text { Volumen pro } \\
\text { Monat in MB }\end{array}$ & $\begin{array}{l}\text { Urheberrechtlich } \\
\text { relevanter Anteil }\end{array}$ & $\begin{array}{l}\text { Anteil von Buch- } \\
\text { downloads am Ge- } \\
\text { samtvolumen }\end{array}$ & $\begin{array}{l}\text { MB } \\
\text { pro } \\
\text { Buch }\end{array}$ \\
\hline 12.500 & 27.300 .000 & 341.250 .000 .000 & $5 \%$ & $0,10 \%$ & 2 \\
\hline
\end{tabular}

\begin{tabular}{|l|l|l|l|l|l|}
\hline $\begin{array}{l}\text { Anzahl } \\
\text { Bücher }\end{array}$ & $\begin{array}{l}\text { Preis pro } \\
\text { E-Book }\end{array}$ & $\begin{array}{l}\text { Anteil der Ver- } \\
\text { triebskosten }\end{array}$ & Preis monatlich & Substitutionsrate & $\begin{array}{l}\text { Preis umgelegt auf An- } \\
\text { schlussinhaber monatlich }\end{array}$ \\
\hline 8.531 .250 & $8,07 €$ & $50 \%$ & $34.423 .593,75 €$ & $30 \%$ & $\mathbf{0 , 3 8} €$ \\
\hline
\end{tabular}




\section{Insgesamt:}

\begin{tabular}{|l|l|l|l|}
\hline Musikindustrie & Filmindustrie & Buchindustrie & Gesamt \\
\hline $3,75 €$ & $1,26 €$ & $0,38 €$ & $\mathbf{5 , 3 9}$ \\
\hline
\end{tabular}

\section{Gestaffelt nach Breitbandgeschwindigkeiten (pro Monat/pro Anschluss):}

\begin{tabular}{|l|l|l|}
\hline $\begin{array}{l}\text { Kategorie } \mathbf{1} \text { (bis einschließlich } 2 \\
\text { MBit/s) }\end{array}$ & Kategorie 2 (2-10 MBit/s) & Kategorie $\mathbf{3}$ (10 MBit/s und mehr) \\
\hline $2,05 €$ & $5,13 €$ & $8,20 €$ \\
\hline
\end{tabular}

(2) Substitutionsrate von 20\%

714 Für die Musikindustrie:

\begin{tabular}{|l|l|l|l|l|l|}
\hline $\begin{array}{l}\text { Volumen } \\
\text { pro Nut- } \\
\text { zer in MB } \\
\text { monatlich }\end{array}$ & $\begin{array}{l}\text { Zahl der Breitband- } \\
\text { anschlüsse }\end{array}$ & $\begin{array}{l}\text { Volumen pro Mo- } \\
\text { nat in MB }\end{array}$ & $\begin{array}{l}\text { Urheberrechtlich } \\
\text { relevanter Anteil }\end{array}$ & $\begin{array}{l}\text { Anteil von Mu- } \\
\text { sikdownloads am } \\
\text { Gesamtvolumen }\end{array}$ & $\begin{array}{l}\text { Durchschnittliche } \\
\text { Größe einer Mu- } \\
\text { sikdatei in MB }\end{array}$ \\
\hline 12.500 & 27.300 .000 & 341.250 .000 .000 & $5 \%$ & $12,6 \%$ & 5 \\
\hline
\end{tabular}

\begin{tabular}{|l|l|l|l|l|l|}
\hline $\begin{array}{l}\text { Musikdownloads } \\
\text { in Stück }\end{array}$ & $\begin{array}{l}\text { Preis pro } \\
\text { Album }\end{array}$ & $\begin{array}{l}\text { Anteil der } \\
\text { Rechteinhaber } \\
\text { am Erlös }\end{array}$ & Preis monatlich & Substitutionsrate & $\begin{array}{l}\text { Preis umgelegt auf } \\
\text { Anschlussinhaber } \\
\text { monatlich }\end{array}$ \\
\hline 429.975 .000 & $0,99 €$ & $80,25 \%$ & $341.604 .388,13 €$ & $20 \%$ & $\mathbf{2 , 5 0 €}$ \\
\hline
\end{tabular}

715 Für die Filmindustrie:

\begin{tabular}{|l|l|l|l|l|l|}
\hline $\begin{array}{l}\text { Volumen } \\
\text { pro Nut- } \\
\text { zer in MB } \\
\text { monatlich }\end{array}$ & $\begin{array}{l}\text { Zahl der Breit- } \\
\text { bandanschlüsse }\end{array}$ & $\begin{array}{l}\text { Volumen pro Mo- } \\
\text { nat in MB }\end{array}$ & $\begin{array}{l}\text { Urheberrechtlich } \\
\text { relevanter Anteil }\end{array}$ & $\begin{array}{l}\text { Anteil von Film- } \\
\text { downloads am } \\
\text { Gesamtvolumen }\end{array}$ & $\begin{array}{l}\text { Durchschnittliche } \\
\text { Größe eines Films }\end{array}$ \\
\hline 12.500 & 27.300 .000 & 341.250 .000 .000 & $5 \%$ & $87,3 \%$ & 700 \\
\hline
\end{tabular}

\begin{tabular}{|l|l|l|l|l|l|}
\hline $\begin{array}{l}\text { Heruntergeladene } \\
\text { Filme in Stück }\end{array}$ & $\begin{array}{l}\text { Preis } \\
\text { pro } \\
\text { Film }\end{array}$ & $\begin{array}{l}\text { Anteil der } \\
\text { Rechteinhaber } \\
\text { am Erlös }\end{array}$ & Preis monatlich & Substitutionsrate & $\begin{array}{l}\text { Preis umgelegt auf } \\
\text { Anschlussinhaber } \\
\text { monatlich }\end{array}$ \\
\hline 21.279 .375 & $7,71 €$ & $70 \%$ & $114.844 .786,88 €$ & $20 \%$ & $\mathbf{0 , 8 4} €$ \\
\hline
\end{tabular}

\section{Für die Buchindustrie:}

\begin{tabular}{|l|l|l|l|l|l|}
\hline $\begin{array}{l}\text { Volumen pro } \\
\text { Nutzer in MB } \\
\text { monatlich }\end{array}$ & $\begin{array}{l}\text { Zahl der Breitbandan- } \\
\text { schlüsse }\end{array}$ & $\begin{array}{l}\text { Volumen pro } \\
\text { Monat in MB }\end{array}$ & $\begin{array}{l}\text { Urheberrechtlich } \\
\text { relevanter Anteil }\end{array}$ & $\begin{array}{l}\text { Anteil von Buch- } \\
\text { downloads am Ge- } \\
\text { samtvolumen }\end{array}$ & $\begin{array}{l}\text { MB } \\
\text { pro } \\
\text { Buch }\end{array}$ \\
\hline 12.500 & 27.300 .000 & 341.250 .000 .000 & $5 \%$ & $0,10 \%$ & 2 \\
\hline
\end{tabular}




\begin{tabular}{|l|l|l|l|l|l|}
\hline $\begin{array}{l}\text { Anzahl } \\
\text { Bücher }\end{array}$ & $\begin{array}{l}\text { Preis pro } \\
\text { E-Book }\end{array}$ & $\begin{array}{l}\text { Anteil der Ver- } \\
\text { triebskosten }\end{array}$ & Preis monatlich & Substitutionsrate & $\begin{array}{l}\text { Preis umgelegt auf An- } \\
\text { schlussinhaber monatlich }\end{array}$ \\
\hline 8.531 .250 & $8,07 €$ & $50 \%$ & $34.423 .593,75 €$ & $20 \%$ & $\mathbf{0 , 2 5} €$ \\
\hline
\end{tabular}

\section{Insgesamt:}

\begin{tabular}{|l|l|l|l|}
\hline Musikindustrie & Filmindustrie & Buchindustrie & Gesamt \\
\hline $2,50 €$ & $0,84 €$ & $0,25 €$ & $3,60 €$ \\
\hline
\end{tabular}

718 Gestaffelt nach Breitbandgeschwindigkeiten (pro Monat/pro Anschluss):

\begin{tabular}{|l|l|l|}
\hline $\begin{array}{l}\text { Kategorie } \mathbf{1} \text { (bis einschließlich } \mathbf{2} \\
\mathbf{M B i t} / \mathbf{s})\end{array}$ & Kategorie 2 (2-10 MBit/s) & Kategorie $\mathbf{3}$ (10 MBit/s und $\mathbf{~ m e h r ) ~}$ \\
\hline $1,37 €$ & $3,42 €$ & $5,47 €$ \\
\hline
\end{tabular}

(3) Substitutionsrate von 10\%

719 Für die Musikindustrie:

\begin{tabular}{|l|l|l|l|l|l|}
\hline $\begin{array}{l}\text { Volumen } \\
\text { pro Nut- } \\
\text { zer in MB } \\
\text { monatlich }\end{array}$ & $\begin{array}{l}\text { Zahl der Breitband- } \\
\text { anschlüsse }\end{array}$ & $\begin{array}{l}\text { Volumen pro Mo- } \\
\text { nat in MB }\end{array}$ & $\begin{array}{l}\text { Urheberrechtlich } \\
\text { relevanter Anteil }\end{array}$ & $\begin{array}{l}\text { Anteil von Mu- } \\
\text { sikdownloads am } \\
\text { Gesamtvolumen }\end{array}$ & $\begin{array}{l}\text { Durchschnittliche } \\
\text { Größe einer Mu- } \\
\text { sikdatei in MB }\end{array}$ \\
\hline 12.500 & 27.300 .000 & 341.250 .000 .000 & $5 \%$ & $12,6 \%$ & 5 \\
\hline
\end{tabular}

\begin{tabular}{|l|l|l|l|l|l|}
\hline $\begin{array}{l}\text { Musikdownloads } \\
\text { in Stück }\end{array}$ & $\begin{array}{l}\text { Preis pro } \\
\text { Album }\end{array}$ & $\begin{array}{l}\text { Anteil der } \\
\text { Rechteinhaber } \\
\text { am Erlös }\end{array}$ & Preis monatlich & Substitutionsrate & $\begin{array}{l}\text { Preis umgelegt auf } \\
\text { Anschlussinhaber } \\
\text { monatlich }\end{array}$ \\
\hline 429.975 .000 & $0,99 €$ & $80,25 \%$ & $341.604 .388,13 €$ & $10 \%$ & $\mathbf{1 , 2 5} €$ \\
\hline
\end{tabular}

\section{Für die Filmindustrie:}

\begin{tabular}{|l|l|l|l|l|l|}
\hline $\begin{array}{l}\text { Volumen } \\
\text { pro Nut- } \\
\text { zer in MB } \\
\text { monatlich }\end{array}$ & $\begin{array}{l}\text { Zahl der Breitband- } \\
\text { anschlüsse }\end{array}$ & $\begin{array}{l}\text { Volumen pro Mo- } \\
\text { nat in MB }\end{array}$ & $\begin{array}{l}\text { Urheberrechtlich } \\
\text { relevanter Anteil }\end{array}$ & $\begin{array}{l}\text { Anteil von Film- } \\
\text { downloads am } \\
\text { Gesamtvolumen }\end{array}$ & $\begin{array}{l}\text { Durchschnittliche } \\
\text { Größe eines Films }\end{array}$ \\
\hline 12.500 & 27.300 .000 & 341.250 .000 .000 & $5 \%$ & $87,3 \%$ & 700 \\
\hline
\end{tabular}

\begin{tabular}{|l|l|l|l|l|l|}
\hline $\begin{array}{l}\text { Heruntergeladene } \\
\text { Filme in Stück }\end{array}$ & $\begin{array}{l}\text { Preis } \\
\text { pro } \\
\text { Film }\end{array}$ & $\begin{array}{l}\text { Anteil der } \\
\text { Rechteinhaber } \\
\text { am Erlös }\end{array}$ & Preis monatlich & Substitutionsrate & $\begin{array}{l}\text { Preis umgelegt auf } \\
\text { Anschlussinhaber } \\
\text { monatlich }\end{array}$ \\
\hline 21.279 .375 & $7,71 €$ & $70 \%$ & $114.844 .786,88 €$ & $10 \%$ & $\mathbf{0 , 4 2} €$ \\
\hline
\end{tabular}


721 Für die Buchindustrie:

\begin{tabular}{|l|l|l|l|l|l|}
\hline $\begin{array}{l}\text { Volumen pro } \\
\begin{array}{l}\text { Nutzer in MB } \\
\text { monatlich }\end{array}\end{array}$ & $\begin{array}{l}\text { Zahl der Breitbandan- } \\
\text { schlüsse }\end{array}$ & $\begin{array}{l}\text { Volumen pro } \\
\text { Monat in MB }\end{array}$ & $\begin{array}{l}\text { Urheberrechtlich } \\
\text { relevanter Anteil }\end{array}$ & $\begin{array}{l}\text { Anteil von Buch- } \\
\text { downloads am Ge- } \\
\text { samtvolumen }\end{array}$ & $\begin{array}{l}\text { MB } \\
\text { pro } \\
\text { Buch }\end{array}$ \\
\hline 12.500 & 27.300 .000 & 341.250 .000 .000 & $5 \%$ & $0,10 \%$ & 2 \\
\hline
\end{tabular}

\begin{tabular}{|l|l|l|l|l|l|}
\hline $\begin{array}{l}\text { Anzahl } \\
\text { Bücher }\end{array}$ & $\begin{array}{l}\text { Preis pro } \\
\text { E-Book }\end{array}$ & $\begin{array}{l}\text { Anteil der Ver- } \\
\text { triebskosten }\end{array}$ & Preis monatlich & Substitutionsrate & $\begin{array}{l}\text { Preis umgelegt auf An- } \\
\text { schlussinhaber monatlich }\end{array}$ \\
\hline 8.531 .250 & $8,07 €$ & $50 \%$ & $34.423 .593,75 €$ & $10 \%$ & $\mathbf{0 , 1 3} €$ \\
\hline
\end{tabular}

722 Insgesamt:

\begin{tabular}{|l|l|l|l|}
\hline Musikindustrie & Filmindustrie & Buchindustrie & Gesamt \\
\hline $1,25 €$ & $0,42 €$ & $0,13 €$ & $\mathbf{1 , 8 0} €$ \\
\hline
\end{tabular}

723 Gestaffelt nach Breitbandgeschwindigkeiten (pro Monat/pro Anschluss):

\begin{tabular}{|l|l|l|}
\hline $\begin{array}{l}\text { Kategorie } \mathbf{1} \text { (bis einschließlich } \mathbf{2} \\
\text { MBit/s) }\end{array}$ & Kategorie 2 (2-10 MBit/s) & Kategorie $\mathbf{3}$ (10 MBit/s und mehr) \\
\hline $0,68 €$ & $1,71 €$ & $2,73 €$ \\
\hline
\end{tabular}




\section{Literaturverzeichnis}

\begin{tabular}{|c|c|}
\hline Adermon, Adrian / Liang, Che-Yuan & $\begin{array}{l}\text { Piracy, Music and Movies: A Natural Experiment, IFN Working } \\
\text { Paper No. 854, 2010, abrufbar unter: } \\
\text { http://www.ifn.se/BinaryLoader.axd?OwnerID=e726617b- } \\
\text { 402a-4a8a-812d- } \\
\text { 8ad11882b14e\&OwnerType=0\&PropertyName=File1\&FileNam } \\
\text { e=Wp854.pdf\&Attachment=True }\end{array}$ \\
\hline Aguiar, Louis / Martens, Bertin & $\begin{array}{l}\text { Digital Music Consumption on the Internet: Evidence from } \\
\text { Clickstream Data, Institute for Prospective Technological Stud- } \\
\text { ies - Digital Economy Working Paper 2013/04, abrufbar unter: } \\
\text { http://ftp.jrc.es/EURdoc/JRC79605.pdf } \\
\text { (zitiert als Aguiar/Martens, 2013) }\end{array}$ \\
\hline $\begin{array}{l}\text { Ahlberg, Hartwig / Götting, Horst-Peter } \\
\text { (Hrsg.) }\end{array}$ & $\begin{array}{l}\text { Beck'scher Online-Kommentar Urheberrecht, Stand 15.9.2012 } \\
\text { (zitiert als BeckOK UrhR) }\end{array}$ \\
\hline Aigrain, Philippe & $\begin{array}{l}\text { Internet \& Création, In Libro Veritas 2008, abrufbar unter: } \\
\text { http://www.ilv- } \\
\text { bibliotheca.net/pdf_ebook_gratuit/internet_et_creation.pdf }\end{array}$ \\
\hline Aigrain, Philippe & $\begin{array}{l}\text { Sharing: Culture and the Economy in the Internet Age, Amster- } \\
\text { dam University Press 2012, abrufbar unter: } \\
\text { http://www.oapen.org/download?type=document\&docid=409 } \\
602\end{array}$ \\
\hline Akerlof, George A. & $\begin{array}{l}\text { The Market for "Lemons": Quality Uncertainty and the Market } \\
\text { Mechanism, Quarterly Journal of Economics 1970, S. 488-500 }\end{array}$ \\
\hline Allot Communications & $\begin{array}{l}\text { Digging deeper into deep packet inspection (DPI), 2007, abruf- } \\
\text { bar unter: http://www.cxo.eu.com/article/Digging-Deeper-Into- } \\
\text { Deep-Packet-Inspection-DPI }\end{array}$ \\
\hline American Assembly, The & $\begin{array}{l}\text { Copyright Infringement and Enforcement in the USA, Research } \\
\text { Note, 2011, abrufbar unter: } \\
\text { http://piracy.americanassembly.org/wp- } \\
\text { content/uploads/2011/11/AA-Research-Note-Infringement- } \\
\text { and-Enforcement-November-2011.pdf }\end{array}$ \\
\hline Andersen, Birgitte / Frenz, Marion & $\begin{array}{l}\text { The Impact of Music Downloads and P2P File-Sharing on the } \\
\text { Purchase of Music: A Study for Industry Canada, 2007, abrufbar } \\
\text { unter: http://www.ic.gc.ca/eic/site/ippd- } \\
\text { dppi.nsf/vwapj/IndustryCanadaPaperMay4_2007_en.pdf/\$FILE } \\
\text { /IndustryCanadaPaperMay4_2007_en.pdf } \\
\text { (zitiert als Andersen/Frenz, 2007) }\end{array}$ \\
\hline Anderson, Nate & $\begin{array}{l}\text { Deep packet inspection meets 'net neutrality, CALEA', 2007, } \\
\text { abrufbar unter: } \\
\text { http://arstechnica.com/gadgets/2007/07/deep-packet- } \\
\text { inspection-meets-net-neutrality/ }\end{array}$ \\
\hline $\begin{array}{l}\text { Arbeitsgemeinschaft der öffentlich- } \\
\text { rechtlichen Rundfunkanstalten der Bun- } \\
\text { desrepublik Deutschland (ARD) / Zweites } \\
\text { Deutsches Fernsehen (ZDF) }\end{array}$ & $\begin{array}{l}\text { ARD/ZDF-Onlinestudie 1998-2012, abrufbar unter: } \\
\text { http://www.ard-zdf- } \\
\text { onlinestudie.de/index.php?id=onlinenutzung-mio0 }\end{array}$ \\
\hline $\begin{array}{l}\text { Arbeitskreis Urheberrecht der SPD- } \\
\text { Bundestagsfraktion }\end{array}$ & $\begin{array}{l}\text { Zwölf Thesen für ein faires und zeitgemäßes Urheberrecht, } 21 . \\
\text { Mai 2012, abrufbar unter: } \\
\text { http://www.spdfraktion.de/sites/default/files/thesenpapier_z } \\
\text { woelf_thesen_fuer_ein_faires_und_zeitgemaesses_urheberrec } \\
\text { ht.pdf }\end{array}$ \\
\hline ARD-Forschungsdienst & $\begin{array}{l}\text { Die Bedeutung des Radios im Alltag, Media Perspektiven 2011, } \\
\text { S. } 617-622\end{array}$ \\
\hline Arlt, Christian & $\begin{array}{l}\text { Anm. zu BVerfG, Beschluss vom 21.12.2010 - } 1 \text { BvR 506/09 - } \\
\text { (Keine) Gerätevergütung für PCs, MMR 2011, S. 749-751 }\end{array}$ \\
\hline
\end{tabular}




\begin{tabular}{|c|c|}
\hline Arlt, Christian & $\begin{array}{l}\text { BVerfG: Verfassungswidrigkeit der BGH-Entscheidung „Drucker } \\
\text { und Plotter“, MMR-Aktuell 2011, } 313015\end{array}$ \\
\hline Arndt, Claus & $\begin{array}{l}\text { Grundrechtsschutz bei der Fernmeldeüberwachung, DÖV 1996, } \\
\text { S. } 459-463\end{array}$ \\
\hline Aschenbrenner, Jo Beatrix & $\begin{array}{l}\text { Leitlinien aus Europa für die Umsetzung der Privatkopieschran- } \\
\text { ke im Zweiten Korb der Urheberrechtsnovelle, ZUM 2005, S. } \\
\text { 145-154 }\end{array}$ \\
\hline Augenstein, Christof & $\begin{array}{l}\text { Rechtliche Grundlagen des Verteilungsplans urheberrechtlicher } \\
\text { Verwertungsgesellschaften, Nomos-Verlag, Baden-Baden } 2004\end{array}$ \\
\hline Bahanovich, David / Collopy, Dennis & $\begin{array}{l}\text { Music Experience and Behaviour in Young People, 2009, abruf- } \\
\text { bar unter: } \\
\text { http://www.academia.edu/238038/Music_Experience_and_Be } \\
\text { haviour_in_Young_People } \\
\text { (zitiert als Bahanovich/Collopy, 2009) }\end{array}$ \\
\hline Balázs, Bodó / Lakatos, Zoltán & $\begin{array}{l}\text { Theatrical Distribution and P2P Movie Piracy: A Survey of P2P } \\
\text { Networks in Hungary Using Transactional Data, International } \\
\text { Journal of Communication, Vol. } 6,2012, \text { S. } 413-445\end{array}$ \\
\hline Bauer, Sebastian & $\begin{array}{l}\text { Das Konzept Musikflatrate. Legalisierung und Vergütung von } \\
\text { Musik-File-Sharing, Dipl. Arbeit (Wirtschaftsuniversität Wien), } \\
\text { 2011, abrufbar unter: } \\
\text { http://ebookbrowse.com/gdoc.php?id=354698097\&url=105ea } \\
\text { b846a3e6ea3765200bb8a43cb97 } \\
\text { (zitiert als Bauer, Dipl. Arb., 2011) }\end{array}$ \\
\hline $\begin{array}{l}\text { Bäumler, Helmut / v. Mutius, Albert } \\
\text { (Hrsg.) }\end{array}$ & $\begin{array}{l}\text { Anonymität im Internet. Grundlagen, Methoden und Tools zur } \\
\text { Realisierung eines Grundrechts, Vieweg Verlag, Braunschweig } \\
2003\end{array}$ \\
\hline Bayaan, Ibrahiim & $\begin{array}{l}\text { Technology and the Music Industry: Effects on Profits, Variety } \\
\text { and Welfare, Emory University, March } 2004 \\
\text { (zitiert als Bayaan, 2004) }\end{array}$ \\
\hline Beck, Simon Markus / Kreißig, Wolfgang & $\begin{array}{l}\text { Tauschbörsen-Nutzer im Fadenkreuz der Strafverfolgungsbe- } \\
\text { hörden, NStZ 2007, S. 304-310 }\end{array}$ \\
\hline Becker, Jürgen / Müller, Stefan & $\begin{array}{l}\text { Die Bestimmung der Höhe der angemessenen Vergütung für } \\
\text { private Vervielfältigung, in: Schierholz, Anke / Melichar, Ferdi- } \\
\text { nand, Kunst, Recht und Geld, Festschrift für Gerhard Pfennig } \\
\text { zum 65. Geburtstag, Beck-Verlag, München 2012, S. 373-386 } \\
\text { (zitiert als Becker/Müller, in: FS Pfennig) }\end{array}$ \\
\hline Bedner, Mark & $\begin{array}{l}\text { "Deep Packet Inspection" - Technologie und rechtliche Initiati- } \\
\text { ven, CR 2010, S. 339-345 }\end{array}$ \\
\hline Beisel, Daniel & $\begin{array}{l}\text { Die Kunstfreiheitsgarantie des Grundgesetzes und ihre straf- } \\
\text { rechtlichen Grenzen, Verlag v. Decker, Heidelberg } 1997\end{array}$ \\
\hline Bell, Tom W. & $\begin{array}{l}\text { Fair Use vs. Fared Use: The Impact of Automated Rights Man- } \\
\text { agement on Copyright's Fair Use Doctrine, North Carolina Law } \\
\text { Review, Vol. 76, 1997-1998, S. 557-620 }\end{array}$ \\
\hline $\begin{array}{l}\text { Belsky, Leah / Kahr, Byron / Berkelham- } \\
\text { mer, Max / Benkler, Yochai }\end{array}$ & $\begin{array}{l}\text { Everything In Its Right Place: Social Cooperation and Artist } \\
\text { Compensation, Michigan Telecommunications \& Technology } \\
\text { Law Review, Vol. 17, 2010-2011, S. 1-66 }\end{array}$ \\
\hline Bernault, Carine / Lebois, Audrey & $\begin{array}{l}\text { Peer-to-peer File Sharing and Literary and Artistic Property. A } \\
\text { Feasibility Study regarding a system of compensation for the } \\
\text { exchange of works via the Internet, 2006, engl. Übersetzung } \\
\text { von Smith, Leigh / Palazzetti, Cédric, hrsg. von Grassmuck, Vol- } \\
\text { ker, abrufbar unter: http://privatkopie.net/files/Feasibility- } \\
\text { Study-p2p-acs_Nantes.pdf } \\
\text { (zitiert als Bernault/Lebois, 2006) }\end{array}$ \\
\hline Bethge, Herbert & $\begin{array}{l}\text { Der Grundrechtsstatus privater Rundfunkveranstalter, NVwZ } \\
\text { 1997, S. 1-6 }\end{array}$ \\
\hline $\begin{array}{l}\text { Bhatia, G. Krishan / Gay, Richard C. / Hon- } \\
\text { ey, W. Ross }\end{array}$ & $\begin{array}{l}\text { Windows Into the Future: How Lessons From Hollywood Will } \\
\text { Shape the Music Industry, Journal of Interactive Marketing, Vol. }\end{array}$ \\
\hline
\end{tabular}




\begin{tabular}{|c|c|}
\hline & 17, No. 2,2003, S. $70-80$ \\
\hline $\begin{array}{l}\text { Bhattacharjee, Sudip / Gopal, Ram D. / } \\
\text { Lertwachara, Kaveepan / Marsden, James } \\
\text { R. / Telang, Rahul }\end{array}$ & $\begin{array}{l}\text { The effect of digital sharing technologies on music markets: A } \\
\text { survival analysis of albums on ranking charts, Management } \\
\text { Science, Vol. 53, 2007, S. 1359-1374 } \\
\text { (zitiert als Bhattacharjee et al.) }\end{array}$ \\
\hline $\begin{array}{l}\text { BigChampagne Online Media Measure- } \\
\text { ment }\end{array}$ & $\begin{array}{l}\text { Monitoring and Identifying P2P Media, abrufbar unter: } \\
\text { http://de.scribd.com/doc/98013570/Monitoring-and- } \\
\text { Identifying-P2P-Media }\end{array}$ \\
\hline Blackburn, David & $\begin{array}{l}\text { On-line Piracy and Recorded Music Sales, 2004, abrufbar unter: } \\
\text { http://citeseerx.ist.psu.edu/viewdoc/download?doi=10.1.1.117 } \\
.2922 \& \text { rep=rep1\&type=pdf }\end{array}$ \\
\hline $\begin{array}{l}\text { Blaufus, Kay / Freyer, Thomas / Trinks, } \\
\text { Matthias }\end{array}$ & $\begin{array}{l}\text { Umsatzbesteuerung elektronischer Dienstleistungen, DStR } \\
2011 \text {, S. 2269-2276 }\end{array}$ \\
\hline $\begin{array}{l}\text { Bayerische Landeszentrale für neue Me- } \\
\text { dien (BLM) }\end{array}$ & $\begin{array}{l}\text { BLM-Webradiomonitor 2011, abrufbar unter: } \\
\text { http://de.slideshare.net/goldmedia/webradiomonitor-2011- } \\
\text { blm-und-goldmedia }\end{array}$ \\
\hline $\begin{array}{l}\text { Bayerische Landeszentrale für neue Me- } \\
\text { dien (BLM) }\end{array}$ & $\begin{array}{l}\text { BLM-Webradiomonitor 2012, abrufbar unter: } \\
\text { http://de.slideshare.net/goldmedia/120716-goldmedia- } \\
\text { webradiomonitor-2012-langfassung }\end{array}$ \\
\hline Blanke-Roeser, Constantin & $\begin{array}{l}\text { Three Strikes against the Bay oder freie Fahrt für Netzpiraten? } \\
\text { Zu Lösungsansätzen für die Krise des Urheberrechts im digitalen } \\
\text { Umfeld unter besonderer Berücksichtigung des Konzepts der } \\
\text { Kulturflatrate, in: Stiftung der Hessischen Rechtsanwaltschaft, } \\
\text { Kulturflatrate, Kulturwertmark oder Three Strikes and you are } \\
\text { out: Wie soll mit Kreativität im Internet umgegangen werden?, } \\
\text { S. 29-73, Optimus Verlag, Göttingen } 2013\end{array}$ \\
\hline Böckenförde, Thomas & $\begin{array}{l}\text { Auf dem Weg zur elektronischen Privatsphäre, JZ 2008, S. 925- } \\
939\end{array}$ \\
\hline Bohne, Daniel & $\begin{array}{l}\text { Zum Erfordernis eines gewerblichen Ausmaßes der Rechtsver- } \\
\text { letzung in } \S 101 \text { Abs. } 2 \text { UrhG, CR 2010, S. 104-109 }\end{array}$ \\
\hline Bornkamm, Joachim & $\begin{array}{l}\text { Die Reprographievergütung im digitalen Zeitalter, in: Loewen- } \\
\text { heim, Ulrich (Hrsg.), Urheberrecht im Informationszeitalter, } \\
\text { Festschrift für Wilhelm Nordemann zum 70. Geburtstag am } 8 . \\
\text { Januar 2004, Verlag C.H. Beck, München 2004, S. 299-311 } \\
\text { (zitiert als Bornkamm, in: FS Nordemann) }\end{array}$ \\
\hline Borowski, Martin & $\begin{array}{l}\text { Grundrechte als Prinzipien, 2. Aufl., Nomos-Verlag, Baden- } \\
\text { Baden } 2007\end{array}$ \\
\hline $\begin{array}{l}\text { Börsenverein des Deutschen Buchhandels } \\
\text { e.V. }\end{array}$ & $\begin{array}{l}\text { Illegal aber egal?, 2010, abrufbar unter: } \\
\text { http://www.boersenverein.de/sixcms/media.php/976/Illegal_a } \\
\text { ber_egal.pdf }\end{array}$ \\
\hline $\begin{array}{l}\text { Börsenverein des Deutschen Buchhandels } \\
\text { e.V. }\end{array}$ & $\begin{array}{l}\text { Fragenkatalog zur Kulturflatrate, 23. Juli 2009, abrufbar unter: } \\
\text { http://www.boersenverein.de/sixcms/media.php/976/Fragenk } \\
\text { atalog_Kulturflatrate.pdf }\end{array}$ \\
\hline $\begin{array}{l}\text { Börsenverein des Deutschen Buchhandels } \\
\text { e.V. }\end{array}$ & $\begin{array}{l}\text { Umsatz- und Preisentwicklung, 2011, abrufbar unter: } \\
\text { http://www.boersenverein.de/de/182716 }\end{array}$ \\
\hline Bosman, Wieland & $\begin{array}{l}\text { Paradigmenwechsel in der Rundfunkfinanzierung: Von der } \\
\text { Rundfunkgebühr zum Rundfunkbeitrag, K\&R 2012, S. 5-11 }\end{array}$ \\
\hline $\begin{array}{l}\text { Bounie, David / Bourreau, Marc / } \\
\text { Waelbroeck, Patrick }\end{array}$ & $\begin{array}{l}\text { Pirates or Explorers? Analysis of Music Consumption in French } \\
\text { Graduate Schools, 2005, abrufbar unter: } \\
\text { http://papers.ssrn.com/sol3/Delivery.cfm/SSRN_ID739284_cod } \\
\text { e485818.pdf?abstractid=739284\&mirid=3 } \\
\text { (zitiert als Bounie/Bourreau/Waelbroeck, 2005) }\end{array}$ \\
\hline Brandhorst, Jürgen & $\begin{array}{l}\text { Die Verteilung - Teil I: Allgemeine Informationen, virtuos, März } \\
\text { 2010, S. 55-57 }\end{array}$ \\
\hline Braun, Thorsten & $\begin{array}{l}\text { „Filesharing"-Netze und deutsches Urheberrecht - Zugleich eine } \\
\text { Entgegnung auf Kreutzer, GRUR 2001, } 193 \text { ff. und } 307 \text { ff. -, } \\
\text { GRUR 2001, S. 1106-1111 }\end{array}$ \\
\hline
\end{tabular}




\begin{tabular}{|c|c|}
\hline Bräutigam, Peter & $\begin{array}{l}\text { Das Nutzungsverhältnis bei sozialen Netzwerken. Zivilrechtli- } \\
\text { cher Austausch von IT-Leistung gegen personenbezogene Da- } \\
\text { ten, MMR 2012, S. } 635-641\end{array}$ \\
\hline Brinkel, Guido & $\begin{array}{l}\text { Filesharing. Verantwortlichkeit in Peer-to-Peer- } \\
\text { Tauschplattformen, Mohr Siebeck Verlag, Tübingen } 2006\end{array}$ \\
\hline British Recorded Music Industry (BPI) & $\begin{array}{l}\text { Digital Music Nation } 2010 \text { - The UK's legal and illegal digital } \\
\text { music landscape, abrufbar unter: } \\
\text { http://www.bpi.co.uk/assets/files/Digital\%20Music\%20Nation } \\
\% 202010 . p d f\end{array}$ \\
\hline Brunst, Phillip W. & $\begin{array}{l}\text { Anonymität im Internet - rechtliche und tatsächliche Rahmen- } \\
\text { bedingungen: zum Spannungsfeld zwischen einem Recht auf } \\
\text { Anonymität bei der elektronischen Kommunikation und den } \\
\text { Möglichkeiten zur Identifizierung und Strafverfolgung, Verlag } \\
\text { Duncker \& Humblot, Berlin } 2009\end{array}$ \\
\hline Bundesnetzagentur (BNetzA) & $\begin{array}{l}\text { Jahresbericht 2011, abrufbar unter: } \\
\text { http://www.bundesnetzagentur.de/SharedDocs/Downloads/DE } \\
\text { /BNetzA/Presse/Berichte/2012/Jahresbericht2011pdf.pdf?_bl } \\
\text { ob=publicationFile }\end{array}$ \\
\hline $\begin{array}{l}\text { Bundesverband Audiovisuelle Medien e.V. } \\
\text { (BVV) / Gesellschaft für Konsumforschung } \\
\text { (GfK) }\end{array}$ & $\begin{array}{l}\text { Der deutsche Videomarkt 2011, abrufbar unter: } \\
\text { http://www.bvv-medien.de/index.php?content_id=19 }\end{array}$ \\
\hline $\begin{array}{l}\text { Bundesverband Informationswirtschaft, } \\
\text { Telekommunikation und neue Medien } \\
\text { e.V. (BITKOM) }\end{array}$ & $\begin{array}{l}\text { Studie Netzgesellschaft, 2011, abrufbar unter: } \\
\text { http://www.bitkom.org/files/documents/BITKOM_Publikation_ } \\
\text { Netzgesellschaft.pdf }\end{array}$ \\
\hline Bundesverband Musikindustrie & $\begin{array}{l}\text { Jahreswirtschaftsbericht 2009, Kap. 3, Umsatz, abrufbar unter: } \\
\text { http://www.musikindustrie.de/uploads/media/Kap3_Umsatz.p } \\
\text { df }\end{array}$ \\
\hline Bundesverband Musikindustrie & $\begin{array}{l}\text { Jahreswirtschaftsbericht 2011, abrufbar unter: } \\
\text { http://www.musikindustrie.de/jahrbuch-umsatz-2011/ }\end{array}$ \\
\hline Bundesverband Musikindustrie & $\begin{array}{l}\text { Musik im digitalen Wandel, 2012, abrufbar unter: } \\
\text { http://www.musikindustrie.de/fileadmin/news/publikationen/ } \\
\text { Kompendium_Musik_im_digitalen_Wandel_FINAL.pdf }\end{array}$ \\
\hline Bundesverband Musikindustrie & $\begin{array}{l}\text { Musikindustrie in Zahlen 2009, abrufbar unter: } \\
\text { http://www.musikindustrie.de/uploads/media/MiZ_2009_gesa } \\
\text { mt_01.pdf }\end{array}$ \\
\hline Bundesverband Musikindustrie & $\begin{array}{l}\text { Musikindustrie in Zahlen 2010, abrufbar unter: } \\
\text { http://www.musikindustrie.de/uploads/media/BVMI-Jahrbuch- } \\
\text { 2010_02.pdf }\end{array}$ \\
\hline Bundesverband Musikindustrie & $\begin{array}{l}\text { Positionspapier zur Kulturflatrate v. 25.1.2010, abrufbar unter: } \\
\text { http://www.musikindustrie.de/fileadmin/news/presse/100125 } \\
\text { _Kulturflatrate_10_Argumente_FINAL.pdf }\end{array}$ \\
\hline Chaos-Computer-Club (CCC) & $\begin{array}{l}\text { Ein Vorschlag zur Güte - die Kulturwertmark, 26.4.2011, abruf- } \\
\text { bar unter: } \\
\text { http://ccc.de/system/uploads/65/original/kulturwertmark- } \\
\text { neu.pdf }\end{array}$ \\
\hline $\begin{array}{l}\text { Chatziathanasiou, Konstantin / Hartmann, } \\
\text { Constantin }\end{array}$ & $\begin{array}{l}\text { Gallisches Dorf „Kulturflatrate“? Zur Vereinbarkeit einer deut- } \\
\text { schen Reform mit internationalem Recht, in: Stiftung der Hes- } \\
\text { sischen Rechtsanwaltschaft, Kulturflatrate, Kulturwertmark } \\
\text { oder Three Strikes and you are out: Wie soll mit Kreativität im } \\
\text { Internet umgegangen werden?, S. 153-176, Optimus Verlag, } \\
\text { Göttingen } 2013\end{array}$ \\
\hline Chen, Yeh-ning / Png, Ivan & $\begin{array}{l}\text { Information Goods Pricing and Copyright Enforcement: Welfare } \\
\text { Analysis, Information Systems Research, Vol. 14, No. 1, March } \\
\text { 2003, S. } 107-123\end{array}$ \\
\hline Cremer, Wolfram & $\begin{array}{l}\text { Freiheitsgrundrechte. Funktionen und Strukturen, Mohr- } \\
\text { Siebeck Verlag, Tübingen } 2003\end{array}$ \\
\hline Cremer, Wolfram & Gewinnstreben als öffentliche Unternehmen legitimierender \\
\hline
\end{tabular}




\begin{tabular}{|c|c|}
\hline & Zweck: Die Antwort des Grundgesetzes, DÖV 2003, S. 921-932 \\
\hline $\begin{array}{l}\text { Cuevas, Ruben / Kryczka, Michal / Cuevas, } \\
\text { Angel / Kaune, Sebastian / Guerrero, } \\
\text { Carmen / Rejaie, Reza }\end{array}$ & $\begin{array}{l}\text { Is Content Publishing in BitTorrent Altruistic or Profit-Driven?, } \\
\text { 2010, abrufbar unter: http://conferences.sigcomm.org/co- } \\
\text { next/2010/CoNEXT_papers/11-Cuevas.pdf } \\
\text { (zitiert als Cuevas et al., 2010) }\end{array}$ \\
\hline Czajka, Sebastian & $\begin{array}{l}\text { Internetnutzung in privaten Haushalten in Deutschland, Statisti- } \\
\text { sches Bundesamt, Wirtschaft und Statistik, August } 2011 \text { S. 709- } \\
\text { 717, abrufbar unter: } \\
\text { https://www.destatis.de/DE/Publikationen/WirtschaftStatistik/ } \\
\text { Informationsgesell- } \\
\text { schaft/InternetnutzungHaushalte_82011.pdf?_blob=publicatio } \\
\text { nFile }\end{array}$ \\
\hline $\begin{array}{l}\text { Czychowski, Christian / Nordemann, Jan } \\
\text { Bernd }\end{array}$ & $\begin{array}{l}\text { Die Entwicklung der unter- und obergerichtlichen Rechtspre- } \\
\text { chung zum Urheberrecht in den Jahren } 2010 \text { und } 2011 \text { (Teil II), } \\
\text { GRUR-RR 2012, S. 233-242 }\end{array}$ \\
\hline $\begin{array}{l}\text { Czychowski, Christian / Nordemann, Jan } \\
\text { Bernd }\end{array}$ & $\begin{array}{l}\text { Vorratsdaten und Urheberrecht - Zulässige Nutzung gespei- } \\
\text { cherter Daten, NJW 2008, S. 3095-3099 }\end{array}$ \\
\hline Czychowski, Christian & $\begin{array}{l}\text { Auskunftsansprüche gegenüber Internetzugangsprovidern } \\
\text { „vor" dem 2. Korb und „,nach" der Enforcement-Richtlinie der } \\
\text { EU, MMR 2004, S. 514-519 }\end{array}$ \\
\hline $\begin{array}{l}\text { Danaher, Brett / Smith, Michael D. / Te- } \\
\text { lang, Rahul / Chen, Siwen }\end{array}$ & $\begin{array}{l}\text { The Effect on Graduated Response Anti-Piracy Laws on Music } \\
\text { Sales: Evidence from an Event Study in France, 2012, abrufbar } \\
\text { unter: } \\
\text { http://papers.ssrn.com/sol3/papers.cfm?abstract_id=1989240 } \\
\text { (zitiert als Danaher et al., 2012) }\end{array}$ \\
\hline Day, John D. / Zimmermann, Hubert & $\begin{array}{l}\text { The OSI Reference Model, Proceedings of the IEEE, Vol. 71, } \\
\text { 1983, S. 1334-1340 }\end{array}$ \\
\hline $\begin{array}{l}\text { Dialog Consult GmbH / Verband der An- } \\
\text { bieter von Telekommunikations- und } \\
\text { Mehrwertdiensten (VATM) }\end{array}$ & $\begin{array}{l}\text { 14. Marktstudie zum Telekommunikationsmarkt Deutschland } \\
\text { 2012, Dialog Consult-Newsletter, Nr. 2/2012, abrufbar unter: } \\
\text { http://www.dialog-consult.com/DCNL/PDF/DCNL032.PDF }\end{array}$ \\
\hline Dieselhorst, Jochen & $\begin{array}{l}\text { Anwendbares Recht bei Internationalen Online-Diensten, ZUM } \\
\text { 1998, S. 293-300 }\end{array}$ \\
\hline $\begin{array}{l}\text { Djordjevic, Valentina / Gehring, Robert A. } \\
\text { / Grassmuck, Volker / Kreutzer, Till / Otto, } \\
\text { Philipp / Spielkamp, Matthias }\end{array}$ & $\begin{array}{l}\text { Arbeit } 2.0 \text { Urheberrecht und kreatives Schaffen in der digitalen } \\
\text { Welt, 2009, abrufbar unter: http://edoc.hu- } \\
\text { berlin.de/oa/books/reSC4iUMGjll/PDF/23SOEj46HKHDU.pdf } \\
\text { (zitiert als Djordjevic et al., 2009) }\end{array}$ \\
\hline Dowell, Jonathan & $\begin{array}{l}\text { Bytes and Pieces: Fragmented Copies, Licensing, and Fair Use in } \\
\text { a Digital World, California Law Review, Vol. 86, 1998, S. 843-878 }\end{array}$ \\
\hline Dreier, Horst (Hrsg.) & $\begin{array}{l}\text { Grundgesetz: Kommentar, Band I, 3. Aufl., Verlag Mohr Siebeck, } \\
\text { Tübingen } 2013 \\
\text { (zitiert als Dreier, GG) }\end{array}$ \\
\hline Dreier, Thomas / Schulze, Gernot (Hrsg.) & $\begin{array}{l}\text { Urheberrechtsgesetz. Urheberrechtswahrnehmungsgesetz, } \\
\text { Kunsturhebergesetz, 4. Aufl., Verlag C.H. Beck, München } 2013 \\
\text { (zitiert als Dreier/Schulze, UrhG) }\end{array}$ \\
\hline Dreier, Thomas & $\begin{array}{l}\text { Padawan und die Folgen für die deutsche Kopiervergütung, } \\
\text { ZUM 2011, S. 281-291 }\end{array}$ \\
\hline $\begin{array}{l}\text { Dreyer, Gunda / Kotthoff, Jost / Meckel, } \\
\text { Astrid (Hrsg.) }\end{array}$ & $\begin{array}{l}\text { Urheberrecht. Urheberrechtsgesetz, Urheberrechtswahrneh- } \\
\text { mungsgesetz, Kunsturhebergesetz, 2. Aufl., Verlag C.F. Müller, } \\
\text { Heidelberg } 2009 \\
\text { (zitiert als Dreyer/Kotthoff/Meckel, UrhR) }\end{array}$ \\
\hline Elberse, Anita & $\begin{array}{l}\text { Bye-Bye Bundles:The Unbundling of Music in Digital Channels } \\
\text { Journal of Marketing, Vol. } 74 \text { (May 2010), S. } 108-123\end{array}$ \\
\hline Enders, Theodor & $\begin{array}{l}\text { Digital Rights Management Systeme (DRMS) als besondere } \\
\text { Herausforderung an das Urheberrecht, ZUM 2004, S. 593-605 }\end{array}$ \\
\hline $\begin{array}{l}\text { Epping, Volker / Hillgruber, Christian } \\
\text { (Hrsg.) }\end{array}$ & $\begin{array}{l}\text { Beck'scher Online-Kommentar GG, Stand: 1.10.2012, Verlag } \\
\text { C.H. Beck } \\
\text { (zitiert als Epping/Hillgruber, BeckOK GG) }\end{array}$ \\
\hline
\end{tabular}




\begin{tabular}{|c|c|}
\hline Erbel, Günter & $\begin{array}{l}\text { Inhalt und Auswirkungen der verfassungsrechtlichen Kunstfrei- } \\
\text { heitsgarantie, Springer Verlag, Berlin } 1966\end{array}$ \\
\hline Erdmann, Gerald / Stanek, Charlotte & $\begin{array}{l}\text { Der iTunes Store für Musiker, 2. Aufl. 2006, abrufbar unter: } \\
\text { www.ipodbuch.de/bonusmaterial/iTunesStore-fuer- } \\
\text { Musiker.pdf }\end{array}$ \\
\hline Fechner, Frank & $\begin{array}{l}\text { Geistiges Eigentum und Verfassung, Mohr Siebeck, Tübingen, } \\
2007 \\
\text { (zitiert als Fechner, 2007) }\end{array}$ \\
\hline $\begin{array}{l}\text { Federrath, Hannes / Golembiewski, Clau- } \\
\text { dia }\end{array}$ & $\begin{array}{l}\text { Speicherung von Nutzungsdaten durch Anonymisierungsdienste } \\
\text { im Internet, DuD 2004, S. 486-490 }\end{array}$ \\
\hline Federrath, Hannes & Gateway, DuD 2003, S. 169 \\
\hline Ferguson, David & $\begin{array}{l}\text { Trends and Statistivs in Peer-to-Peer, Workshop on Technical } \\
\text { and legal aspects of peer-to-peer television, 2006, abrufbar } \\
\text { unter: } \\
\text { http://www.noticiasdot.com/publicaciones/2006/0406/1804/n } \\
\text { oti- } \\
\text { cias/images/CacheLogic_AmsterdamWorkshop_Presentation_v } \\
\text { 1.0.ppt }\end{array}$ \\
\hline Fisher III, William W. & $\begin{array}{l}\text { Promises to Keep. Technology, Law, and the Future of Enter- } \\
\text { tainment, Stanford University Press, Stanford, California } 2004 \\
\text { (zitiert als Fisher, 2004) }\end{array}$ \\
\hline Fitzner, Julia & $\begin{array}{l}\text { Von Digital-Rights-Management zu Content Identification, No- } \\
\text { mos Verlag, Baden-Baden } 2011\end{array}$ \\
\hline Formatt-Institut & $\begin{array}{l}\text { Film- und Fernsehproduktion in Nordrhein-Westfalen im Ver- } \\
\text { gleich zu anderen Bundesländern } 2009 \text { und 2010, Juni 2012, } \\
\text { abrufbar unter: } \\
\text { http://www.mbem.nrw.de/web/media_get.php?mediaid=2472 } \\
\text { 4\&fileid=78336\&sprachid=1 }\end{array}$ \\
\hline Frank, Christian & Urheberabgaben nach Padawan, CR 2011, S. 1-6 \\
\hline Frevert, Tobias & $\begin{array}{l}\text { Netzneutralität 2012. Aktueller Stand der Gesetzgebung, MMR } \\
2012 \text {, S. 510-515 }\end{array}$ \\
\hline $\begin{array}{l}\text { Fromm, Friedrich Karl / Nordemann, Wil- } \\
\text { helm (Hrsg.) }\end{array}$ & $\begin{array}{l}\text { Urheberrecht. Kommentar zum Urheberrechtsgesetz, Verlags- } \\
\text { gesetz, Urheberrechtswahrnehmungsgesetz, 10. Aufl., Kohl- } \\
\text { hammer Verlag, Stuttgart } 2008 \\
\text { (zitiert als Fromm/Nordemann, UrhR) }\end{array}$ \\
\hline Galwass, Hans-Ullrich & $\begin{array}{l}\text { Faktische Beeinträchtigungen im Bereich der Grundrechte, } \\
\text { Duncker \& Humblot, Berlin } 1970\end{array}$ \\
\hline $\begin{array}{l}\text { Garland, Eric / BigChampagne Online } \\
\text { Media Measurement }\end{array}$ & $\begin{array}{l}\text { From Napster to Now: File Sharing Trends, Präsentation bei } \\
\text { International Association of Music Business Research, Vienna } \\
\text { Music Business Research Days, 2010, abrufbar unter: } \\
\text { http://musikwirtschaftsforschung.files.wordpress.com/2010/06 } \\
\text { /eric_garland_vienna_music_business_research_end- } \\
\text { revised.pdf }\end{array}$ \\
\hline $\begin{array}{l}\text { Geiger, Christophe / Griffiths, Jonathan / } \\
\text { Hilty, Reto }\end{array}$ & $\begin{array}{l}\text { Erklärung für eine ausgewogene Auslegung des Drei-Stufen- } \\
\text { Tests im Urheberrecht, GRUR Int. 2008, S. 822-825 }\end{array}$ \\
\hline Gesellschaft für Konsumforschung (GfK) & $\begin{array}{l}\text { DCN-Studie 2011, Presseversion, abrufbar unter: } \\
\text { http://www.musikindustrie.de/uploads/media/DCN- } \\
\text { Studie_2011_Presseversion_FINAL_02.pdf }\end{array}$ \\
\hline Gesellschaft für Konsumforschung (GfK) & DCN-Studie 2012, Vollversion \\
\hline Gesellschaft für Konsumforschung (GfK) & $\begin{array}{l}\text { Pressemitteilung vom 3.7.2009, abrufbar unter: } \\
\text { http://www.gfk.com/imperia/md/content/presse/pm_tc_score } \\
\text { _uli_2009_dfin.pdf }\end{array}$ \\
\hline Gesellschaft für Konsumforschung (GfK) & $\begin{array}{l}\text { Verbraucherpanel Media*Scope Buch, Durchschnittspreis von } \\
\text { E-Books in Deutschland im Jahr } 2010 \text { und 2011, abrufbar unter: } \\
\text { http://de.statista.com/statistik/daten/studie/232200/umfrage/ } \\
\text { durchschnittspreis-von-e-books-in-deutschland/ }\end{array}$ \\
\hline Gesellschaft für musikalische Auffüh- & Geschäftsbericht 2011, abrufbar unter: \\
\hline
\end{tabular}


rungs- und mechanische Vervielfältigungsrechte (GEMA)

Gesellschaft für musikalische Aufführungs- und mechanische Vervielfältigungsrechte (GEMA)

Gesellschaft für musikalische Aufführungs- und mechanische Vervielfältigungsrechte (GEMA)

Gesellschaft für musikalische Aufführungs- und mechanische Vervielfältigungsrechte (GEMA)
https://www.gema.de/fileadmin/user_upload/Presse/Publikati onen/Geschaeftsbericht/geschaeftsbericht_2011.pdf

INKA FAQs, Fragen und Antworten zur „Inkassobezogenen Abrechnung im Bereich U-Musik“ (INKA) (Antrag 28 zur Mitgliederversammlung 2012), 2012, abrufbar unter: https://www.gema.de/fileadmin/user_upload/Musikurheber/ Mitgliederbereich/MGV_2012/INKA_FAQs.pdf (zitiert als GEMA, INKA FAQs, 2012)

Jahrbuch 2011/2012, abrufbar unter:

https://www.gema.de/fileadmin/user_upload/Presse/Publikati onen/Jahrbuch/gema_jahrbuch_2011-12.pdf

Präsentation INKA, Inkassobezogene Abrechnung im Bereich UMusik („Alternatives Abrechnungsmodell U“), 2012, abrufbar unter:

https://www.gema.de/fileadmin/user_upload/Musikurheber/ Mitgliederbereich/MGV_2012/12_06_13_INKA-

Prasentation.pdf

(zitiert als GEMA, Präsentation INKA, 2012)

Gesellschaft für musikalische Aufführungs- und mechanische Vervielfältigungsrechte (GEMA) Gesellschaft zur Verfolgung von Urheberrechtsverletzungen e.V. (GVU)

Tagesordnung Mitgliederversammlung 2012, abrufbar unter: https://www.gema.de/fileadmin/user_upload/Musikurheber/ Mitgliederbereich/MGV_2012/Tagesordnung_MGV_2012.pdf Pressemitteilung vom 26.11.2012, abrufbar unter: http://www.gvu.de/25_207_Kein_negativer_Effekt_fuer_Kinos _durch_Schliessung_von_Megaupload_in_Deutschland_Besuch szah-

len_von_Filmen_unter_200_Kopien_in_2012_gegenueber_Vorj ahren_sogar_gestiegen.htm

Geuer, Ermano „Eine Wohnung, ein Beitrag." - Überlegungen zur Popularklage gegen die neuen Rundfunkbeiträge, MMR-Aktuell 2012, 335995

Gola, Peter / Klug, Christoph / Reif, Yvette Datenschutz- und presserechtliche Bewertung der „Vorratsdatenspeicherung", NJW 2007, S. 2599-2602

\begin{tabular}{|l|l|}
\hline Görisch, Christoph & $\begin{array}{l}\text { Netzneutralität - ein Grundsatz des europäischen Regulierungs- } \\
\text { rechts?, EuZW 2012, S. 494-498 }\end{array}$ \\
\hline Grabenwarter, Christoph (Hrsg.) & $\begin{array}{l}\text { Allgemeinheit der Grundrechte und Vielfalt der Gesellschaft. } \\
\text { 34. Tagung der Wissenschaftlichen Mitarbeiterinnen und Mit- } \\
\text { arbeiter der Fachrichtung "Öffentliches Recht", Wien 1994, } \\
\text { Boorberg Verlag, Stuttgart 1994 }\end{array}$ \\
\hline Grassmuck, Volker & $\begin{array}{l}\text { Academic Studies on the Effect of File-Sharing on the Recorded } \\
\text { Music Industry: A Literature Overview, 2010, abrufbar unter: } \\
\text { http://papers.ssrn.com/sol3/Delivery.cfm/SSRN_ID1749579_co } \\
\text { de1607300.pdf?abstractid=1749579\&mirid=3 } \\
\text { (zitiert als Grassmuck, 2010) }\end{array}$ \\
\hline Grassmuck, Volker & $\begin{array}{l}\text { Ein Plädoyer für durchsetzbare Schrankenbestimmungen für } \\
\text { Privatkopie, Zitat und Filesharing, ZUM 2005, 104-109 }\end{array}$ \\
\hline Grassmuck, Volker & $\begin{array}{l}\text { Erwiderung auf das Musikindustrie-Positionspapier zur Kultur- } \\
\text { flatrate, 10.02.2010, abrufbar unter: } \\
\text { https://netzpolitik.org/2010/erwiderung-auf-das- } \\
\text { musikindustrie-positionspapier-zur-kulturflatrate/ }\end{array}$ \\
\hline Grassmuck, Volker & $\begin{array}{l}\text { The World is Going Flat(-Rate), 2009, abrufbar unter: } \\
\text { http://waste.informatik.hu-berlin.de/Grassmuck/Texts/09-05- } \\
\text { 11_World_is_going_Flatrate_IP-Watch.pdf } \\
\text { (zitiert als Grassmuck, 2009) }\end{array}$ \\
\hline Graulich, Kurt & $\begin{array}{l}\text { Vorschlag zum Besseren - die Tauschlizenz, 2011, abrufbar } \\
\text { unter: http://www.vgrass.de/wp- } \\
\text { content/uploads/2011/10/Kulturwertmark-Replik_v1.0.pdf } \\
\text { (zitiert als Grassmuck, 2011) }\end{array}$ \\
\hline Telekommunikationsgesetz und Vorratsdatenspeicherung, \\
\hline
\end{tabular}




\begin{tabular}{|c|c|}
\hline & NVwZ 2008, 485-492 \\
\hline Grzeszick, Bernd & Geistiges Eigentum und Art. 14 GG, ZUM 2007, S. 344-353 \\
\hline Guggemos, Werner-Christian & $\begin{array}{l}\text { Digital Rights Management im praktischen Einsatz, ZUM 2004, } \\
\text { S. 183-188 }\end{array}$ \\
\hline Hahn, Werner / Vesting, Thomas (Hrsg.) & $\begin{array}{l}\text { Beck'scher Kommentar zum Rundfunkrecht, 3. Aufl., Verlag C.H. } \\
\text { Beck } 2012 \\
\text { (zitiert als Hahn/Vesting, Rundfunkrecht) }\end{array}$ \\
\hline Hammond, Robert G. & $\begin{array}{l}\text { Profit Leak? Pre-Release File Sharing and the Music Industry, } \\
\text { 2012, abrufbar unter: } \\
\text { http://papers.ssrn.com/sol3/papers.cfm ?abstract_id=2059356 } \\
\text { (zitiert als Hammond, 2012) }\end{array}$ \\
\hline Hansen, Markus & DRM-Desaster: Das Sony BMG-Rootkit, DuD 2006, S. 95-97 \\
\hline Hargreaves, Ian & $\begin{array}{l}\text { Digital Opportunity. A Review of Intellectual Property and } \\
\text { Growth, 2011, abrufbar unter: } \\
\text { http://www.ipo.gov.uk/ipreview-finalreport.pdf } \\
\text { (zitiert als Hargreaves, Digital Opportunity, 2011) }\end{array}$ \\
\hline Hargreaves, Ian & $\begin{array}{l}\text { Digital Opportunity. A Review of Intellectual Property and } \\
\text { Growth, Supporting Document CC, Data on the Prevalence and } \\
\text { Impact of Piracy and Counterfeiting, 2011, abrufbar unter: } \\
\text { http://www.ipo.gov.uk/ipreview-doc-cc.pdf }\end{array}$ \\
\hline Härting, Niko & Internetrecht, 4. Aufl., Schmidt Verlag, Köln 2010 \\
\hline $\begin{array}{l}\text { Haute Autorité pour la diffusion des } \\
\text { oeuvres et la protection des droits sur } \\
\text { I'Internet (HADOPI) }\end{array}$ & $\begin{array}{l}\text { Hadopi, } 11 / 2 \text { year after the launch, 2012, abrufbar unter: } \\
\text { http://de.scribd.com/doc/87387866/Hadopi-Report } \\
\text { (zitiert als HADOPI, 2012) }\end{array}$ \\
\hline $\begin{array}{l}\text { Haute Autorité pour la diffusion des } \\
\text { oeuvres et la protection des droits sur } \\
\text { l'Internet (HADOPI) }\end{array}$ & $\begin{array}{l}\text { Hadopi, cultural assets and internet use: practices and percep- } \\
\text { tions of French internet users, 2011, abrufbar unter: } \\
\text { http://www.hadopi.fr/download/HADOPI_T0_version_long.pdf }\end{array}$ \\
\hline He, Huaiwen & $\begin{array}{l}\text { Seeking a Balanced Interpretation of the Three-Step Test - An } \\
\text { Adjusted Structure in View of Divergent Approaches, IIC 2009, } \\
\text { S. 274-308 }\end{array}$ \\
\hline Heckmann, Dirk & $\begin{array}{l}\text { Geltungskraft und Geltungsverlust von Rechtsnormen. Elemen- } \\
\text { te einer Theorie der autoritativen Normgeltungsbeendigung, } \\
\text { Verlag Mohr Siebeck, Tübingen } 1997\end{array}$ \\
\hline $\begin{array}{l}\text { Heinemeyer, Dennis / Kreitlow, Matthias } \\
\text { / Nordmeyer, Arne / Sabellek, André }\end{array}$ & $\begin{array}{l}\text { Kampf gegen Filesharing als Modell verfehlter Mehrfachkom- } \\
\text { pensation? Fragen zur Schadenshöhe, zu Gesamtschuldnern } \\
\text { und Beweisen bei Tauschbörsen, MMR 2012, S. 279-284 }\end{array}$ \\
\hline Hendricks, Ken / Sorensen, Alan & $\begin{array}{l}\text { Information and the Skewness of Music Sales, Journal of Politi- } \\
\text { cal Economy, Vol. 117, No. 2, 2009, S. 324-369 }\end{array}$ \\
\hline Hennemann, Moritz & $\begin{array}{l}\text { Urheberrechtsdurchsetzung und Internet, Nomos Verlag, Ba- } \\
\text { den-Baden } 2011\end{array}$ \\
\hline Heun, Werner & $\begin{array}{l}\text { Funktionell-rechtliche Schranken der Verfassungsgerichtsbar- } \\
\text { keit, Nomos Verlag, Baden-Baden, } 1992\end{array}$ \\
\hline $\begin{array}{l}\text { Hoeren, Thomas / Sieber, Ulrich / Holzna- } \\
\text { gel, Bernd (Hrsg.) }\end{array}$ & $\begin{array}{l}\text { Handbuch Multimedia-Recht, Stand: 32. EL, Verlag C.H. Beck, } \\
\text { München } 2012 \\
\text { (zitiert als Hoeren/Sieber, Multimedia-Recht) }\end{array}$ \\
\hline Hoeren, Thomas & $\begin{array}{l}\text { Anonymität im Web - Grundfragen und aktuelle Entwicklun- } \\
\text { gen, ZRP 2010, S. 251-253 }\end{array}$ \\
\hline Hoeren, Thomas & $\begin{array}{l}\text { Der Zweite Korb - Eine Übersicht zu den geplanten Änderungen } \\
\text { im Urheberrechtsgesetz, MMR 2007, S. 615-620 }\end{array}$ \\
\hline Hoeren, Thomas & $\begin{array}{l}\text { Geolokalisation und Glücksspielrecht (Teil 2), ZfWG 2008, S. } \\
311-315\end{array}$ \\
\hline Hoeren, Thomas & $\begin{array}{l}\text { Zoning und Geolocation - Technische Ansätze zu einer Reterri- } \\
\text { torialisierung des Internet, MMR 2007, S. 3-6 }\end{array}$ \\
\hline Hohagen, Gisbert & $\begin{array}{l}\text { Die Freiheit der Vervielfältigung zum eigenen Gebrauch, Verlag } \\
\text { C.H. Beck, München, } 2004\end{array}$ \\
\hline Hong, Seung-Hyun & $\begin{array}{l}\text { Measuring the Effect of Napster on Recorded Music Sales: Dif- } \\
\text { ference-in-differences Estimates under Compositional Changes, }\end{array}$ \\
\hline
\end{tabular}




\begin{tabular}{|c|c|}
\hline & $\begin{array}{l}\text { 2011, abrufbar unter: } \\
\text { http://netfiles.uiuc.edu/hyunhong/www/napster.pdf } \\
\text { (zitiert als Hong, 2011) }\end{array}$ \\
\hline $\begin{array}{l}\text { Huygen, Annelies / Rutten, Paul / Hu- } \\
\text { veneers, Sanne / Limonard, Sander / } \\
\text { Poort, Joost / Leenheer, Jorna / Janssen, } \\
\text { Kieja / van Eijk, Nico / Helberger, Natali }\end{array}$ & $\begin{array}{l}\text { Ups and downs. Economic and cultural effects of file sharing on } \\
\text { music, film and games, 2009, abrufbar unter: } \\
\text { http://www.ivir.nl/publicaties/vaneijk/Ups_And_Downs_autho } \\
\text { rised_translation.pdf } \\
\text { (zitiert als Huygen et al., 2009) }\end{array}$ \\
\hline $\begin{array}{l}\text { International Federation of the Phono- } \\
\text { graphic Industry (IFPI) }\end{array}$ & $\begin{array}{l}\text { Digital Music Report 2009, abrufbar unter: } \\
\text { http://www.ifpi.org/content/library/dmr2009.pdf }\end{array}$ \\
\hline $\begin{array}{l}\text { International Federation of the Phono- } \\
\text { graphic Industry (IFPI) }\end{array}$ & $\begin{array}{l}\text { Digital Music Report 2010, abrufbar unter: } \\
\text { http://www.ifpi.org/content/library/dmr2010.pdf }\end{array}$ \\
\hline $\begin{array}{l}\text { International Federation of the Phono- } \\
\text { graphic Industry (IFPI) }\end{array}$ & $\begin{array}{l}\text { Digital Music Report 2012, abrufbar unter: } \\
\text { http://www.ifpi.org/content/library/DMR2012.pdf }\end{array}$ \\
\hline IPSOS & $\begin{array}{l}\text { Digital \& Physical Piracy in GB, 2007, abrufbar unter: } \\
\text { http://industry.bfi.org.uk/media/pdf/g/m/Ipsos_Piracy_UK_20 } \\
\text { 07.pdf }\end{array}$ \\
\hline Isensee, Josef / Kirchhof, Paul (Hrsg.) & $\begin{array}{l}\text { Handbuch des Staatsrechts, Bd. IV, 3. Aufl., Verlag C.F. Müller, } \\
\text { Heidelberg } 2006\end{array}$ \\
\hline Isensee, Josef & $\begin{array}{l}\text { Menschenwürde - die säkulare Gesellschaft auf der Suche nach } \\
\text { dem Absoluten, AöR } 131 \text { (2006), S. 173-218 }\end{array}$ \\
\hline Jani, Ole / Ebbinghaus, Amélie & $\begin{array}{l}\text { "Gerechter“ Ausgleich für Privatkopien: Tarif-Festsetzung nach } \\
\text { dem „Padawan“-Urteil des EuGH, GRUR-Prax 2011, S. 71-74 }\end{array}$ \\
\hline Joecks, Holger / Miebach, Wulf (Hrsg.) & $\begin{array}{l}\text { Münchener Kommentar zum StGB, 2. Aufl., Verlag C.H. Beck, } \\
\text { München } 2012\end{array}$ \\
\hline Johansson, Daniel / Larsson, Markus & $\begin{array}{l}\text { The Swedish Music Industry in Graphs. Economic Development } \\
\text { Report 2000-2008, 2009, abrufbar unter: } \\
\text { http://ec.europa.eu/avpolicy/docs/other_actions/col_2009/pu } \\
\text { b/kth_annex.pdf } \\
\text { (zitiert als Johansson/Larsson, 2009) }\end{array}$ \\
\hline Katzenberger, Paul & $\begin{array}{l}\text { Urheberrechtsfragen der elektronischen Textkommunikation, } \\
\text { GRUR Int. 1983, S. 895-919 }\end{array}$ \\
\hline $\begin{array}{l}\text { Kerner, Hans-Jürgen / Bott, Klaus / Reich, } \\
\text { Kerstin }\end{array}$ & $\begin{array}{l}\text { Die Entwicklung von Kriminalitätsvorstellungen bei jungen } \\
\text { Menschen, in: Feltes, Thomas / Pfeiffer, Christian / Steinhilper, } \\
\text { Gernot (Hrsg.), Kriminalpolitik und ihre wissenschaftlichen } \\
\text { Grundlagen, Festschrift für Professor Dr. Hans-Dieter Schwind } \\
\text { zum 70. Geburtstag, S. 963-993, Verlag C.F. Müller, Heidelberg, } \\
\text { München, Landsberg, Berlin 2006 } \\
\text { (zitiert als Kerner/Bott/Reich, in: FS Schwind) }\end{array}$ \\
\hline Kilian, Wolfgang / Heussen, Benno (Hrsg.) & $\begin{array}{l}\text { Computerrechts-Handbuch, Stand: 31. EL, Verlag C.H. Beck, } \\
\text { München } 2012\end{array}$ \\
\hline Kilian, Wolfgang & $\begin{array}{l}\text { Entwicklungsgeschichte und Perspektiven des Rechtsschutzes } \\
\text { von Computersoftware in Europa, GRUR Int. 2011, S. 895-901 }\end{array}$ \\
\hline Kirchberg, Elena & $\begin{array}{l}\text { Die Störerhaftung von Internetanschlussinhabern auf dem } \\
\text { Prüfstand, ZUM 2012, S. 544-550 }\end{array}$ \\
\hline Klimanek, Sophia & $\begin{array}{l}\text { Kulturflatrate. Frieden für die Copyright Wars?, in: Stiftung der } \\
\text { Hessischen Rechtsanwaltschaft, Kulturflatrate, Kulturwertmark } \\
\text { oder Three Strikes and you are out: Wie soll mit Kreativität im } \\
\text { Internet umgegangen werden?, S. 75-114, Optimus Verlag, } \\
\text { Göttingen } 2013\end{array}$ \\
\hline Knies, Wolfgang & $\begin{array}{l}\text { Schranken der Kunstfreiheit als verfassungsrechtliches Prob- } \\
\text { lem, Verlag C.H. Beck, München } 1967\end{array}$ \\
\hline Knights, Roger & $\begin{array}{l}\text { Limitations and Exceptions Under the „Three-Step-Test“ and in } \\
\text { National Legislation - Differences Between the Analog and Digi- } \\
\text { tal Environment, WIPO/CR/MOW/01/2, 2001, abrufbar unter: } \\
\text { http://www.wipo.int/edocs/mdocs/copyright/en/wipo_cr_mo } \\
\text { w_01/wipo_cr_mow_01_2.pdf }\end{array}$ \\
\hline
\end{tabular}




\begin{tabular}{|c|c|}
\hline & (zitiert als Knights, 2001) \\
\hline Koch, Franz & $\begin{array}{l}\text { Internationale Gerichtszuständigkeit und Internet, CR 1999, S. } \\
121-129\end{array}$ \\
\hline Köhntopp, Marit / Köhntopp, Christian & Datenspuren im Internet, CR 2000, S. 248-257 \\
\hline $\begin{array}{l}\text { Kreile, Reinhold / Becker, Jürgen / Rie- } \\
\text { senhuber, Karl (Hrsg.) }\end{array}$ & $\begin{array}{l}\text { Recht und Praxis der GEMA. Handbuch und Kommentar, } 2 . \\
\text { Aufl., Verlag de Gruyter Recht, Berlin } 2008\end{array}$ \\
\hline Kreile, Reinhold & $\begin{array}{l}\text { Einnahme und Verteilung der gesetzlichen Geräte- und Leerkas- } \\
\text { settenvergütung für private Vervielfältigung in Deutschland, } \\
\text { GRUR Int. 1992, S. 24-36 }\end{array}$ \\
\hline Kretschmer, Martin & $\begin{array}{l}\text { Private Copying and Fair Compensation: An Empirical Study of } \\
\text { Copyright Levies in Europe, 2011, abrufbar unter: } \\
\text { http://papers.ssrn.com/sol3/Delivery.cfm/SSRN_ID2124942_co } \\
\text { de1042378.pdf?abstractid=2063809\&mirid=3 } \\
\text { (zitiert als Kretschmer, 2011) }\end{array}$ \\
\hline Krüger, Christof & $\begin{array}{l}\text { Anpassung der Höhe der Urhebervergütung für die Privatkopie } \\
\text { durch einen neuen § } 54 \text { III } 1 \text { UrhG?, GRUR 2005, S. 206-209 }\end{array}$ \\
\hline Kühn, Ulrich & $\begin{array}{l}\text { Geolokalisierung mit anonymisierten IP-Adressen, DuD 2009, S. } \\
747-751\end{array}$ \\
\hline Kur, Annette & $\begin{array}{l}\text { Haftung für Rechtsverletzungen Dritter: Reformbedarf im euro- } \\
\text { päischen IPR?, WRP 2011, S. 971-982 }\end{array}$ \\
\hline Landes, William M. / Posner, Richard A. & $\begin{array}{l}\text { The Economic Structure of Intellectual Property Law, The Belk- } \\
\text { nap Press of Harvard Univ. Press, Cambridge } 2003\end{array}$ \\
\hline Langston, Marc / Tyler, James & $\begin{array}{l}\text { Linking to journal articles in an online teaching environment: } \\
\text { The persistent link, DOI, and OpenURL, Internet and Higher } \\
\text { Education, Vol. 7, 2004, S. 51-58 }\end{array}$ \\
\hline Laufhütte, Friedrich Wilhelm (Hrsg.), & $\begin{array}{l}\text { Strafgesetzbuch. Leipziger Kommentar, 12. Aufl., Verlag de } \\
\text { Gruyter Recht, Berlin } 2010\end{array}$ \\
\hline Leeb, Leonhard & $\begin{array}{l}\text { Der Wert künstlerischer Arbeit. Urheberrecht, Rechtewahr- } \\
\text { nehmung und Administration durch Verwertungsgesellschaf- } \\
\text { ten, Verlag facultas.wuv, Wien } 2009\end{array}$ \\
\hline Leible, Stefan (Hrsg.) & $\begin{array}{l}\text { Die Bedeutung des Internationalen Privatrechts im Zeitalter der } \\
\text { neuen Medien, Richard Boorberg Verlag, Stuttgart, München, } \\
\text { Hannover, Berlin, Weimar, Dresden, } 2003\end{array}$ \\
\hline Leistner, Matthias / Hansen, Gerd & $\begin{array}{l}\text { Die Begründung des Urheberrechts im digitalen Zeitalter, GRUR } \\
2008 \text {, S. 479-490 }\end{array}$ \\
\hline Leistner, Matthias / Stang, Felix & $\begin{array}{l}\text { Die Neuerung der wettbewerbsrechtlichen Verkehrspflichten - } \\
\text { Ein Siegeszug der Prüfungspflichten?, WRP 2008, S. 533-555 }\end{array}$ \\
\hline Lessig, Lawrence & $\begin{array}{l}\text { Free Culture, The Nature and Future of Creativity, New York } \\
2004 .\end{array}$ \\
\hline Lessig, Lawrence & $\begin{array}{l}\text { Remix. Making art and commerce thrive in the hybrid economy, } \\
\text { Bloomsbury Press, London } 2008\end{array}$ \\
\hline Leung, Tin Cheuk & $\begin{array}{l}\text { Should the Music Industry Sue Its Own Customers? Impacts of } \\
\text { Music Piracy and Policy Suggestions, 2009, abrufbar unter: } \\
\text { http://faculty.washington.edu/bajari/metricssp10/ipod.pdf } \\
\text { (zitiert als Leung, 2009) }\end{array}$ \\
\hline Leupold, Andreas / Glossner, Silke (Hrsg.) & $\begin{array}{l}\text { Münchener Anwaltshandbuch IT-Recht, 2. Aufl., Verlag C.H. } \\
\text { Beck, München } 2011\end{array}$ \\
\hline Liebowitz, Stan J. / Watt, Richard & $\begin{array}{l}\text { How to Best Ensure Remuneration For Creators in the Market } \\
\text { For Music? Copyright and Its Alternatives, Journal of Economic } \\
\text { Surveys, Vol. 20, No. 4, 2006, S. 513-545 }\end{array}$ \\
\hline Liebowitz, Stan J. & $\begin{array}{l}\text { Copyright Issues, Copying and MP3 File-Sharing, 2011, abrufbar } \\
\text { unter: http://www.utdallas.edu/ liebowit/intprop/main.htm } \\
\text { (zitiert als Liebowitz, 2011) }\end{array}$ \\
\hline Liebowitz, Stan J. & $\begin{array}{l}\text { File-Sharing: Creative Destruction or just Plain Destruction?, } \\
\text { 2005, abrufbar unter: } \\
\text { http://www.utdallas.edu/ liebowit/intprop/destruction4.pdf } \\
\text { (zitiert als Liebowitz, 2005) }\end{array}$ \\
\hline
\end{tabular}




\begin{tabular}{|c|c|}
\hline Liebowitz, Stan J. & $\begin{array}{l}\text { Testing File Sharing's Impact on Music Album Sales in Cities, } \\
\text { Management Science, Vol. 54, 2008, S. 852-859 }\end{array}$ \\
\hline Liebowitz, Stan J. & $\begin{array}{l}\text { Testing File-Sharing's Impact by Examining Record Sales in Cit- } \\
\text { ies, 2006, abrufbar unter: } \\
\text { http://papers.ssrn.com/sol3/papers.cfm?abstract_id=829245 } \\
\text { (zitiert als Liebowitz, 2006) }\end{array}$ \\
\hline Liebowitz, Stan J. & $\begin{array}{l}\text { Will MP3 downloads Annihilate the Record Industry? The Evi- } \\
\text { dence so Far, 2003, abrufbar unter: } \\
\text { http://www.utdallas.edu/ liebowit/intprop/records.pdf } \\
\text { (zitiert als Liebowitz 2003) }\end{array}$ \\
\hline Loewenheim, Ulrich (Hrsg.) & $\begin{array}{l}\text { Handbuch des Urheberrechts, 2. Aufl., Verlag C.H. Beck, Mün- } \\
\text { chen } 2010\end{array}$ \\
\hline Love, James & $\begin{array}{l}\text { Artists want to be paid: The Blur/Banff proposal, 2002, abrufbar } \\
\text { unter: http://www.vgrass.de/wp-content/uploads/2011/04/02- } \\
\text { 04_Love_blur-banff.pdf }\end{array}$ \\
\hline Lüft, Stefan & $\begin{array}{l}\text { BVerfG: Entscheidung des BGH zur Gerätevergütung für PCs } \\
\text { verstößt gegen Garantie des gesetzlichen Richters, GRUR-Prax } \\
\text { 2011, S. } 62\end{array}$ \\
\hline Madden, Mary & $\begin{array}{l}\text { Artists, Musicians and the Internet, 2004, S. 24, abrufbar unter: } \\
\text { http://www.pewinternet.org/ /media//Files/Reports/2004/PIP } \\
\text { _Artists.Musicians_Report.pdf.pdf } \\
\text { (zitiert als Madden, 2004) }\end{array}$ \\
\hline Malcher, Arno & $\begin{array}{l}\text { Personalisierte Webradios - Sendung oder Abruf. Die urheber- } \\
\text { rechtliche Einordnung internetbasierter Musikdienste am Bei- } \\
\text { spiel personalisierter Webradios, Verlag Dr. Kovac, Hamburg } \\
2011\end{array}$ \\
\hline Mantz, Reto & $\begin{array}{l}\text { Anm. zu BGH: Haftung des Internetanschlussinhabers mit } \\
\text { WLAN - Sommer unseres Lebens, MMR 2010, S. 565-570 }\end{array}$ \\
\hline $\begin{array}{l}\text { Martens, Dirk / Herfert, Jan / Karbe, } \\
\text { Tobias }\end{array}$ & $\begin{array}{l}\text { Auswirkungen digitaler Piraterie auf die Ökonomie der Medien, } \\
\text { 2012, abrufbar unter: } \\
\text { http://www.medienboard.de/WebObjects/Medienboard.woa/ } \\
\text { media/20587 } \\
\text { (zitiert als Martens/Herfert/Karbe, 2012) }\end{array}$ \\
\hline Martin, Ali & $\begin{array}{l}\text { Digital Rights Management (DRM) in Online Music Stores: DRM- } \\
\text { Encumbered Music Downloads' Inevitable Demise as a Result of } \\
\text { the Negative Effects of Heavy-Handed Copyright Law, Loyola of } \\
\text { Los Angeles Entertainment Law Review, Vol. 28, No. 3, 2007- } \\
\text { 2008, S. 265-294 }\end{array}$ \\
\hline $\begin{array}{l}\text { Maunz, Theodor (Begr.) / Schmidt- } \\
\text { Bleibtreu, Bruno / Klein, Franz / Bethge, } \\
\text { Herbert }\end{array}$ & $\begin{array}{l}\text { Bundesverfassungsgerichtsgesetz, Kommentar, Stand. 38. EL, } \\
\text { Verlag C.H. Beck, Müchen } 2012 \\
\text { (zitiert als Maunz/Schmidt-Bleibtreu/Klein/Bethge, BVerfGG) }\end{array}$ \\
\hline Maunz, Theodor / Dürig, Günter (Begr.) & $\begin{array}{l}\text { Grundgesetz, Kommentar, Stand: 68. EL, Verlag C.H. Beck, } \\
\text { München } 2013 \\
\text { (zitiert als Maunz/Dürig, GG) }\end{array}$ \\
\hline Mengel, Anja & $\begin{array}{l}\text { Compliance und Arbeitsrecht. Implementierung, Durchsetzung, } \\
\text { Organisation, Verlag C.H. Beck, München } 2009\end{array}$ \\
\hline Merges, Robert P. & $\begin{array}{l}\text { End of Friction - Property Rights and Contract in the Newton } \\
\text { World of on-Line Commerce, Berkeley Technology Law Journal, } \\
\text { Vol. 12, 1997, S. 115-136 }\end{array}$ \\
\hline Merten, Detlef / Papier, Hans-Jürgen & $\begin{array}{l}\text { Handbuch der Grundrechte in Deutschland und Europa, Bd. II, } \\
\text { Verlag C.F. Müller, Heidelberg } 2006 \\
\text { (zitiert als Merten/Papier, Handbuch der Grundrechte) }\end{array}$ \\
\hline Merten, Detlef / Papier, Hans-Jürgen & $\begin{array}{l}\text { Handbuch der Grundrechte in Deutschland und Europa, Bd. IV, } \\
\text { Verlag C.F. Müller, Heidelberg } 2011 \\
\text { (zitiert als Merten/Papier, Handbuch der Grundrechte) }\end{array}$ \\
\hline Meschede, Thomas & $\begin{array}{l}\text { Der Schutz digitaler Musik- und Filmwerke vor privater Verviel- } \\
\text { fältigung nach den zwei Gesetzen zur Regelung des Urheber- }\end{array}$ \\
\hline
\end{tabular}




\begin{tabular}{|c|c|}
\hline & $\begin{array}{l}\text { rechts in der Informationsgesellschaft, Peter Lang, Frankfurt am } \\
\text { Main, } 2007 \\
\text { (zitiert als Meschede, 2007) }\end{array}$ \\
\hline $\begin{array}{l}\text { Mestmäcker, Ernst-Joachim / Schweitzer, } \\
\text { Heike (Hrsg.) }\end{array}$ & $\begin{array}{l}\text { Europäisches Wettbewerbsrecht, 2. Aufl., Verlag C.H. Beck, } \\
\text { München } 2004\end{array}$ \\
\hline Meyerdierks, Per & $\begin{array}{l}\text { Anm. zu EuGH, Urt. vom 24. November } 2011 \text { - C-70/10, ZD } \\
\text { 2012, S. } 29\end{array}$ \\
\hline Möhring, Philipp / Nicolini, Käte & $\begin{array}{l}\text { Urheberrechtsgesetz, Kommentar, 2. Aufl., Vahlen Verlag, } \\
\text { München } 2000 \\
\text { (zitiert als Möhring/Nicolini, UrhG) }\end{array}$ \\
\hline $\begin{array}{l}\text { Mortimer, Julie Holland / Nosko, Chris / } \\
\text { Sorensen, Alan }\end{array}$ & $\begin{array}{l}\text { Supply Responses to Digital Distribution: Recorded Music and } \\
\text { Live Performances. Working Paper 16507, 2010, abrufbar un- } \\
\text { ter: http://www.nber.org/papers/w16507.pdf?new_window=1 } \\
\text { (zitiert als Mortimer/Nosko/Sorensen, 2010) }\end{array}$ \\
\hline Möstl, Markus & $\begin{array}{l}\text { Vorratsdatenspeicherung - wie geht es weiter?, ZRP 2011, S. } \\
225-229\end{array}$ \\
\hline $\begin{array}{l}\text { Motion Picture Association of America } \\
\text { (MPAA) }\end{array}$ & $\begin{array}{l}\text { Theatrical Market Statistics 2011, abrufbar unter: } \\
\text { http://www.mpaa.org/Resources/5bec4ac9-a95e-443b-987b- } \\
\text { bff6fb5455a9.pdf }\end{array}$ \\
\hline Muguet, Francis & $\begin{array}{l}\text { Le mécénat global, septembre 2008.version 0.3, abrufbar un- } \\
\text { ter : http://mecenat-global.org/index-fr.html }\end{array}$ \\
\hline Müller, Stefan & $\begin{array}{l}\text { Festlegung und Inkasso von Vergütungen für die private Ver- } \\
\text { vielfältigung auf der Grundlage des "Zweiten Korbs", ZUM 2007, } \\
\text { S. 777-791 }\end{array}$ \\
\hline Müller, Stefan & $\begin{array}{l}\text { Verbesserung des gesetzlichen Instrumentariums zur Durchset- } \\
\text { zung von Vergütungsansprüchen für private Vervielfältigung, } \\
\text { ZUM 2008, S. 377-384 }\end{array}$ \\
\hline Music Matters/Synovate/MidemNet & Global Survey of 8,500, aged $18+$ in 13 countries, 2010 \\
\hline Nägele, Thomas / Nitsche, Christina & $\begin{array}{l}\text { Gesetzentwurf der Bundesregierung zur Verbesserung der } \\
\text { Durchsetzung von Rechten des geistigen Eigentums, WRP 2007, } \\
\text { S. 1047-1058 }\end{array}$ \\
\hline Netanel, Neil Weinstock & $\begin{array}{l}\text { Impose A Noncommercial Use Levy To Allow Free Peer-To-Peer } \\
\text { File Sharing, Harvard Journal of Law \& Technology, Vol. 17, } \\
\text { 2003, S. 1-84 }\end{array}$ \\
\hline Neumann, Volker & $\begin{array}{l}\text { Legislative Einschätzungsprärogative und gerichtliche Kontroll- } \\
\text { dichte bei Eingriffen in die Tarifautonomie, RdA 2007, S. 71-76 }\end{array}$ \\
\hline Nielsen Company, The / Billboard & $\begin{array}{l}2011 \text { Music Industry Report, abrufbar unter: } \\
\text { http://narm.com/PDF/NielsenMusic2011YEUpdate.pdf }\end{array}$ \\
\hline Niemann, Fabian & $\begin{array}{l}\text { Urheberrechtsabgaben und Verfassungsrecht: o tempora, o } \\
\text { mores, CR 2011, S. 69-76 }\end{array}$ \\
\hline Nordemann, Jan Bernd & $\begin{array}{l}\text { Haftung von Providern im Urheberrecht, GRUR 2011, S. 977- } \\
981\end{array}$ \\
\hline O'Leary, Brian & $\begin{array}{l}\text { Impact of P2P and Free Distribution on Book Sales, O'Reilly } \\
\text { Media, } 2009\end{array}$ \\
\hline Oberholzer-Gee, Felix / Strumpf, Koleman & $\begin{array}{l}\text { File-Sharing and Copyright, } 2010 \text { (überarbeitete Fassung von } \\
\text { Working Paper 09-132, 2009), abrufbar unter: } \\
\text { http://musicbusinessresearch.files.wordpress.com/2010/06/pa } \\
\text { per-felix-oberholzer-gee.pdf } \\
\text { (zitiert als Oberholzer-Gee/Strumpf, 2010) }\end{array}$ \\
\hline Oberholzer-Gee, Felix / Strumpf, Koleman & $\begin{array}{l}\text { File-Sharing and Copyright, Working Paper 09-132, 2009, abruf- } \\
\text { bar unter: http://www.hbs.edu/research/pdf/09-132.pdf } \\
\text { (zitiert als Oberholzer-Gee/Strumpf, 2009) }\end{array}$ \\
\hline Oberholzer-Gee, Felix / Strumpf, Koleman & $\begin{array}{l}\text { The Effect of File Sharing on Record Sales. An Empirical Analy- } \\
\text { sis, 2004, abrufbar unter: } \\
\text { http://www.unc.edu/ cigar/papers/FileSharing_March2004.pd } \\
\text { f } \\
\text { (zitiert als Oberholzer-Gee/Strumpf, } 2004 \text { - Studie) }\end{array}$ \\
\hline
\end{tabular}




\begin{tabular}{|c|c|}
\hline Oberholzer-Gee, Felix / Strumpf, Koleman & $\begin{array}{l}\text { The Effect of File Sharing on Record Sales. An Empirical Analy- } \\
\text { sis, Journal of Political Economy, Vol. 115, 2007, S. 1-42 } \\
\text { (zitiert als Oberholzer-Gee/Strumpf, 2007) }\end{array}$ \\
\hline Ohne Autor & $\begin{array}{l}\text { BMJ: Gesetzentwurf zu Quick Freeze, MMR-Aktuell 2011, } \\
319439\end{array}$ \\
\hline $\begin{array}{l}\text { Organisation für wirtschaftliche Zusam- } \\
\text { menarbeit und Entwicklung (OECD) }\end{array}$ & $\begin{array}{l}\text { OECD Report on Digital Music: Opportunities and Challenges, } \\
\text { OECD Digital Economy Papers, No. 100, 2005, abrufbar unter: } \\
\text { http://www.oecd- } \\
\text { ili- } \\
\text { brary.org/docserver/download/5kz9hcfddr48.pdf?expires=135 } \\
\text { 5702820\&id=id\&accname=guest\&checksum=A4CECC34333890 } \\
\text { 387772F0227F29AD74 }\end{array}$ \\
\hline Osenberg, Ralph & $\begin{array}{l}\text { Die Unverzichtbarkeit des Urheberpersönlichkeitsrechts, Hoch- } \\
\text { schulverlag, Freiburg } 1985\end{array}$ \\
\hline Ott, Sieghart & $\begin{array}{l}\text { Kunst und Staat: Der Künstler zwischen Freiheit und Zensur, Dt. } \\
\text { Taschenbuch-Verlag, München } 1968 \\
\text { (zitiert als Ott, Kunst und Staat) }\end{array}$ \\
\hline Ott, Stephan & $\begin{array}{l}\text { Haftung für Embedded Videos von YouTube und anderen Vi- } \\
\text { deoplattformen im Internet, ZUM 2008, 556-564 }\end{array}$ \\
\hline Palm, Wolfgang & $\begin{array}{l}\text { Öffentliche Kunstförderung zwischen Kunstfreiheitsgarantie } \\
\text { und Kulturstaat, Verlag Duncker \& Humblot, Berlin } 1997\end{array}$ \\
\hline Paulus, Andreas / Wesche, Steffen & Urheberrecht und Verfassung, ZGE 2010, S. 385-397 \\
\hline Paulus, Andreas & $\begin{array}{l}\text { Die Immaterialgüterrechte im Zeitalter der Neuen Medien, in: } \\
\text { Durner, Wolfgang/Peine, Franz-Joseph/Shirvani, Foroud (Hrsg.), } \\
\text { Freiheit und Sicherheit in Deutschland und Europa, Festschrift } \\
\text { für Hans-Jürgen Papier zum 70. Geburtstag, Verlag Duncker \& } \\
\text { Humblot, Berlin 2013, S. 561-586 } \\
\text { (zitiert als Paulus, in: FS Papier, 2013) }\end{array}$ \\
\hline Peitz, Martin / Waelbroeck, Patrick & $\begin{array}{l}\text { The Effect of Internet Piracy on CD Sales: Cross-Section Evi- } \\
\text { dence, CESIFO Working Paper No. 1122, 2004, abrufbar unter: } \\
\text { http://papers.ssrn.com/sol3/Delivery.cfm/SSRN_ID511763_cod } \\
\text { e102458.pdf?abstractid=511763\&mirid=3 } \\
\text { (zitiert als Peitz/Waelbroeck, 2004) }\end{array}$ \\
\hline Peitz, Martin / Waelbroeck, Patrick & $\begin{array}{l}\text { Why the Music Industry May Gain from Free Downloading - } \\
\text { The Role of Sampling, 2006, abrufbar unter: } \\
\text { http://papers.ssrn.com/sol3/Delivery.cfm/SSRN_ID829544_cod } \\
\text { e57579.pdf?abstractid=829544\&mirid=3 } \\
\text { (zitiert als Peitz/Waelbroeck, 2006) }\end{array}$ \\
\hline Pense, Andreas & $\begin{array}{l}\text { Erlösverteilung - Erlösprognose bei der Film- und Fernsehpro- } \\
\text { duktion, 19.4.2010, abrufbar unter: } \\
\text { http://www.ffhsh.de/art/MediaCenter/Downloads/Sonstiges/H } \\
\text { andout_Film_und_Recht_190410.pdf }\end{array}$ \\
\hline Peter, Markus & $\begin{array}{l}\text { Störer im Internet - Haften Eltern für ihre Kinder?, K\&R 2007, S. } \\
371-375\end{array}$ \\
\hline Petschulat, Tim O. & $\begin{array}{l}\text { Kulturflatrate. Auf der Suche nach einem internetkompatiblen } \\
\text { Urheberrecht, 2010, abrufbar unter: http://library.fes.de/pdf- } \\
\text { files/do/07256.pdf } \\
\text { (zitiert als Petschulat, Kulturflatrate) }\end{array}$ \\
\hline Peukert, Christian / Claussen, Jörg & $\begin{array}{l}\text { Piracy and Movie Revenues: Evidence from Megaupload, 2012, } \\
\text { abrufbar unter: http://ssrn.com/abstract=2176246 }\end{array}$ \\
\hline Pfennig, Gerhard & $\begin{array}{l}\text { Digital Rights Management Systeme aus der Sicht von Verwer- } \\
\text { tungs- gesellschaften, ZUM 2004, S. 198-203 }\end{array}$ \\
\hline Porter, Thomas & $\begin{array}{l}\text { The Perils of Deep Packet Inspection, 2010, abrufbar unter: } \\
\text { http://www.symantec.com/connect/articles/perils-deep- } \\
\text { packet-inspection }\end{array}$ \\
\hline PricewaterhouseCoopers & $\begin{array}{l}\text { E-Books in Deutschland, 2010, abrufbar unter: } \\
\text { http://www.pwc.de/de_DE/de/technologie-medien-und- }\end{array}$ \\
\hline
\end{tabular}




\begin{tabular}{|c|c|}
\hline & $\begin{array}{l}\text { telekommunikation/assets/E-books_in_Deutschland_- } \\
\text { _Beginn_einer_neuen_Gutenberg-Aera.pdf }\end{array}$ \\
\hline PricewaterhouseCoopers & $\begin{array}{l}\text { German Entertainment and Media Outlook: 2011-2015, Fach- } \\
\text { verlag Moderne Wirtschaft, Frankfurt } 2012\end{array}$ \\
\hline Prill, Aileen & $\begin{array}{l}\text { Webradio-Streamripping: Eine neue Form der Musikpiraterie?, } \\
\text { erscheint demnächst }\end{array}$ \\
\hline $\begin{array}{l}\text { privatkopie.net / Forum Informatikerin- } \\
\text { nen für Frieden und gesellschaftliche } \\
\text { Verantwortung e.V.(FIfF)/ Netzwerk Neue } \\
\text { Medien Chaos Computer Club / FoeBuD } \\
\text { e.V. / Attac / AG Wissensallmende und } \\
\text { freier Informationsfluss / ODEM -- Online- } \\
\text { Demonstrations-Plattform für Menschen- } \\
\text { und Bürgerrechte im digitalen Zeitalter }\end{array}$ & $\begin{array}{l}\text { Kompensation ohne Kontrolle, Stellungnahme zum Zweiten } \\
\text { Korb der Novellierung des Urheberrechtsgesetzes v. 21.4.2004, } \\
\text { abrufbar unter: http://privatkopie.net/files/Stellungnahme- } \\
\text { ACS.pdf } \\
\text { (zitier als: privatkopie.net u.a., Kompensation ohne Kontrolle, } \\
\text { 2004) }\end{array}$ \\
\hline Ramsauer, Ulrich & $\begin{array}{l}\text { Die faktischen Beeinträchtigungen des Eigentums, Duncker \& } \\
\text { Humblot, Berlin } 1980\end{array}$ \\
\hline Rau, Marco / Behrens, Martin & $\begin{array}{l}\text { Catch me if you can ... Anonymisierungsdienste und die Haftung } \\
\text { für mittelbare Rechtsverletzungen, K\&R 2009, S. 766-771 }\end{array}$ \\
\hline Renner, Kai-Hinrich / Renner, Tim & $\begin{array}{l}\text { Digital ist besser. Warum das Abendland auch durch das Inter- } \\
\text { net nicht untergehen wird, Campus Verlag, Frankfurt/New York } \\
2011\end{array}$ \\
\hline Ricketson, Sam / Ginsburg, Jane C. & $\begin{array}{l}\text { International Copyright and Neighbouring Rights, The Berne } \\
\text { Convention and Beyond Volume I and Volumee II, Oxford, New } \\
\text { York, 2. Aufl. } 2006\end{array}$ \\
\hline Ridder, Helmut & $\begin{array}{l}\text { Freiheit der Kunst nach dem Grundgesetz, Vahlen Verlag, Berlin } \\
1963\end{array}$ \\
\hline Riesenhuber, Karl / Klöhn, Lars & $\begin{array}{l}\text { Das Urhebervertragsrecht im Lichte der Verhaltensökonomik, } \\
\text { INTERGU-Tagung 2009, Verlag de Gruyter, Berlin } 2010\end{array}$ \\
\hline Riesenhuber, Karl & Priorität als Verteilungsprinzip?, ZUM 2012, S. 746-758 \\
\hline Riesenhuber, Karl & $\begin{array}{l}\text { Grundlagen der „angemessenen Vergütung“, GRUR 2013, S. } \\
\text { 582-590 }\end{array}$ \\
\hline Rob, Rafael / Waldfogel, Joel & $\begin{array}{l}\text { Piracy On the High C's: Music Downloading, Sales Displacement, } \\
\text { and Social Welfare in a Sample of College Students, 2004, } \\
\text { abrufbar unter: } \\
\text { http://www.nber.org/papers/w10874.pdf?new_window=1 } \\
\text { (zitiert als Rob/Waldfogel, 2004) }\end{array}$ \\
\hline Röhl, Christoph / Bosch, Andreas & $\begin{array}{l}\text { Musiktauschbörsen im Internet - Eine Analyse der aktuellen } \\
\text { tatsächlichen und rechtlichen Entwicklungen, NJOZ 2008, S. } \\
1197-1215\end{array}$ \\
\hline Rosen, Sherwin & $\begin{array}{l}\text { The Economics of Superstars, The American Economic Review, } \\
\text { Vol. } 71 \text {, No. } 5,1981, \text { S. } 845-858\end{array}$ \\
\hline Roßnagel, Alexander (Hrsg.) & $\begin{array}{l}\text { Handbuch Datenschutzrecht. Die neuen Grundlagen für Wirt- } \\
\text { schaft und Verwaltung, Verlag C.H. Beck, München } 2003\end{array}$ \\
\hline $\begin{array}{l}\text { Roßnagel, Alexander / Jandt, Silke / } \\
\text { Schnabel, Christoph / Yliniva-Hoffmann, } \\
\text { Anne }\end{array}$ & $\begin{array}{l}\text { Die Zulässigkeit einer Kulturflatrate nach nationalem und euro- } \\
\text { päischem Recht, Kurzgutachten, 2009, abrufbar unter: } \\
\text { http://www.gruene- } \\
\text { bundes- } \\
\text { tag.de/fileadmin/media/gruenebundestag_de/themen_az/netz } \\
\text { poli- } \\
\text { tik/16_fragen_und_16_antworten/kurzgutachten_zur_kulturfla } \\
\text { trate.pdf } \\
\text { (zitiert als Roßnagel et al., Gutachten, 2009) }\end{array}$ \\
\hline $\begin{array}{l}\text { Roßnagel, Alexander / Jandt, Silke / } \\
\text { Schnabel, Christoph }\end{array}$ & $\begin{array}{l}\text { Kulturflatrate. Ein verfassungsrechtlich zulässiges alternatives } \\
\text { Modell zur Künstlervergütung?, MMR 2010, S. 8-12 }\end{array}$ \\
\hline Runge, Philipp & $\begin{array}{l}\text { Die Vereinbarkeit einer Content-Flatrate für Musik mit dem } \\
\text { Drei-Stufen-Test, GRUR Int. 2007, S. 130-137 }\end{array}$ \\
\hline Säcker, Franz Jürgen / Rixecker, Roland & Münchener Kommentar zum BGB, Bd. 2, 6. Aufl., Verlag C.H. \\
\hline
\end{tabular}




\begin{tabular}{|c|c|}
\hline (Hrsg.) & Beck, München 2012 \\
\hline Sandvine & $\begin{array}{l}2008 \text { Global Broadband Phenomena, 2008, abrufbar unter: } \\
\text { http://www.sandvine.com/downloads/documents/2008\%20GI } \\
\text { obal\%20Broadband\%20Phenomena\%20-\%20Full\%20Report.pdf }\end{array}$ \\
\hline Sandvine & $\begin{array}{l}\text { Fall } 2010 \text { Global Internet Phenomena Report, 2010, abrufbar } \\
\text { unter: } \\
\text { http://www.sandvine.com/downloads/documents/2010\%20GI } \\
\text { obal\%20Internet\%20Phenomena\%20Report.pdf }\end{array}$ \\
\hline Sandvine & $\begin{array}{l}\text { Global Internet Phenomena Report } 1 \mathrm{H} \text { 2013, 2013, abrufbar } \\
\text { unter: } \\
\text { http://www.sandvine.com/downloads/documents/Phenomena } \\
\text { 2013_2013/Sandvine_Global_Internet_Phenomena_Report_1H_ }\end{array}$ \\
\hline Schaar, Peter & $\begin{array}{l}\text { Datenschutz im Internet: Die Grundlagen, Verlag C.H. Beck, } \\
\text { München } 2002\end{array}$ \\
\hline Schapiro, Leo & $\begin{array}{l}\text { Die neuen Musiktauschbörsen unter »Freunden«, ZUM 2008, S. } \\
\text { 273-282 }\end{array}$ \\
\hline Schenke, Wolf-Rüdiger & $\begin{array}{l}\text { Der Umfang der bundesverfassungsgerichtlichen Überprüfung, } \\
\text { NJW 1979, S. 1321-1329 }\end{array}$ \\
\hline Schippan, Martin & $\begin{array}{l}\text { Rechtsfragen bei der Implementierung von Digital Rights Ma- } \\
\text { nagement-Systemen, ZUM 2004, S. 188-198 }\end{array}$ \\
\hline Schlaich, Klaus / Korioth, Stefan & $\begin{array}{l}\text { Das Bundesverfassungsgericht. Stellung, Verfahren, Entschei- } \\
\text { dungen, 9. Aufl., Verlag C.H. Beck, München } 2012\end{array}$ \\
\hline Schmalz, Gisela & $\begin{array}{l}\text { No Economy - Wie der Gratiswahn das Internet zerstört, Eich- } \\
\text { born Verlag, Frankfurt a.M. } 2009\end{array}$ \\
\hline $\begin{array}{l}\text { Schönke, Adolf (Begr.) / Schröder, Horst } \\
\text { (Fortg.) }\end{array}$ & $\begin{array}{l}\text { Strafgesetzbuch, Kommentar, 28. Aufl., Verlag C.H. Beck, Mün- } \\
\text { chen } 2010\end{array}$ \\
\hline Schramm, Marc / Wegener, Christoph & $\begin{array}{l}\text { Neue Anforderungen an eine anlasslose Speicherung von Vor- } \\
\text { ratsdaten, MMR 2011, S. 9-13 }\end{array}$ \\
\hline Schreibauer, Marcus & $\begin{array}{l}\text { Das Pornographieverbot des § } 184 \text { StGB. Grundlagen, Tatbe- } \\
\text { standsprobleme, Reformvorschläge, Roderer Verlag, Regens- } \\
\text { burg } 1999\end{array}$ \\
\hline $\begin{array}{l}\text { Schricker, Gerhard / Loewenheim, Ulrich } \\
\text { (Hrsg.) }\end{array}$ & $\begin{array}{l}\text { Urheberrecht, Kommentar, 4. Aufl., Verlag C.H. Beck, München } \\
2010 \\
\text { (zitiert als Schricker/Loewenheim, UrhR) }\end{array}$ \\
\hline Schroeder, Friedrich-Christian & $\begin{array}{l}\text { Pornographie, Jugendschutz und Kunstfreiheit, Verlag C.F. Mül- } \\
\text { ler, Heidelberg } 1992\end{array}$ \\
\hline Schulz, Daniela & $\begin{array}{l}\text { Der Bedeutungswandel des Urheberrechts durch Digital Rights } \\
\text { Management - Paradigmenwechsel im deutschen Urheber- } \\
\text { recht?, GRUR 2006, S. 470-477 }\end{array}$ \\
\hline $\begin{array}{l}\text { Schwartmann, Rolf / Hentsch, Christian- } \\
\text { Henner }\end{array}$ & $\begin{array}{l}\text { Die verfassungsrechtlichen Grenzen der Urheberrechtsdebatte, } \\
\text { ZUM 2012, S. 759-771 }\end{array}$ \\
\hline Schwartmann, Rolf & Filesharing, Sharehosting \& Co., K\&R Beihefter 2/2011, S. 1-23 \\
\hline Schwetter, Holger / Volz, Jakob & $\begin{array}{l}\text { Preiszusammensetzung bei Hardware und Software Tonträgern, } \\
\text { 2004, abrufbar unter: http://www.schwetter.de/copy/ }\end{array}$ \\
\hline Senftleben, Martin & $\begin{array}{l}\text { Copyright, Limitations and the Three-Step Test, Verlag Kluwer } \\
\text { Law International, The Hague/London/New York } 2004\end{array}$ \\
\hline Senftleben, Martin & $\begin{array}{l}\text { Grundprobleme des urheberrechtlichen Dreistufentests, GRUR } \\
\text { Int. 2004, S. 200-211 }\end{array}$ \\
\hline Senftleben, Martin & $\begin{array}{l}\text { Privates digitales Kopieren im Spiegel des Dreistufentests, CR } \\
\text { 2003, S. 914-919 }\end{array}$ \\
\hline $\begin{array}{l}\text { Shalunov, Stanislav / Teitelbaum, Benja- } \\
\text { min }\end{array}$ & $\begin{array}{l}\text { TCP Use and Performance on Internet2, ACM SIGCOMM Inter- } \\
\text { net Measurement Workshop, 2001, abrufbar unter: } \\
\text { http://ben.teitelbaum.us/internet2/papers/i2tcp- } \\
\text { imeas2001.pdf }\end{array}$ \\
\hline Smith, Michael D. / Telang, Rahul & $\begin{array}{l}\text { Piracy or Promotion? The Impact of Broadband Internet Pene- } \\
\text { tration on DVD Sales, 2009, abrufbar unter: }\end{array}$ \\
\hline
\end{tabular}




\begin{tabular}{|c|c|}
\hline & $\begin{array}{l}\text { http://papers.ssrn.com/sol3/Delivery.cfm/SSRN_ID1395781_co } \\
\text { de291479.pdf?abstractid=918240\&mirid=3 } \\
\text { (zitiert als Smith/Telang, 2009) }\end{array}$ \\
\hline Smith, Michael D./ Telang, Rahul & $\begin{array}{l}\text { Competing with Free: The Impact of Movie Broadcasts on DVD } \\
\text { Sales and Internet Piracy, 2008, abrufbar unter: } \\
\text { http://ssrn.com/abstract=1028306 (=Management Information } \\
\text { Systems Quarterly (MIS Quarterly), Vol. } 33 \text { No. 2, 2009, S. 321- } \\
\text { 338) } \\
\text { (zitiert als Smith/Telang, 2008) }\end{array}$ \\
\hline Sodan, Helge & $\begin{array}{l}\text { Gesundheitsbehördliche Informationstätigkeit und Grund- } \\
\text { rechtsschutz, DÖV 1987, S. 858-866 }\end{array}$ \\
\hline Solmecke, Christian / Bärenfänger, Jan & $\begin{array}{l}\text { Urheberrechtliche Schutzfähigkeit von Dateifragmenten, MMR } \\
2011 \text {, S. 567-573 }\end{array}$ \\
\hline Solmecke, Christian & $\begin{array}{l}\text { Rechtliche Beurteilung der Nutzung von Musiktauschbörsen, } \\
\text { K\&R 2007, S. 138-143 }\end{array}$ \\
\hline Spies, Axel & $\begin{array}{l}\text { USA: Neues FCC-Verfahren zu VoIP (lokale Rufnummernportie- } \\
\text { rung), MMR 2008, XII }\end{array}$ \\
\hline Spindler, Gerald / Schuster, Fabian (Hrsg.) & $\begin{array}{l}\text { Recht der elektronischen Medien, 2. Aufl., Verlag C.H. Beck, } \\
\text { München } 2011\end{array}$ \\
\hline Spindler, Gerald & $\begin{array}{l}\text { Anm. zu EuGH, Urt. v. 24.11.2011 - C-70/10 - Scarlet/SABAM, JZ } \\
\text { 2012, S. 311-313 }\end{array}$ \\
\hline Spindler, Gerald & $\begin{array}{l}\text { Der Auskunftsanspruch gegen Verletzer und Dritte im Urheber- } \\
\text { recht nach neuem Recht, ZUM 2008, S. 640-648 }\end{array}$ \\
\hline Spindler, Gerald & $\begin{array}{l}\text { Die kollisionsrechtliche Behandlung von Urheberrechtsverlet- } \\
\text { zungen im Internet, IPRax 2003, S. 412-421 }\end{array}$ \\
\hline Spindler, Gerald & $\begin{array}{l}\text { Europäisches Urheberrecht in der Informationsgesellschaft, } \\
\text { GRUR 2002, S. 105-120 }\end{array}$ \\
\hline Spindler, Gerald & $\begin{array}{l}\text { Europarechtliche Rahmenbedingungen der Störerhaftung im } \\
\text { Internet, MMR 2011, S. 703-707 }\end{array}$ \\
\hline Spindler, Gerald & $\begin{array}{l}\text { Geräteabgaben im Lichte europarechtlicher Vorgaben - die } \\
\text { jüngste EuGH-Rechtsprechung und die Konsequenzen für das } \\
\text { deutsche Recht, in: Schierholz, Anke / Melichar, Ferdinand, } \\
\text { Kunst, Recht und Geld, Festschrift für Gerhard Pfennig zum } 65 . \\
\text { Geburtstag, Beck-Verlag, München 2012, S. 387-402 } \\
\text { (zitiert als Spindler, in: FS Pfennig) }\end{array}$ \\
\hline Spindler, Gerald & $\begin{array}{l}\text { Haftung für private WLANs im Delikts- und Urheberrecht, CR } \\
2010, \text { S. 592-600 }\end{array}$ \\
\hline Spindler, Gerald & $\begin{array}{l}\text { Morpheus, Napster \& Co. - Die kollisionsrechtliche Behandlung } \\
\text { von Urheberrechtsverletzungen im Internet, in: Leible (Hrsg.), } \\
\text { Die Bedeutung des internationalen Privatrechts im Zeitalter der } \\
\text { neuen Medien, S. 155-180 }\end{array}$ \\
\hline Spindler, Gerald & $\begin{array}{l}\text { Persönlichkeitsschutz im Internet - Anforderungen und Grenzen } \\
\text { einer Regulierung. Gutachten F zum 69. Deutschen Juristentag, } \\
\text { Verlag C.H. Beck, München } 2012\end{array}$ \\
\hline Spindler, Gerald & $\begin{array}{l}\text { Reformen der Vergütungsregeln im Urhebervertragsrecht, ZUM } \\
\text { 2012, S. 921-933 }\end{array}$ \\
\hline Spitz, Malte / Dittrich, Simon Edwin & $\begin{array}{l}\text { Schöne neue kreative Welt, 2010, abrufbar unter: } \\
\text { http://events.ccc.de/sigint/2010/wiki/Fahrplan/events/3843.de } \\
\text {.html }\end{array}$ \\
\hline Stallmann, Richard M. & $\begin{array}{l}\text { Freedom - or Copyright?, 2009, abrufbar unter: } \\
\text { http://www.gnu.org/philosophy/freedom-or-copyright.html }\end{array}$ \\
\hline Stern, Klaus & $\begin{array}{l}\text { Das Staatsrecht der Bundesrepublik Deutschland, Band IV/1, } \\
\text { Verlag C.H. Beck, München } 2006\end{array}$ \\
\hline Stettner, Rupert & $\begin{array}{l}\text { Verfassungsbindungen des experimentierenden Gesetzgebers, } \\
\text { NVwZ 1989, S. 806-812 }\end{array}$ \\
\hline Stickelbrock, Barbara & $\begin{array}{l}\text { Die Zukunft der Privatkopie im digitalen Zeitalter, GRUR 2004, } \\
\text { S. 736-743 }\end{array}$ \\
\hline
\end{tabular}




\begin{tabular}{|c|c|}
\hline Stieper, Malte & $\begin{array}{l}\text { Rechtfertigung, Rechtsnatur und Disponibilität der Schranken } \\
\text { des Urheberrechts, Verlag Mohr Siebeck, Tübingen } 2009\end{array}$ \\
\hline $\begin{array}{l}\text { Stiftung der Hessischen Rechtsan- } \\
\text { waltschaft }\end{array}$ & $\begin{array}{l}\text { Kulturflatrate, Kulturwertmark oder Three strikes and you are } \\
\text { out: Wie soll mit Kreativität im Internet umgegangen werden?, } \\
\text { Optimus Verlag, Göttingen } 2013 \\
\text { (zitiert als: Stiftung der Hessischen Rechtsanwaltschaft, Kultur- } \\
\text { flatrate) }\end{array}$ \\
\hline STIM & $\begin{array}{l}\text { Pirates, file-sharers and music users. A survey of the conditions } \\
\text { for new music services on the Internet, 2009, abrufbar unter: } \\
\text { http://mpa.weblobe.net/files/pdf/STIM_survey_on_the_condit } \\
\text { ions_for_new_online_music_services.pdf }\end{array}$ \\
\hline Tanaka, Tatsuo & $\begin{array}{l}\text { Does file sharing reduce music CD sales? A case of Japan, IIR } \\
\text { Working Paper WP\#05-08, 2004, abrufbar unter: } \\
\text { http://hermes-ir.lib.hit- } \\
\text { u.ac.jp/rs/bitstream/10086/15965/1/070iirWP05-08.pdf } \\
\text { (zitiert als Tanaka, 2004) }\end{array}$ \\
\hline TERA Consultants & $\begin{array}{l}\text { Building a Digital Economy: The Importance of Saving Jobs In } \\
\text { the EU's Creative Industries, March 2010, abrufbar unter: } \\
\text { http://www.droit-technologie.org/upload/dossier/doc/219- } \\
\text { 1.pdf } \\
\text { (zitiert als TERA Consultants, 2010) }\end{array}$ \\
\hline Tettinger, Peter J. & Steine aus dem Glashaus, JZ 2004, S. 1144-1146 \\
\hline Toner, Alan & $\begin{array}{l}\text { Blur Banff proposal. P2P Foundation, 2008, abrufbar unter: } \\
\text { http://p2pfoundation.net/Blur_Banff_Proposal }\end{array}$ \\
\hline Tschmuck, Peter & $\begin{array}{l}\text { Beantwortung der Fragen zur „Entwicklung des Urheberrechts } \\
\text { in der digitalen Gesellschaft“ der Enquete-Kommission Internet } \\
\text { und digitale Gesellschaft des Deutschen Bundestags, 2010, } \\
\text { Ausschussdrucksache 17(24)009-C, abrufbar unter: } \\
\text { http://www.bundestag.de/internetenquete/dokumentation/Sit } \\
\text { zungen/20101129/A-Drs_17_24_009_C_- } \\
\text { Stellungnahme_Prof_Tschmuck.pdf } \\
\text { (zitiert als Tschmuck, Stellungnahme, 2010) }\end{array}$ \\
\hline Tschmuck, Peter & $\begin{array}{l}\text { Creativity and Innovation in the Music Industry, 2. Aufl., Sprin- } \\
\text { ger Verlag, Berlin } 2012 \\
\text { (zitiert als Tschmuck, Creativity, 2012) }\end{array}$ \\
\hline Tschmuck, Peter & $\begin{array}{l}\text { Die Erklärungsansätze für die Wirkung von File-Sharing im } \\
\text { Überblick, 2009, } \\
\text { http://musikwirtschaftsforschung.files.wordpress.com/2009/05 } \\
\text { /ansatze-zu-file-sharing-wirkungen.doc } \\
\text { (zitiert als Tschmuck, Ansätze, 2009) }\end{array}$ \\
\hline Tschmuck, Peter & $\begin{array}{l}\text { Die ökonomischen Folgen der Musik-„Piraterie“, 2011, abrufbar } \\
\text { unter: } \\
\text { http://musikwirtschaftsforschung.wordpress.com/2011/02/10/ } \\
\text { die-okonomischen-folgen-der-musik-piraterie/ } \\
\text { (zitiert als Tschmuck, Ökonomische Folgen, 2011) }\end{array}$ \\
\hline Tschmuck, Peter & $\begin{array}{l}\text { Die Rezession in der Musikindustrie - eine Ursachenanalyse, } \\
\text { 2009, abrufbar unter: } \\
\text { http://musikwirtschaftsforschung.wordpress.com/2009/06/25/ } \\
\text { die-rezession-in-der-musikindustrie-eine-ursachenanalyse } \\
\text { (zitiert als Tschmuck, Ursachenanalyse, 2009) }\end{array}$ \\
\hline Tschmuck, Peter & $\begin{array}{l}\text { Kreativität und Innovation in der Musikindustrie, StudienVerlag, } \\
\text { Innsbruck } 2003 \\
\text { (zitiert als Tschmuck, Kreativität, 2003) }\end{array}$ \\
\hline Tschmuck, Peter & $\begin{array}{l}\text { The Economics of Music File Sharing - A Literature Overview. } \\
\text { Conference paper presented at the first Vienna Music Business } \\
\text { Research Days, 2010, abrufbar unter: } \\
\text { http://musikwirtschaftsforschung.files.wordpress.com/2010/06 }\end{array}$ \\
\hline
\end{tabular}




\begin{tabular}{|c|c|}
\hline & $\begin{array}{l}\text { /tschmuck-the-economics-of-file-sharing-end.doc } \\
\text { (zitiert als Tschmuck, Economics, 2010) }\end{array}$ \\
\hline Tschmuck, Peter & $\begin{array}{l}\text { wie böse ist das file-sharing? - teil 3, 2009, abrufbar unter: } \\
\text { http://musikwirtschaftsforschung.wordpress.com/2009/04/09/ } \\
\text { wie-bose-ist-das-file-sharing-\%E2\%80\%93-teil-3/ } \\
\text { (zitiert als Tschmuck, Teil 3, 2009) }\end{array}$ \\
\hline Tschmuck, Peter & $\begin{array}{l}\text { wie böse ist das file-sharing? - Teil 4, 2009, abrufbar unter: } \\
\text { http://musikwirtschaftsforschung.wordpress.com/2009/04/14/ } \\
\text { wie-bose-ist-das-file-sharing-teil-4/ } \\
\text { (zitiert als Tschmuck, Teil 4, 2009) }\end{array}$ \\
\hline Tschmuck, Peter & $\begin{array}{l}\text { wie böse ist das file-sharing? - Teil 8, 2009, abrufbar unter: } \\
\text { http://musikwirtschaftsforschung.wordpress.com/2009/04/17/ } \\
\text { wie-bose-ist-das-file-sharing-teil-8/ } \\
\text { (zitiert als Tschmuck, Teil 8, 2009) }\end{array}$ \\
\hline Tschmuck, Peter & $\begin{array}{l}\text { wie böse ist das file-sharing? - teil 18, 2009, abrufbar unter: } \\
\text { http://musikwirtschaftsforschung.wordpress.com/2009/05/25/ } \\
\text { wie-bose-ist-das-file-sharing-teil-18/ } \\
\text { (zitiert als Tschmuck, Teil 18, 2009) }\end{array}$ \\
\hline Ueberfeldt, Lara & $\begin{array}{l}\text { Kulturflatrate, Kulturwertmark oder Three Strikes and you are } \\
\text { out, in: Stiftung der Hessischen Rechtsanwaltschaft, Kulturflat- } \\
\text { rate, Kulturwertmark oder Three Strikes and you are out: Wie } \\
\text { soll mit Kreativität im Internet umgegangen werden?, S. 115- } \\
\text { 151, Optimus Verlag, Göttingen } 2013\end{array}$ \\
\hline Ünlü, Vural & $\begin{array}{l}\text { Content Protection, economic analysis and techno-legal imple- } \\
\text { mentation, Utz Verlag, München } 2005\end{array}$ \\
\hline $\begin{array}{l}\text { US Government Accountability Office } \\
\text { (GAO) }\end{array}$ & $\begin{array}{l}\text { Intellectual Property - Observations on Efforts to Quantify the } \\
\text { Economic Effects of Counterfeit and Pirated Good, 2010, abruf- } \\
\text { bar unter: http://www.gao.gov/new.items/d10423.pdf }\end{array}$ \\
\hline US Social Science Research Council & $\begin{array}{l}\text { Media piracy in emerging economies, 2011, abrufbar unter: } \\
\text { http://piracy.americanassembly.org/wp- } \\
\text { content/uploads/2011/06/MPEE-PDF-1.0.4.pdf }\end{array}$ \\
\hline v. Lewinski, Silke & $\begin{array}{l}\text { Mandatory Collective Administration of Exclusive Rights - A } \\
\text { Case Study On Its Compatibility With International and EC Cop- } \\
\text { yright Law, 2004, abrufbar unter: } \\
\text { http://portal.unesco.org/culture/en/files/19552/11515904771s } \\
\text { vl_e.pdf/svl_e.pdf } \\
\text { (zitiert als v. Lewinski, 2004) }\end{array}$ \\
\hline v. Lewinski, Silke & International Copyright Law and Policy, Oxford, 2008. \\
\hline $\begin{array}{l}\text { v. Mangoldt, Hermann (Begr.) / Klein, } \\
\text { Friedrich (Fortg.) / Starck, Christian (Hrsg.) }\end{array}$ & $\begin{array}{l}\text { Kommentar zum Grundgesetz, 6. Aufl., Vahlen Verlag, München } \\
2010 \\
\text { (zitiert als v. Mangoldt/Klein/Starck, GG) }\end{array}$ \\
\hline v. Zimmermann, Georg & Recording-Software für Internetradios, MMR 2007, S. 553-558 \\
\hline v. Münch, Ingo / Kunig, Philip (Hrsg.) & $\begin{array}{l}\text { Grundgesetz-Kommentar, 6. Aufl., Verlag C.H. Beck, München } \\
2012 \\
\text { (zitiert als v. Münch/Kunig, GG) }\end{array}$ \\
\hline van Eijk, Nico / Poort, Joost, / Rutten, Paul & $\begin{array}{l}\text { Legal, Economic and Cultural Aspects of File Sharing, Communi- } \\
\text { cation \& Strategies, Vol. 77, 1. Quart. 2010, S. } 35\end{array}$ \\
\hline Ventroni, Stefan & $\begin{array}{l}\text { Erleichtert die EU-Richtlinie zur kollektiven Rechtewahrneh- } \\
\text { mung den Erwerb von Online-Musikrechten?, MMR 2012, S. } \\
\text { 565-566 }\end{array}$ \\
\hline $\begin{array}{l}\text { Verband Privater Rundfunk und Tele- } \\
\text { medien e.V. (VPRT) }\end{array}$ & $\begin{array}{l}\text { VPRT-Marktprognose 2012, abrufbar unter: } \\
\text { http://www.vprt.de/thema/marktentwicklung/marktdaten/um } \\
\text { s\%C3\%A4tze/pay-tv-ums\%C3\%A4tze/content/pay-tv- } \\
\text { ums\%C3\%A4tze-steigen-um-rund-12-p?c=0 }\end{array}$ \\
\hline Verweyen, Urs & Geräteabgaben: Eins vor, Zwei zurück, GRUR 2012, S. 875-881 \\
\hline VG Bild-Kunst & $\begin{array}{l}\text { Geschäftsbericht 2011, abrufbar unter: } \\
\text { http://www.bildkunst.de/html/geschaeftsbericht.html }\end{array}$ \\
\hline
\end{tabular}




\begin{tabular}{|c|c|}
\hline VG Bild-Kunst & $\begin{array}{l}\text { Verteilungsplan, abrufbar unter: } \\
\text { http://www.bildkunst.de/uploads/media/verteilungsplan.pdf }\end{array}$ \\
\hline VG WORT & $\begin{array}{l}\text { Auszahlungen Belletristik und Kinderbücher, abrufbar unter: } \\
\text { http://www.vgwort.de/auszahlungen/belletristik-und- } \\
\text { kinderbuecher.html }\end{array}$ \\
\hline VG WORT & $\begin{array}{l}\text { Geschäftsbericht 2011, abrufbar unter: } \\
\text { http://www.vgwort.de/fileadmin/pdf/geschaeftsberichte/Gesc } \\
\text { h\%C3\%A4ftsbericht_2011_final_1.pdf }\end{array}$ \\
\hline VG WORT & $\begin{array}{l}\text { Verteilungplan v. 8.6.2013, abrufbar unter: } \\
\text { http://www.vgwort.de/fileadmin/pdf/verteilungsplan/Verteilu } \\
\text { ngsplan_Fassung_8.6.2013.pdf }\end{array}$ \\
\hline Vianello, Mirk & $\begin{array}{l}\text { Lizenzierung von Musik in nutzergenerierten Videos, MMR } \\
\text { 2009, S. 90-95 }\end{array}$ \\
\hline Vitorino, António & $\begin{array}{l}\text { Recommendations resulting from the Mediation on Private } \\
\text { Copying and Reprography Levies vom } 31 \text {. Janurar } 2013 \\
\text { abrufbar unter: } \\
\text { http://ec.europa.eu/internal_market/copyright/docs/levy_refo } \\
\text { rm/130131_levies-vitorino-recommendations_en.pdf } \\
\text { (zitiert als Vitorino, Recommendations) }\end{array}$ \\
\hline Vogel, Martin & $\begin{array}{l}\text { Wahrnehmungsrecht und Verwertungsgesellschaften in der } \\
\text { Bundesrepublik Deutschland - Eine Bestandsaufnahme im Hin- } \\
\text { blick auf die Harmonisierung des Urheberrechts in der Europäi- } \\
\text { schen Gemeinschaft, GRUR 1993, S. 513-531 }\end{array}$ \\
\hline Waldfogel, Joel & $\begin{array}{l}\text { Bye, Bye, Miss American Pie? The Supply of New Recorded } \\
\text { Music Since Napster, NBER Working Paper 16882, abrufbar } \\
\text { unter: } \\
\text { http://www.nber.org/papers/w16882.pdf?new_window=1 } \\
\text { (zitiert als Waldfogel, 2011) }\end{array}$ \\
\hline Waldfogel, Joel & $\begin{array}{l}\text { Copyright Protection, Technological Change, and the Quality of } \\
\text { New Products: Evidence from Recorded Music since Napster, } \\
\text { Journal of Law \& Economics 2013, Vol. } 55 \text { No. } 4 \text { (November } \\
\text { 2012), S. } 715-740\end{array}$ \\
\hline Walter, Michel / von Lewinsky, Silke & $\begin{array}{l}\text { European Copyright Law, Oxford University Press, Oxford, New } \\
\text { York } 2012 \\
\text { (zitiert als Walter/Lewinsky, European Copyright Law) }\end{array}$ \\
\hline $\begin{array}{l}\text { Wandtke, Arthur-Axel / Bullinger, } \\
\text { Winfried }\end{array}$ & $\begin{array}{l}\text { Praxiskommentar zum Urheberrecht, 3. Aufl., Verlag C.H. Beck, } \\
\text { München } 2009 \\
\text { (zitiert als Wandtke/Bullinger, UrhR) }\end{array}$ \\
\hline Wang, Avery & $\begin{array}{l}\text { The Shazam Music Recognition Service, Communications of the } \\
\text { ACM, Vol. } 49 \text {, No. } 8,2006 \text {, S. } 44-48\end{array}$ \\
\hline Wegener, Christoph / Heidrich, Joerg & $\begin{array}{l}\text { Neuer Standard - Neue Herausforderungen: IPv6 und Daten- } \\
\text { schutzCR 2011, S. 479-484 }\end{array}$ \\
\hline Wegmann, Jürgen & $\begin{array}{l}\text { Die wirtschaftliche Bewertung von Filmrechten - ein praxiser- } \\
\text { probter Ansatz, in: Hering, Thomas / Klingelhöfer, Heinz Eckart } \\
\text { / Koch, Wolfgang (Hrsg.), Unternehmungswert und Rech- } \\
\text { nungswesen, Festschrift für Univ.-Prof. Dr. Manfred Jürgen } \\
\text { Matschke zum 65. Geburtstag, Verlag Gabler, Wiesbaden 2008, } \\
\text { S. 149-170 } \\
\text { (zitiert als Wegmann, in: FS Matschke) }\end{array}$ \\
\hline Wehr, Christina / Ujica, Matei & $\begin{array}{l}\text { „Alles muss raus!” - Datenspeicherungs- und Auskunftsplichten } \\
\text { der Access-Provider nach dem Urteil des BVerfG zur Vorratsda- } \\
\text { tenspeicherung, MMR 2010, S. 667-671 }\end{array}$ \\
\hline Wiebe, Andreas & $\begin{array}{l}\text { Providerhaftung in Europa: Neue Denkanstöße durch den EuGH } \\
\text { (Teil 1), WRP 2012, S. 1182-1189 }\end{array}$ \\
\hline Wiebe, Andreas & $\begin{array}{l}\text { Providerhaftung in Europa: Neue Denkanstöße durch den EuGH } \\
\text { (Teil 2), WRP 2012, S. 1336-1341 }\end{array}$ \\
\hline Wikström, Patrik & The Music Industry. Music in the Cloud, Polity Press, Cambridge \\
\hline
\end{tabular}




\begin{tabular}{|l|l|}
\hline $\begin{array}{l}\text { Winkelmüller, Michael / Kessler, Hans- } \\
\text { Wolfram }\end{array}$ & $\begin{array}{l}\text { Territorialisierung von Internet-Angeboten - Technische Mög- } \\
\text { lichkeiten, völker-, wirtschaftsverwaltungs- und ordnungsrecht- } \\
\text { liche Aspekte, GewA 2009, S. 181-183 }\end{array}$ \\
\hline Wirtz, Bernd W. & $\begin{array}{l}\text { Medien- und Internetmanagement, 4. Aufl., Gabler Verlag, } \\
\text { Wiesbaden 2004 }\end{array}$ \\
\hline Wischenbart, Rüdiger & $\begin{array}{l}\text { The Global eBook Market: Currtent Conditions \& Future Projec- } \\
\text { tions, February 2013, Sebastopol 2013 (nur elektronisch veröf- } \\
\text { fentlicht, zitiert ist die Version im .pdf Format) } \\
\text { (zitiert als Wischenbart, The Global eBook Market) }\end{array}$ \\
\hline Wolter & $\begin{array}{l}\text { Buchkalkulation - Was verdienen Autor und Verlag an Büchern, } \\
21.6 .2012, \text { abrufbar unter: http://www.voland- } \\
\text { quist.de/verlagsblog/buchkalkulation-was-verdienen-autor- } \\
\text { und-verlag-an-buchern/ }\end{array}$ \\
\hline Wronka, Georg & $\begin{array}{l}\text { Das Verhältnis zwischen dem allgemeinen Persönlichkeitsrecht } \\
\text { und den sogenannten besonderen Persönlichkeitsrechten, } \\
\text { UFITA 69 (1973), S. 71 }\end{array}$ \\
\hline Zentner, Alejandro & $\begin{array}{l}\text { Measuring the Effect of File Sharing on Music Purchases, Jour- } \\
\text { nal of Law \& Economics, Vol. 49, 2006, S. 63-90 }\end{array}$ \\
\hline Zombik, Peter & $\begin{array}{l}\text { Der Kampf gegen Musikdiebstahl im Internet, ZUM 2006, S. } \\
\text { 450-456 }\end{array}$ \\
\hline Zwengel, Wolfgang & Kulturflatrates, Nomos Verlagsgesellschaft, Baden-Baden 2012 \\
\hline
\end{tabular}


n diesem von der Bundestagsfraktion Bündnis 90/Die Grünen in Auftrag gegebenem lergebnisoffenem Gutachten untersucht Prof. Spindler die rechtliche und ökonomische Umsetzbarkeit sowie Ausgestaltung eines Ansatzes zur sog. „Kulturflatrate“. Die Fragestellung war, ob ein Pauschalvergütungsansatz auf Breitbandanschlüsse ein Baustein für ein gerechteres, modernes Urheberrecht, für angemessene Vergütung für die Urheberlnnen und einen gesellschaftlichen Interessensausgleich sein kann.

Das umfassende und detailreiche Gutachten leistet einen wesentlichen Beitrag zur Diskussion um die Zukunft des Urheberrechts, die in Zeiten des globalen Internets in praktisch jedem Land geführt wird. Es wird vollumfänglich der Öffentlichkeit zugänglich gemacht, damit die Ergebnisse von allen Interessierten genutzt und weiterentwickelt werden können.

Prof. Spindler zeigt in seinem Gutachten auf, dass ein Pauschalvergütungsansatz verfassungsrechtlich zulässig und ein attraktives Modell zur angemessenen Vergütung für Kreative sein kann. Für die Situation der Nutzerlnnen bietet er eine geeignete Alternative zu repressiven und gesellschaftlich höchst konfliktträchtigen Formen der Rechtsdurchsetzung. Gleichzeitig wird aber auch auf gewichtige Schwierigkeiten und Hürden bei der Umsetzung eines solchen Modells hingewiesen.

Das Gutachten und dieser Text erschienen zunächst unter der Creative Commons Lizenz BY-NC-ND auf der Internetpräsenz der Bundestagsfraktion von Bündnis 90/ Die Grünen. Diese Überarbeitung hat den Stand 08. Januar 2014. 